\title{
Total Synthesis of the Oxopolyene Macrolide (-)-Marinisporolide C
}

Luiz C. Dias* and Emílio C. de Lucca Jr.

Institute of Chemistry, University of Campinas, 13083-970, C.P. 6154, Campinas, SP, Brasil

*Idias@iqm.unicamp.br

\section{Table of Contents}

General Information

Synthesis of C23-C30 Fragment of Marinisporolide C S3

Synthesis of C31-C35 Fragment of Marinisporolide C S11

Synthesis of C23-C35 Fragment of Marinisporolide C S14

Synthesis of C10-C18 Fragment of Marinisporolide C S22

Synthesis of C19-C22 Fragment of Marinisporolide C S30

Synthesis of C10-C22 Fragment of Marinisporolide C S33

Synthesis of C8-C35 Fragment of Marinisporolide C

Synthesis of C3-C7 Fragment of Marinisporolide C

Completion of the Total Synthesis of (-)-Marinisporolide C

Comparison for Natural and Synthetic Marinisporolide C

\section{General Information}

All reactions were carried out under an atmosphere of argon with dry solvents under anhydrous conditions unless otherwise stated. Yields refer to chromatographically and spectroscopically ( ${ }^{1} \mathrm{H}$ NMR) homogeneous material, unless otherwise stated.

Tetrahydrofuran (THF), diethyl ether $\left(\mathrm{Et}_{2} \mathrm{O}\right)$, and dioxane were distilled from sodium/benzophenone prior to use. Triethylamine $\left(\mathrm{Et}_{3} \mathrm{~N}\right)$, pyridine (py), 2,6-lutidine, dichloromethane $\left(\mathrm{CH}_{2} \mathrm{Cl}_{2}\right)$, benzene $(\mathrm{PhH})$, toluene $(\mathrm{PhMe})$, and hexane were distilled from $\mathrm{CaH}_{2}$ prior to use. Oxalyl chloride $\left((\mathrm{COCl})_{2}\right)$, acetonitrile $(\mathrm{MeCN})$, isobutiraldehyde $(i-\mathrm{PrCHO})$, and crotonaldehyde were distilled prior to use. Acetic acid $(\mathrm{AcOH})$ was fractionally distilled from acetic anhydride and chromium ( $\mathrm{VI})$ oxide prior to use. Methanol $(\mathrm{MeOH})$ was distilled from $\mathrm{Mg}(\mathrm{OMe})_{2}$ and stored over molecular sieves. $N, N$-dimethylformamide (DMF) and 
dimethyl sulfoxide (DMSO) were distilled under reduced pressure from $\mathrm{CaH}_{2}$ and stored over molecular sieves. Camphorsulfonic acid (CSA) was recrystallized from ethyl acetate. The other reagents were used without further purification, unless otherwise stated.

The purification of reaction products was performed by flash column chromatography using silica gel (230-400 mesh). Reactions were monitored by thin layer chromatography carried out on silica-gel 60 and GF (5-40 $\mu$ m thickness) plates, and visualization was accomplished using UV light and phosphomolybdic acid staining followed by heating.

Optical rotations were measured on a Perkin Elmer 341 polarimeter with a sodium lamp using a $1.0 \mathrm{~cm}$ cell and are reported as follows: $[\alpha]_{D}{ }^{\top\left({ }^{\circ} \mathrm{C}\right)}(c(\mathrm{~g} / 100 \mathrm{~mL})$, solvent).

Melting points were measured with a Buchi M-565 equipment and are uncorrected.

${ }^{1} \mathrm{H}$ and proton-decoupled ${ }^{13} \mathrm{C}$ NMR spectra were acquired on a Bruker DPX250 (250 $\mathrm{MHz}$ for ${ }^{1} \mathrm{H}$ NMR and $62.5 \mathrm{MHz}$ for ${ }^{13} \mathrm{C}$ NMR), Bruker Avance 400 (400 MHz for ${ }^{1} \mathrm{H}$ NMR and $100 \mathrm{MHz}$ for ${ }^{13} \mathrm{C}$ NMR), Bruker Avance $500\left(500 \mathrm{MHz}\right.$ for ${ }^{1} \mathrm{H}$ and $125 \mathrm{MHz}$ for ${ }^{13} \mathrm{C} \mathrm{NMR}$ ), or Bruker Avance 600 (600 MHz for ${ }^{1} \mathrm{H}$ and $150 \mathrm{MHz}$ for ${ }^{13} \mathrm{C}$ NMR). Chemical shifts $(\delta)$ are reported in ppm using residual undeuterated solvent as an internal standard $\left(\mathrm{CHCl}_{3}\right.$ at 7.25 ppm, $\mathrm{C}_{6} \mathrm{H}_{6}$ at $7.16 \mathrm{ppm}, \mathrm{MeOH}$ at $3.30 \mathrm{ppm}, \mathrm{DMSO}$ at $2.49 \mathrm{ppm}$ and TMS at $0.00 \mathrm{ppm}$ for ${ }^{1} \mathrm{H}$ NMR spectra and $\mathrm{CDCl}_{3}$ at $77.0 \mathrm{ppm}, \mathrm{C}_{6} \mathrm{D}_{6}$ at $128.0 \mathrm{ppm}, \mathrm{CD}_{3} \mathrm{OD}$ at $49.0 \mathrm{ppm}$ and DMSO- $d_{6}$ at $39.5 \mathrm{ppm}$ for ${ }^{13} \mathrm{C}$ NMR spectra). Multiplicity data are reported as follows: $s=$ singlet, $d=$ doublet, $\mathrm{t}=$ triplet, $\mathrm{q}=$ quartet, quint $=$ quintet, sext $=$ sextet, $\mathrm{br} \mathrm{s}=$ broad singlet, $\mathrm{dd}=$ doublet of doublets, $\mathrm{dt}=$ doublet of triplets, $\mathrm{ddd}=$ doublet of doublet of doublets, $\mathrm{ddt}=$ doublet of doublet of triplets, $\mathrm{dtd}=$ doublet of triplet of doublets, $\mathrm{dqd}=$ doublet of quartet of doublets, and $\mathrm{m}=$ multiplet. The multiplicity is followed by the coupling constant(s) in $\mathrm{Hz}$ and integration.

Infrared spectra were recorded on Perkin Elmer Spectrum Two spectrometer. Wavelengths of maximum absorbance (max) are quoted in wavenumbers $\left(\mathrm{cm}^{-1}\right)$.

High-resolution mass spectrometry (HRMS) were measured using electrospray ionization (ESI) (Waters Xevo Q-Tof, Thermo LTQ - FT Ultra, or Thermo Q Exactive) or using electron ionization (EI) (GCT Premier Waters).

The UV/Vis spectrum was obtained from Agilent HP8453 spectrophotometer.

The CD spectrum was obtained with Jasco J-720 spectropolarimeter using a $1.0 \mathrm{~cm}$ cell. 


\section{Synthesis of C23-C30 Fragment of Marinisporolide C}

\section{3-((tert-butyldimethylsilyl)oxy)propan-1-ol (S2):}

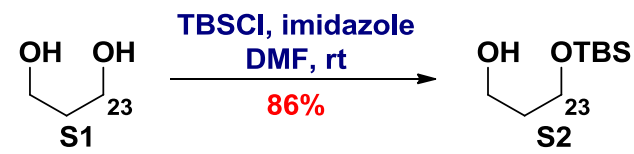

Compound $\mathbf{S 2}$ was prepared according to the literature procedure: Nilewski, C.; Deprez, N. R.; Fessard, T. C.; Li, D. B.; Geisser, R. W.; Carreira, E. M. Angew. Chem., Int. Ed. 2011, 50, 7940.

TLC: $R_{f}=0.38$ (80:20 hexane:EtOAc)

${ }^{1} \mathrm{H}$ NMR (250 MHz, CDCl 3 ) $\delta 0.04(\mathrm{~s}, 6 \mathrm{H}), 0.86(\mathrm{~s}, 9 \mathrm{H}), 1.73$ (quint, $J=5.7 \mathrm{~Hz}, 2 \mathrm{H}$ ), $2.76(\mathrm{br}$ $\mathrm{s}, 1 \mathrm{H}), 3.72-3.81(\mathrm{~m}, 4 \mathrm{H})$.

${ }^{13} \mathrm{C}$ NMR $\left(62.5 \mathrm{MHz}, \mathrm{CDCl}_{3}\right) \delta-5.6,18.1,25.8,34.2,62.0,62.6$.

IR (film) $v_{\text {max }} / \mathrm{cm}^{-1} 3398,2955,2930,2885,2858,1472,1464,1389,1362,1257,1090,1007$, 966, 837, 777, 714, 663.

3-((tert-butyldimethylsilyl)oxy)propanal (12):

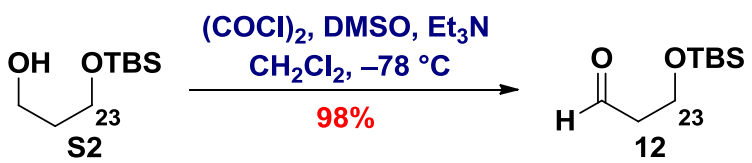

Compound 12 was prepared according to the literature procedure: Mortensen, M. S.; Osbourn, J. M.; O’Doherty, G. A. Org. Lett. 2007, 9, 3105.

TLC: $R_{f}=0.64$ (80:20 hexane:EtOAc)

${ }^{1} \mathrm{H}$ NMR $\left(250 \mathrm{MHz}, \mathrm{CDCl}_{3}\right) \delta 0.03(\mathrm{~s}, 6 \mathrm{H}), 0.85(\mathrm{~s}, 9 \mathrm{H}), 2.56(\mathrm{td}, J=6.0$ and $2.0 \mathrm{~Hz}, 2 \mathrm{H}), 3.96$ (t, $J=6.0 \mathrm{~Hz}, 2 \mathrm{H}), 9.77(\mathrm{t}, J=2.0 \mathrm{~Hz}, 1 \mathrm{H})$.

${ }^{13} \mathrm{C}$ NMR $\left(62.5 \mathrm{MHz}, \mathrm{CDCl}_{3}\right) \delta-5.5,18.2,25.8,46.5,57.4,201.9$.

IR (film) $v_{\text {max }} / \mathrm{cm}^{-1}$ 2955, 2930, 2885, 2858, 2739, 2712, 1726, 1472, 1464, 1389, 1362, 1256 , $1101,939,837,775,665$. 
$(-)-(R)-4-((4-m e t h o x y b e n z y l) o x y)$ pentan-2-one (11):

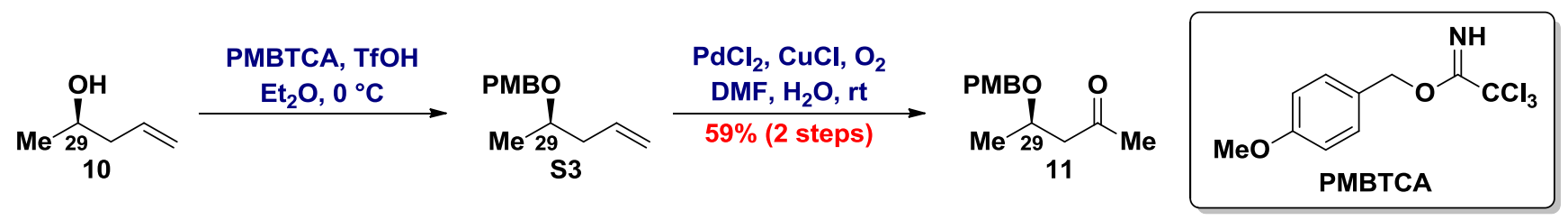

NOTE: Compound S3 is volatile and its permanence for more than 5 minutes on a high vacuum pump (approximately $0.5 \mathrm{mmHg}$ ) drastically reduces the reaction yield.

To a solution of homoallylic alcohol $10(2.0 \mathrm{~g}, 23.2 \mathrm{mmol}, 100 \mathrm{~mol} \%)$ and p-methoxybenzyl2,2,2-trichloroacetimidate $(9.83 \mathrm{~g}, 34.8 \mathrm{mmol}, 150 \mathrm{~mol} \%)$ in $\mathrm{Et}_{2} \mathrm{O}(110 \mathrm{~mL}, 0.20 \mathrm{M})$ at $0{ }^{\circ} \mathrm{C}$

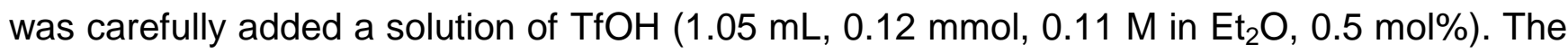
reaction mixture was stirred under the same conditions for 30 min before being quenched by the addition of saturated aqueous solution of $\mathrm{NaHCO}_{3}(25 \mathrm{~mL})$. The layers were separated, and the aqueous layer was extracted with $\mathrm{Et}_{2} \mathrm{O}(3 \times 25 \mathrm{~mL})$. The combined organic layers were washed with $\mathrm{H}_{2} \mathrm{O}(50 \mathrm{~mL})$, brine $(50 \mathrm{~mL})$, dried over $\mathrm{MgSO}_{4}$, filtered, and concentrated under reduced pressure. Compound $\mathbf{S 3}$ was partially purified by flash column chromatography using a solution of hexane/ethyl acetate $(80: 20)$ as the eluent.

A mixture of $\mathrm{PdCl}_{2}$ (411 mg, $2.32 \mathrm{mmol}, 10 \mathrm{~mol} \%$ ) and $\mathrm{CuCl}(2.30 \mathrm{~g}, 23.2 \mathrm{mmol}, 100 \mathrm{~mol} \%)$ in DMF: $\mathrm{H}_{2} \mathrm{O}(7: 1)(440 \mathrm{~mL}, 0.05 \mathrm{M})$ was purged with $\mathrm{O}_{2}$ with vigorous stirring to activate the reaction medium. The reaction mixture was stirred for $30 \mathrm{~min}$, yielding a deep-green mixture. After this period, a solution of olefin S3 (theor. $23.2 \mathrm{mmol}, 100 \mathrm{~mol} \%$ ) in DMF (24 mL, $0.97 \mathrm{M}$ ) was added, and the reaction medium was stirred vigorously for $16 \mathrm{~h}$ under an $\mathrm{O}_{2}$ atmosphere. The reaction was diluted with $\mathrm{Et}_{2} \mathrm{O}(300 \mathrm{~mL})$ and quenched by the addition of $\mathrm{H}_{2} \mathrm{O}(90 \mathrm{~mL})$. The layers were separated and the aqueous layer was extracted with $\mathrm{Et}_{2} \mathrm{O}(3 \times 100 \mathrm{~mL})$. The combined organic layers were washed with $\mathrm{H}_{2} \mathrm{O}(3 \times 100 \mathrm{~mL})$, brine $(100 \mathrm{~mL})$, dried over $\mathrm{MgSO}_{4}$, filtered, and concentrated under reduced pressure. The residue was purified by flash column chromatography using a solution of hexane/ethyl acetate $(80: 20)$ as the eluent to provide methylketone 11 ( $3.06 \mathrm{~g}, 13.8 \mathrm{mmol}, 59 \%$ over 2 steps).

Physical state: pale yellow oil

TLC: $R_{f}=0.31$ (80:20 hexane:EtOAc)

Optical rotation: $[\alpha]_{D}^{20}-30\left(c 1.0, \mathrm{CHCl}_{3}\right)$ 
${ }^{1} \mathrm{H}$ NMR (250 MHz, $\left.\mathrm{C}_{6} \mathrm{D}_{6}\right) \delta 1.04(\mathrm{~d}, J=6.2 \mathrm{~Hz}, 3 \mathrm{H}), 1.74(\mathrm{~s}, 3 \mathrm{H}), 2.04(\mathrm{dd}, J=15.8$ and 5.4 $\mathrm{Hz}, 1 \mathrm{H}), 2.44(\mathrm{dd}, J=15.8$ and $7.3 \mathrm{~Hz}, 1 \mathrm{H}), 3.34(\mathrm{~s}, 3 \mathrm{H}), 3.82-3.95(\mathrm{~m}, 1 \mathrm{H}), 4.23(\mathrm{~d}, J=11.4$ $\mathrm{Hz}, 1 \mathrm{H}), 4.35(\mathrm{~d}, J=11.4 \mathrm{~Hz}, 1 \mathrm{H}), 6.75-6.81(\mathrm{~m}, 2 \mathrm{H}), 7.17-7.21(\mathrm{~m}, 2 \mathrm{H})$.

${ }^{13} \mathrm{C}$ NMR $\left(62.5 \mathrm{MHz}, \mathrm{C}_{6} \mathrm{D}_{6}\right) \delta 19.8,30.5,50.6,54.8,70.5,71.4,114.0,129.3,131.4,159.6$, 205.4.

IR (film) $v_{\text {max }} / \mathrm{cm}^{-1} 2970,2934,2905,2870,2837,1715,1614,1587,1514,1466,1373,1302$, 1248, 1175, 1136, 1090, 1036, 822.

(-)-(2R,6R)-8-((tert-butyldimethylsilyl)oxy)-6-hydroxy-2-((4-methoxybenzyl)oxy)octan-4one (13):
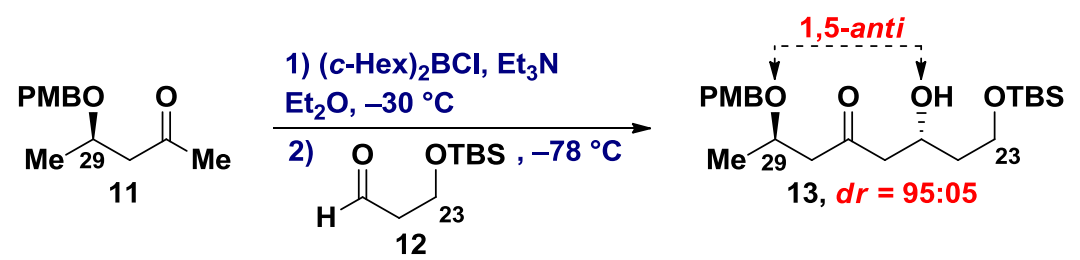

To a solution of methylketone $11(1.12 \mathrm{~g}, 5.02 \mathrm{mmol}, 100 \mathrm{~mol} \%)$ in $\mathrm{Et}_{2} \mathrm{O}(50 \mathrm{~mL}, 0.10 \mathrm{M})$ at $-30{ }^{\circ} \mathrm{C}$ was added $(\mathrm{c}-\mathrm{Hex})_{2} \mathrm{BCl}(2.17 \mathrm{~mL}, 10.0 \mathrm{mmol}, 200 \mathrm{~mol} \%)$ dropwise, followed by the addition of $\mathrm{Et}_{3} \mathrm{~N}(1.47 \mathrm{~mL}, 10.5 \mathrm{mmol}, 210 \mathrm{~mol} \%)$ dropwise, which resulted in the formation of a white cloud. The mixture was stirred under the same conditions for $30 \mathrm{~min}$. The reaction medium was then cooled to $-78^{\circ} \mathrm{C}$, and a solution of aldehyde $12(1.23 \mathrm{~g}, 6.53 \mathrm{mmol}, 130$ $\mathrm{mol} \%)$ in $\mathrm{Et}_{2} \mathrm{O}(8.0 \mathrm{~mL}, 0.80 \mathrm{M})$ was added over 30 min using a syringe pump. The resulting mixture was stirred for $3 \mathrm{~h}$ at $-78{ }^{\circ} \mathrm{C}$, followed by quenching via the dropwise addition of $\mathrm{MeOH}(20 \mathrm{~mL})$. The volatiles were removed under reduced pressure, and the residue was partially purified by flash column chromatography using a solution of hexane $/ \mathrm{CH}_{2} \mathrm{Cl}_{2} / \mathrm{ethyl}$ acetate (55:35:10) as the eluent to provide the aldol adduct 13 ( $d r=95: 05,1,5$-anti:1,5-syn) and cyclohexanol as impurity. Diastereoisomeric ratio was determined by ${ }^{13} \mathrm{C} N \mathrm{NR}$ analysis of the diastereoisomeric mixture of aldol adducts.

Physical state: colorless oil

TLC: $R_{f}=0.59$ (55:35:10 hexane: $\left.\mathrm{CH}_{2} \mathrm{Cl}_{2}: \mathrm{EtOAc}\right)$

Optical rotation: $[\alpha]_{D}^{20}-24\left(c 1.5, \mathrm{CH}_{2} \mathrm{Cl}_{2}\right)$

${ }^{1} \mathrm{H}$ NMR (500 MHz, $\left.\mathrm{C}_{6} \mathrm{D}_{6}\right) \delta 0.03(\mathrm{~s}, 3 \mathrm{H}), 0.04(\mathrm{~s}, 3 \mathrm{H}), 0.94(\mathrm{~s}, 9 \mathrm{H}), 1.02(\mathrm{~d}, J=6.1 \mathrm{~Hz}, 3 \mathrm{H})$, $1.49-1.54(\mathrm{~m}, 1 \mathrm{H}), 1.59-1.64(\mathrm{~m}, 1 \mathrm{H}), 2.08(\mathrm{dd}, J=15.7$ and $4.9 \mathrm{~Hz}, 1 \mathrm{H}), 2.25(\mathrm{dd}, J=16.8$ 
and $3.8 \mathrm{~Hz}, 1 \mathrm{H}$ ), $2.38(\mathrm{dd}, J=16.8$ and $8.5 \mathrm{~Hz}, 1 \mathrm{H}), 2.52(\mathrm{dd}, J=15.7$ and $7.7 \mathrm{~Hz}, 1 \mathrm{H}), 3.31$ (s, 3H), 3.46 (d, $J=2.5 \mathrm{~Hz}, 1 \mathrm{H}$ ), 3.66 (ddd, $J=10.2,6.4$ and $5.4 \mathrm{~Hz}, 1 \mathrm{H}$ ), 3.72 (ddd, $J=10.2$, 6.8 and $5.2 \mathrm{~Hz}, 1 \mathrm{H}), 3.91-3.98(\mathrm{~m}, 1 \mathrm{H}), 4.23(\mathrm{~d}, J=11.2 \mathrm{~Hz}, 1 \mathrm{H}), 4.29-4.34(\mathrm{~m}, 1 \mathrm{H}), 4.35(\mathrm{~d}$, $J=11.2 \mathrm{~Hz}, 1 \mathrm{H}), 6.79-6.82(\mathrm{~m}, 2 \mathrm{H}), 7.20-7.22(\mathrm{~m}, 2 \mathrm{H})$.

${ }^{13} \mathrm{C}$ NMR $\left(125 \mathrm{MHz}, \mathrm{C}_{6} \mathrm{D}_{6}\right) \delta-5.4\left(2 \times \mathrm{CH}_{3}\right), 18.4,19.8,26.0,39.2,50.7,51.1,54.7,61.1$, $66.3,70.6,71.4,114.0,129.5,131.2,159.7,209.0$.

IR (film) $v_{\max } / \mathrm{cm}^{-1} 3460,2955,2930,2856,1711,1614,1514,1472,1375,1250,1094,1036$, 837, 777, 737.

HRMS (ESI TOF-MS) $\mathrm{m} / \mathrm{z}$ calcd for $\mathrm{C}_{22} \mathrm{H}_{38} \mathrm{O}_{5} \mathrm{SiK}[\mathrm{M}+\mathrm{K}]^{+}: 449.2126$, found: 449.2157 .

\section{(-)-(3R,5R,7R)-1-((tert-butyldimethylsilyl)oxy)-7-((4-methoxybenzyl)oxy)octane-3,5-diol} (S4):

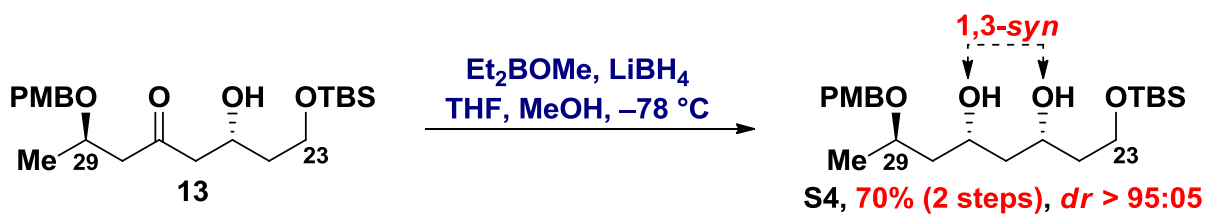

To a solution of aldol adduct 13 (theor. $5.02 \mathrm{mmol}, 100 \mathrm{~mol} \%$ ) in THF:MeOH (4:1) (24 mL, $0.21 \mathrm{M})$ at $-78{ }^{\circ} \mathrm{C}$ was added $\mathrm{Et}_{2} \mathrm{BOMe}(0.84 \mathrm{~mL}, 6.02 \mathrm{mmol}, 120 \mathrm{~mol} \%)$. The solution was stirred for $15 \mathrm{~min}$ under these conditions, and $\mathrm{LiBH}_{4}(3.0 \mathrm{~mL}, 6.00 \mathrm{mmol}, 2.0 \mathrm{M}$ in THF, 120 mol\%) was added over $1 \mathrm{~h}$ using a syringe pump. The reaction was stirred for $1 \mathrm{~h}$ and then warmed to $-40^{\circ} \mathrm{C}$. The reaction was quenched by the addition of $\mathrm{pH} 7$ phosphate buffer (58 $\mathrm{mL}$ ) and $\mathrm{MeOH}(110 \mathrm{~mL})$. The reaction was warmed to $0 \stackrel{\circ}{\circ}$, and $30 \% \mathrm{H}_{2} \mathrm{O}_{2}(44 \mathrm{~mL})$ was added dropwise. The mixture was stirred for $1 \mathrm{~h}$, and the volatiles were removed under reduced pressure. The aqueous layer was extracted with EtOAc $(3 \times 100 \mathrm{~mL})$. The combined organic layers were washed with saturated aqueous solution of $\mathrm{NaHCO}_{3}(2 \times 100 \mathrm{~mL})$, brine $(100 \mathrm{~mL})$, dried over $\mathrm{MgSO}_{4}$, filtered, and concentrated under reduced pressure. The residue was dissolved in $\mathrm{MeOH}(50 \mathrm{~mL})$, and the solvent was removed under reduced pressure in a $60{ }^{\circ} \mathrm{C}$ bath to remove chelated boron species. This procedure was repeated 6 times to provide the diol S4 (1.45 g, $3.51 \mathrm{mmol}, 70 \%$ over 2 steps, $d r>95: 05,1,3-$ syn:1,3-anti), which was used in the next step without further purification.

Physical state: yellow oil

TLC: $R_{f}=0.59(60: 40$ hexane:EtOAc) 
Optical rotation: $[\alpha]_{D}^{20}-32\left(c 2.2, \mathrm{CH}_{2} \mathrm{Cl}_{2}\right)$

${ }^{1} \mathrm{H}$ NMR (600 MHz, CDCl $\left.)_{3}\right) 0.09(\mathrm{~s}, 6 \mathrm{H}), 0.91(\mathrm{~s}, 9 \mathrm{H}), 1.24(\mathrm{~d}, J=6.0 \mathrm{~Hz}, 3 \mathrm{H}), 1.50(\mathrm{dt}, J=$ 14.1 and $2.6 \mathrm{~Hz}, 1 \mathrm{H}), 1.58-1.76(\mathrm{~m}, 5 \mathrm{H}), 3.79-3.83(\mathrm{~m}, 1 \mathrm{H}), 3.81(\mathrm{~s}, 3 \mathrm{H}), 3.84-3.89(\mathrm{~m}, 2 \mathrm{H})$, $3.94(\mathrm{br} \mathrm{s}, 1 \mathrm{H}), 4.06-4.10(\mathrm{~m}, 1 \mathrm{H}), 4.15-4.18(\mathrm{~m}, 2 \mathrm{H}), 4.41(\mathrm{~d}, J=11.2 \mathrm{~Hz}, 1 \mathrm{H}), 4.56(\mathrm{~d}, J=$ $11.2 \mathrm{~Hz}, 1 \mathrm{H}), 6.88-6.89(\mathrm{~m}, 2 \mathrm{H}), 7.27-7.29(\mathrm{~m}, 2 \mathrm{H})$.

${ }^{13} \mathrm{C}$ NMR (150 MHz, $\left.\mathrm{CDCl}_{3}\right) \delta-5.5,-5.5,18.1,19.6,25.8,39.0,43.6,44.0,55.2,61.9,69.2$, 70.4, $72.1(2 \times \mathrm{CH}), 113.8,129.4,130.6,159.2$.

IR (film) $v_{\text {max }} / \mathrm{cm}^{-1} 3454,2955,2932,2858,1641,1614,1514,1265,1250,1086,1036,837$, 739.

HRMS (ESI TOF-MS) $\mathrm{m} / z$ calcd for $\mathrm{C}_{22} \mathrm{H}_{41} \mathrm{O}_{5} \mathrm{Si}[\mathrm{M}+\mathrm{H}]^{+}:$413.2723, found: 413.2719 .

(-)-tert-butyl(2-((4R,6S)-6-((R)-2-((4-methoxybenzyl)oxy)propyl)-2,2-dimethyl-1,3-dioxan4-yl)ethoxy)dimethylsilane (14):

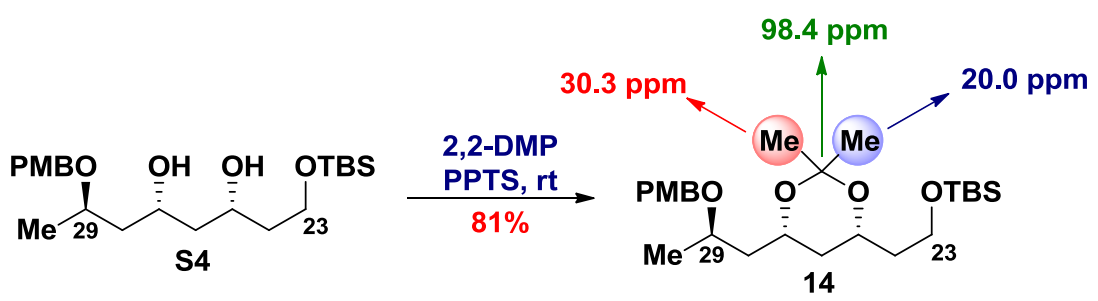

To a solution of diol S4 (1.36 g, $3.29 \mathrm{mmol}, 100 \mathrm{~mol} \%)$ in 2,2-DMP (37 mL, $0.09 \mathrm{M})$ at room temperature was added PPTS (416 $\mathrm{mg}, 1.65 \mathrm{mmol}, 50 \mathrm{~mol} \%$ ). The reaction medium was stirred for $20 \mathrm{~h}$. The mixture was filtered through silica and Celite, and the residue was washed with $\mathrm{CH}_{2} \mathrm{Cl}_{2}(5 \times 50 \mathrm{~mL})$ and concentrated under reduced pressure. The residue was purified by flash column chromatography using a solution of hexane/ethyl acetate (90:10) as the eluent to provide acetonide $14(1.21 \mathrm{~g}, 2.68 \mathrm{mmol}, 81 \%)$. This compound was used to confirm the 1,3-syn relative stereochemistry between C25 and C27 on the basis of Rychnovsky ${ }^{13} \mathrm{C}$ NMR analysis: a) Rychnovsky, S. D.; Skalitzky, D. J. Tetrahedron Lett. 1990, 31, 945. b) Rychnovsky, S. D.; Rogers, B.; Yang, G. J. Org. Chem. 1993, 58, 3511. c) Rychnovsky, S. D.; Rogers, B. N.; Richardson, T. I. Acc. Chem. Res. 1998, 31, 9. For a theoretical work, see: d) Tormena, C. F.; Dias, L. C.; Rittner, R. J. Phys. Chem. A 2005, 109, 6077.

Physical state: colorless oil

TLC: $R_{f}=0.46$ (90:10 hexane:EtOAc) 
Optical rotation: $[\alpha]_{D}^{20}-27\left(c 0.9, \mathrm{CH}_{2} \mathrm{Cl}_{2}\right)$

${ }^{1} \mathrm{H}$ NMR (250 MHz, CDCl 3$) \delta 0.04(\mathrm{~s}, 6 \mathrm{H}), 0.89(\mathrm{~s}, 9 \mathrm{H}), 1.18(\mathrm{~d}, J=6.0 \mathrm{~Hz}, 3 \mathrm{H}), 1.36(\mathrm{~s}, 3 \mathrm{H})$, $1.40(\mathrm{~s}, 3 \mathrm{H}), 1.42-1.69(\mathrm{~m}, 6 \mathrm{H}), 3.60-3.77(\mathrm{~m}, 3 \mathrm{H}), 3.80(\mathrm{~s}, 3 \mathrm{H}), 3.97-4.15(\mathrm{~m}, 2 \mathrm{H}), 4.35(\mathrm{~d}$, $J=11.2 \mathrm{~Hz}, 1 \mathrm{H}), 4.52(\mathrm{~d}, J=11.2 \mathrm{~Hz}, 1 \mathrm{H}), 6.84-6.90(\mathrm{~m}, 2 \mathrm{H}), 7.24-7.28(\mathrm{~m}, 2 \mathrm{H})$.

${ }^{13} \mathrm{C}$ NMR (62.5 MHz, $\left.\mathrm{CDCl}_{3}\right) \delta-5.4\left(2 \times \mathrm{CH}_{3}\right), 18.3,20.0,20.1,25.9,30.3,37.7,39.5,44.5$, $55.2,58.9,65.5,65.7,70.5,70.7,98.4,113.8,129.3,131.1,159.1$.

IR (film) $v_{\text {max }} / \mathrm{cm}^{-1}$ 2993, 2951, 2935, 2856, 1614, 1514, 1472, 1464, 1379, 1250, 1200, 1171, $1101,1038,960,835,775,739$.

HRMS (ESI TOF-MS) $\mathrm{m} / z$ calcd for $\mathrm{C}_{25} \mathrm{H}_{45} \mathrm{O}_{5} \mathrm{Si}[\mathrm{M}+\mathrm{H}]^{+}: 453.3036$, found: 453.3026 .

\section{(+)-(R)-4-((tert-butyldimethylsilyl)oxy)-1-((2R,4R,6R)-2-(4-methoxyphenyl)-6-methyl-1,3-} dioxan-4-yl)butan-2-ol (S5):
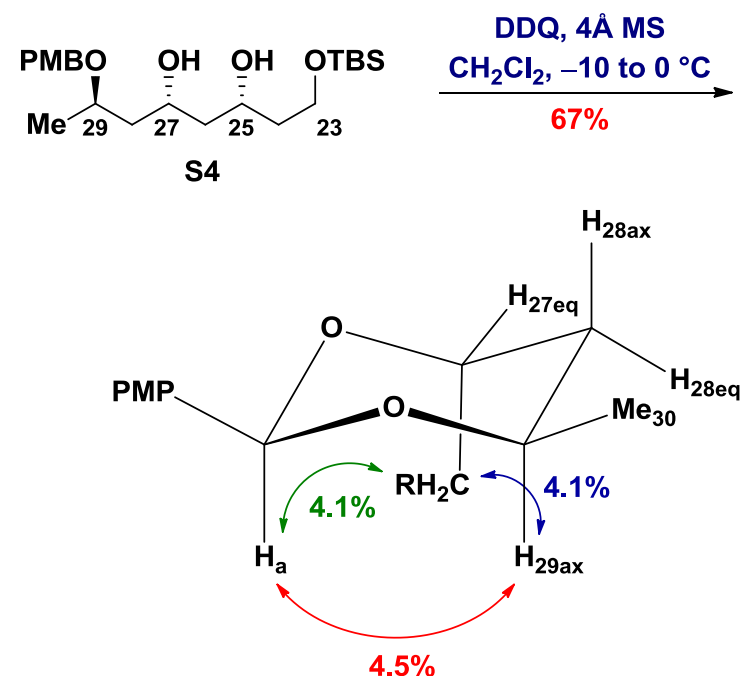

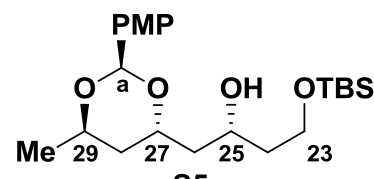

S5

$J_{29 \mathrm{ax}, 28 \mathrm{eq}}=2.5 \mathrm{~Hz}$

$J_{29 a x, 28 a x}=12.1 \mathrm{~Hz}$

$J_{28 \mathrm{ax}, 27 \mathrm{eq}}=6.2 \mathrm{~Hz}$

$J_{28 \mathrm{ax}, 28 \mathrm{eq}}=13.6 \mathrm{~Hz}$

To a solution of diol S4 (50 mg, $0.12 \mathrm{mmol}, 100 \mathrm{~mol} \%)$ in $\mathrm{CH}_{2} \mathrm{Cl}_{2}(2.5 \mathrm{~mL}, 0.05 \mathrm{M})$ at room temperature was added activated $4 \AA$ molecular sieves $(37 \mathrm{mg})$. After $15 \mathrm{~min}$, the mixture was cooled to $-10{ }^{\circ} \mathrm{C}$, and DDQ ( $34 \mathrm{mg}, 0.15 \mathrm{mmol}, 125 \mathrm{~mol} \%$ ) was added. The reaction medium was stirred for $5 \mathrm{~min}$ at $-10^{\circ} \mathrm{C}$ and warmed to $0{ }^{\circ} \mathrm{C}$. After $2 \mathrm{~h}$, the reaction mixture was loaded directly onto a flash column chromatography using a solution of hexane/ethyl acetate (80:20) as the eluent to provide the PMP acetal S5 (33 mg, $80 \mu \mathrm{mol}, 67 \%$ ). This compound was used to confirm the 1,3-anti relative stereochemistry between C27 and C29 on the basis of ${ }^{1} \mathrm{H}$ NMR and 1D selective NOE spectra analyses. 
Physical state: pale yellow solid

TLC: $R_{f}=0.39$ (70:30 hexane:EtOAc)

$\mathrm{mp} 39-41^{\circ} \mathrm{C}$

Optical rotation: $[\alpha]_{D}^{20}+10\left(c 1.5, \mathrm{CH}_{2} \mathrm{Cl}_{2}\right)$

${ }^{1} \mathrm{H}$ NMR (600 MHz, $\left.\mathrm{CDCl}_{3}\right) \delta 0.06(\mathrm{~s}, 6 \mathrm{H}), 0.88(\mathrm{~s}, 9 \mathrm{H}), 1.26(\mathrm{~d}, J=6.0 \mathrm{~Hz}, 3 \mathrm{H}), 1.46-1.49$ $(\mathrm{m}, 1 \mathrm{H}), 1.61$ (dt, $J=14.3$ and $4.9 \mathrm{~Hz}, 1 \mathrm{H}), 1.69-1.74(\mathrm{~m}, 2 \mathrm{H}), 1.98$ (ddd, $J=13.6,11.8$ and $6.2 \mathrm{~Hz}, 1 \mathrm{H}$ ), 2.42 (ddd, $J=14.4,10.2$ and $7.6 \mathrm{~Hz}, 1 \mathrm{H}$ ), 3,60 (br s, $1 \mathrm{H}$ ), 3.77 (s, 3H), 3.77-3.83 (m, $1 \mathrm{H}$ ), 3.84-3.89 (m, $1 \mathrm{H}$ ), 4.05 (quint, $J=6.0 \mathrm{~Hz}, 1 \mathrm{H}$ ), 4.13 (dqd, $J=12.1,6.2$ and $2.5 \mathrm{~Hz}$, $1 \mathrm{H}$ ), 4.42 (quint, $J=5.3 \mathrm{~Hz}, 1 \mathrm{H}), 5.81(\mathrm{~s}, 1 \mathrm{H}), 6.85-6.87(\mathrm{~m}, 2 \mathrm{H}), 7.38-7.41(\mathrm{~m}, 2 \mathrm{H})$.

${ }^{13} \mathrm{C}$ NMR $\left(100 \mathrm{MHz}, \mathrm{CDCl}_{3}\right) \delta-5.5,-5.5,18.1,21.9,25.8,35.9,37.6,38.4,55.2,61.8,68.6$, $69.8,71.4,94.4,113.6,127.4,131.3,159.9$.

IR (film) $v_{\max } / \mathrm{cm}^{-1} 3468,2953,2932,2856,1616,1518,1464,1377,1304,1250,1173,1122$, 1094, 1036, 837, 777, 737.

HRMS (ESI TOF-MS) $\mathrm{m} / \mathrm{z}$ calcd for $\mathrm{C}_{22} \mathrm{H}_{38} \mathrm{O}_{5} \mathrm{SiNa}[\mathrm{M}+\mathrm{Na}]^{+}: 433.2386$, found: 433.2373 .

\section{(-)-(R)-1-((4S,6R)-6-(2-((tert-butyldimethylsilyl)oxy)ethyl)-2,2-dimethyl-1,3-dioxan-4-} yl)propan-2-ol (S6):

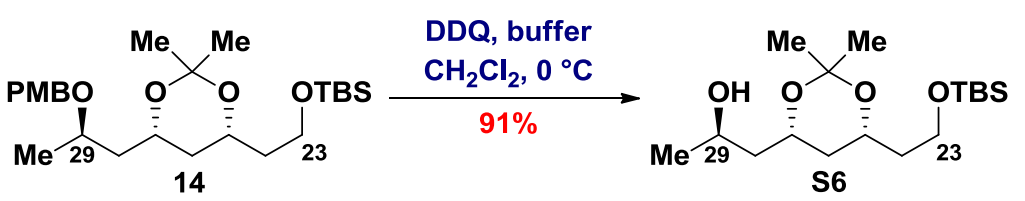

To a solution of PMB ether 14 (2.03 g, $4.48 \mathrm{mmol}, 100 \mathrm{~mol} \%$ ) in $\mathrm{CH}_{2} \mathrm{Cl}_{2}$ :phosphate buffer $\mathrm{pH}$ $7(9: 1)(75 \mathrm{~mL}, 0,06 \mathrm{M})$ at $0 \stackrel{\circ}{\circ} \mathrm{C}$ was added DDQ $(1.21 \mathrm{~g}, 5.33 \mathrm{mmol}, 120 \mathrm{~mol} \%)$. The mixture was stirred for $45 \mathrm{~min}$ under the same conditions, followed by quenching via the addition of a solution of $\mathrm{H}_{2} \mathrm{O}$ :saturated aqueous solution of $\mathrm{NaHCO}_{3}(1: 1)(16 \mathrm{~mL})$. The resulting mixture was filtered over Celite, washed with $\mathrm{CH}_{2} \mathrm{Cl}_{2}(5 \times 50 \mathrm{~mL})$ and concentrated under reduced pressure. The residue was purified by flash column chromatography using hexane/ethyl acetate $(80: 20)$ as the eluent to provide alcohol $\mathbf{S 6}(1.36 \mathrm{~g}, 4.09 \mathrm{mmol}, 91 \%)$.

Physical state: pale yellow oil

TLC: $R_{f}=0.33$ (80:20 hexane:EtOAc)

Optical rotation: $[\alpha]_{D}^{20}-1.6\left(c 3.7, \mathrm{CHCl}_{3}\right)$ 
${ }^{1} \mathrm{H}$ NMR (250 MHz, $\left.\mathrm{C}_{6} \mathrm{D}_{6}\right) \delta 0.07(\mathrm{~s}, 3 \mathrm{H}), 0.07(\mathrm{~s}, 3 \mathrm{H}), 0.99(\mathrm{~s}, 9 \mathrm{H}), 1.05-1.12(\mathrm{~m}, 1 \mathrm{H}), 1.10$ (d, $J=6.3 \mathrm{~Hz}, 3 \mathrm{H}), 1.17-1.31(\mathrm{~m}, 1 \mathrm{H}), 1.34(\mathrm{~s}, 3 \mathrm{H}), 1.39-1.77(\mathrm{~m}, 4 \mathrm{H}), 1.43(\mathrm{~s}, 3 \mathrm{H}), 2.17(\mathrm{br}$ $\mathrm{s}, 1 \mathrm{H}), 3.59-3.67(\mathrm{~m}, 1 \mathrm{H}), 3.72-3.82(\mathrm{~m}, 1 \mathrm{H}), 3.93-4.05(\mathrm{~m}, 3 \mathrm{H})$.

${ }^{13} \mathrm{C}$ NMR $\left(62.5 \mathrm{MHz}, \mathrm{C}_{6} \mathrm{D}_{6}\right) \delta-5.3,-5.2,18.5,19.8,24.0,26.1,30.5,37.3,40.0,44.8,59.1$, 64.3, 65.8, 67.0, 98.6.

IR (film) $v_{\max } / \mathrm{cm}^{-1} 3443,2993,2955,2930,2883,2858,1637,1472,1381,1265,1257,1200$, 1167, 1101, 1020, 1005, 959, 837, 777, 739.

HRMS (ESI TOF-MS) $\mathrm{m} / z$ calcd for $\mathrm{C}_{17} \mathrm{H}_{37} \mathrm{O}_{4} \mathrm{Si}[\mathrm{M}+\mathrm{H}]^{+}: 333.2461$, found: 333.2472 .

\section{(+)-1-((4R,6R)-6-(2-((tert-butyldimethylsilyl)oxy)ethyl)-2,2-dimethyl-1,3-dioxan-4-} yl)propan-2-one (15):

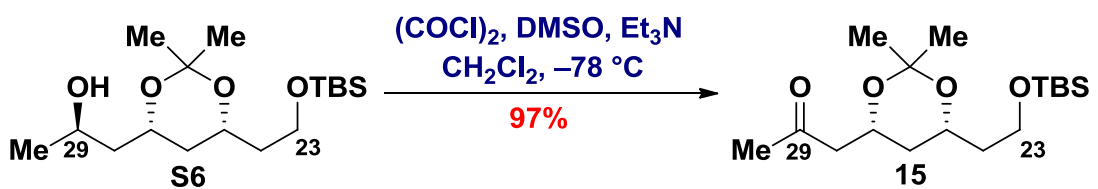

To a solution of oxalyl chloride $(0.43 \mathrm{~mL}, 4.81 \mathrm{mmol}, 120 \mathrm{~mol} \%)$ in $\mathrm{CH}_{2} \mathrm{Cl}_{2}(23 \mathrm{~mL}, 0.21 \mathrm{M})$ at $-78{ }^{\circ} \mathrm{C}$ was added DMSO $(0.66 \mathrm{~mL}, 9.35 \mathrm{mmol}, 240 \mathrm{~mol} \%)$ dropwise. The reaction medium was stirred for $30 \mathrm{~min}$, followed by the dropwise addition of a solution of alcohol $\mathbf{S 6}(1.30 \mathrm{~g}$, $3.91 \mathrm{mmol}, 100 \mathrm{~mol} \%)$ in $\mathrm{CH}_{2} \mathrm{Cl}_{2}(9.5 \mathrm{~mL}, 0.41 \mathrm{M})$. After $30 \mathrm{~min}, \mathrm{Et}_{3} \mathrm{~N}(2.8 \mathrm{~mL}, 20.0 \mathrm{mmol}$, $510 \mathrm{~mol} \%$ ) was added dropwise, and the resulting slurry was warmed to $0{ }^{\circ} \mathrm{C}$ and stirred for 1 h. The reaction was then diluted with $\mathrm{Et}_{2} \mathrm{O}(20 \mathrm{~mL})$ and saturated aqueous solution of $\mathrm{NH}_{4} \mathrm{Cl}$ $(20 \mathrm{~mL})$. The layers were separated, and the aqueous layer was extracted with $\mathrm{Et}_{2} \mathrm{O}(3 \times 50$ $\mathrm{mL})$. The combined organic layers were washed with $\mathrm{H}_{2} \mathrm{O}(3 \times 50 \mathrm{~mL})$, brine $(50 \mathrm{~mL})$, dried over $\mathrm{MgSO}_{4}$, filtered, and concentrated under reduced pressure. The residue was purified by flash column chromatography using hexane/ethyl acetate $(80: 20)$ as the eluent to provide methylketone 15 (1.25 g, $3.78 \mathrm{mmol}, 97 \%)$.

Physical state: pale yellow oil

TLC: $R_{f}=0.61$ (80:20 hexane:EtOAc)

Optical rotation: $[\alpha]_{D}^{20}+18\left(c 1.4, \mathrm{CH}_{2} \mathrm{Cl}_{2}\right)$

${ }^{1} \mathrm{H}$ NMR (250 MHz, $\mathrm{C}_{6} \mathrm{D}_{6}$ ) $\delta 0.06(\mathrm{~s}, 6 \mathrm{H}), 0.98(\mathrm{~s}, 9 \mathrm{H}), 1.04$ (q, $J=12.6 \mathrm{~Hz}, 1 \mathrm{H}$ ), 1.22 (dt, $J=$ 12.6 and $2.6 \mathrm{~Hz}, 1 \mathrm{H}), 1.35(\mathrm{~s}, 3 \mathrm{H}), 1.45(\mathrm{~s}, 3 \mathrm{H}), 1.52-1.70(\mathrm{~m}, 2 \mathrm{H}), 1.73(\mathrm{~s}, 3 \mathrm{H}), 1.97(\mathrm{dd}, J=$ 
15.9 and $4.9 \mathrm{~Hz}, 1 \mathrm{H}), 2.38(\mathrm{dd}, J=15.9$ and $7.4 \mathrm{~Hz}, 1 \mathrm{H}), 3.58-3.67(\mathrm{~m}, 1 \mathrm{H}), 3.70-3.80(\mathrm{~m}$, $1 \mathrm{H}), 3.91-4.01(\mathrm{~m}, 1 \mathrm{H}), 4.16-4.27(\mathrm{~m}, 1 \mathrm{H})$.

${ }^{13} \mathrm{C}$ NMR $\left(62.5 \mathrm{MHz}, \mathrm{C}_{6} \mathrm{D}_{6}\right) \delta-5.3,-5.2,18.4,19.8,26.1,30.4,30.5,37.3,40.0,50.0,59.1$, 65.6, 65.9, 98.7, 204.5.

IR (film) $v_{\max } / \mathrm{cm}^{-1}$ 2995, 2955, 2930, 2858, 1717, 1472, 1381, 1362, 1265, 1257, 1200, 1171 , 1097, 957, 837, 777, 739.

HRMS (ESI TOF-MS) $\mathrm{m} / \mathrm{z}$ calcd for $\mathrm{C}_{17} \mathrm{H}_{34} \mathrm{O}_{4} \mathrm{SiK}[\mathrm{M}+\mathrm{K}]^{+}: 369.1863$, found: 369.1852 .

\section{Synthesis of C31-C35 Fragment of Marinisporolide C}

(-)-(R)-4-benzyl-3-((2R,3S)-3-hydroxy-2,4-dimethylpentanoyl)oxazolidin-2-one (S9):
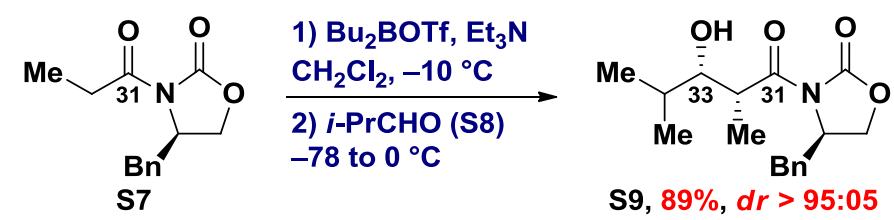

Compound $\mathbf{S 9}$ was prepared according to the literature procedure: a) Evans, D. A.; Allison, B. D.; Yang, M. G.; Masse, C. E. J. Am. Chem. Soc. 2001, 123, 10840. b) Evans, D. A.; Connell, B. T. J. Am. Chem. Soc. 2003, 125, 10899.

TLC: $R_{f}=0.46(70: 30$ hexane:EtOAc)

mp $58-60^{\circ} \mathrm{C}$

Optical rotation: $[\alpha]_{D}{ }^{20}-39\left(c 0.9, \mathrm{CHCl}_{3}\right)$

${ }^{1} \mathrm{H}$ NMR $\left(250 \mathrm{MHz}, \mathrm{CDCl}_{3}\right) \delta 0.90(\mathrm{~d}, J=6.8 \mathrm{~Hz}, 3 \mathrm{H}), 1.02(\mathrm{~d}, J=6.5 \mathrm{~Hz}, 3 \mathrm{H}), 1.24(\mathrm{~d}, J=$ $6.8 \mathrm{~Hz}, 3 \mathrm{H}$ ), 1.72 (oct, $J=6.8 \mathrm{~Hz}, 1 \mathrm{H}$ ), 2.80 (dd, $J=13.4$ and $9.4 \mathrm{~Hz}, 1 \mathrm{H}$ ), $3.02(\mathrm{~d}, J=2.2 \mathrm{~Hz}$, $1 \mathrm{H}$ ), $3.23(\mathrm{dd}, J=13.4$ and $3.2 \mathrm{~Hz}, 1 \mathrm{H}$ ), 3.53-3.57 (m, $1 \mathrm{H}$ ), 3.96 (qd, $J=7.0$ and $2.8 \mathrm{~Hz}, 1 \mathrm{H}$ ), 4.13-4.24 (m, 2H), 4.64-4.74 (m, 1H), 7.18-7.35 (m, 5H).

${ }^{13} \mathrm{C}$ NMR $\left(62.5 \mathrm{MHz}, \mathrm{CDCl}_{3}\right) \delta 9.9,18.7,18.9,30.6,37.5,39.6,54.9,65.9,76.4,127.2,128.7$, 129.2, 134.9, 152.7, 177.4 .

IR (film) $v_{\text {max }} / \mathrm{cm}^{-1} 3508,3063,3030,2964,2876,1780,1695,1456,1387,1354,1236,1211$, $1117,1097,984,974,764,737,702$. 
(-)-(2R,4S,5S)-4-isopropyl-2-(4-methoxyphenyl)-5-methyl-1,3-dioxane (S11):

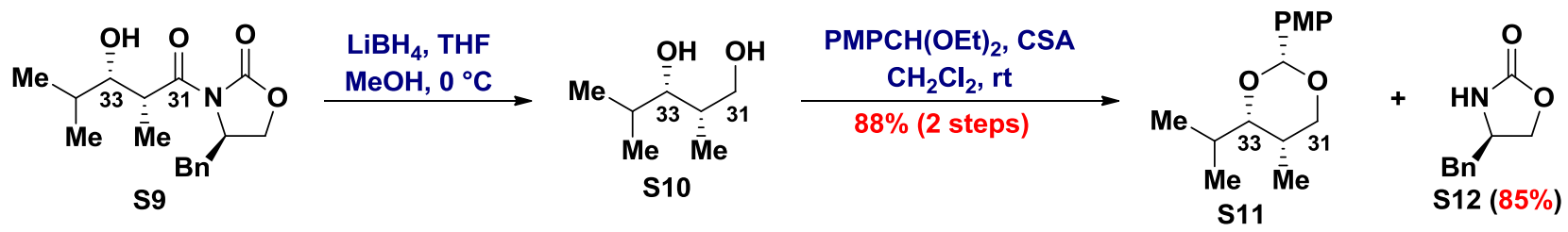

Compound S11 was prepared according to the literature procedure: a) Evans, D. A.; Allison, B. D.; Yang, M. G.; Masse, C. E. J. Am. Chem. Soc. 2001, 123, 10840. b) Evans, D. A.; Connell, B. T. J. Am. Chem. Soc. 2003, 125, 10899.

TLC: $R_{f}=0.50$ (95:05 hexane:EtOAc)

$\mathrm{mp} 60-62^{\circ} \mathrm{C}$

Optical rotation: $[\alpha]_{D}{ }^{20}-29\left(c 1.0, \mathrm{CHCl}_{3}\right)$

${ }^{1} \mathrm{H}$ NMR (250 MHz, $\left.\mathrm{CDCl}_{3}\right) \delta 0.84(\mathrm{~d}, J=6.8 \mathrm{~Hz}, 3 \mathrm{H}), 1.01(\mathrm{~d}, J=6.5 \mathrm{~Hz}, 3 \mathrm{H}), 1.14(\mathrm{~d}, J=$ $7.0 \mathrm{~Hz}, 3 \mathrm{H}), 1.61-1.85(\mathrm{~m}, 2 \mathrm{H}), 3.35(\mathrm{dd}, J=9.9$ and $2.2 \mathrm{~Hz}, 1 \mathrm{H}), 3.77(\mathrm{~s}, 3 \mathrm{H}), 3.97-4.06(\mathrm{~m}$, $2 \mathrm{H}), 5.42(\mathrm{~s}, 1 \mathrm{H}), 6.86-6.89(\mathrm{~m}, 2 \mathrm{H}), 7.41-7.44(\mathrm{~m}, 2 \mathrm{H})$.

${ }^{13} \mathrm{C}$ NMR $\left(62.5 \mathrm{MHz}, \mathrm{CDCl}_{3}\right) \delta 10.9,17.3,19.5,29.4,30.0,55.2,73.9,85.7,101.5,113.4$, 127.2, 131.7, 159.7 .

IR (film) $v_{\max } / \mathrm{cm}^{-1} 3053,2964,2937,2916,2839,1616,1518,1466,1385,1265,1250,1167$, 1103, 1034, 1003, 831, 739, 704.

(+)-(2S,3S)-3-((4-methoxybenzyl)oxy)-2,4-dimethylpentan-1-ol (S13):

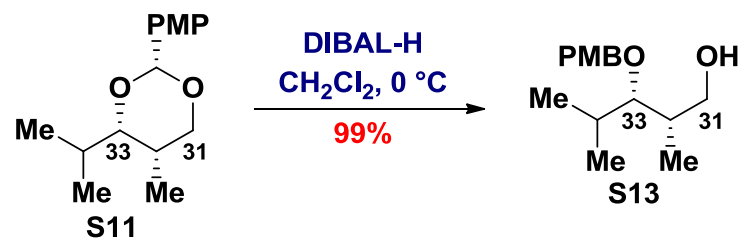

Compound S13 was prepared according to the literature procedure: a) Evans, D. A.; Allison, B. D.; Yang, M. G.; Masse, C. E. J. Am. Chem. Soc. 2001, 123, 10840. b) Evans, D. A.; Connell, B. T. J. Am. Chem. Soc. 2003, 125, 10899.

TLC: $R_{f}=0.16(90: 10$ hexane:EtOAc)

Optical rotation: $[\alpha]_{D}^{20}+12\left(c 1.1, \mathrm{CHCl}_{3}\right)$ 
${ }^{1} \mathrm{H}$ NMR $\left(250 \mathrm{MHz}, \mathrm{CDCl}_{3}\right) \delta 0.92(\mathrm{~d}, J=7.0 \mathrm{~Hz}, 6 \mathrm{H}), 1.04(\mathrm{~d}, J=6.6 \mathrm{~Hz}, 3 \mathrm{H}), 1.81-1.99(\mathrm{~m}$, $3 \mathrm{H}), 3.22(\mathrm{dd}, J=7.6$ and $3.2 \mathrm{~Hz}, 1 \mathrm{H}), 3.50-3.64(\mathrm{~m}, 2 \mathrm{H}), 3.79(\mathrm{~s}, 3 \mathrm{H}), 4.49(\mathrm{~d}, J=10.7 \mathrm{~Hz}$, $1 \mathrm{H}), 4.56(\mathrm{~d}, J=10.7 \mathrm{~Hz}, 1 \mathrm{H}), 6.84-6.89(\mathrm{~m}, 2 \mathrm{H}), 7.26-7.29(\mathrm{~m}, 2 \mathrm{H})$.

${ }^{13} \mathrm{C}$ NMR $\left(62.5 \mathrm{MHz}, \mathrm{CDCl}_{3}\right) \delta 10.9,19.4,19.8,30.8,37.6,55.2,66.3,74.2,85.4,113.7$, 129.2, 131.0, 159.1 .

IR (film) $v_{\text {max }} / \mathrm{cm}^{-1} 3414,2962,2935,2914,2874,1612,1514,1466,1302,1265,1250,1175$, $1068,1034,825,739,704$.

(-)-(2R,3S)-3-((4-methoxybenzyl)oxy)-2,4-dimethylpentanal (16):

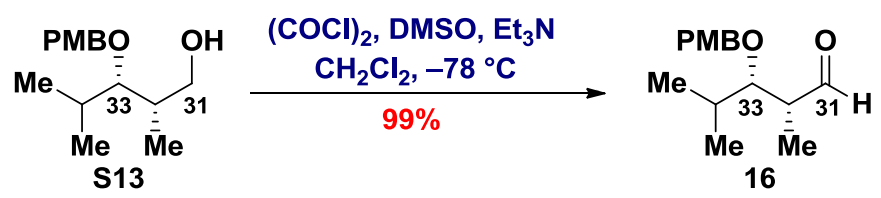

Compound 16 was prepared according to the literature procedure: Evans, D. A.; Connell, B. T. J. Am. Chem. Soc. 2003, 125, 10899.

TLC: $R_{f}=0.68(80: 20$ hexane:EtOAc)

Optical rotation: $[\alpha]_{D}^{20}-36\left(c 1.4, \mathrm{CHCl}_{3}\right)$

${ }^{1} \mathrm{H}$ NMR $\left(400 \mathrm{MHz}, \mathrm{CDCl}_{3}\right) \delta 0.93(\mathrm{~d}, J=6.8 \mathrm{~Hz}, 3 \mathrm{H}), 1.02(\mathrm{~d}, J=6.5 \mathrm{~Hz}, 3 \mathrm{H}), 1.16(\mathrm{~d}, J=$ 6.8 Hz, 3H), 1.89 (oct, $J=6.8 \mathrm{~Hz}, 1 \mathrm{H}$ ), 2.58 (qdd, $J=7.0,3.5$ and $1.0 \mathrm{~Hz}, 1 \mathrm{H}$ ), 3.58 (dd, $J=$ 7.3 and $3.5 \mathrm{~Hz}, 1 \mathrm{H}), 3.78(\mathrm{~s}, 3 \mathrm{H}), 4.37-4.43(\mathrm{~m}, 2 \mathrm{H}), 6.83-6.87(\mathrm{~m}, 2 \mathrm{H}), 7.19-7.23(\mathrm{~m}, 2 \mathrm{H})$, $9.77(\mathrm{~d}, J=1.0 \mathrm{~Hz}, 1 \mathrm{H})$.

${ }^{13} \mathrm{C}$ NMR $\left(100 \mathrm{MHz}, \mathrm{CDCl}_{3}\right) \delta 8.1,19.0,19.6,31.2,49.2,55.2,73.5,83.3,113.7,129.3$, 130.4, 159.2, 204.9.

IR (film) $v_{\text {max }} / \mathrm{cm}^{-1}$ 2962, 2874, 1722, 1613, 1514, 1465, 1248, 1088, 1066, 1035, 823, 736. 


\section{Synthesis of C23-C35 Fragment of Marinisporolide C}

\section{(+)-(4S,5S,6S)-1-((4R,6R)-6-(2-((tert-butyldimethylsilyl)oxy)ethyl)-2,2-dimethyl-1,3-}

dioxan-4-yl)-4-hydroxy-6-((4-methoxybenzyl)oxy)-5,7-dimethyloctan-2-one (S14):
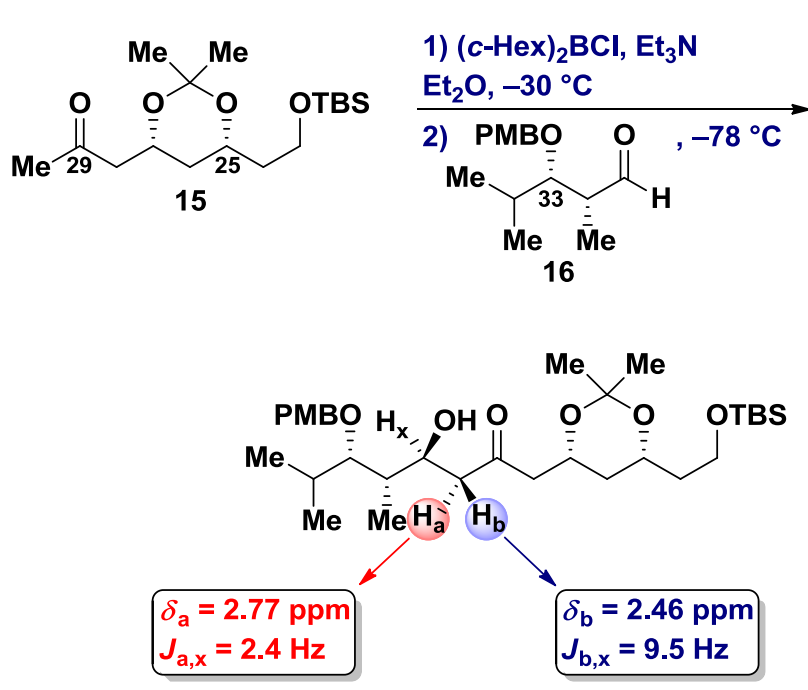

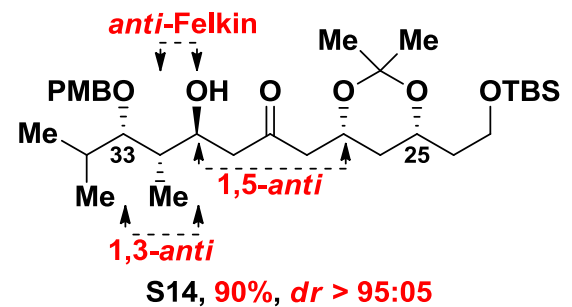

$\mathrm{S} 14,90 \%, d r>95: 05$

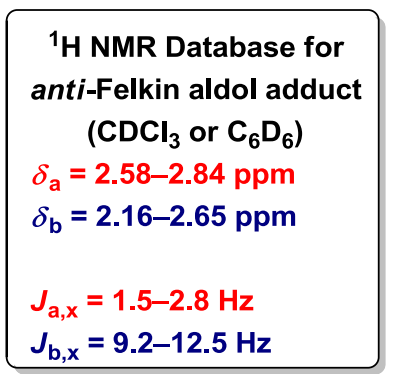

To a solution of methylketone $15(1.20 \mathrm{~g}, 3.63 \mathrm{mmol}, 100 \mathrm{~mol} \%)$ in $\mathrm{Et}_{2} \mathrm{O}(50 \mathrm{~mL}, 0.07 \mathrm{M})$ at $-30{ }^{\circ} \mathrm{C}$ was added $(\mathrm{c}-\mathrm{Hex})_{2} \mathrm{BCl}(1.7 \mathrm{~mL}, 7.26 \mathrm{mmol}, 200 \mathrm{~mol} \%)$ dropwise, followed by the addition of $\mathrm{Et}_{3} \mathrm{~N}(2.3 \mathrm{~mL}, 9.20 \mathrm{mmol}, 250 \mathrm{~mol} \%)$ dropwise, which resulted in the formation of a white cloud. The mixture was stirred under the same conditions for $30 \mathrm{~min}$. The reaction medium was then cooled to $-78^{\circ} \mathrm{C}$, and a solution of aldehyde $16(1.18 \mathrm{~g}, 4.72 \mathrm{mmol}, 130$ mol\%) in $\mathrm{Et}_{2} \mathrm{O}(5.0 \mathrm{~mL}, 0.94 \mathrm{M})$ was added over $30 \mathrm{~min}$ using a syringe pump. The resulting mixture was stirred for $3 \mathrm{~h}$ at $-78{ }^{\circ} \mathrm{C}$, followed by quenching via the dropwise addition of $\mathrm{MeOH}(20 \mathrm{~mL})$. The volatiles were removed under reduced pressure, and the residue was purified by flash column chromatography using a solution of hexane/ethyl acetate (80:20) as the eluent to provide the aldol adduct $\mathbf{S} 14(1.90 \mathrm{~g}, 3.26 \mathrm{mmol}, 90 \%, d r>95: 05,1,5$-anti:1,5syn). This compound was used to confirm the anti-Felkin relative stereochemistry between C31 and $\mathrm{C} 32$ on the basis of Roush ${ }^{1} \mathrm{H}$ NMR ABX pattern analysis: a) Roush, W. R.; Bannister, T. D.; Wendt, M. D.; VanNieuwenhze, M. S.; Gustin, D. J.; Dilley, G. J.; Lane, G. C.; Scheidt, K. A.; Smith, W. J., III J. Org. Chem. 2002, 67, 4284. b) Dias, L. C.; Aguilar, A. M.; Salles, A. G., Jr.; Steil, L. J.; Roush, W. R. J. Org. Chem. 2005, 70, 10461.

Physical state: pale yellow oil

TLC: $R_{f}=0.36(80: 20$ hexane:EtOAc) 
Optical rotation: $[\alpha]_{D}^{20}+2\left(c 2.3, \mathrm{CHCl}_{3}\right)$

${ }^{1} \mathrm{H}$ NMR $\left(600 \mathrm{MHz}, \mathrm{CDCl}_{3}\right) \delta 0.03(\mathrm{~s}, 6 \mathrm{H}), 0.84(\mathrm{~d}, J=7.0 \mathrm{~Hz}, 3 \mathrm{H}), 0.86(\mathrm{~d}, J=6.8 \mathrm{~Hz}, 3 \mathrm{H})$, $0.88(\mathrm{~s}, 9 \mathrm{H}), 1.04(\mathrm{~d}, J=6.6 \mathrm{~Hz}, 3 \mathrm{H}), 1.18(\mathrm{q}, J=12.7 \mathrm{~Hz}, 1 \mathrm{H}), 1.33(\mathrm{~s}, 3 \mathrm{H}), 1.42(\mathrm{~s}, 3 \mathrm{H})$, $1.54(\mathrm{dt}, J=12.7$ and $2.4 \mathrm{~Hz}, 1 \mathrm{H}), 1.58-1.65(\mathrm{~m}, 2 \mathrm{H}), 1.71-1.76(\mathrm{~m}, 1 \mathrm{H}), 1.81-1.89(\mathrm{~m}, 1 \mathrm{H})$, 2.37 (dd, $J=15.6$ and $4.4 \mathrm{~Hz}, 1 \mathrm{H}$ ), 2.46 (dd, $J=16.8$ and $9.5 \mathrm{~Hz}, 1 \mathrm{H}$ ), 2.69 (dd, $J=15.6$ and $8.1 \mathrm{~Hz}, 1 \mathrm{H}$ ), $2.77(\mathrm{dd}, J=16.8$ and $2.4 \mathrm{~Hz}, 1 \mathrm{H}), 3.42(\mathrm{~d}, J=3.6 \mathrm{~Hz}, 1 \mathrm{H}), 3.47(\mathrm{dd}, J=8.7$ and $2.1 \mathrm{~Hz}, 1 \mathrm{H}$ ), $3.63(\mathrm{dt}, J=10.4$ and $5.5 \mathrm{~Hz}, 1 \mathrm{H}$ ), 3.70 (ddd, $J=10.2,8.1$ and $5.3 \mathrm{~Hz}, 1 \mathrm{H}$ ), 3.78 (s, 3H), 3.99-4.07 (m, 2H), 4.32-4.37 (m, 1H), $4.56(\mathrm{~d}, J=10.9 \mathrm{~Hz}, 1 \mathrm{H}), 4.58(\mathrm{~d}, J=10.9 \mathrm{~Hz}$, $1 \mathrm{H}), 6.85-6.87(\mathrm{~m}, 2 \mathrm{H}), 7.27-7.28(\mathrm{~m}, 2 \mathrm{H})$.

${ }^{13} \mathrm{C}$ NMR $\left(125 \mathrm{MHz}, \mathrm{CDCl}_{3}\right) \delta-5.4,-5.4,10.0,18.3,19.6,19.7,20.0,25.9,30.0,31.1,36.9$, $39.3,40.3,49.1,49.5,55.2,58.7,65.4,66.1,69.4,74.2,83.8,98.8,113.7,129.2,131.4$, 159.0, 210.6 .

IR (film) $v_{\text {max }} / \mathrm{cm}^{-1} 3441,2957,2930,2856,1705,1645,1614,1514,1464,1381,1265,1250$, 1094, 1036, 837, 739.

HRMS (ESI TOF-MS) $\mathrm{m} / \mathrm{z}$ calcd for $\mathrm{C}_{32} \mathrm{H}_{56} \mathrm{O}_{7} \mathrm{SiNa}[\mathrm{M}+\mathrm{Na}]^{+}: 603.3693$, found: 603.3720 .

(-)-1-((4R,6R)-6-(2-((tert-butyldimethylsilyl)oxy)ethyl)-2,2-dimethyl-1,3-dioxan-4-yl)-3((2R,4S,5S,6S)-6-isopropyl-2-(4-methoxyphenyl)-5-methyl-1,3-dioxan-4-yl)propan-2-one (S15):
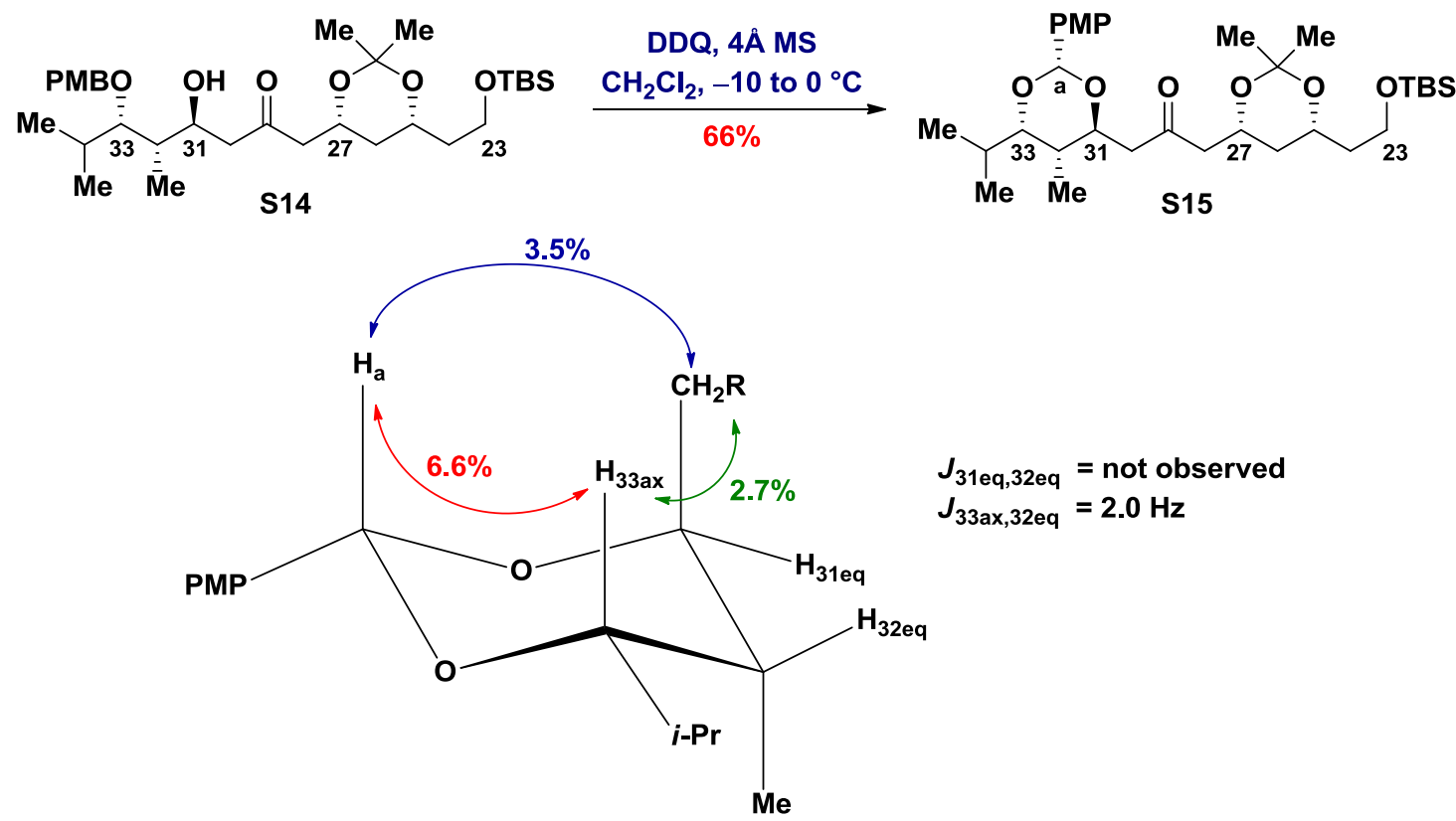

To a solution of aldol adduct S14 (50 mg, $86 \mu \mathrm{mol}, 100 \mathrm{~mol} \%$ ) in $\mathrm{CH}_{2} \mathrm{Cl}_{2}(1.8 \mathrm{~mL}, 0.05 \mathrm{M}$ ) at room temperature was added activated $4 \AA$ molecular sieves $(27 \mathrm{mg})$. After $15 \mathrm{~min}$, the S15 
mixture was cooled to $-10{ }^{\circ} \mathrm{C}$, and DDQ (25 mg, $\left.0.11 \mathrm{mmol}, 130 \mathrm{~mol} \%\right)$ was added. The reaction medium was stirred for $5 \mathrm{~min}$ at $-10^{\circ} \mathrm{C}$ and warmed to $0{ }^{\circ} \mathrm{C}$. After $1.5 \mathrm{~h}$, the reaction mixture was loaded directly onto a flash column chromatography using a solution of hexane/ethyl acetate (80:20) as the eluent to provide the PMP acetal S15 (33 mg, $57 \mu \mathrm{mol}$, $66 \%$ ). This compound was used to confirm the 1,3-anti relative stereochemistry between C31 and $\mathrm{C} 33$ on the basis of ${ }^{1} \mathrm{H}$ NMR and $1 \mathrm{D}$ selective NOE spectra analyses.

Physical state: pale yellow oil

TLC: $R_{f}=0.76$ (70:30 hexane:EtOAc)

Optical rotation: $[\alpha]_{D}^{20}-21\left(c 3.3, \mathrm{CHCl}_{3}\right)$

${ }^{1} \mathrm{H}$ NMR $\left(500 \mathrm{MHz}, \mathrm{CDCl}_{3}\right) \delta 0.03(\mathrm{~s}, 6 \mathrm{H}), 0.80(\mathrm{~d}, J=6.8 \mathrm{~Hz}, 3 \mathrm{H}), 0.88(\mathrm{~s}, 9 \mathrm{H}), 1.00(\mathrm{~d}, J=$ $6.4 \mathrm{~Hz}, 3 \mathrm{H}), 1.14(\mathrm{q}, J=11.9 \mathrm{~Hz}, 1 \mathrm{H}), 1.20(\mathrm{~d}, J=6.9 \mathrm{~Hz}, 3 \mathrm{H}), 1.34(\mathrm{~s}, 3 \mathrm{H}), 1.42(\mathrm{~s}, 3 \mathrm{H})$, 1.52-1.62 (m, 4H), 1.75-1.82 (m, 1H), $2.47(\mathrm{dd}, J=15.6$ and $5.1 \mathrm{~Hz}, 1 \mathrm{H}), 2.70(\mathrm{dd}, J=15.6$ and 7.2 and $\mathrm{Hz}, 1 \mathrm{H}$ ), $2.92(\mathrm{dd}, J=16.0$ and $7.2 \mathrm{~Hz}, 1 \mathrm{H}$ ), $3.17(\mathrm{dd}, J=16.0$ and $7.1 \mathrm{~Hz}, 1 \mathrm{H}$ ), $3.44(\mathrm{dd}, J=9.7$ and $2.0 \mathrm{~Hz}, 1 \mathrm{H}$ ), $3.62(\mathrm{dt}, J=10.2$ and $5.2 \mathrm{~Hz}, 1 \mathrm{H}$ ), 3.70 (ddd, $J=10.2,8.0$ and $5.4 \mathrm{~Hz}, 1 \mathrm{H}), 3.78(\mathrm{~s}, 3 \mathrm{H}), 4.01-4.06(\mathrm{~m}, 1 \mathrm{H}), 4.30-4.35(\mathrm{~m}, 1 \mathrm{H}), 4.42(\mathrm{t}, J=7.1 \mathrm{~Hz}, 1 \mathrm{H})$, $5.66(\mathrm{~s}, 1 \mathrm{H}), 6.85-6.87(\mathrm{~m}, 2 \mathrm{H}), 7.37-7.39(\mathrm{~m}, 2 \mathrm{H})$.

${ }^{13} \mathrm{C}$ NMR $\left(125 \mathrm{MHz}, \mathrm{CDCl}_{3}\right) \delta-5.4,-5.4,12.9,17.4,18.2,19.8\left(2 \times \mathrm{CH}_{3}\right), 25.9,29.2,30.1$, 32.3, 36.9, 39.3, 45.5, 49.8, 55.3, 58.7, 65.4, 65.9, 75.8, 80.8, 95.4, 98.7, 113.5, 127.3, 131.5, 159.8, 206.7.

IR (film) $v_{\text {max }} / \mathrm{cm}^{-1}$ 2957, 2930, 2856, 1715, 1616, 1518, 1472, 1464, 1381, 1250, 1171, 1101 , $1038,960,835,777,739$.

HRMS (ESI TOF-MS) $\mathrm{m} / z$ calcd for $\mathrm{C}_{32} \mathrm{H}_{55} \mathrm{O}_{7} \mathrm{Si}[\mathrm{M}+\mathrm{H}]^{+}: 579.3717$, found: 579.3720 .

\section{(+)-(2R,4S,5S,6S)-1-((4S,6R)-6-(2-((tert-butyldimethylsilyl)oxy)ethyl)-2,2-dimethyl-1,3-} dioxan-4-yl)-6-((4-methoxybenzyl)oxy)-5,7-dimethyloctane-2,4-diol (S16):

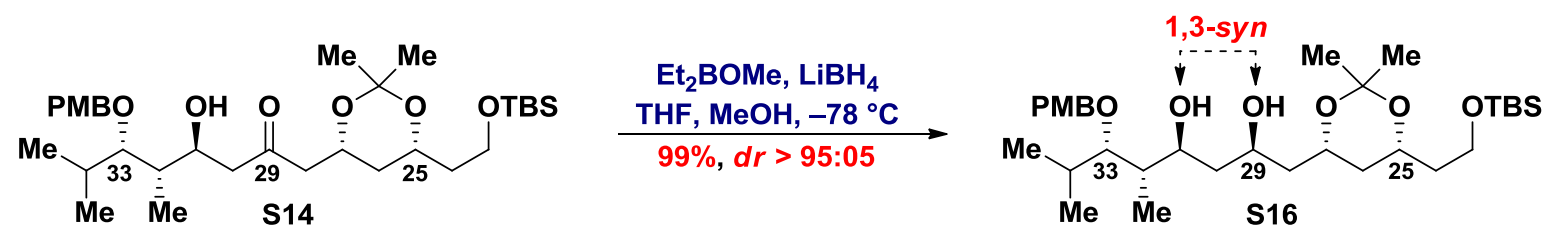

To a solution of aldol adduct $\mathbf{S 1 4}(1.20 \mathrm{~g}, 2.06 \mathrm{mmol}, 100 \mathrm{~mol} \%)$ in THF:MeOH (4:1) (10 mL, $0.21 \mathrm{M})$ at $-78{ }^{\circ} \mathrm{C}$ was added $\mathrm{Et}_{2} \mathrm{BOMe}(0.35 \mathrm{~mL}, 2.50 \mathrm{mmol}, 120 \mathrm{~mol} \%)$. The solution was stirred for $15 \mathrm{~min}$ under these conditions, and $\mathrm{LiBH}_{4}(1.26 \mathrm{~mL}, 2.52 \mathrm{mmol}, 2.0 \mathrm{M}$ in THF, 120 
mol\%) was added over $1 \mathrm{~h}$ using a syringe pump. The reaction was stirred for $1 \mathrm{~h}$ and then warmed to $-40{ }^{\circ} \mathrm{C}$. The reaction was quenched by the addition of $\mathrm{pH} 7$ phosphate buffer ( 28 $\mathrm{mL}$ ) and $\mathrm{MeOH}(52 \mathrm{~mL})$. The reaction was warmed to $0 \stackrel{\circ}{\circ} \mathrm{C}$, and $30 \% \mathrm{H}_{2} \mathrm{O}_{2}(21 \mathrm{~mL})$ was added dropwise. The mixture was stirred for $1 \mathrm{~h}$, and the volatiles were removed under reduced pressure. The aqueous layer was extracted with $\mathrm{EtOAc}(3 \times 50 \mathrm{~mL})$. The combined organic layers were washed with saturated aqueous solution of $\mathrm{NaHCO}_{3}(2 \times 50 \mathrm{~mL})$, brine (100 mL), dried over $\mathrm{MgSO}_{4}$, filtered, and concentrated under reduced pressure. The residue was dissolved in $\mathrm{MeOH}(50 \mathrm{~mL})$, and the solvent was removed under reduced pressure in a $60 \stackrel{\circ}{ } \mathrm{C}$ bath to remove chelated boron species. This procedure was repeated 6 times to provide the diol S16 (1.20 g, $2.06 \mathrm{mmol}, 99 \%, d r>95: 05,1,3-s y n: 1,3-$ anti), which was used in the next step without further purification.

\section{Physical state: white gum}

TLC: $R_{f}=0.30$ (80:20 hexane:EtOAc)

Optical rotation: $[\alpha]_{D}^{20}+7\left(c 2.0, \mathrm{CHCl}_{3}\right)$

${ }^{1} \mathrm{H}$ NMR $\left(500 \mathrm{MHz}, \mathrm{CDCl}_{3}\right) \delta 0.03(\mathrm{~s}, 6 \mathrm{H}), 0.86-0.88(\mathrm{~m}, 6 \mathrm{H}), 0.88(\mathrm{~s}, 9 \mathrm{H}), 1.02(\mathrm{~d}, J=6.4$ $\mathrm{Hz}, 3 \mathrm{H}), 1.30-1.50(\mathrm{~m}, 3 \mathrm{H}), 1.37(\mathrm{~s}, 3 \mathrm{H}), 1.45(\mathrm{~s}, 3 \mathrm{H}), 1.58-1.67(\mathrm{~m}, 5 \mathrm{H}), 1.70-1.76(\mathrm{~m}, 1 \mathrm{H})$, 1.88 (oct, $J=6.9 \mathrm{~Hz}, 1 \mathrm{H}$ ), 3.44 (dd, $J=7.9$ and $1.9 \mathrm{~Hz}, 1 \mathrm{H}$ ), 3.64 (dt, $J=10.3$ and $5.3 \mathrm{~Hz}$, $1 \mathrm{H}), 3.69-3.74(\mathrm{~m}, 1 \mathrm{H}), 3.78(\mathrm{~s}, 3 \mathrm{H}), 3.78-3.83(\mathrm{~m}, 1 \mathrm{H}), 3.91-4.22(\mathrm{~m}, 5 \mathrm{H}), 4.54(\mathrm{~d}, J=10.8$ $\mathrm{Hz}, 1 \mathrm{H}), 4.62(\mathrm{~d}, J=10.8 \mathrm{~Hz}, 1 \mathrm{H}), 6.85-6.86(\mathrm{~m}, 2 \mathrm{H}), 7.26-7.28(\mathrm{~m}, 2 \mathrm{H})$.

${ }^{13} \mathrm{C}$ NMR (62.5 MHz, $\left.\mathrm{CDCl}_{3}\right) \delta-5.4\left(2 \times \mathrm{CH}_{3}\right), 10.8,18.3,19.8\left(2 \times \mathrm{CH}_{3}\right), 19.9,25.9,30.2$, $30.8,36.8,39.4,41.0,41.1,43.0,55.3,58.7,65.6,67.0,70.2,73.7,75.0,84.4,98.7,113.7$, 129.2, 131.3, 159.0 .

IR (film) $v_{\text {max }} / \mathrm{cm}^{-1} 3441,3055,2986,2957,2930,2856,1659,1645,1614,1514,1421,1265$, 1095, 897, 837, 739, 706.

HRMS (ESI TOF-MS) $\mathrm{m} / z$ calcd for $\mathrm{C}_{32} \mathrm{H}_{59} \mathrm{O}_{7} \mathrm{Si}[\mathrm{M}+\mathrm{H}]^{+}: 583.4030$, found: 583.4047 . 
(+)-tert-butyl(2-((4R,6R)-6-(((4S,6S)-6-((2S,3S)-3-((4-methoxybenzyl)oxy)-4methylpentan-2-yl)-2,2-dimethyl-1,3-dioxan-4-yl)methyl)-2,2-dimethyl-1,3-dioxan-4yl)ethoxy)dimethylsilane (17):
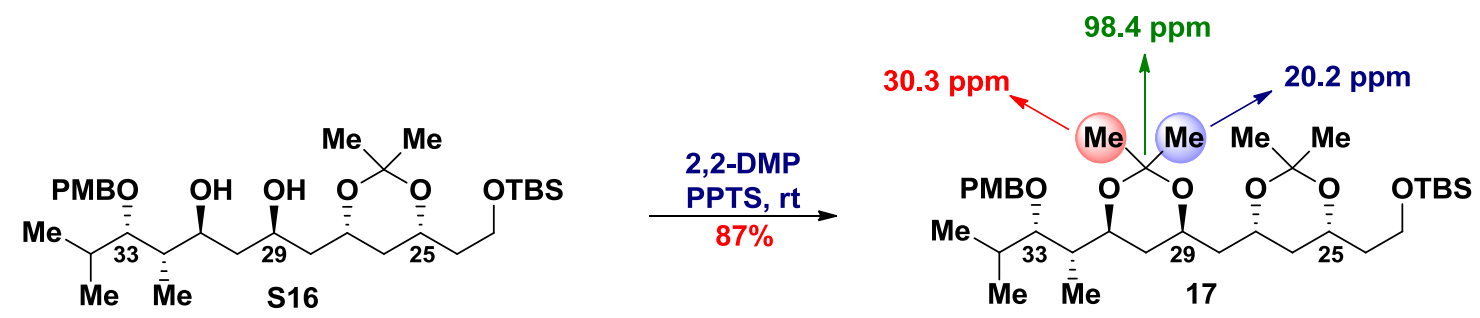

To a solution of diol S16 (1.20 g, $2.06 \mathrm{mmol}, 100 \mathrm{~mol} \%)$ in 2,2-DMP (25 mL, $0.08 \mathrm{M})$ at room temperature was added PPTS (260 $\mathrm{mg}, 1.03 \mathrm{mmol}, 50 \mathrm{~mol} \%$ ). The reaction medium was stirred for $24 \mathrm{~h}$. The mixture was filtered through silica and Celite, and the residue was washed with $\mathrm{CH}_{2} \mathrm{Cl}_{2}(5 \times 50 \mathrm{~mL})$ and concentrated under reduced pressure. The residue was purified by flash column chromatography using a solution of hexane/ethyl acetate (80:20) as the eluent to provide acetonide $17(1.12 \mathrm{~g}, 1.80 \mathrm{mmol}, 87 \%)$. This compound was used to confirm the 1,3-syn relative stereochemistry between C29 and C31 on the basis of Rychnovsky ${ }^{13} \mathrm{C}$ NMR analysis: a) Rychnovsky, S. D.; Skalitzky, D. J. Tetrahedron Lett. 1990, 31, 945. b) Rychnovsky, S. D.; Rogers, B.; Yang, G. J. Org. Chem. 1993, 58, 3511. c) Rychnovsky, S. D.; Rogers, B. N.; Richardson, T. I. Acc. Chem. Res. 1998, 31, 9. For a theoretical work, see: d) Tormena, C. F.; Dias, L. C.; Rittner, R. J. Phys. Chem. A 2005, 109, 6077 .

Physical state: pale yellow oil

TLC: $R_{f}=0.74$ (80:20 hexane:EtOAc)

Optical rotation: $[\alpha]_{D}^{20}+10\left(c 1.6, \mathrm{CHCl}_{3}\right)$

${ }^{1} \mathrm{H}$ NMR (600 MHz, $\left.\mathrm{CDCl}_{3}\right) \delta 0.03(\mathrm{~s}, 6 \mathrm{H}), 0.82(\mathrm{~d}, J=7.2 \mathrm{~Hz}, 3 \mathrm{H}), 0.84(\mathrm{~d}, J=6.8 \mathrm{~Hz}, 3 \mathrm{H})$, $0.88(\mathrm{~s}, 9 \mathrm{H}), 1.02$ (d, $J=6.6 \mathrm{~Hz}, 3 \mathrm{H}), 1.06(\mathrm{q}, J=12.2 \mathrm{~Hz}, 1 \mathrm{H}), 1.15(\mathrm{q}, J=12.2 \mathrm{~Hz}, 1 \mathrm{H}$ ), $1.36(\mathrm{~s}, 3 \mathrm{H}), 1.37(\mathrm{~s}, 3 \mathrm{H}), 1.39(\mathrm{~s}, 3 \mathrm{H}), 1.40(\mathrm{~s}, 3 \mathrm{H}), 1.45(\mathrm{dt}, J=12.8$ and $2.3 \mathrm{~Hz}, 1 \mathrm{H}), 1.50$ (dd, $J=6.4$ and $5.8 \mathrm{~Hz}, 2 \mathrm{H}), 1.57-1.68(\mathrm{~m}, 4 \mathrm{H}), 1.79-1.85(\mathrm{~m}, 1 \mathrm{H}), 3.41(\mathrm{dd}, J=9.0$ and 1.7 $\mathrm{Hz}, 1 \mathrm{H}$ ), 3.64 (dt, $J=10.3$ and $5.3 \mathrm{~Hz}, 1 \mathrm{H}$ ), 3.71 (ddd, $J=10.3,8.3$ and $5.3 \mathrm{~Hz}, 1 \mathrm{H}$ ), 3.79 (s, $3 \mathrm{H}), 3.79-3.83(\mathrm{~m}, 1 \mathrm{H}), 4.00-4.09(\mathrm{~m}, 3 \mathrm{H}), 4.50(\mathrm{~d}, J=11.8 \mathrm{~Hz}, 1 \mathrm{H}), 4.52(\mathrm{~d}, J=11.8 \mathrm{~Hz}$, $1 \mathrm{H}), 6.85-6.87(\mathrm{~m}, 2 \mathrm{H}), 7.25-7.26(\mathrm{~m}, 2 \mathrm{H})$. 
${ }^{13} \mathrm{C}$ NMR (150 MHz, $\left.\mathrm{CDCl}_{3}\right) \delta-5.4\left(2 \times \mathrm{CH}_{3}\right), 8.8,18.3,19.4,19.9,20.1,20.2,25.9,30.3$, $30.4,31.2,35.9,37.7,39.5,41.3,43.4,55.2,58.9,64.9,64.9,65.8,70.1,74.0,82.7,98.4$, 98.4, 113.7, 128.7, 131.8, 158.9.

IR (film) $v_{\max } / \mathrm{cm}^{-1} 2991,2953,2937,2858,1614,1514,1472,1464,1379,1248,1202,1171$, 1107, 1092, 959, 835, 775, 739 .

HRMS (ESI TOF-MS) $\mathrm{m} / z$ calcd for $\mathrm{C}_{35} \mathrm{H}_{63} \mathrm{O}_{7} \mathrm{Si}[\mathrm{M}+\mathrm{H}]^{+}: 623.4343$, found: 623.4360 .

\section{(+)-2-((4R,6R)-6-(((4S,6S)-6-((2S,3S)-3-((4-methoxybenzyl)oxy)-4-methylpentan-2-yl)-2,2-} dimethyl-1,3-dioxan-4-yl)methyl)-2,2-dimethyl-1,3-dioxan-4-yl)ethanol (S17):

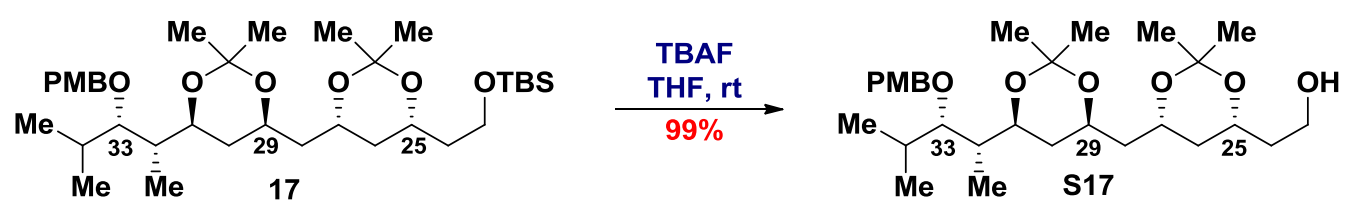

To a solution of compound 17 (300 mg, $0.48 \mathrm{mmol}, 100 \mathrm{~mol} \%$ ) in THF (3.2 mL, $0.15 \mathrm{M}$ ) at room temperature was added TBAF $(0.96 \mathrm{~mL}, 0.96 \mathrm{mmol}, 1 \mathrm{M}$ in THF, $200 \mathrm{~mol} \%)$. After 2.5 $\mathrm{h}$, the volatiles were removed under reduced pressure, and the residue was purified by flash column chromatography using a solution of hexane/ethyl acetate (50:50) as the eluent to provide alcohol S17 (244 mg, $0.48 \mathrm{mmol}, 99 \%$ ).

Physical state: colorless oil

TLC: $R_{f}=0.50$ (50:50 hexane:EtOAc)

Optical rotation: $[\alpha]_{D}^{20}+5\left(c 1.4, \mathrm{CHCl}_{3}\right)$

${ }^{1} \mathrm{H}$ NMR $\left(500 \mathrm{MHz}, \mathrm{CDCl}_{3}\right) \delta 0.83(\mathrm{~d}, J=6.8 \mathrm{~Hz}, 3 \mathrm{H}), 0.84(\mathrm{~d}, J=6.6 \mathrm{~Hz}, 3 \mathrm{H}), 1.02(\mathrm{~d}, J=$ $6.6 \mathrm{~Hz}, 3 \mathrm{H}), 1.07(\mathrm{q}, J=12.5 \mathrm{~Hz}, 1 \mathrm{H}), 1.25-1.32(\mathrm{~m}, 2 \mathrm{H}), 1.37(\mathrm{~s}, 3 \mathrm{H}), 1.38(\mathrm{~s}, 3 \mathrm{H}), 1.39(\mathrm{~s}$, $3 \mathrm{H}), 1.42-1.45(\mathrm{~m}, 1 \mathrm{H}), 1.44(\mathrm{~s}, 3 \mathrm{H}), 1.50-1.53(\mathrm{~m}, 2 \mathrm{H}), 1.60(\mathrm{dt}, J=12.5$ and $2.3 \mathrm{~Hz}, 1 \mathrm{H})$, 1.63-1.77 (m, 2H), 1.80-1.87 (m, 1H), $2.60(\mathrm{br} \mathrm{s}, 1 \mathrm{H}), 3.42(\mathrm{dd}, J=9.0$ and $1.9 \mathrm{~Hz}, 1 \mathrm{H})$, 3.73-3.84 (m, 3H), $3.80(\mathrm{~s}, 3 \mathrm{H}), 4.03-4.14(\mathrm{~m}, 3 \mathrm{H}), 4.52-4.54(\mathrm{~m}, 2 \mathrm{H}), 6.86-6.88(\mathrm{~m}, 2 \mathrm{H})$, 7.26-7.27 (m, 2H).

${ }^{13} \mathrm{C}$ NMR (125 MHz, $\left.\mathrm{CDCl}_{3}\right) \delta 8.7,19.4,19.9,20.1,20.2,30.3,30.4,31.2,35.9,37.1,38.0$, 41.3, 43.2, 55.3, 61.0, $64.8(2 \times \mathrm{CH}), 69.8,70.1,74.0,82.7,98.4,98.7,113.7,128.7,131.7$, 158.9 .

IR (film) $v_{\text {max }} / \mathrm{cm}^{-1} 3463,2991,2944,2916,2874,1614,1515,1465,1380,1248,1203,1170$, $1105,1037,956,917,874,831,738$. 
HRMS (ESI TOF-MS) $\mathrm{m} / z$ calcd for $\mathrm{C}_{29} \mathrm{H}_{48} \mathrm{O}_{7} \mathrm{Na}[\mathrm{M}+\mathrm{Na}]^{+}: 531.3298$, found: 531.3328 .

(+)-5-((2-((4S,6S)-6-(((4S,6S)-6-((2S,3S)-3-((4-methoxybenzyl)oxy)-4-methylpentan-2-yl)2,2-dimethyl-1,3-dioxan-4-yl)methyl)-2,2-dimethyl-1,3-dioxan-4-yl)ethyl)thio)-1-phenyl$1 \mathrm{H}$-tetrazole (S19):

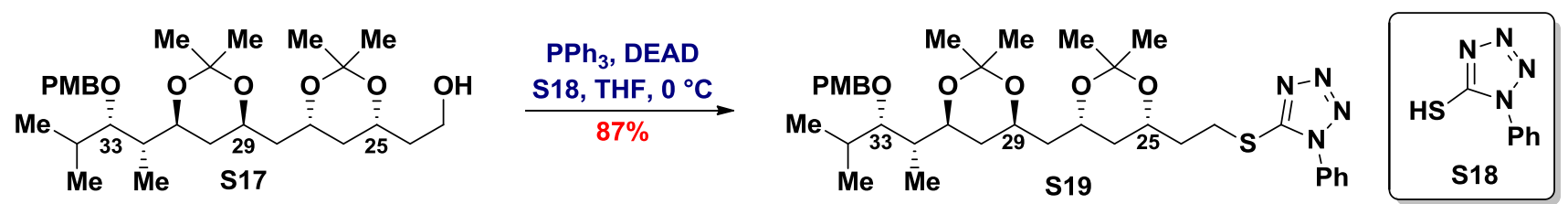

To a solution of compound $\mathbf{S} 17(115 \mathrm{mg}, 0.23 \mathrm{mmol}, 100 \mathrm{~mol} \%)$ in THF $(2.0 \mathrm{~mL}, 0.11 \mathrm{M})$ at 0 ${ }^{\circ} \mathrm{C}$ were added $\mathrm{PPh}_{3}(89 \mathrm{mg}, 0.34 \mathrm{mmol}, 150 \mathrm{~mol} \%)$ and 1-phenyl-1 $\mathrm{H}$-tetrazole-5-thiol (S18) (81 $\mathrm{mg}, 0.45 \mathrm{mmol}, 200 \mathrm{~mol} \%)$, followed by the addition of DEAD (64 $\mu \mathrm{L}, 0.41 \mathrm{mmol}, 180$ mol\%) dropwise. Ater $30 \mathrm{~min}$ at $0{ }^{\circ} \mathrm{C}$, the reaction was diluted with $\mathrm{Et}_{2} \mathrm{O}(5 \mathrm{~mL})$ and quenched by the addition of saturated aqueous solution of $\mathrm{NaHCO}_{3}(5 \mathrm{~mL})$. The layers were separated and the aqueous layer was extracted with $\mathrm{Et}_{2} \mathrm{O}(3 \times 10 \mathrm{~mL})$. The combined organic layers were washed with brine $(10 \mathrm{~mL})$, dried over $\mathrm{MgSO}_{4}$, filtered, and concentrated under reduced pressure. The residue was purified by flash column chromatography using a solution of hexane/ethyl acetate (80:20) as the eluent to provide sulfide $\mathbf{S} 19$ (131 mg, $0.196 \mathrm{mmol}, 87 \%)$.

Physical state: viscous colorless oil

TLC: $R_{f}=0.33$ (80:20 hexane:EtOAc)

Optical rotation: $[\alpha]_{D}^{20}+20\left(c 1.2, \mathrm{CHCl}_{3}\right)$

${ }^{1} \mathrm{H}$ NMR $\left(500 \mathrm{MHz}, \mathrm{CDCl}_{3}\right) \delta 0.82(\mathrm{~d}, J=6.8 \mathrm{~Hz}, 3 \mathrm{H}), 0.84(\mathrm{~d}, J=6.0 \mathrm{~Hz}, 3 \mathrm{H}), 1.02(\mathrm{~d}, J=$ $6.6 \mathrm{~Hz}, 3 \mathrm{H}$ ), $1.06(\mathrm{q}, J=12.5 \mathrm{~Hz}, 1 \mathrm{H}), 1.20(\mathrm{q}, J=12.5 \mathrm{~Hz}, 1 \mathrm{H}), 1.37(\mathrm{~s}, 3 \mathrm{H}), 1.37(\mathrm{~s}, 3 \mathrm{H})$, $1.38(\mathrm{~s}, 3 \mathrm{H}), 1.40(\mathrm{~s}, 3 \mathrm{H}), 1.46(\mathrm{dt}, J=12.5$ and $2.2 \mathrm{~Hz}, 1 \mathrm{H}), 1.49-1.52(\mathrm{~m}, 2 \mathrm{H}), 1.60(\mathrm{dt}, J=$ 12.5 and $2.2 \mathrm{~Hz}, 1 \mathrm{H}), 1.64-1.68(\mathrm{~m}, 1 \mathrm{H}), 1.80-1.87(\mathrm{~m}, 1 \mathrm{H}), 1.88-1.96(\mathrm{~m}, 1 \mathrm{H}), 1.99-2.07$ $(\mathrm{m}, 1 \mathrm{H}), 3.41-3.54(\mathrm{~m}, 3 \mathrm{H}), 3.79-3.84(\mathrm{~m}, 1 \mathrm{H}), 3.80(\mathrm{~s}, 3 \mathrm{H}), 3.97-4.11(\mathrm{~m}, 3 \mathrm{H}), 4.52-4.54$ $(\mathrm{m}, 2 \mathrm{H}), 6.86-6.88(\mathrm{~m}, 2 \mathrm{H}), 7.25-7.27(\mathrm{~m}, 2 \mathrm{H}), 7.52-7.59(\mathrm{~m}, 5 \mathrm{H})$.

${ }^{13} \mathrm{C}$ NMR (125 MHz, $\left.\mathrm{CDCl}_{3}\right) \delta 8.7,19.4,19.8,20.1,20.2,29.2,30.2,30.4,31.2,35.5,35.8$, $37.1,41.3,43.2,55.2,64.8,64.8,67.5,70.1,74.0$, 82.7, 98.3, 98.7, 113.7, 123.8, 128.7, $129.8,130.1,131.7,133.7,154.4,158.8$.

IR (film) $v_{\text {max }} / \mathrm{cm}^{-1} 2991,2953,2943,2915,2873,1613,1598,1514,1501,1464,1381,1248$, 1203, 1170, 1036, 979, 943, 873, 829, 761, 738, 695. 
HRMS (ESI TOF-MS) $\mathrm{m} / \mathrm{z}$ calcd for $\mathrm{C}_{36} \mathrm{H}_{53} \mathrm{O}_{6} \mathrm{~N}_{4} \mathrm{~S}[\mathrm{M}+\mathrm{H}]^{+}: 669.3686$, found: 669.3727 .

(+)-5-((2-((4S,6S)-6-(((4S,6S)-6-((2S,3S)-3-((4-methoxybenzyl)oxy)-4-methylpentan-2-yl)2,2-dimethyl-1,3-dioxan-4-yl)methyl)-2,2-dimethyl-1,3-dioxan-4-yl)ethyl)sulfonyl)-1phenyl-1H-tetrazole (9):
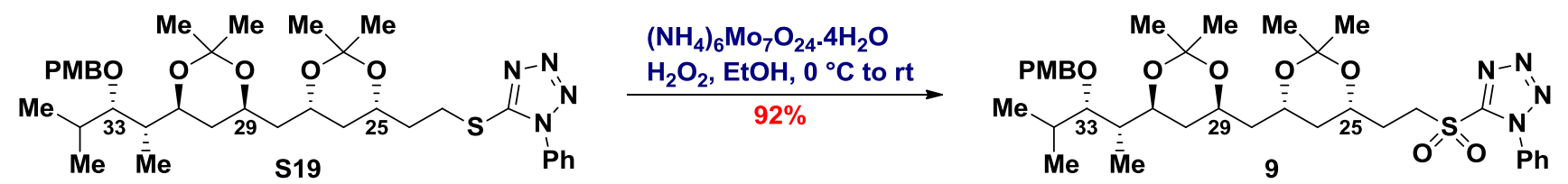

To a solution of sulfide $\mathbf{S} 19$ (107 mg, $0.16 \mathrm{mmol}, 100 \mathrm{~mol} \%)$ in $\mathrm{EtOH}(1.2 \mathrm{~mL}, 0.13 \mathrm{M})$ at $0{ }^{\circ} \mathrm{C}$ was added a solution of $\left(\mathrm{NH}_{4}\right)_{6} \mathrm{Mo}_{7} \mathrm{O}_{24} \cdot 4 \mathrm{H}_{2} \mathrm{O}(20 \mathrm{mg}, 16 \mu \mathrm{mol}, 10 \mathrm{~mol} \%)$ in $\mathrm{H}_{2} \mathrm{O}_{2}(0.3 \mathrm{~mL}$, $0.05 \mathrm{M})$ dropwise. The reaction was slowly warmed to room temperature. After $15 \mathrm{~h}$, the reaction was diluted with $\mathrm{Et}_{2} \mathrm{O}(10 \mathrm{~mL})$ and quenched by the addition of brine $(10 \mathrm{~mL})$. The layers were separated and the aqueous layer was extracted with $\mathrm{Et}_{2} \mathrm{O}(3 \times 10 \mathrm{~mL})$. The combined organic layers were washed with brine $(10 \mathrm{~mL})$, dried over $\mathrm{MgSO}_{4}$, filtered, and concentrated under reduced pressure. The residue was purified by flash column chromatography using a solution of hexane/ethyl acetate (80:20) as the eluent to provide sulfone 9 (104 mg, $0.15 \mathrm{mmol}, 92 \%)$.

Physical state: white solid

TLC: $R_{f}=0.43(80: 20$ hexane:EtOAc)

mp $46-48^{\circ} \mathrm{C}$

Optical rotation: $[\alpha]_{D}^{20}+11\left(c 2.3, \mathrm{CHCl}_{3}\right)$

${ }^{1} \mathrm{H}$ NMR $\left(600 \mathrm{MHz}, \mathrm{CDCl}_{3}\right) \delta 0.82(\mathrm{~d}, J=7.0 \mathrm{~Hz}, 3 \mathrm{H}), 0.84(\mathrm{~d}, J=6.6 \mathrm{~Hz}, 3 \mathrm{H}), 1.02(\mathrm{~d}, J=$ 6.6 Hz, 3H), 1.07 (q, J = $12.0 \mathrm{~Hz}, 1 \mathrm{H}), 1.19(\mathrm{q}, J=12.0 \mathrm{~Hz}, 1 \mathrm{H}), 1.36(\mathrm{~s}, 3 \mathrm{H}), 1.37(\mathrm{~s}, 3 \mathrm{H})$, $1.39(\mathrm{~s}, 3 \mathrm{H}), 1.39(\mathrm{~s}, 3 \mathrm{H}), 1.47-1.52(\mathrm{~m}, 3 \mathrm{H}), 1.59-1.61(\mathrm{~m}, 1 \mathrm{H}), 1.64-1.69(\mathrm{~m}, 1 \mathrm{H})$, 1.79-1.87 (m, 1H), 1.99-2.06 (m, 1H), 2.12-2.18 (m, 1H), $3.42(\mathrm{dd}, J=9.0$ and $1.5 \mathrm{~Hz}, 1 \mathrm{H})$, 3.78-3.83 (m, 2H), $3.80(\mathrm{~s}, 3 \mathrm{H}), 3.92(\mathrm{ddd}, J=14.8,10.1$ and $5.1 \mathrm{~Hz}, 1 \mathrm{H}), 4.01-4.11(\mathrm{~m}, 3 \mathrm{H})$, $4.52(\mathrm{~s}, 2 \mathrm{H}), 6.86-6.88(\mathrm{~m}, 2 \mathrm{H}), 7.26-7.27(\mathrm{~m}, 2 \mathrm{H})$, 7.59-7.64 (m, 3H), 7.68-7.69 (m, 2H).

${ }^{13} \mathrm{C}$ NMR (150 MHz, $\left.\mathrm{CDCl}_{3}\right) \delta 8.8,19.4,19.8,20.1,20.2,28.6,30.1,30.4,31.2,35.8,37.0$, $41.3,43.1,52.4,55.3,64.6,64.8,66.9,70.1,74.0,82.7,98.4,98.9,113.7,125.1,128.6$, $129.7,131.5,131.7,133.0,153.4,158.9$. 
IR (film) $v_{\max } / \mathrm{cm}^{-1} 2992,2955,2943,2916,2873,1614,1514,1499,1464,1381,1346,1248$, 1204, 1171, 1153, 1104, 1077, 1036, 981, 941, 873, 830, 763, 738, 689.

HRMS (ESI TOF-MS) $\mathrm{m} / \mathrm{z}$ calcd for $\mathrm{C}_{36} \mathrm{H}_{53} \mathrm{O}_{8} \mathrm{~N}_{4} \mathrm{~S}$ [M + H] $]^{+}: 701.3584$, found: 701.3583 .

\section{Synthesis of C10-C18 Fragment of Marinisporolide C}

octa-1,7-diene-4,5-diol (S22):

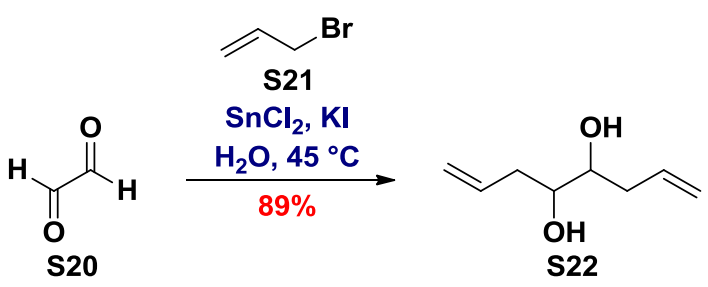

Compound S22 was prepared according to the literature procedure: Airiau, E.; Spangenberg, T.; Girard, N.; Breit, B.; Mann, A. Org. Lett. 2010, 12, 528.

TLC: $R_{f}=0.22(80: 20$ hexane:EtOAc)

${ }^{1} \mathrm{H}$ NMR (250 MHz, $\left.\mathrm{CDCl}_{3}\right) \delta$ 2.18-2.37 (m, 6H), 3.49-3.69 (m, 2H), 5.10-5.18 (m, 4H), $5.76-5.92(\mathrm{~m}, 2 \mathrm{H})$.

${ }^{13} \mathrm{C}$ NMR (62.5 MHz, $\mathrm{CDCl}_{3}$ ) $\delta$ (36.4), 38.2, 72.7, (72.8), 118.1, (118.3), 134.4, (134.7). ${ }^{1}$

IR (film) $v_{\text {max }} / \mathrm{cm}^{-1} 3404,3078,3003,2980,2935,2910,1641,1435,1418,1207,1053,995$, 916, 868.

but-3-enal (18):

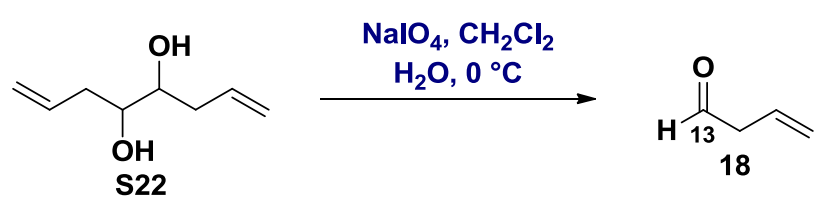

NOTE: This procedure should be performed immediately prior to enolization of methylketone 11.

Compound 18 was prepared according to the literature procedure: Airiau, E.; Spangenberg, T.; Girard, N.; Breit, B.; Mann, A. Org. Lett. 2010, 12, 528.

\footnotetext{
${ }^{1}$ The signals stated in brackets refer to the minor isomer.
} 
(-)-(2R,6R)-6-hydroxy-2-((4-methoxybenzyl)oxy)non-8-en-4-one (19):

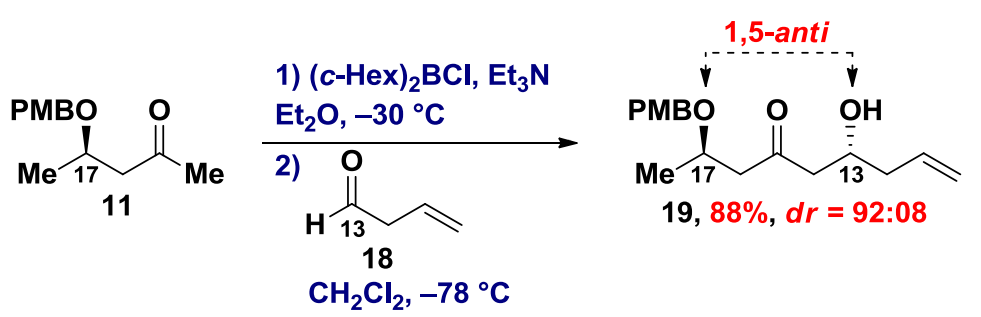

To a solution of methylketone $11(2.53 \mathrm{~g}, 11.4 \mathrm{mmol}, 100 \mathrm{~mol} \%)$ in $\mathrm{Et}_{2} \mathrm{O}(110 \mathrm{~mL}, 0.10 \mathrm{M})$ at $-30{ }^{\circ} \mathrm{C}$ was added $(c-\mathrm{Hex})_{2} \mathrm{BCl}(4.9 \mathrm{~mL}, 22.7 \mathrm{mmol}, 200 \mathrm{~mol} \%)$ dropwise, followed by the addition of $\mathrm{Et}_{3} \mathrm{~N}(3.4 \mathrm{~mL}, 24.4 \mathrm{mmol}, 210 \mathrm{~mol} \%)$ dropwise, which resulted in the formation of a white cloud. The mixture was stirred under the same conditions for $30 \mathrm{~min}$. The reaction medium was then cooled to $-78{ }^{\circ} \mathrm{C}$, and a solution of aldehyde 18 (theor. $34.0 \mathrm{mmol}, 300$ mol\%) in $\mathrm{CH}_{2} \mathrm{Cl}_{2}(6.0 \mathrm{~mL}, 5.7 \mathrm{M})$ was added over 30 min using a syringe pump. The resulting mixture was stirred for $1 \mathrm{~h}$ at $-78{ }^{\circ} \mathrm{C}$, followed by quenching via the addition of $\mathrm{pH} 7$ phosphate buffer $(22 \mathrm{~mL})$. The mixture was warmed to $0{ }^{\circ} \mathrm{C}$, and $\mathrm{MeOH}(67 \mathrm{~mL})$ and a solution of $30 \% \mathrm{H}_{2} \mathrm{O}_{2}(22 \mathrm{~mL})$ in $\mathrm{MeOH}(45 \mathrm{~mL})$ were added dropwise. The reaction medium was stirred for $1 \mathrm{~h}$ under the same conditions. The volatiles were removed under reduced pressure, and the aqueous layer was extracted with $\mathrm{Et}_{2} \mathrm{O}(3 \times 50 \mathrm{~mL})$. The combined organic layers were washed with saturated aqueous solution of $\mathrm{NaHCO}_{3}(2 \times 50 \mathrm{~mL})$, brine $(100 \mathrm{~mL})$, dried over $\mathrm{MgSO}_{4}$, filtered, and concentrated under reduced pressure. The residue was purified by flash column chromatography using a solution of hexane/ethyl acetate (70:30) as the eluent to provide the aldol adduct $19(2.93 \mathrm{~g}, 10.0 \mathrm{mmol}, 88 \%, d r=92: 08,1,5$-anti:1,5syn) as a colorless oil. Diastereoisomeric ratio was determined by ${ }^{13} \mathrm{C}$ NMR analysis of the diastereoisomeric mixture of aldol adducts.

Physical state: pale yellow oil

TLC: $R_{f}=0.46(60: 40$ hexane:EtOAc)

Optical rotation: $[\alpha]_{D}{ }^{20}-49\left(c 2.5, \mathrm{CHCl}_{3}\right)$

${ }^{1} \mathbf{H}$ NMR (500 MHz, $\left.\mathbf{C}_{6} \mathrm{D}_{6}\right) \delta 1.01(\mathrm{~d}, J=6.1 \mathrm{~Hz}, 3 \mathrm{H}), 1.98-2.08(\mathrm{~m}, 2 \mathrm{H}), 2.11-2.27(\mathrm{~m}, 3 \mathrm{H})$, 2.46 (ddd, $J=15.5,7.7$ and $4.1 \mathrm{~Hz}, 1 \mathrm{H}), 2.97-3.04(\mathrm{~m}, 1 \mathrm{H}), 3.30(\mathrm{~s}, 3 \mathrm{H}), 3.88-3.94(\mathrm{~m}, 1 \mathrm{H})$, 4.00-4.09 (m, 1H), $4.21(\mathrm{~d}, J=11.2 \mathrm{~Hz}, 1 \mathrm{H}), 4.35(\mathrm{~d}, J=11.2 \mathrm{~Hz}, 1 \mathrm{H}), 4.98-5.01(\mathrm{~m}, 2 \mathrm{H})$, 5.77 (ddt, $J=16.7,9.6$ and $7.1 \mathrm{~Hz}, 1 \mathrm{H}), 6.79-6.81(\mathrm{~m}, 2 \mathrm{H}), 7.19-7.21(\mathrm{~m}, 2 \mathrm{H})$.

${ }^{13} \mathrm{C}$ NMR (125 MHz, $\left.\mathrm{C}_{6} \mathrm{D}_{6}\right) \delta 19.7,41.4,50.0,50.6,54.7,67.2,70.6,71.4,114.0,117.3$, 129.5, 131.1, 135.1, 159.7, 209.4. 
IR (film) $v_{\text {max }} / \mathrm{cm}^{-1} 3448,3076,2972,2932,2907,2837,1709,1641,1614,1587,1514,1466$, $1375,1302,1248,1175,1080,1034,918,824,754$.

HRMS (ESI TOF-MS) $\mathrm{m} / z$ calcd for $\mathrm{C}_{17} \mathrm{H}_{24} \mathrm{O}_{4} \mathrm{~K}[\mathrm{M}+\mathrm{K}]^{+}: 331.1312$, found: 331.1299 .

\section{(-)-(4R,6S,8R)-8-((4-methoxybenzyl)oxy)non-1-ene-4,6-diol (S23):}

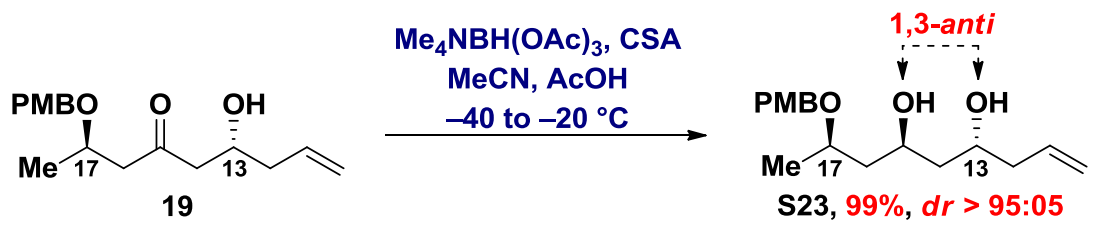

To a slurry of $\mathrm{Me}_{4} \mathrm{NBH}(\mathrm{OAc})_{3}(16.7 \mathrm{~g}, 63.6 \mathrm{mmol}, 400 \mathrm{~mol} \%)$ in $\mathrm{MeCN}(45 \mathrm{~mL}, 1.4 \mathrm{M})$ was added $\mathrm{AcOH}(45 \mathrm{~mL})$. The mixture was stirred at room temperature for $30 \mathrm{~min}$ and then cooled to $-40 \stackrel{\circ}{\circ}$. A solution of aldol adduct 19 (4.66 g, $15.9 \mathrm{mmol}, 100 \mathrm{~mol} \%)$ in MeCN (45 $\mathrm{mL}, 0.35 \mathrm{M}$ ) was added dropwise, followed by the addition of a solution of CSA (1.85 g, 8.0 $\mathrm{mmol}, 50 \mathrm{~mol} \%)$ in $\mathrm{MeCN}: \mathrm{AcOH}(1: 1)(90 \mathrm{~mL}, 0.09 \mathrm{M})$. The reaction medium was warmed to $-20{ }^{\circ} \mathrm{C}$ and stirred for $20 \mathrm{~h}$. The mixture was poured into an Erlenmeyer flask containing saturated aqueous solution of $\mathrm{NaHCO}_{3}(1400 \mathrm{~mL})$. After gas liberation ceased, saturated aqueous solution of sodium potassium tartrate $(800 \mathrm{~mL})$ and $\mathrm{Et}_{2} \mathrm{O}(800 \mathrm{~mL})$ were added. The mixture was stirred vigorously for $8 \mathrm{~h}$. The layers were separated, and the aqueous layer was extracted with $\mathrm{Et}_{2} \mathrm{O}(3 \times 500 \mathrm{~mL})$. The combined organic layers were washed with brine (500 $\mathrm{mL}$ ), dried over $\mathrm{MgSO}_{4}$, filtered, and concentrated under reduced pressure to provide diol S23 (4.68 g, $15.9 \mathrm{mmol}, 99 \%, d r>95: 05,1,3-a n t i: 1,3-s y n)$, which was used in the next step without further purification.

Physical state: yellow oil

TLC: $R_{f}=0.47$ (50:50 hexane:EtOAc)

Optical rotation: $[\alpha]_{D}{ }^{20}-49\left(c 2.5, \mathrm{CHCl}_{3}\right)$

${ }^{1} \mathrm{H}$ NMR $\left(500 \mathrm{MHz}, \mathrm{CDCl}_{3}\right) \delta 1.24(\mathrm{~d}, J=6.1 \mathrm{~Hz}, 3 \mathrm{H}), 1.51-1.58(\mathrm{~m}, 3 \mathrm{H}), 1.80(\mathrm{dt}, J=14.6$ and $10.1 \mathrm{~Hz}, 1 \mathrm{H}), 2.19-2.29(\mathrm{~m}, 2 \mathrm{H}), 3.17(\mathrm{br} \mathrm{s}, 1 \mathrm{H}), 3.79(\mathrm{~s}, 3 \mathrm{H}), 3.79-3.85(\mathrm{~m}, 1 \mathrm{H}), 3.96$ (quint, $J=6.2 \mathrm{~Hz}, 1 \mathrm{H}), 4.11-4.15(\mathrm{~m}, 1 \mathrm{H}), 4.18(\mathrm{br} \mathrm{s}, 1 \mathrm{H}), 4.34(\mathrm{~d}, J=11.0 \mathrm{~Hz}, 1 \mathrm{H}), 4.60(\mathrm{~d}, J$ $=11.0 \mathrm{~Hz}, 1 \mathrm{H}), 5.07-5.11(\mathrm{~m}, 2 \mathrm{H}), 5.82(\mathrm{ddt}, J=17.3,10.2$ and $7.1 \mathrm{~Hz}, 1 \mathrm{H}), 6.87-6.88(\mathrm{~m}$, $2 \mathrm{H}), 7.24-7.25(\mathrm{~m}, 2 \mathrm{H})$.

${ }^{13} \mathrm{C}$ NMR $\left(62.5 \mathrm{MHz}, \mathrm{CDCl}_{3}\right) \delta 19.6,42.0,42.3,43.5,55.2,67.9,69.7,70.0,75.9,113.9$, $117.4,129.4,129.9,135.0,159.3$. 
IR (film) $v_{\text {max }} / \mathrm{cm}^{-1} 3437,3053,2972,2937,2914,2872,2839,1641,1614,1587,1514,1466$, $1441,1377,1302,1265,1250,1175,1090,1034,918,824,739,704$.

HRMS (ESI TOF-MS) $\mathrm{m} / z$ calcd for $\mathrm{C}_{17} \mathrm{H}_{26} \mathrm{O}_{4} \mathrm{Na}[\mathrm{M}+\mathrm{Na}]^{+}: 317.1729$, found: 317.1718 .

\section{(-)-(4R,6R)-4-allyl-6-((R)-2-((4-methoxybenzyl)oxy)propyl)-2,2-dimethyl-1,3-dioxane} (S24):

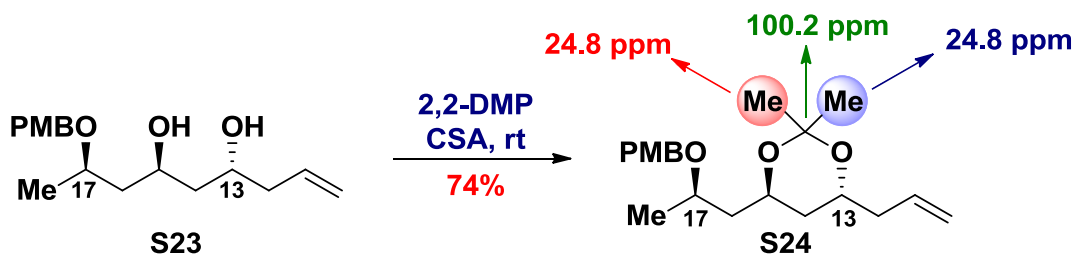

To a solution of diol S23 (1.07 g, $3.64 \mathrm{mmol}, 100 \mathrm{~mol} \%$ ) in 2,2-DMP (43 mL, $0.08 \mathrm{M}$ ) at room temperature was added CSA ( $85 \mathrm{mg}, 0.36 \mathrm{mmol}, 10 \mathrm{~mol} \%$ ). After $17 \mathrm{~h}$, the reaction was quenched by the addition of $\mathrm{NaHCO}_{3}(100 \mathrm{mg})$, filtered, and concentrated under reduced pressure. The residue was purified by flash column chromatography using a solution of hexane/ethyl acetate (90:10) as the eluent to provide acetonide $\mathbf{S} 24$ (895 mg, $2.68 \mathrm{mmol}$, $74 \%$ ). This compound was used to confirm the 1,3-syn relative stereochemistry between $\mathrm{C} 13$ and $\mathrm{C} 15$ on the basis of Rychnovsky ${ }^{13} \mathrm{C}$ NMR analysis: a) Rychnovsky, S. D.; Skalitzky, D. J. Tetrahedron Lett. 1990, 31, 945. b) Rychnovsky, S. D.; Rogers, B.; Yang, G. J. Org. Chem. 1993, 58, 3511. c) Rychnovsky, S. D.; Rogers, B. N.; Richardson, T. I. Acc. Chem. Res. 1998, 31, 9. For a theoretical work, see: d) Tormena, C. F.; Dias, L. C.; Rittner, R. J. Phys. Chem. A 2005, 109, 6077.

Physical state: colorless oil

TLC: $R_{f}=0.77$ (80:20 hexane:EtOAc)

Optical rotation: $[\alpha]_{D}{ }^{20}-43\left(c 2.4, \mathrm{CHCl}_{3}\right)$

${ }^{1} \mathrm{H}$ NMR $\left(500 \mathrm{MHz}, \mathrm{CDCl}_{3}\right) \delta 1.20(\mathrm{~d}, J=6.1 \mathrm{~Hz}, 3 \mathrm{H}), 1.33(\mathrm{~s}, 6 \mathrm{H}), 1.48$ (dt, $J=13.9$ and 6.2 $\mathrm{Hz}, 1 \mathrm{H}), 1.51-1.58(\mathrm{~m}, 2 \mathrm{H}), 1.94(\mathrm{dt}, J=14.0$ and $6.9 \mathrm{~Hz}, 1 \mathrm{H}), 2.16(\mathrm{dt}, J=14.0$ and $6.8 \mathrm{~Hz}$, $1 \mathrm{H}$ ), 2.28 (dt, $J=14.0$ and $6.9 \mathrm{~Hz}, 1 \mathrm{H}$ ), 3.62 (sext, $J=6.3 \mathrm{~Hz}, 1 \mathrm{H}$ ), $3.80(\mathrm{~s}, 3 \mathrm{H}$ ), 3.81-3.87 (m, $1 \mathrm{H}), 3.90-3.96(\mathrm{~m}, 1 \mathrm{H}), 4.36(\mathrm{~d}, J=11.3 \mathrm{~Hz}, 1 \mathrm{H}), 4.49(\mathrm{~d}, J=11.3 \mathrm{~Hz}, 1 \mathrm{H}), 5.03-5.10$ (m, 2H), 5.78 (ddt, $J=17.1,10.2$ and $6.8 \mathrm{~Hz}, 1 \mathrm{H}), 6.86-6.89(\mathrm{~m}, 2 \mathrm{H}), 7.25-7.26(\mathrm{~m}, 2 \mathrm{H})$.

${ }^{13} \mathrm{C}$ NMR (125 MHz, $\left.\mathrm{CDCl}_{3}\right) \delta 19.5,24.8,24.8,38.0,40.1,42.8,55.2,63.7,66.1,69.9,71.1$, $100.2,113.7,116.8,129.3,131.0,134.4,159.1$. 
IR (film) $v_{\max } / \mathrm{cm}^{-1} 3074,2984,2937,2837,1643,1614,1587,1514,1464,1443,1379,1302$, 1248, 1225, 1173, 1117, 1036, 995, 947, 912, 822, 754.

HRMS (ESI TOF-MS) $\mathrm{m} / z$ calcd for $\mathrm{C}_{20} \mathrm{H}_{30} \mathrm{O}_{4} \mathrm{Na}[\mathrm{M}+\mathrm{Na}]^{+}: 357.2042$, found: 357.2037 .

(-)-(R)-1-((2R,4S,6R)-2-(4-methoxyphenyl)-6-methyl-1,3-dioxan-4-yl)pent-4-en-2-ol (S25):
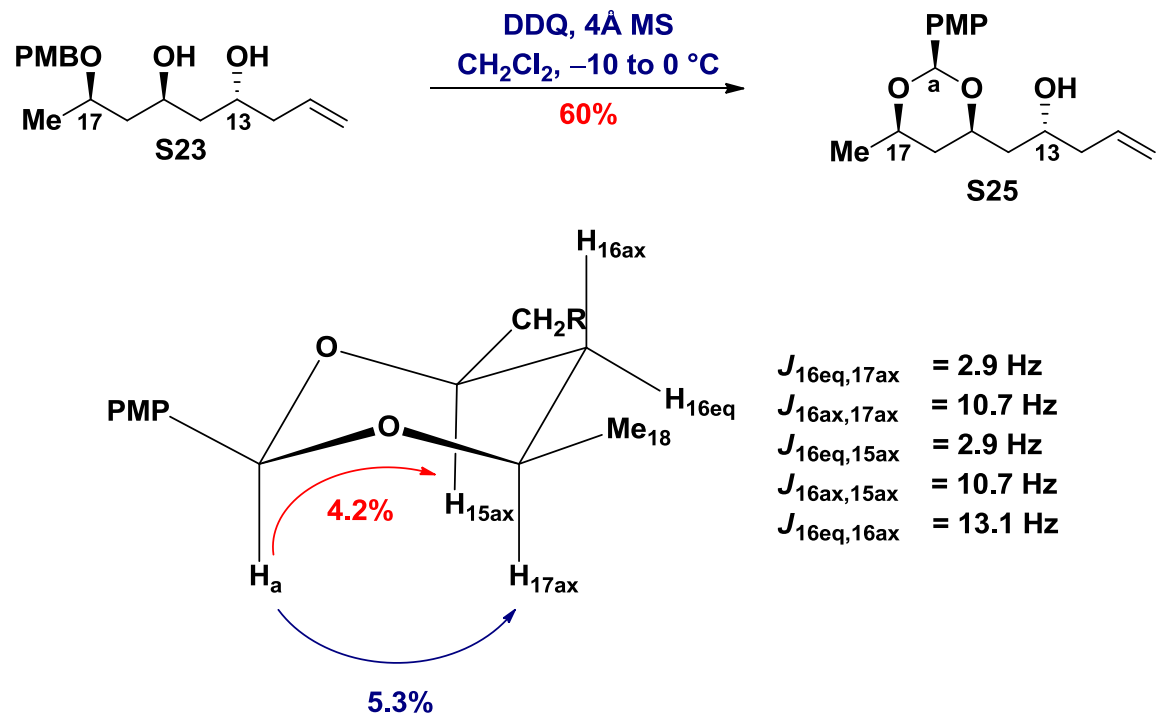

To a solution of diol S23 (52 mg, $0.18 \mathrm{mmol}, 100 \mathrm{~mol} \%$ ) in $\mathrm{CH}_{2} \mathrm{Cl}_{2}(3.8 \mathrm{~mL}, 0.05 \mathrm{M})$ at room temperature was added activated $4 \AA$ molecular sieves $(56 \mathrm{mg}$ ). After $15 \mathrm{~min}$, the mixture was cooled to $-10^{\circ} \mathrm{C}$, and DDQ (51 $\mathrm{mg}, 0.22 \mathrm{mmol}, 120 \mathrm{~mol} \%$ ) was added. The reaction medium was stirred for $5 \mathrm{~min}$ at $-10^{\circ} \mathrm{C}$ and warmed to $0{ }^{\circ} \mathrm{C}$. After $1.5 \mathrm{~h}$, the reaction mixture was loaded directly onto a flash column chromatography using a solution of hexane/ethyl acetate $(60: 40)$ as the eluent to provide the PMP acetal S25 (32 mg, $0.11 \mathrm{mmol}, 60 \%)$. This compound was used to confirm the 1,3-syn relative stereochemistry between $\mathrm{C} 15$ and $\mathrm{C} 17$ on the basis of ${ }^{1} \mathrm{H}$ NMR and $1 \mathrm{D}$ selective NOE spectra analyses.

Physical state: pale yellow oil

TLC: $R_{f}=0.57$ (60:40 hexane:EtOAc)

Optical rotation: $[\alpha]_{D}{ }^{20}-35\left(c 3.2, \mathrm{CHCl}_{3}\right)$

${ }^{1} \mathrm{H}$ NMR $\left(500 \mathrm{MHz}, \mathrm{CDCl}_{3}\right) \delta 1.30(\mathrm{~d}, J=6.1 \mathrm{~Hz}, 3 \mathrm{H}), 1.50(\mathrm{dt}, J=13.1$ and $10.7 \mathrm{~Hz}, 1 \mathrm{H})$, 1.56 (dt, $J=13.1$ and $2.7 \mathrm{~Hz}, 1 \mathrm{H}$ ), 1.66 (ddd, $J=14.5,9.3$ and $3.4 \mathrm{~Hz}, 1 \mathrm{H}$ ), 1.76 (ddd, $J=$ 14.5, 8.2 and $2.6 \mathrm{~Hz}, 1 \mathrm{H}), 2.19-2.30(\mathrm{~m}, 2 \mathrm{H}), 2.39(\mathrm{br} \mathrm{s}, 1 \mathrm{H}), 3.78(\mathrm{~s}, 3 \mathrm{H}), 3.92-3.97(\mathrm{~m}, 1 \mathrm{H})$, 3.99-4.03 (m, 1H), 4.10-4.15 (m, 1H), 5.10-5.14 (m, 2H), $5.49(\mathrm{~s}, 1 \mathrm{H}), 5.82$ (ddt, $J=17.2$, 10.3 and $7.2 \mathrm{~Hz}, 1 \mathrm{H}), 6.86-6.88(\mathrm{~m}, 2 \mathrm{H}), 7.40-7.42(\mathrm{~m}, 2 \mathrm{H})$. 
${ }^{13} \mathrm{C}$ NMR (125 MHz, $\mathrm{CDCl}_{3}$ ) $\delta 21.6,38.4,42.0,42.2,55.2,67.0,72.9,74.0,100.7,113.6$, 117.9, 127.4, 131.2, 134.7, 159.8 .

IR (film) $v_{\text {max }} / \mathrm{cm}^{-1} 3439,3076,2974,2935,2914,2851,1641,1616,1589,1518,1340,1304$, 1250, 1155, 1111, 1061, 1034, 1011, 912, 825, 775, 737.

HRMS (ESI FTMS) $\mathrm{m} / z$ calcd for $\mathrm{C}_{17} \mathrm{H}_{25} \mathrm{O}_{4}[\mathrm{M}+\mathrm{H}]^{+}:$: 293.1747, found: 293.1746 .

\section{(-)-(5R,7R)-5-allyl-7-((S)-2-((4-methoxybenzyl)oxy)propyl)-2,2,3,3,9,9,10,10-octamethyl-}

4,8-dioxa-3,9-disilaundecane (20):
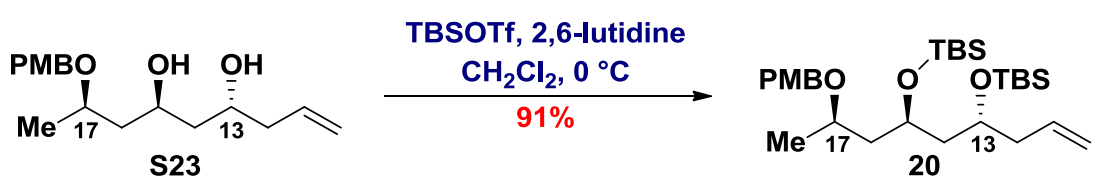

To a solution of diol S23 (1.19 g, $4.04 \mathrm{mmol}, 100 \mathrm{~mol} \%)$ in $\mathrm{CH}_{2} \mathrm{Cl}_{2}(58 \mathrm{~mL}, 0.07 \mathrm{M})$ at $0{ }^{\circ} \mathrm{C}$ were added 2,6-lutidine (2.82 mL, $24.2 \mathrm{mmol}, 600 \mathrm{~mol} \%$ ) and TBSOTf (2.8 mL, $12.1 \mathrm{mmol}$, $300 \mathrm{~mol} \%$ ) dropwise. After $1.5 \mathrm{~h}$, the reaction was quenched by the addition of saturated aqueous solution of $\mathrm{NaHCO}_{3}(30 \mathrm{~mL})$. The layers were separated, and the aqueous layer was extracted with $\mathrm{CH}_{2} \mathrm{Cl}_{2}(3 \times 50 \mathrm{~mL})$. The combined organic layers were washed with brine (50 $\mathrm{mL}$ ), dried over $\mathrm{MgSO}_{4}$, filtered, and concentrated under reduced pressure. The residue was purified by flash column chromatography using a solution of hexane/ethyl acetate (90:10) as the eluent to provide compound 20 (1.93 g, $3.69 \mathrm{mmol}, 91 \%)$.

Physical state: colorless oil

TLC: $R_{f}=0.81$ (90:10 hexane:EtOAc)

Optical rotation: $[\alpha]_{D}^{20}-12\left(c 2.4, \mathrm{CHCl}_{3}\right)$

${ }^{1} \mathrm{H}$ NMR (250 MHz, $\left.\mathrm{CDCl}_{3}\right) \delta 0.02(\mathrm{~s}, 3 \mathrm{H}), 0.05(\mathrm{~s}, 9 \mathrm{H}), 0.85(\mathrm{~s}, 9 \mathrm{H}), 0.87(\mathrm{~s}, 9 \mathrm{H}), 1.18(\mathrm{~d}, J=$ $6.0 \mathrm{~Hz}, 3 \mathrm{H}$ ), 1.48 (dt, $J=13.8$ and $6.1 \mathrm{~Hz}, 1 \mathrm{H}), 1.60-1.65(\mathrm{~m}, 2 \mathrm{H}), 1.86(\mathrm{dt}, J=13.8$ and 6.4 $\mathrm{Hz}, 1 \mathrm{H}$ ), 2.12-2.31 (m, 2H), 3.63 (sext, $J=6.2 \mathrm{~Hz}, 1 \mathrm{H}$ ), $3.79(\mathrm{~s}, 3 \mathrm{H}), 3.79-3.93(\mathrm{~m}, 2 \mathrm{H}), 4.38$ (d, $J=11.3 \mathrm{~Hz}, 1 \mathrm{H}$ ), 4.47 (d, $J=11.3 \mathrm{~Hz}, 1 \mathrm{H}$ ), 5.00-5.06 (m, 2H), 5.81 (ddt, $J=17.8,9.5$ and $7.0 \mathrm{~Hz}, 1 \mathrm{H}), 6.83-6.87(\mathrm{~m}, 2 \mathrm{H}), 7.24-7.27(\mathrm{~m}, 2 \mathrm{H})$.

${ }^{13} \mathrm{C}$ NMR (62.5 MHz, $\left.\mathrm{CDCl}_{3}\right) \delta-4.3,-4.1,-4.0,-3.9,18.0,18.1,19.8,25.9\left(2 \times\left(\mathrm{CH}_{3}\right)_{3}\right)$, $42.3,45.2,45.5,55.2,67.5,69.5,69.9,71.7,113.7,116.9,129.1,131.1,135.0,159.0$.

IR (film) $v_{\text {max }} / \mathrm{cm}^{-1} 3076,2955,2930,2888,2857,1614,1514,1472,1463,1374,1249,1061$, 913, 836, 774.

HRMS (ESI TOF-MS) $\mathrm{m} / z$ calcd for $\mathrm{C}_{29} \mathrm{H}_{54} \mathrm{O}_{4} \mathrm{Si}_{2} \mathrm{Na}[\mathrm{M}+\mathrm{Na}]^{+}: 545.3458$, found: 545.3439 . 
(+)-(2R,4R,6R)-4,6-bis((tert-butyldimethylsilyl)oxy)non-8-en-2-ol (S26):

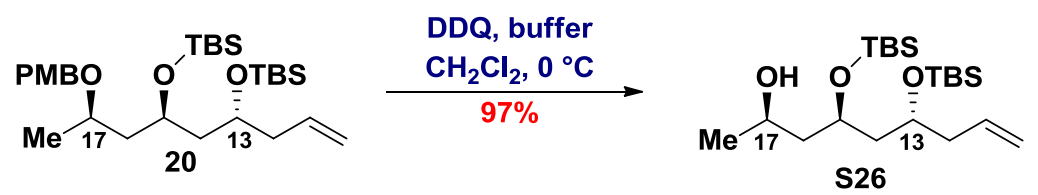

To a solution of PMB ether 20 (7.40 g, $14.1 \mathrm{mmol}, 100 \mathrm{~mol} \%)$ in $\mathrm{CH}_{2} \mathrm{Cl}_{2}$ :phosphate buffer $\mathrm{pH}$ $7(9: 1)(235 \mathrm{~mL}, 0.06 \mathrm{M})$ at $0 \stackrel{\circ}{\circ}$ was added DDQ (3.84 g, $16.9 \mathrm{mmol}, 120 \mathrm{~mol} \%)$. The mixture was stirred for $1 \mathrm{~h}$ under the same conditions, followed by quenching via the addition of a solution of $\mathrm{H}_{2} \mathrm{O}$ :saturated aqueous solution of $\mathrm{NaHCO}_{3}(1: 1)(60 \mathrm{~mL})$. The resulting mixture was filtered over Celite, washed with $\mathrm{CH}_{2} \mathrm{Cl}_{2}(5 \times 100 \mathrm{~mL})$ and concentrated under reduced pressure. The residue was purified by flash column chromatography using hexane/ethyl acetate (90:10) as the eluent to provide alcohol $\mathbf{S} 26$ (5.50 g, $13.7 \mathrm{mmol}, 97 \%)$.

Physical state: pale yellow oil

TLC: $R_{f}=0.59$ (90:10 hexane:EtOAc)

Optical rotation: $[\alpha]_{D}^{20}+11\left(c 2.3, \mathrm{CHCl}_{3}\right)$

${ }^{1} \mathrm{H}$ NMR $\left(500 \mathrm{MHz}, \mathrm{CDCl}_{3}\right) \delta 0.05(\mathrm{~s}, 3 \mathrm{H}), 0.05(\mathrm{~s}, 3 \mathrm{H}), 0.08(\mathrm{~s}, 3 \mathrm{H}), 0.09(\mathrm{~s}, 3 \mathrm{H}), 0.87$ (s, $9 \mathrm{H}), 0.88(\mathrm{~s}, 9 \mathrm{H}), 1.16(\mathrm{~d}, J=6.3 \mathrm{~Hz}, 3 \mathrm{H}), 1.52(\mathrm{dt}, J=14.2$ and $8.9 \mathrm{~Hz}, 1 \mathrm{H}), 1.58-1.74(\mathrm{~m}$, $3 \mathrm{H}$ ), 2.12-2.24 (m, 2H), $3.11(\mathrm{br} \mathrm{s}, 1 \mathrm{H}), 3.74$ (quint, $J=6.1 \mathrm{~Hz}, 1 \mathrm{H}), 3.87-3.95(\mathrm{~m}, 2 \mathrm{H})$, 5.02-5.06 (m, 2H), 5.75-5.83 (m, 1H).

${ }^{13} \mathrm{C}$ NMR (125 MHz, $\left.\mathrm{CDCl}_{3}\right) \delta-4.5,-4.4,-4.2,-3.8,17.9,18.0,23.5,25.8,25.8,42.0,45.9$ $\left(2 \times \mathrm{CH}_{2}\right), 67.0,69.7,71.4,117.3,134.6$.

IR (film) $v_{\text {max }} / \mathrm{cm}^{-1} 3445,3078,2957,2931,2896,2858,1642,1473,1463,1362,1256,1070$, 1005, 913, 836, 807, 774.

HRMS (ESI TOF-MS) $\mathrm{m} / z$ calcd for $\mathrm{C}_{21} \mathrm{H}_{47} \mathrm{O}_{3} \mathrm{Si}_{2}[\mathrm{M}+\mathrm{H}]^{+}:$403.3064, found: 403.3055 .

(+)-(4S,6R)-4,6-bis((tert-butyldimethylsilyl)oxy)non-8-en-2-one (S27):

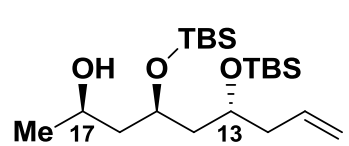

S26

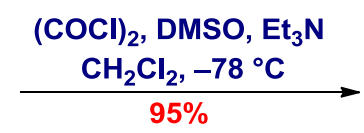

$95 \%$

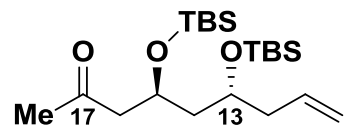

S27

To a solution of oxalyl chloride (1.42 mL, $16.5 \mathrm{mmol}, 120 \mathrm{~mol} \%)$ in $\mathrm{CH}_{2} \mathrm{Cl}_{2}(80 \mathrm{~mL}, 0.21 \mathrm{M})$ at $-78{ }^{\circ} \mathrm{C}$ was added DMSO $(2.34 \mathrm{~mL}, 33.0 \mathrm{mmol}, 240 \mathrm{~mol} \%)$ dropwise. The reaction medium was stirred for $30 \mathrm{~min}$, followed by the dropwise addition of a solution of alcohol S26 $(5.50 \mathrm{~g}$, 
$13.7 \mathrm{mmol}, 100 \mathrm{~mol} \%)$ in $\mathrm{CH}_{2} \mathrm{Cl}_{2}(33 \mathrm{~mL}, 0.42 \mathrm{M})$. After $30 \mathrm{~min}, \mathrm{Et}_{3} \mathrm{~N}(11.5 \mathrm{~mL}, 82.5 \mathrm{mmol}$, $600 \mathrm{~mol} \%$ ) was added dropwise, and the resulting slurry was warmed to $0{ }^{\circ} \mathrm{C}$ and stirred for 1 h. The reaction was then diluted with $\mathrm{Et}_{2} \mathrm{O}(100 \mathrm{~mL})$ and saturated aqueous solution of $\mathrm{NH}_{4} \mathrm{Cl}$ $(100 \mathrm{~mL})$. The layers were separated, and the aqueous layer was extracted with $\mathrm{Et}_{2} \mathrm{O}(3 \times$ $100 \mathrm{~mL})$. The combined organic layers were washed with $\mathrm{H}_{2} \mathrm{O}(3 \times 100 \mathrm{~mL})$, brine $(100 \mathrm{~mL})$, dried over $\mathrm{MgSO}_{4}$, filtered, and concentrated under reduced pressure. The residue was purified by flash column chromatography using hexane/ethyl acetate (90:10) as the eluent to provide methylketone $\mathbf{S} 27$ (5.23 g, $13.1 \mathrm{mmol}, 95 \%)$.

Physical state: colorless oil

TLC: $R_{f}=0.68$ (90:10 hexane:EtOAc)

Optical rotation: $[\alpha]_{D}^{20}+1\left(c 2.7, \mathrm{CHCl}_{3}\right)$

${ }^{1} \mathrm{H}$ NMR $\left(500 \mathrm{MHz}, \mathrm{CDCl}_{3}\right) \delta 0.01(\mathrm{~s}, 3 \mathrm{H}), 0.05(\mathrm{~s}, 3 \mathrm{H}), 0.06(\mathrm{~s}, 6 \mathrm{H}), 0.84(\mathrm{~s}, 9 \mathrm{H}), 0.87(\mathrm{~s}$, 9H), $1.54(\mathrm{dt}, J=13.7$ and $6.8 \mathrm{~Hz}, 1 \mathrm{H}), 1.66(\mathrm{dt}, J=13.7$ and $5.8 \mathrm{~Hz}, 1 \mathrm{H}), 2.13(\mathrm{~s}, 3 \mathrm{H})$, 2.16-2.26 (m, 2H), $2.51(\mathrm{dd}, J=15.0$ and $5.0 \mathrm{~Hz}, 1 \mathrm{H}), 2.59(\mathrm{dd}, J=15.0$ and $7.1 \mathrm{~Hz}, 1 \mathrm{H}$ ), 3.76 (quint, $J=6.0 \mathrm{~Hz}, 1 \mathrm{H}$ ), 4.21 (quint, $J=6.2 \mathrm{~Hz}, 1 \mathrm{H}$ ), 5.02-5.05 (m, 2H), 5.78 (ddt, $J=$ 17.7, 9.5 and $7.1 \mathrm{~Hz}, 1 \mathrm{H})$.

${ }^{13} \mathrm{C}$ NMR (125 MHz, $\left.\mathrm{CDCl}_{3}\right) \delta-4.4\left(2 \times \mathrm{CH}_{3}\right),-4.3,-4.1,18.0,18.0,25.8,25.9,31.5,42.2$, 45.3, 51.9, 67.2, 69.6, 117.2, 134.6, 207.5.

IR (film) $v_{\max } / \mathrm{cm}^{-1} 3079,2956,2930,2896,2858,1721,1642,1473,1464,1361,1256,1092$, 1005, 914, 836, 775.

HRMS (ESI TOF-MS) $\mathrm{m} / z$ calcd for $\mathrm{C}_{21} \mathrm{H}_{45} \mathrm{O}_{3} \mathrm{Si}_{2}[\mathrm{M}+\mathrm{H}]^{+}:$401.2907, found: 401.2891 .

(6S,8R)-8-allyl-6-((tert-butyldimethylsilyl)oxy)-2,2,10,10,11,11-hexamethyl-4-methylene3,9-dioxa-2,10-disiladodecane (21):

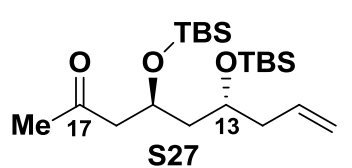

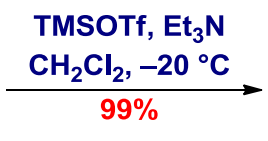

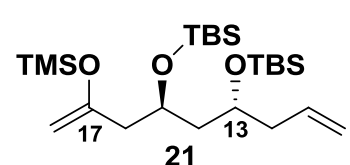

21

To a solution of methylketone $\mathbf{S} 27(1.0 \mathrm{~g}, 2.50 \mathrm{mmol}, 100 \mathrm{~mol} \%)$ in $\mathrm{CH}_{2} \mathrm{Cl}_{2}(20 \mathrm{~mL}, 0.12 \mathrm{M})$ at room temperature was added $\mathrm{Et}_{3} \mathrm{~N}(1.0 \mathrm{~mL}, 7.50 \mathrm{mmol}, 300 \mathrm{~mol} \%)$. After $5 \mathrm{~min}$, the reaction medium was cooled to $-20^{\circ} \mathrm{C}$ and TMSOTf $(1.0 \mathrm{~mL}, 5.00 \mathrm{mmol}, 200 \mathrm{~mol} \%)$ was added dropwise. After $30 \mathrm{~min}$, the reaction was diluted with $\mathrm{Et}_{2} \mathrm{O}(20 \mathrm{~mL})$, transferred to a separatory 
funnel, washed with saturated aqueous solution of $\mathrm{NaHCO}_{3}(10 \mathrm{~mL})$, brine $(10 \mathrm{~mL})$, dried over $\mathrm{MgSO}_{4}$, filtered, and concentrated under reduced pressure to provide the enolsilane 21 $(1.18 \mathrm{~g}, 2.50 \mathrm{mmol}, 99 \%)$, which was used in the next step without further purification.

Physical state: yellow oil

TLC: $R_{f}=0.81$ (95:05 hexane:EtOAc)

${ }^{1} \mathrm{H}$ NMR (250 MHz, $\left.\mathrm{C}_{6} \mathrm{D}_{6}\right) \delta 0.13(\mathrm{~s}, 3 \mathrm{H}), 0.17(\mathrm{~s}, 6 \mathrm{H}), 0.19(\mathrm{~s}, 3 \mathrm{H}), 0.21(\mathrm{~s}, 9 \mathrm{H}), 1.02(\mathrm{~s}$, $18 \mathrm{H}$ ), 1.77 (ddd, $J=14.1,8.1$ and $4.4 \mathrm{~Hz}, 1 \mathrm{H}$ ), 1.93 (ddd, $J=14.1,7.4$ and $4.1 \mathrm{~Hz}, 1 \mathrm{H}$ ), 2.22 (dd, $J=13.5$ and $7.6 \mathrm{~Hz}, 1 \mathrm{H}), 2.33-2.38(\mathrm{~m}, 2 \mathrm{H}), 2.44(\mathrm{dd}, J=13.5$ and $5.4 \mathrm{~Hz}, 1 \mathrm{H})$, 4.03-4.12 (m, 1H), 4.14-4.15 (m, 2H), 4.23-4.33 (m, 1H), 5.06-5.14 (m, 2H), 5.94 (ddt, $J=$ $17.2,10.1$ and $7.0 \mathrm{~Hz}, 1 \mathrm{H})$.

\section{Synthesis of C19-C22 Fragment of Marinisporolide C}

(+)-(R)-methyl 3-hydroxy-4-(trityloxy)butanoate (S30):

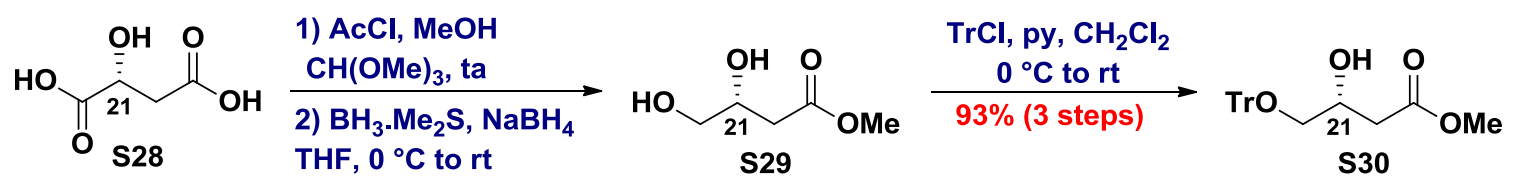

Compound S30 was prepared according to the literature procedure: a) Tararov, V. I.; König, G.; Börner, A. Adv. Synth. Catal. 2006, 348, 2633. b) White, J. D.; Martin, W. H. C.; Lincoln, C.; Yang, J. Org. Lett. 2007, 9, 3481. c) White, J. D.; Lincoln, C. M.; Yang, J.; Martin, W. H. C.; Chan, D. B. J. Org. Chem. 2008, 73, 4139.

TLC: $R_{f}=0.55$ (60:40 hexane:EtOAc)

Optical rotation: $[\alpha]_{D}^{20}+4\left(c 2.1, \mathrm{CHCl}_{3}\right)$

${ }^{1} \mathrm{H}$ NMR $\left(500 \mathrm{MHz}, \mathrm{CDCl}_{3}\right) \delta 2.51(\mathrm{dd}, J=16.2$ and $8.1 \mathrm{~Hz}, 1 \mathrm{H}), 2.57(\mathrm{dd}, J=16.2$ and 4.4 $\mathrm{Hz}, 1 \mathrm{H}), 3.00(\mathrm{~d}, J=4.1 \mathrm{~Hz}, 1 \mathrm{H}), 3.14-3.19(\mathrm{~m}, 2 \mathrm{H}), 3.64(\mathrm{~s}, 3 \mathrm{H}), 4.20-4.23(\mathrm{~m}, 1 \mathrm{H}), 7.22(\mathrm{t}$, $J=7.3 \mathrm{~Hz}, 3 \mathrm{H}), 7.28(\mathrm{t}, J=7.3 \mathrm{~Hz}, 6 \mathrm{H}), 7.42(\mathrm{~d}, J=7.3 \mathrm{~Hz}, 6 \mathrm{H})$.

${ }^{13} \mathrm{C}$ NMR (125 MHz, $\left.\mathrm{CDCl}_{3}\right) \delta 38.3,51.7,66.5,67.5,86.7,127.0,127.8,128.6,143.6,172.6$.

IR (film) $v_{\max } / \mathrm{cm}^{-1} 3461,3087,3059,3028,3024,2951,2925,2874,1732,1637,1598,1491$, 1449, 1266, 1219, 1173, 1073, 1033, 1002, 900, 765, 747, 707, 700, 633. 
(+)-(R)-methyl 3-((tert-butyldimethylsilyl)oxy)-4-(trityloxy)butanoate (S31):
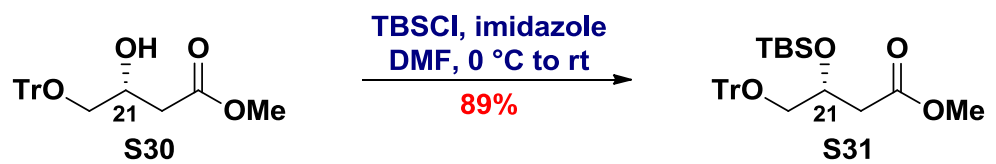

Compound S31 was prepared according to the literature procedure: a) White, J. D.; Martin, W. H. C.; Lincoln, C.; Yang, J. Org. Lett. 2007, 9, 3481. b) White, J. D.; Lincoln, C. M.; Yang, J.; Martin, W. H. C.; Chan, D. B. J. Org. Chem. 2008, 73, 4139.

TLC: $R_{f}=0.70$ (80:20 hexane:EtOAc)

mp $67-69^{\circ} \mathrm{C}$

Optical rotation: $[\alpha]_{D}^{20}+20\left(c 1.0, \mathrm{CHCl}_{3}\right)$

${ }^{1} \mathrm{H}$ NMR (500 MHz, $\mathrm{CDCl}_{3}$ ) $\delta 0.01(\mathrm{~s}, 3 \mathrm{H}), 0.07(\mathrm{~s}, 3 \mathrm{H}), 0.89(\mathrm{~s}, 9 \mathrm{H}), 2.56(\mathrm{dd}, J=14.8$ and $8.0 \mathrm{~Hz}, 1 \mathrm{H}$ ), 2.84 (dd, $J=14.8$ and $4.4 \mathrm{~Hz}, 1 \mathrm{H}$ ), 3.10 (dd, $J=9.2$ and $6.9 \mathrm{~Hz}, 1 \mathrm{H}$ ), 3.22 (dd, $J$ $=9.2$ and $4.7 \mathrm{~Hz}, 1 \mathrm{H}), 3.71(\mathrm{~s}, 3 \mathrm{H}), 4.31-4.36(\mathrm{~m}, 1 \mathrm{H}), 7.31(\mathrm{t}, J=7.3 \mathrm{~Hz}, 3 \mathrm{H}), 7.37(\mathrm{t}, J=$ $7.3 \mathrm{~Hz}, 6 \mathrm{H}), 7.52(\mathrm{~d}, J=7.3 \mathrm{~Hz}, 6 \mathrm{H})$.

${ }^{13} \mathrm{C}$ NMR $\left(125 \mathrm{MHz}, \mathrm{CDCl}_{3}\right) \delta-5.2,-4.6,17.9,25.6,40.7,51.4,67.2,68.9,86.6,127.0$, 127.7, 128.7, 143.9, 172.2.

IR (film) $v_{\max } / \mathrm{cm}^{-1} 3087,3060,3033,3024,2953,2930,2885,2857,1741,1598,1491,1472$, 1449, 1256, 1172, 1123, 1076, 1003, 837, 777, 745, 706, 633.

(+)-(R)-3-((tert-butyldimethylsilyl)oxy)-4-(trityloxy)butan-1-ol (S32):
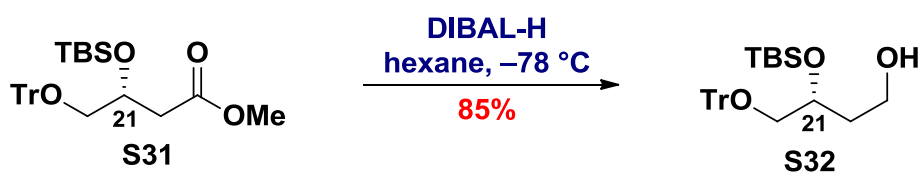

Compound S32 was prepared according to the literature procedure: a) White, J. D.; Martin, W. H. C.; Lincoln, C.; Yang, J. Org. Lett. 2007, 9, 3481. b) White, J. D.; Lincoln, C. M.; Yang, J.; Martin, W. H. C.; Chan, D. B. J. Org. Chem. 2008, 73, 4139.

TLC: $R_{f}=0.44$ (90:10 hexane:EtOAc)

Optical rotation: $[\alpha]_{D}^{20}+16\left(c 1.4, \mathrm{CHCl}_{3}\right)$

${ }^{1} \mathrm{H}$ NMR $\left(500 \mathrm{MHz}, \mathrm{CDCl}_{3}\right) \delta-0.06(\mathrm{~s}, 3 \mathrm{H}), 0.02(\mathrm{~s}, 3 \mathrm{H}), 0.84(\mathrm{~s}, 9 \mathrm{H}), 1.78-1.84(\mathrm{~m}, 1 \mathrm{H})$, 2.00 (ddt, $J=14.1,8.1$ and $4.7 \mathrm{~Hz}, 1 \mathrm{H}), 2.37(\mathrm{~m}, 1 \mathrm{H}), 3.08(\mathrm{dd}, J=9.3$ and $7.2 \mathrm{~Hz}, 1 \mathrm{H}$ ), 3.15 
(dd, $J=9.3$ and $4.7 \mathrm{~Hz}, 1 \mathrm{H}), 3.67-3.75(\mathrm{~m}, 2 \mathrm{H}), 3.98-4.03(\mathrm{~m}, 1 \mathrm{H}), 7.22(\mathrm{t}, J=7.3 \mathrm{~Hz}, 3 \mathrm{H})$, 7.29 (t, $J=7.3 \mathrm{~Hz}, 6 \mathrm{H}), 7.44(\mathrm{~d}, J=7.3 \mathrm{~Hz}, 6 \mathrm{H})$.

${ }^{13} \mathrm{C}$ NMR (125 MHz, $\left.\mathrm{CDCl}_{3}\right) \delta-5.1,-4.6,17.9,25.7,36.6,59.9,66.8,70.8,86.7,127.0$, $127.8,128.6,143.9$.

IR (film) $v_{\max } / \mathrm{cm}^{-1} 3436,3087,3060,3034,3028,2954,2929,2884,2857,1598,1491,1472$, 1449, 1256, 1075, 1032, 837, 776, 745, 706, 633.

\section{(+)-(R)-3-((tert-butyldimethylsilyl)oxy)-4-(trityloxy)butanal (22):}

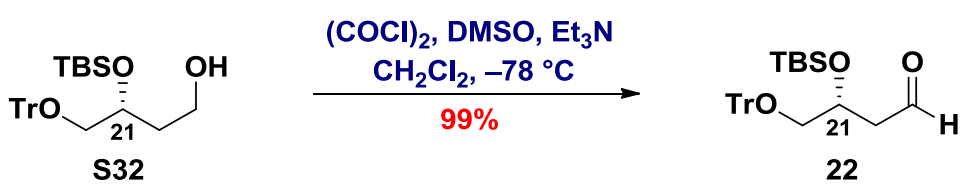

To a solution of oxalyl chloride $(0.50 \mathrm{~mL}, 5.80 \mathrm{mmol}, 150 \mathrm{~mol} \%)$ in $\mathrm{CH}_{2} \mathrm{Cl}_{2}(23 \mathrm{~mL}, 0.25 \mathrm{M})$ at $-78{ }^{\circ} \mathrm{C}$ was added DMSO $(0.82 \mathrm{~mL}, 11.6 \mathrm{mmol}, 300 \mathrm{~mol} \%)$ dropwise. The reaction medium was stirred for $30 \mathrm{~min}$, followed by the dropwise addition of a solution of alcohol S32 $(1.80 \mathrm{~g}$, $3.89 \mathrm{mmol}, 100 \mathrm{~mol} \%)$ in $\mathrm{CH}_{2} \mathrm{Cl}_{2}(10 \mathrm{~mL}, 0.39 \mathrm{M})$. After $30 \mathrm{~min}, \mathrm{Et}_{3} \mathrm{~N}(4.0 \mathrm{~mL}, 29.0 \mathrm{mmol}, 750$ mol\%) was added dropwise, and the resulting slurry was warmed to $0{ }^{\circ} \mathrm{C}$ and stirred for $1 \mathrm{~h}$. The reaction was then diluted with $\mathrm{Et}_{2} \mathrm{O}(50 \mathrm{~mL})$ and saturated aqueous solution of $\mathrm{NH}_{4} \mathrm{Cl}(25$ $\mathrm{mL})$. The layers were separated, and the aqueous layer was extracted with $\mathrm{Et}_{2} \mathrm{O}(3 \times 50 \mathrm{~mL})$. The combined organic layers were washed with $\mathrm{H}_{2} \mathrm{O}(3 \times 50 \mathrm{~mL})$, brine $(50 \mathrm{~mL})$, dried over $\mathrm{MgSO}_{4}$, filtered, and concentrated under reduced pressure to provide the aldehyde 22 (1.77 $\mathrm{g}, 3.85 \mathrm{mmol}, 99 \%$ ), which was used in the next step without further purification.

Physical state: white solid

TLC: $R_{f}=0.33$ (95:05 hexane:EtOAc)

$\mathrm{mp} 70-72^{\circ} \mathrm{C}$

Optical rotation: $[\alpha]_{D}^{20}+10\left(c 1.3, \mathrm{CHCl}_{3}\right)$

${ }^{1} \mathrm{H}$ NMR $\left(500 \mathrm{MHz}, \mathrm{C}_{6} \mathrm{D}_{6}\right) \delta-0.05(\mathrm{~s}, 3 \mathrm{H}),-0.01(\mathrm{~s}, 3 \mathrm{H}), 0.88(\mathrm{~s}, 9 \mathrm{H}), 2.38$ (ddd, $J=16.0,6.6$ and $2.0 \mathrm{~Hz}, 1 \mathrm{H}$ ), 2.44 (ddd, $J=16.0,4.8$ and $2.0 \mathrm{~Hz}, 1 \mathrm{H}$ ), 3.15 (dd, $J=9.3$ and $6.6 \mathrm{~Hz}, 1 \mathrm{H}$ ), $3.22(\mathrm{dd}, J=9.3$ and $4.8 \mathrm{~Hz}, 1 \mathrm{H}), 4.17(\mathrm{tt}, J=6.6$ and $4.8 \mathrm{~Hz}, 1 \mathrm{H}), 7.03(\mathrm{t}, J=7.3 \mathrm{~Hz}, 3 \mathrm{H}$ ), $7.12(\mathrm{t}, J=7.3 \mathrm{~Hz}, 6 \mathrm{H}), 7.50-7.52(\mathrm{~m}, 6 \mathrm{H}), 9.53(\mathrm{t}, J=2.0 \mathrm{~Hz}, 1 \mathrm{H})$.

${ }^{13} \mathrm{C}$ NMR (125 MHz, $\left.\mathbf{C}_{6} \mathrm{D}_{6}\right) \delta-4.9,-4.4,18.1,25.9,49.3,67.8,67.8,87.3,127.3,128.1$, 129.1, 144.4, 199.7. 
IR (film) $v_{\text {max }} / \mathrm{cm}^{-1}$ 3087, 3060, 3033, 3024, 2955, 2929, 2885, 2857, 2724, 1727, 1648, 1634, 1491, 1472, 1449, 1254, 1088, 1077, 1016, 836, 776, 762, 746, 706, 632.

HRMS (ESI FT-ICR-MS) $\mathrm{m} / z$ calcd for $\mathrm{C}_{30} \mathrm{H}_{40} \mathrm{O}_{4} \mathrm{SiNa}[\mathrm{M}+\mathrm{Na}+\mathrm{MeOH}]^{+}: 515.2594$, found: 515.2584 .

\section{Synthesis of C10-C22 Fragment of Marinisporolide C}

\section{(+)-(5R,7S,11R,13R)-5-allyl-7-((tert-butyldimethylsilyl)oxy)-11-hydroxy-}

\section{2,2,3,3,15,15,16,16-octamethyl-13-((trityloxy)methyl)-4,14-dioxa-3,15-disilaheptadecan-} 9-one (23):
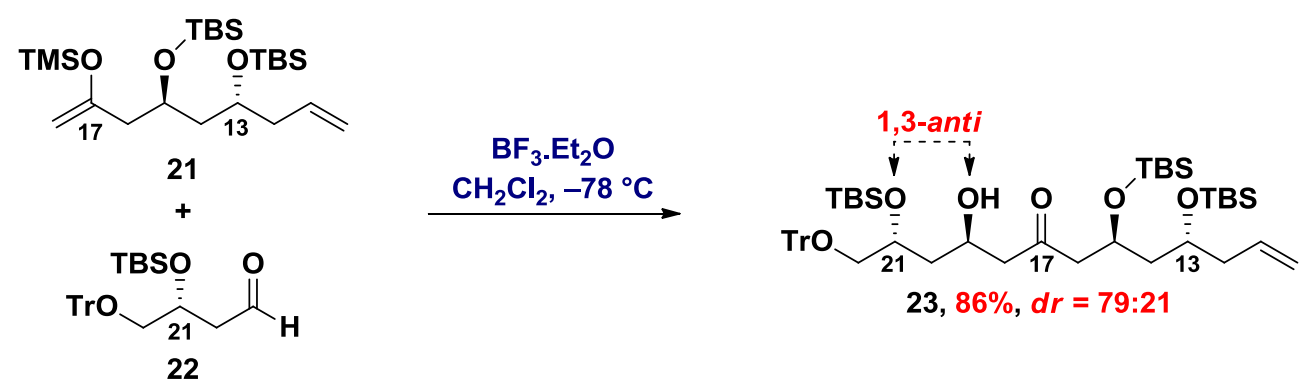

To a solution of enolsilane 21 (946 mg, $2.00 \mathrm{mmol}, 100 \mathrm{~mol} \%)$ and aldehyde $22(1.13 \mathrm{~g}, 2.46$ mmol, $125 \mathrm{~mol} \%)$ in $\mathrm{CH}_{2} \mathrm{Cl}_{2}(25 \mathrm{~mL}, 0.08 \mathrm{M})$ at $-78{ }^{\circ} \mathrm{C}$ was added $\mathrm{BF}_{3} . \mathrm{Et}_{2} \mathrm{O}(0.30 \mathrm{~mL}, 2.40$ mmol, $120 \mathrm{~mol} \%)$ dropwise. After $1 \mathrm{~h}$, the reaction was quenched by the addition of phosphate buffer $\mathrm{pH} 7(10 \mathrm{~mL})$ and warmed to room temperature. The layers were separated, and the aqueous layer was extracted with $\mathrm{CH}_{2} \mathrm{Cl}_{2}(3 \times 25 \mathrm{~mL})$. The combined organic layers were washed with brine $(10 \mathrm{~mL})$, dried over $\mathrm{MgSO}_{4}$, filtered, and concentrated under reduced pressure. The residue was purified by flash column chromatography using hexane/ethyl acetate (90:10) as the eluent to provide aldol adduct $23(1.48 \mathrm{~g}, 1.72 \mathrm{mmol}, 86 \%, d r=79: 21$, 1,3-anti:1,3-syn).

Physical state: colorless oil

TLC: $R_{f}=0.49$ (90:10 hexane:EtOAc)

Optical rotation: $[\alpha]_{D}^{20}+6\left(c 1.0, \mathrm{CHCl}_{3}\right)$

${ }^{1} \mathrm{H}$ NMR $\left(500 \mathrm{MHz}, \mathrm{CDCl}_{3}\right) \delta-0.08(\mathrm{~s}, 3 \mathrm{H}), 0.02(\mathrm{~s}, 3 \mathrm{H}), 0.03(\mathrm{~s}, 3 \mathrm{H}), 0.06(\mathrm{~s}, 9 \mathrm{H}), 0.81(\mathrm{~s}$, $9 \mathrm{H}), 0.84(\mathrm{~s}, 9 \mathrm{H}), 0.87(\mathrm{~s}, 9 \mathrm{H}), 1.51-1.61(\mathrm{~m}, 2 \mathrm{H}), 1.65-1.70(\mathrm{~m}, 1 \mathrm{H}), 1.77$ (ddd, $J=13.6$, 10.4 and $3.0 \mathrm{~Hz}, 1 \mathrm{H}), 2.16-2.27(\mathrm{~m}, 2 \mathrm{H}), 2.49-2.63(\mathrm{~m}, 4 \mathrm{H}), 3.04(\mathrm{dd}, J=9.2$ and $6.8 \mathrm{~Hz}$, $1 \mathrm{H}$ ), $3.12(\mathrm{dd}, J=9.2$ and $4.7 \mathrm{~Hz}, 1 \mathrm{H}$ ), $3.38-3.40(\mathrm{~m}, 1 \mathrm{H}$ ), 3.77 (quint, $J=5.8 \mathrm{~Hz}, 1 \mathrm{H}$ ), 
4.05-4.10 (m, 1H), 4.20-4.25 (m, 2H), 5.03-5.06 (m, 2H), 5.75-5.83 (m, 1H), $7.22(\mathrm{t}, J=7.5$ $\mathrm{Hz}, 3 \mathrm{H}), 7.28$ (t, $J=7.5 \mathrm{~Hz}, 6 \mathrm{H}), 7.44(\mathrm{~d}, J=7.5 \mathrm{~Hz}, 6 \mathrm{H})$.

${ }^{13} \mathrm{C}$ NMR (125 MHz, $\left.\mathrm{CDCl}_{3}\right) \delta-5.0,-4.6,-4.4\left(2 \times \mathrm{CH}_{3}\right),-4.3,-4.1,17.9,18.0,18.0,25.8$, 25.8, 25.9, 40.9, 42.2, 45.2, 51.5, 51.6, 64.1, 66.9, 67.2, 69.1, 69.6, 86.6, 117.2, 126.9, 127.7, 128.7, 134.6, 144.0, 209.8.

IR (film) $v_{\text {max }} / \mathrm{cm}^{-1} 3449,3087,3062,3034,2954,2929,2888,2857,1706,1641,1472,1256$, 1076, 836, 775, 706, 633.

HRMS (ESI FT-ICR-MS) $\mathrm{m} / z$ calcd for $\mathrm{C}_{50} \mathrm{H}_{80} \mathrm{O}_{6} \mathrm{Si}_{3} \mathrm{Na}[\mathrm{M}+\mathrm{Na}]^{+}: 883.5160$, found: 883.5152 .

\section{(+)-(5R,7S,11R,13R)-5-allyl-7,11-bis((tert-butyldimethylsilyl)oxy)-2,2,3,3,15,15,16,16-} octamethyl-13-((trityloxy)methyl)-4,14-dioxa-3,15-disilaheptadecan-9-one (S33):

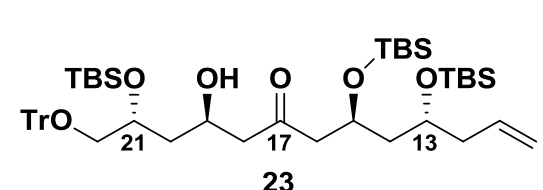

23
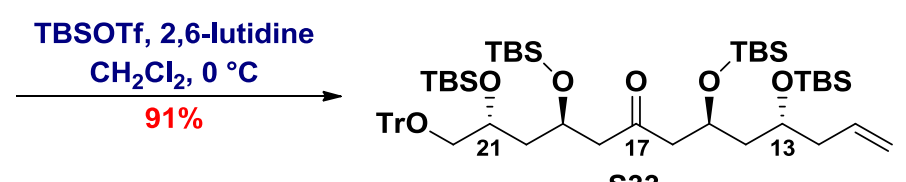

To a solution of aldol adduct $23(1.26 \mathrm{~g}, 1.46 \mathrm{mmol}, 100 \mathrm{~mol} \%)$ in $\mathrm{CH}_{2} \mathrm{Cl}_{2}(19 \mathrm{~mL}, 0.08 \mathrm{M})$ at $0{ }^{\circ} \mathrm{C}$ were added 2,6-lutidine $(0.51 \mathrm{~mL}, 4.38 \mathrm{mmol}, 300 \mathrm{~mol} \%)$ and TBSOTf $(0.50 \mathrm{~mL}, 2.19$ mmol, $150 \mathrm{~mol} \%$ ) dropwise. After $1 \mathrm{~h}$, the reaction was quenched by the addition of saturated aqueous solution of $\mathrm{NaHCO}_{3}(10 \mathrm{~mL})$. The layers were separated, and the aqueous layer was extracted with $\mathrm{CH}_{2} \mathrm{Cl}_{2}(3 \times 25 \mathrm{~mL})$. The combined organic layers were washed with brine $(25$ $\mathrm{mL}$ ), dried over $\mathrm{MgSO}_{4}$, filtered, and concentrated under reduced pressure. The residue was purified by flash column chromatography using a solution of hexane/ethyl acetate (95:05) as the eluent to provide compound $\mathbf{S} 33$ as a $79: 21$ mixture of diastereoisomers $(1.30 \mathrm{~g}, 1.33$ mmol, 91\%).

Physical state: colorless oil

TLC: $R_{f}=0.74$ (95:05 hexane:EtOAc)

Optical rotation: $[\alpha]_{D}^{20}+5\left(c 1.0, \mathrm{CHCl}_{3}\right)$

${ }^{1} \mathrm{H}$ NMR $\left(500 \mathrm{MHz}, \mathrm{CDCl}_{3}\right) \delta-0.08(\mathrm{~s}, 3 \mathrm{H}), 0.00(\mathrm{~s}, 3 \mathrm{H}), 0.01(\mathrm{~s}, 3 \mathrm{H}), 0.03(\mathrm{~s}, 3 \mathrm{H}), 0.06$ (s, $9 \mathrm{H}), 0.07(\mathrm{~s}, 3 \mathrm{H}), 0.84(\mathrm{~s}, 9 \mathrm{H}), 0.84(\mathrm{~s}, 9 \mathrm{H}), 0.86(\mathrm{~s}, 9 \mathrm{H}), 0.88(\mathrm{~s}, 9 \mathrm{H}), 1.50-1.57(\mathrm{~m}, 2 \mathrm{H})$, $1.63(\mathrm{dt}, J=13.4$ and $5.8 \mathrm{~Hz}, 1 \mathrm{H}), 1.94(\mathrm{dt}, J=13.7$ and $5.8 \mathrm{~Hz}, 1 \mathrm{H}), 2.16-2.27(\mathrm{~m}, 2 \mathrm{H})$, 2.41-2.63 (m, 4H), $2.90(\mathrm{dd}, J=9.0$ and $6.4 \mathrm{~Hz}, 1 \mathrm{H}$ ), $3.03(\mathrm{dd}, J=9.0$ and $4.7 \mathrm{~Hz}, 1 \mathrm{H}$ ), 3.76 (quint, $J=5.8 \mathrm{~Hz}, 1 \mathrm{H}$ ), 3.92 (quint, $J=5.8 \mathrm{~Hz}, 1 \mathrm{H}$ ), 4.21 (quint, $J=5.8 \mathrm{~Hz}, 1 \mathrm{H}$ ), $5.02-5.05$ 
(m, 2H), 5.75-5.83 (m, 1H), $7.22(\mathrm{t}, J=7.4 \mathrm{~Hz}, 3 \mathrm{H}), 7.28(\mathrm{t}, J=7.4 \mathrm{~Hz}, 6 \mathrm{H}), 7.44(\mathrm{~d}, J=7.4$ $\mathrm{Hz}, 6 \mathrm{H})$.

${ }^{13} \mathrm{C}$ NMR $\left(125 \mathrm{MHz}, \mathrm{CDCl}_{3}\right) \delta-4.6,-4.3,-4.3,-4.2,-4.2,-4.2,-4.2,-4.1,18.0,18.0,18.0$, 18.1, $25.9\left(3 \times\left(\mathrm{CH}_{3}\right)_{3}\right), 25.9,42.2,43.9,45.4,52.2,52.4,66.7,66.7,67.9,69.5(2 \times \mathrm{CH})$, $86.5,117.1,126.9,127.7,128.7,134.8,144.1,207.0$.

IR (film) $v_{\text {max }} / \mathrm{cm}^{-1}$ 3086, 3062, 3034, 2955, 2929, 2891, 2857, 1717, 1641, 1491, 1472, 1463, 1449, 1387, 1361, 1256, 1099, 1076, 1005, 836, 808, 775, 745, 705, 633.

HRMS (ESI FT-ICR-MS) $\mathrm{m} / z$ calcd for $\mathrm{C}_{56} \mathrm{H}_{94} \mathrm{O}_{6} \mathrm{Si}_{4} \mathrm{Na}[\mathrm{M}+\mathrm{Na}]^{+}: 997.6025$, found: 997.6029 .

\section{(-)-(5R,7S,11R,13R)-5-allyl-7,11-bis((tert-butyldimethylsilyl)oxy)-13-(hydroxymethyl)-}

\section{2,2,3,3,15,15,16,16-octamethyl-4,14-dioxa-3,15-disilaheptadecan-9-one (S34):}
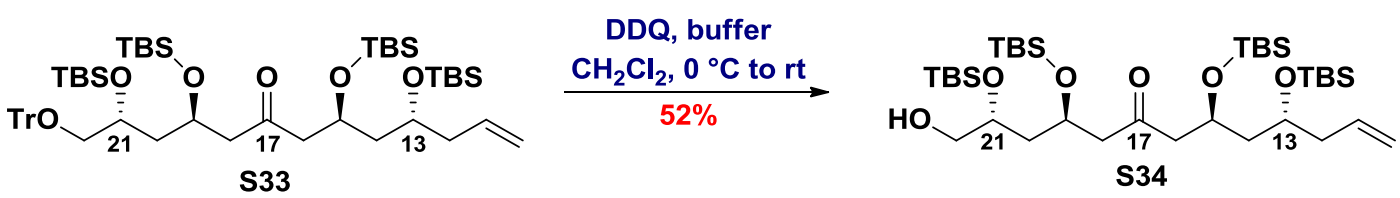

To a solution of trityl ether $\mathbf{S} 33$ (800 $\mathrm{mg}, 0.82 \mathrm{mmol}, 100 \mathrm{~mol} \%$ ) in $\mathrm{CH}_{2} \mathrm{Cl}_{2}$ :phosphate buffer $\mathrm{pH} 7(9: 1)(76 \mathrm{~mL}, 0.01 \mathrm{M})$ at $0{ }^{\circ} \mathrm{C}$ was added DDQ (617 mg, $\left.2.72 \mathrm{mmol}, 330 \mathrm{~mol} \%\right)$. After 5 min, the reaction was warmed to room temperature. After $24 \mathrm{~h}$, the reaction was quenched via the addition of a solution of $\mathrm{H}_{2} \mathrm{O}$ :saturated aqueous solution of $\mathrm{NaHCO}_{3}(1: 1)(11 \mathrm{~mL})$. The resulting mixture was filtered over Celite, washed with $\mathrm{CH}_{2} \mathrm{Cl}_{2}(5 \times 50 \mathrm{~mL})$ and concentrated under reduced pressure. The residue was purified by flash column chromatography using hexane/ethyl acetate $(90: 10)$ as the eluent to provide alcohol S34 as only one diastereoisomer (309 mg, $0.42 \mathrm{mmol}, 52 \%$ ).

\section{Physical state: colorless oil}

TLC: $R_{f}=0.43$ (90:10 hexane:EtOAc)

Optical rotation: $[\alpha]_{D}^{20}-5\left(c 1.7, \mathrm{CHCl}_{3}\right)$

${ }^{1} \mathrm{H}$ NMR $\left(500 \mathrm{MHz}, \mathrm{CDCl}_{3}\right) \delta 0.01(\mathrm{~s}, 3 \mathrm{H}), 0.03(\mathrm{~s}, 3 \mathrm{H}), 0.05(\mathrm{~s}, 3 \mathrm{H}), 0.05(\mathrm{~s}, 6 \mathrm{H}), 0.07$ (s, $3 \mathrm{H}), 0.08(\mathrm{~s}, 3 \mathrm{H}), 0.09(\mathrm{~s}, 3 \mathrm{H}), 0.84(\mathrm{~s}, 9 \mathrm{H}), 0.85(\mathrm{~s}, 9 \mathrm{H}), 0.87(\mathrm{~s}, 9 \mathrm{H}), 0.89(\mathrm{~s}, 9 \mathrm{H}), 1.54(\mathrm{dt}, J$ $=13.9$ and $6.5 \mathrm{~Hz}, 1 \mathrm{H}), 1.61-1.73(\mathrm{~m}, 3 \mathrm{H}), 2.10(\mathrm{br} \mathrm{s}, 1 \mathrm{H}), 2.15-2.26(\mathrm{~m}, 2 \mathrm{H}), 2.50(\mathrm{dd}, J=$ 15.6 and $5.2 \mathrm{~Hz}, 1 \mathrm{H}), 2.55-2.59(\mathrm{~m}, 2 \mathrm{H}), 2.62(\mathrm{dd}, J=16.2$ and $6.3 \mathrm{~Hz}, 1 \mathrm{H}), 3.44-3.46(\mathrm{~m}$, $1 \mathrm{H}$ ), 3.57-3.59 (m, 1H), 3.76 (quint, $J=6.0 \mathrm{~Hz}, 1 \mathrm{H}$ ), 3.80 (quint, $J=5.2 \mathrm{~Hz}, 1 \mathrm{H}$ ), $4.22(\mathrm{~m}$, $2 \mathrm{H}$ ), 5.02-5.05 (m, 1H), 5.78 (ddt, $J=17.7,9.5$ and $7.1 \mathrm{~Hz}, 1 \mathrm{H}$ ). 
${ }^{13} \mathrm{C}$ NMR (125 MHz, $\left.\mathrm{CDCl}_{3}\right) \delta-4.5,-4.4\left(3 \times \mathrm{CH}_{3}\right),-4.3,-4.3,-4.3,-4.1,17.9,18.0,18.1(2$ $\left.\times \mathrm{C}_{0}\right), 25.8,25.8\left(2 \times\left(\mathrm{CH}_{3}\right)_{3}\right), 25.9,42.0,42.2,45.3,52.1,52.3,66.6,66.6,66.8,69.5,70.9$, 117.2, 134.7, 207.3.

IR (film) $v_{\max } / \mathrm{cm}^{-1} 3445,2955,2930,2888,2858,1715,1641,1473,1463,1256,1101,836$, $808,775$.

HRMS (ESI FT-ICR-MS) $\mathrm{m} / z$ calcd for $\mathrm{C}_{37} \mathrm{H}_{80} \mathrm{O}_{6} \mathrm{Si}_{4} \mathrm{Na}[\mathrm{M}+\mathrm{Na}]^{+}:$755.4930, found: 755.4917.

\section{(+)-(2R,4R,8S,10R)-2,4,8,10-tetrakis((tert-butyldimethylsilyl)oxy)-6-oxotridec-12-enal (8):}
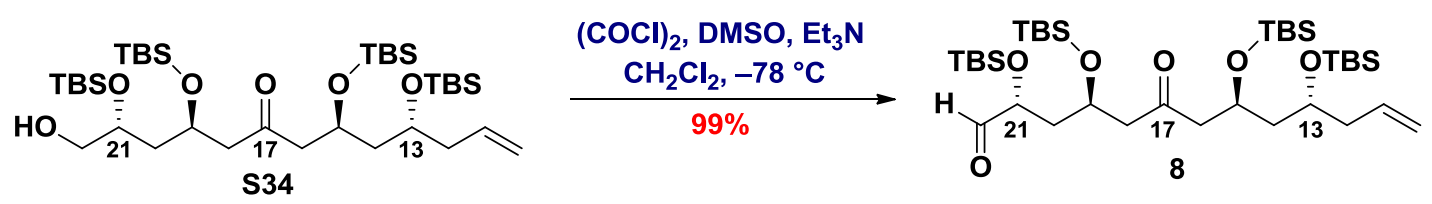

To a solution of oxalyl chloride $(0.12 \mathrm{~mL}, 1.38 \mathrm{mmol}, 300 \mathrm{~mol} \%)$ in $\mathrm{CH}_{2} \mathrm{Cl}_{2}(6.6 \mathrm{~mL}, 0.21 \mathrm{M})$ at $-78{ }^{\circ} \mathrm{C}$ was added DMSO $(0.20 \mathrm{~mL}, 2.88 \mathrm{mmol}, 610 \mathrm{~mol} \%)$ dropwise. The reaction medium was stirred for $30 \mathrm{~min}$, followed by the dropwise addition of a solution of alcohol S34 (343 $\mathrm{mg}$, $0.47 \mathrm{mmol}, 100 \mathrm{~mol} \%)$ in $\mathrm{CH}_{2} \mathrm{Cl}_{2}(2.9 \mathrm{~mL}, 0.16 \mathrm{M})$. After $30 \mathrm{~min}, \mathrm{Et}_{3} \mathrm{~N}(0.98 \mathrm{~mL}, 7.04 \mathrm{mmol}$, $1500 \mathrm{~mol} \%$ ) was added dropwise, and the resulting slurry was warmed to $0{ }^{\circ} \mathrm{C}$ and stirred for $1 \mathrm{~h}$. The reaction was then diluted with $\mathrm{Et}_{2} \mathrm{O}(25 \mathrm{~mL})$ and saturated aqueous solution of $\mathrm{NH}_{4} \mathrm{Cl}$ $(25 \mathrm{~mL})$. The layers were separated, and the aqueous layer was extracted with $\mathrm{Et}_{2} \mathrm{O}(3 \times 50$ $\mathrm{mL})$. The combined organic layers were washed with $\mathrm{H}_{2} \mathrm{O}(3 \times 50 \mathrm{~mL})$, brine $(50 \mathrm{~mL})$, dried over $\mathrm{MgSO}_{4}$, filtered, and concentrated under reduced pressure to provide the aldehyde 8 (344 mg, $0.47 \mathrm{mmol}, 99 \%$ ), which was used in the next step without further purification.

\section{Physical state: yellow oil}

TLC: $R_{f}=0.60$ (90:10 hexane:EtOAc)

Optical rotation: $[\alpha]_{D}^{20}+7\left(c 2.2, \mathrm{CHCl}_{3}\right)$

${ }^{1} \mathrm{H}$ NMR $\left(500 \mathrm{MHz}, \mathrm{CDCl}_{3}\right) \delta 0.00(\mathrm{~s}, 3 \mathrm{H}), 0.02(\mathrm{~s}, 3 \mathrm{H}), 0.04(\mathrm{~s}, 3 \mathrm{H}), 0.05$ (s, 3H), 0.05 (s, $6 \mathrm{H}), 0.06(\mathrm{~s}, 3 \mathrm{H}), 0.09(\mathrm{~s}, 3 \mathrm{H}), 0.83(\mathrm{~s}, 9 \mathrm{H}), 0.84(\mathrm{~s}, 9 \mathrm{H}), 0.87(\mathrm{~s}, 9 \mathrm{H}), 0.91(\mathrm{~s}, 9 \mathrm{H}), 1.53(\mathrm{dt}, J$ $=13.7$ and $6.6 \mathrm{~Hz}, 1 \mathrm{H}), 1.64(\mathrm{dt}, J=13.7$ and $6.1 \mathrm{~Hz}, 1 \mathrm{H}), 1.86(\mathrm{dt}, J=14.2$ and $6.0 \mathrm{~Hz}, 1 \mathrm{H})$, $1.92(\mathrm{dt}, J=14.2$ and $5.3 \mathrm{~Hz}, 1 \mathrm{H}), 2.15-2.26(\mathrm{~m}, 2 \mathrm{H}), 2.46(\mathrm{dd}, J=15.6$ and $4.9 \mathrm{~Hz}, 1 \mathrm{H})$, $2.56(\mathrm{dd}, J=15.6$ and $7.2 \mathrm{~Hz}, 1 \mathrm{H}$ ), 2.59 (dd, $J=16.7$ and $5.5 \mathrm{~Hz}, 1 \mathrm{H}$ ), 2.78 (dd, $J=16.7$ and $7.2 \mathrm{~Hz}, 1 \mathrm{H}$ ), 3.74 (quint, $J=5.9 \mathrm{~Hz}, 1 \mathrm{H}$ ), 4.13-4.15 (m, 1H), 4.21 (quint, $J=5.3 \mathrm{~Hz}, 1 \mathrm{H}$ ), 4.31-4.36 (m, 1H), 5.02-5.05 (m, 2H), 5.74-5.82 (m, 1H), $9.58(\mathrm{~d}, J=1,4 \mathrm{~Hz}, 1 \mathrm{H})$. 
${ }^{13} \mathrm{C}$ NMR (125 MHz, $\left.\mathrm{CDCl}_{3}\right) \delta-4.8,-4.5,-4.4,-4.4\left(3 \times \mathrm{CH}_{3}\right),-4.3,-4.1,18.0\left(2 \times \mathrm{C}_{0}\right), 18.1$, 18.2, $25.8\left(2 \times\left(\mathrm{CH}_{3}\right)_{3}\right), 25.8,25.9,40.2,42.1,45.4,51.9\left(2 \times \mathrm{CH}_{2}\right), 65.8,66.6,69.5,75.9$, 117.2, 134.7, 202.8, 207.0.

IR (film) $v_{\max } / \mathrm{cm}^{-1} 2955,2930,2896,2858,2710,1737,1717,1472,1463,1362,1256,1103$, 1005, 836, 808, 776.

HRMS (ESI FT-ICR-MS) $\mathrm{m} / z$ calcd for $\mathrm{C}_{37} \mathrm{H}_{78} \mathrm{O}_{6} \mathrm{Si}_{4} \mathrm{Na}[\mathrm{M}+\mathrm{Na}]^{+}:$753.4773, found: 753.4768 .

\section{Synthesis of C8-C35 Fragment of Marinisporolide C}

(+)-(5R,7S,11R,13R)-5-allyl-7,11-bis((tert-butyldimethylsilyl)oxy)-13-((E)-3-((4R,6R)-6(((4S,6S)-6-((2S,3S)-3-((4-methoxybenzyl)oxy)-4-methylpentan-2-yl)-2,2-dimethyl-1,3dioxan-4-yl)methyl)-2,2-dimethyl-1,3-dioxan-4-yl)prop-1-en-1-yl)-2,2,3,3,15,15,16,16octamethyl-4,14-dioxa-3,15-disilaheptadecan-9-one (24):

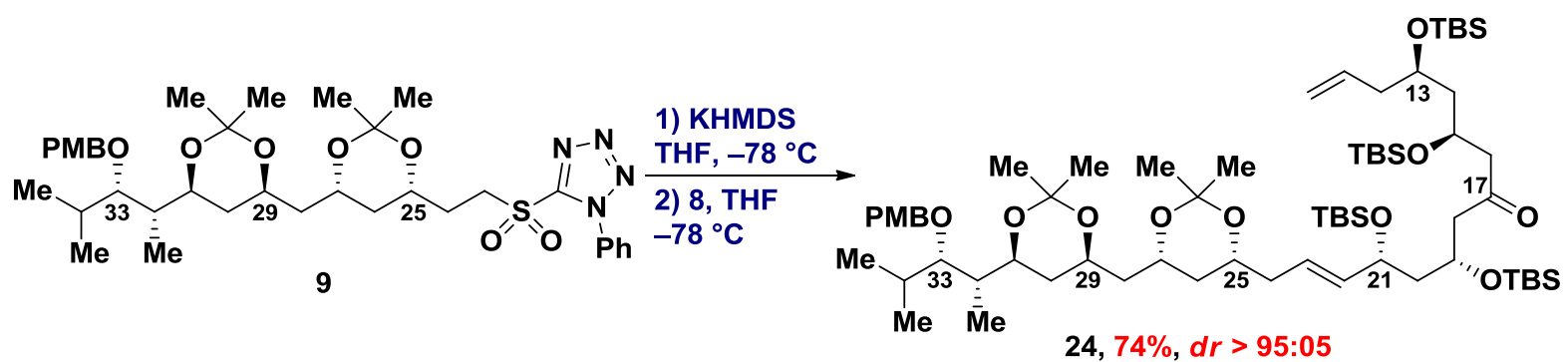

To a solution of sulfone 9 (296 mg, $0.42 \mathrm{mmol}, 100 \mathrm{~mol} \%)$ in THF $(5.0 \mathrm{~mL}, 0.08 \mathrm{M})$ at $-78^{\circ} \mathrm{C}$ was added KHMDS $(0.46 \mathrm{~mL}, 0.46 \mathrm{mmol}, 1 \mathrm{M}$ in THF, $110 \mathrm{~mol} \%)$ dropwise. The reaction medium was stirred for $5 \mathrm{~min}$, followed by the dropwise addition of a solution of aldehyde 8 (344 mg, $0.47 \mathrm{mmol}, 110 \mathrm{~mol} \%$ ) in THF $(2.6 \mathrm{~mL}, 0.18 \mathrm{M})$. After $20 \mathrm{~min}$, the reaction was diluted with $\mathrm{Et}_{2} \mathrm{O}(10 \mathrm{~mL})$ and quenched by the addition of brine $(10 \mathrm{~mL})$. The layers were separated, and the aqueous layer was extracted with $\mathrm{Et}_{2} \mathrm{O}(3 \times 25 \mathrm{~mL})$. The combined organic layers were dried over $\mathrm{MgSO}_{4}$, filtered, and concentrated under reduced pressure. The residue was purified by flash column chromatography using hexane/ethyl acetate (90:10) as the eluent to provide compound 24 (370 $\mathrm{mg}, 0.31 \mathrm{mmol}, 74 \%, d r>95: 05, E: Z)$.

Physical state: colorless oil

TLC: $R_{f}=0.48$ (90:10 hexane:EtOAc)

Optical rotation: $[\alpha]_{D}^{20}+2\left(c 0.7, \mathrm{CHCl}_{3}\right)$ 
${ }^{1} \mathrm{H}$ NMR (500 MHz, CDCl $) \delta 0.01(\mathrm{~s}, 9 \mathrm{H}), 0.03(\mathrm{~s}, 3 \mathrm{H}), 0.05(\mathrm{~s}, 3 \mathrm{H}), 0.06(\mathrm{~s}, 9 \mathrm{H}), 0.82(\mathrm{~d}, J=$ $6.6 \mathrm{~Hz}, 3 \mathrm{H}), 0.83(\mathrm{~d}, J=7.0 \mathrm{~Hz}, 3 \mathrm{H}), 0.84(\mathrm{~s}, 9 \mathrm{H}), 0.85(\mathrm{~s}, 9 \mathrm{H}), 0.87(\mathrm{~s}, 9 \mathrm{H}), 0.87(\mathrm{~s}, 9 \mathrm{H})$, $1.00(\mathrm{~d}, J=6.6 \mathrm{~Hz}, 3 \mathrm{H}), 1.06(\mathrm{q}, J=12.2 \mathrm{~Hz}, 1 \mathrm{H}), 1.06(\mathrm{q}, J=12.2 \mathrm{~Hz}, 1 \mathrm{H}), 1.35(\mathrm{~s}, 3 \mathrm{H})$, $1.36(\mathrm{~s}, 3 \mathrm{H}), 1.38(\mathrm{~s}, 3 \mathrm{H}), 1.39(\mathrm{~s}, 3 \mathrm{H}), 1.42(\mathrm{dt}, J=12.2$ and $2.0 \mathrm{~Hz}, 1 \mathrm{H}), 1.48-1.70(\mathrm{~m}, 8 \mathrm{H})$, 1.78-1.85 (m, 1H), $2.09(\mathrm{dt}, J=13.8$ and $6.4 \mathrm{~Hz}, 1 \mathrm{H}), 2.15-2.26(\mathrm{~m}, 3 \mathrm{H}), 2.48-2.59(\mathrm{~m}, 4 \mathrm{H})$, 3.41 (dd, $J=8.9$ and $1.7 \mathrm{~Hz}, 1 \mathrm{H}$ ), 3.76 (quint, $J=5.8 \mathrm{~Hz}, 1 \mathrm{H}$ ), $3.79(\mathrm{~s}, 3 \mathrm{H}$ ), 3.79-3.87 (m, 2H), 4.03-4.09 (m, 2H), $4.10(\mathrm{q}, J=7.2 \mathrm{~Hz}, 1 \mathrm{H}), 4.18-4.23(\mathrm{~m}, 2 \mathrm{H}), 4.50(\mathrm{~d}, J=11.6 \mathrm{~Hz}$, $1 \mathrm{H}), 4.52(\mathrm{~d}, J=11.6 \mathrm{~Hz}, 1 \mathrm{H}), 5.02-5.05(\mathrm{~m}, 2 \mathrm{H}), 5.43(\mathrm{dd}, J=15.4$ and $7.2 \mathrm{~Hz}, 1 \mathrm{H}), 5.52$ (dt, $J=15.4$ and $6.9 \mathrm{~Hz}, 1 \mathrm{H}), 5.74-5.82(\mathrm{~m}, 1 \mathrm{H}), 6.84-6.87(\mathrm{~m}, 2 \mathrm{H}), 7.24-7.26(\mathrm{~m}, 2 \mathrm{H})$.

${ }^{13} \mathrm{C}$ NMR $\left(125 \mathrm{MHz}, \mathrm{CDCl}_{3}\right) \delta-4.6,-4.3\left(3 \times \mathrm{CH}_{3}\right),-4.2\left(2 \times \mathrm{CH}_{3}\right),-4.1,-3.6,8.8,18.0(2 \times$ $\left.\mathrm{C}_{0}\right), 18.1,18.2,19.5,19.8,20.1,20.2,25.9\left(2 \times\left(\mathrm{CH}_{3}\right)_{3}\right), 25.9,26.0,30.2,30.4,31.2,35.9$, $37.1,39.3,41.3,42.2,43.3,45.4,46.8,52.1,52.3,55.3,64.7,64.8,66.4,66.8,69.0,69.5$, $70.2,71.2,74.0,82.7,98.4,98.5,113.7,117.1,126.5,128.7,131.8,134.8,135.9,158.9$, 207.3.

IR (film) $v_{\max } / \mathrm{cm}^{-1}$ 2991, 2954, 2930, 2857, 1717, 1641, 1615, 1515, 1472, 1463, 1379, 1250, 1203, 1169, 1088, 1040, 836, 775.

HRMS (ESI FT-ICR-MS) $\mathrm{m} / z$ calcd for $\mathrm{C}_{66} \mathrm{H}_{124} \mathrm{O}_{11} \mathrm{Si}_{4} \mathrm{Na}[\mathrm{M}+\mathrm{Na}]^{+}: 1227.8118$, found: 1227.8110 .

(-)-(5R,7S,11R,13R)-5-allyl-7,11-bis((tert-butyldimethylsilyl)oxy)-13-((E)-3-((4R,6R)-6(((4S,6S)-6-((2R,3S)-3-hydroxy-4-methylpentan-2-yl)-2,2-dimethyl-1,3-dioxan-4-

yl)methyl)-2,2-dimethyl-1,3-dioxan-4-yl)prop-1-en-1-yl)-2,2,3,3,15,15,16,16-octamethyl4,14-dioxa-3,15-disilaheptadecan-9-one (S35):

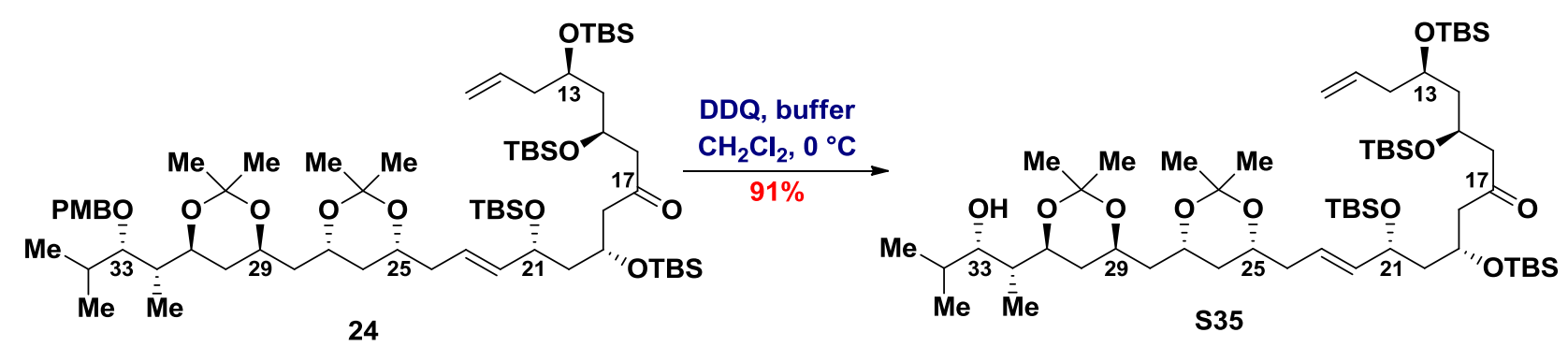

To a solution of PMB ether 24 ( $370 \mathrm{mg}, 0.31 \mathrm{mmol}, 100 \mathrm{~mol} \%$ ) in $\mathrm{CH}_{2} \mathrm{Cl}_{2}$ :phosphate buffer $\mathrm{pH}$ $7(9: 1)(5.0 \mathrm{~mL}, 0.06 \mathrm{M})$ at $0{ }^{\circ} \mathrm{C}$ was added DDQ (104 mg, $\left.0.46 \mathrm{mmol}, 150 \mathrm{~mol} \%\right)$. The mixture was stirred for $1 \mathrm{~h}$ under the same conditions, followed by quenching via the addition of a solution of $\mathrm{H}_{2} \mathrm{O}$ :saturated aqueous solution of $\mathrm{NaHCO}_{3}(1: 1)(5 \mathrm{~mL})$. The resulting 
mixture was filtered over Celite, washed with $\mathrm{CH}_{2} \mathrm{Cl}_{2}(5 \times 25 \mathrm{~mL})$ and concentrated under reduced pressure. The residue was purified by flash column chromatography using hexane/ethyl acetate (80:20) as the eluent to provide alcohol S35 (305 mg, $0.28 \mathrm{mmol}, 91 \%)$.

Physical state: pale yellow oil

TLC: $R_{f}=0.72$ (80:20 hexane:EtOAc)

Optical rotation: $[\alpha]_{D}^{20}-5\left(c 1.0, \mathrm{CHCl}_{3}\right)$

${ }^{1} \mathrm{H}$ NMR (500 MHz, CDCl $) \delta 0.01(\mathrm{~s}, 9 \mathrm{H}), 0.03(\mathrm{~s}, 3 \mathrm{H}), 0.05(\mathrm{~s}, 3 \mathrm{H}), 0.05(\mathrm{~s}, 9 \mathrm{H}), 0.78(\mathrm{~d}, J=$ $6.8 \mathrm{~Hz}, 3 \mathrm{H}), 0.84(\mathrm{~s}, 9 \mathrm{H}), 0.85(\mathrm{~s}, 9 \mathrm{H}), 0.87(\mathrm{~s}, 9 \mathrm{H}), 0.87(\mathrm{~s}, 9 \mathrm{H}), 0.92(\mathrm{~d}, J=7.1 \mathrm{~Hz}, 3 \mathrm{H})$, $1.01(\mathrm{~d}, J=6.4 \mathrm{~Hz}, 3 \mathrm{H}), 1.13(\mathrm{q}, J=12.0 \mathrm{~Hz}, 1 \mathrm{H}), 1.32-1.44(\mathrm{~m}, 3 \mathrm{H}), 1.34(\mathrm{~s}, 3 \mathrm{H}), 1.35$ (s, $3 \mathrm{H}), 1.39(\mathrm{~s}, 6 \mathrm{H}), 1.47-1.70(\mathrm{~m}, 8 \mathrm{H}), 2.09(\mathrm{dt}, J=14.0$ and $6.4 \mathrm{~Hz}, 1 \mathrm{H}), 2.15-2.26(\mathrm{~m}, 3 \mathrm{H})$, 2.48-2.58 (m, 4H), $2.99(\mathrm{br} \mathrm{s}, 1 \mathrm{H}), 3.51(\mathrm{~d}, J=9.3 \mathrm{~Hz}, 1 \mathrm{H}$ ), 3.76 (quint, $J=5.7 \mathrm{~Hz}, 1 \mathrm{H}$ ), 3.82-3.87 (m, 1H), 3.90-3.93 (m, 1H), 4.02-4.12 (m, 3H), 4.19-4.23 (m, 2H), 5.02-5.05 (m, 2H), $5.43(\mathrm{dd}, J=15.4$ and $7.1 \mathrm{~Hz}, 1 \mathrm{H}), 5.52(\mathrm{dt}, J=15.4$ and $6.7 \mathrm{~Hz}, 1 \mathrm{H}), 5.74-5.82(\mathrm{~m}, 1 \mathrm{H})$. ${ }^{13} \mathrm{C}$ NMR (125 MHz, $\left.\mathrm{CDCl}_{3}\right) \delta-4.6,-4.3\left(3 \times \mathrm{CH}_{3}\right),-4.2\left(2 \times \mathrm{CH}_{3}\right),-4.1,-3.6,10.0,18.0(2 \times$ $\left.\mathrm{C}_{0}\right), 18.1,18.2,19.0,19.5,19.8,19.9,25.9\left(2 \times\left(\mathrm{CH}_{3}\right)_{3}\right), 25.9,26.0,30.2\left(2 \times \mathrm{CH}_{3}\right), 30.9$, $35.1,37.1,38.7,39.2,42.2,43.0,45.4,46.8,52.1,52.3,64.6,65.0,66.4,66.8,69.0,69.5$, 71.2, 74.0, 76.1, 98.5, 98.9, 117.1, 126.5, 134.8, 136.0, 207.3.

IR (film) $v_{\max } / \mathrm{cm}^{-1} 3526,2991,2954,2930,2858,1717,1473,1468,1380,1256,1203,1168$, 1095, 836, 775.

HRMS (ESI FT-ICR-MS) $\mathrm{m} / \mathrm{z}$ calcd for $\mathrm{C}_{58} \mathrm{H}_{116} \mathrm{O}_{10} \mathrm{Si}_{4} \mathrm{Na}[\mathrm{M}+\mathrm{Na}]^{+}: 1107.7543$, found: 1107.7534.

(-)-(2E,5R,7S,11R,13R,14E)-5,7,11,13-tetrakis((tert-butyldimethylsilyl)oxy)-16-((4R,6R)-6(((4S,6S)-6-((2R,3S)-3-hydroxy-4-methylpentan-2-yl)-2,2-dimethyl-1,3-dioxan-4-

yl)methyl)-2,2-dimethyl-1,3-dioxan-4-yl)-9-oxohexadeca-2,14-dienal (25):

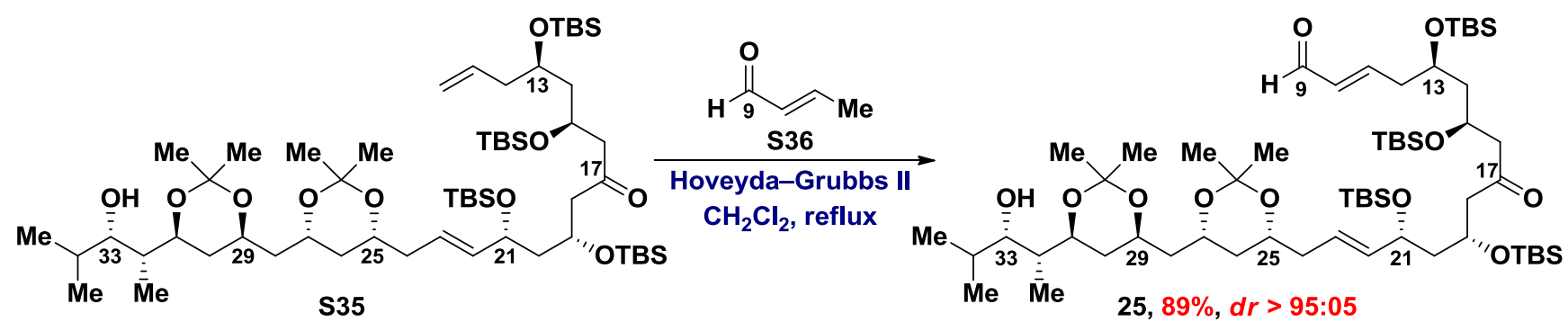

A solution of olefin S35 (200 mg, $0.184 \mathrm{mmol}, 100 \mathrm{~mol} \%$ ) and crotonaldehyde (S36) (0.05 
$\mathrm{mL}, 0.550 \mathrm{mmol}, 300 \mathrm{~mol} \%)$ in $\mathrm{CH}_{2} \mathrm{Cl}_{2}(1.8 \mathrm{~mL}, 0.10 \mathrm{M})$ at room temperature was purged with argon for $10 \mathrm{~min}$. After this period, Hoveyda-Grubbs $2^{\text {nd }}$ generation catalyst $(11 \mathrm{mg}, 18.4$ $\mu \mathrm{mol}, 10 \mathrm{~mol} \%$ ) was added, and the reaction medium was warmed to $50^{\circ} \mathrm{C}$. After $24 \mathrm{~h}$, the volatiles were removed under reduced pressure, and the residue was purified by flash column chromatography using a solution of hexane/ethyl acetate (80:20) as the eluent to provide the aldehyde 25 (181 mg, $0.163 \mathrm{mmol}, 89 \%, d r>95: 05, E: Z$ ).

\section{Physical state: colorless oil}

TLC: $R_{f}=0.66$ (80:20 hexane:EtOAc)

Optical rotation: $[\alpha]_{D}^{20}-8\left(c 1.0, \mathrm{CHCl}_{3}\right)$

${ }^{1} \mathrm{H}$ NMR $\left(500 \mathrm{MHz}, \mathrm{CDCl}_{3}\right) \delta 0.01(\mathrm{~s}, 6 \mathrm{H}), 0.01(\mathrm{~s}, 3 \mathrm{H}), 0.03(\mathrm{~s}, 3 \mathrm{H}), 0.04(\mathrm{~s}, 3 \mathrm{H}), 0.05(\mathrm{~s}$, $6 \mathrm{H}), 0.07(\mathrm{~s}, 3 \mathrm{H}), 0.78(\mathrm{~d}, J=6.7 \mathrm{~Hz}, 3 \mathrm{H}), 0.84(\mathrm{~s}, 18 \mathrm{H}), 0.86(\mathrm{~s}, 9 \mathrm{H}), 0.87(\mathrm{~s}, 9 \mathrm{H}), 0.92(\mathrm{~d}, J$ $=6.7 \mathrm{~Hz}, 3 \mathrm{H}), 1.00(\mathrm{~d}, J=6.7 \mathrm{~Hz}, 3 \mathrm{H}), 1.12(\mathrm{q}, J=12.2 \mathrm{~Hz}, 1 \mathrm{H}), 1.34(\mathrm{~s}, 3 \mathrm{H}), 1.36(\mathrm{~s}, 3 \mathrm{H})$, $1.38(\mathrm{~s}, 6 \mathrm{H}), 1.38-1.68(\mathrm{~m}, 11 \mathrm{H}), 2.08(\mathrm{dt}, J=14.0$ and $6.7 \mathrm{~Hz}, 1 \mathrm{H}), 2.20(\mathrm{dt}, J=14.0$ and 6.7 $\mathrm{Hz}, 1 \mathrm{H}), 2.41-2.62(\mathrm{~m}, 6 \mathrm{H}), 2.99(\mathrm{br} \mathrm{s}, 1 \mathrm{H}), 3.50(\mathrm{~d}, J=9.2 \mathrm{~Hz}, 1 \mathrm{H}), 3.82-3.86(\mathrm{~m}, 1 \mathrm{H})$, 3.88-3.92 (m, 2H), 4.03-4.12 (m, 3H), 4.19 (quint, $J=6.1 \mathrm{~Hz}, 2 \mathrm{H}$ ), 5.42 (dd, $J=15.3$ and 7.0 $\mathrm{Hz}, 1 \mathrm{H}), 5.52(\mathrm{dt}, J=15.3$ and $6.7 \mathrm{~Hz}, 1 \mathrm{H}), 6.13(\mathrm{dd}, J=15.9$ and $7.9 \mathrm{~Hz}, 1 \mathrm{H}), 6.84(\mathrm{dt}, J=$ 15.9 and $7.9 \mathrm{~Hz}, 1 \mathrm{H}), 9.48(\mathrm{~d}, J=7.9 \mathrm{~Hz}, 1 \mathrm{H})$.

${ }^{13} \mathrm{C}$ NMR (125 MHz, $\left.\mathrm{CDCl}_{3}\right) \delta-4.6,-4.3,-4.3,-4.3\left(2 \times \mathrm{CH}_{3}\right),-4.3,-4.2,-3.6,10.0,17.9$, 18.0, 18.0, 18.2, 19.0, 19.5, 19.8, 19.9, $25.8\left(2 \times\left(\mathrm{CH}_{3}\right)_{3}\right), 25.9,26.0,30.2\left(2 \times \mathrm{CH}_{3}\right), 30.9$, $35.1,37.0,38.7,39.2,41.0,43.0,45.7,46.7,52.1,52.3,64.6,65.0,66.5(2 \times \mathrm{CH}), 68.8,68.9$, $71.2,74.0,76.1,98.5,98.9,126.5,135.0,135.9,154.8,193.8,207.1$.

IR (film) $v_{\max } / \mathrm{cm}^{-1} 3452,2954,2929,2857,1691,1650,1633,1472,1463,1379,1256,1202$, 1167, 1095, 836, 776.

HRMS (ESI FT-ICR-MS) $\mathrm{m} / \mathrm{z}$ calcd for $\mathrm{C}_{59} \mathrm{H}_{116} \mathrm{O}_{11} \mathrm{Si}_{4} \mathrm{Na}[\mathrm{M}+\mathrm{Na}]^{+}:$1135.7492, found: 1135.7488. 
(-)-(5R,7R,11S,13R)-7,11-bis((tert-butyldimethylsilyl)oxy)-5-((E)-3-((4R,6R)-6-(((4S,6S)-6((2R,3S)-3-hydroxy-4-methylpentan-2-yl)-2,2-dimethyl-1,3-dioxan-4-yl)methyl)-2,2-

dimethyl-1,3-dioxan-4-yl)prop-1-en-1-yl)-13-((2E,4E)-5-iodopenta-2,4-dien-1-yl)-

2,2,3,3,15,15,16,16-octamethyl-4,14-dioxa-3,15-disilaheptadecan-9-one (S37):

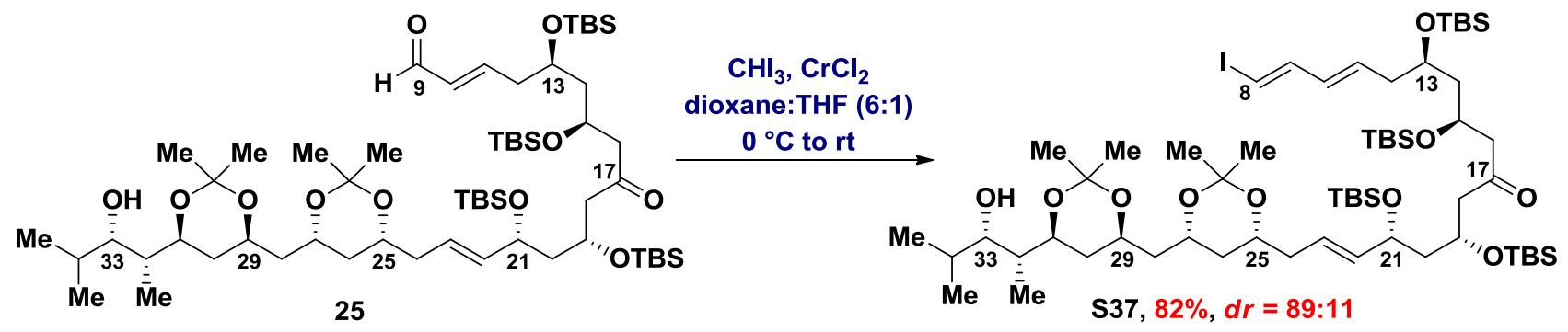

To a suspension of $\mathrm{CrCl}_{2}(141 \mathrm{mg}, 1.15 \mathrm{mmol}, 3000 \mathrm{~mol} \%$.) in dioxane:THF (6:1) (1.8 mL, $0.64 \mathrm{M}$ ) at $0{ }^{\circ} \mathrm{C}$ was added a solution of the aldehyde 25 (42 mg, $38.3 \mu \mathrm{mol}, 100 \mathrm{~mol} \%$ ) and $\mathrm{CH}_{3}$ (122 mg, $\left.0.31 \mathrm{mmol}, 800 \mathrm{~mol} \%\right)$ in dioxane:THF (6:1) (1.2 mL, $\left.0.03 \mathrm{M}\right)$. The reaction medium was stirred for $30 \mathrm{~min}$ at $0{ }^{\circ} \mathrm{C}$ and $1.5 \mathrm{~h}$ at $0{ }^{\circ} \mathrm{C}$. After this period, the reaction was diluted with EtOAc $(5 \mathrm{~mL})$ and quenched by the addition of saturated aqueous solution of $\mathrm{NH}_{4} \mathrm{Cl}(5 \mathrm{~mL})$ and saturated aqueous solution of $\mathrm{Na}_{2} \mathrm{~S}_{2} \mathrm{O}_{3}(5 \mathrm{~mL})$. The layers were separated and the aqueous layer was extracted with EtOAc $(3 \times 10 \mathrm{~mL})$. The combined organic layers were washed with brine $(10 \mathrm{~mL})$, dried over $\mathrm{Na}_{2} \mathrm{SO}_{4}$, filtered, and concentrated under reduced pressure. The residue was purified by flash column chromatography using a solution of hexane/ethyl acetate (90:10) as the eluent to provide vinyl iodide S37 (39 mg, $31.5 \mu \mathrm{mol}$, $82 \%, d r=89: 11, E: Z$ ). Diastereoisomeric ratio was determined by ${ }^{1} \mathrm{H}$ NMR analysis of the diastereoisomeric mixture of compounds.

Physical state: pale yellow oil

TLC: $R_{f}=0.30$ (90:10 hexane:EtOAc)

Optical rotation: $[\alpha]_{D}^{20}-6\left(c 0.5, \mathrm{CHCl}_{3}\right)$

${ }^{1} \mathrm{H}$ NMR (500 MHz, CDCl $) \delta 0.01(\mathrm{~s}, 3 \mathrm{H}), 0.01(\mathrm{~s}, 3 \mathrm{H}), 0.01(\mathrm{~s}, 3 \mathrm{H}), 0.03(\mathrm{~s}, 6 \mathrm{H}), 0.04(\mathrm{~s}$, $3 \mathrm{H}), 0.05(\mathrm{~s}, 3 \mathrm{H}), 0.06(\mathrm{~s}, 3 \mathrm{H}), 0.78(\mathrm{~d}, J=6.7 \mathrm{~Hz}, 3 \mathrm{H}), 0.84(\mathrm{~s}, 9 \mathrm{H}), 0.85(\mathrm{~s}, 9 \mathrm{H}), 0.87$ (s, $18 \mathrm{H}$ ), 0.92 (d, $J=7.2 \mathrm{~Hz}, 3 \mathrm{H}$ ), 1.02 (d, $J=6.4 \mathrm{~Hz}, 3 \mathrm{H}$ ), 1.13 (q, $J=12.0 \mathrm{~Hz}, 1 \mathrm{H}$ ), 1.34-1.46 $(\mathrm{m}, 3 \mathrm{H}), 1.35(\mathrm{~s}, 3 \mathrm{H}), 1.36(\mathrm{~s}, 3 \mathrm{H}), 1.39(\mathrm{~s}, 6 \mathrm{H}), 1.47-1.70(\mathrm{~m}, 8 \mathrm{H}), 2.07-2.27(\mathrm{~m}, 4 \mathrm{H})$, 2.24-2.64 (m, 4H), $2.99(\mathrm{~s}, 1 \mathrm{H}), 3.51(\mathrm{~d}, J=9.3 \mathrm{~Hz}, 1 \mathrm{H}), 3.73-3.79(\mathrm{~m}, 1 \mathrm{H}), 3.82-3.87(\mathrm{~m}$, $1 \mathrm{H}), 3.90-3.94(\mathrm{~m}, 1 \mathrm{H}), 4.02-4.13(\mathrm{~m}, 3 \mathrm{H}), 4.16-4.22(\mathrm{~m}, 2 \mathrm{H}), 5.43(\mathrm{dd}, J=15.4$ and $7.2 \mathrm{~Hz}$, 
1H), $5.52(\mathrm{dt}, J=15.4$ and $6.9 \mathrm{~Hz}, 1 \mathrm{H}$ ), 5.69 (dt, $J=15.2$ and $7.2 \mathrm{~Hz}, 1 \mathrm{H}$ ), $5.98(\mathrm{dd}, J=15.2$ and $10.5 \mathrm{~Hz}, 1 \mathrm{H}), 6.18(\mathrm{~d}, J=14.3 \mathrm{~Hz}, 1 \mathrm{H}), 6.98(\mathrm{dd}, J=14.3$ and $10.5 \mathrm{~Hz}, 1 \mathrm{H})$.

${ }^{13} \mathrm{C}$ NMR $\left(125 \mathrm{MHz}, \mathrm{CDCl}_{3}\right) \delta-4.6,-4.3,-4.3\left(2 \times \mathrm{CH}_{3}\right),-4.3,-4.2,-4.1,-3.6,10.0,18.0$, 18.0, 18.0, 18.2, 19.0, 19.5, 19.8, 19.9, $25.9\left(3 \times\left(\mathrm{CH}_{3}\right)_{3}\right), 26.0,30.2\left(2 \times \mathrm{CH}_{3}\right), 30.9,35.1$, $37.0,38.7,39.2,40.7,43.0,45.5,46.7,52.2,52.2,64.6,65.0,66.5,66.7,69.0,69.3,71.2$, $74.1,76.1,77.0,98.5,98.9,126.5,131.8,132.6,136.0,145.3,207.3$.

IR (film) $v_{\text {max }} / \mathrm{cm}^{-1} 3508,2991,2954,2930,2897,2857,1716,1642,1472,1463,1380,1256$, 1202, 1168, 1094, 979, 939, 836, 808, 776, 740.

HRMS (ESI FTMS) $\mathrm{m} / z$ calcd for $\mathrm{C}_{60} \mathrm{H}_{117} \mathrm{O}_{10} \mathrm{Si}_{4} \mathrm{INa}[\mathrm{M}+\mathrm{Na}]^{+}: 1259.6666$, found: 1259.6663 .

\section{2-(2S,3S)-2-((4S,6S)-6-(((4R,6R)-2,2-dimethyl-6-((2E,4R,6R,10S,12R,14E,16E)-4,6,10,12-} tetrakis((tert-butyldimethylsilyl)oxy)-17-iodo-8-oxoheptadeca-2,14,16-trien-1-yl)-1,3dioxan-4-yl)methyl)-2,2-dimethyl-1,3-dioxan-4-yl)-4-methylpentan-3-yl (diethoxyphosphoryl)propanoate (26):

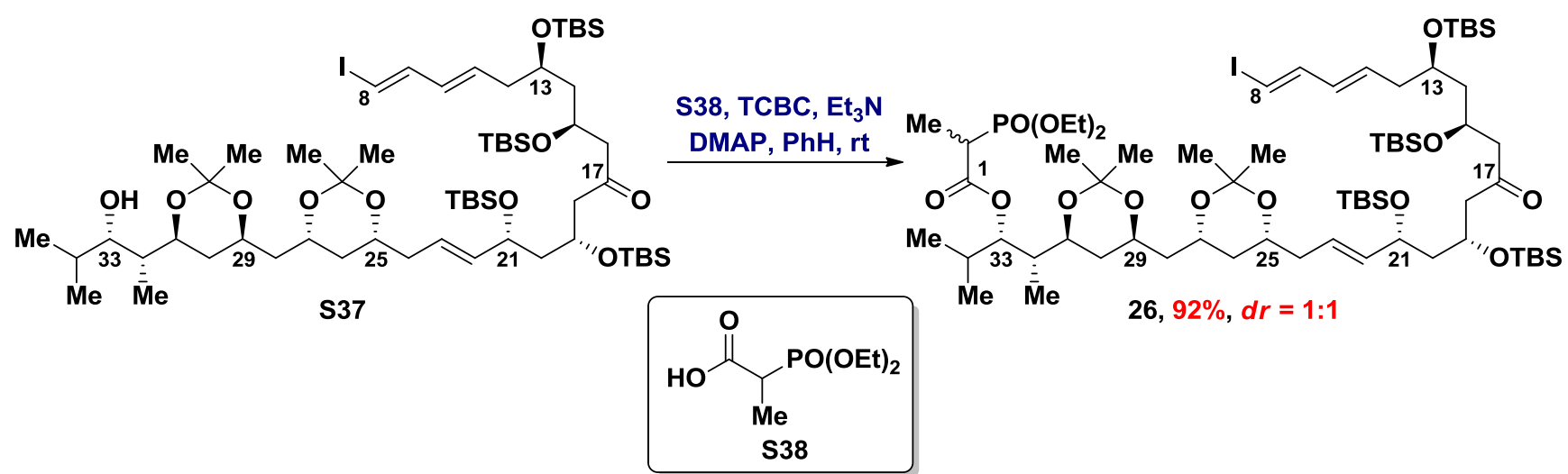

To a solution of alcohol S37 (62 mg, $50.1 \mu \mathrm{mol}, 100 \mathrm{~mol} \%$ ) and acid S38 (111 mg, 0.53 $\mathrm{mmol}, 1060 \mathrm{~mol} \%)$ in benzene $(2.8 \mathrm{~mL}, 0.02 \mathrm{M})$ at room temperature were added $\mathrm{Et}_{3} \mathrm{~N}(0.24$ $\mathrm{mL}, 1.71 \mathrm{mmol}, 3400 \mathrm{~mol} \%)$, TCBC $(0.13 \mathrm{~mL}, 0.80 \mathrm{mmol}, 1600 \mathrm{~mol} \%)$, and DMAP (131 $\mathrm{mg}$, $1.07 \mathrm{mmol}, 2100 \mathrm{~mol} \%)$. After $1 \mathrm{~h}$, the reaction was quenched by the addition of saturated aqueous solution of $\mathrm{NaHCO}_{3}(5 \mathrm{~mL})$. The layers were separated and the aqueous layer was extracted with $\mathrm{Et}_{2} \mathrm{O}(3 \times 10 \mathrm{~mL})$. The combined organic layers were washed with brine $(10$ $\mathrm{mL}$ ), dried over $\mathrm{Na}_{2} \mathrm{SO}_{4}$, filtered, and concentrated under reduced pressure. The residue was purified by flash column chromatography using a solution of hexane/ethyl acetate (60:40) as the eluent to provide compound 26 (66 mg, $46.2 \mu \mathrm{mol}, 92 \%, d r=1: 1, \mathrm{C} 2 R: \mathrm{C} 2 S$ ). Diastereoisomeric ratio was determined by ${ }^{1} \mathrm{H}$ NMR analysis of the diastereoisomeric mixture of compounds. 
Physical state: pale yellow oil

TLC for compound less polar: $R_{f}=0.60$ (60:40 hexane:EtOAc)

TLC for compound more polar: $R_{f}=0.48$ (60:40 hexane:EtOAc)

Optical rotation: $[\alpha]_{D}^{20}+2\left(c 0,5, \mathrm{CHCl}_{3}\right)$

${ }^{1} \mathrm{H}$ NMR $\left(500 \mathrm{MHz}, \mathrm{CDCl}_{3}\right) \delta 0.00(\mathrm{~s}, 3 \mathrm{H}), 0.01(\mathrm{~s}, 6 \mathrm{H}), 0.03(\mathrm{~s}, 6 \mathrm{H}), 0.04(\mathrm{~s}, 3 \mathrm{H}), 0.05(\mathrm{~s}$, $3 \mathrm{H}), 0.05(\mathrm{~s}, 3 \mathrm{H}), 0.81-0.89(\mathrm{~m}, 42 \mathrm{H}), 0.92(\mathrm{~d}, J=6.6 \mathrm{~Hz}, 3 \mathrm{H}), 1.04(\mathrm{q}, J=12.0 \mathrm{~Hz}, 1 \mathrm{H}), 1.12$ $(\mathrm{q}, J=12.0 \mathrm{~Hz}, 1 \mathrm{H}), 1.30-1.48(\mathrm{~m}, 27 \mathrm{H}), 1.50-1.69(\mathrm{~m}, 6 \mathrm{H}), 1.71-1.78(\mathrm{~m}, 1 \mathrm{H}), 1.83-1.90$ $(\mathrm{m}, 1 \mathrm{H}), 2.06-2.26(\mathrm{~m}, 4 \mathrm{H}), 2.46-2.62(\mathrm{~m}, 4 \mathrm{H}), 3.00(\mathrm{dq}, J=23.3$ and $7.3 \mathrm{~Hz}, 1 \mathrm{H}), 3.48-3.59$ $(\mathrm{m}, 1 \mathrm{H}), 3.70-3.86(\mathrm{~m}, 2 \mathrm{H}), 3.99-4.06(\mathrm{~m}, 2 \mathrm{H}), 4.08-4.22(\mathrm{~m}, 7 \mathrm{H}), 5.10(\mathrm{dd}, J=8.9$ and 2.1 $\mathrm{Hz}, 1 \mathrm{H}$ ), $5.42(\mathrm{dd}, J=15.4$ and $7.2 \mathrm{~Hz}, 1 \mathrm{H}), 5.51$ (dt, $J=15.4$ and $6.8 \mathrm{~Hz}, 1 \mathrm{H}$ ), 5.69 (dt, $J=$ 15.3 and $7.3 \mathrm{~Hz}, 1 \mathrm{H}$ ), $5.98(\mathrm{dd}, J=15.2$ and $10.6 \mathrm{~Hz}, 1 \mathrm{H}$ ), $6.18(\mathrm{~d}, J=14.3 \mathrm{~Hz}, 1 \mathrm{H}$ ), 6.98 (dd, $J=14.3$ and $10.6 \mathrm{~Hz}, 1 \mathrm{H})$.

${ }^{13} \mathrm{C}$ NMR $\left(125 \mathrm{MHz}, \mathrm{CDCl}_{3}\right) \delta-4.6,-4.3,-4.3\left(2 \times \mathrm{CH}_{3}\right),-4.3,-4.2,-4.1,-3.6,8.5,12.2$ (d, $J=6.4 \mathrm{~Hz}), 16.4\left(\mathrm{~d}, J=5.4 \mathrm{~Hz}, 2 \times \mathrm{CH}_{3}\right), 18.0,18.0,18.1,18.2,19.0,19.5,19.8,19.9,25.9$ $\left(3 \times\left(\mathrm{CH}_{3}\right)_{3}\right), 26.0,30.2,30.2,30.9,35.3,37.1,39.3,39.7(\mathrm{~d}, J=134.4 \mathrm{~Hz}), 39.9,40.7,43.3$, 45.6, 46.7, 52.2, 52.2, 62.4 (d, $J=7.3 \mathrm{~Hz}$ ), 62.5 (d, $J=6.4 \mathrm{~Hz}$ ), 64.8, 64.8, 66.5, 66.7, 68.9, 69.3, 71.2, 77.0, 78.4, 98.5, 98.6, 126.5, 131.8, 132.6, 135.9, 145.3, 168.9 (d, $J=4.5 \mathrm{~Hz}$ ), 207.3.

IR (film) $v_{\text {max }} / \mathrm{cm}^{-1}$ 2990, 2954, 2930, 2907, 2857, 1732, 1644, 1634, 1472, 1463, 1380, 1256, 1204, 1168, 1098, 1053, 1027, 977, 836, 776.

HRMS (ESI FTMS) $\mathrm{m} / z$ calcd for $\mathrm{C}_{67} \mathrm{H}_{130} \mathrm{O}_{14} \mathrm{Si}_{4} \mathrm{IPNa}[\mathrm{M}+\mathrm{Na}]^{+}: 1451.7212$, found: 1451.7208 .

\section{Synthesis of C3-C7 Fragment of Marinisporolide C}

(E)-3-(tributylstannyl)prop-2-en-1-ol (S41):
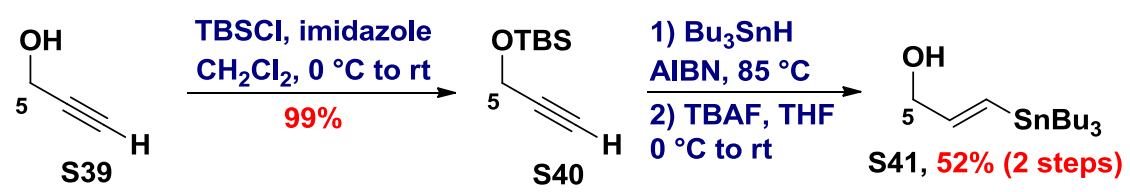

Compound $\mathbf{S} 41$ was prepared according to the literature procedure: a) Li, P.; Li, J.; Arikan, F.; Ahlbrecht, W.; Dieckmann, M.; Menche, D. J. Am. Chem. Soc. 2009, 131, 11678. b) Li, P.; Li, J.; Arikan, F.; Ahlbrecht, W.; Dieckmann, M.; Menche, D. J. Org. Chem. 2010, 75, 2429. 
Physical state: pale yellow oil

TLC: $R_{f}=0.34$ (90:10 hexane:EtOAc)

${ }^{1} \mathrm{H}$ NMR $\left(600 \mathrm{MHz}, \mathrm{CDCl}_{3}\right) \delta 0.86-0.90(\mathrm{~m}, 15 \mathrm{H}), 1.30$ (sext, $\left.J=7.4 \mathrm{~Hz}, 6 \mathrm{H}\right), 1.43-1.55(\mathrm{~m}$, $6 \mathrm{H}), 4.16(\mathrm{t}, J=5.0 \mathrm{~Hz}, 2 \mathrm{H}), 6.14(\mathrm{dt}, J=19.4$ and $4.1 \mathrm{~Hz}, 1 \mathrm{H}), 6.20(\mathrm{~d}, J=19.4 \mathrm{~Hz}, 1 \mathrm{H})$.

${ }^{13} \mathrm{C}$ NMR (150 MHz, $\mathrm{CDCl}_{3}$ ) $\delta 9.4,13.7,27.3,29.0,66.4,128.4,147.0$.

IR (film) $v_{\text {max }} / \mathrm{cm}^{-1} 3308,2956,2925,2871,2853,1604,1464,1418,1376,1357,1341,1292$, 1180, 1072, 1002, 991, 874, 690, 665, 596.

(E)-3-(tributylstannyl)acrylaldehyde (S42):

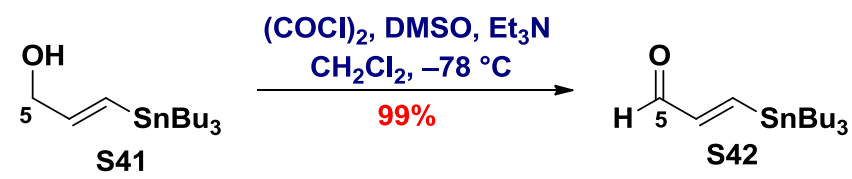

Compound S42 was prepared according to the literature procedure: Oda, H.; Kobayashi, T.; Kosugi, M.; Migita, T. Tetrahedron 1995, 51, 695.

Physical state: yellow oil

TLC: $R_{f}=0.88$ (90:10 hexane:EtOAc)

${ }^{1} \mathrm{H}$ NMR $\left(600 \mathrm{MHz}, \mathrm{CDCl}_{3}\right) \delta 0.88(\mathrm{t}, J=7.3 \mathrm{~Hz}, 9 \mathrm{H}), 0.95-1.06(\mathrm{~m}, 6 \mathrm{H}), 1.30$ (sext, $J=7.3$ $\mathrm{Hz}, 6 \mathrm{H}), 1.44-1.53(\mathrm{~m}, 6 \mathrm{H}), 6.62(\mathrm{dd}, J=19.2$ and $7.6 \mathrm{~Hz}, 1 \mathrm{H}), 7.78(\mathrm{~d}, J=19.2 \mathrm{~Hz}, 1 \mathrm{H})$, $9.40(\mathrm{~d}, J=7.5 \mathrm{~Hz}, 1 \mathrm{H})$.

${ }^{13} \mathrm{C}$ NMR (150 MHz, $\left.\mathrm{CDCl}_{3}\right) \delta 9.8,13.6,27.2,28.9,147.6,163.3,193.7$.

IR (film) $v_{\max } / \mathrm{cm}^{-1}$ 2958, 2927, 2872, 2854, 2787, 2696, 1693, 1464, 1377, 1191, 1073, 991, 666.

(2E,4E)-ethyl 5-(tributylstannyl)penta-2,4-dienoate (S44):

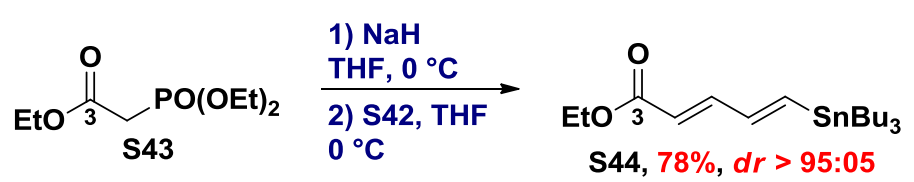

Compound S44 was prepared according to the literature procedure: Kim, H. J.; Choi, S.-h.; Jeon, B.-s.; Kim, N.; Pongdee, R.; Wu, Q.; Liu, H.-w. Angew. Chem., Int. Ed. 2014, 53, 13553.

Physical state: pale yellow oil

TLC: $R_{f}=0.68(95: 05 \%$ hexane:EtOAc) 
${ }^{1} \mathrm{H}$ NMR (600 MHz, $\left.\mathrm{CDCl}_{3}\right) \delta 0.88(\mathrm{t}, J=7.2 \mathrm{~Hz}, 9 \mathrm{H}), 0.92-1.00(\mathrm{~m}, 6 \mathrm{H}), 1.28(\mathrm{t}, J=7.2 \mathrm{~Hz}$, $3 \mathrm{H}$ ), 1.30 (sext, $J=7.2 \mathrm{~Hz}, 6 \mathrm{H}$ ), 1.42-1.55 (m, 6H), 4.19 (q, $J=7.2 \mathrm{~Hz}, 2 \mathrm{H}), 5.79$ (d, J = 15.2 $\mathrm{Hz}, 1 \mathrm{H}$ ), 6.64 (dd, $J=18.8$ and $10.4 \mathrm{~Hz}, 1 \mathrm{H}), 6.80(\mathrm{~d}, J=18.8 \mathrm{~Hz}, 1 \mathrm{H}), 7.17$ (dd, $J=15.2$ and $10.4 \mathrm{~Hz}, 1 \mathrm{H})$.

${ }^{13} \mathrm{C}$ NMR (150 MHz, $\left.\mathrm{CDCl}_{3}\right) \delta 9.6,13.7,14.3,27.2,29.0,60.3,119.9,144.2,146.3,147.2$, 167.4 .

IR (film) $v_{\max } / \mathrm{cm}^{-1} 2957,2927,2872,2853,1718,1626,1559,1464,1366,1294,1274,1211$, $1151,1100,1045,1007,960,871,787,691,663,597$.

(2E,4E)-5-(tributylstannyl)penta-2,4-dien-1-ol (7):

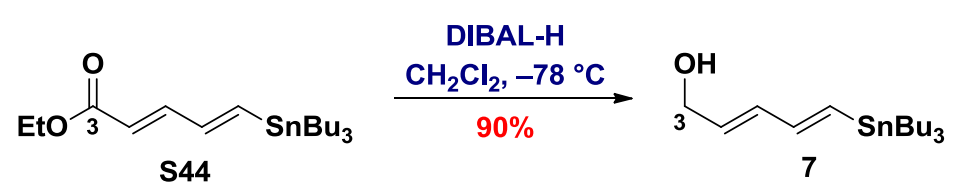

To a solution of ester $\mathbf{S} 44$ (463 mg, $1.12 \mathrm{mmol}, 100 \mathrm{~mol} \%)$ in $\mathrm{CH}_{2} \mathrm{Cl}_{2}(2.4 \mathrm{~mL}, 0.47 \mathrm{M})$ at -78 ${ }^{\circ} \mathrm{C}$ was added DIBAL-H (2.8 mL, $2.8 \mathrm{mmol}, 1 \mathrm{M}$ em hexano, $250 \mathrm{~mol} \%$ ) over 40 min using a syringe pump. After $30 \mathrm{~min}$, the reaction was quenched by the addition of saturated aqueous solution of sodium potassium tartrate $(10 \mathrm{~mL})$ and warmed to room temperature. After $3 \mathrm{~h}$, the layers were separated and the aqueous layer was extracted with $\mathrm{Et}_{2} \mathrm{O}(3 \times 25 \mathrm{~mL})$. The combined organic layers were washed with brine $(25 \mathrm{~mL})$, dried over $\mathrm{MgSO}_{4}$, filtered, and concentrated under reduced pressure. The residue was purified by flash column chromatography using a solution of hexane/ethyl acetate $(80: 20)$ as the eluent to provide alcohol 7 (376 mg, $1.01 \mathrm{mmol}, 90 \%$ ).

Physical state: colorless oil

TLC: $R_{f}=0.53$ (20\% EtOAc in Hexano)

${ }^{1} \mathrm{H}$ NMR $\left(500 \mathrm{MHz}, \mathrm{CDCl}_{3}\right) \delta 0.88(\mathrm{t}, J=7.3 \mathrm{~Hz}, 9 \mathrm{H}), 0.89(\mathrm{t}, J=7.5 \mathrm{~Hz}, 6 \mathrm{H}), 1.26-1.35(\mathrm{~m}$, $6 \mathrm{H}), 1.46-1.52(\mathrm{~m}, 6 \mathrm{H}), 4.19(\mathrm{t}, J=5.8 \mathrm{~Hz}, 2 \mathrm{H}), 5.78(\mathrm{dt}, J=15.1$ and $5.8 \mathrm{~Hz}, 1 \mathrm{H}), 6.22(\mathrm{dd}, J$ $=15.1$ and $10.0 \mathrm{~Hz}, 1 \mathrm{H}), 6.25(\mathrm{~d}, J=18.8 \mathrm{~Hz}, 1 \mathrm{H}), 6.52(\mathrm{dd}, J=18.8$ and $10.0 \mathrm{~Hz}, 1 \mathrm{H})$.

${ }^{13} \mathrm{C}$ NMR (125 MHz, $\left.\mathrm{CDCl}_{3}\right) \delta 9.5,13.7,27.2,29.1,63.4,130.7,134.6,135.1,145.9$.

IR (film) $v_{\max } / \mathrm{cm}^{-1} 3347,2956,2924,2852,1566,1464,1418,1376,1083,999,659$. 


\section{Completion of the Total Synthesis of (-)-Marinisporolide C}

(2S,3S)-2-((4S,6S)-6-(((4R,6R)-6-((E)-3-((2R,4R,8R,10S)-4,10-bis $(($ tert-

butyldimethylsilyl)oxy)-8-((2E,4E)-5-iodopenta-2,4-dien-1-yl)-1,7-

dioxaspiro[5.5] undecan-2-yl)allyl)-2,2-dimethyl-1,3-dioxan-4-yl)methyl)-2,2-dimethyl-1,3dioxan-4-yl)-4-methylpentan-3-yl 2-(diethoxyphosphoryl)propanoate (27):
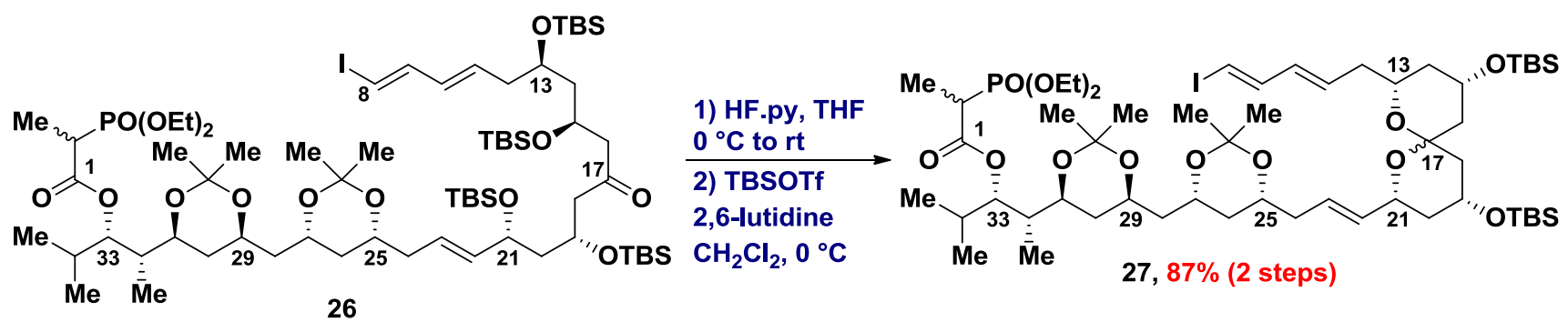

To a solution of compound 26 (50 mg, $35.0 \mu \mathrm{mol}, 100 \mathrm{~mol} \%)$ in THF $(1.9 \mathrm{~mL}, 0.02 \mathrm{M})$ at $0{ }^{\circ} \mathrm{C}$, was added HF.pyridine (50.3 $\mathrm{LL}, 1.94 \mathrm{mmol}, 5540 \mathrm{~mol} \%)$. The reaction medium was stirred for $5 \mathrm{~min}$ at $0 \stackrel{\circ}{\circ} \mathrm{C}$ and warmed to room temperature. After $24 \mathrm{~h}$, the reaction medium was cooled to $0{ }^{\circ} \mathrm{C}$ and HF.pyridine $(50.3 \mu \mathrm{L}, 1.94 \mathrm{mmol}, 5540 \mathrm{~mol} \%)$ was added The reaction medium was stirred for $5 \mathrm{~min}$ at $0 \stackrel{\circ}{\circ} \mathrm{C}$ and warmed to room temperature. After $24 \mathrm{~h}$, the reaction medium was cooled to $0^{\circ} \mathrm{C}$ and quenched by the addition of TMSOMe $(0.50 \mathrm{~mL})$. The reaction medium was stirred for $5 \mathrm{~min}$ at $0 \stackrel{\circ}{\circ}$ and $15 \mathrm{~min}$ at room temperature. After this period, the volatiles were removed under reduced pressure, and the residue was used in the next step without further purification.

To a solution of resulting residue (theor. $35.0 \mu \mathrm{mol}, 100 \mathrm{~mol} \%)$ in $\mathrm{CH}_{2} \mathrm{Cl}_{2}(0.58 \mathrm{~mL}, 0.06 \mathrm{M})$ at $0{ }^{\circ} \mathrm{C}$ were added 2,6-lutidine $(0.04 \mathrm{~mL}, 0.43 \mathrm{mmol}, 1200 \mathrm{~mol} \%)$ and TBSOTf $(0.05 \mathrm{~mL}, 0.22$ $\mathrm{mmol}, 630 \mathrm{~mol} \%$ ) dropwise. After $1 \mathrm{~h}$, the reaction mixture was loaded directly onto a flash column chromatography using a solution of hexane/ $\mathrm{CH}_{2} \mathrm{Cl}_{2}$ /ethyl acetate $(30: 30: 40)$ as the eluent to provide the compound 27 ( $36 \mathrm{mg}, 30.4 \mu \mathrm{mol}, 87 \%$ over 2 steps).

Physical state: pale yellow oil

TLC: $R_{f}=0.67$ (50:50 hexane:EtOAc) 
(2S,3S)-2-((4S,6S)-6-(((4R,6R)-6-((E)-3-((2R,4R,8R,10S)-4,10-bis)((tert-

butyldimethylsilyl)oxy)-8-((2E,4E,6E,8E)-10-hydroxydeca-2,4,6,8-tetraen-1-yl)-1,7-

dioxaspiro[5.5]undecan-2-yl)allyl)-2,2-dimethyl-1,3-dioxan-4-yl)methyl)-2,2-dimethyl-1,3dioxan-4-yl)-4-methylpentan-3-yl 2-(diethoxyphosphoryl)propanoate (S45):

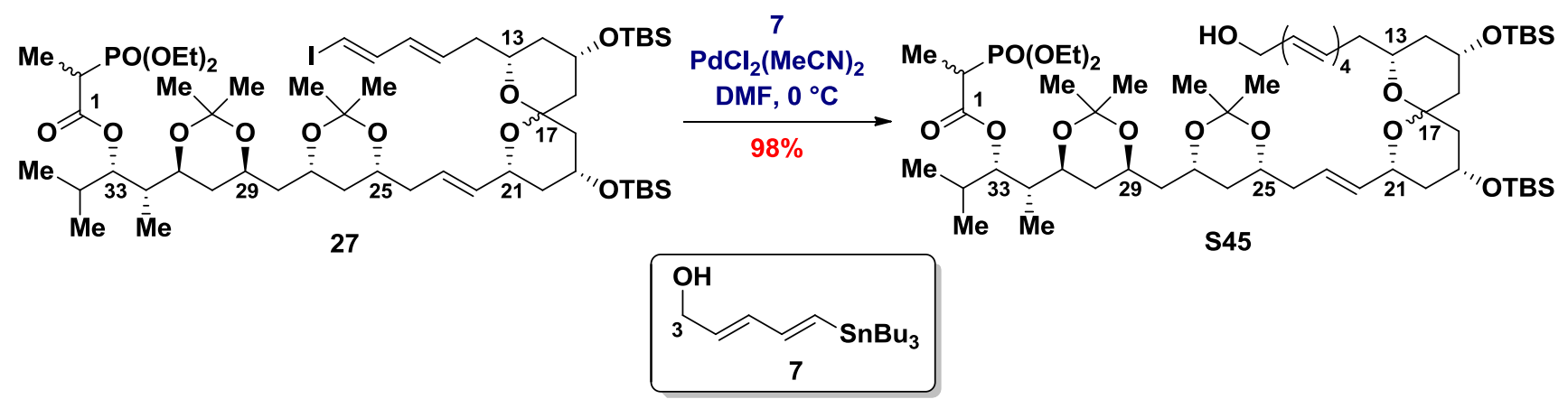

NOTE: This entire experimental procedure was performed in the dark.

To a solution of compound 27 (36 mg, $30.4 \mu \mathrm{mol}, 100 \mathrm{~mol} \%$ ) and stannane 7 (34 mg, 91.2 $\mu \mathrm{mol}, 300 \mathrm{~mol} \%)$ in DMF $(3.4 \mathrm{~mL}, 8.9 \mathrm{mM})$ at $0{ }^{\circ} \mathrm{C}$ was added a solution of $\mathrm{PdCl}_{2}\left(\mathrm{MeCN}_{2}\right.$ (2.4 mg, $9.12 \mu \mathrm{mol}, 30 \mathrm{~mol} \%)$ in DMF $(1.0 \mathrm{~mL}, 9 \mathrm{mM})$ dropwise. After $30 \mathrm{~min}$, the reaction was diluted with $\mathrm{Et}_{2} \mathrm{O}(10 \mathrm{~mL})$ and quenched by the addition of $\mathrm{pH} 7$ phosphate buffer $(5 \mathrm{~mL})$. The layers were separated, and the aqueous layer was extracted with $\mathrm{Et}_{2} \mathrm{O}(3 \times 10 \mathrm{~mL})$. The combined organic layers were washed with brine $(10 \mathrm{~mL})$, dried over $\mathrm{Na}_{2} \mathrm{SO}_{4}$, filtered, and concentrated under reduced pressure. The residue was purified by flash column chromatography using a solution of hexane/ethyl acetate/ $\mathrm{Et}_{3} \mathrm{~N}(50: 48: 02)$ as the eluent to provide compound S45 (34 mg, $29.8 \mu \mathrm{mol}, 98 \%$ ).

Physical state: pale yellow oil

TLC: $R_{f}=0.37$ (50:50 hexane:EtOAc)

${ }^{1} \mathrm{H}$ NMR (400 MHz, $\left.\mathrm{C}_{6} \mathrm{D}_{6}\right) \delta$ 0.01-0.15 (m, 12H), 0.81-2.50 (m, 68H), 2.92-3.04 (m, 1H), 3.41-4.45 (m, 14H), 5.48-6.77 (m, 11H). 
(2S,3S)-2-((4S,6S)-6-(((4R,6R)-6-((E)-3-((2R,4R,8R,10S)-4,10-bis ((tert-

butyldimethylsilyl)oxy)-8-((2E,4E,6E,8E)-10-oxodeca-2,4,6,8-tetraen-1-yl)-1,7-

dioxaspiro[5.5] undecan-2-yl)allyl)-2,2-dimethyl-1,3-dioxan-4-yl)methyl)-2,2-dimethyl-1,3dioxan-4-yl)-4-methylpentan-3-yl 2-(diethoxyphosphoryl)propanoate (28):

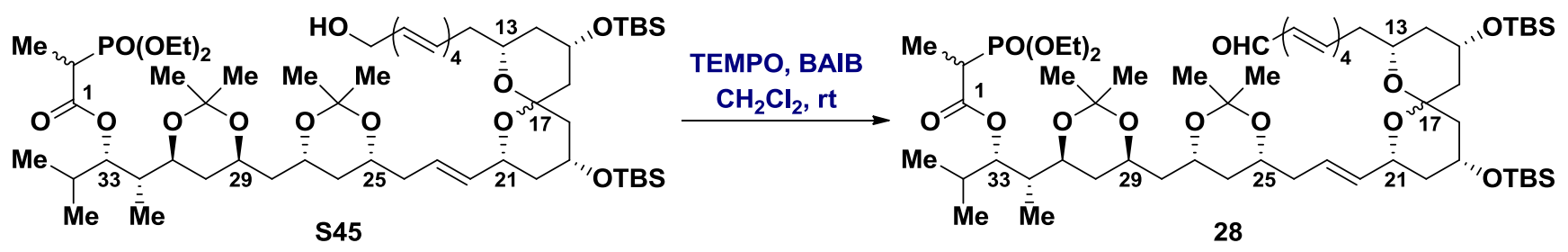

NOTE: This entire experimental procedure was performed in the dark. This compound was used immediately after purification.

To a solution of compound S45 (34 mg, $29.8 \mu \mathrm{mol}, 100 \mathrm{~mol} \%)$ in $\mathrm{CH}_{2} \mathrm{Cl}_{2}(0.3 \mathrm{~mL}, 0.10 \mathrm{M})$ at room temperature were added TEMPO (0.9 mg, $5.96 \mu \mathrm{mol}, 20 \mathrm{~mol} \%)$ and BAIB (29 mg, 89.4 $\mu \mathrm{mol}, 300 \mathrm{~mol} \%)$. After $1.5 \mathrm{~h}$, volatiles were removed in a gentle stream of argon gas. The residue was purified by flash column chromatography using a solution of hexane/ethyl acetate/ $\mathrm{Et}_{3} \mathrm{~N}$ (50:48:02) as the eluent to provide aldehyde 28, which was immediately used in the next step without further purification.

Physical state: pale yellow oil

TLC: $R_{f}=0.46(50: 50$ hexane:EtOAc)

(-)-Marinisporolide C (3):
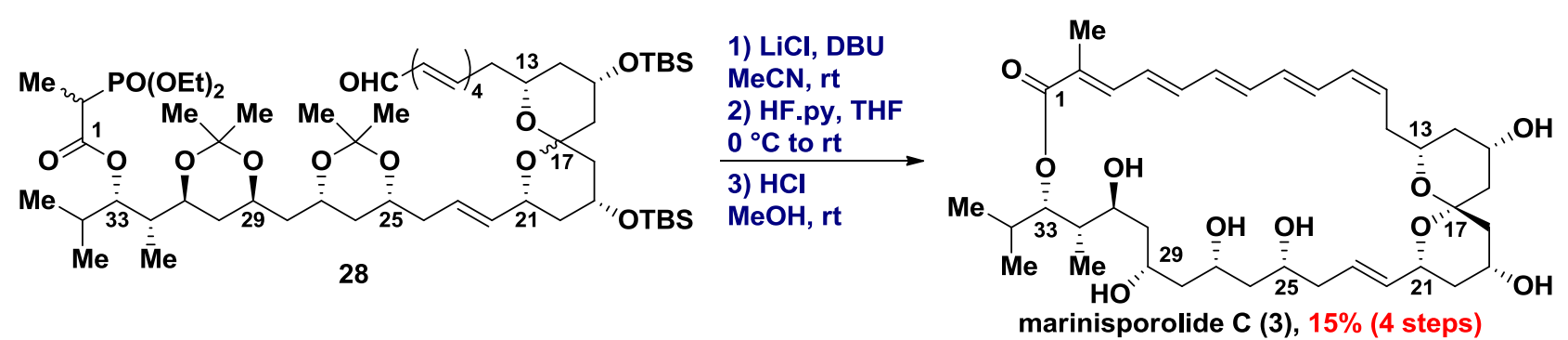

NOTE: This entire experimental procedure was performed in the dark.

A separate dry flask had been charged with $\mathrm{LiCl}(26 \mathrm{mg}, 0.626 \mathrm{mmol}, 2100 \mathrm{~mol} \%$ ) and heated at $140{ }^{\circ} \mathrm{C}$ overnight under high vacuum. ${ }^{2}$ After allowing the flask to cool to room temperature, a solution of aldehyde 28 (theor. $29.8 \mu \mathrm{mol}, 100 \mathrm{~mol} \%$ ) in $\mathrm{MeCN}(24.5 \mathrm{~mL}, 1.2$

\footnotetext{
${ }^{2}$ This procedure was carried out the night before the preparation of aldehyde 28 , so that the activation of $\mathrm{LiCl}$ and preparation of compound $\mathbf{2 8}$ ended at the same time.
} 
$\mathrm{mM}$ ) was added using a cannula. The mixture was stirred for 30 min under these conditions, and DBU (71 $\mu \mathrm{L}, 0.477 \mathrm{mmol}, 1600 \mathrm{~mol} \%)$ was added dropwise. After $48 \mathrm{~h}$, the reaction was diluted with $\mathrm{Et}_{2} \mathrm{O}(50 \mathrm{~mL})$ and quenched by the addition of $\mathrm{pH} 7$ phosphate buffer $(50 \mathrm{~mL})$. The layers were separated and the aqueous layer was extracted with $\mathrm{Et}_{2} \mathrm{O}(3 \times 50 \mathrm{~mL})$. The combined organic layers were washed with brine $(100 \mathrm{~mL})$, dried over $\mathrm{Na}_{2} \mathrm{SO}_{4}$, filtered, and concentrated under reduced pressure, and the residue was used in the next step without further purification.

TLC: $R_{f}=0.69(80: 20$ hexane:EtOAc)

To a solution of resulting residue (theor. $29.8 \mu \mathrm{mol}, 100 \mathrm{~mol} \%$ ) in THF $(2.2 \mathrm{~mL}, 0.01 \mathrm{M})$ at 0 ${ }^{\circ} \mathrm{C}$, was added HF.pyridine $(43.0 \mu \mathrm{L}, 1.65 \mathrm{mmol}, 5540 \mathrm{~mol} \%)$. The reaction medium was stirred for $5 \mathrm{~min}$ at $0{ }^{\circ} \mathrm{C}$ and warmed to room temperature. After $24 \mathrm{~h}$, the reaction medium was cooled to $0{ }^{\circ} \mathrm{C}$ and quenched by the addition of TMSOMe $(0.43 \mathrm{~mL})$. The reaction medium was stirred for $5 \mathrm{~min}$ at $0{ }^{\circ} \mathrm{C}$ and $15 \mathrm{~min}$ at room temperature. After this period, the volatiles were removed under reduced pressure, and the residue was purified by flash column chromatography using a solution of $\mathrm{CHCl}_{3} / \mathrm{MeOH}$ (95:05) as the eluente.

\section{TLC for compound less polar: $R_{f}=0.48\left(95: 05 \mathrm{CHCl}_{3}: \mathrm{MeOH}\right)$}

TLC for compound more polar: $R_{f}=0.31\left(95: 05 \mathrm{CHCl}_{3}: \mathrm{MeOH}\right)$

To a solution of resulting residue (theor. $29.8 \mu \mathrm{mol}, 100 \mathrm{~mol} \%$ ) in $\mathrm{MeOH}(30 \mathrm{~mL}, 1 \mathrm{mM}$ ) at room temperature was added $12 \mathrm{M}$ aqueous solution of $\mathrm{HCl}(210 \mu \mathrm{L}, 2.52 \mathrm{mmol}, 8400 \mathrm{~mol} \%)$. After $21 \mathrm{~h}, \mathrm{Et}_{3} \mathrm{~N}(0.42 \mathrm{~mL}, 3.0 \mathrm{mmol}, 10000 \mathrm{~mol} \%)$ was added and the volatiles were removed under reduced pressure. The residue was purified by flash column chromatography using a solution of $\mathrm{CHCl}_{3} / \mathrm{MeOH}(85: 15)$ as the eluent to provide the (-)-marinisporolide $\mathrm{C}$ (3) (3.0 $\mathrm{mg}, 4.45 \mu \mathrm{mol}, 15 \%$ over 4 steps).

Physical state: yellow solid

TLC: $R_{f}=0.33\left(85: 15 \mathrm{CHCl}_{3}: \mathrm{MeOH}\right)$.

Optical rotation: $[\alpha]_{D}^{20}-7(c 0.03, \mathrm{MeOH})$ 
${ }^{1} \mathrm{H}$ NMR (600 MHz, DMSO-d $)_{6} \delta 0.80(\mathrm{~d}, J=6.8 \mathrm{~Hz}, 3 \mathrm{H}), 0.88(\mathrm{~d}, J=6.8 \mathrm{~Hz}, 3 \mathrm{H}), 0.90(\mathrm{~d}, J=$ $7.0 \mathrm{~Hz}, 3 \mathrm{H}), 0.91(\mathrm{~m}, 1 \mathrm{H}), 1.00(\mathrm{q}, J=11.5 \mathrm{~Hz}, 1 \mathrm{H}), 1.06(\mathrm{q}, J=11.5 \mathrm{~Hz}, 1 \mathrm{H}), 1.17(\mathrm{~m}, 1 \mathrm{H})$, $1.18(\mathrm{~m}, 1 \mathrm{H}), 1.36(\mathrm{~m}, 1 \mathrm{H}), 1.38(\mathrm{~m}, 1 \mathrm{H}), 1.44(\mathrm{~m}, 1 \mathrm{H}), 1.54(\mathrm{~m}, 1 \mathrm{H}), 1.59(\mathrm{~m}, 1 \mathrm{H}), 1.73$ (quint, $J=7.3 \mathrm{~Hz}, 1 \mathrm{H}), 1.80(\mathrm{~m}, 1 \mathrm{H}), 1.81(\mathrm{~m}, 1 \mathrm{H}), 1.82(\mathrm{~m}, 1 \mathrm{H}), 1.89(\mathrm{~s}, 3 \mathrm{H}), 1.89(\mathrm{~m}, 1 \mathrm{H})$, $1.92(\mathrm{~m}, 1 \mathrm{H}), 2.09(\mathrm{dd}, J=12.4$ and $6.4 \mathrm{~Hz}, 1 \mathrm{H}), 2.22(\mathrm{dd}, J=13.0$ and $3.0 \mathrm{~Hz}, 1 \mathrm{H}), 2.49(\mathrm{~m}$, $1 \mathrm{H}), 3.15(\mathrm{~m}, 1 \mathrm{H}), 3.30(\mathrm{~m}, 1 \mathrm{H}), 3.50(\mathrm{~m}, 1 \mathrm{H}), 3.52(\mathrm{~m}, 1 \mathrm{H}), 3.73(\mathrm{~m}, 1 \mathrm{H}), 3.81(\mathrm{~m}, 1 \mathrm{H}), 3.84$ $(\mathrm{m}, 1 \mathrm{H}), 4.17$ (ddd, $J=11.3,7.5$ and $1.7 \mathrm{~Hz}, 1 \mathrm{H}), 4.40(\mathrm{~d}, J=4.3 \mathrm{~Hz}, \mathrm{OH}-25), 4.44(\mathrm{~d}, J=5.1$ $\mathrm{Hz}, \mathrm{OH}-31$ ), 4.49 (br s, OH-19), 4.54 (br s, OH-27), 4.57 (br s, OH-29), 4.74 (br s, OH-15), $4.93(\mathrm{~d}, J=10.0 \mathrm{~Hz}, 1 \mathrm{H}$ ), 5.26 (dd, $J=15.4$ and $7.3 \mathrm{~Hz}, 1 \mathrm{H}$ ), 5.34 (ddd, $J=15.4,7.5$ and 6.0 $\mathrm{Hz}, 1 \mathrm{H}), 5.57(\mathrm{td}, J=10.4$ and $6.4 \mathrm{~Hz}, 1 \mathrm{H}), 6.21(\mathrm{t}, J=11.4 \mathrm{~Hz}, 1 \mathrm{H}), 6.34(\mathrm{dd}, J=14.6$ and $11.2 \mathrm{~Hz}, 1 \mathrm{H}), 6.45(\mathrm{dd}, J=14.6$ and $10.2 \mathrm{~Hz}, 1 \mathrm{H}), 6.62(\mathrm{dd}, J=14.6$ and $11.2 \mathrm{~Hz}, 1 \mathrm{H}), 6.63$ (dd, $J=14.7$ and $9.6 \mathrm{~Hz}, 1 \mathrm{H}$ ), $6.66(\mathrm{dd}, J=14.7$ and $10.2 \mathrm{~Hz}, 1 \mathrm{H}), 6.72(\mathrm{dd}, J=14.6$ and $11.4 \mathrm{~Hz}, 1 \mathrm{H}), 7.05(\mathrm{~d}, J=9.6 \mathrm{~Hz}, 1 \mathrm{H})$.

${ }^{13} \mathrm{C}$ NMR (150 MHz, DMSO- $\boldsymbol{d}_{6}$ ) $\delta 8.9,13.0,18.9,19.9,29.5,34.0,38.2,40.5,40.6,41.0$, $41.3,41.7,42.2,45.0,45.5,61.8,63.8,65.6,66.7,68.1,69.3,69.8,70.7,78.3,98.8,126.3$, $127.2,128.4,130.3,131.0,131.7,131.8,132.1,132.8,137.4,137.6,140.1,167.4$.

IR (film) $v_{\text {max }} / \mathrm{cm}^{-1} 3417,2958,2925,2854,1679,1644,1579,1448,1233,1099,1025,987$.

HRMS (ESI FTMS) $\mathrm{m} / \mathrm{z}$ calcd for $\mathrm{C}_{38} \mathrm{H}_{58} \mathrm{O}_{10} \mathrm{Na}[\mathrm{M}+\mathrm{Na}]^{+}: 697.3922$, found: 697.3923 .

UV-Vis (MeOH) $\lambda_{\max } / \mathbf{n m : ~ 2 6 1 , ~ 3 6 0 , ~} 373$. 


\section{Comparison for Natural and Synthetic Marinisporolide C}

Table S1: ${ }^{1} \mathrm{H}$ and ${ }^{13} \mathrm{C}$ NMR chemical shifts for natural and synthetic marinisporolide $\mathrm{C}(\mathbf{3})$

\begin{tabular}{|c|c|c|c|c|c|c|}
\hline \multirow[b]{2}{*}{ Position } & \multicolumn{3}{|c|}{ Natural Product } & \multicolumn{3}{|c|}{ Synthetic Product } \\
\hline & $\delta^{13} \mathrm{C}$ & $\delta^{1} \mathrm{H}$ & multiplicity ( $\mathrm{J}$ in Hz) & $\delta^{13} \mathrm{C}^{a}$ & $\delta^{1} \mathrm{H}^{a}$ & multiplicity ( $\mathrm{J}$ in $\mathrm{Hz}$ ) \\
\hline 1 & 167.4 & & & 167.4 & & \\
\hline 2 & 126.3 & & & 126.3 & & \\
\hline 3 & 137.5 & 7.05 & $d(9.5)$ & 137.6 & 7.05 & $d(9.6)$ \\
\hline 4 & 127.2 & 6.62 & $\mathrm{dd}(15.0,9.5)$ & 127.2 & 6.63 & dd $(14.7,9.6)$ \\
\hline 5 & 137.5 & 6.66 & $\mathrm{dd}(15.0,10.0)$ & 140.1 & 6.66 & $\mathrm{dd}(14.7,10.2)$ \\
\hline 6 & 131.7 & 6.45 & $\mathrm{dd}(14.5,10.0)$ & 131.8 & 6.45 & $\mathrm{dd}(14.6,10.2)$ \\
\hline 7 & 140.1 & 6.62 & $\mathrm{dd}(14.5,11.0)$ & 137.4 & 6.62 & $\mathrm{dd}(14.6,11.2)$ \\
\hline 8 & 131.7 & 6.34 & $\mathrm{dd}(14.5,11.0)$ & 131.7 & 6.34 & $\mathrm{dd}(14.6,11.2)$ \\
\hline 9 & 132.1 & 6.72 & $\mathrm{dd}(14.5,11.5)$ & 132.1 & 6.72 & $\mathrm{dd}(14.6,11.4)$ \\
\hline 10 & 131.0 & 6.21 & $\mathrm{dd}(11.5,11.5)$ & 131.0 & 6.21 & $\mathrm{t}(11.4)$ \\
\hline 11 & 130.3 & 5.56 & br ddd $(11.5,11.0,6.0)$ & 130.3 & 5.57 & $\operatorname{td}(10.4,6.4)$ \\
\hline $12 \mathrm{a}$ & \multirow{2}{*}{34.1} & 2.09 & br ddd $(6.0,6.0,6.0)$ & \multirow{2}{*}{34.0} & 2.09 & $\mathrm{dd}(12.4,6.4)$ \\
\hline $12 b$ & & 2.45 & $\mathrm{~m}$ & & 2.49 & $\mathrm{~m}$ \\
\hline 13 & 69.3 & 3.30 & $\mathrm{~m}$ & 69.3 & 3.30 & $\mathrm{~m}$ \\
\hline $14 a$ & \multirow{2}{*}{41.5} & 1.07 & $\operatorname{ddd}(11.0,11.0,11.0)$ & \multirow{2}{*}{41.3} & 1.06 & $q(11.5)$ \\
\hline $14 b$ & & 1.80 & $\mathrm{~m}$ & & 1.82 & $\mathrm{~m}$ \\
\hline 15 & 63.8 & 3.71 & br m & 63.8 & 3.73 & $\mathrm{~m}$ \\
\hline $16 a$ & \multirow{2}{*}{45.5} & 1.16 & $\mathrm{~m}$ & \multirow{2}{*}{45.0} & 1.17 & $\mathrm{~m}$ \\
\hline $16 b$ & & 1.80 & $\mathrm{~m}$ & & 1.80 & $\mathrm{~m}$ \\
\hline 17 & 98.8 & & & 98.8 & & \\
\hline $18 a$ & \multirow{2}{*}{38.3} & 0.84 & $\mathrm{~m}$ & \multirow{2}{*}{38.2} & 0.91 & $\mathrm{~m}$ \\
\hline $18 \mathrm{~b}$ & & 2.22 & br dd $(13.0,3.0)$ & & 2.22 & $d d(13.0,3.0)$ \\
\hline 19 & 61.8 & 3.50 & br m & 61.8 & 3.52 & $\mathrm{~m}$ \\
\hline $20 a$ & \multirow{2}{*}{41.5} & 1.00 & $\operatorname{ddd}(11.5,11.5,11.5)$ & \multirow{2}{*}{41.7} & 1.00 & $q(11.5)$ \\
\hline $20 \mathrm{~b}$ & & 1.57 & br d (11.5) & & 1.59 & $\mathrm{~m}$ \\
\hline 21 & 69.9 & 4.15 & br ddd $(11.5,7.5,2.0)$ & 69.8 & 4.17 & ddd $(11.3,7.5,1.7)$ \\
\hline 22 & 132.8 & 5.26 & $\mathrm{dd}(15.5,7.5)$ & 132.8 & 5.26 & $\mathrm{dd}(15.4,7.3)$ \\
\hline 23 & 128.4 & 5.34 & ddd $(15.5,7.5,6.0)$ & 128.4 & 5.34 & ddd $(15.4,7.5,6.0)$ \\
\hline $24 a$ & \multirow{2}{*}{40.5} & 1.82 & $\mathrm{~m}$ & \multirow{2}{*}{40.6} & 1.81 & $\mathrm{~m}$ \\
\hline $24 b$ & & 1.90 & $\mathrm{~m}$ & & 1.92 & $\mathrm{~m}$ \\
\hline 25 & 68.1 & 3.48 & br m & 68.1 & 3.50 & $\mathrm{~m}$ \\
\hline $26 a$ & \multirow{2}{*}{44.9} & 1.16 & $\mathrm{~m}$ & \multirow{2}{*}{45.5} & 1.18 & $\mathrm{~m}$ \\
\hline $26 b$ & & 1.35 & $\mathrm{~m}$ & & 1.38 & $\mathrm{~m}$ \\
\hline 27 & 65.6 & 3.80 & br m & 65.6 & 3.81 & $\mathrm{~m}$ \\
\hline $28 a$ & \multirow{2}{*}{42.1} & 1.35 & $\mathrm{~m}$ & 120 & & $m$ \\
\hline $28 b$ & & 1.39 & $\mathrm{~m}$ & 42.2 & 1,36 & $\mathrm{~m}$ \\
\hline 29 & 66.7 & 3.82 & br m & 66.7 & 3.84 & $\mathrm{~m}$ \\
\hline $30 a$ & 100 & 1.42 & $\mathrm{~m}$ & 105 & 1.44 & $\mathrm{~m}$ \\
\hline $30 \mathrm{~b}$ & 40.8 & 1.53 & br m & 40.5 & 1.54 & $\mathrm{~m}$ \\
\hline 31 & 70.7 & 3.14 & br m & 70.7 & 3.15 & $\mathrm{~m}$ \\
\hline 32 & 40.5 & 1.72 & $\mathrm{dq}(7.0,7.0)$ & 41.0 & 1.73 & quint (7.3) \\
\hline 33 & 78.3 & 4.93 & $d(9.5)$ & 78.3 & 4.93 & $d(10.0)$ \\
\hline 34 & 29.5 & 1.86 & $\mathrm{~m}$ & 29.5 & 1.89 & $\mathrm{~m}$ \\
\hline 35 & 19.9 & 0.80 & $d(6.5)$ & 19.9 & 0.80 & $d(6.8)$ \\
\hline 36 & 18.9 & 0.87 & $d(6.5)$ & 18.9 & 0.88 & $d(6.8)$ \\
\hline 37 & 8.9 & 0.91 & $\mathrm{~d}(7.0)$ & 8.9 & 0.90 & $\mathrm{~d}(7.0)$ \\
\hline 38 & 13.0 & 1.89 & $\mathrm{~s}$ & 13.0 & 1.89 & $\mathrm{~s}$ \\
\hline $\mathrm{OH}-15$ & & 4.73 & $d(5.0)$ & & 4.74 & br s \\
\hline $\mathrm{OH}-19$ & & 4.50 & $\operatorname{brd}(5.0)$ & & 4.49 & br s \\
\hline $\mathrm{OH}-25$ & & 4.40 & $d(5.0)$ & & 4.40 & $\mathrm{~d}(4.3)$ \\
\hline $\mathrm{OH}-27$ & & 4.53 & $\mathrm{~d}(4.0)$ & & 4.54 & br s \\
\hline $\mathrm{OH}-29$ & & 4.56 & $d(4.5)$ & & 4.57 & br s \\
\hline $\mathrm{OH}-31$ & & 4.43 & $d(6.0)$ & & 4.44 & $d(5.1)$ \\
\hline
\end{tabular}

${ }^{a}$ Assignment based on COSY, HSQC, and HMBC experiments.

Carbons C5 and C7 (red), C16 and C26 (blue), and C24, C30, and C32 (green) were probably misassigned in the original paper of isolation. We believe C5 should be: 140.1 ppm, C7 should be: 137.4 ppm, C16 should be: 45.0 ppm, C26 should be: 45.5 ppm, C24 should be: 40.6 ppm, C30 should be: 40.5 ppm, and C32 should be: 41.0 ppm. 


\section{${ }^{1} \mathrm{H}$ NMR, ${ }^{13} \mathrm{C}$ NMR, IR, and HRMS Spectra for New Compounds}

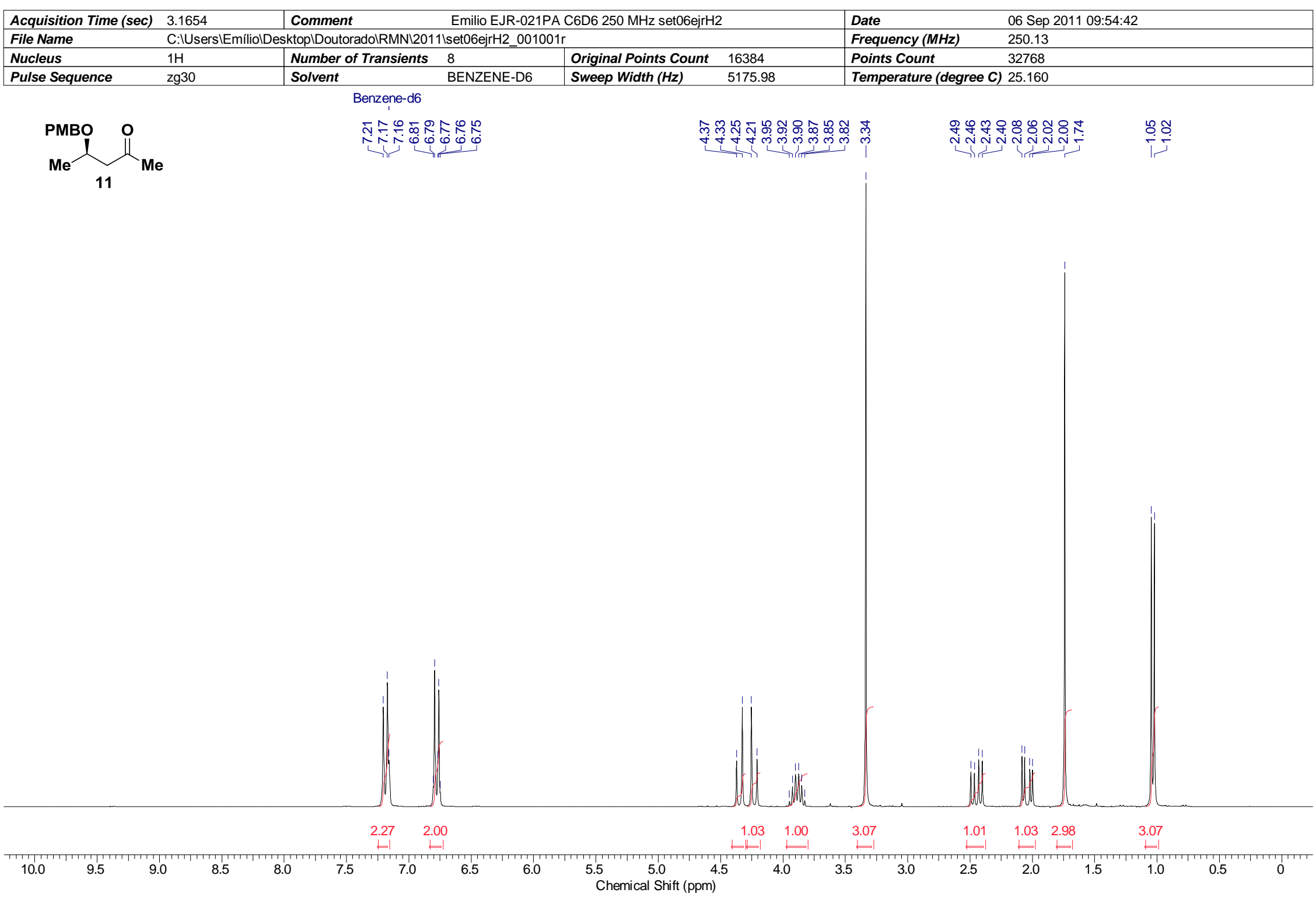

Figure 1: ${ }^{1} \mathrm{H}$ NMR spectrum of compound $11\left(250 \mathrm{MHz}, \mathrm{C}_{6} \mathrm{D}_{6}\right)$. 


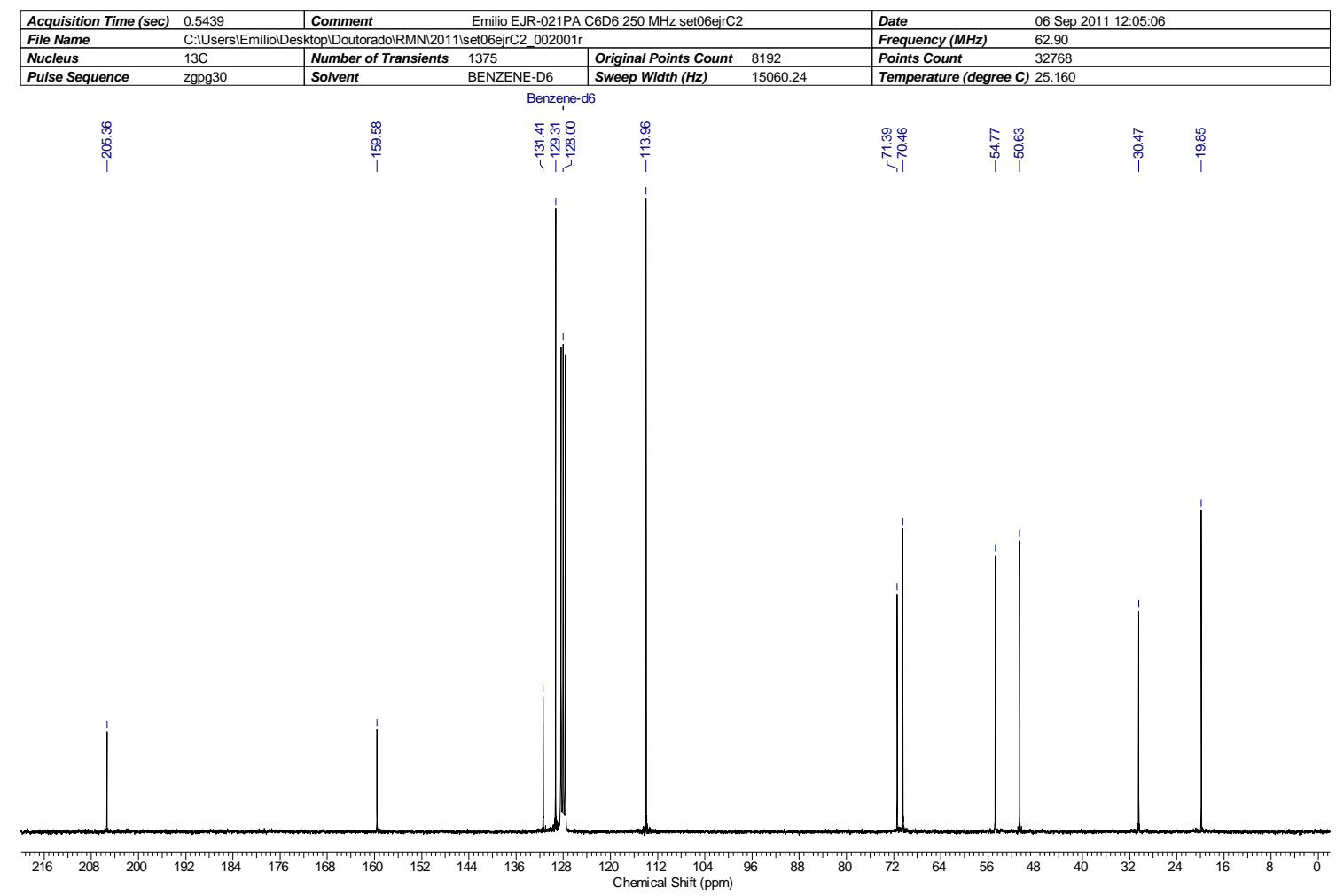

Figure 2: ${ }^{13} \mathrm{C}$ NMR spectrum of compound $11\left(62.5 \mathrm{MHz}, \mathrm{C}_{6} \mathrm{D}_{6}\right)$.

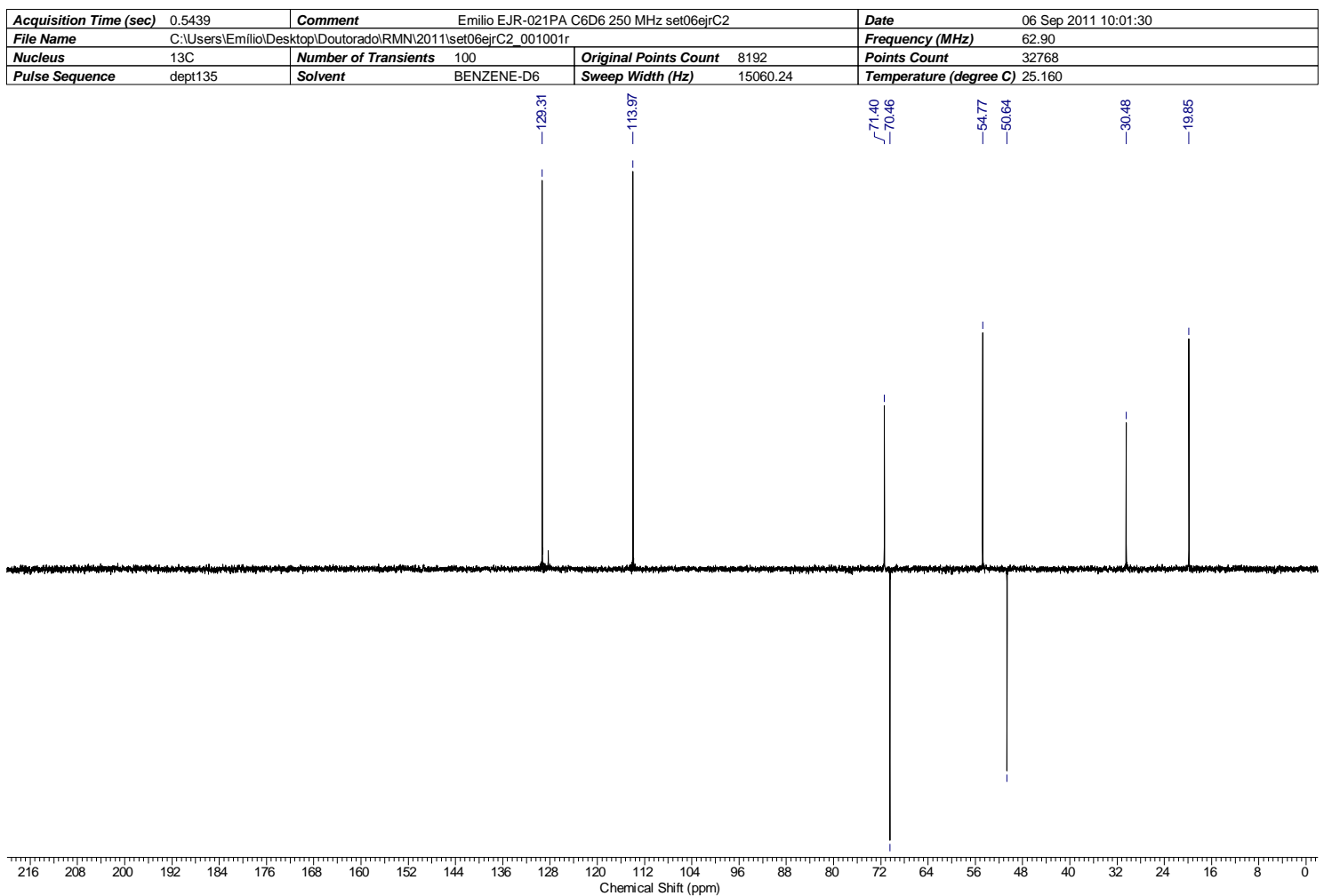

Figure 3: ${ }^{13} \mathrm{C}$ NMR (dept 135) spectrum of compound $11\left(62.5 \mathrm{MHz}, \mathrm{C}_{6} \mathrm{D}_{6}\right)$. 


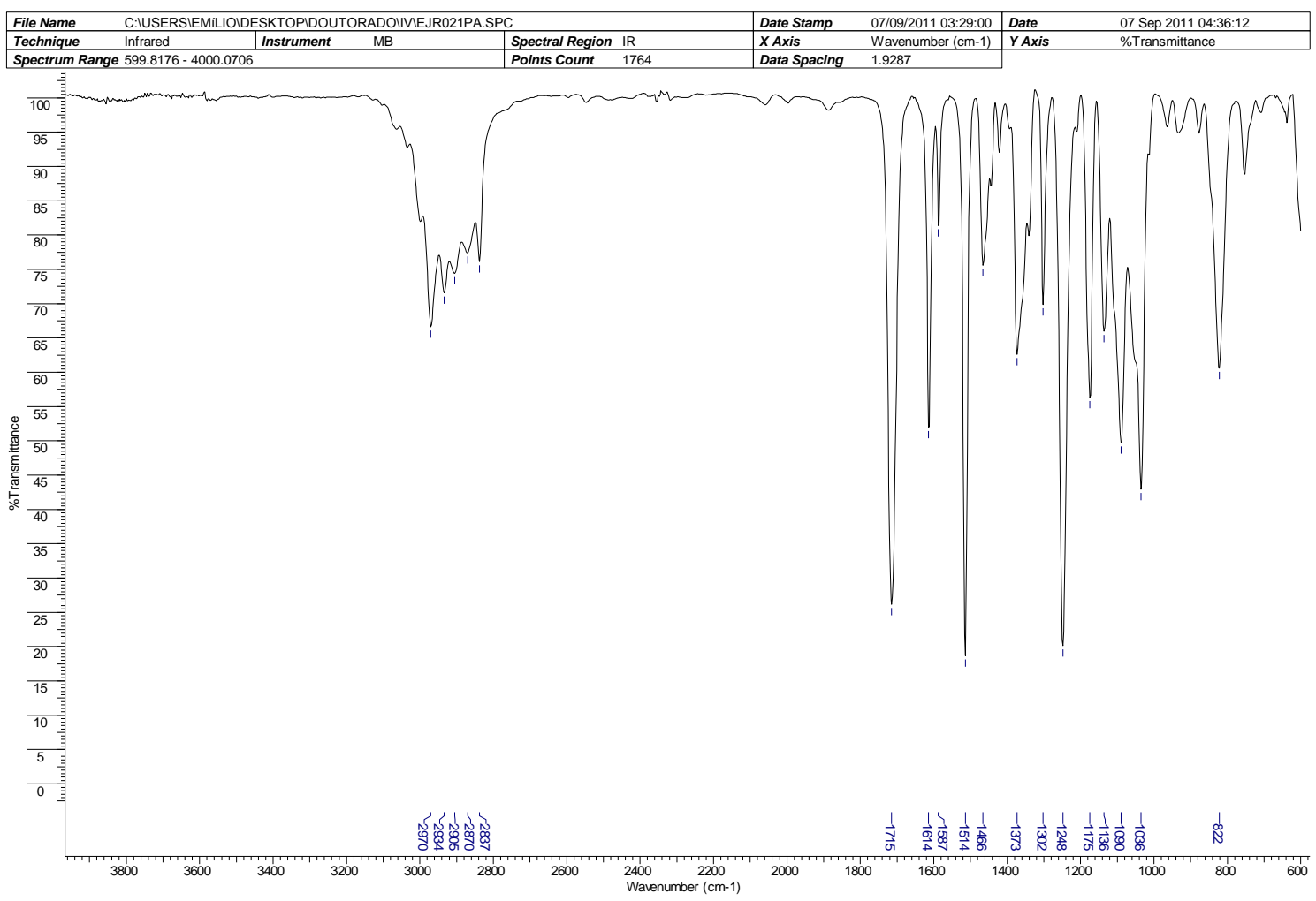

Figure 4: IR spectrum of compound 11. 


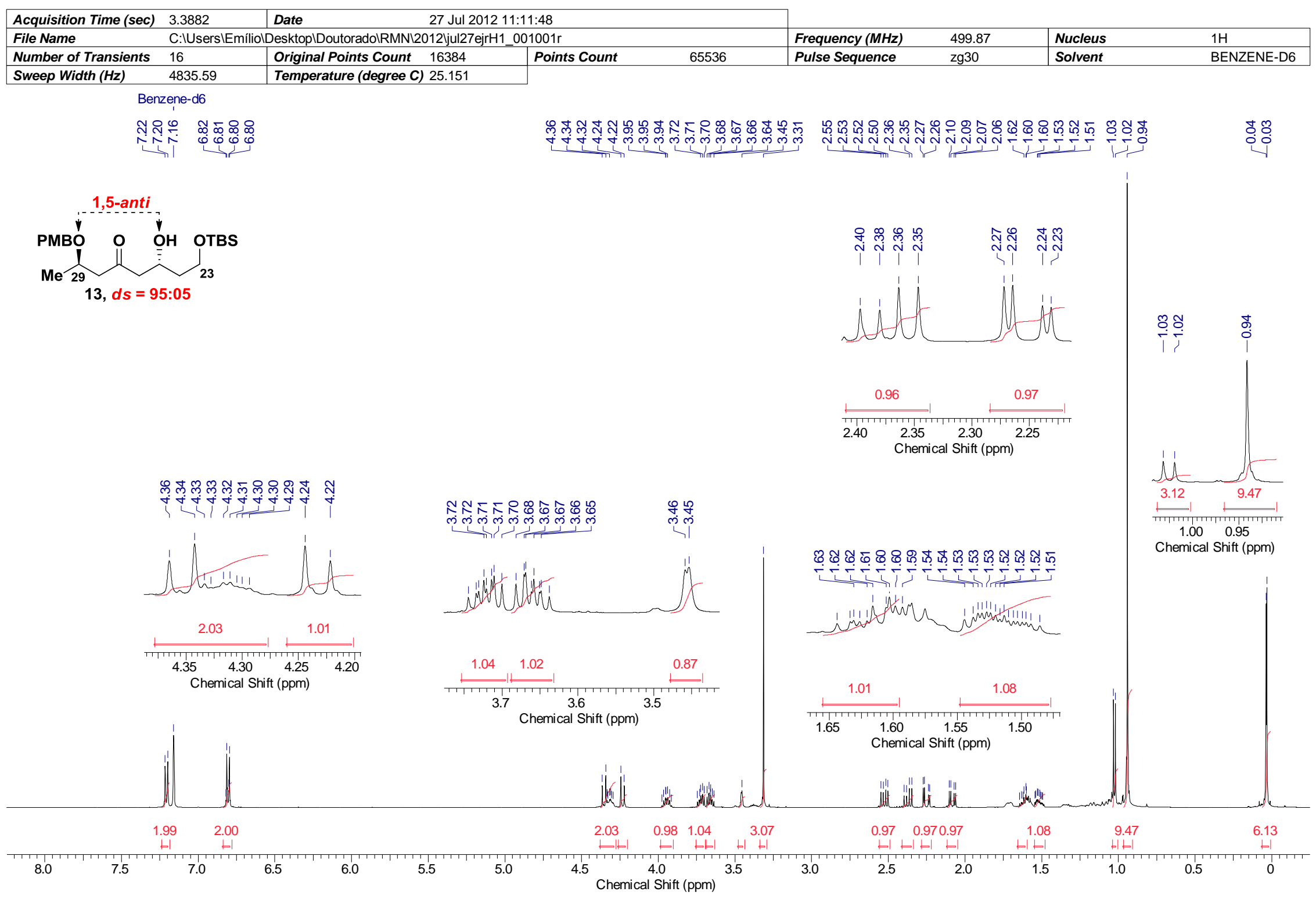

Figure 5: ${ }^{1} \mathrm{H}$ NMR spectrum of compound $13\left(500 \mathrm{MHz}, \mathrm{C}_{6} \mathrm{D}_{6}\right)$. 


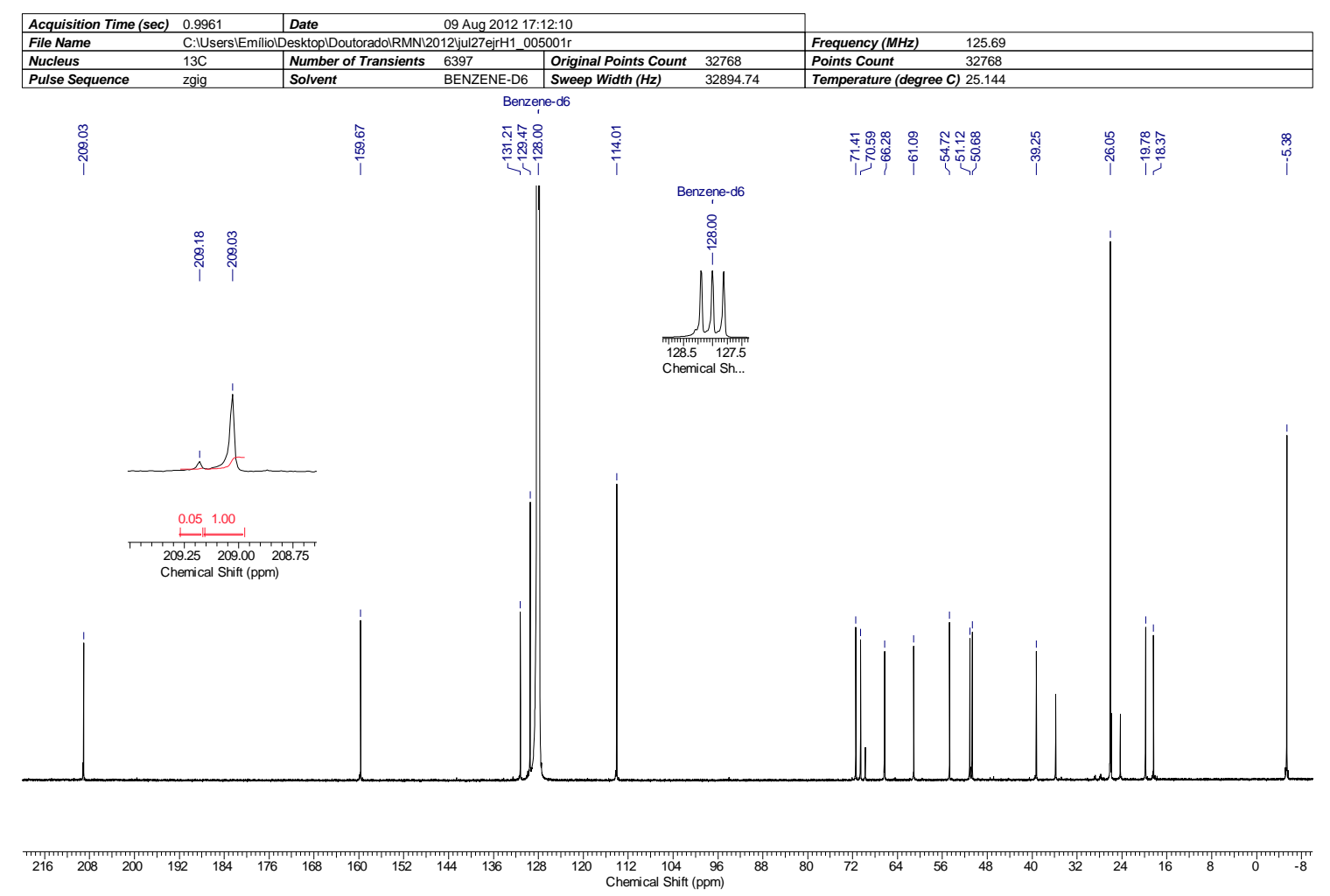

Figure 6: ${ }^{13} \mathrm{C}$ NMR spectrum of compound $13\left(125 \mathrm{MHz}, \mathrm{C}_{6} \mathrm{D}_{6}\right)$.

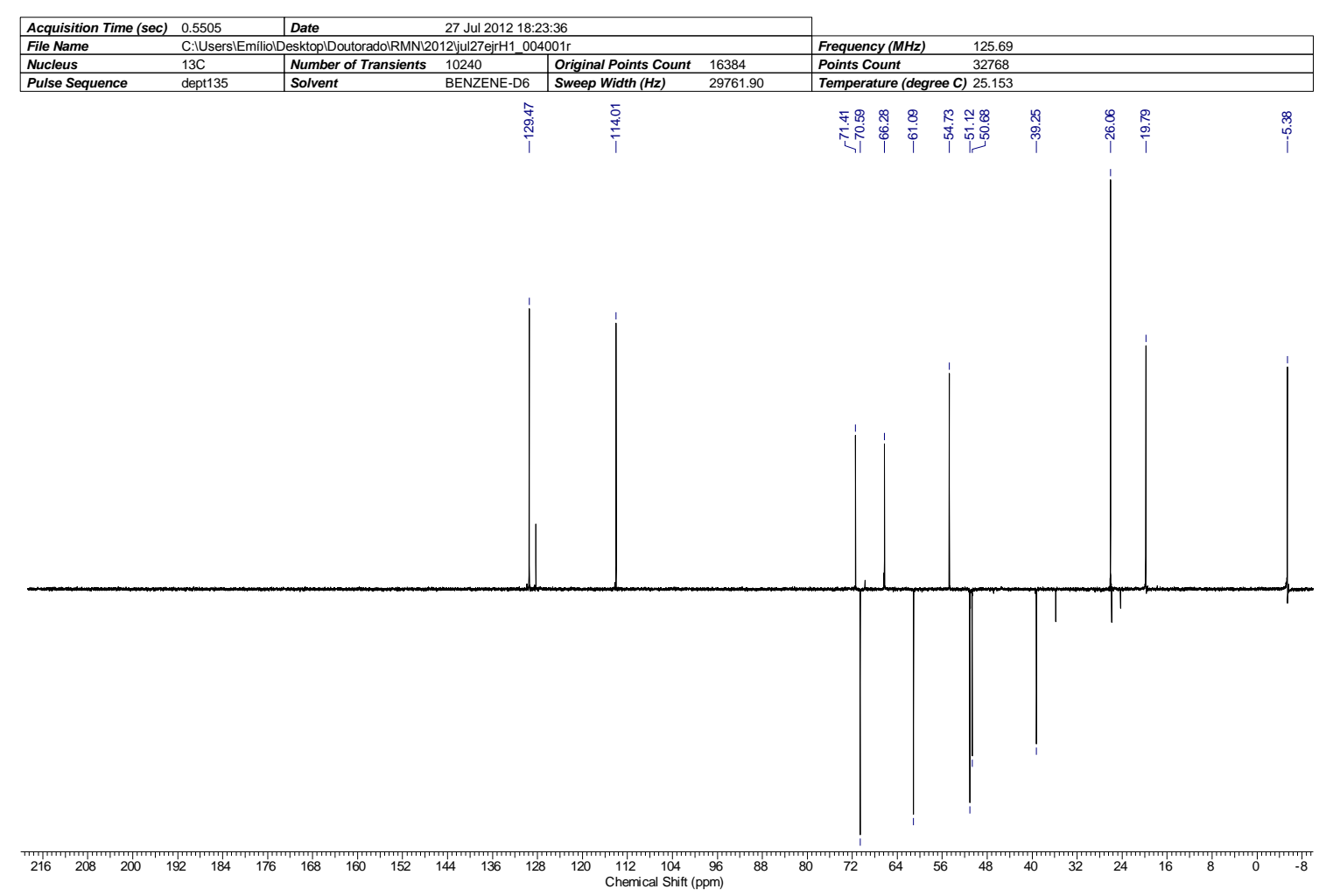

Figure 7: ${ }^{13} \mathrm{C}$ NMR (dept 135) spectrum of compound $13\left(125 \mathrm{MHz}, \mathrm{C}_{6} \mathrm{D}_{6}\right)$. 


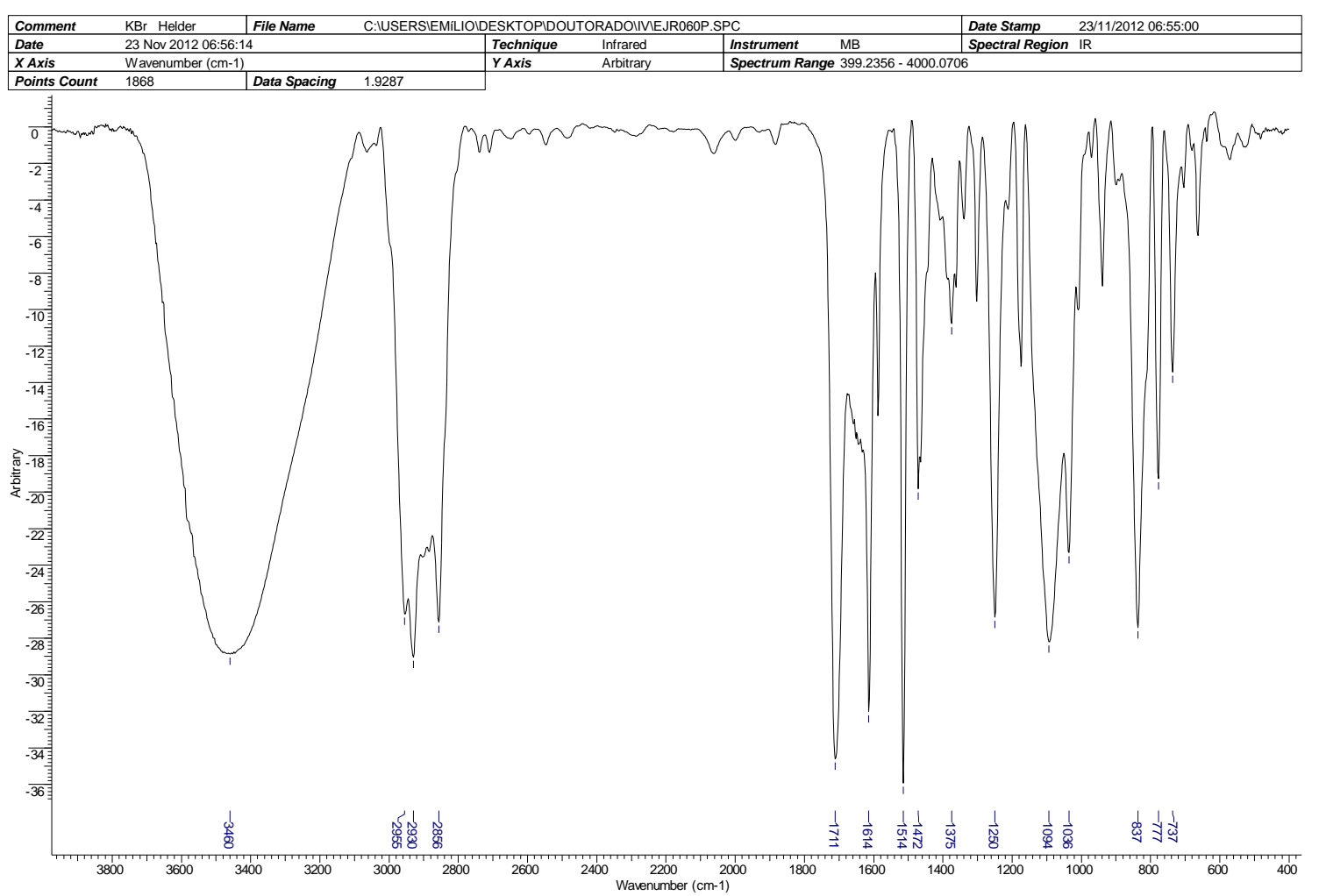

CONE $=25$ COLLISION ENERGY $=6$

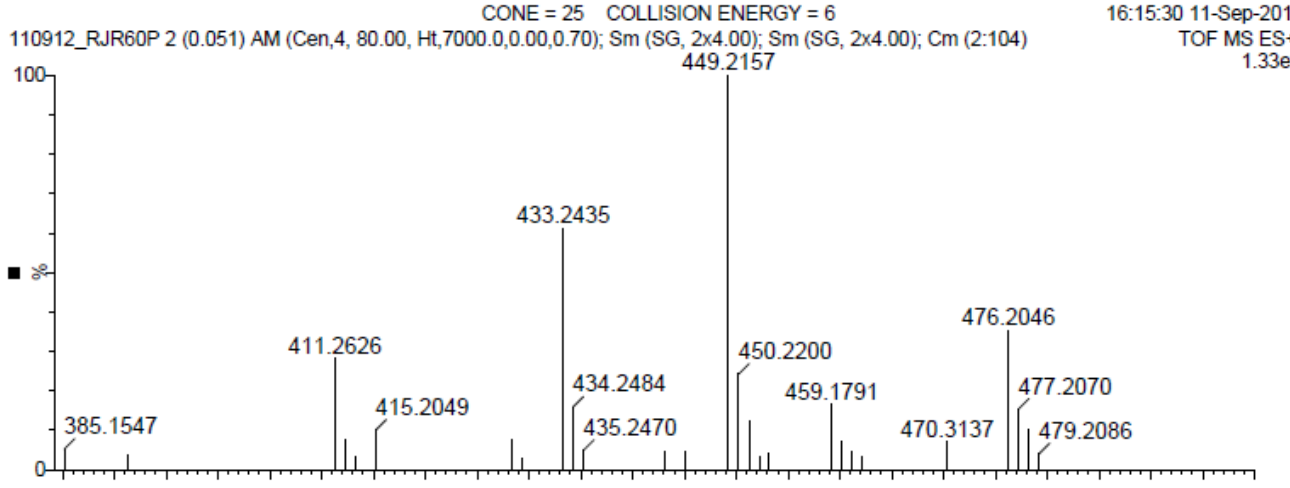

110912_RJR60P $(0.034)$ Is $(1.00,1.00) \mathrm{C} 22 \mathrm{H} 38 \mathrm{O} 5 \mathrm{SiK}$

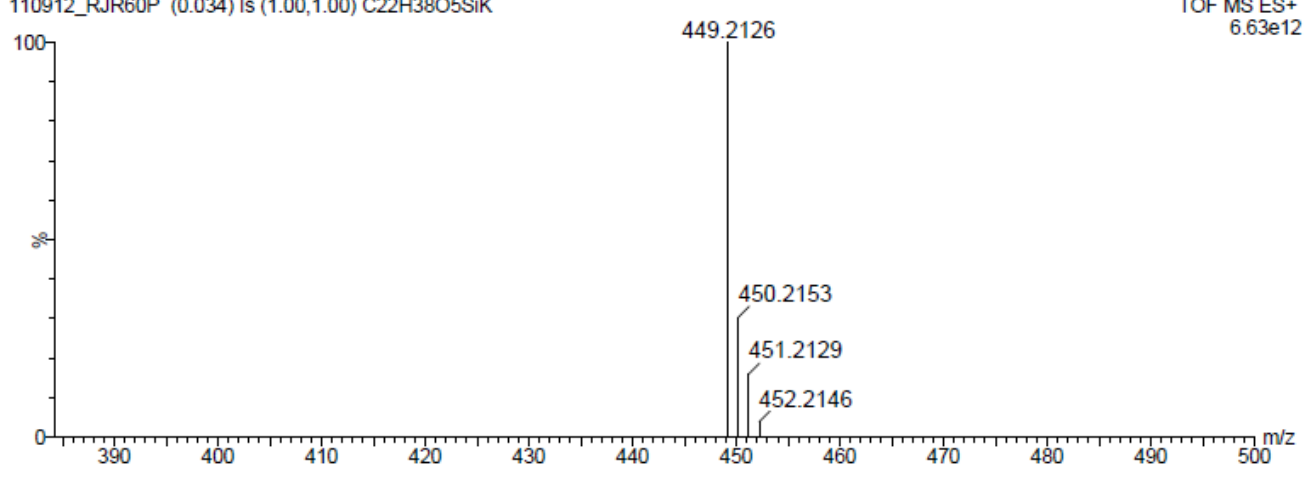

Figure 8: IR and HRMS (ESI TOF-MS) spectra of compound 13. 


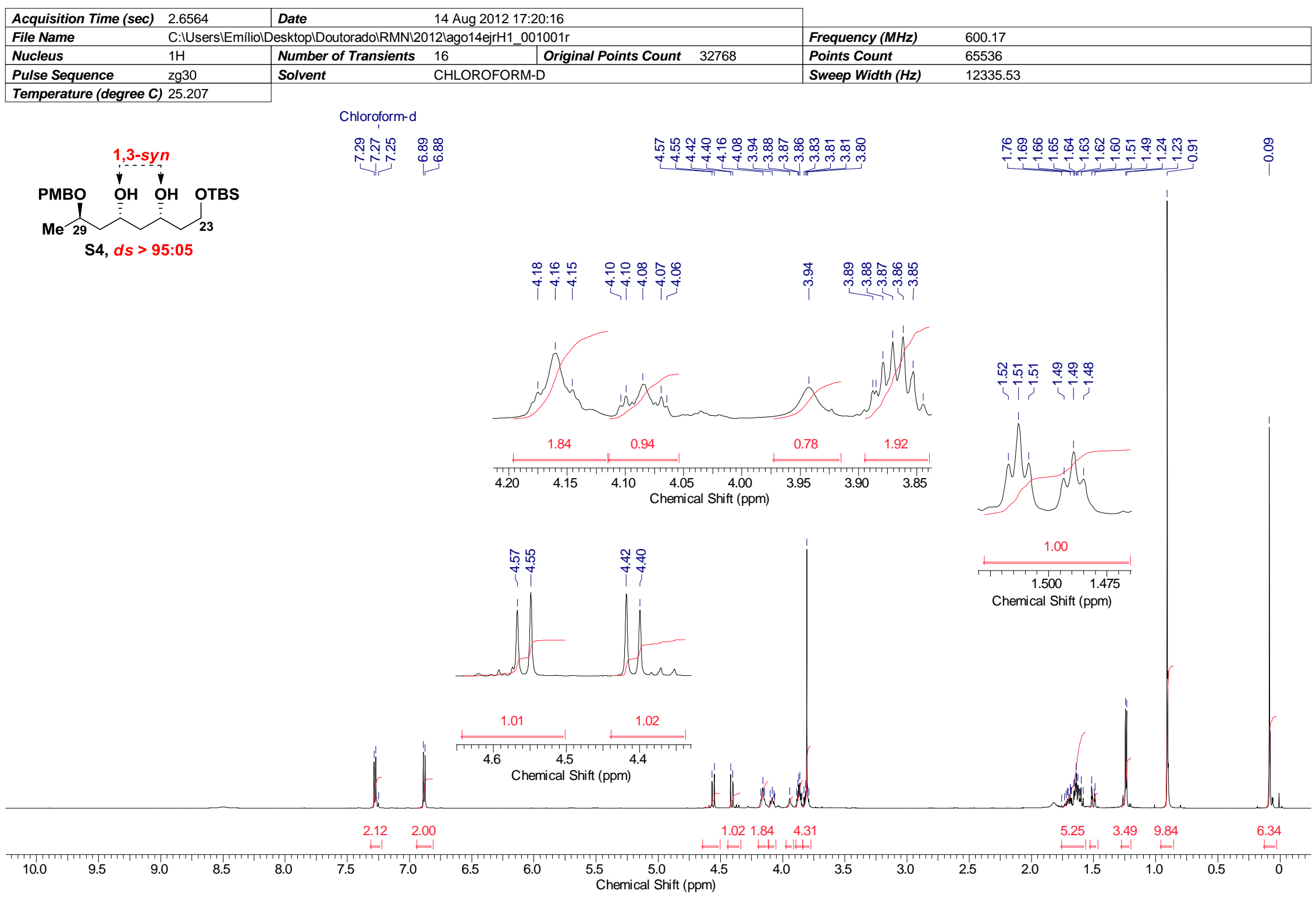

Figure 9: ${ }^{1} \mathrm{H}$ NMR spectrum of compound $\mathbf{S} 4\left(600 \mathrm{MHz}, \mathrm{CDCl}_{3}\right)$. 


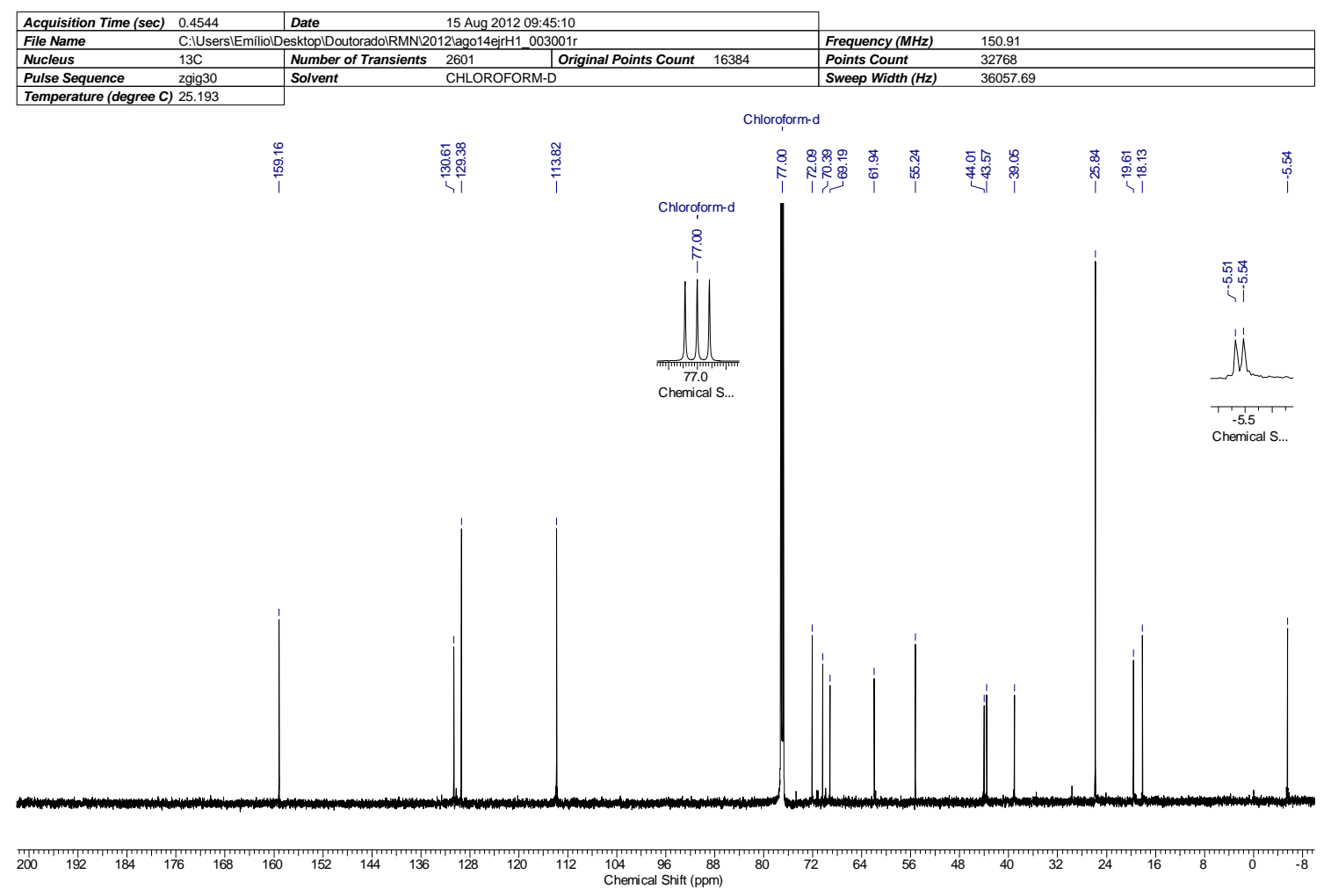

Figure 10: ${ }^{13} \mathrm{C}$ NMR spectrum of compound $\mathbf{S 4}\left(150 \mathrm{MHz}, \mathrm{CDCl}_{3}\right)$.

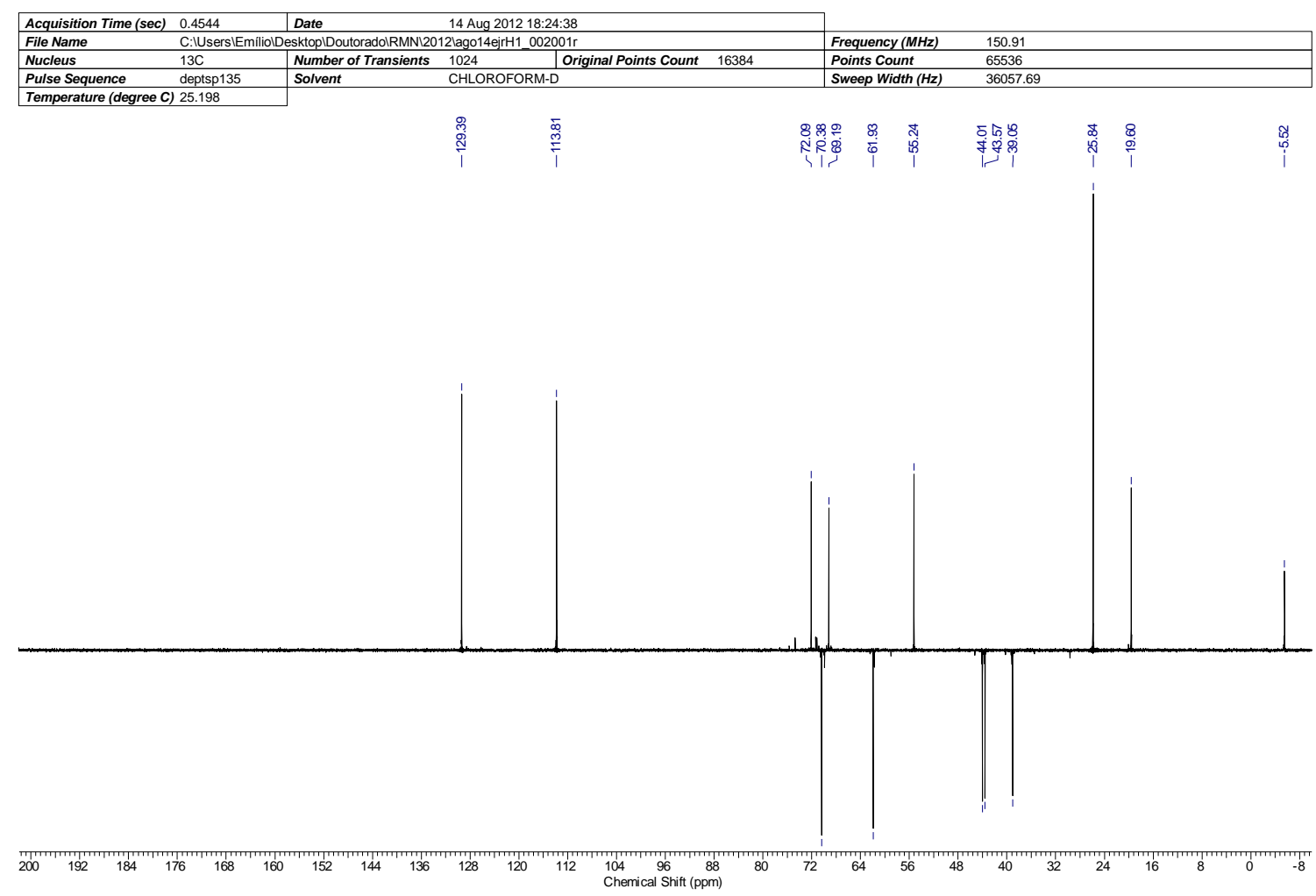

Figure 11: ${ }^{13} \mathrm{C}$ NMR (dept 135) spectrum of compound $\mathbf{S} 4\left(150 \mathrm{MHz}, \mathrm{CDCl}_{3}\right.$ ). 


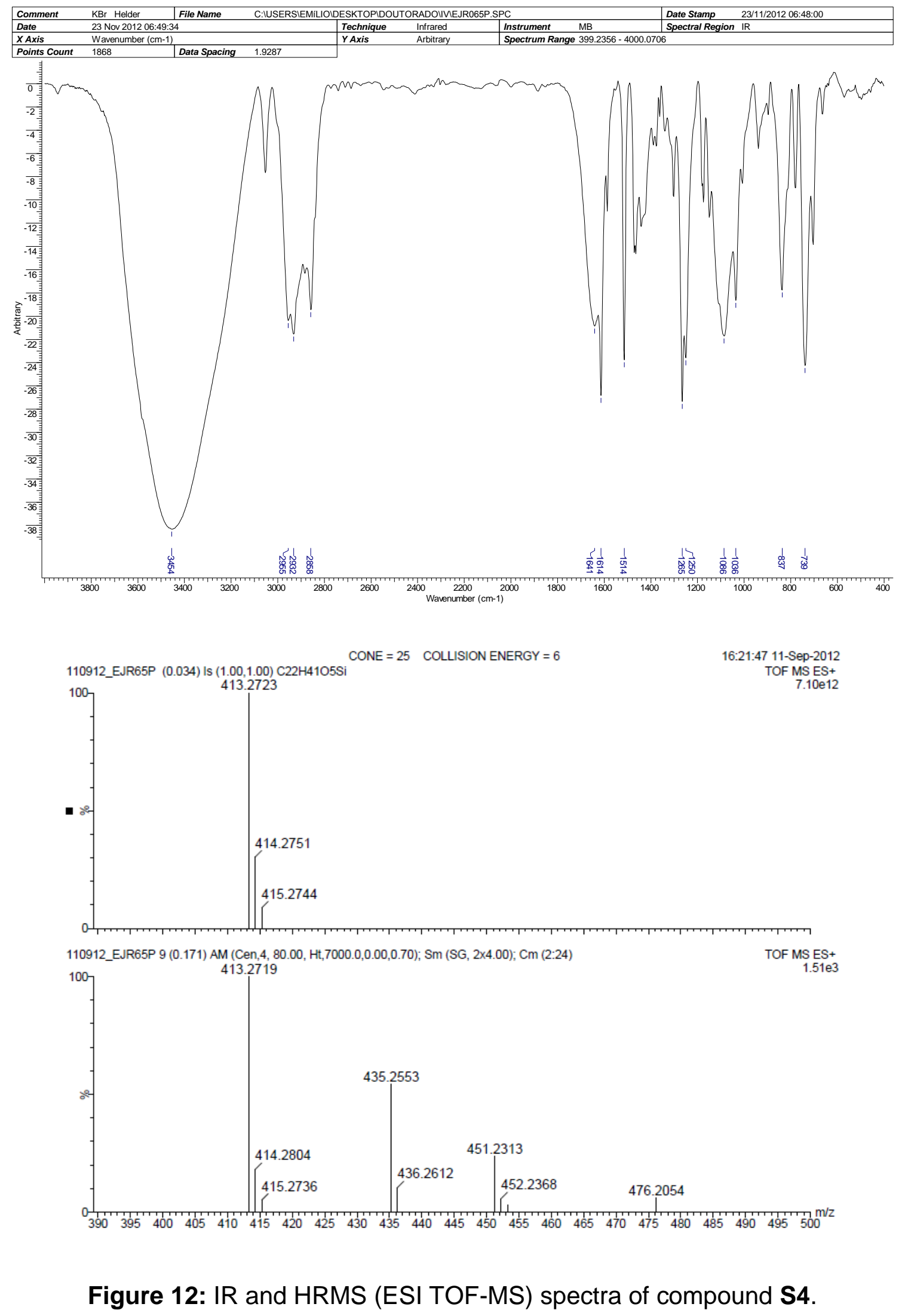




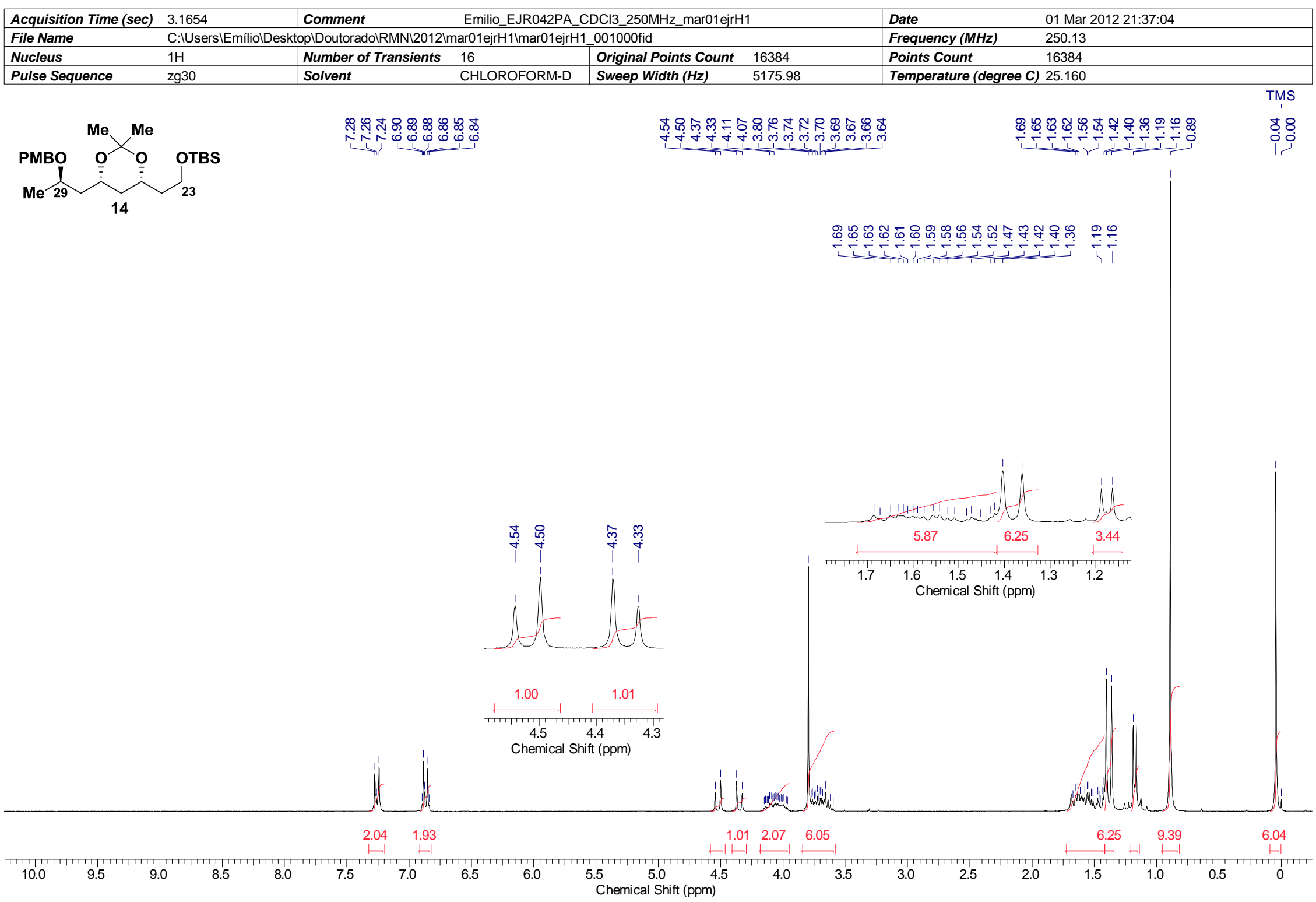

Figure 13: ${ }^{1} \mathrm{H}$ NMR spectrum of compound $14\left(250 \mathrm{MHz}, \mathrm{CDCl}_{3}\right)$. 


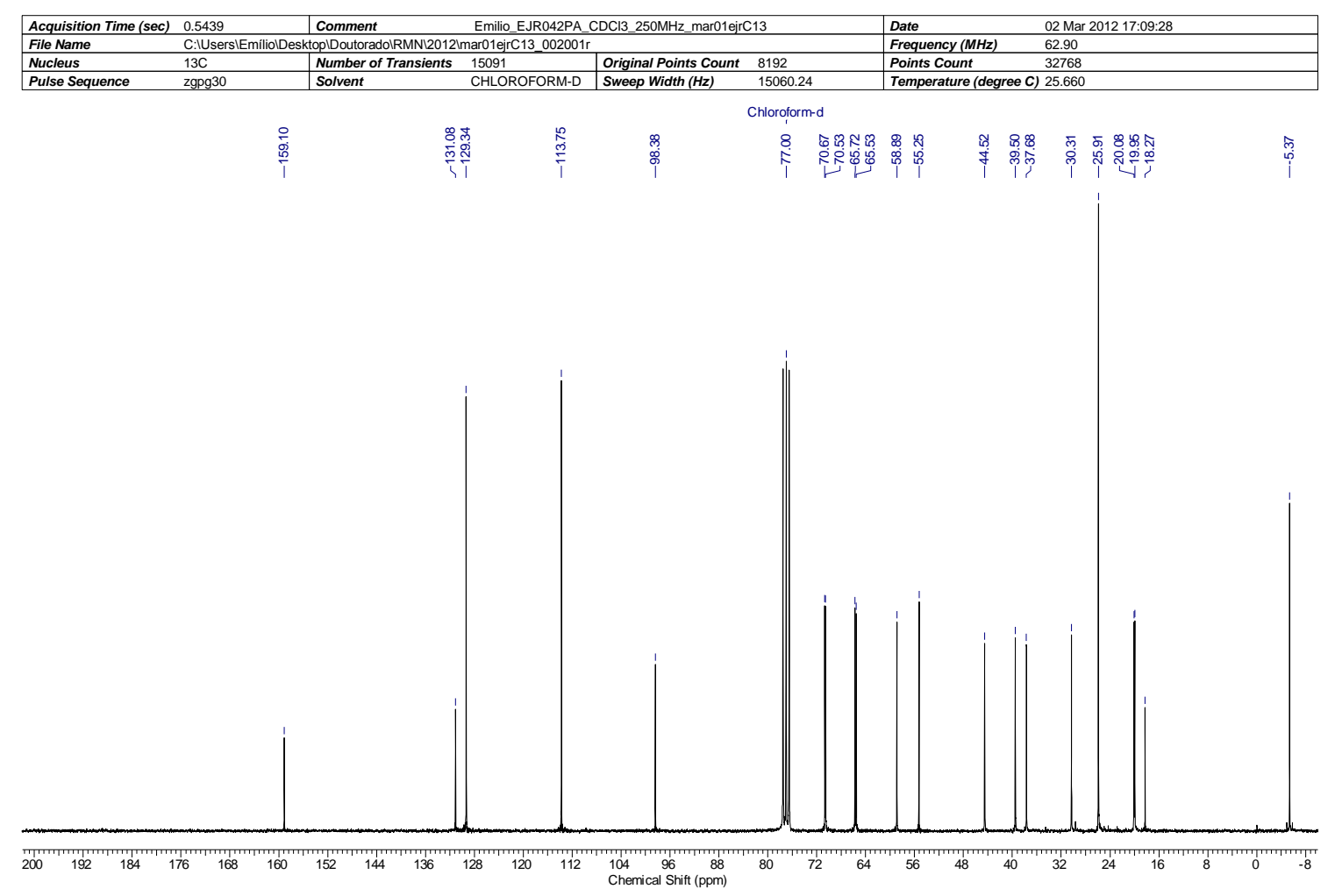

Figure 14: ${ }^{13} \mathrm{C}$ NMR spectrum of compound $14\left(62.5 \mathrm{MHz}, \mathrm{CDCl}_{3}\right)$.

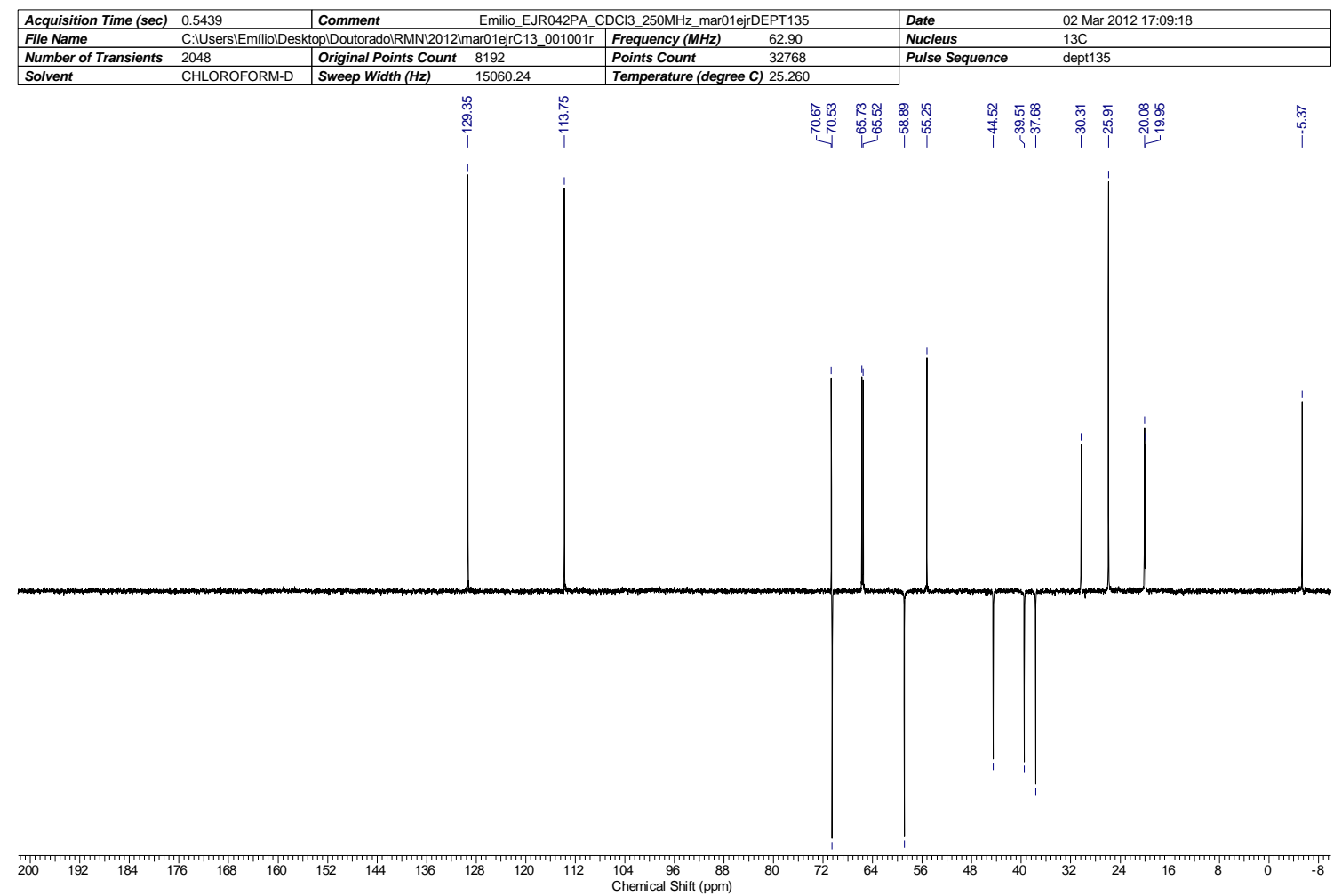

Figure 15: ${ }^{13} \mathrm{C}$ NMR (dept 135) spectrum of compound $14\left(62.5 \mathrm{MHz}, \mathrm{CDCl}_{3}\right)$. 


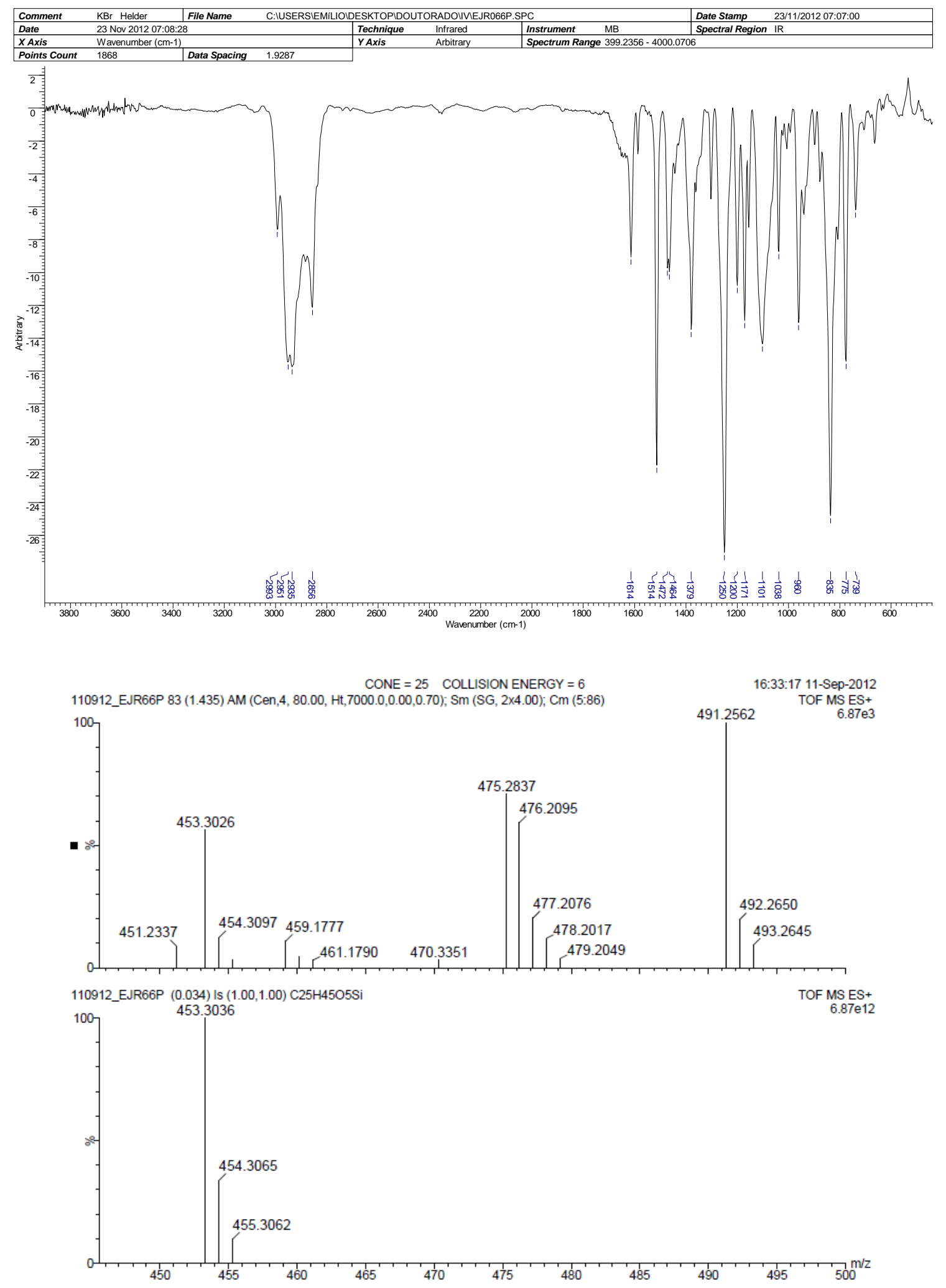

Figure 16: IR and HRMS (ESI TOF-MS) spectra of compound 14. 


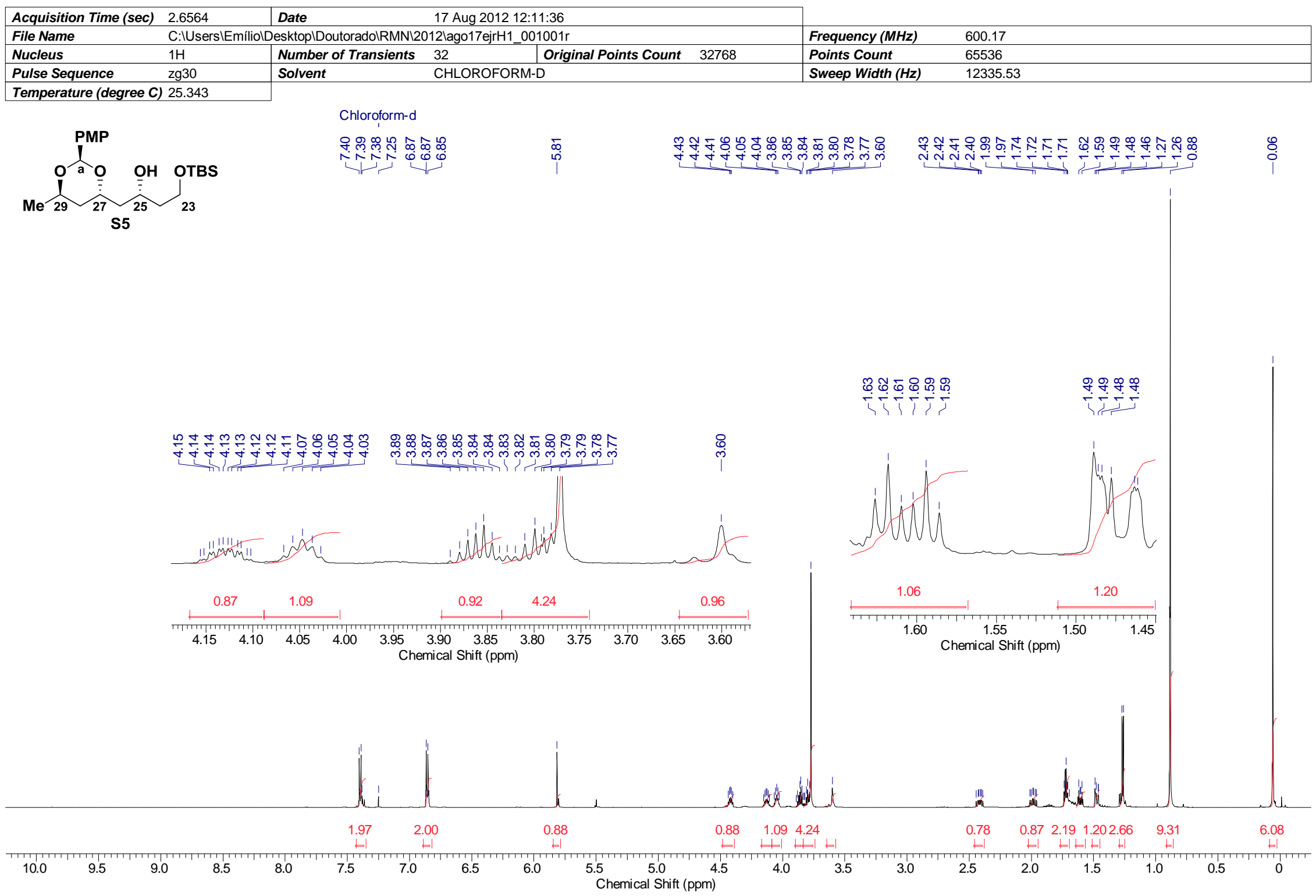

Figure 17: ${ }^{1} \mathrm{H}$ NMR spectrum of compound $\mathbf{S} 5\left(600 \mathrm{MHz}, \mathrm{CDCl}_{3}\right)$. 


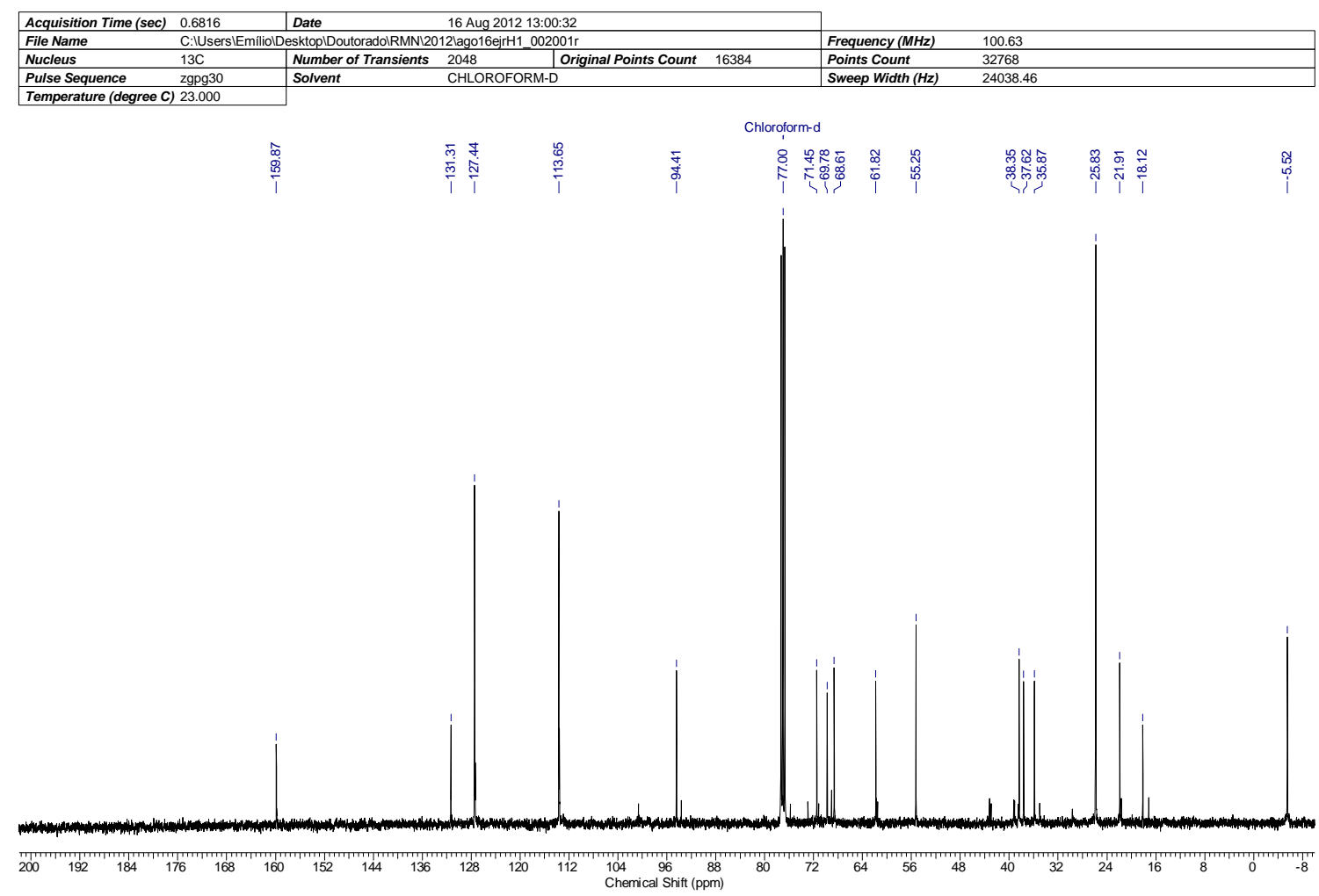

Figure 18: ${ }^{13} \mathrm{C}$ NMR spectrum of compound $\mathbf{S} 5\left(100 \mathrm{MHz}, \mathrm{CDCl}_{3}\right)$.

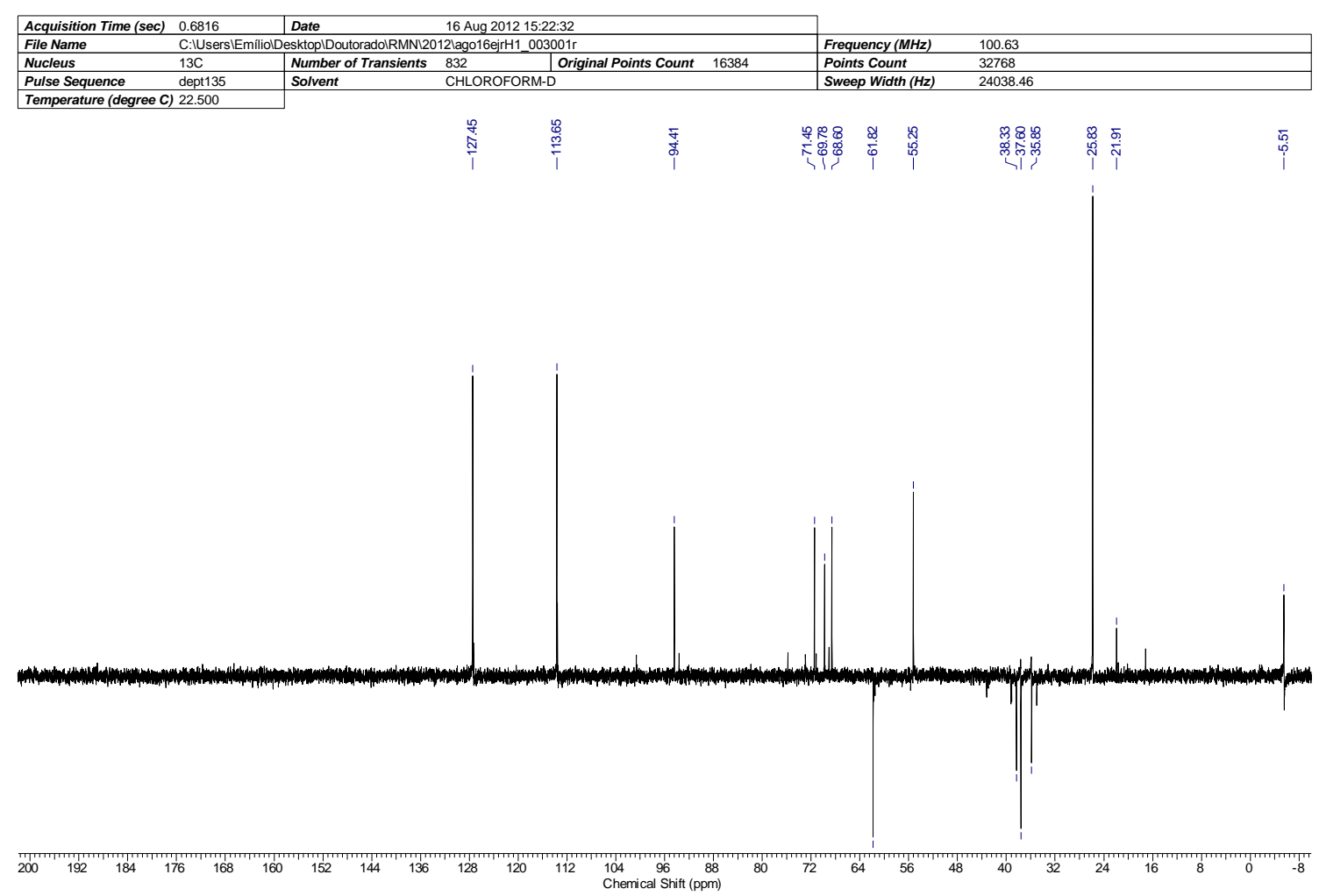

Figure 19: ${ }^{13} \mathrm{C}$ NMR (dept 135) spectrum of compound $\mathbf{S 5}\left(100 \mathrm{MHz}, \mathrm{CDCl}_{3}\right.$ ). 


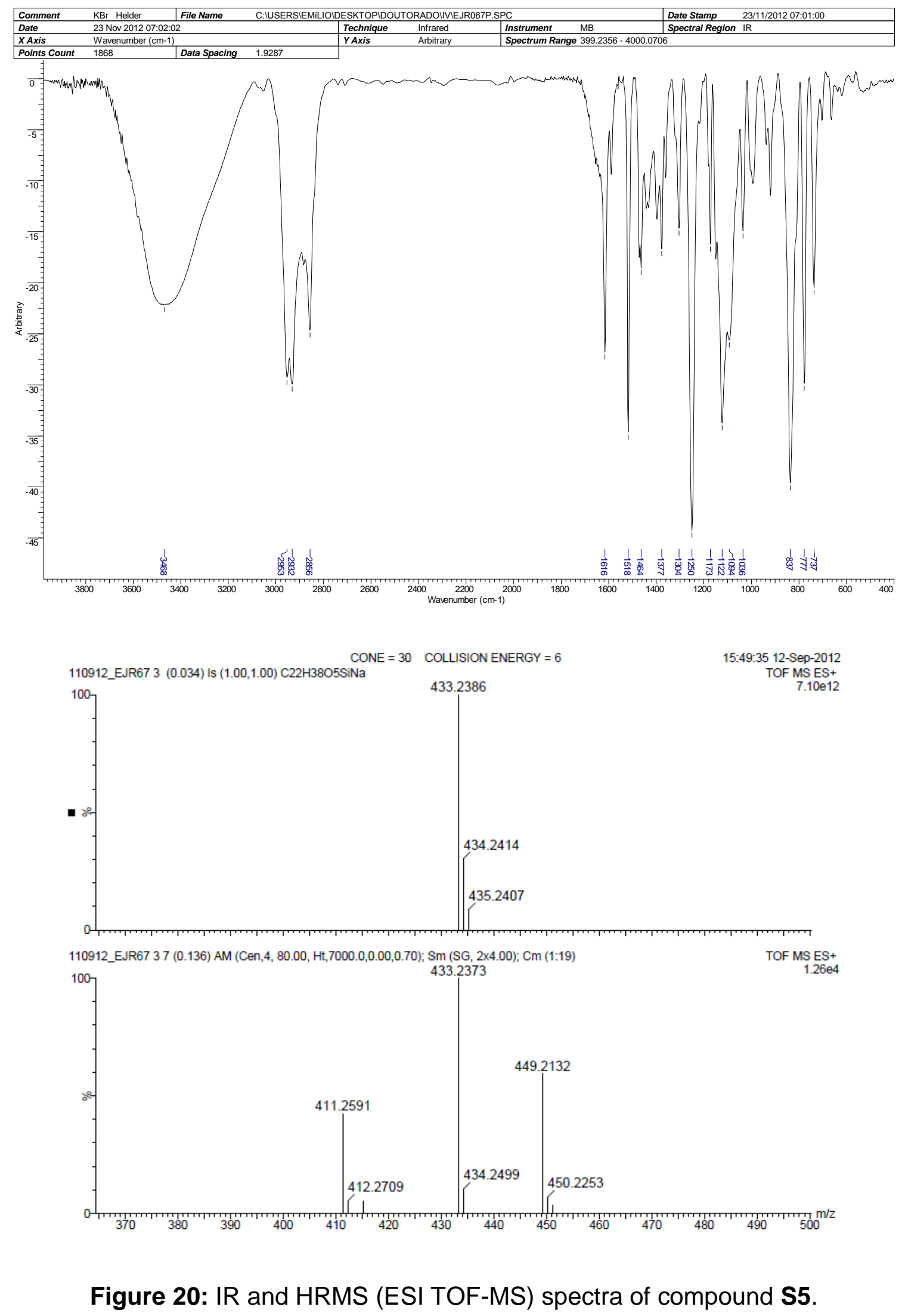




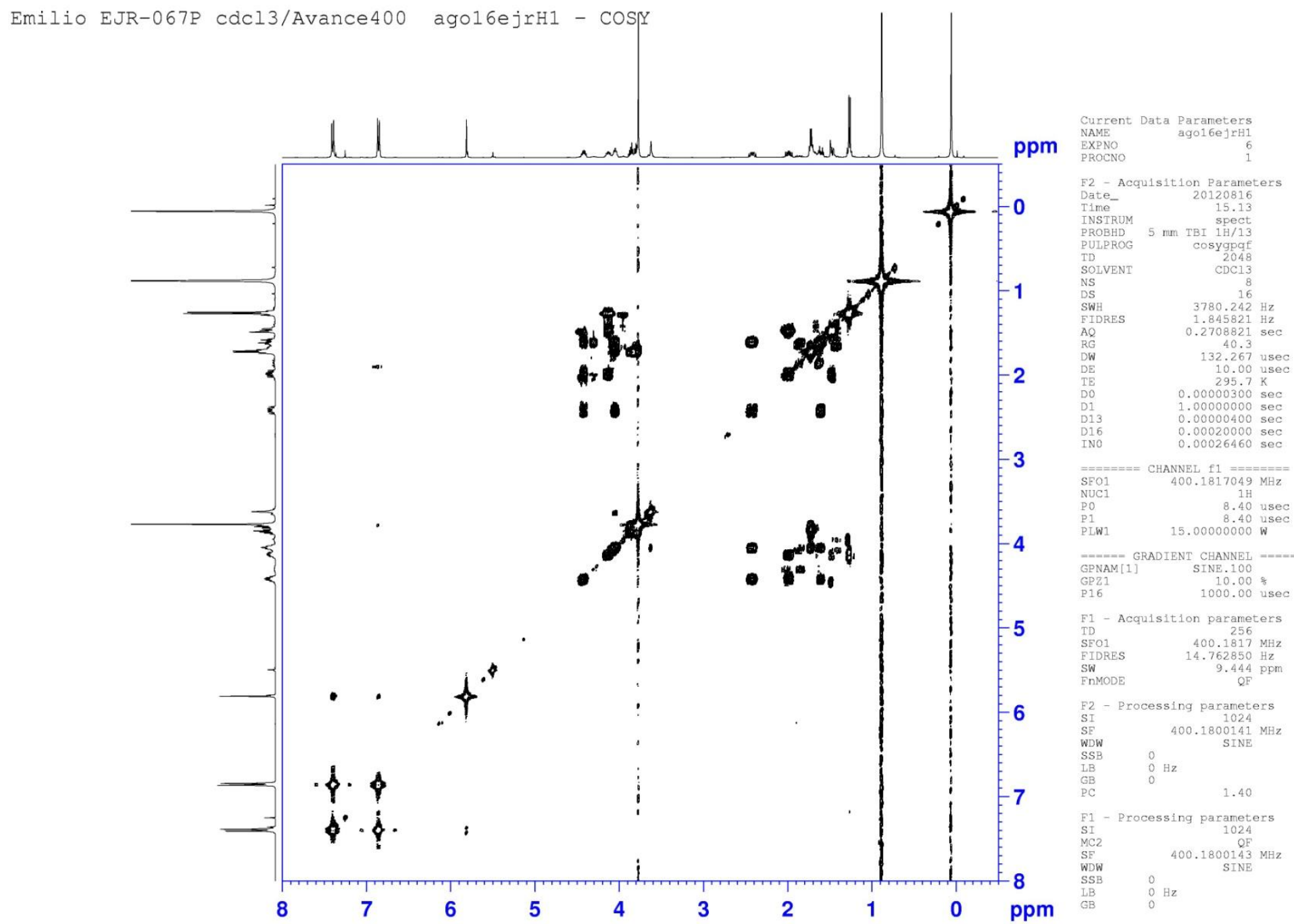

Figure 21: COSY contour map of compound $\mathbf{S} 5\left(400 \mathrm{MHz}, \mathrm{CDCl}_{3}\right)$. 


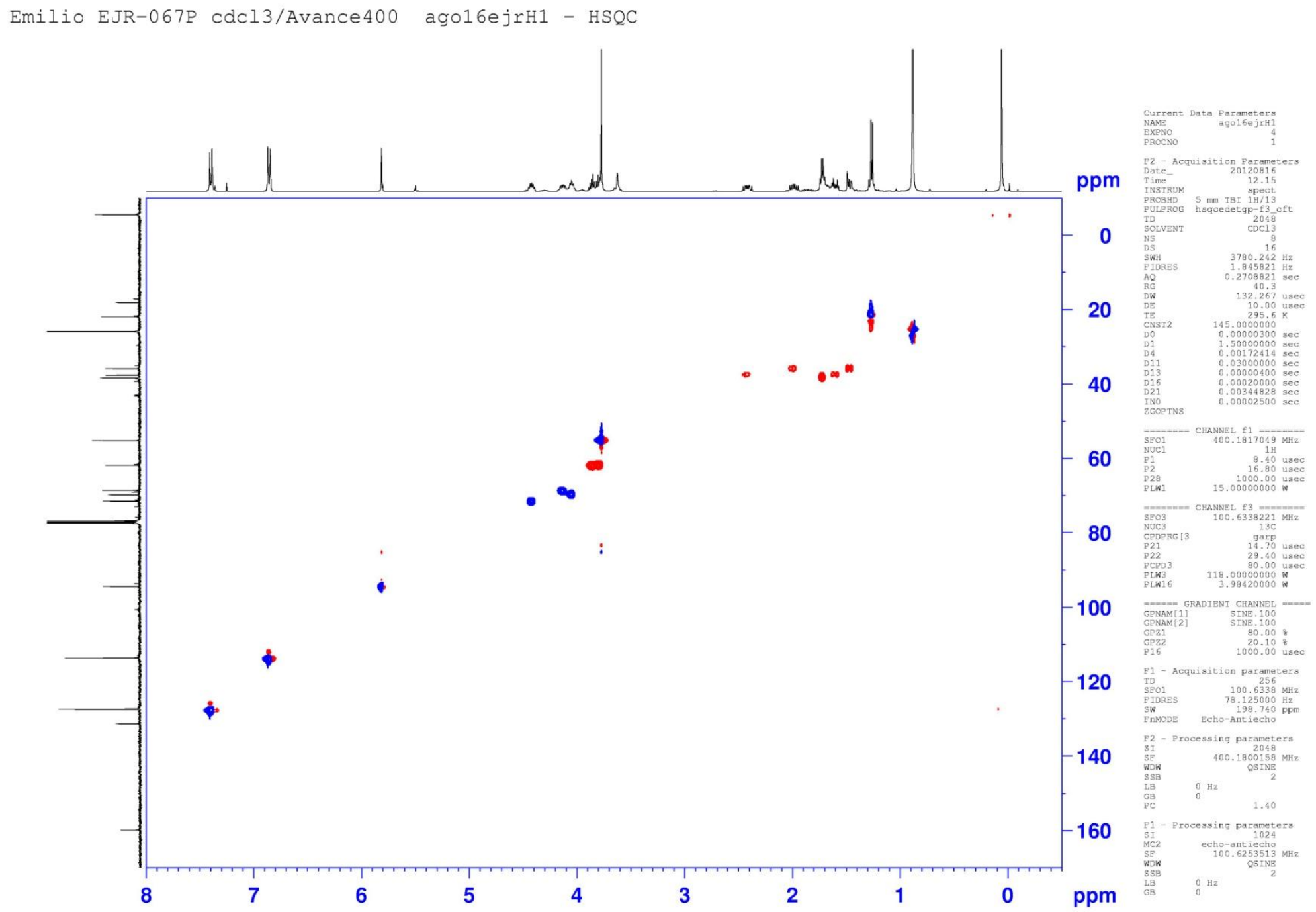

Figure 22: HSQC contour map of compound S5. 


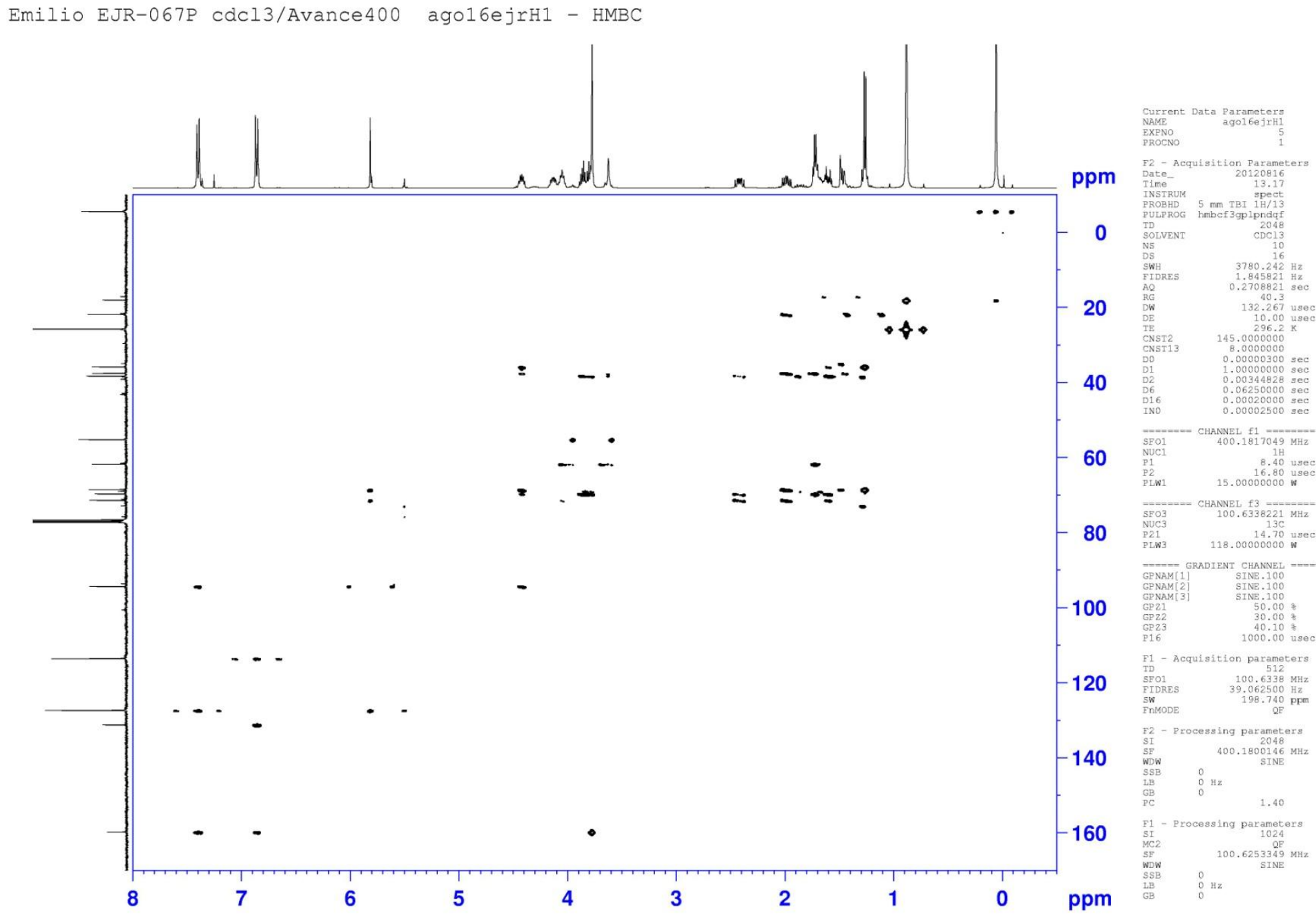

Figure 23: HMBC contour map of compound S5. 


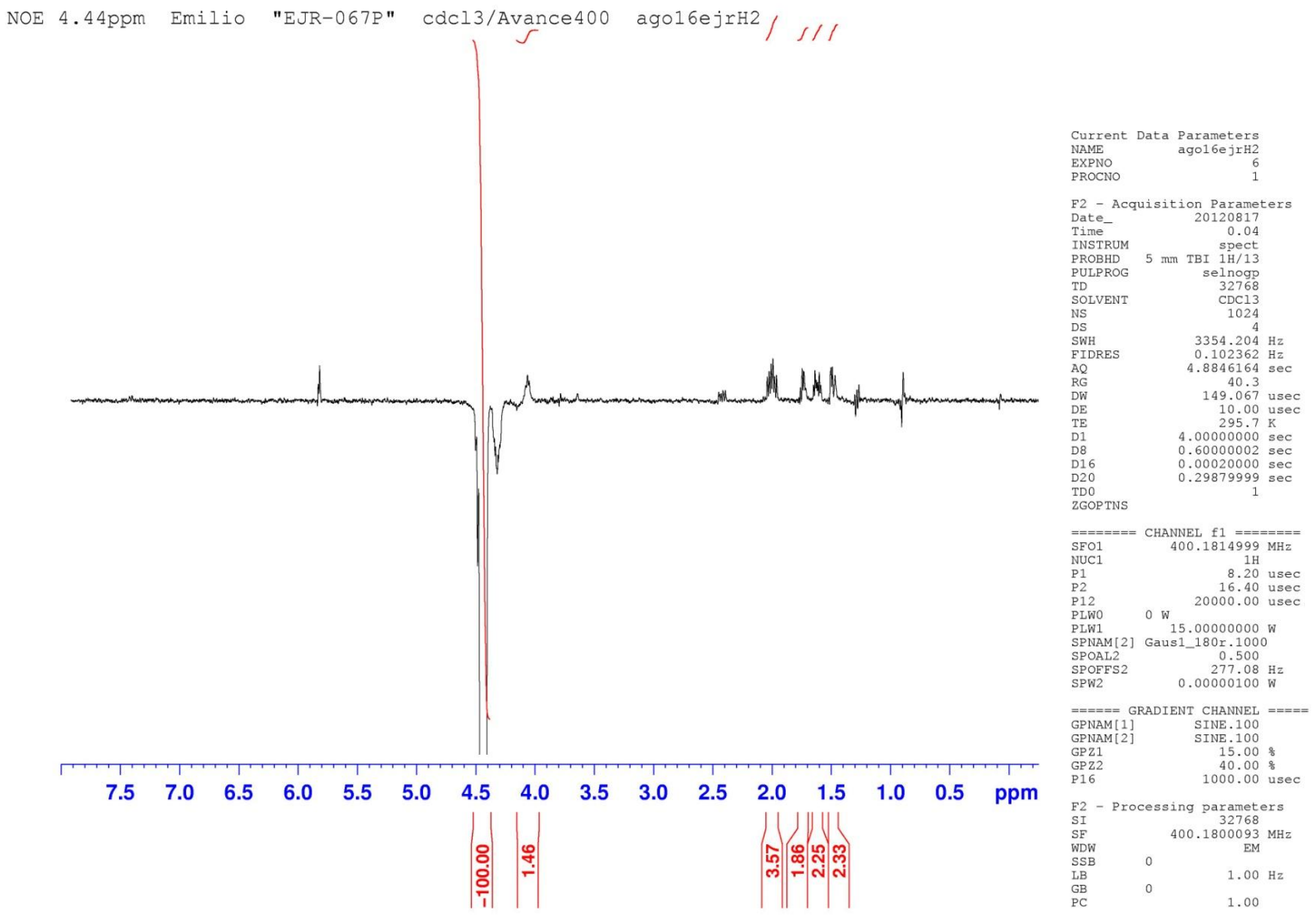

Figure 24: ${ }^{1} \mathrm{H}$ NMR (1D selective NOE) spectrum of compound $\mathbf{S 5}\left(400 \mathrm{MHz}, \mathrm{CDCl}_{3}\right)$. 


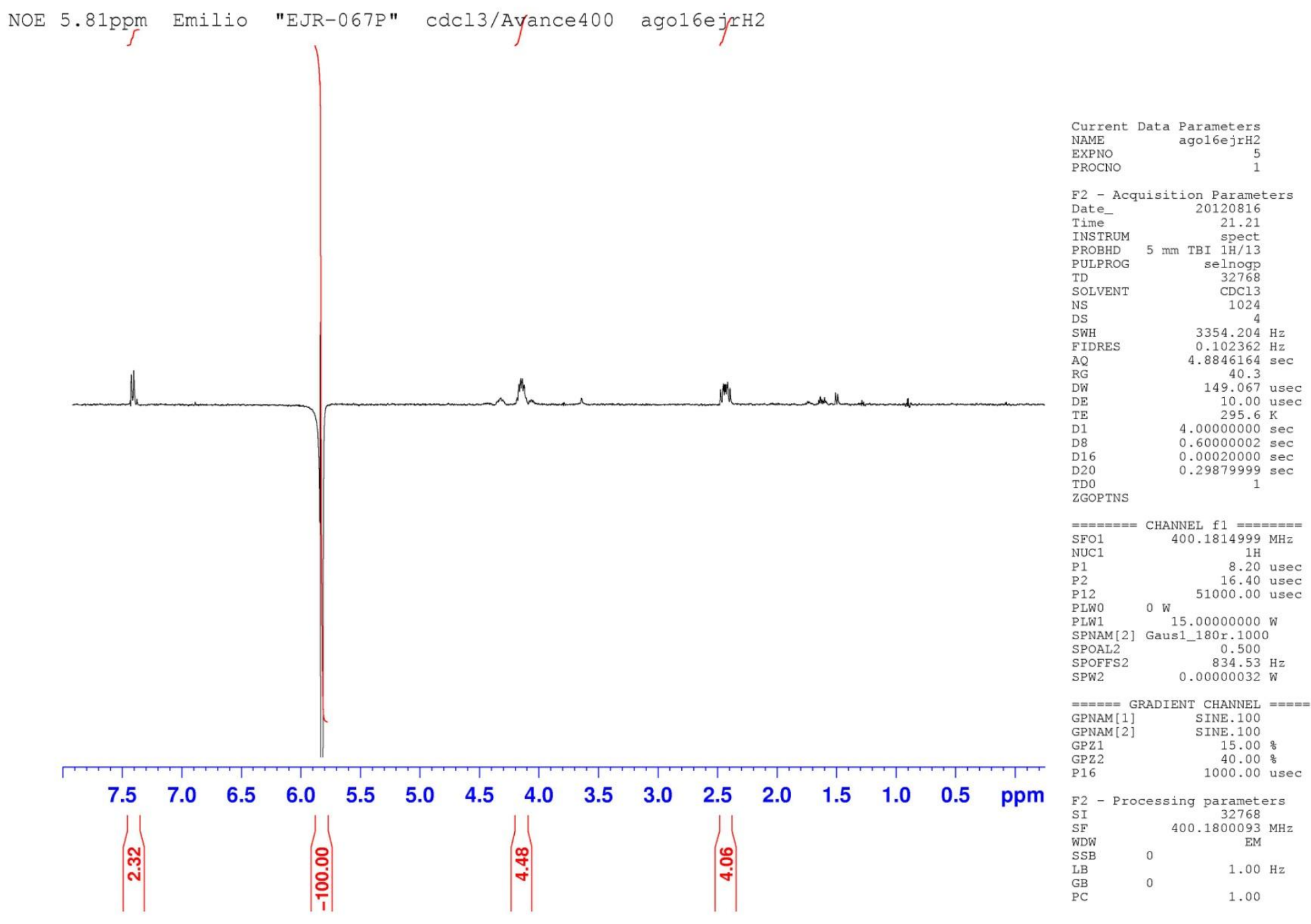

Figure 25: ${ }^{1} \mathrm{H}$ NMR (1D selective NOE) spectrum of compound $\mathbf{S} 5\left(400 \mathrm{MHz}, \mathrm{CDCl}_{3}\right)$. 


\begin{tabular}{|c|c|c|c|c|c|c|}
\hline Acquisition Time & \multirow{2}{*}{\multicolumn{4}{|c|}{\begin{tabular}{l|lc}
3.1654 & Comment & Emílio EJR-068P C6D6 250 MHz ago22ejrH1 \\
C:IUsersIEmíliolDesktoplDoutoradoIRMNI2012lago22ejrH1_001001r
\end{tabular}}} & \multirow{2}{*}{\multicolumn{2}{|c|}{\begin{tabular}{|ll} 
Date & 22 Aug 2012 13:24:08 \\
Frequency $(\mathbf{M H z})$ & 250.13 \\
\end{tabular}}} \\
\hline File Name & & & & & & \\
\hline Nucleus & $1 \mathrm{H}$ & Number of Transients & 8 & Original Points Count 16384 & Points Count & 32768 \\
\hline Pulse Sequence & $\mathrm{zg} 30$ & Solvent & BENZENE-D6 & Sweep Width $(\mathrm{Hz})$ & Temperature (degree $C$ ) & 25.160 \\
\hline
\end{tabular}

Pulse Sequence Benzene-d6

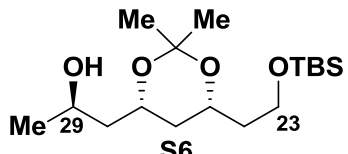

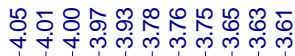

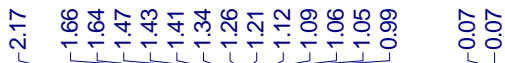
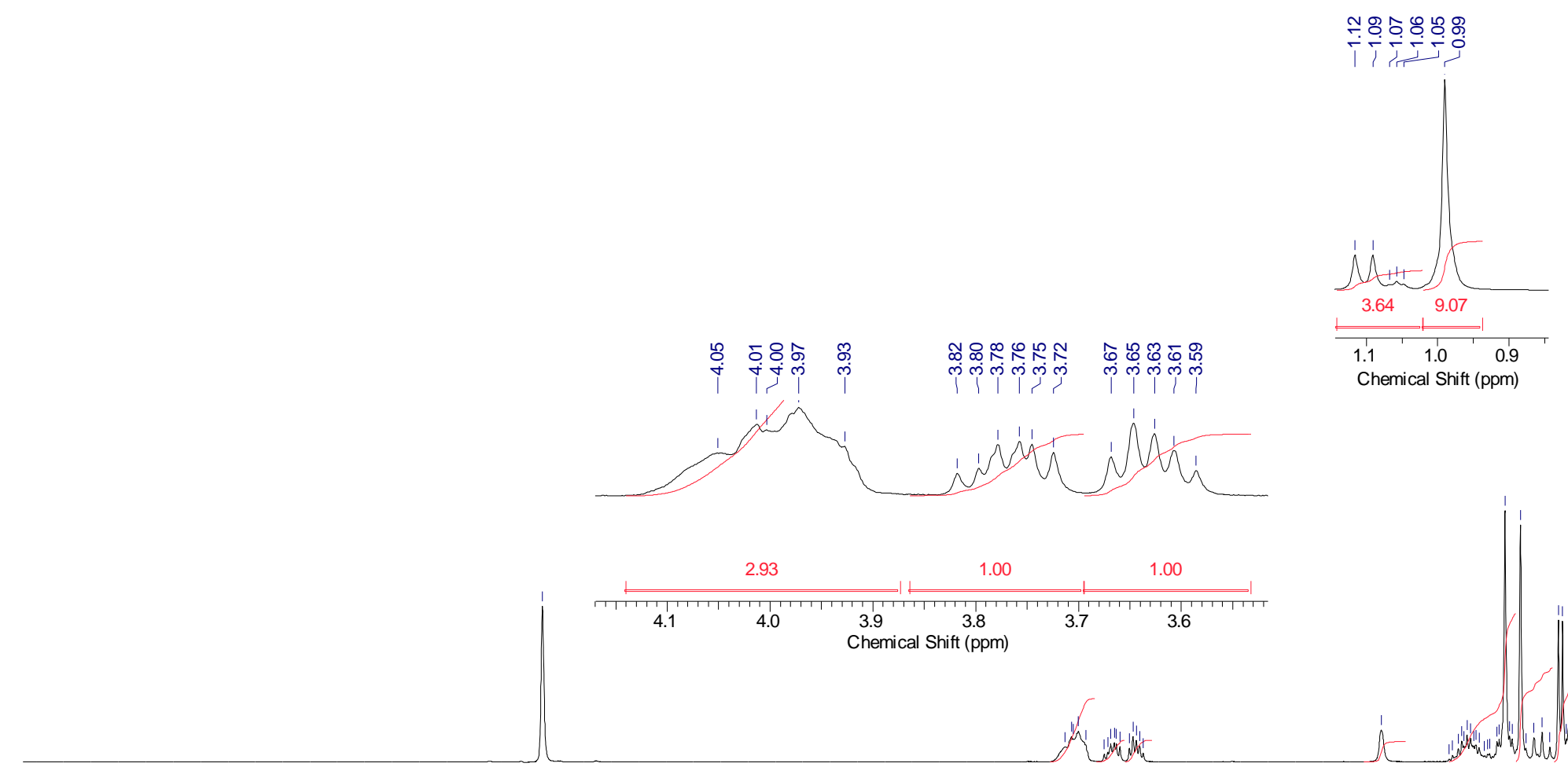

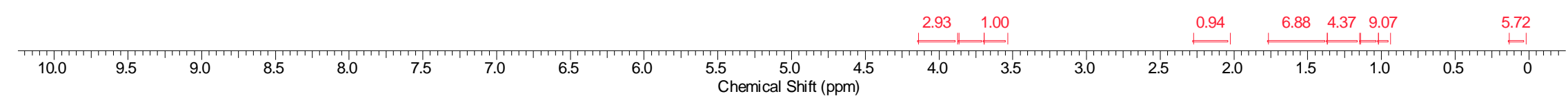

Figure 26: ${ }^{1} \mathrm{H}$ NMR spectrum of compound $\mathbf{S 6}\left(250 \mathrm{MHz}, \mathrm{C}_{6} \mathrm{D}_{6}\right)$. 


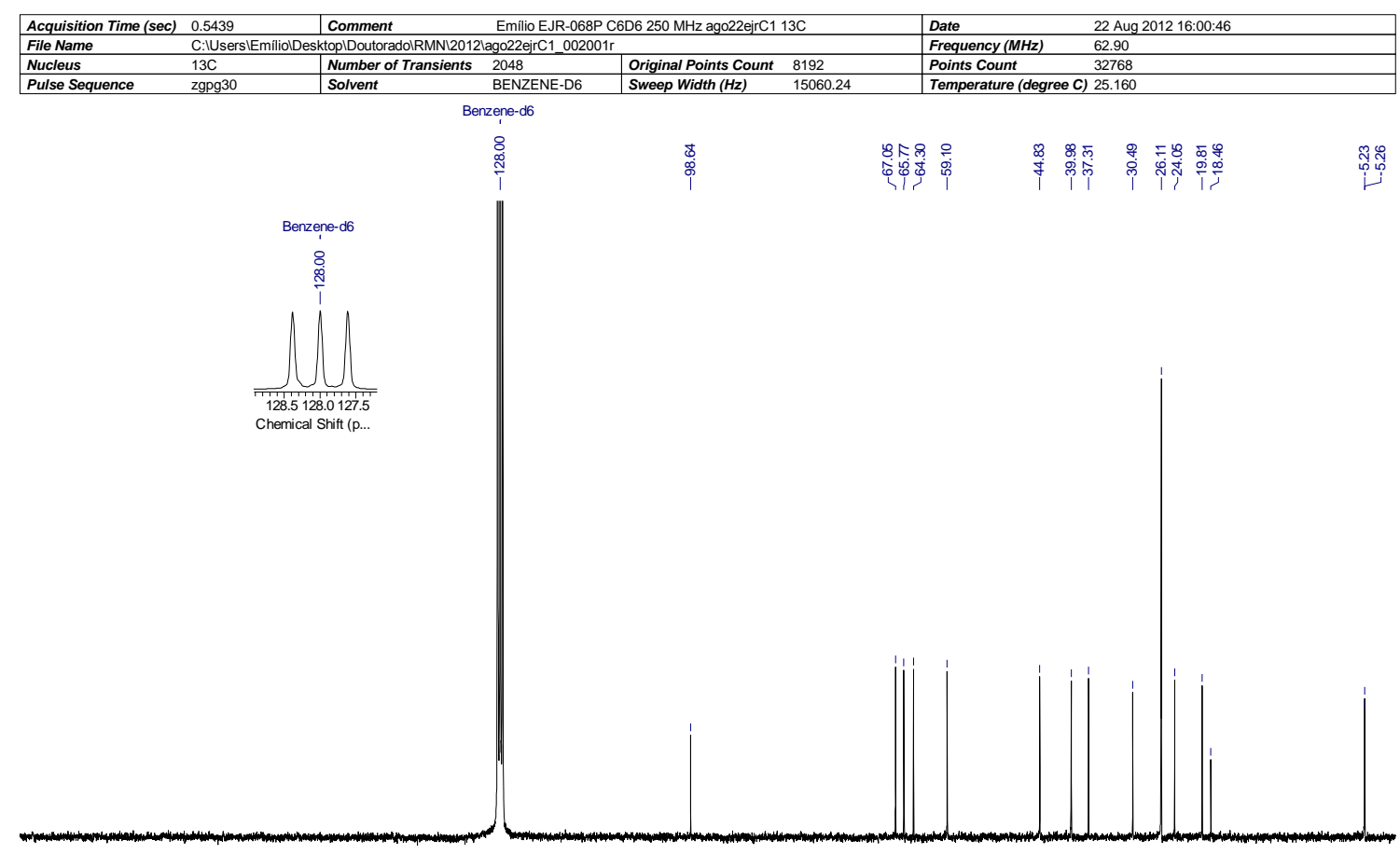

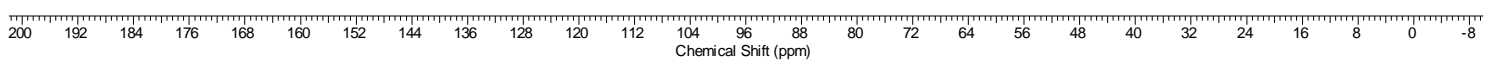

Figure 27: ${ }^{13} \mathrm{C}$ NMR spectrum of compound $\mathbf{S 6}\left(62.5 \mathrm{MHz}, \mathrm{C}_{6} \mathrm{D}_{6}\right)$.

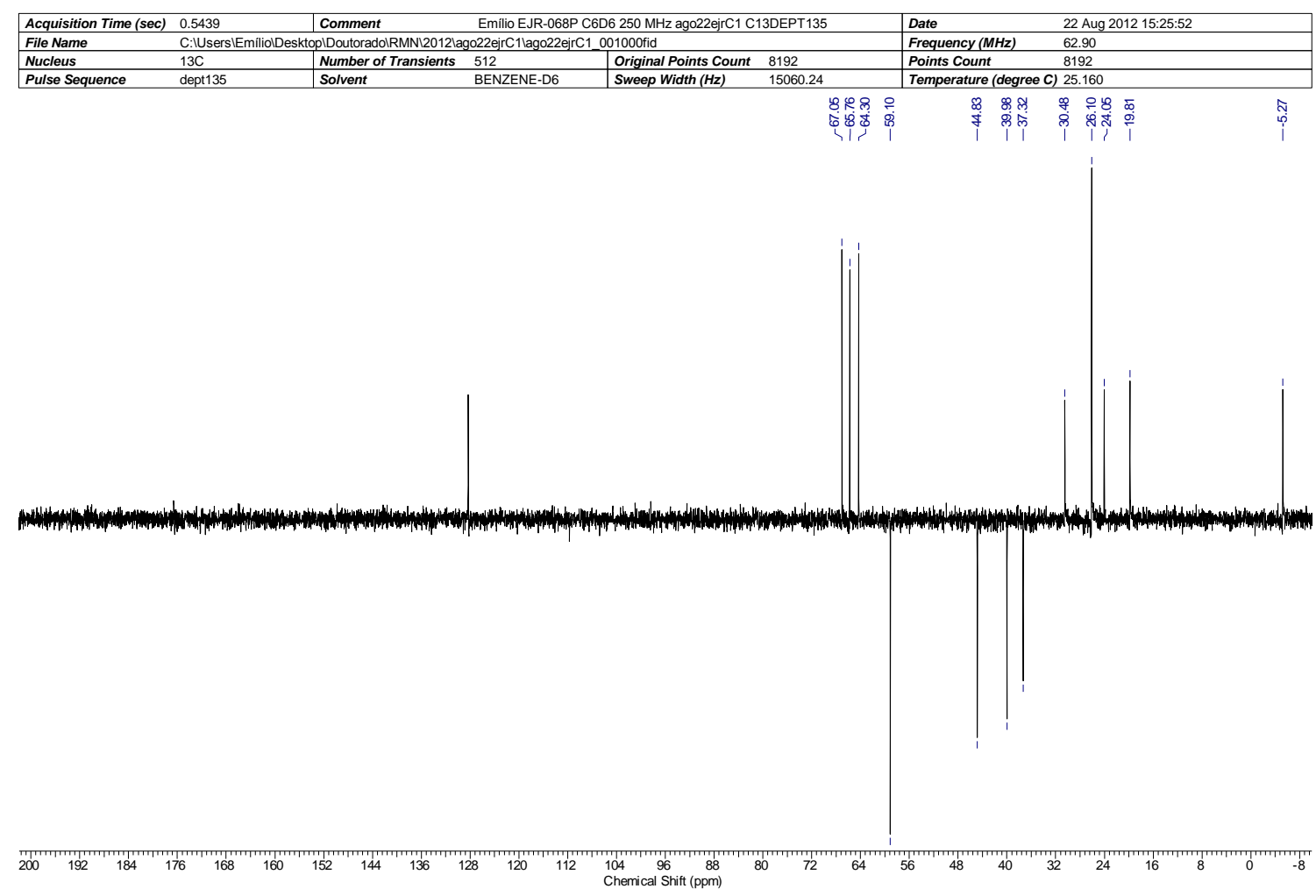

Figure 28: ${ }^{13} \mathrm{C}$ NMR (dept 135) spectrum of compound $\mathbf{S 6}\left(62.5 \mathrm{MHz}, \mathrm{C}_{6} \mathrm{D}_{6}\right)$. 


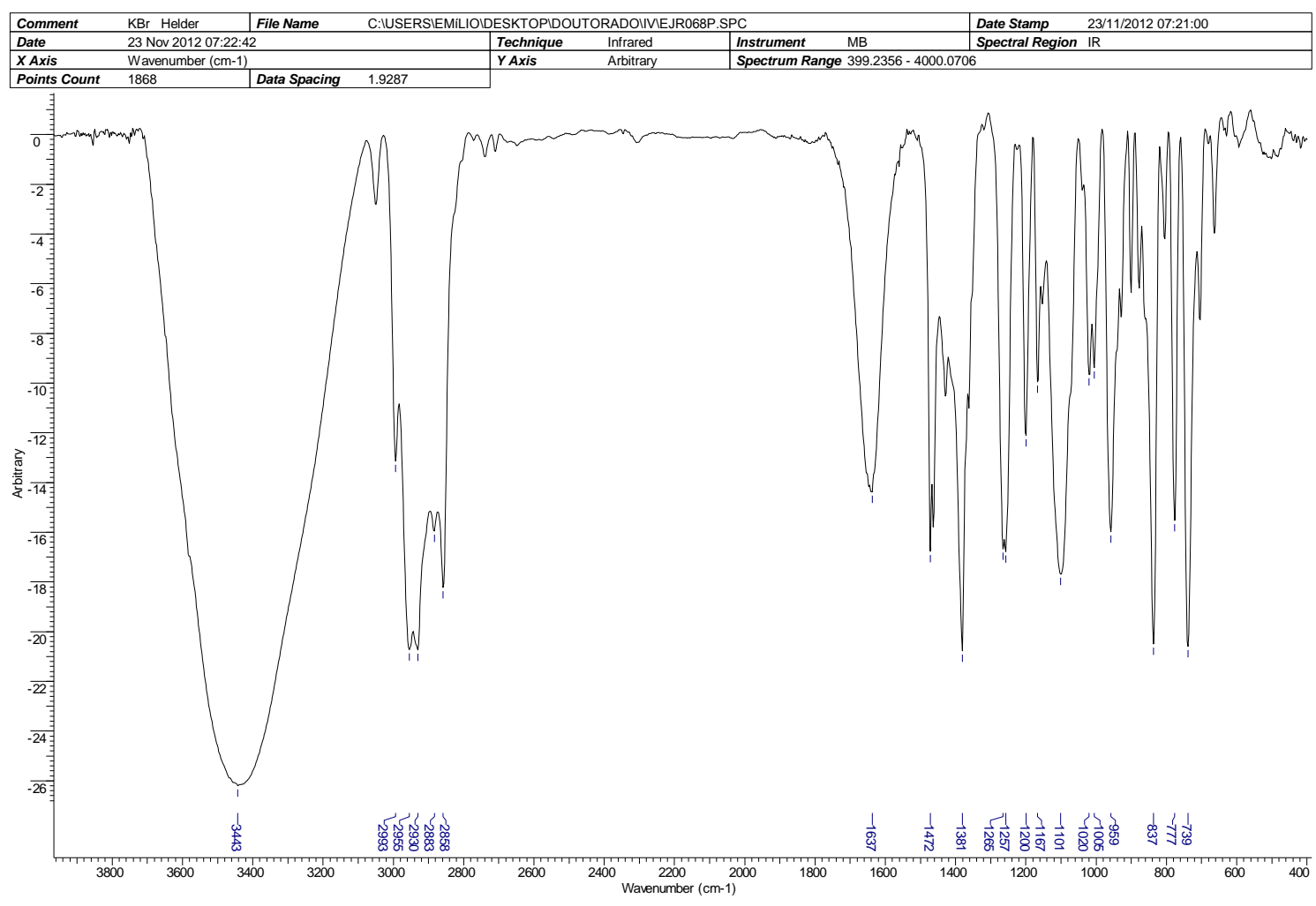

CONE $=10$ COLLISION ENERGY $=6$
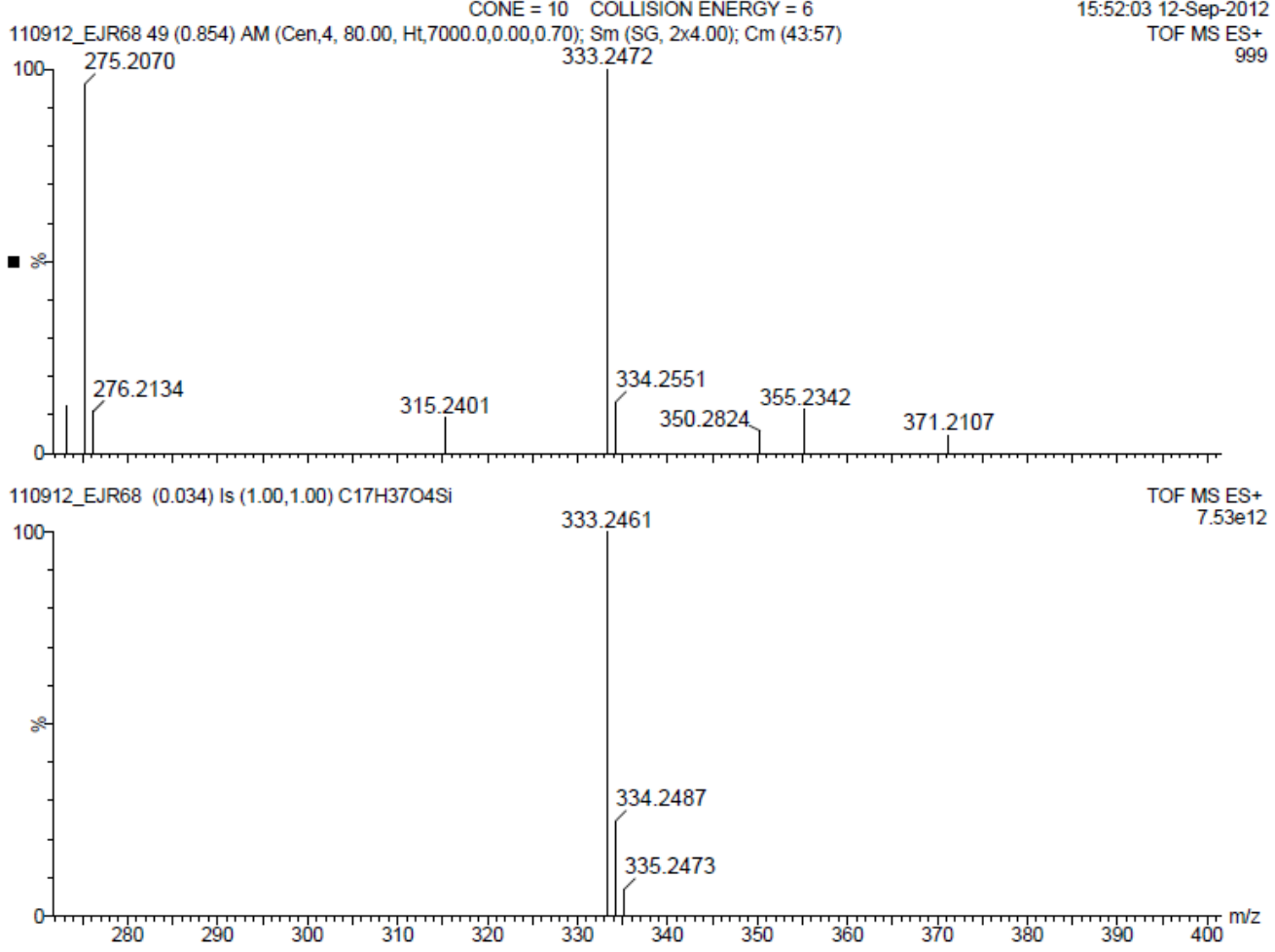

Figure 29: IR and HRMS (ESI TOF-MS) spectra of compound S6. 


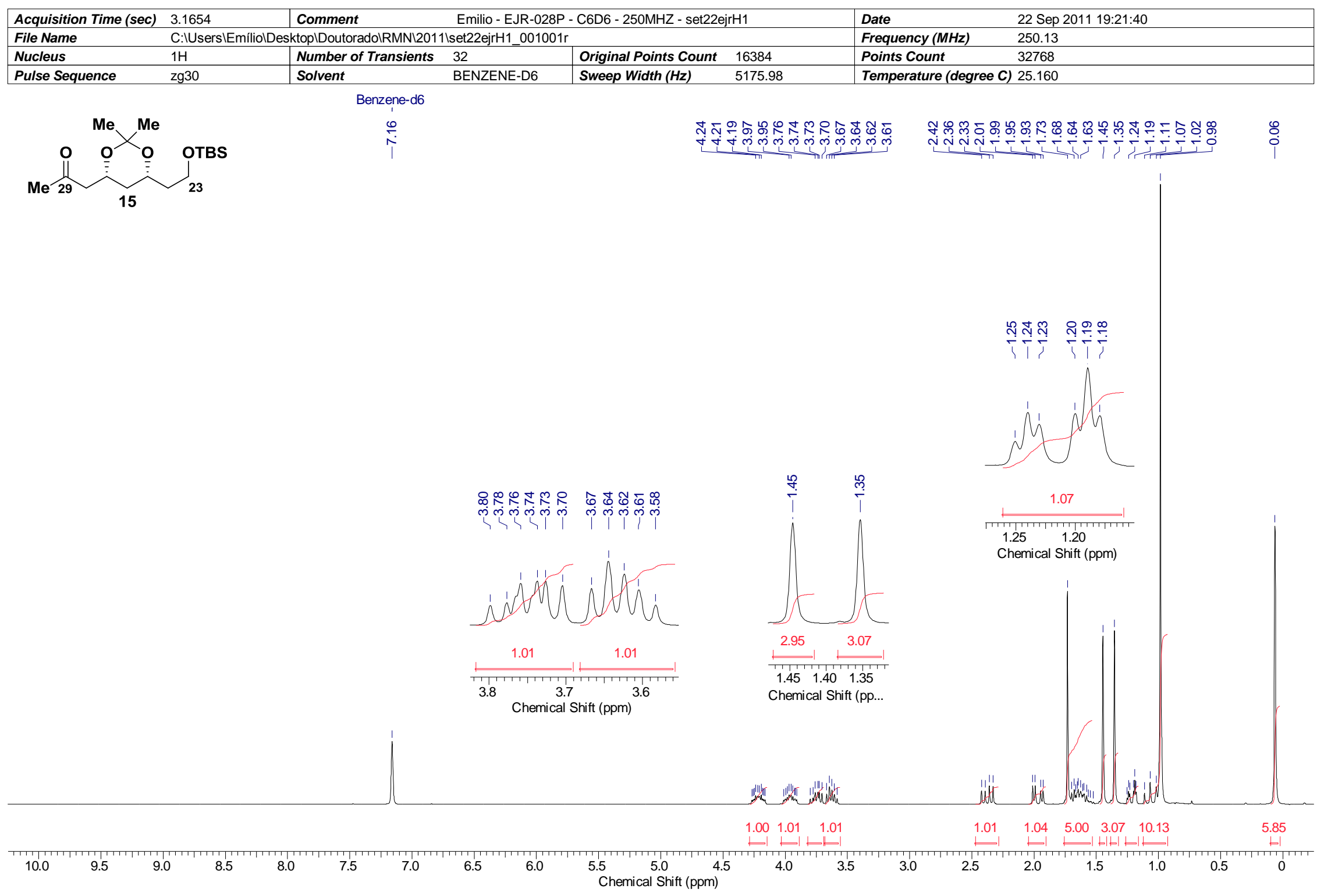

Figure 30: ${ }^{1} \mathrm{H}$ NMR spectrum of compound $15\left(250 \mathrm{MHz}, \mathrm{C}_{6} \mathrm{D}_{6}\right)$. 


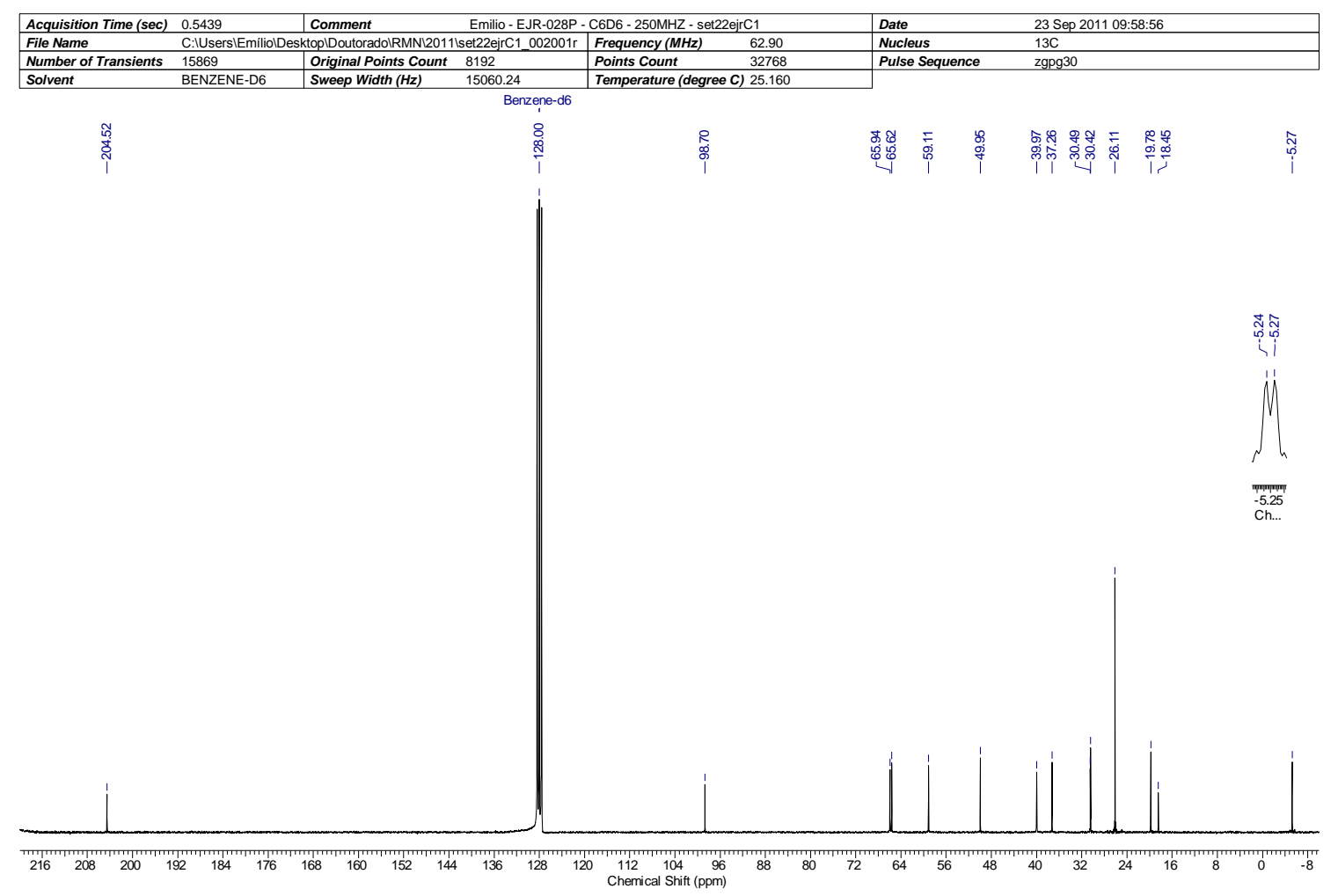

Figure 31: ${ }^{13} \mathrm{C}$ NMR spectrum of compound $15\left(62.5 \mathrm{MHz}, \mathrm{C}_{6} \mathrm{D}_{6}\right)$.

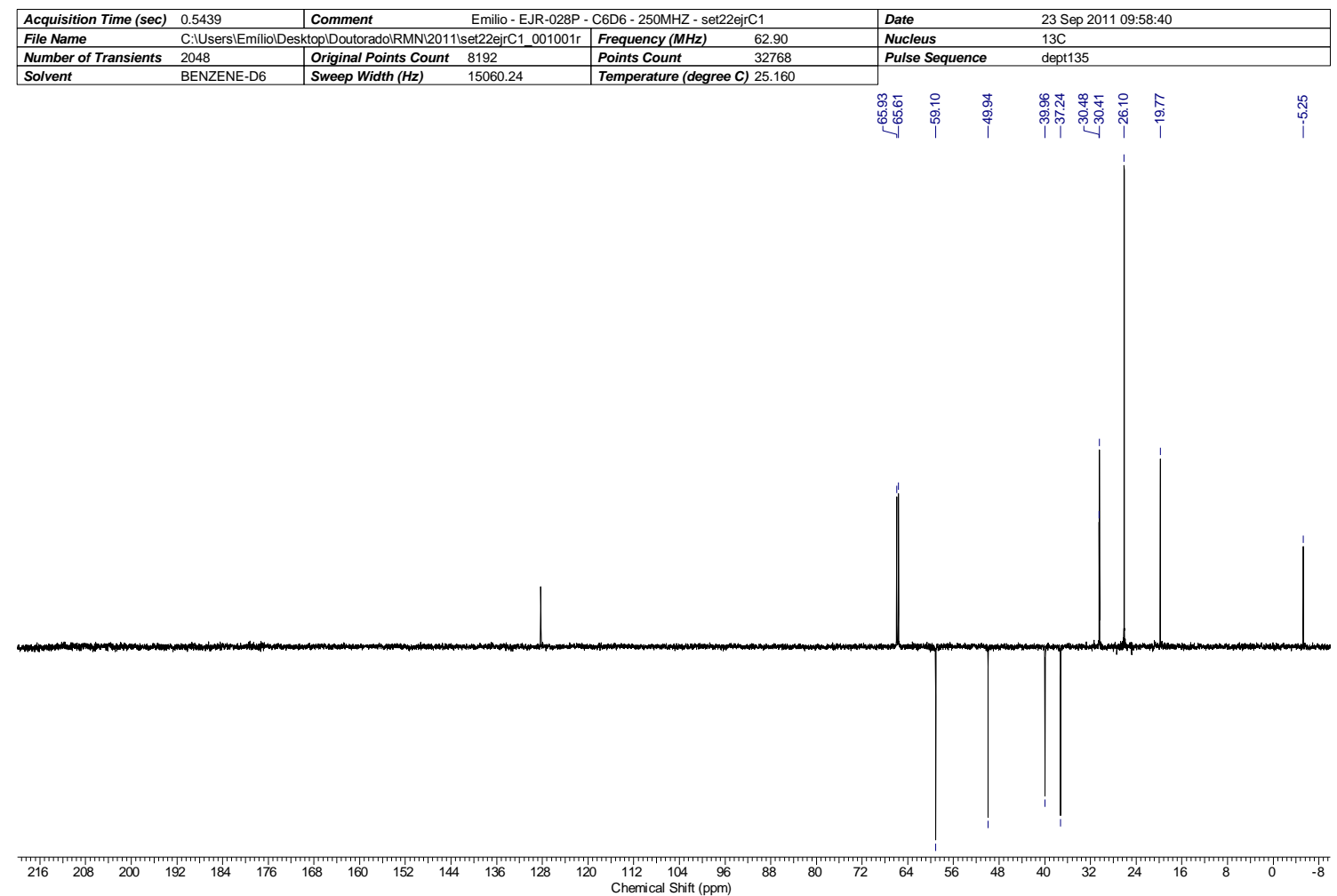

Figure 32: ${ }^{13} \mathrm{C}$ NMR (dept 135) spectrum of compound $15\left(62.5 \mathrm{MHz}, \mathrm{C}_{6} \mathrm{D}_{6}\right)$. 


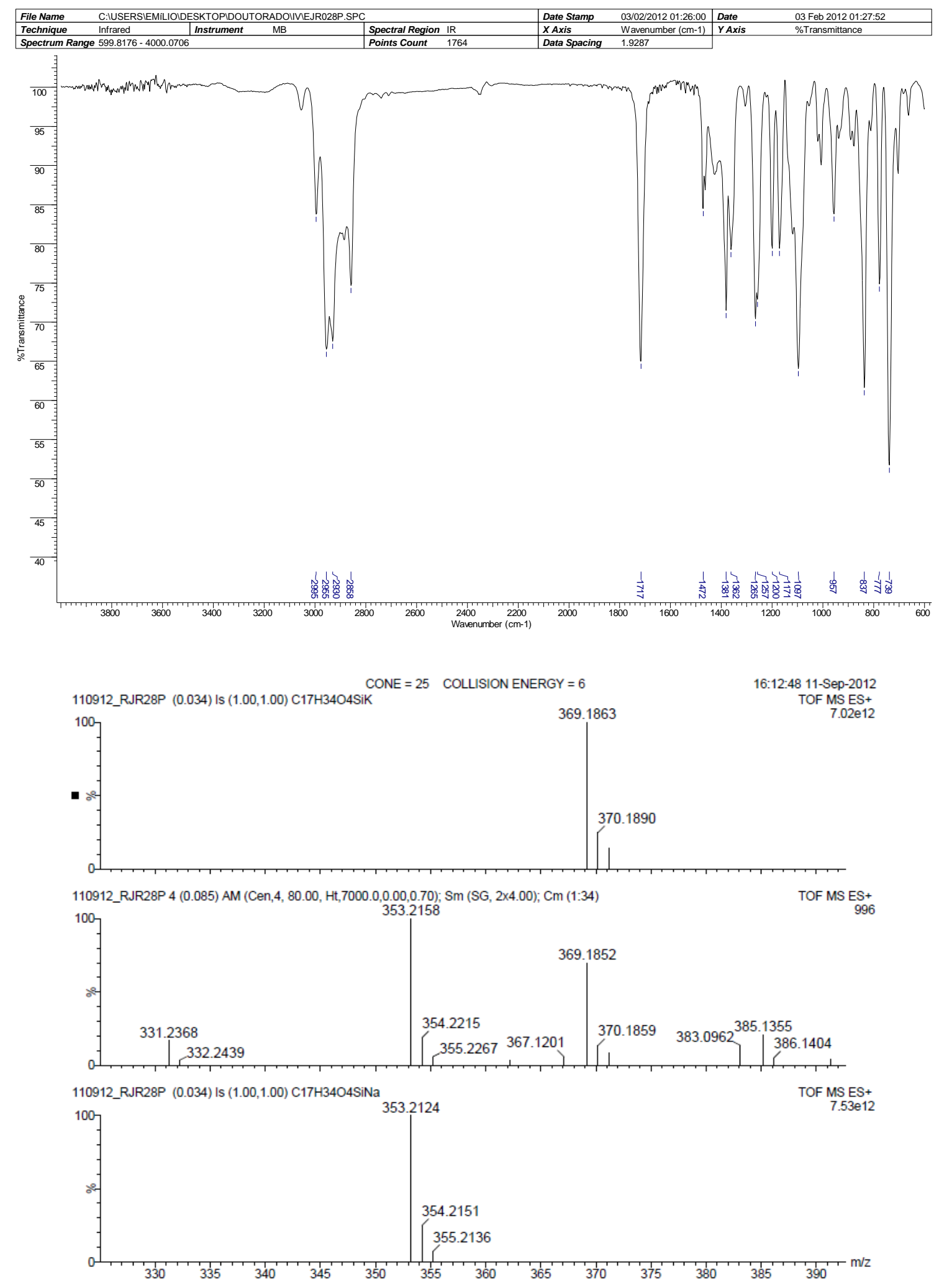

Figure 33: IR and HRMS (ESI TOF-MS) spectra of compound 15. 


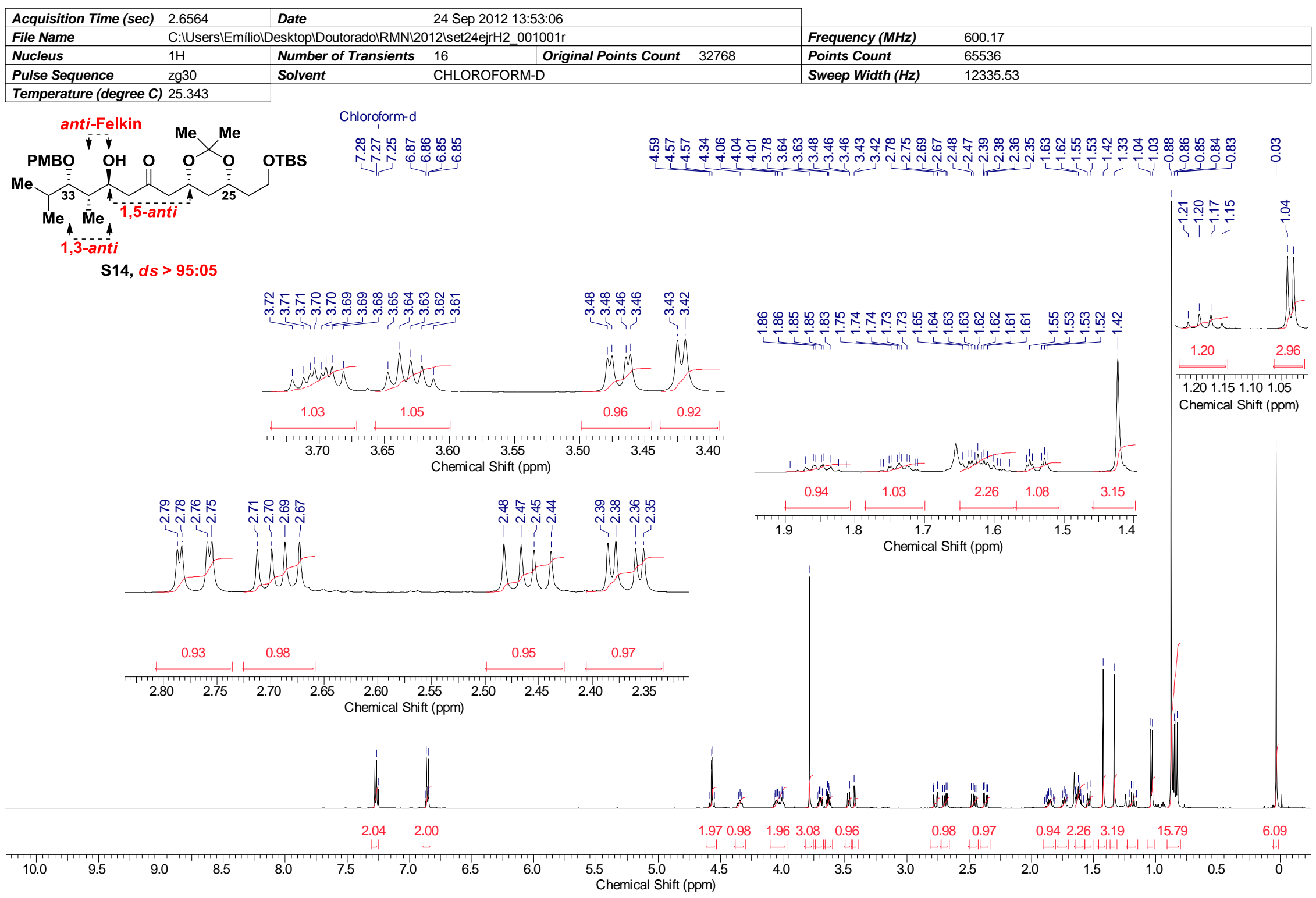

Figure 34: ${ }^{1} \mathrm{H}$ NMR spectrum of compound $\mathbf{S 1 4}\left(600 \mathrm{MHz}, \mathrm{CDCl}_{3}\right)$. 


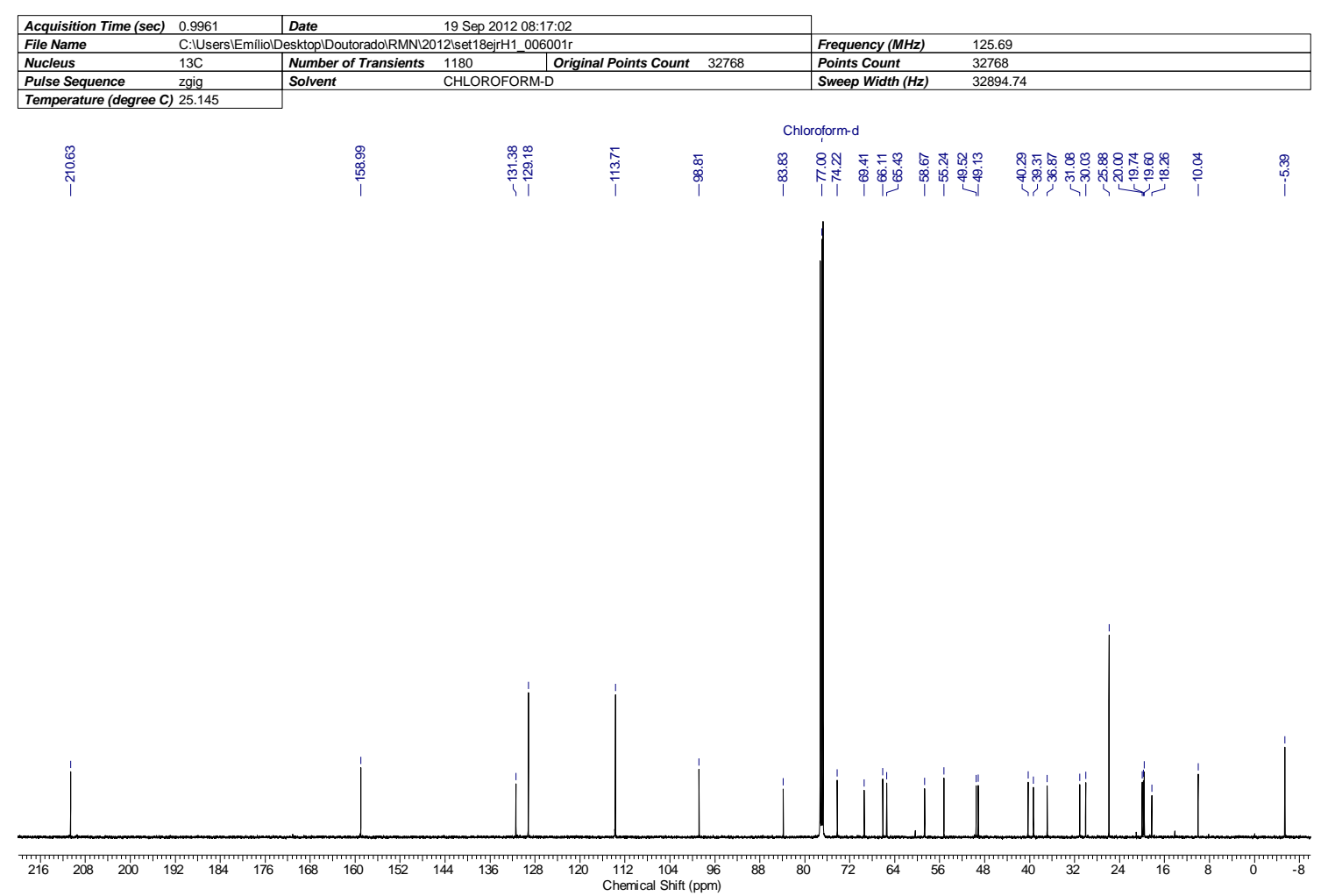

Figure 35: ${ }^{13} \mathrm{C}$ NMR spectrum of compound $\mathbf{S 1 4}\left(125 \mathrm{MHz}, \mathrm{CDCl}_{3}\right)$.
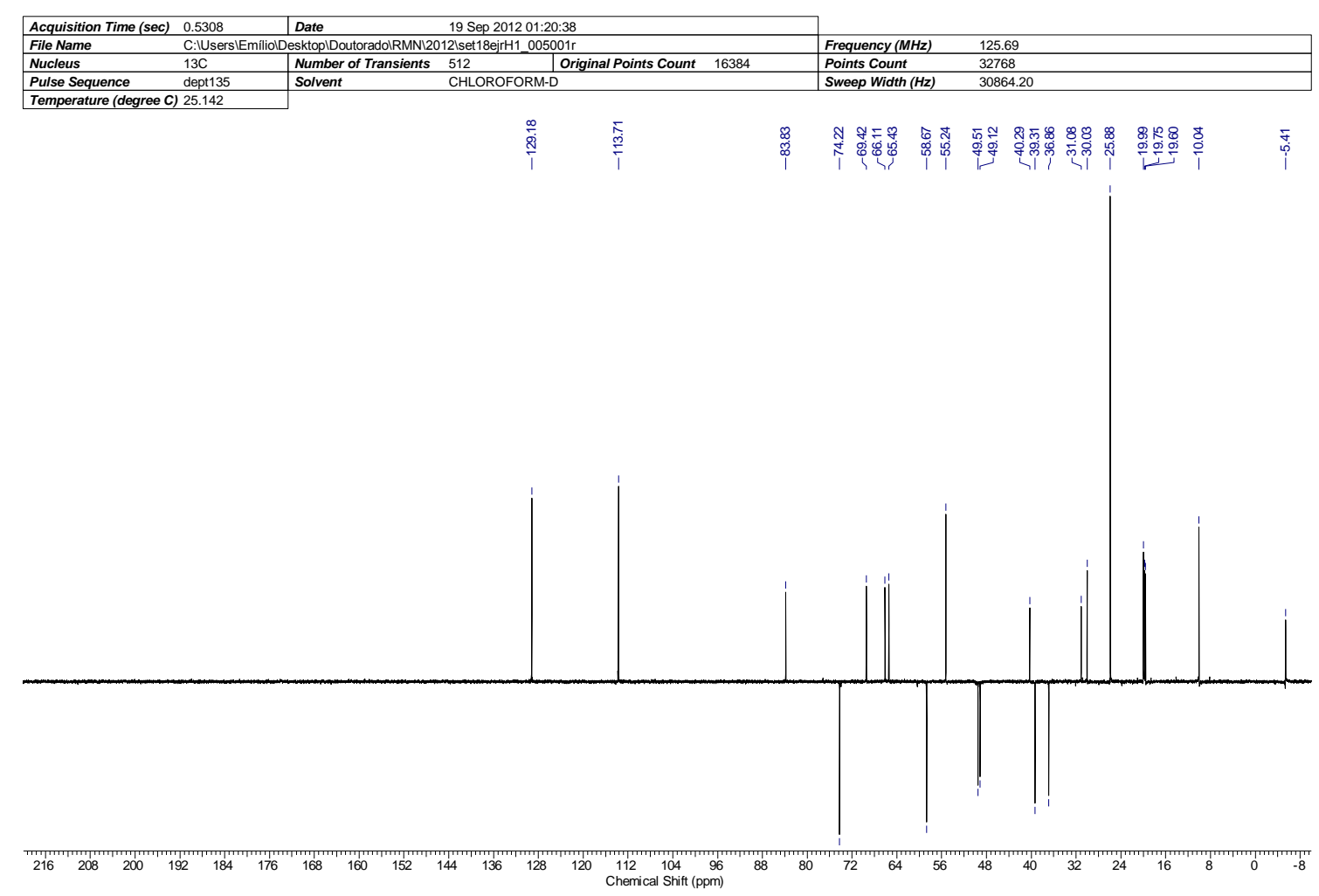

Figure 36: ${ }^{13} \mathrm{C}$ NMR (dept 135) spectrum of compound $\mathbf{S 1 4}\left(125 \mathrm{MHz}, \mathrm{CDCl}_{3}\right)$. 

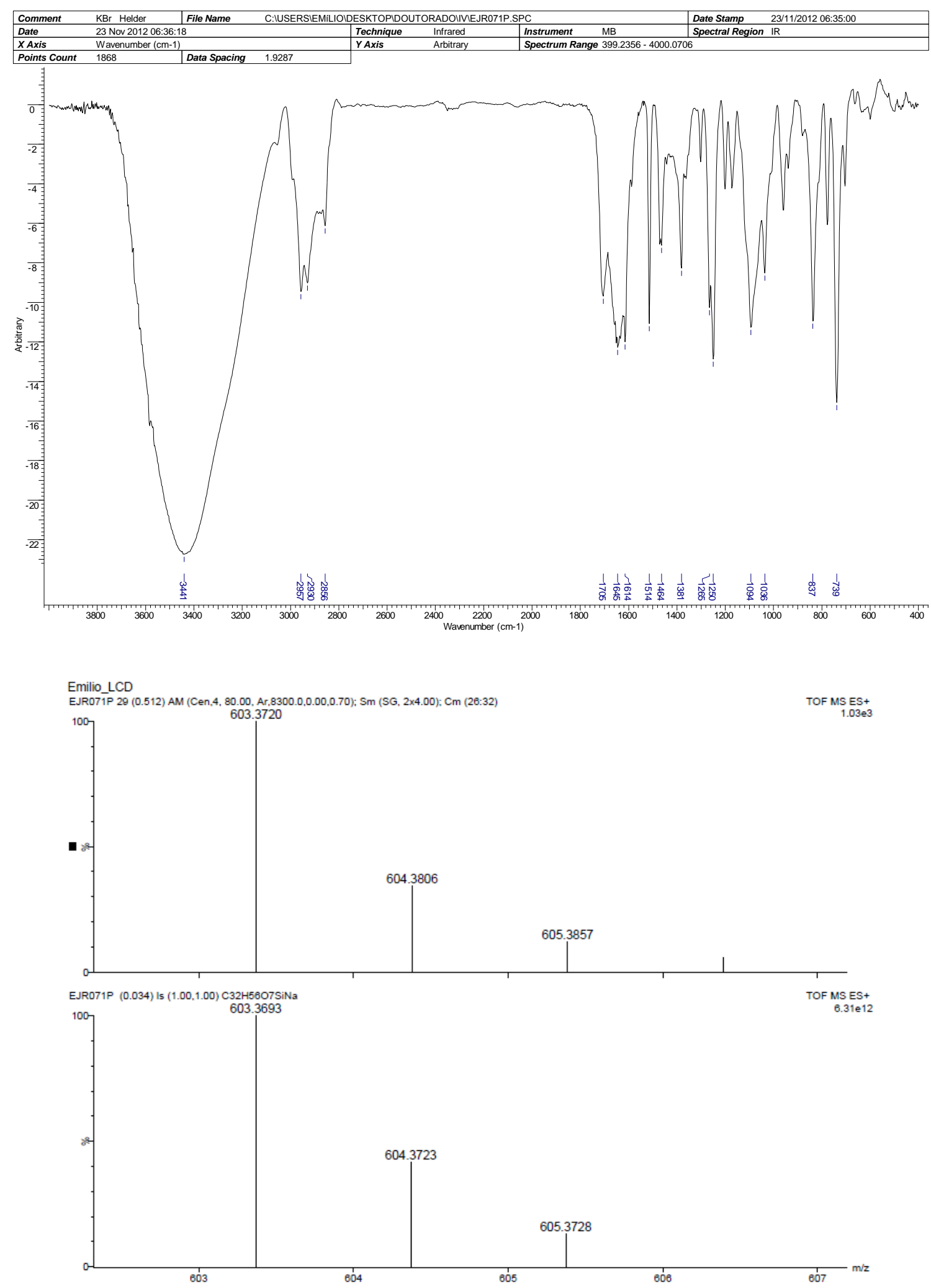

Figure 37: IR and HRMS (ESI TOF-MS) spectra of compound S14. 


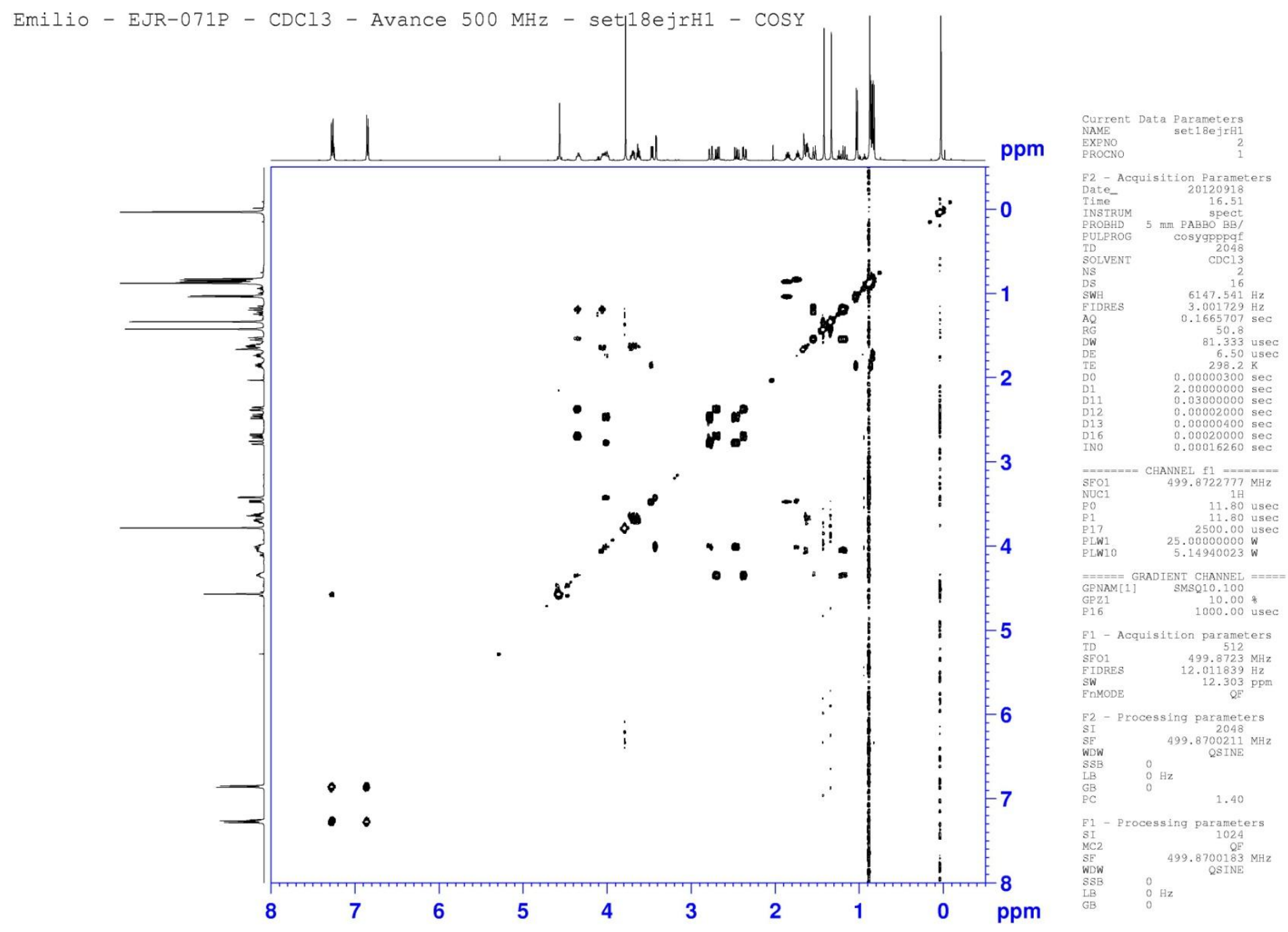

Figure 38: COSY contour map of compound $\mathbf{S} 14\left(500 \mathrm{MHz}, \mathrm{CDCl}_{3}\right)$. 


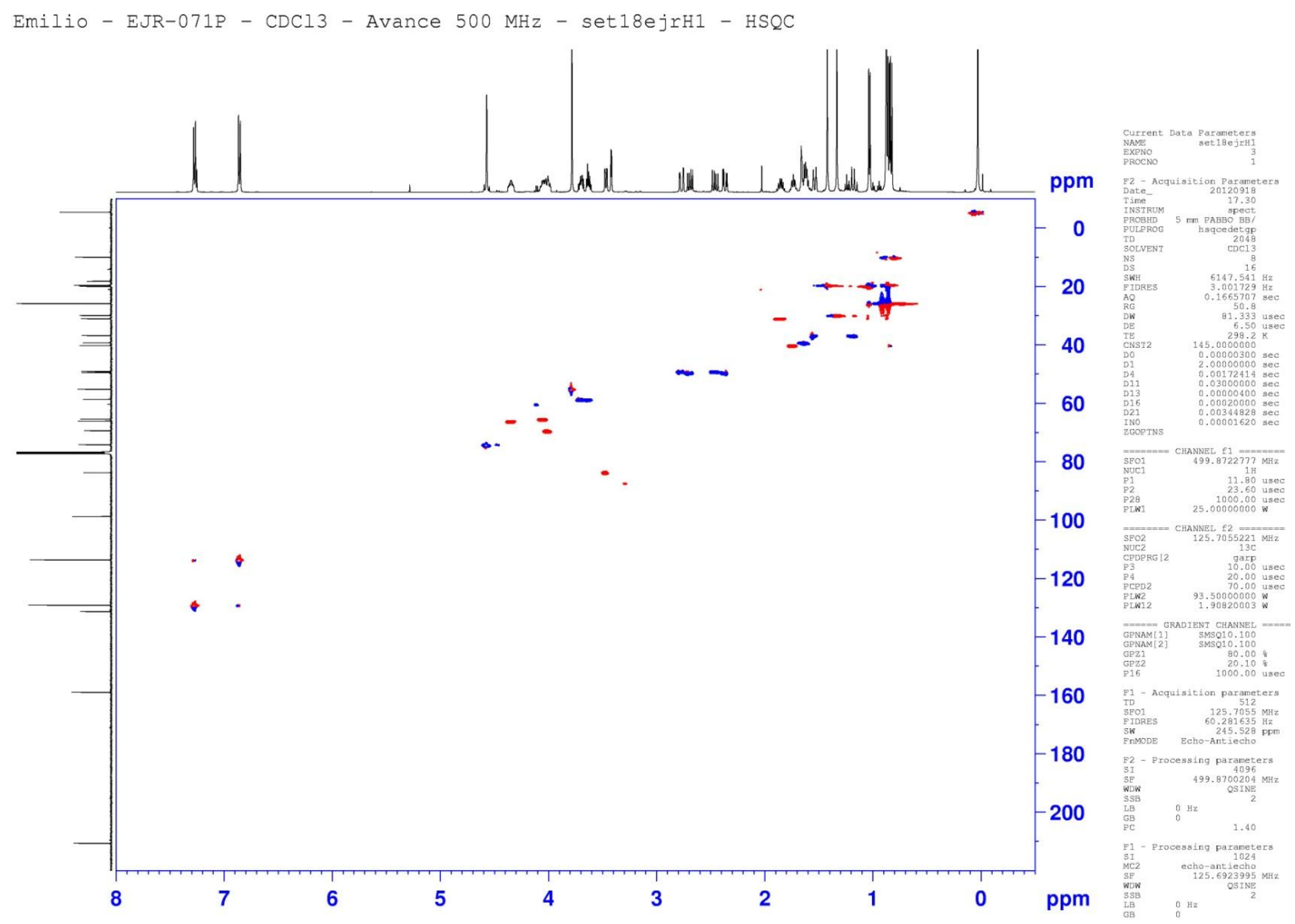

Figure 39: HSQC contour map of compound S14. 


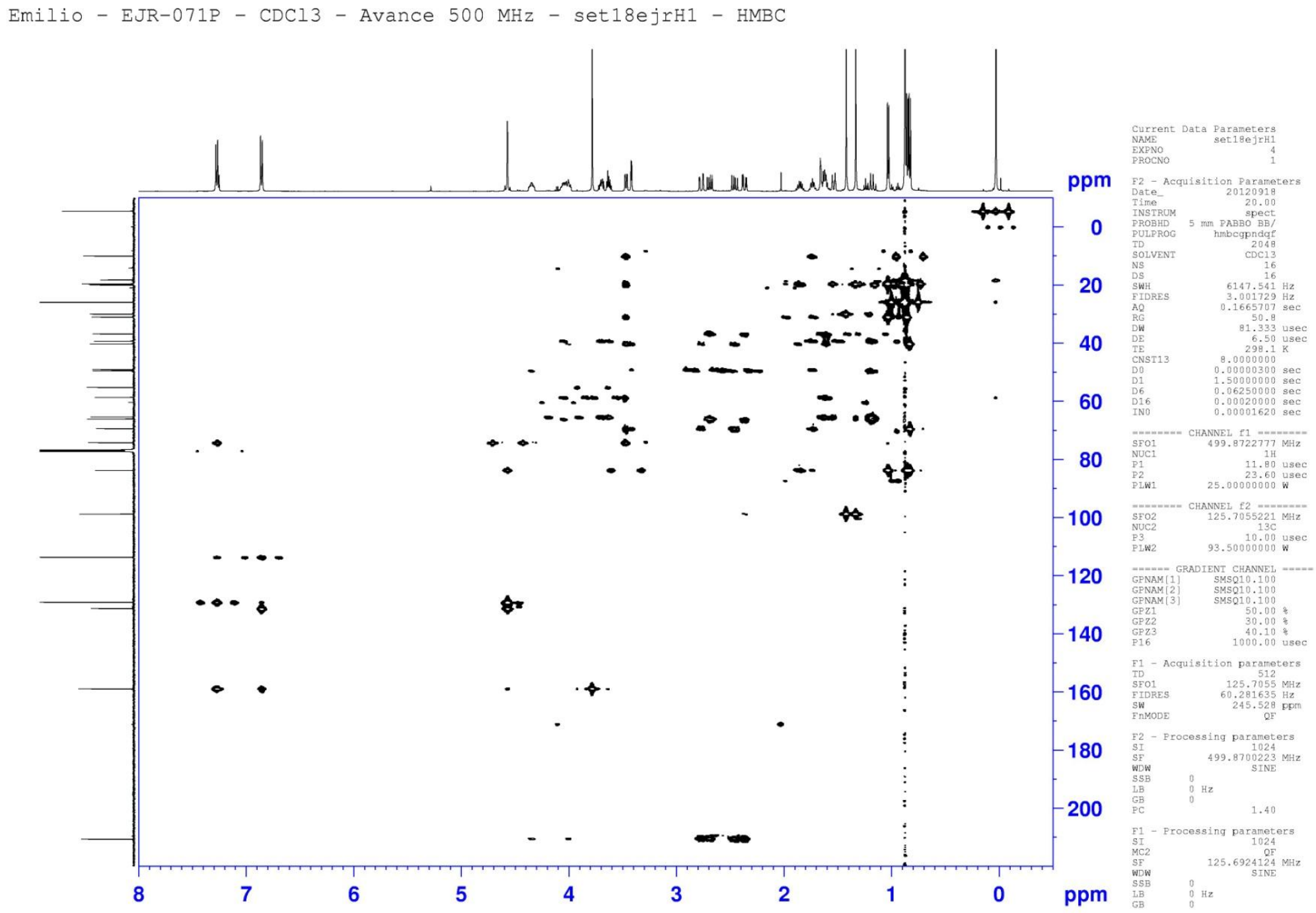

Figure 40: HMBC contour map of compound S14. 


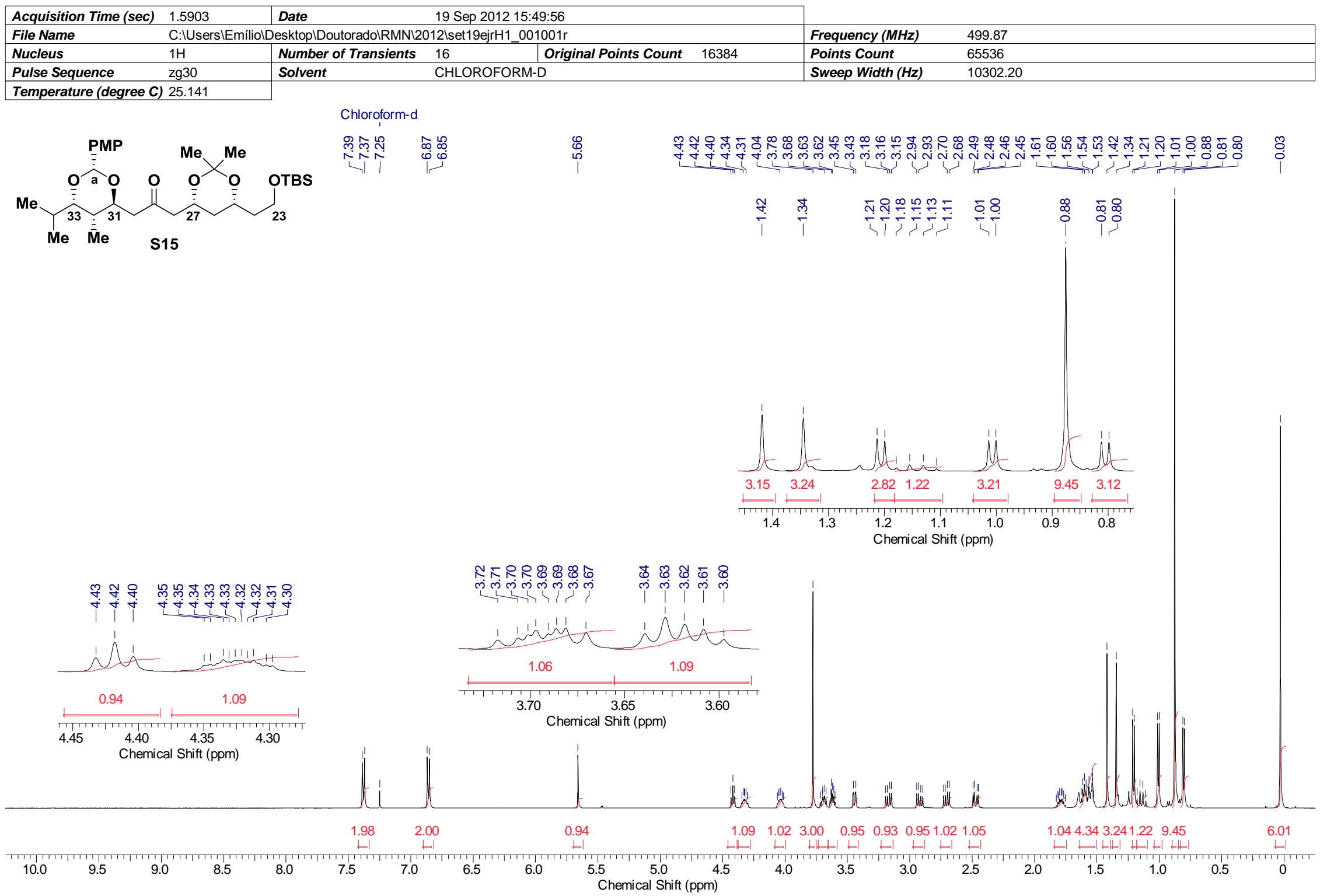

Figure 41: ${ }^{1} \mathrm{H}$ NMR spectrum of compound $\mathbf{S} 15\left(500 \mathrm{MHz}, \mathrm{CDCl}_{3}\right)$. 


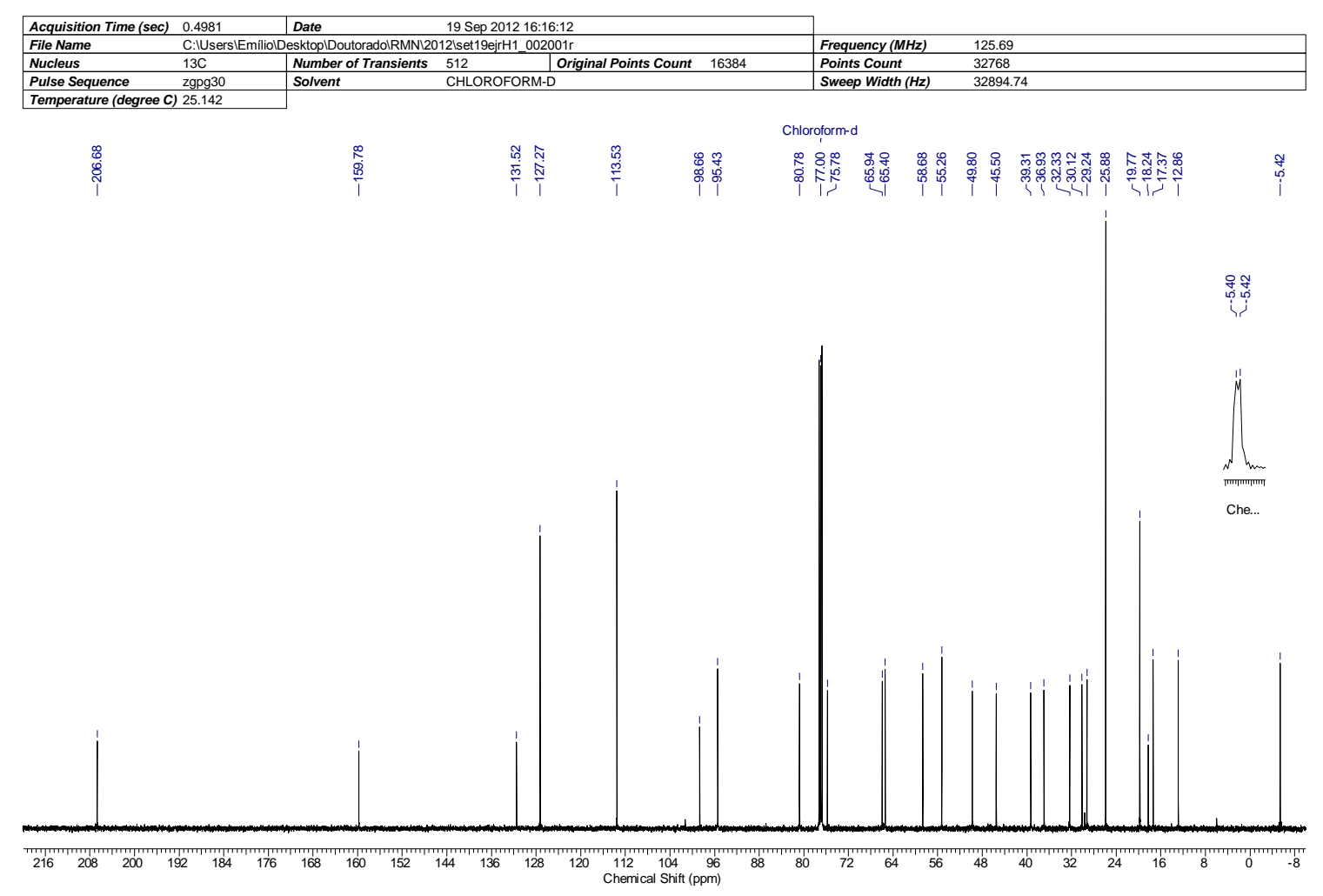

Figure 42: ${ }^{13} \mathrm{C}$ NMR spectrum of compound $\mathbf{S} 15\left(125 \mathrm{MHz}, \mathrm{CDCl}_{3}\right)$.

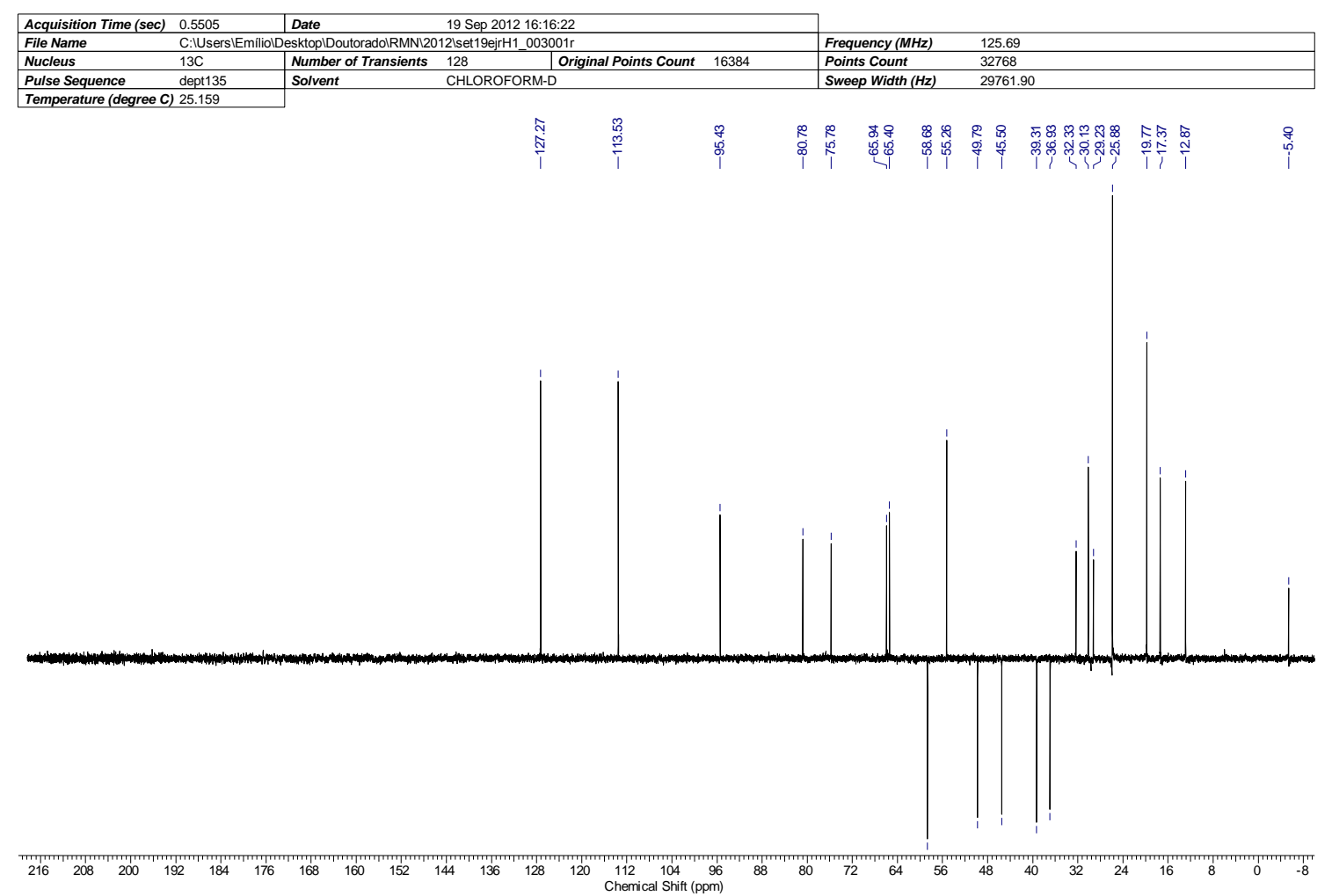

Figure 43: ${ }^{13} \mathrm{C}$ NMR (dept 135) spectrum of compound $\mathbf{S} 15\left(125 \mathrm{MHz}, \mathrm{CDCl}_{3}\right)$. 


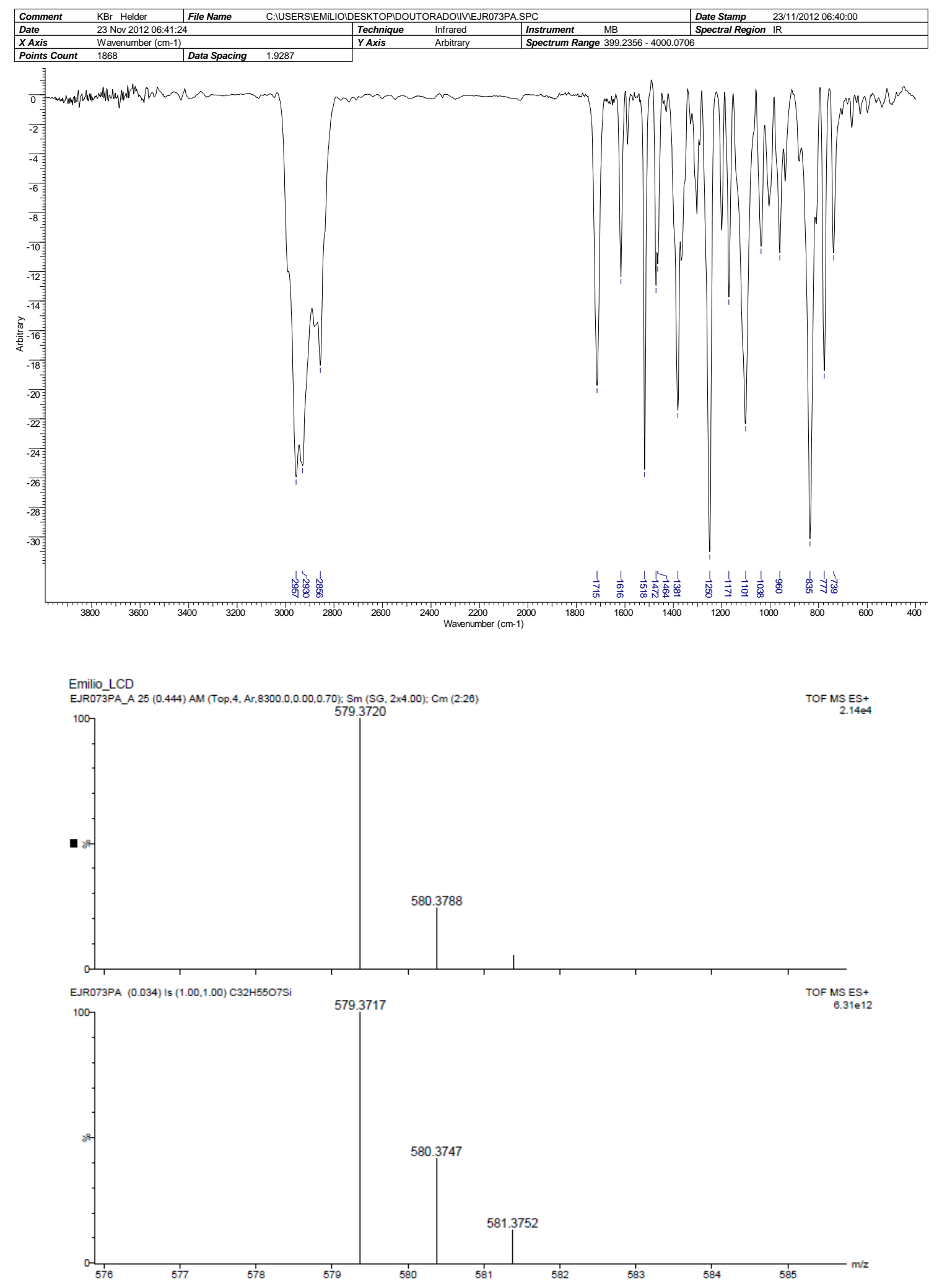

Figure 44: IR and HRMS (ESI TOF-MS) spectra of compound S15. 


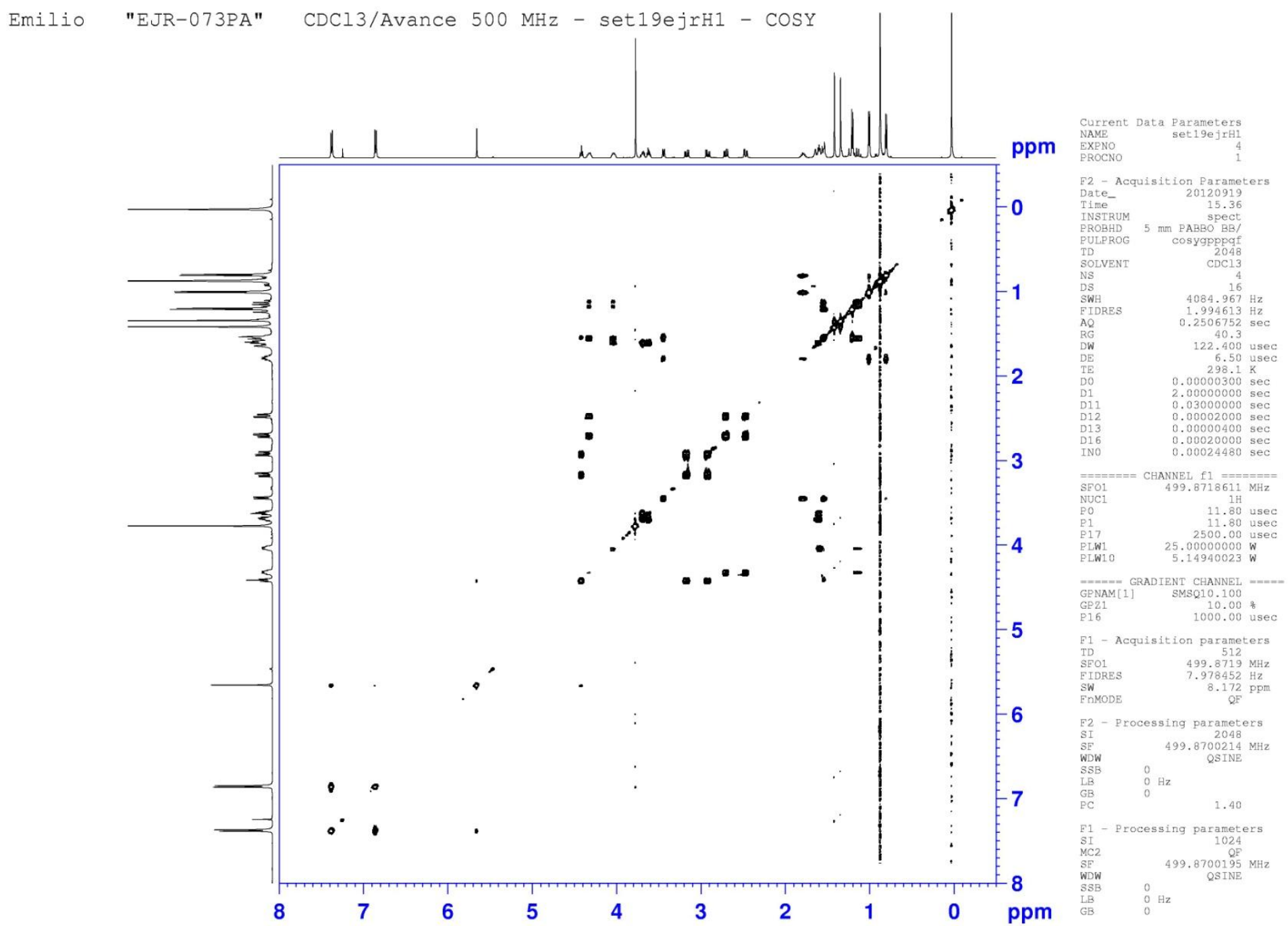

Figure 45: COSY contour map of compound $\mathbf{S} 15\left(500 \mathrm{MHz}, \mathrm{CDCl}_{3}\right)$. 


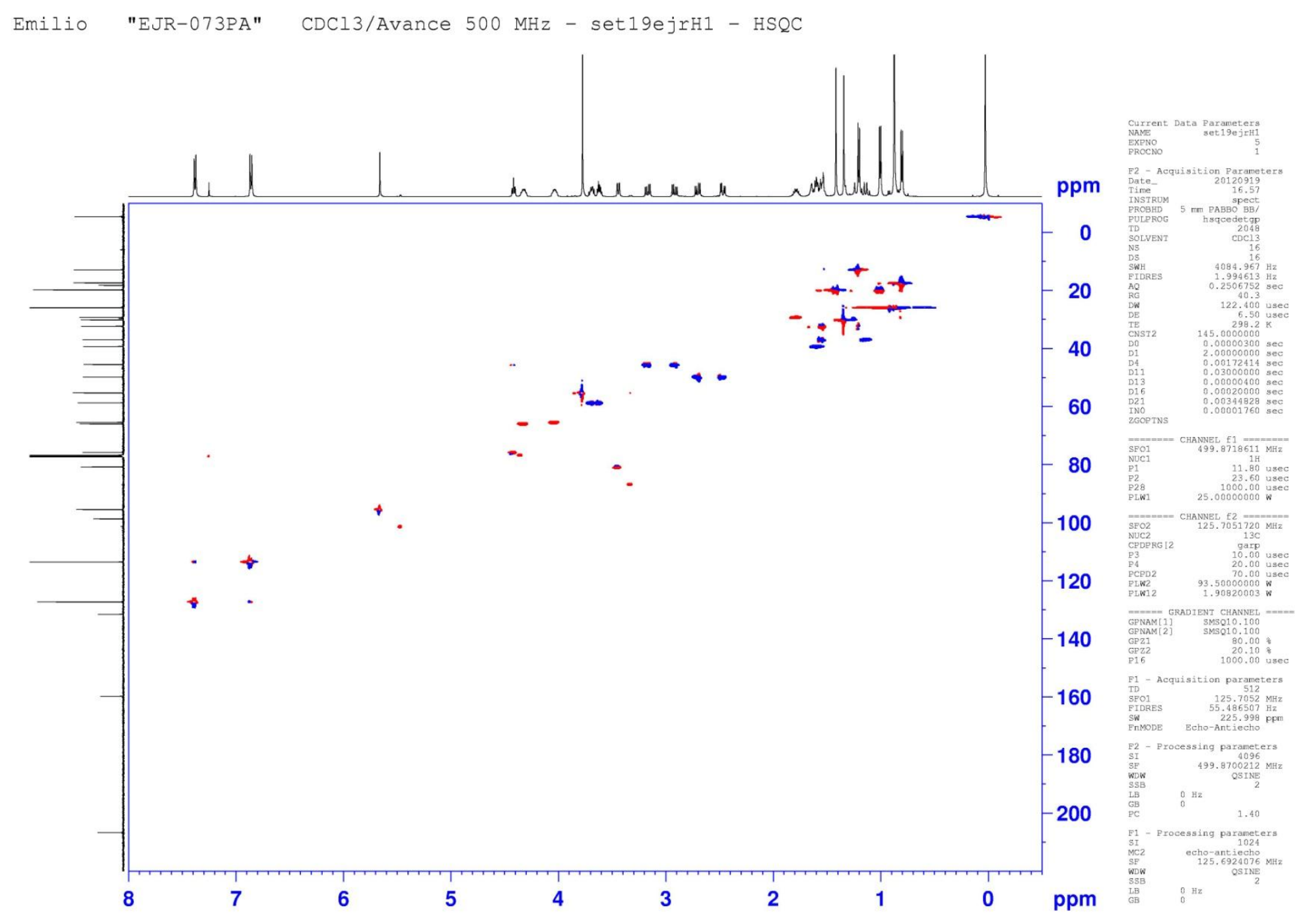

Figure 46: HSQC contour map of compound S15. 


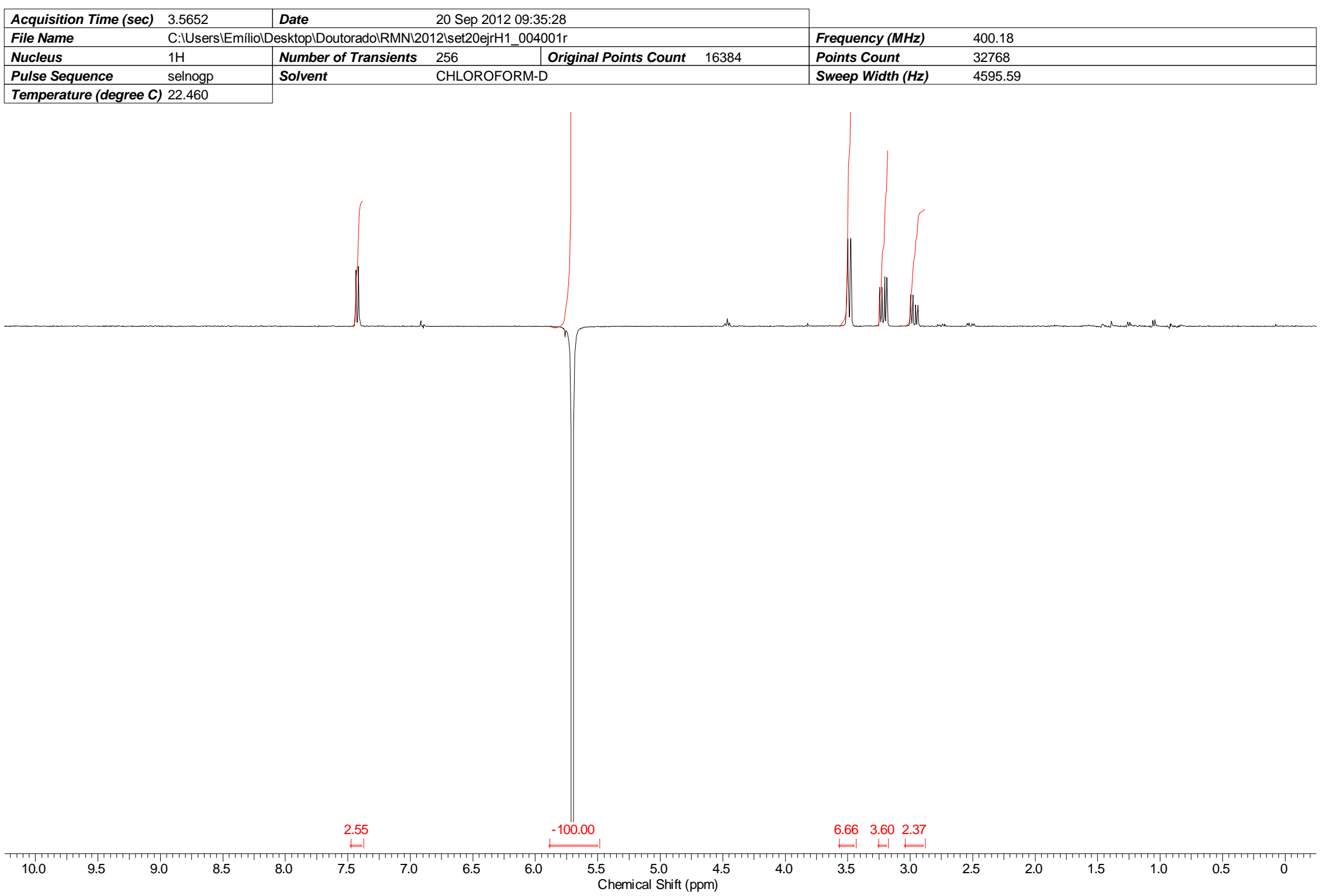

Figure 47: ${ }^{1} \mathrm{H}$ NMR (1D selective NOE) spectrum of compound $\mathbf{S} 15\left(400 \mathrm{MHz}, \mathrm{CDCl}_{3}\right)$. 


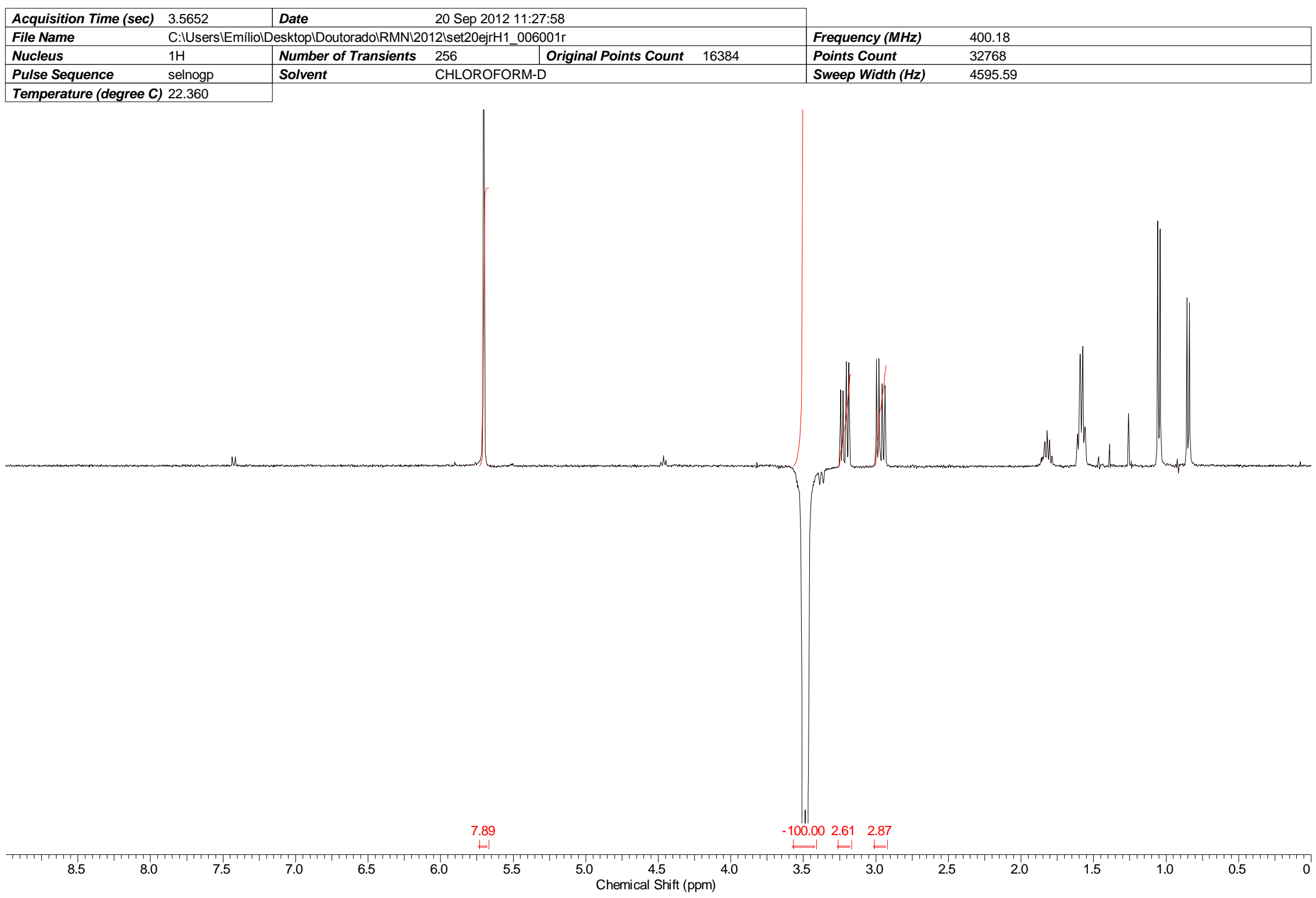

Figure 48: ${ }^{1} \mathrm{H}$ NMR (1D selective NOE) spectrum of compound $\mathbf{S} 15\left(400 \mathrm{MHz}, \mathrm{CDCl}_{3}\right)$. 


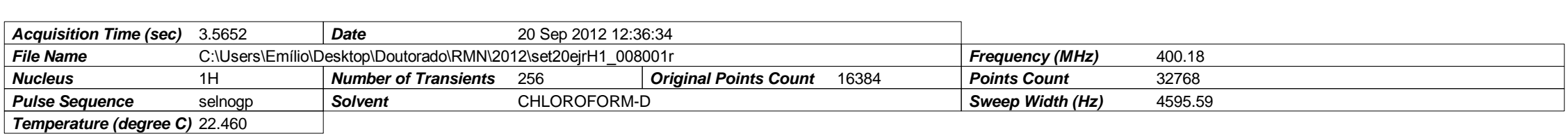

Pulse Sequence selnogp

4595.59

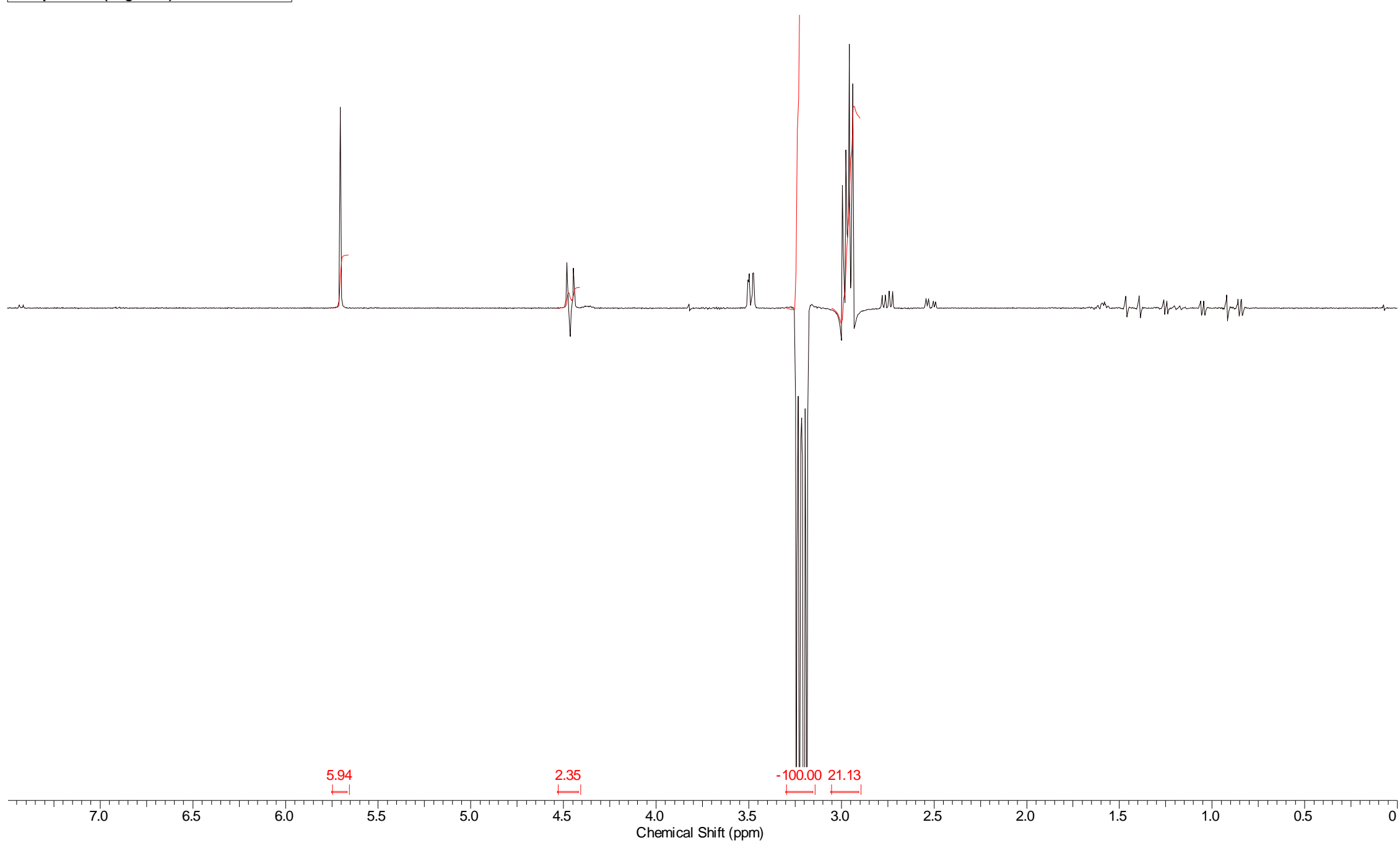

Figure 49: ${ }^{1} \mathrm{H}$ NMR (1D selective NOE) spectrum of compound $\mathbf{S} 15\left(400 \mathrm{MHz}, \mathrm{CDCl}_{3}\right)$. 


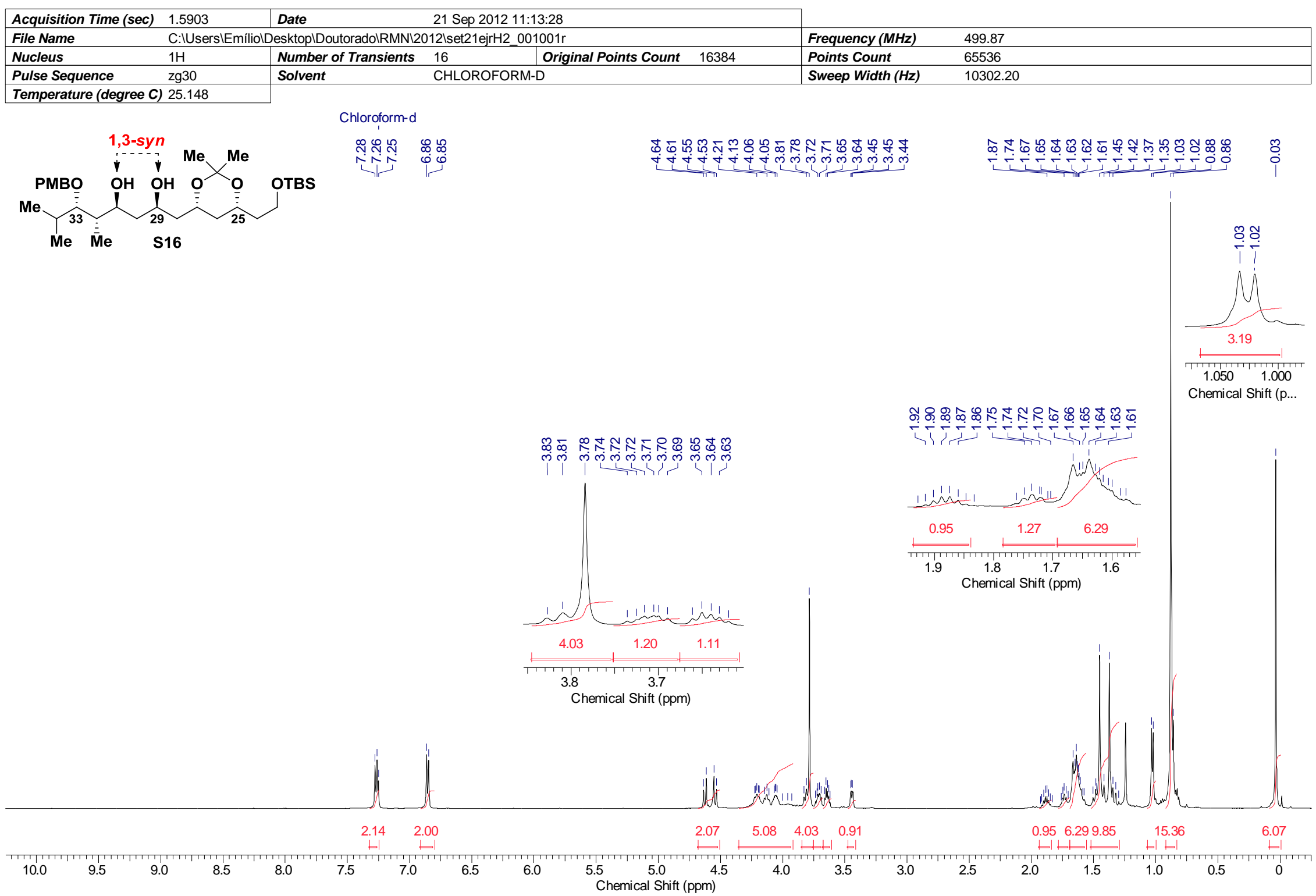

Figure 50: ${ }^{1} \mathrm{H}$ NMR spectrum of compound $\mathbf{S} 16\left(500 \mathrm{MHz}, \mathrm{CDCl}_{3}\right)$. 


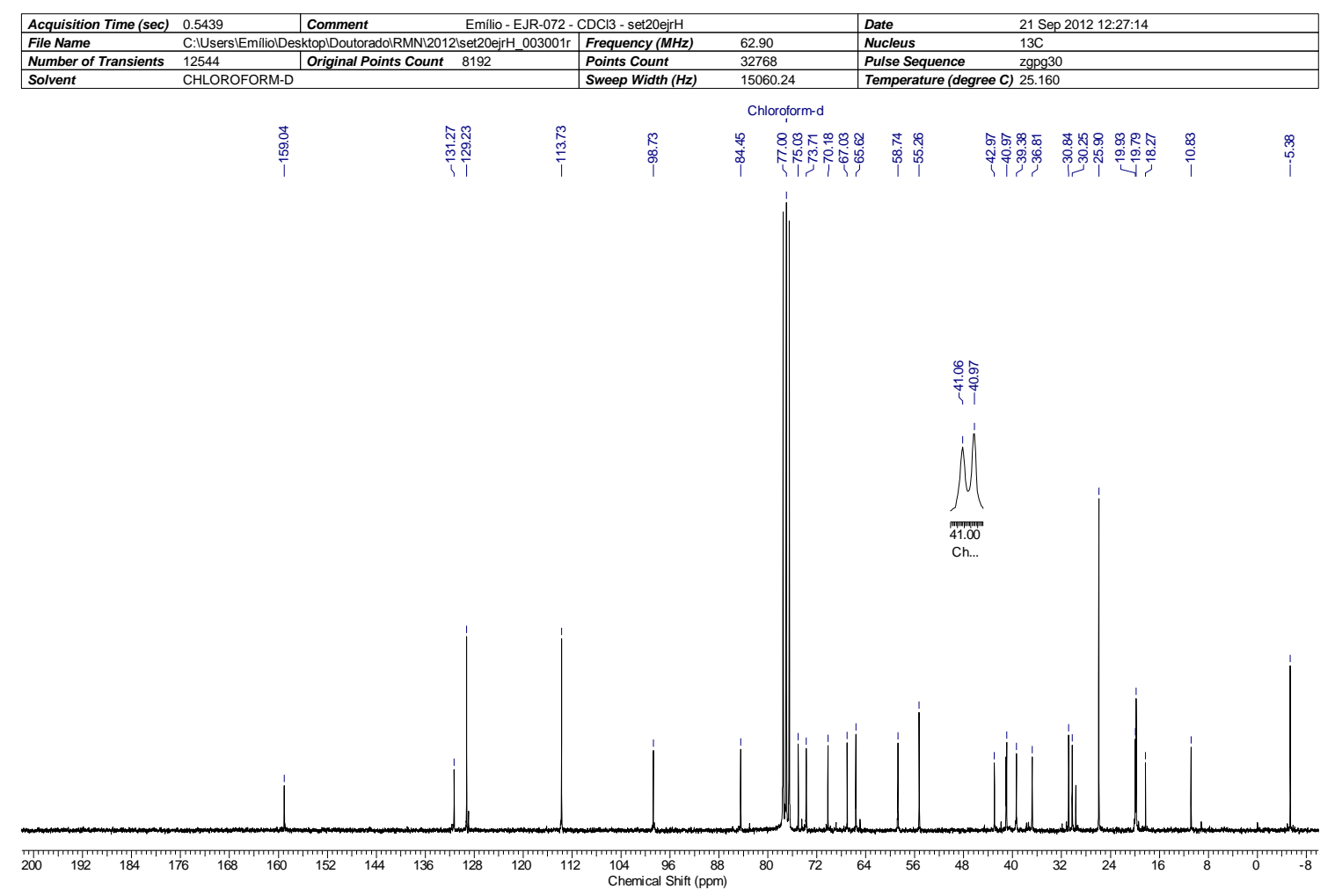

Figure 51: ${ }^{13} \mathrm{C}$ NMR spectrum of compound $\mathbf{S} 16\left(62.5 \mathrm{MHz}, \mathrm{CDCl}_{3}\right)$.

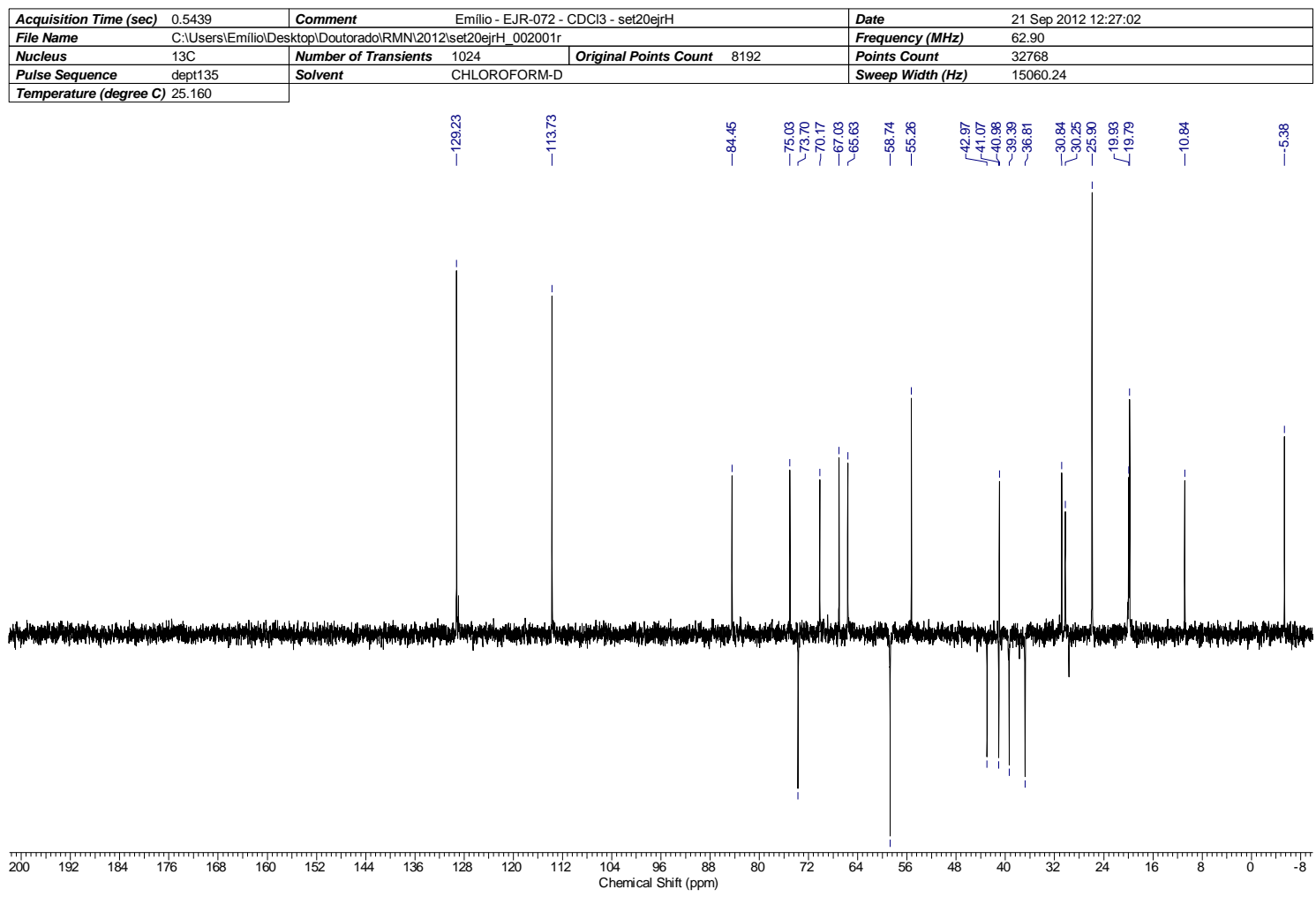

Figure 52: ${ }^{13} \mathrm{C}$ NMR (dept 135) spectrum of compound $\mathbf{S} 16\left(62.5 \mathrm{MHz}, \mathrm{CDCl}_{3}\right)$. 

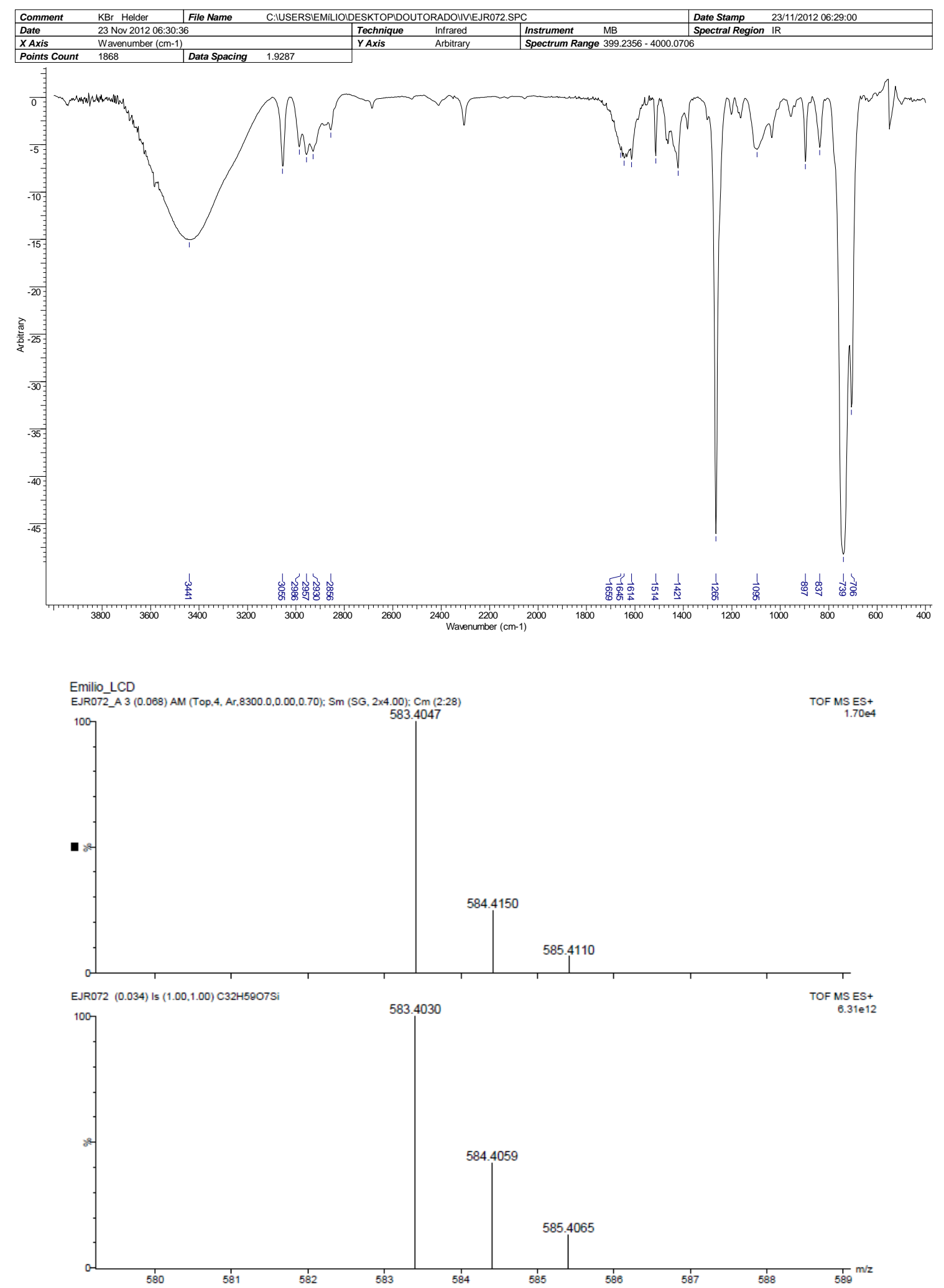

Figure 53: IR and HRMS (ESI TOF-MS) spectra of compound S16. 


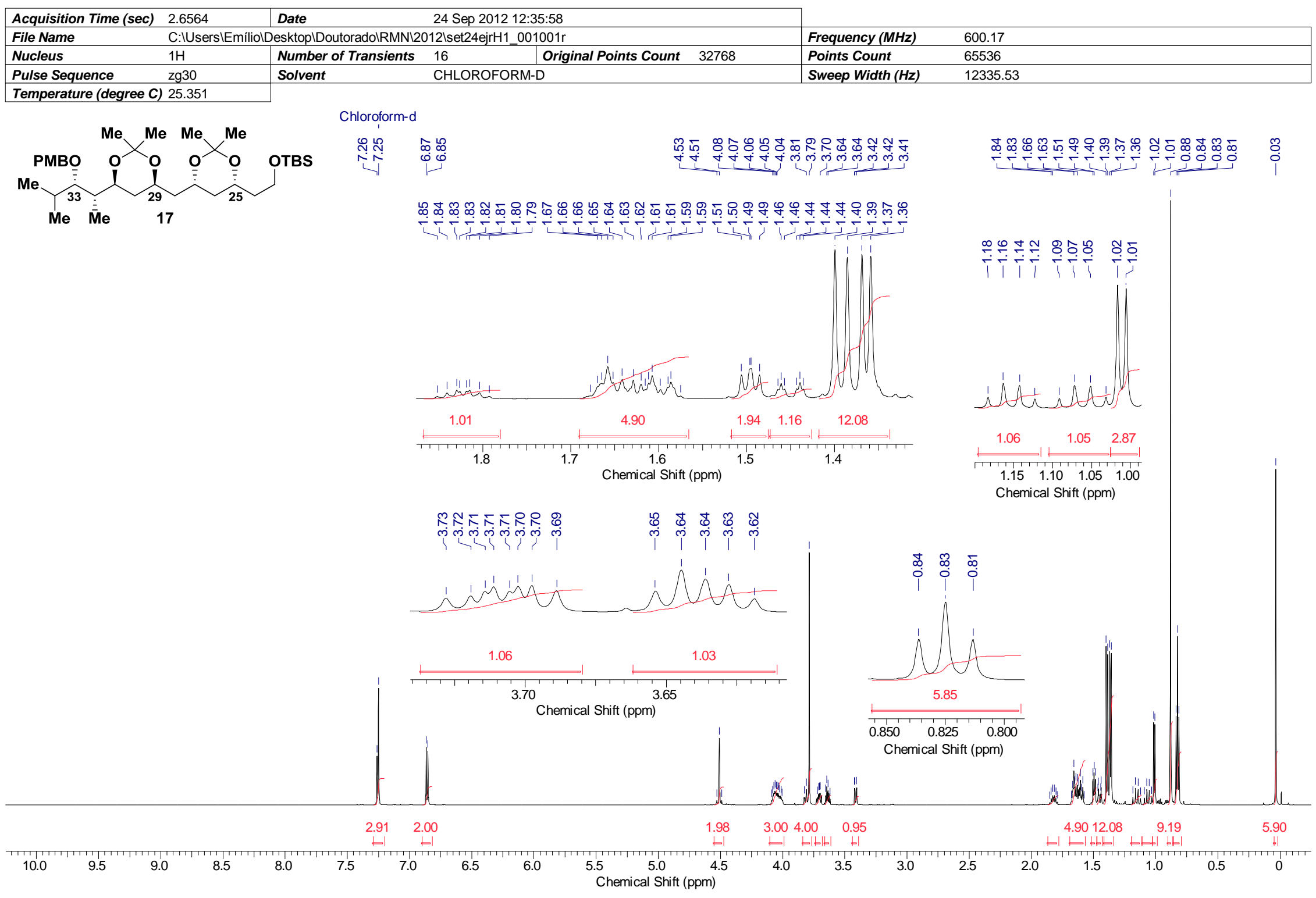

Figure 54: ${ }^{1} \mathrm{H}$ NMR spectrum of compound $17\left(600 \mathrm{MHz}, \mathrm{CDCl}_{3}\right)$. 


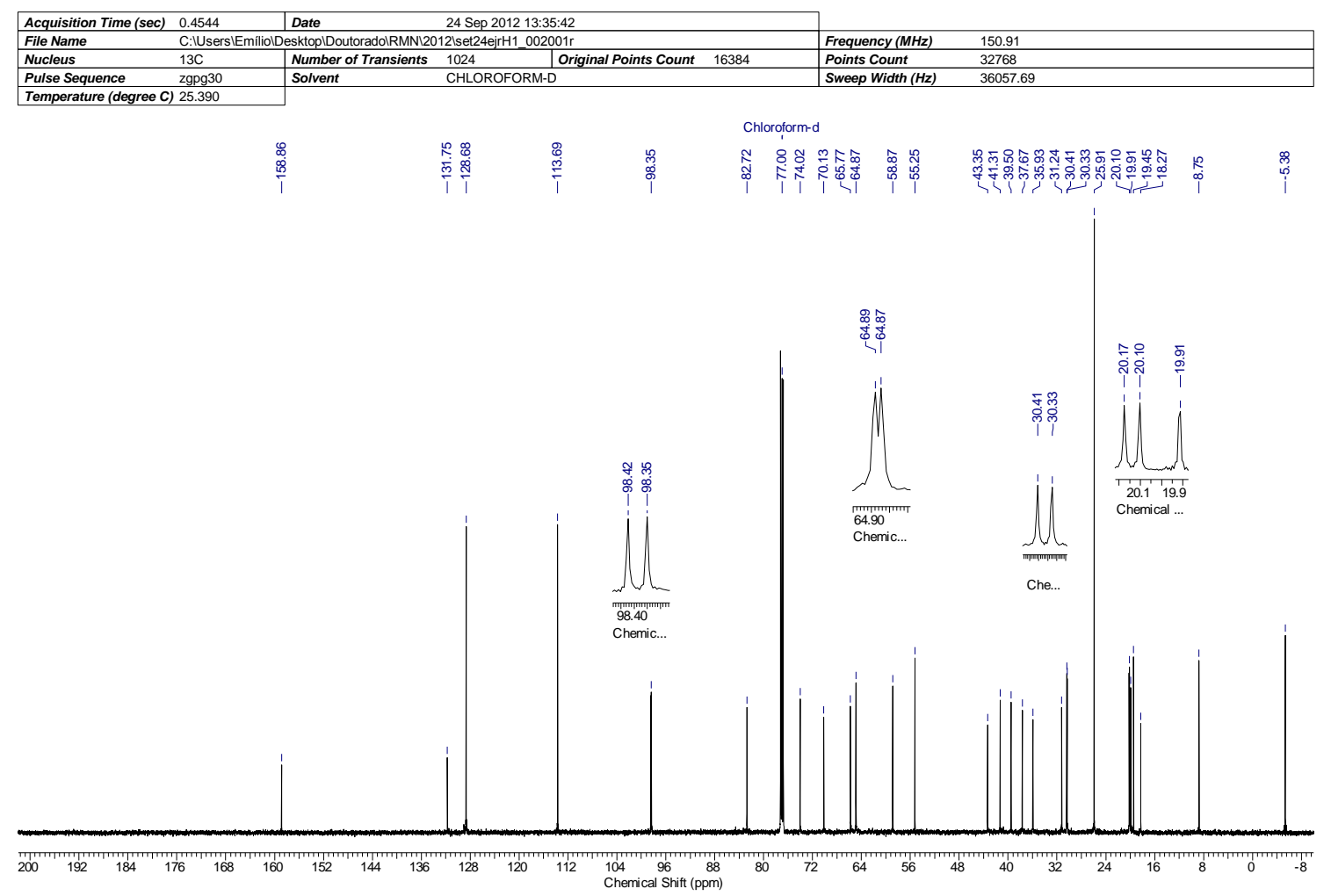

Figure 55: ${ }^{13} \mathrm{C}$ NMR spectrum of compound $17\left(150 \mathrm{MHz}, \mathrm{CDCl}_{3}\right)$.

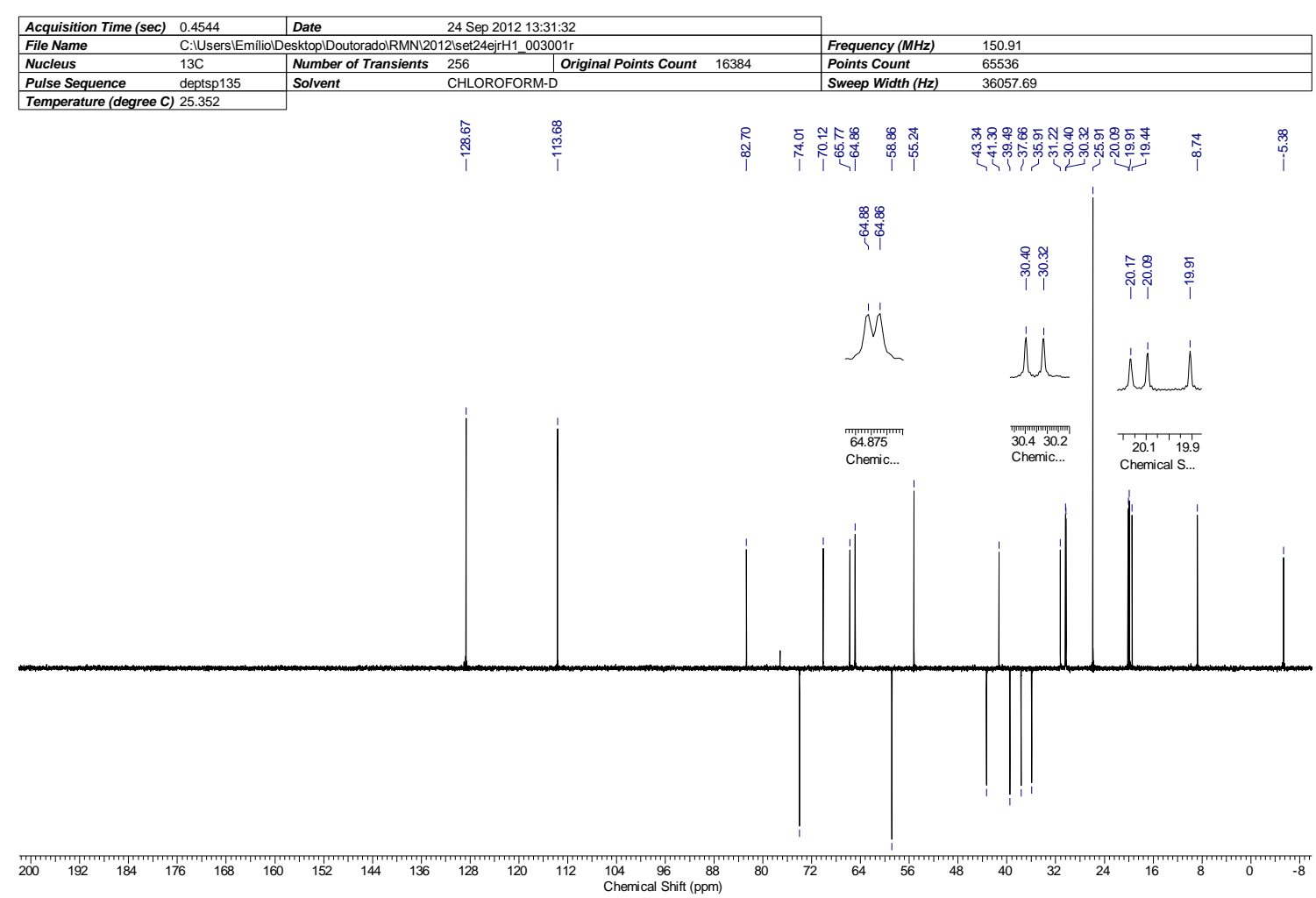

Figure 56: ${ }^{13} \mathrm{C}$ NMR (dept 135) spectrum of compound $17\left(150 \mathrm{MHz}, \mathrm{CDCl}_{3}\right)$. 

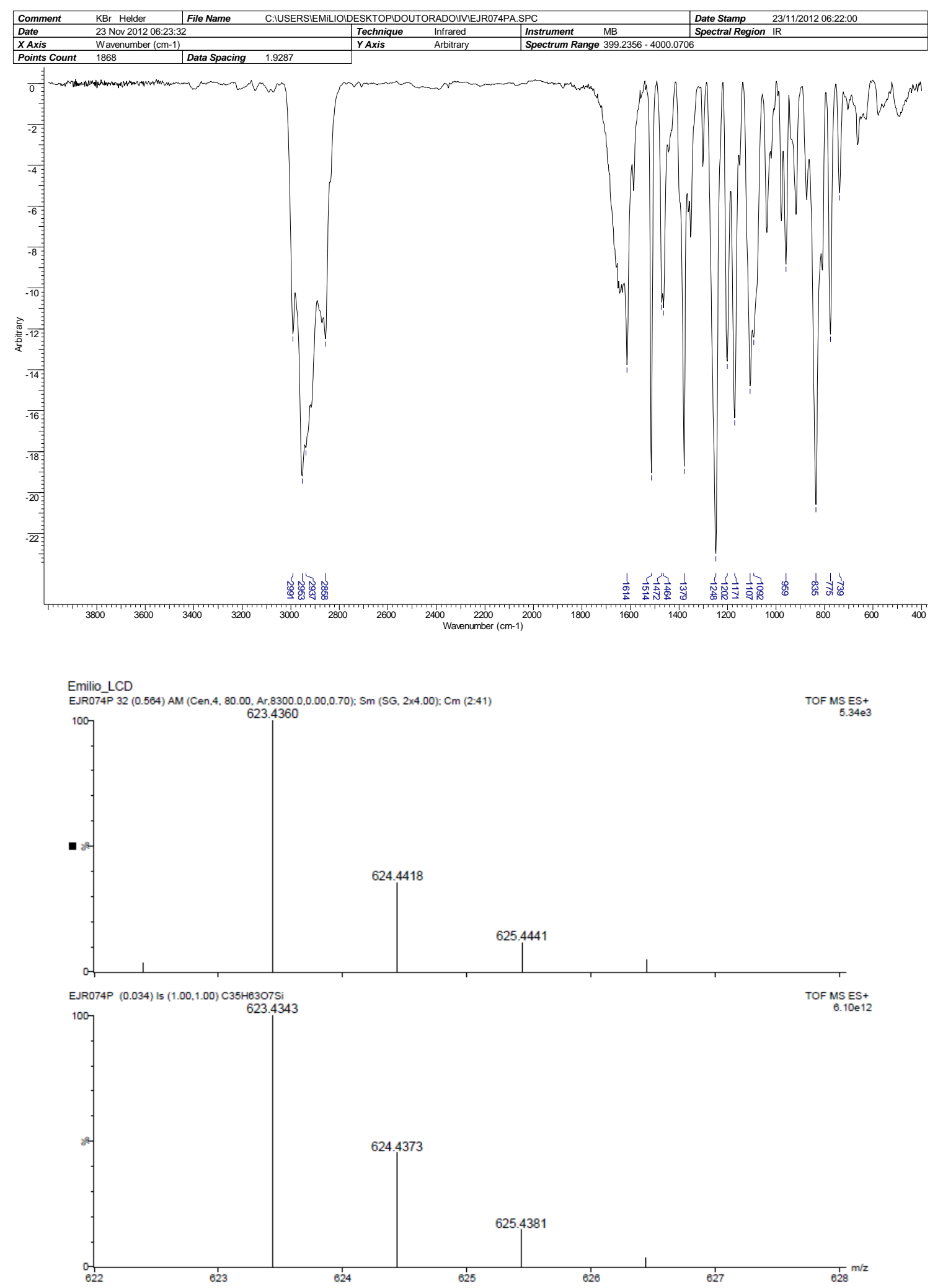

Figure 57: IR and HRMS (ESI TOF-MS) spectra of compound 17. 


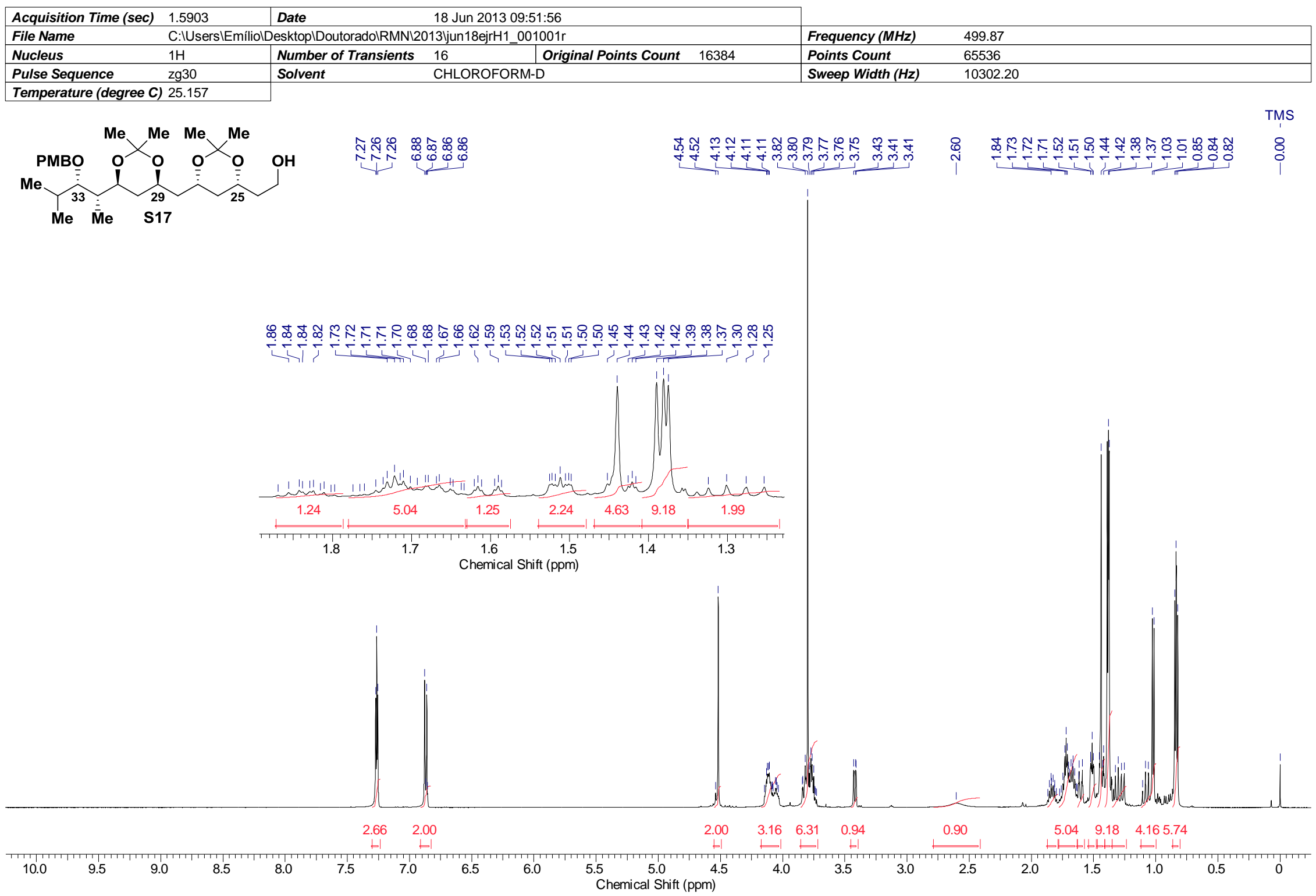

Figure 58: ${ }^{1} \mathrm{H}$ NMR spectrum of compound $\mathbf{S} 17\left(500 \mathrm{MHz}, \mathrm{CDCl}_{3}\right)$. 


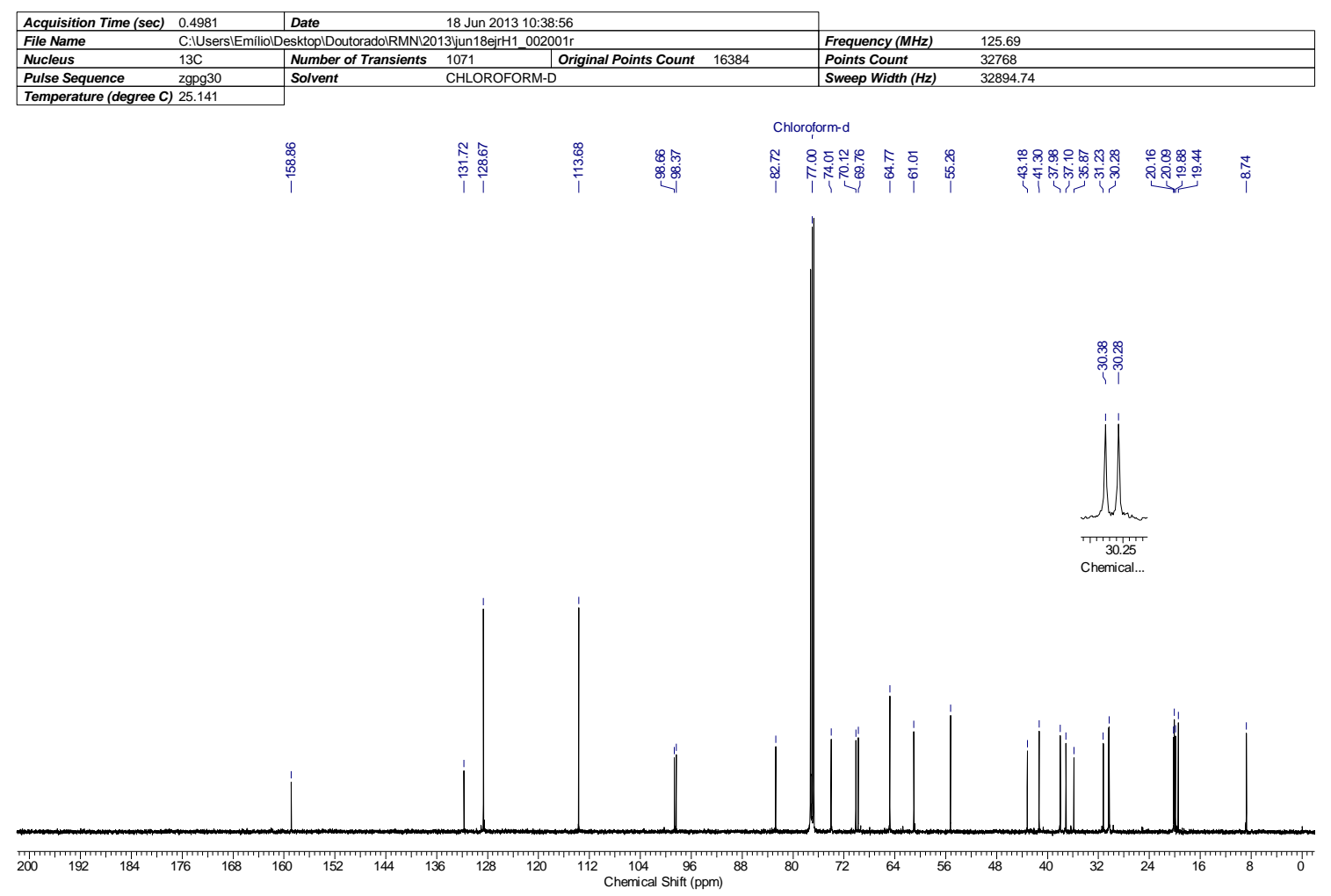

Figure 59: ${ }^{13} \mathrm{C}$ NMR spectrum of compound $\mathbf{S 1 7}\left(125 \mathrm{MHz}, \mathrm{CDCl}_{3}\right)$.

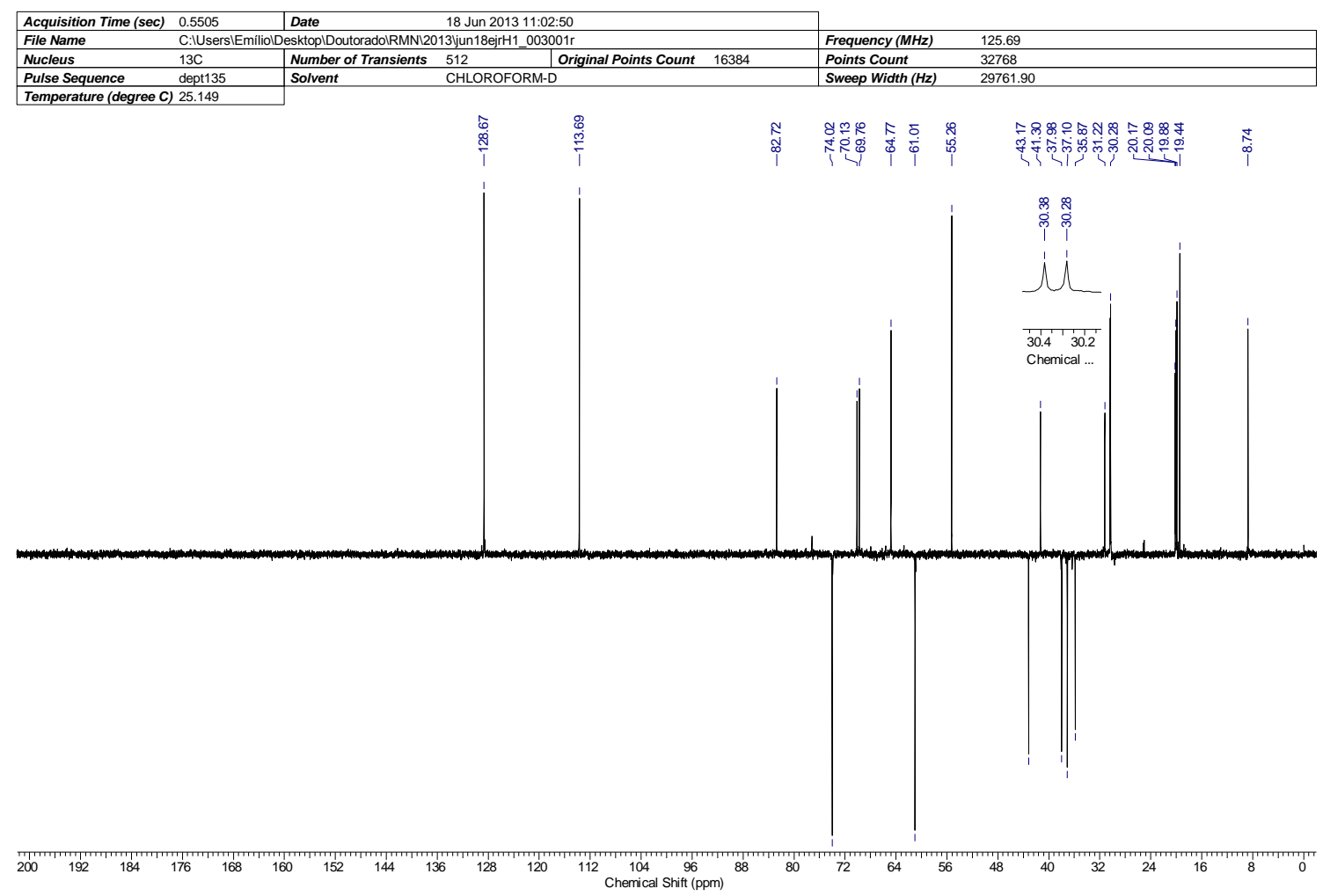

Figure 60: ${ }^{13} \mathrm{C}$ NMR (dept 135) spectrum of compound $\mathbf{S} 17\left(125 \mathrm{MHz}, \mathrm{CDCl}_{3}\right)$. 

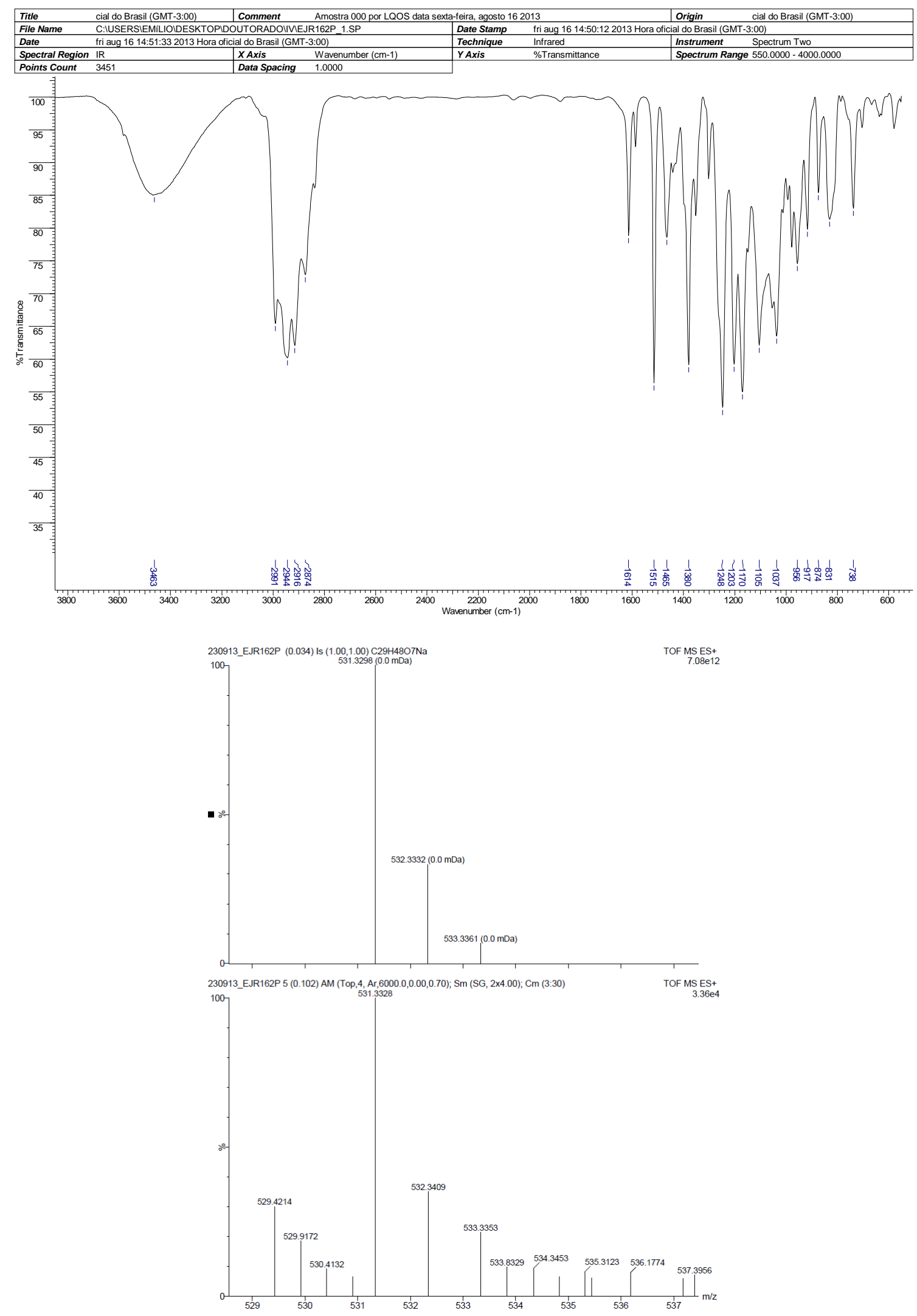

Figure 61: IR and HRMS (ESI TOF-MS) spectra of compound S17. 


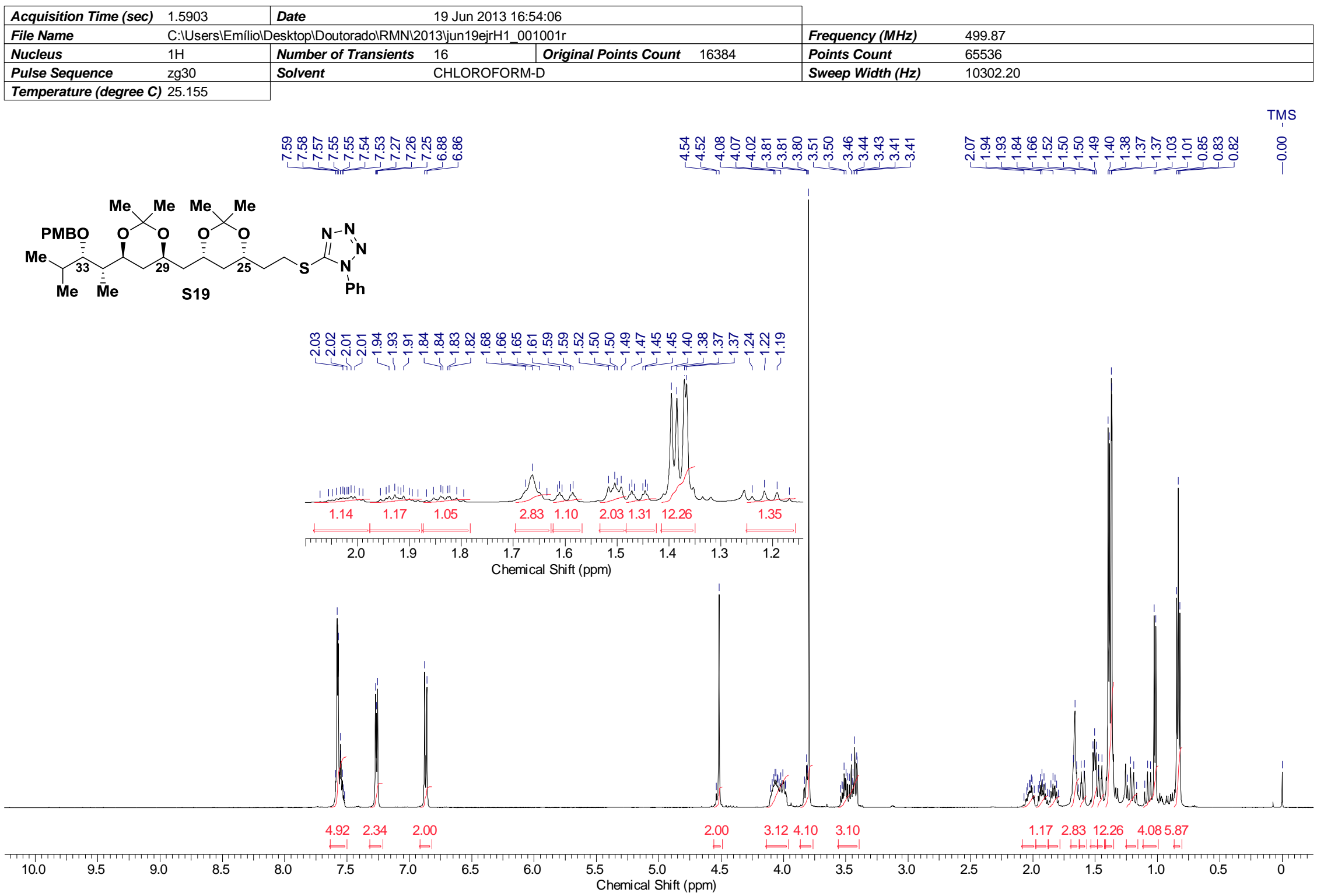

Figure 62: ${ }^{1} \mathrm{H}$ NMR spectrum of compound $\mathbf{S} 19\left(500 \mathrm{MHz}, \mathrm{CDCl}_{3}\right)$. 


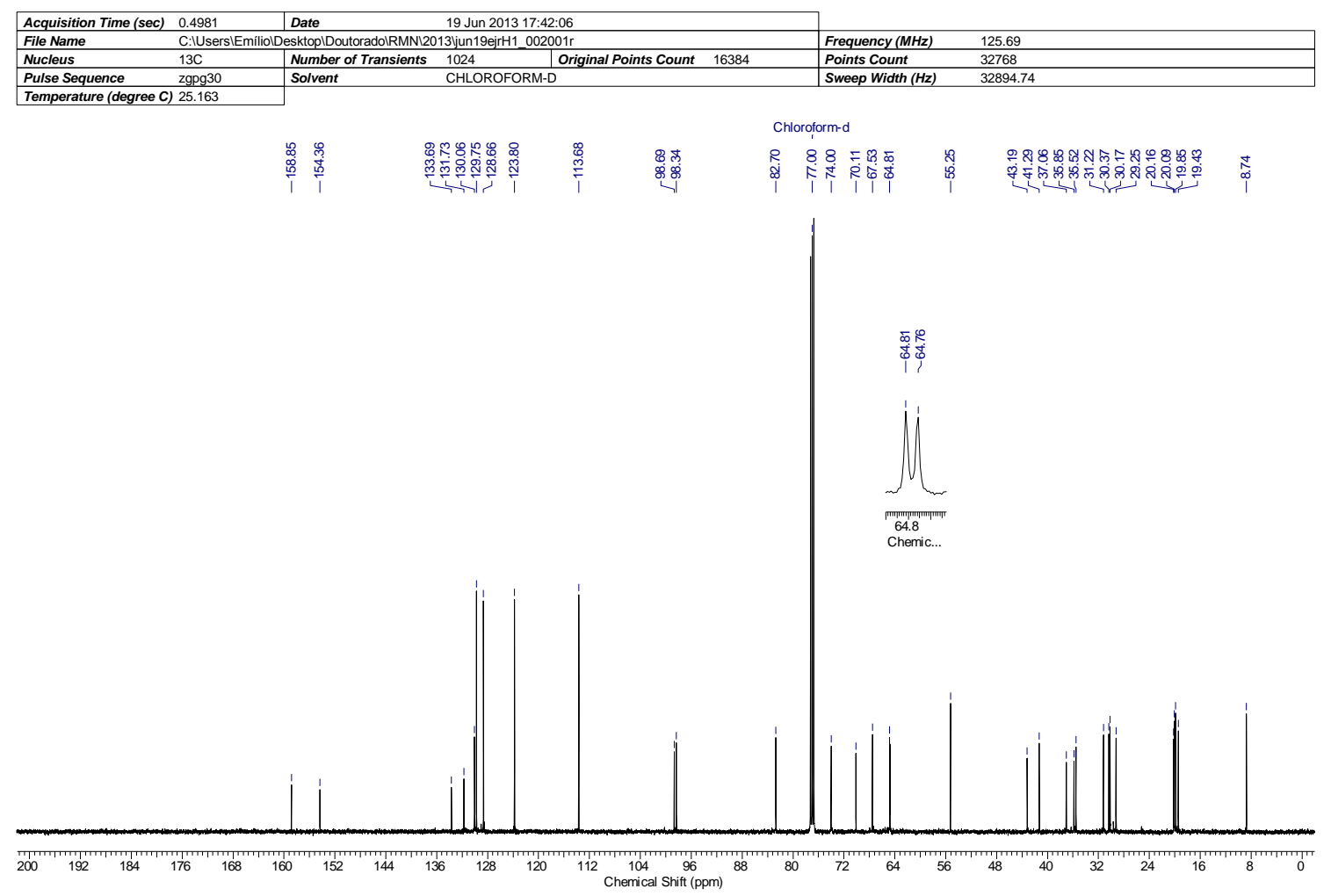

Figure 63: ${ }^{13} \mathrm{C}$ NMR spectrum of compound $\mathbf{S} 19\left(125 \mathrm{MHz}, \mathrm{CDCl}_{3}\right)$.

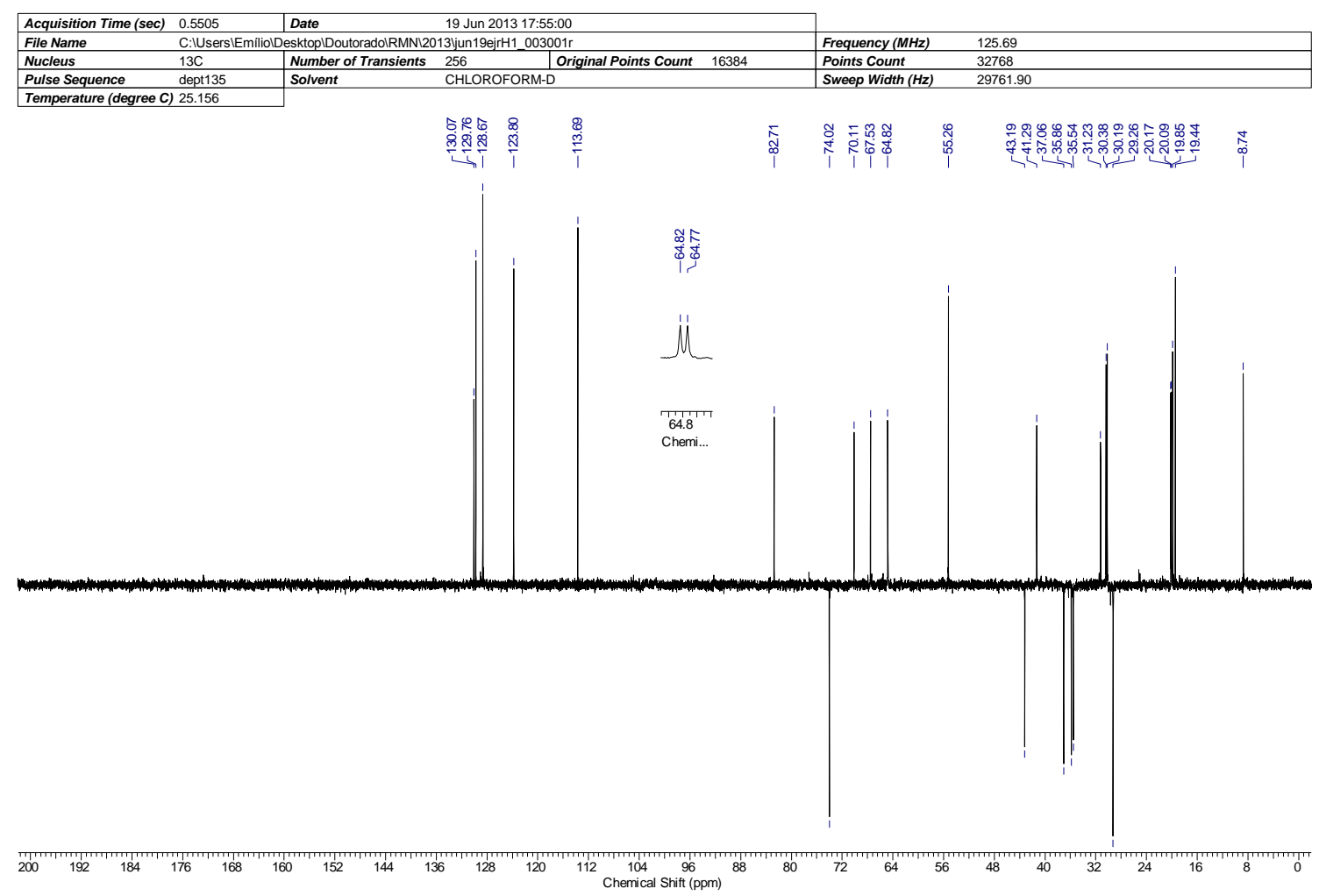

Figure 64: ${ }^{13} \mathrm{C}$ NMR (dept 135) spectrum of compound $\mathbf{S} 19\left(125 \mathrm{MHz}, \mathrm{CDCl}_{3}\right)$. 


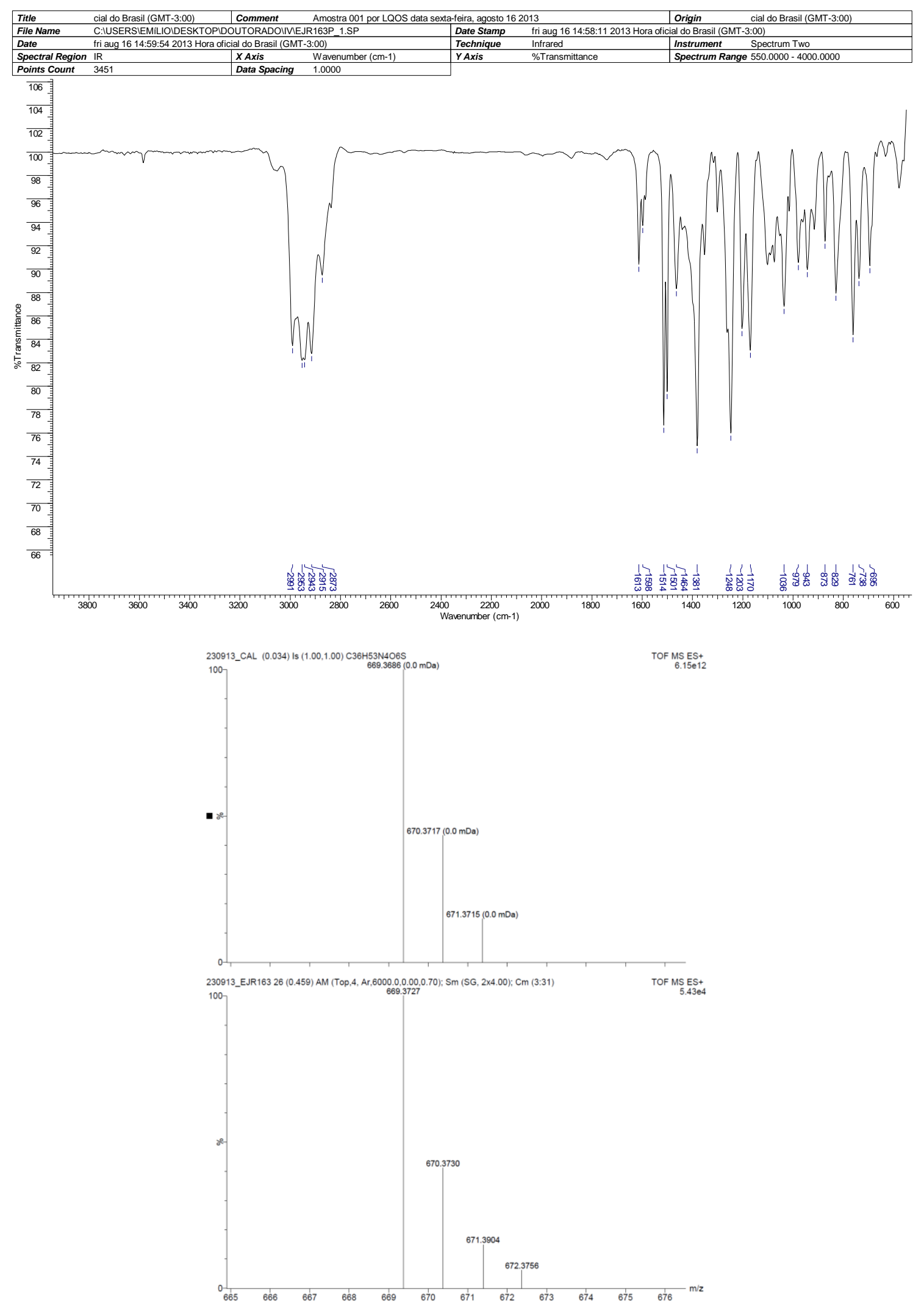

Figure 65: IR and HRMS (ESI TOF-MS) spectra of compound S19. 


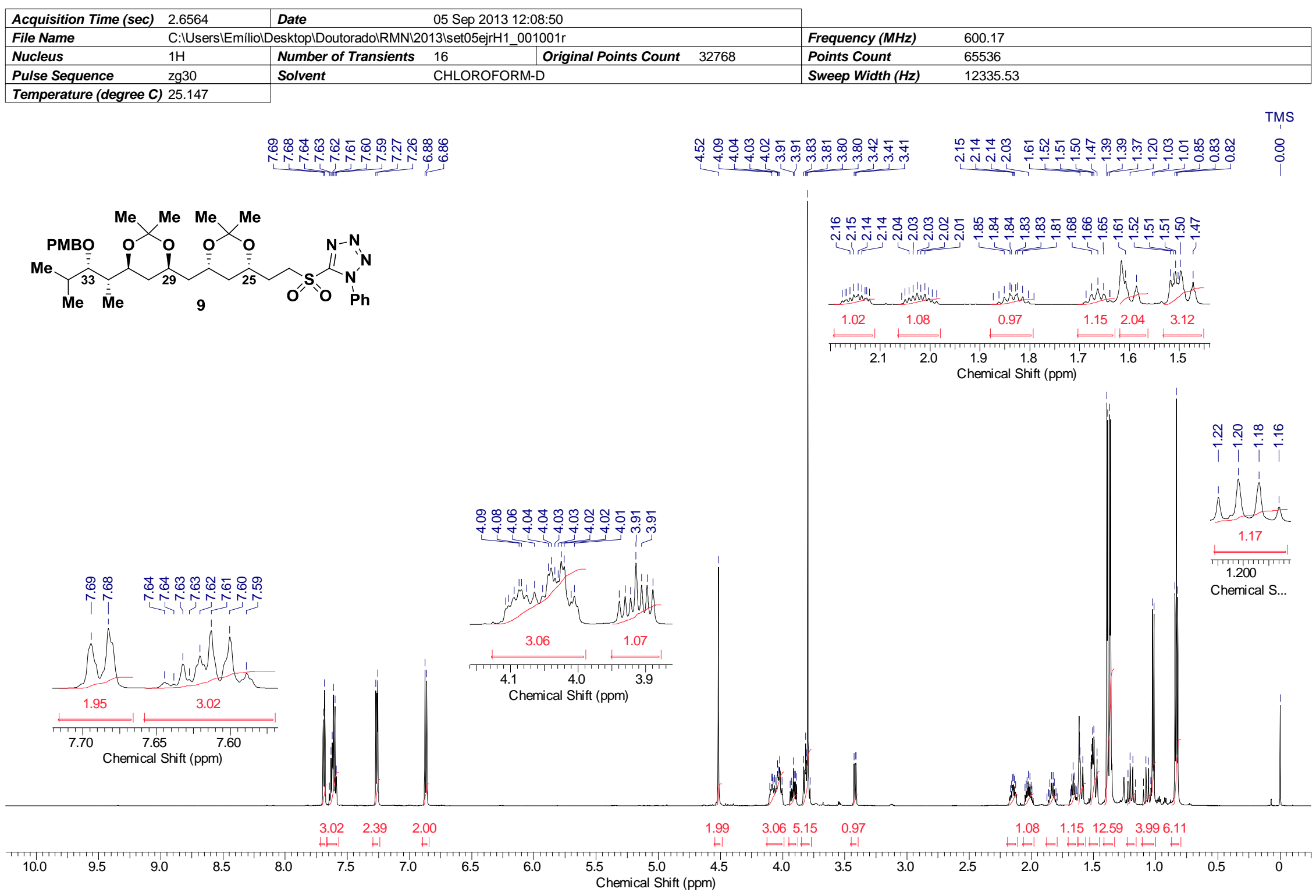

Figure 66: ${ }^{1} \mathrm{H}$ NMR spectrum of compound $9\left(600 \mathrm{MHz}, \mathrm{CDCl}_{3}\right)$. 


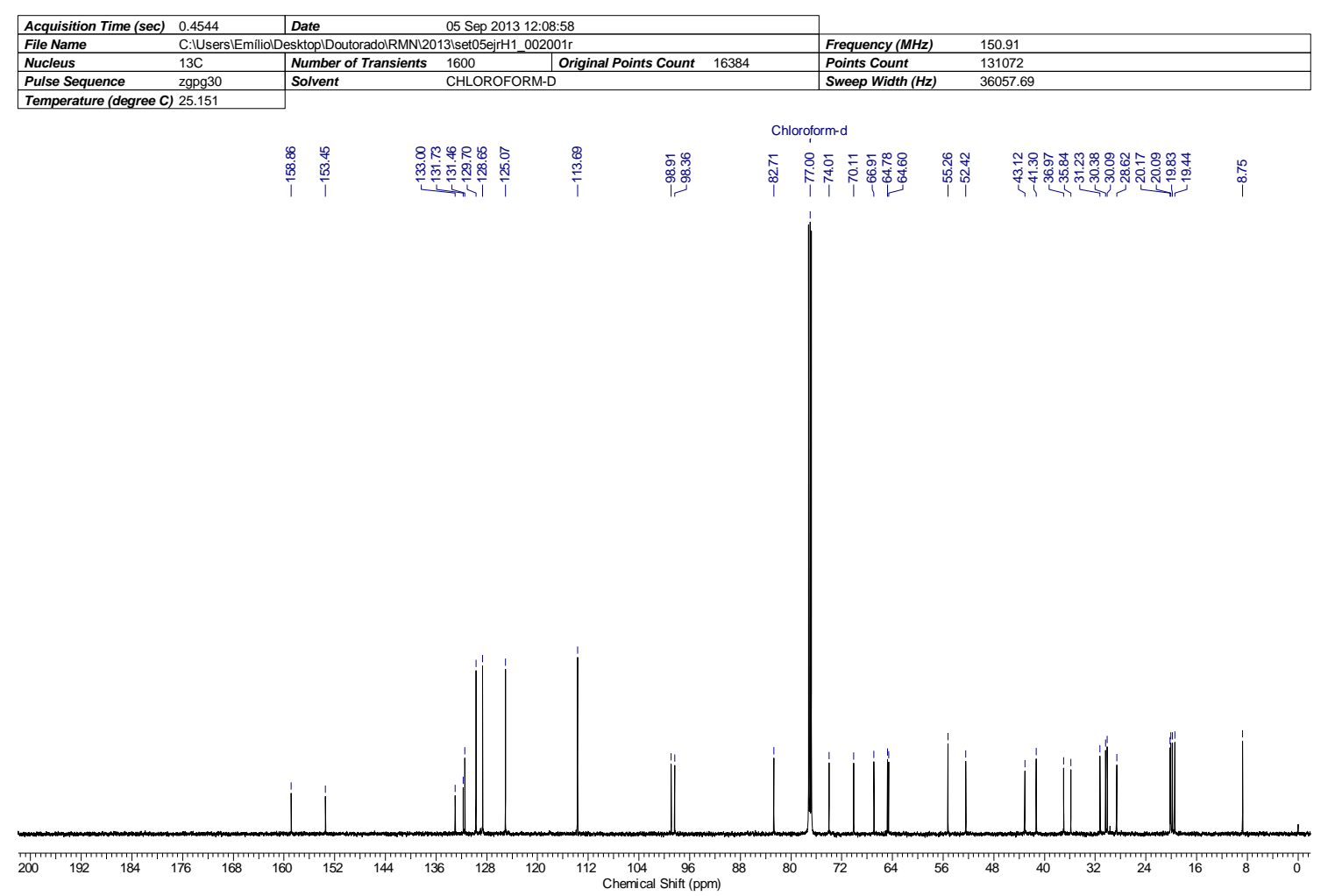

Figure 67: ${ }^{13} \mathrm{C}$ NMR spectrum of compound $9\left(150 \mathrm{MHz}, \mathrm{CDCl}_{3}\right)$.

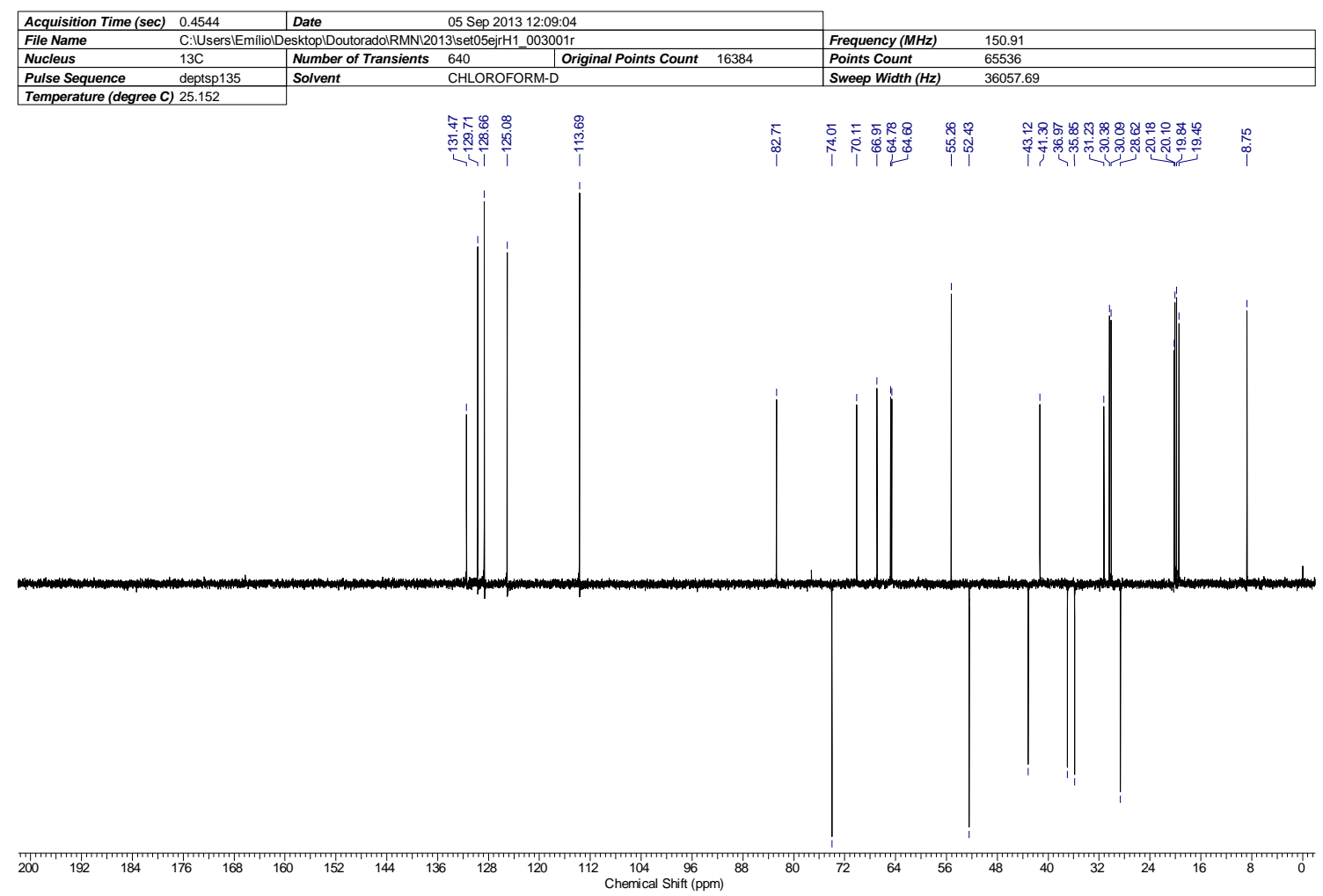

Figure 68: ${ }^{13} \mathrm{C}$ NMR (dept 135) spectrum of compound 9 (150 MHz, $\mathrm{CDCl}_{3}$ ). 


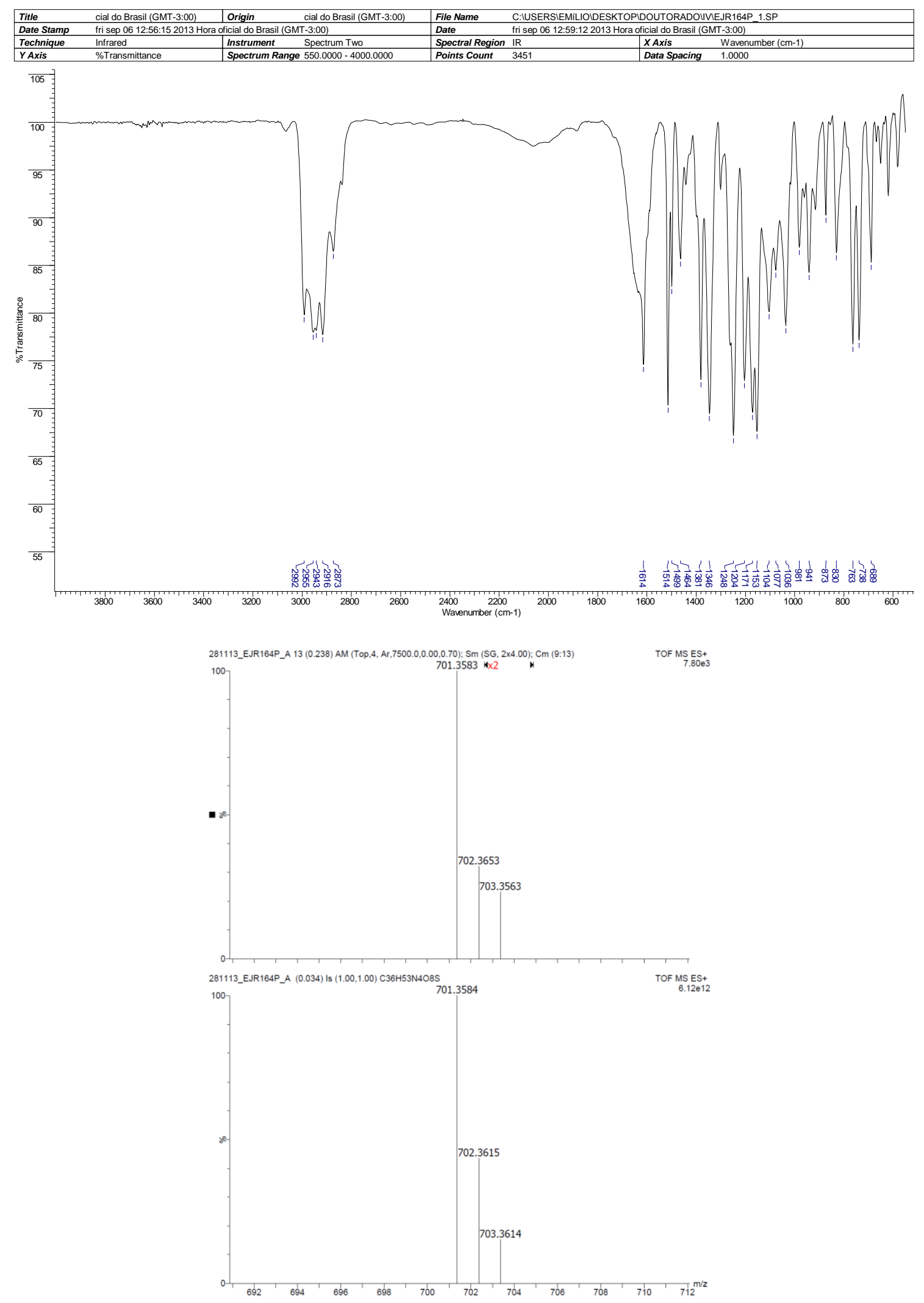

Figure 69: IR and HRMS (ESI TOF-MS) spectra of compound 9. 


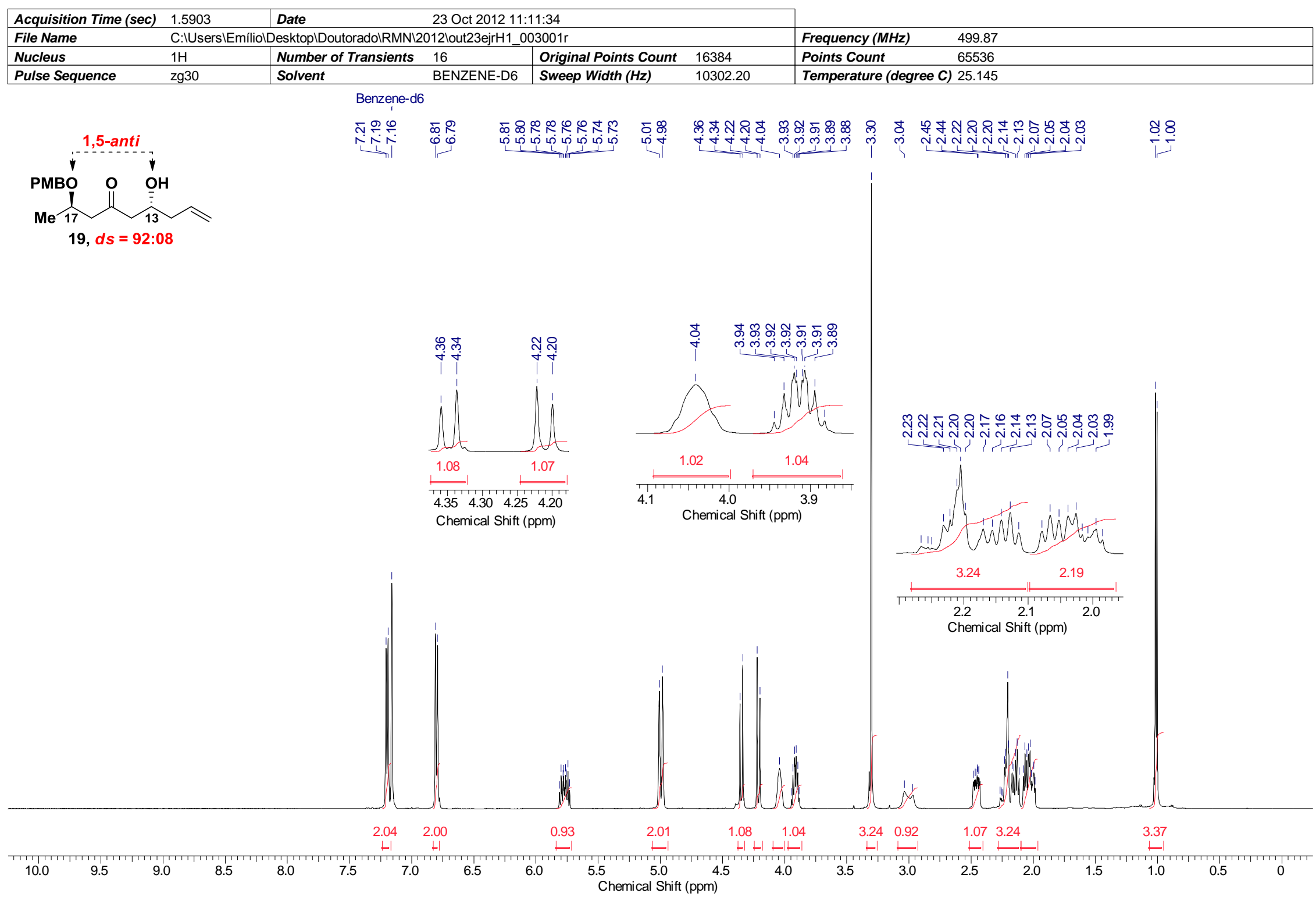

Figure 70: ${ }^{1} \mathrm{H}$ NMR spectrum of compound $19\left(500 \mathrm{MHz}, \mathrm{C}_{6} \mathrm{D}_{6}\right)$. 


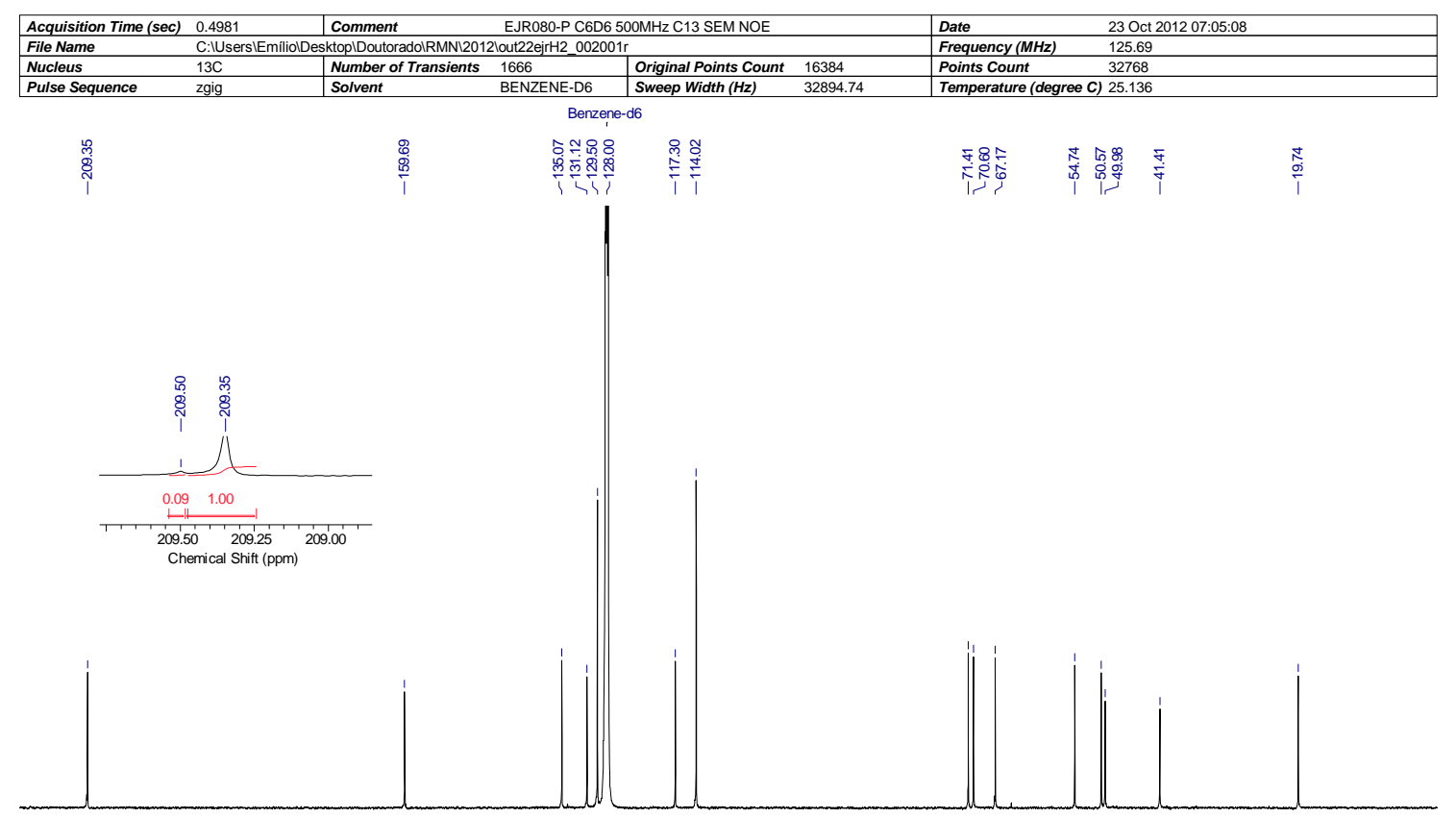

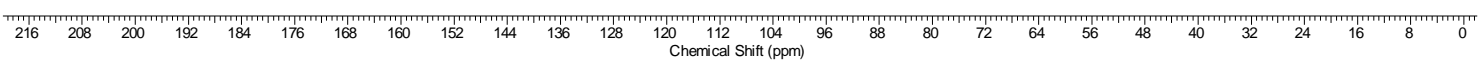

Figure 71: ${ }^{13} \mathrm{C}$ NMR spectrum of compound $19\left(125 \mathrm{MHz}, \mathrm{C}_{6} \mathrm{D}_{6}\right)$.

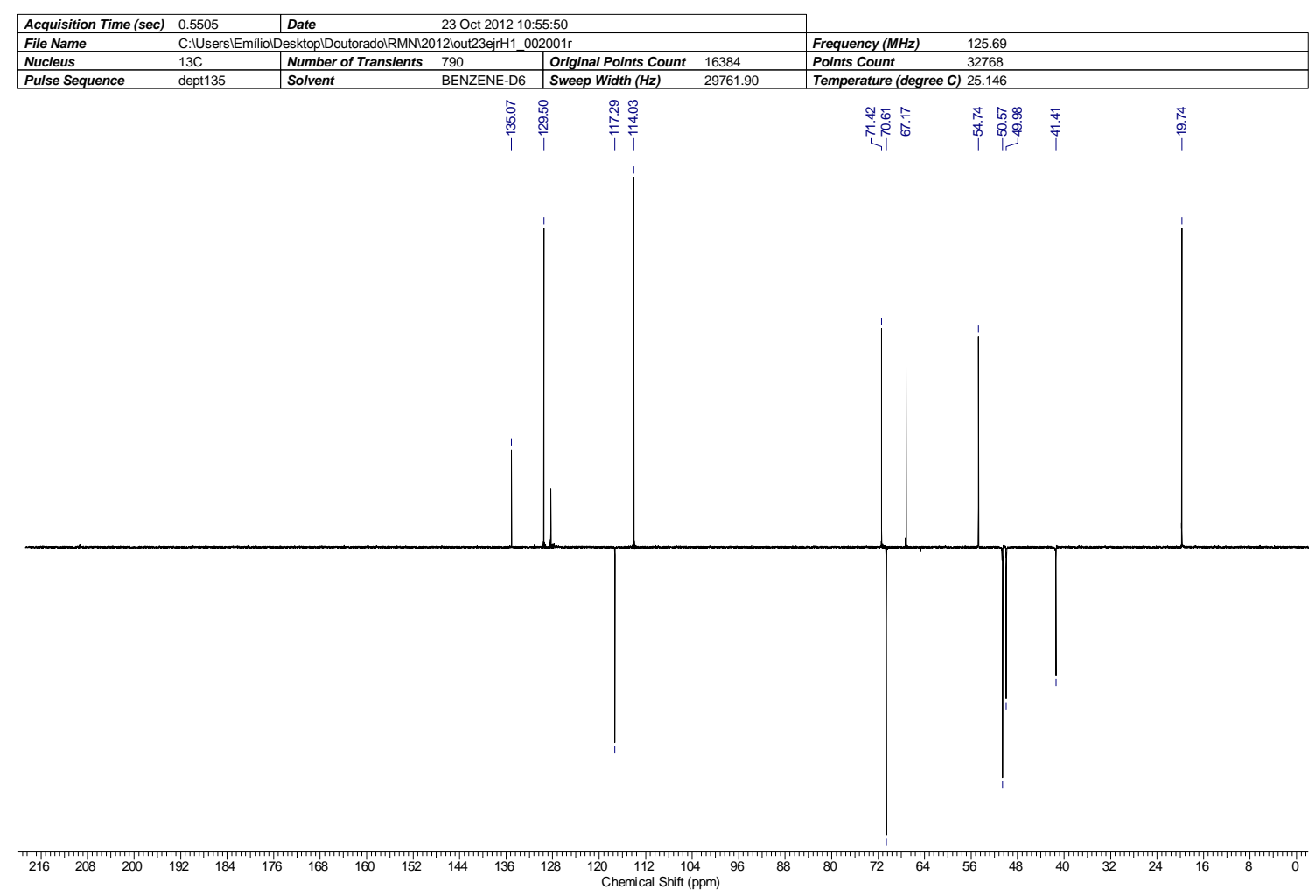

Figure 72: ${ }^{13} \mathrm{C}$ NMR (dept 135) spectrum of compound $19\left(125 \mathrm{MHz}, \mathrm{C}_{6} \mathrm{D}_{6}\right)$. 

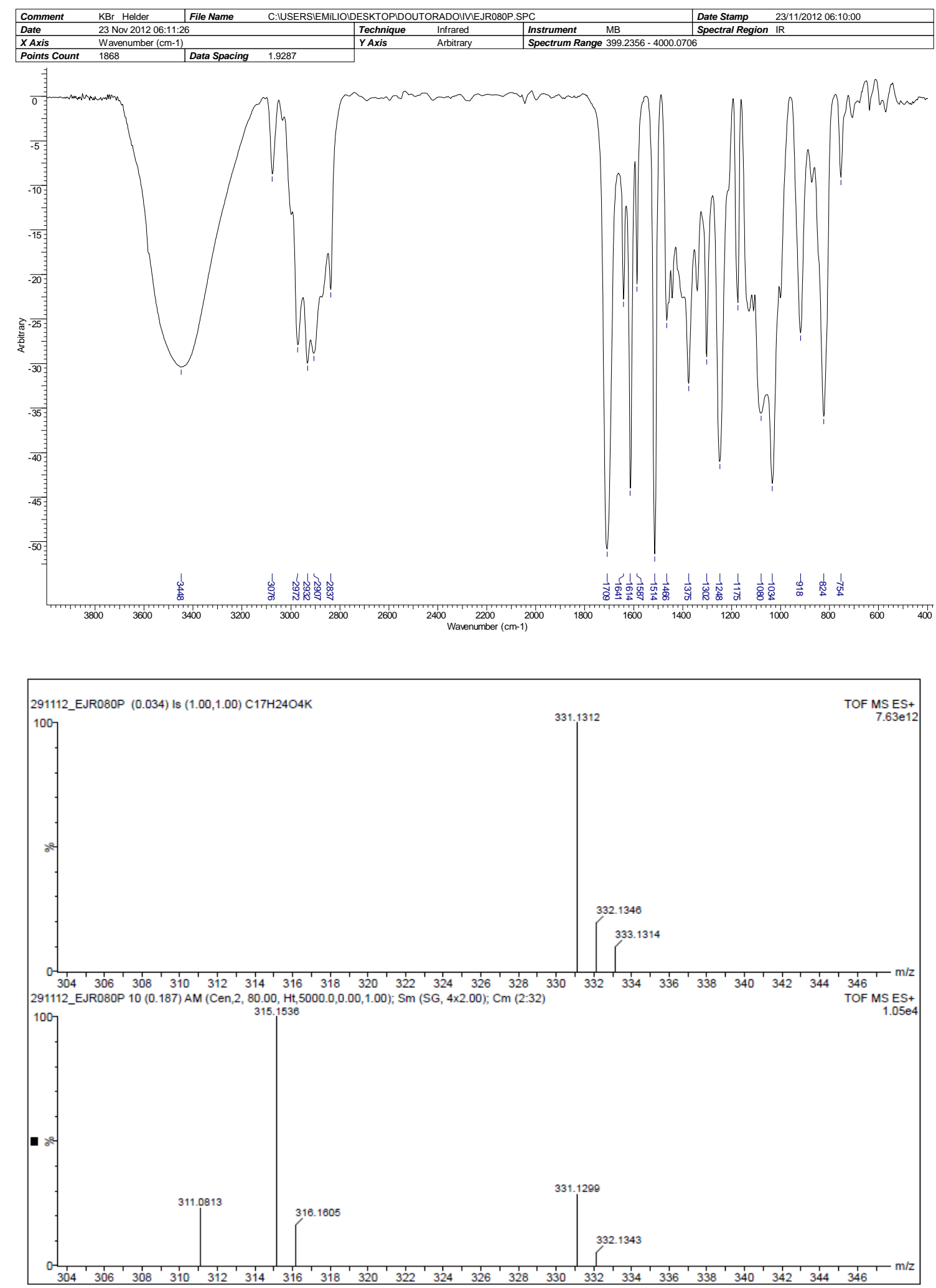

Figure 73: IR and HRMS (ESI TOF-MS) spectra of compound 19. 


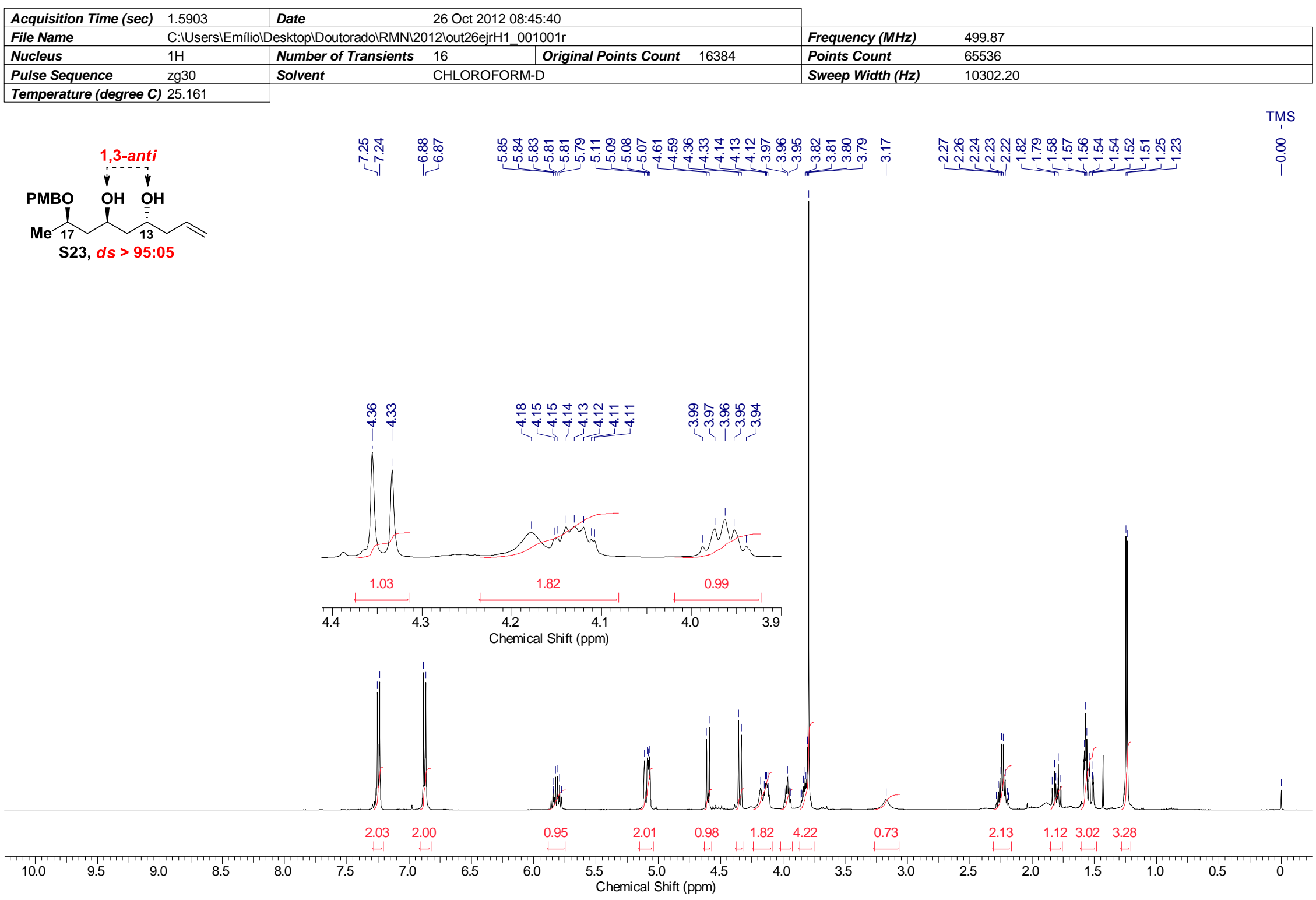

Figure 74: ${ }^{1} \mathrm{H}$ NMR spectrum of compound $\mathbf{S} 23\left(500 \mathrm{MHz}, \mathrm{CDCl}_{3}\right)$. 


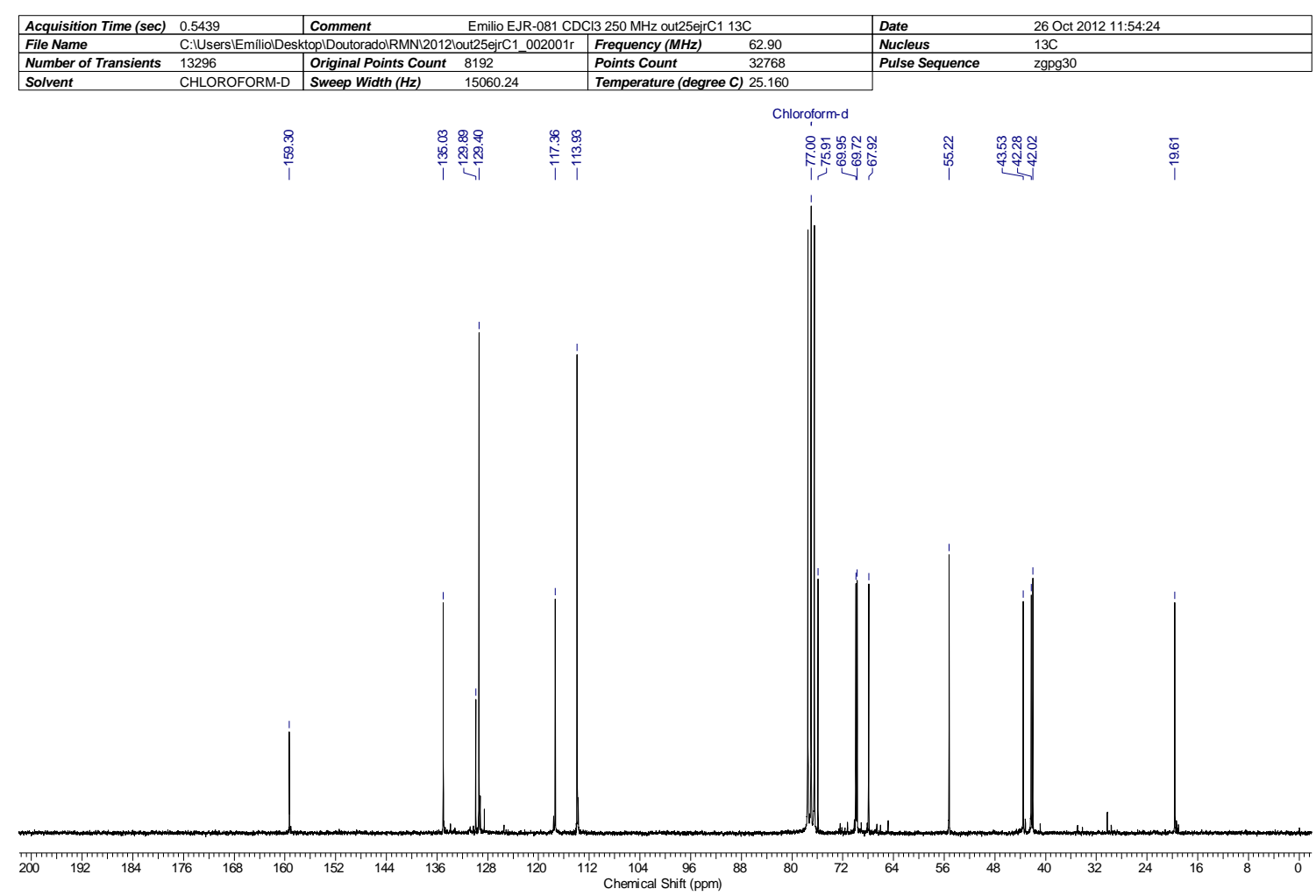

Figure 75: ${ }^{13} \mathrm{C}$ NMR spectrum of compound $\mathbf{S} 23\left(62.5 \mathrm{MHz}, \mathrm{CDCl}_{3}\right)$.

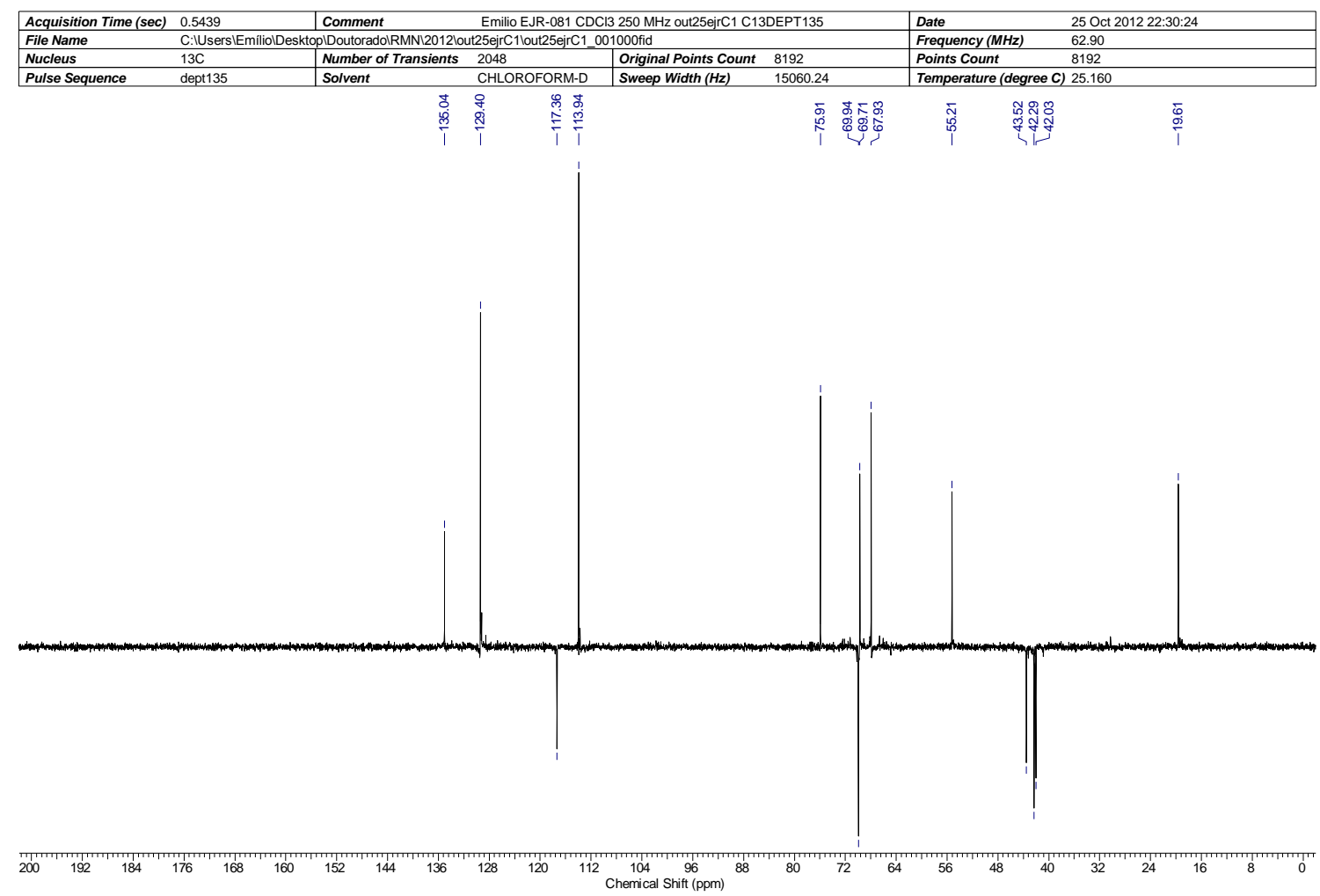

Figure 76: ${ }^{13} \mathrm{C}$ NMR (dept 135) spectrum of compound $\mathbf{S} 23\left(62.5 \mathrm{MHz}, \mathrm{CDCl}_{3}\right)$. 

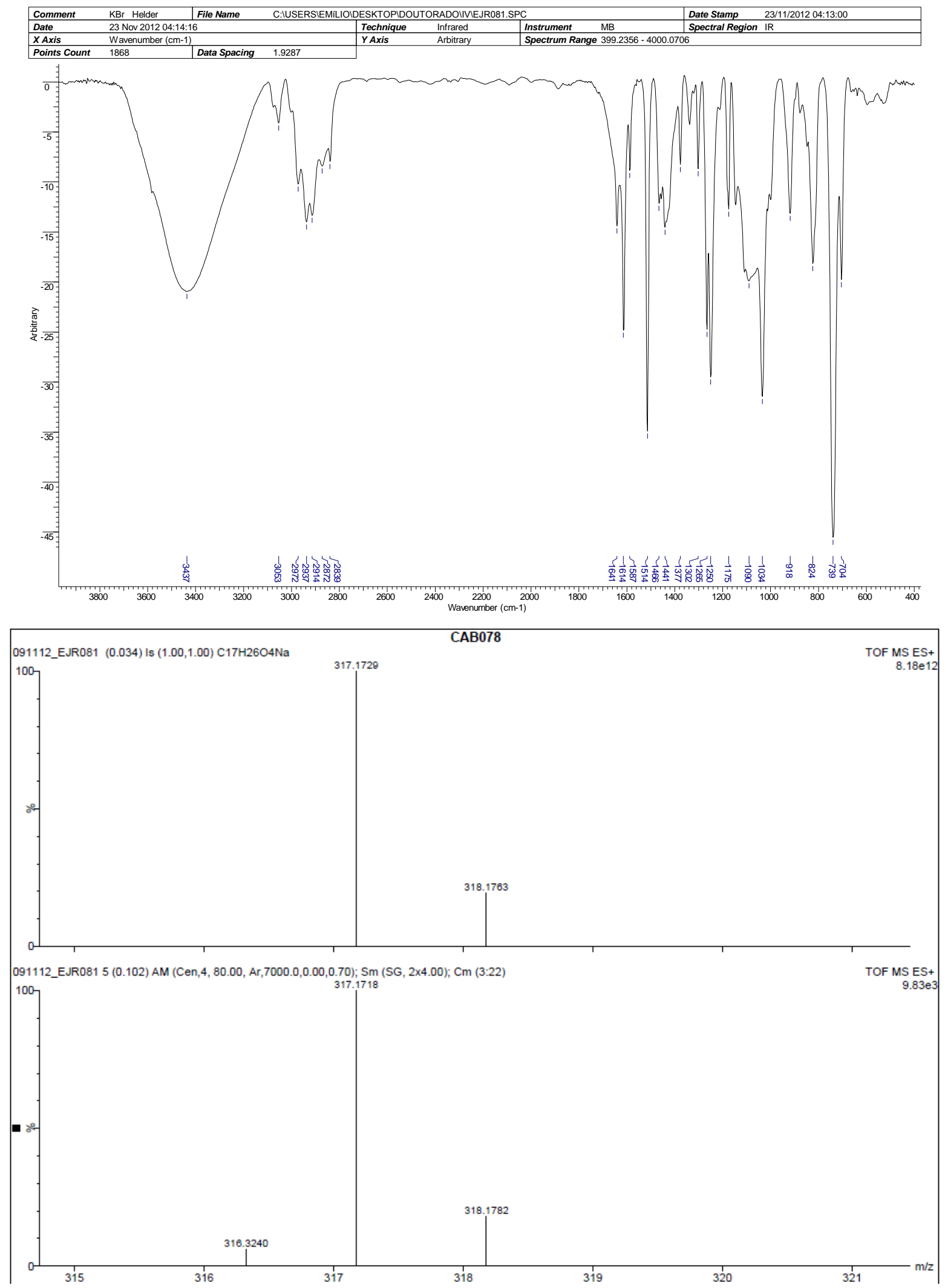

Figure 77: IR and HRMS (ESI TOF-MS) spectra of compound S23. 


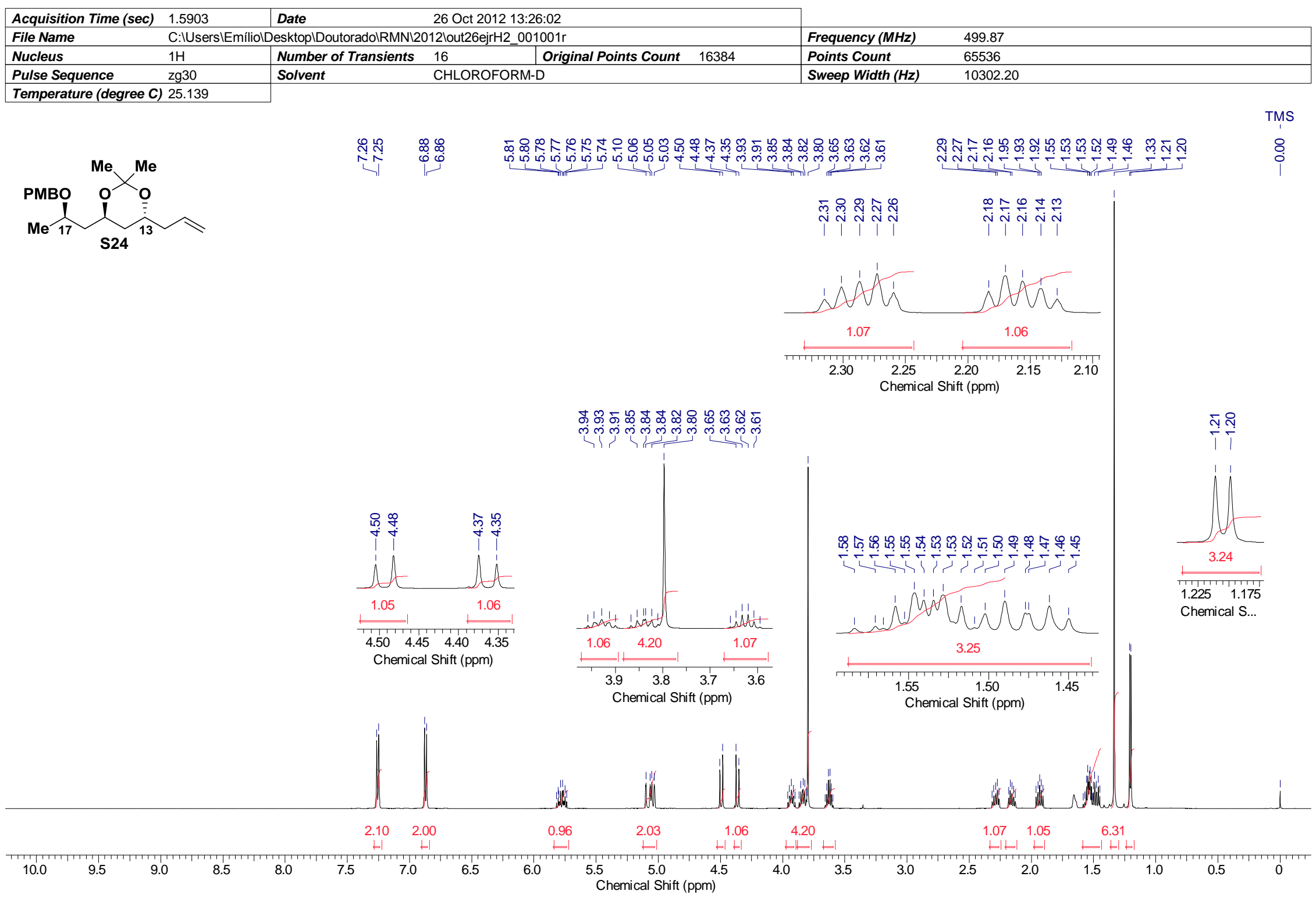

Figure 78: ${ }^{1} \mathrm{H}$ NMR spectrum of compound $\mathbf{S} 24\left(500 \mathrm{MHz}, \mathrm{CDCl}_{3}\right)$. 


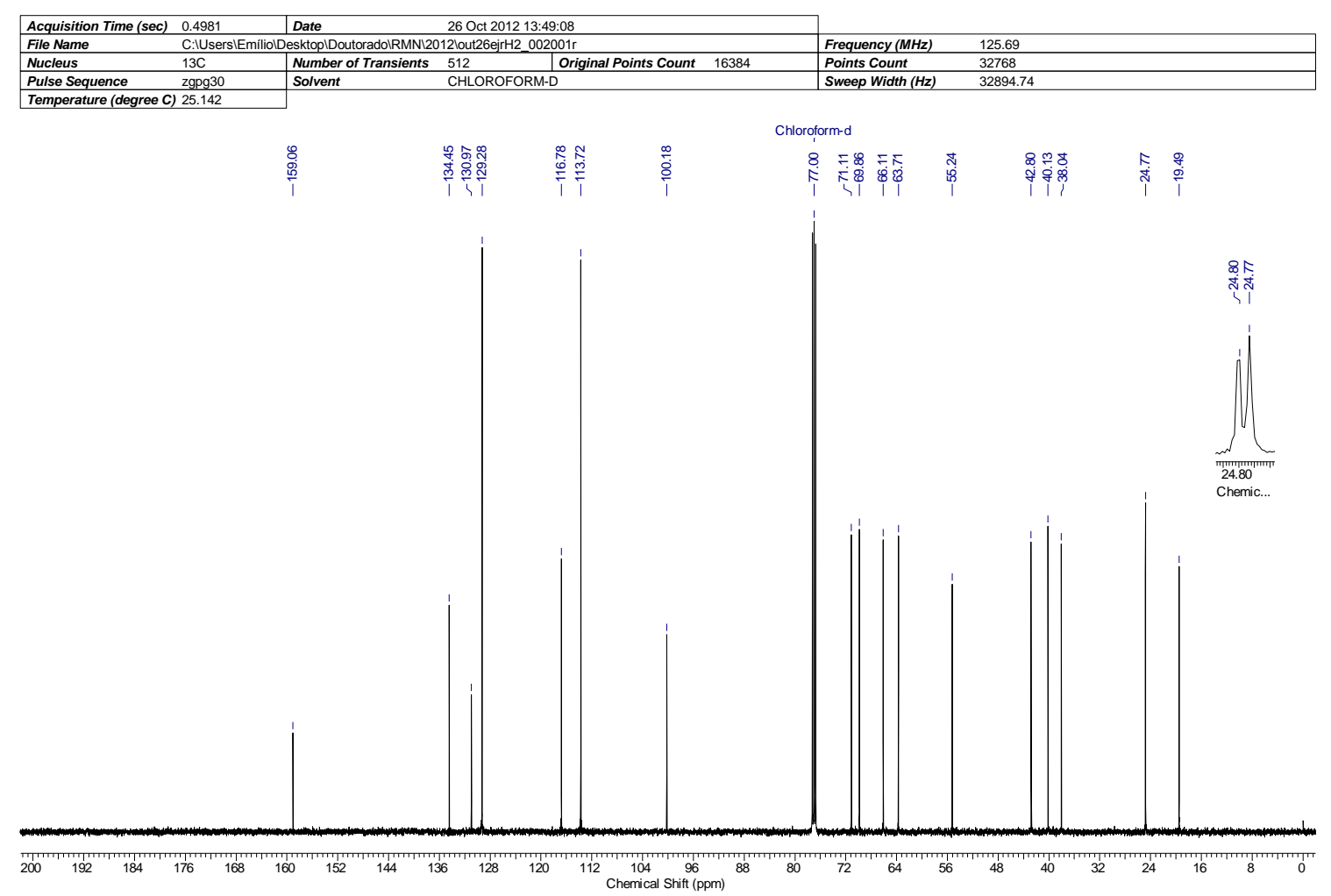

Figure 79: ${ }^{13} \mathrm{C}$ NMR spectrum of compound $\mathbf{S 2 4}\left(125 \mathrm{MHz}, \mathrm{CDCl}_{3}\right)$.

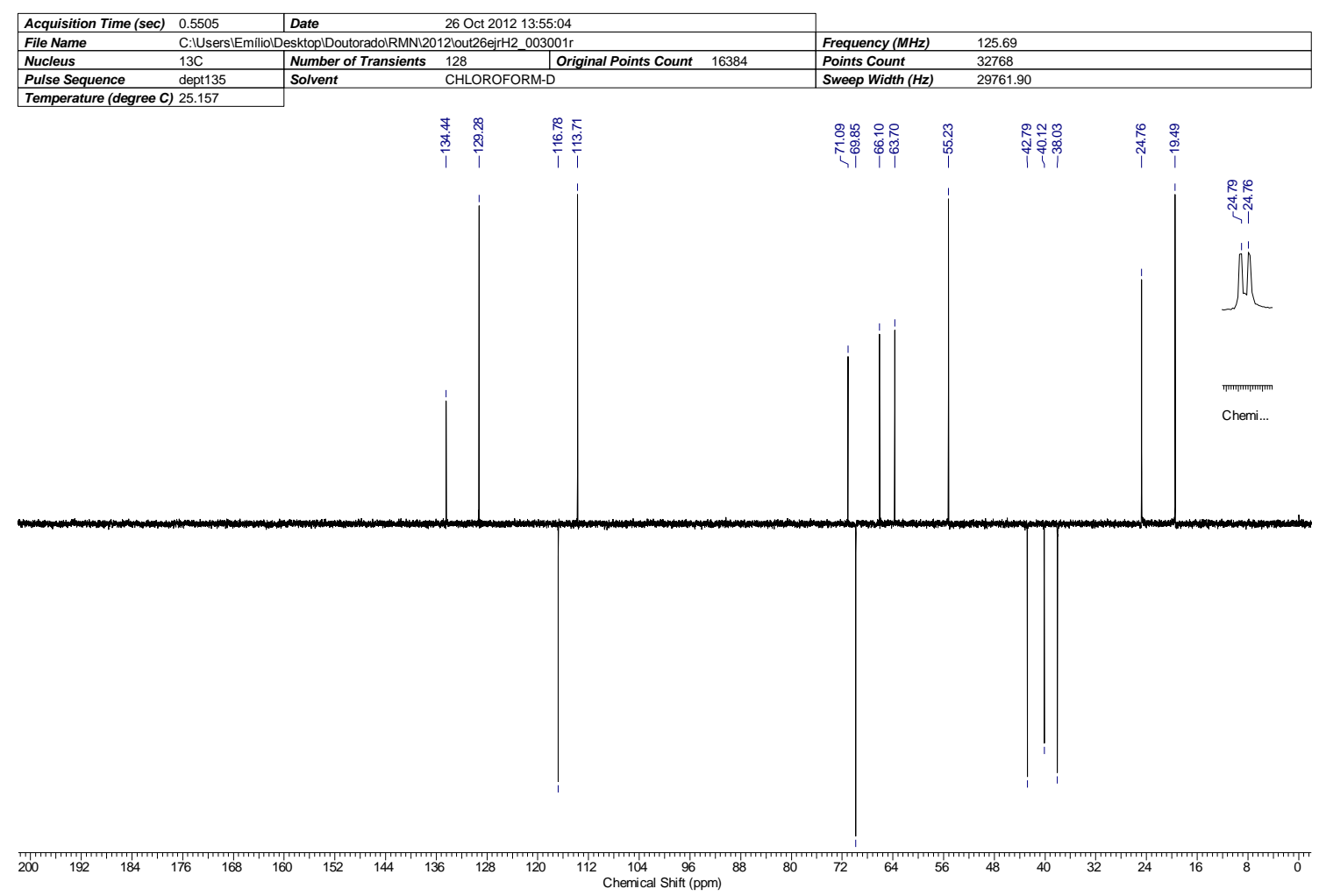

Figure 80: ${ }^{13} \mathrm{C}$ NMR (dept 135) spectrum of compound $\mathbf{S} 24\left(125 \mathrm{MHz}, \mathrm{CDCl}_{3}\right)$. 

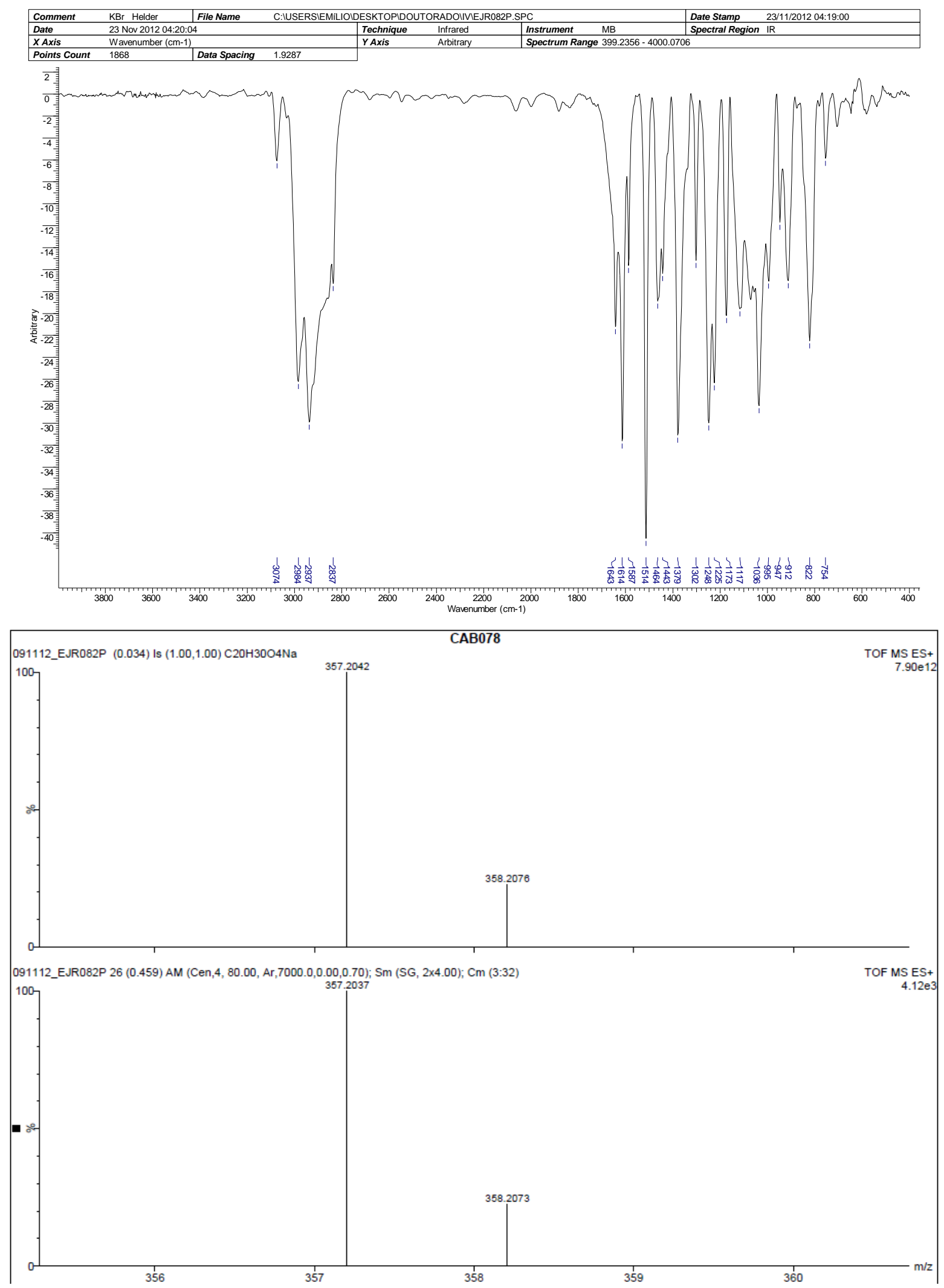

Figure 81: IR and HRMS (ESI TOF-MS) spectra of compound S24. 


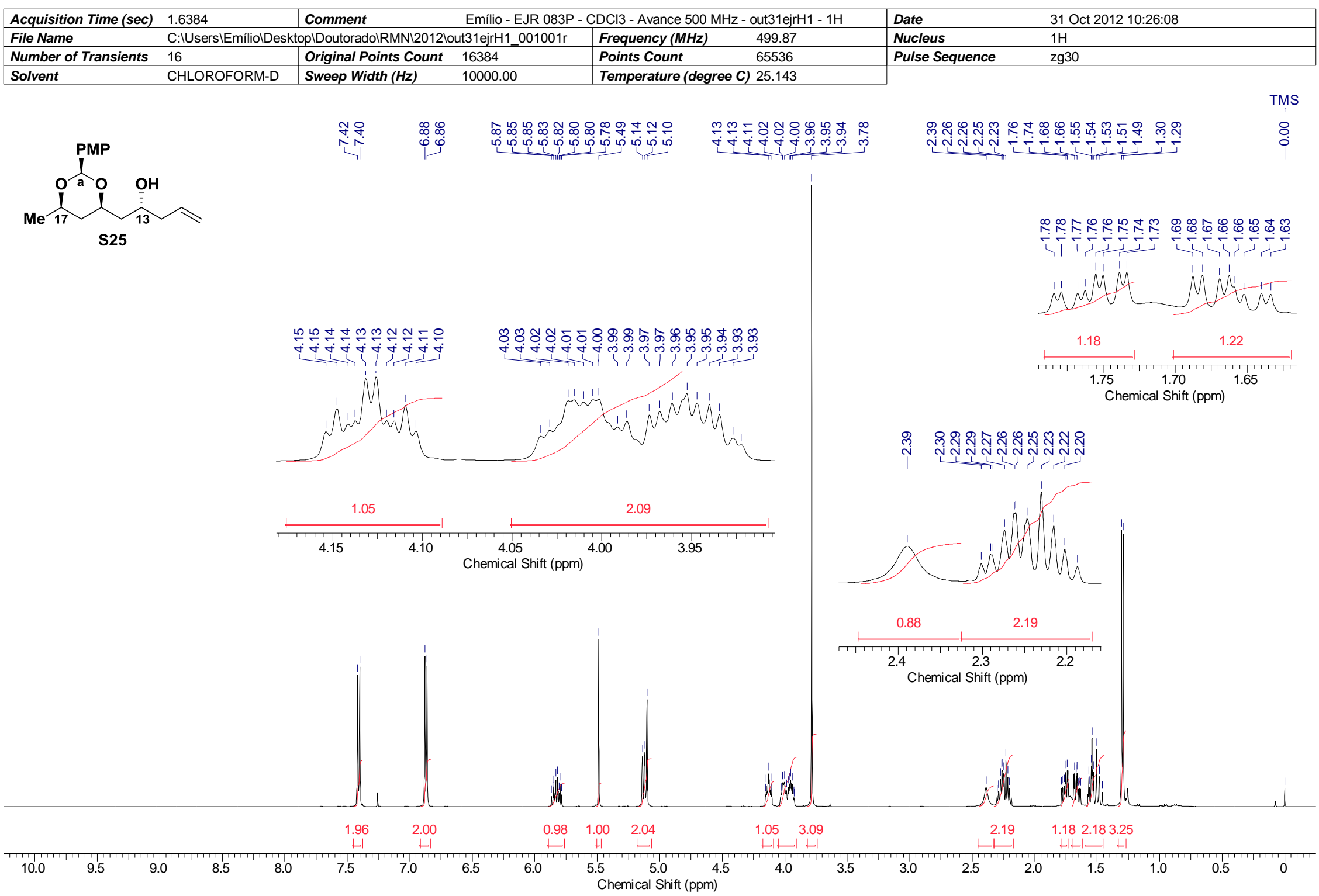

Figure 82: ${ }^{1} \mathrm{H}$ NMR spectrum of compound $\mathbf{S} 25\left(500 \mathrm{MHz}, \mathrm{CDCl}_{3}\right)$. 


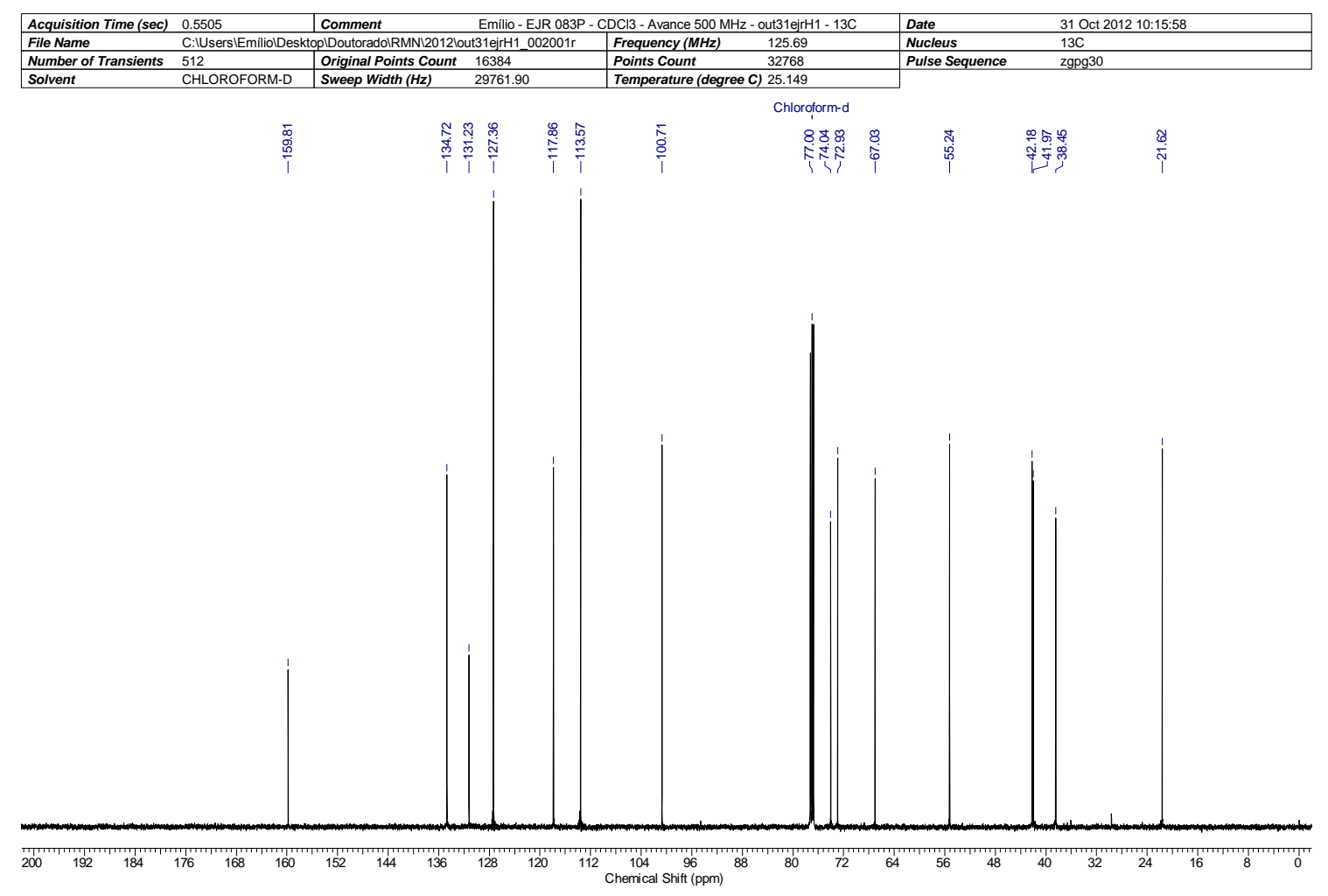

Figure 83: ${ }^{13} \mathrm{C}$ NMR spectrum of compound $\mathbf{S 2 5}\left(125 \mathrm{MHz}, \mathrm{CDCl}_{3}\right)$.

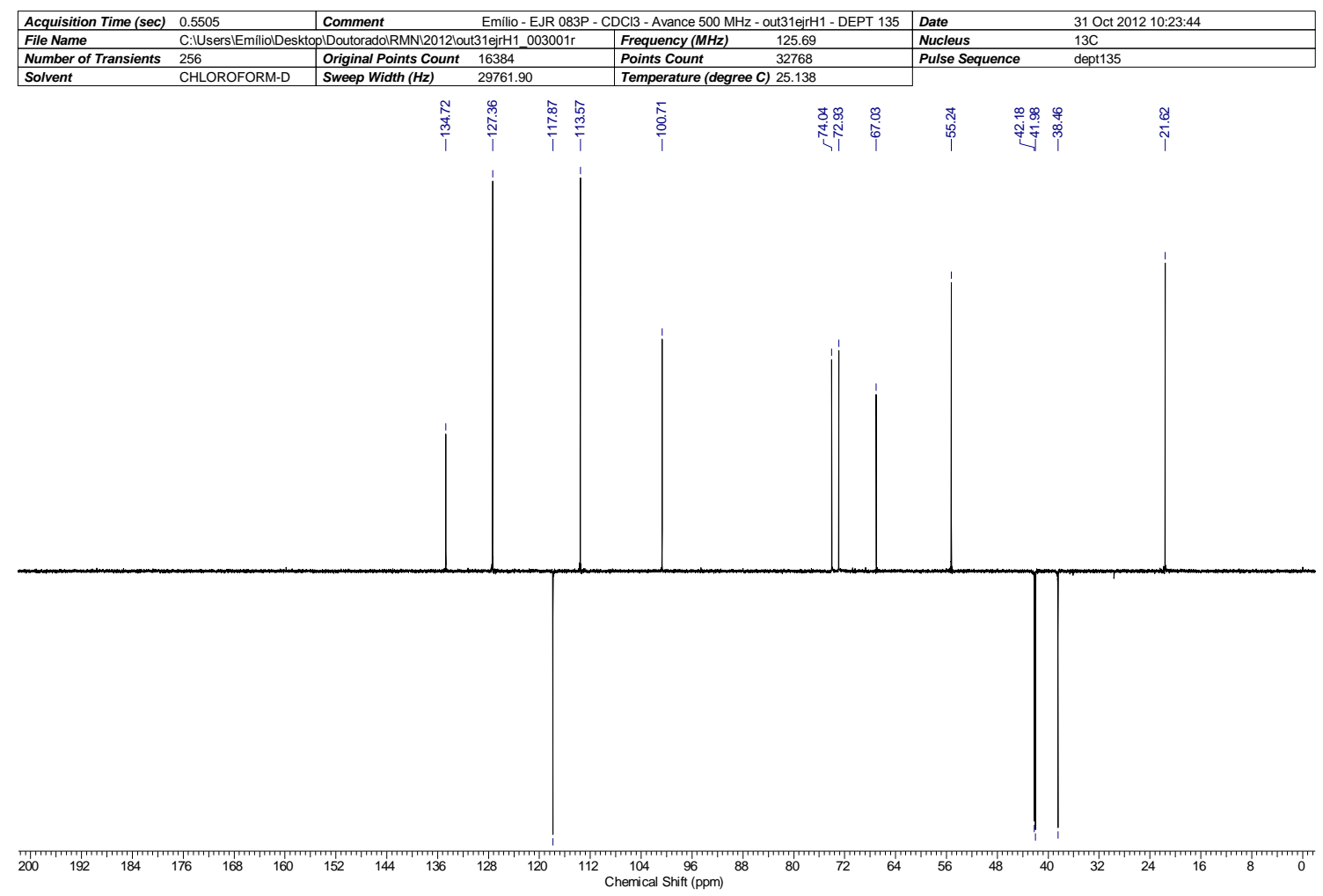

Figure 84: ${ }^{13} \mathrm{C}$ NMR (dept 135) spectrum of compound $\mathbf{S} 25\left(125 \mathrm{MHz}, \mathrm{CDCl}_{3}\right)$. 

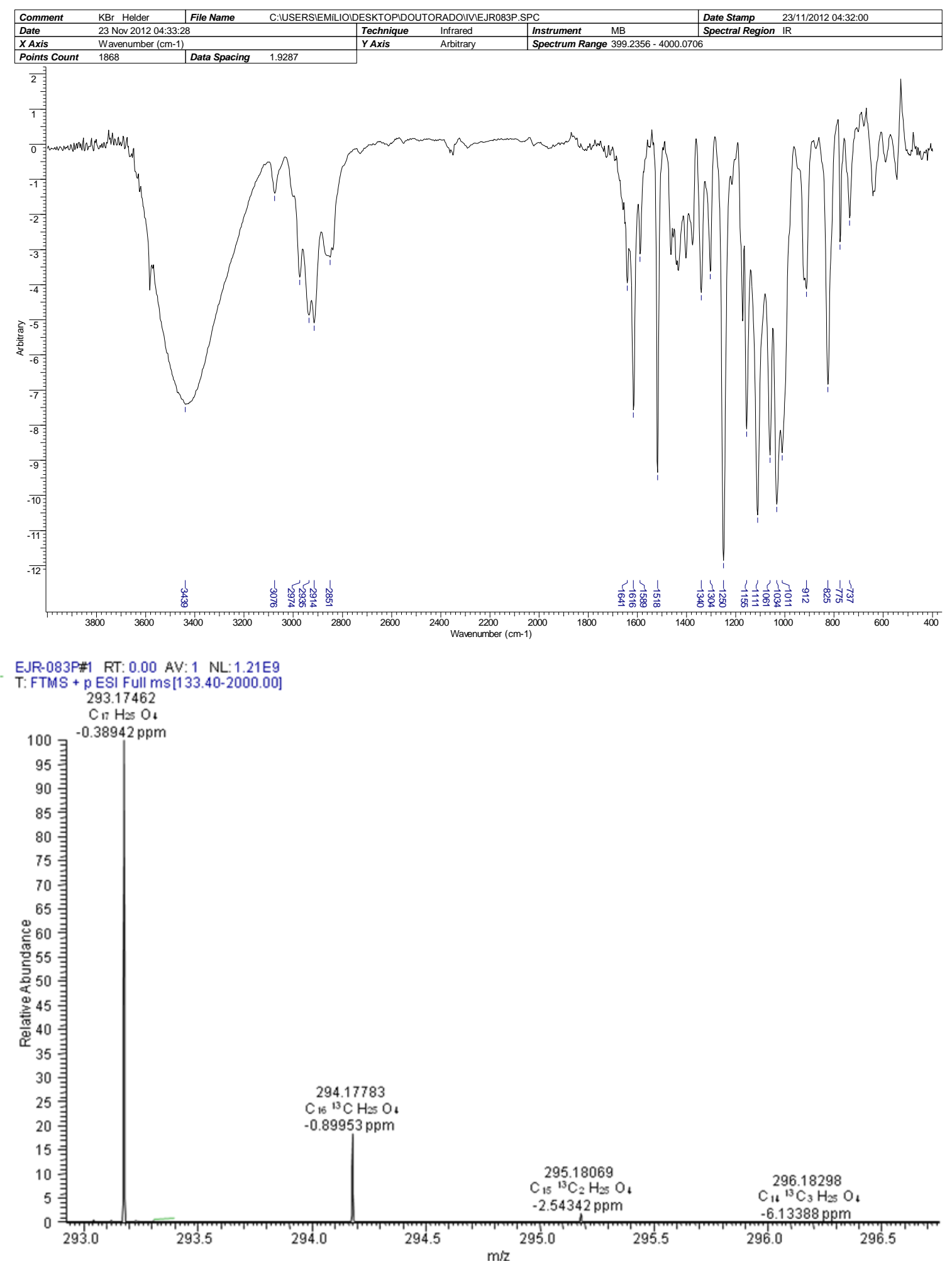

Figure 85: IR and HRMS (ESI FTMS) spectra of compound S25. 


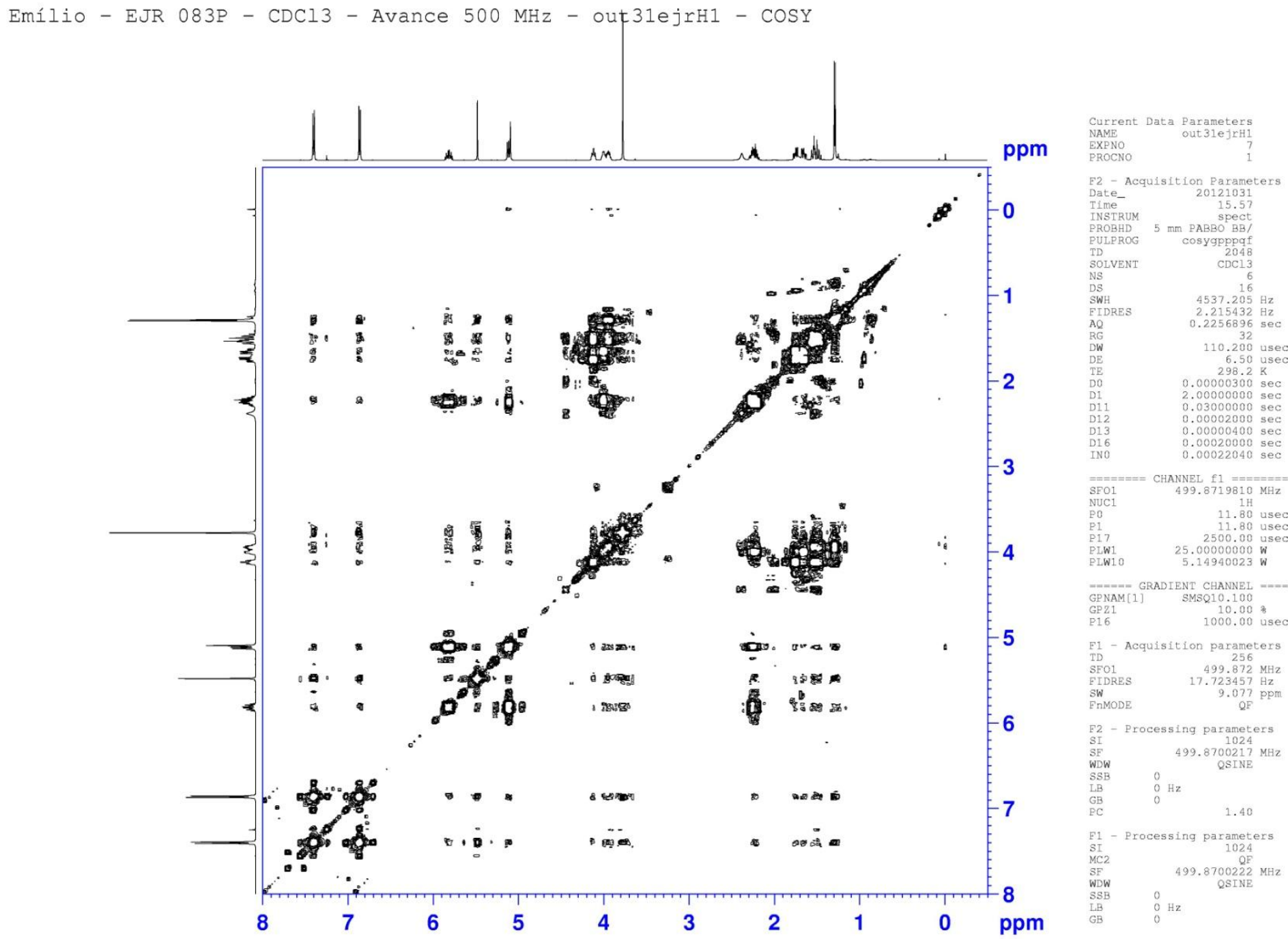

Figure 86: COSY contour map of compound $\mathbf{S} 25\left(500 \mathrm{MHz}, \mathrm{CDCl}_{3}\right)$. 


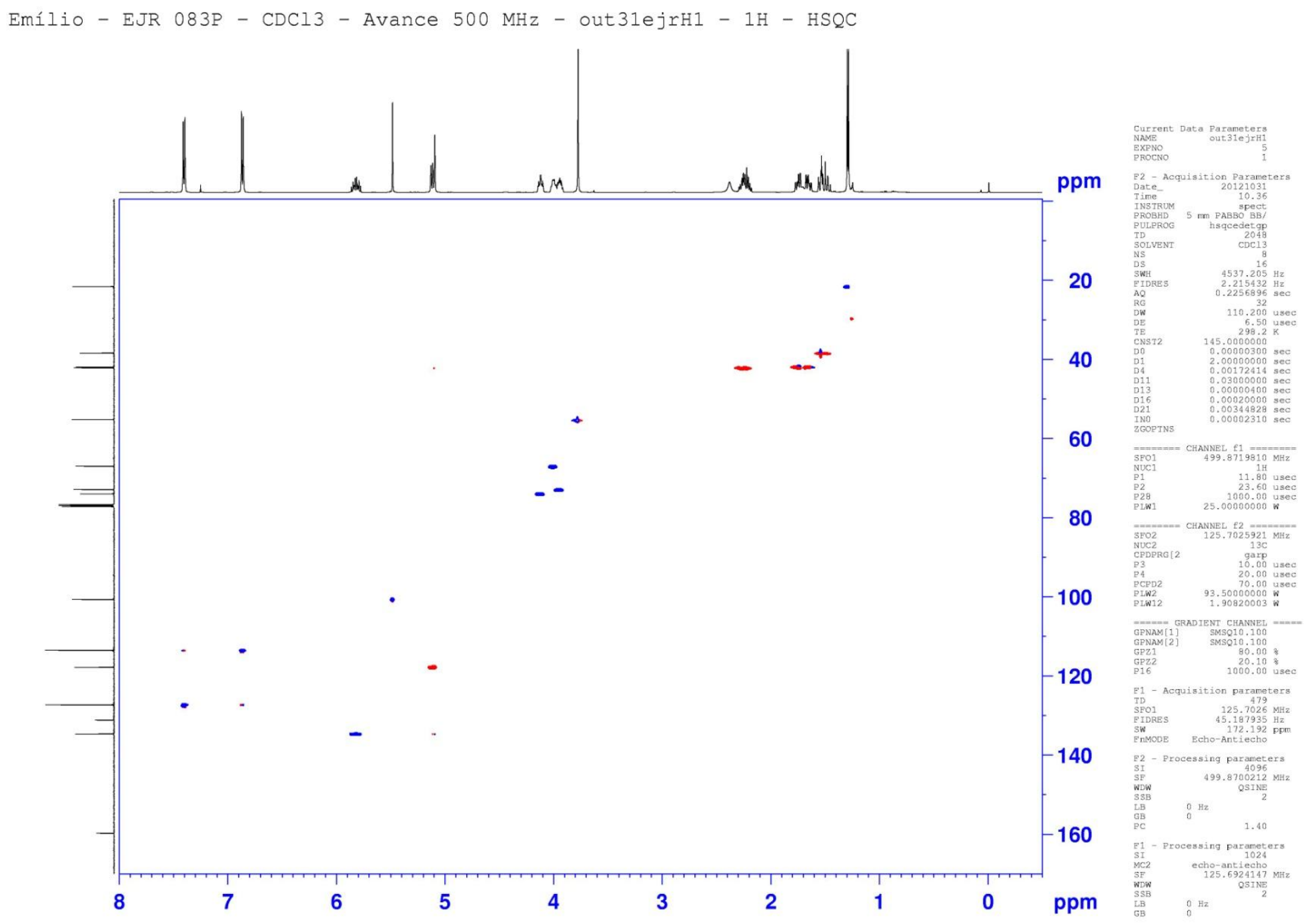

Figure 87: HSQC contour map of compound S25. 


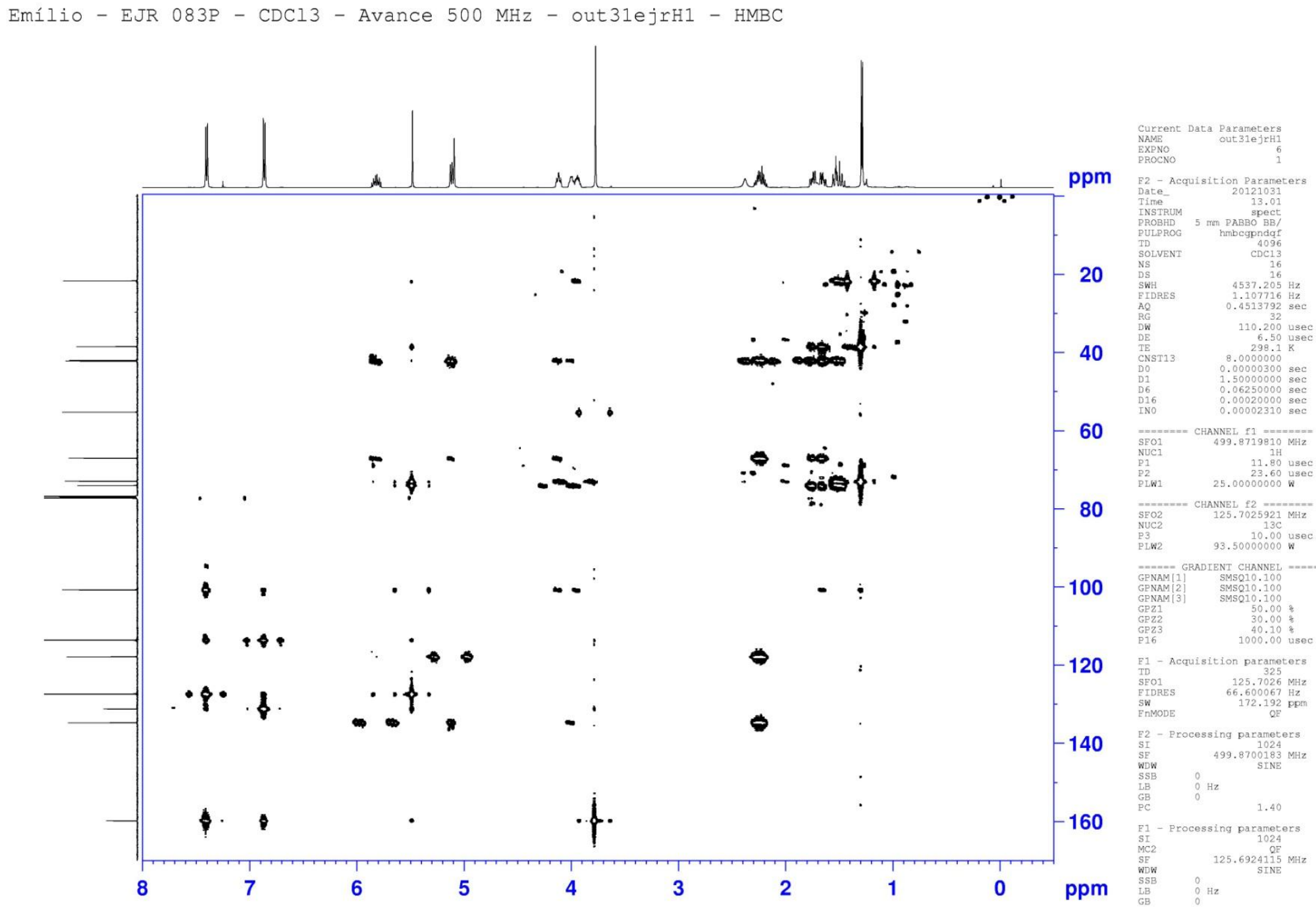

Figure 88: HMBC contour map of compound S25. 


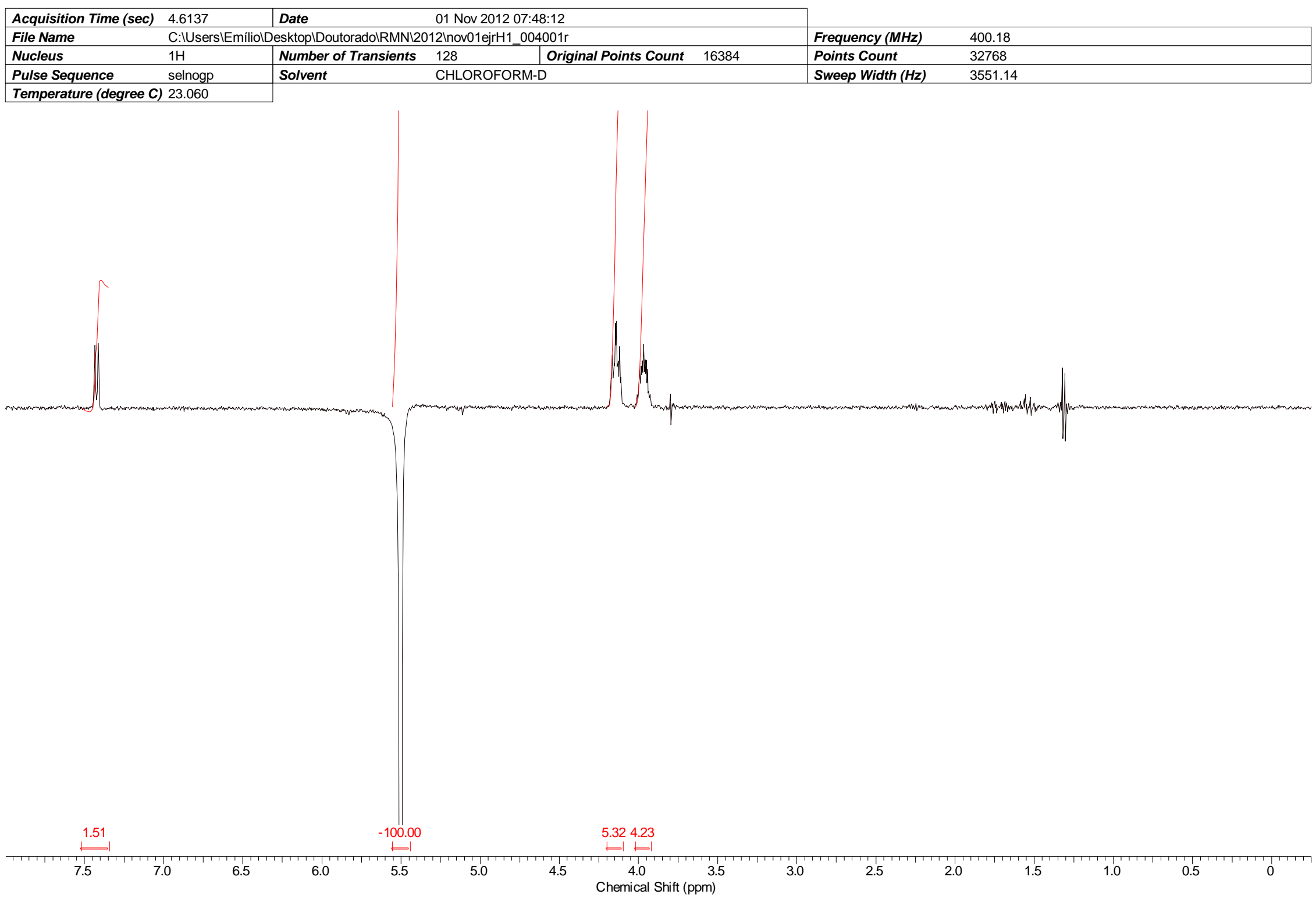

Figure 89: ${ }^{1} \mathrm{H}$ NMR (1D selective NOE) spectrum of compound $\mathbf{S} 25\left(400 \mathrm{MHz}, \mathrm{CDCl}_{3}\right)$. 


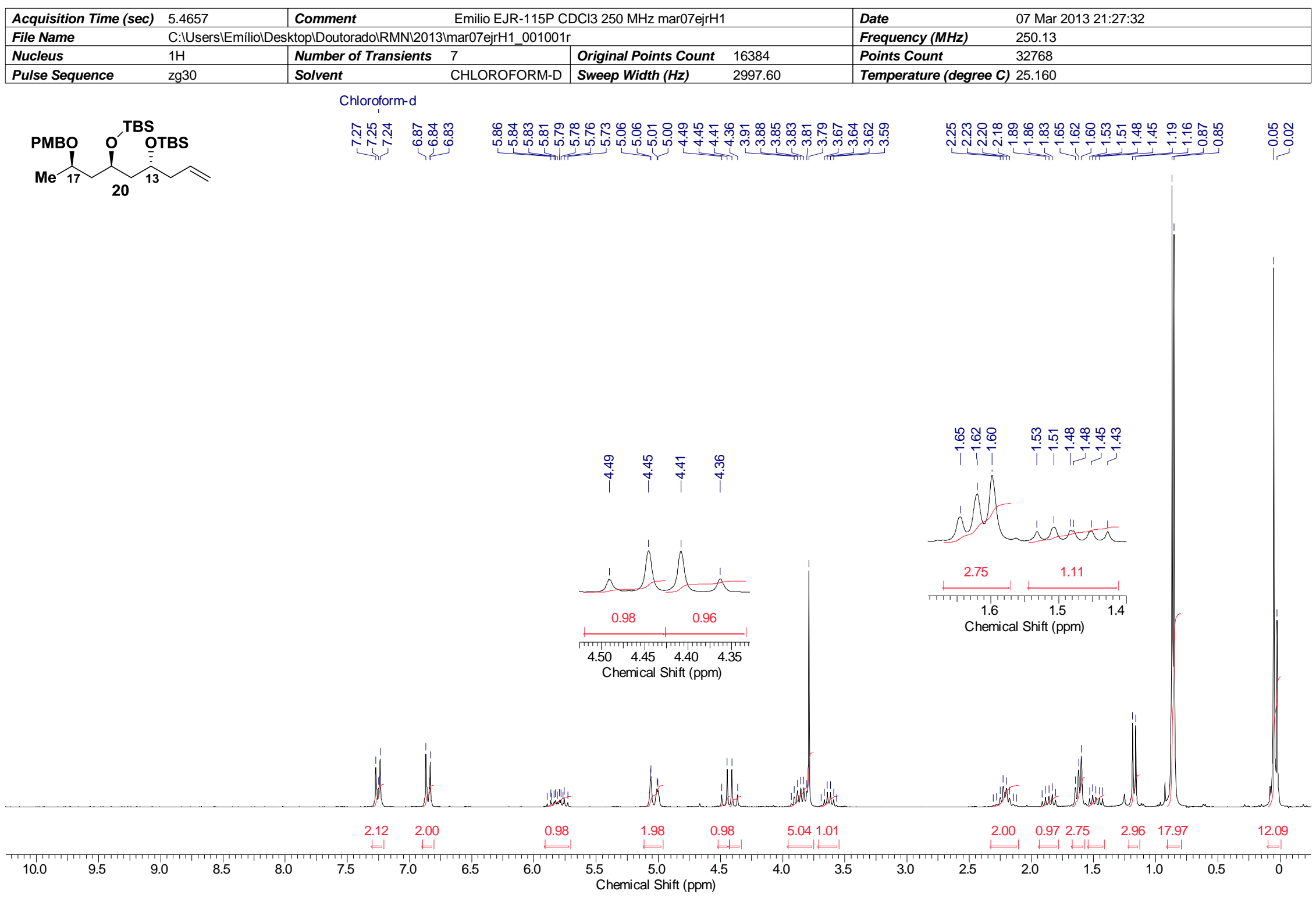

Figure 90: ${ }^{1} \mathrm{H}$ NMR spectrum of compound $20\left(250 \mathrm{MHz}, \mathrm{CDCl}_{3}\right)$. 


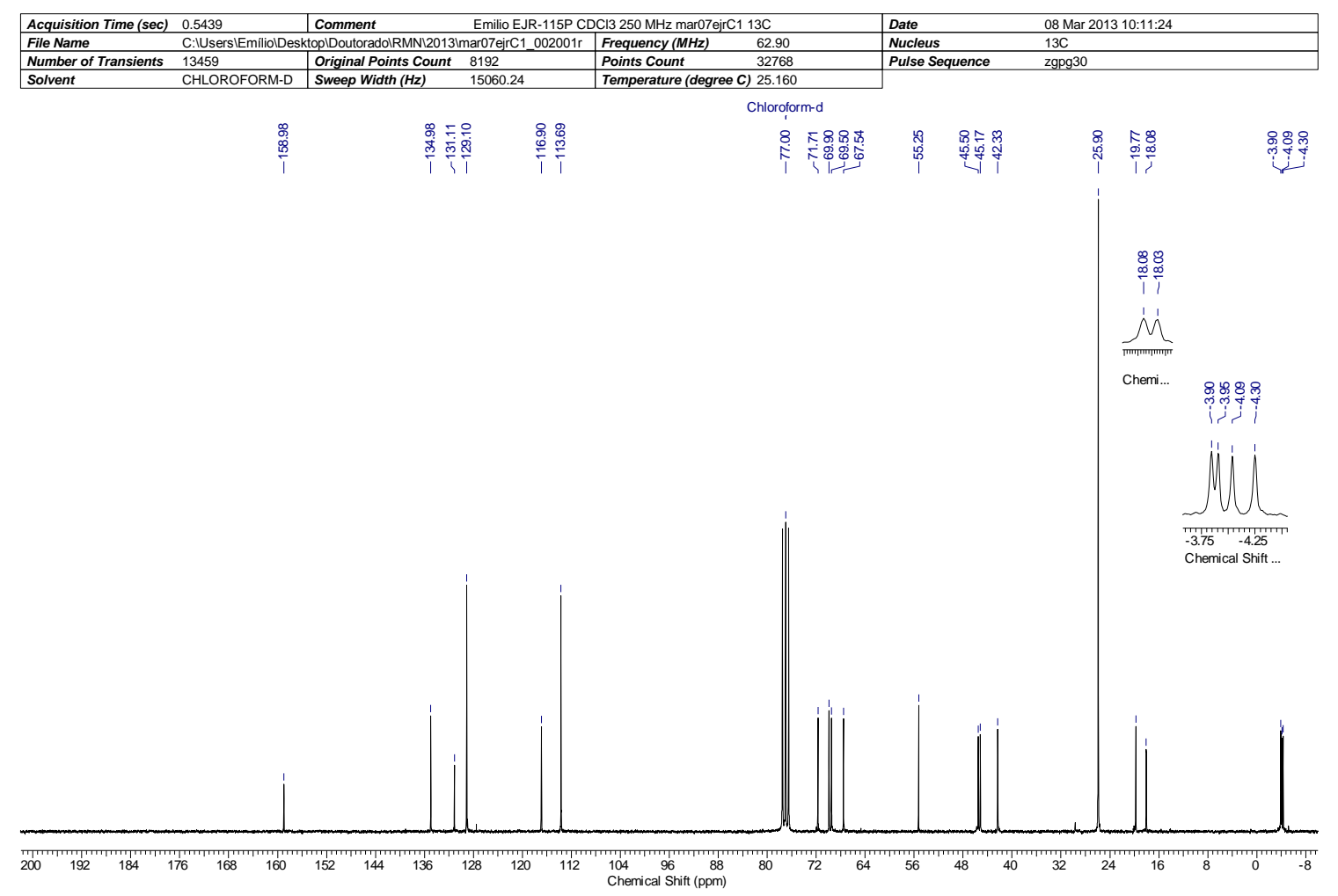

Figure 91: ${ }^{13} \mathrm{C}$ NMR spectrum of compound $20\left(62.5 \mathrm{MHz}, \mathrm{CDCl}_{3}\right)$.
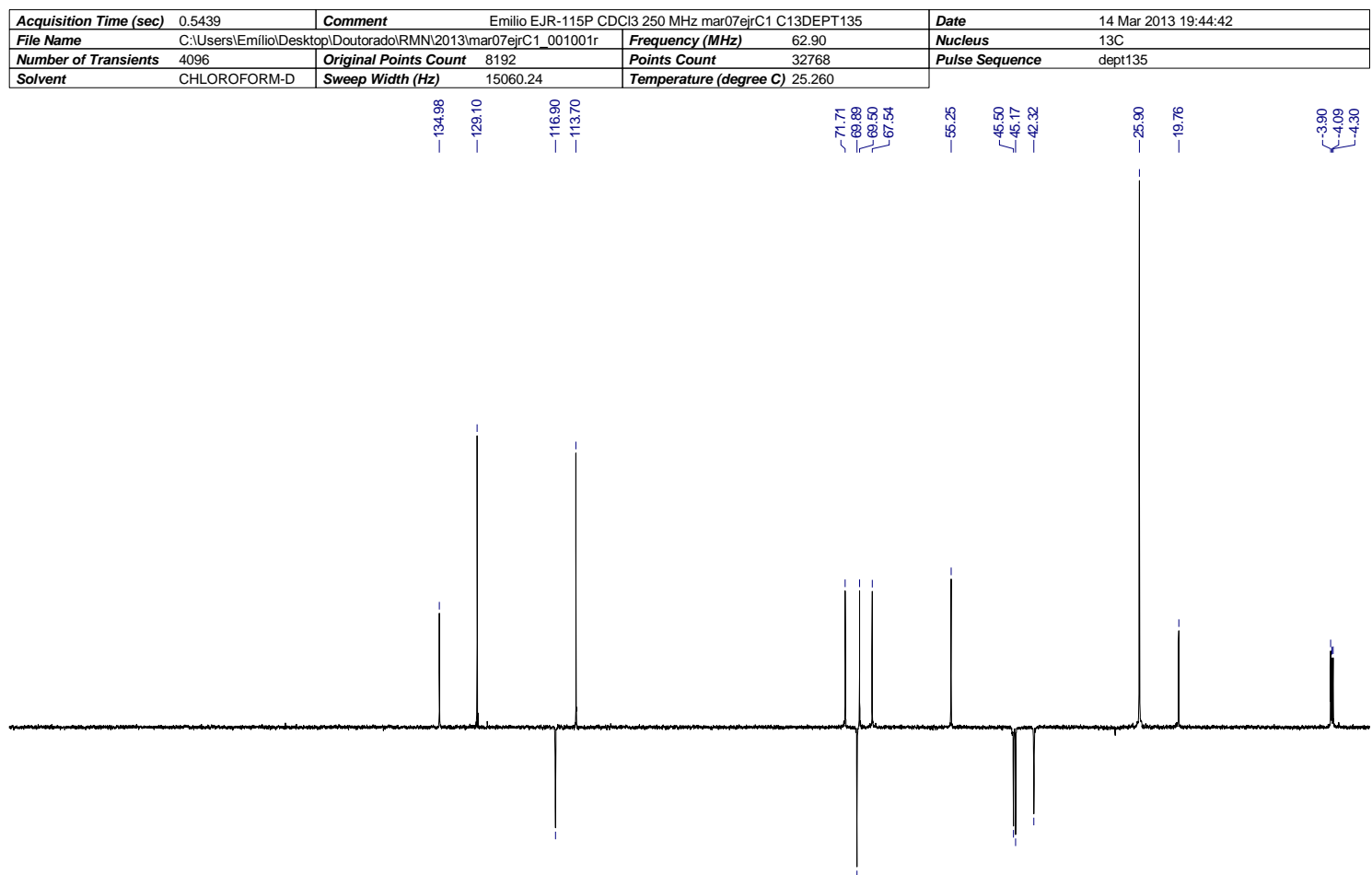

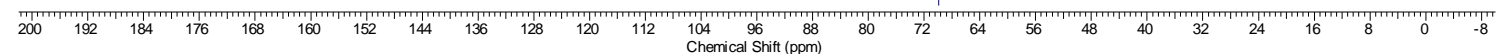

Figure 92: ${ }^{13} \mathrm{C}$ NMR (dept 135) spectrum of compound $20\left(62.5 \mathrm{MHz}, \mathrm{CDCl}_{3}\right)$. 


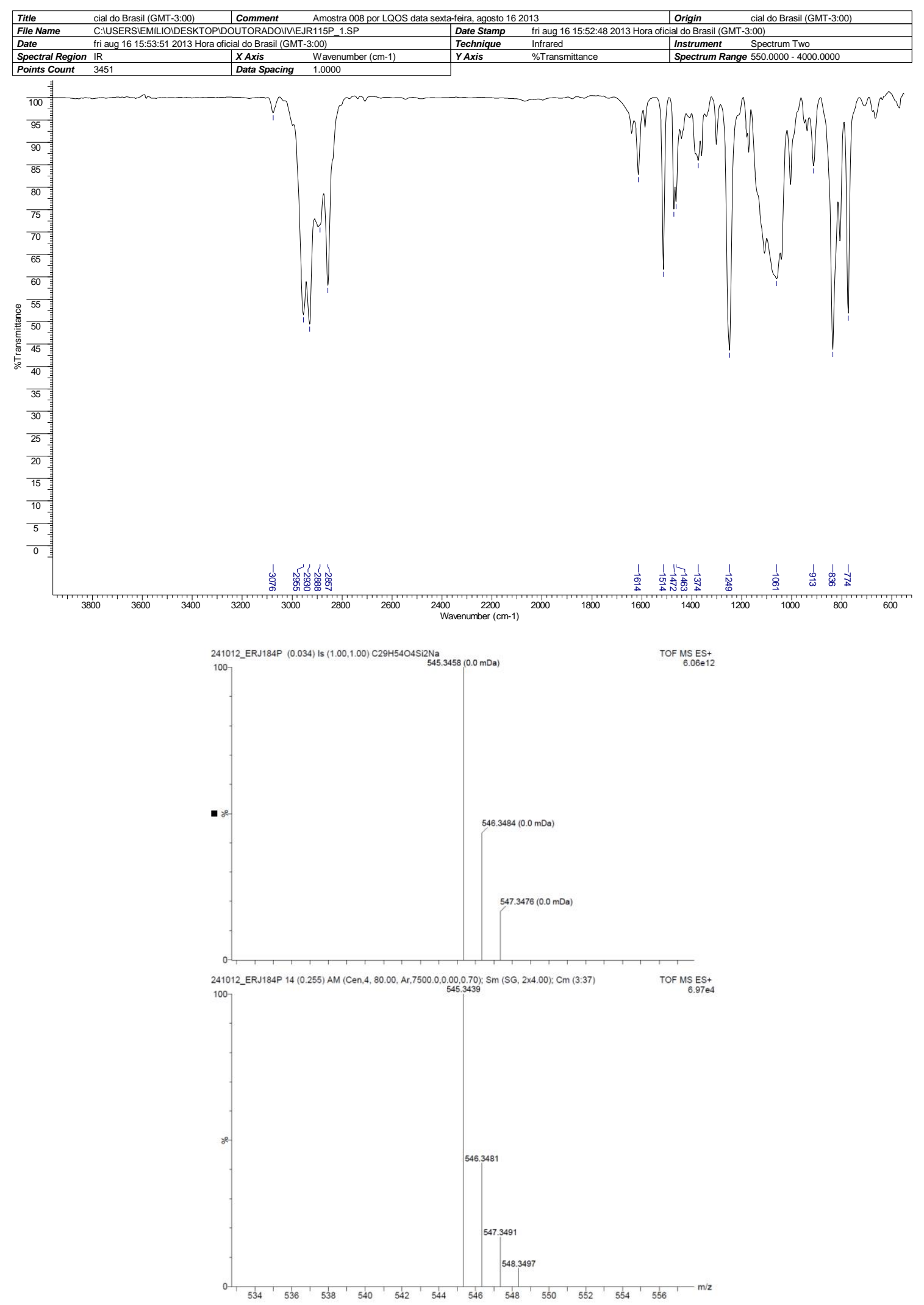

Figure 93: IR and HRMS (ESI TOF-MS) spectra of compound 20. 


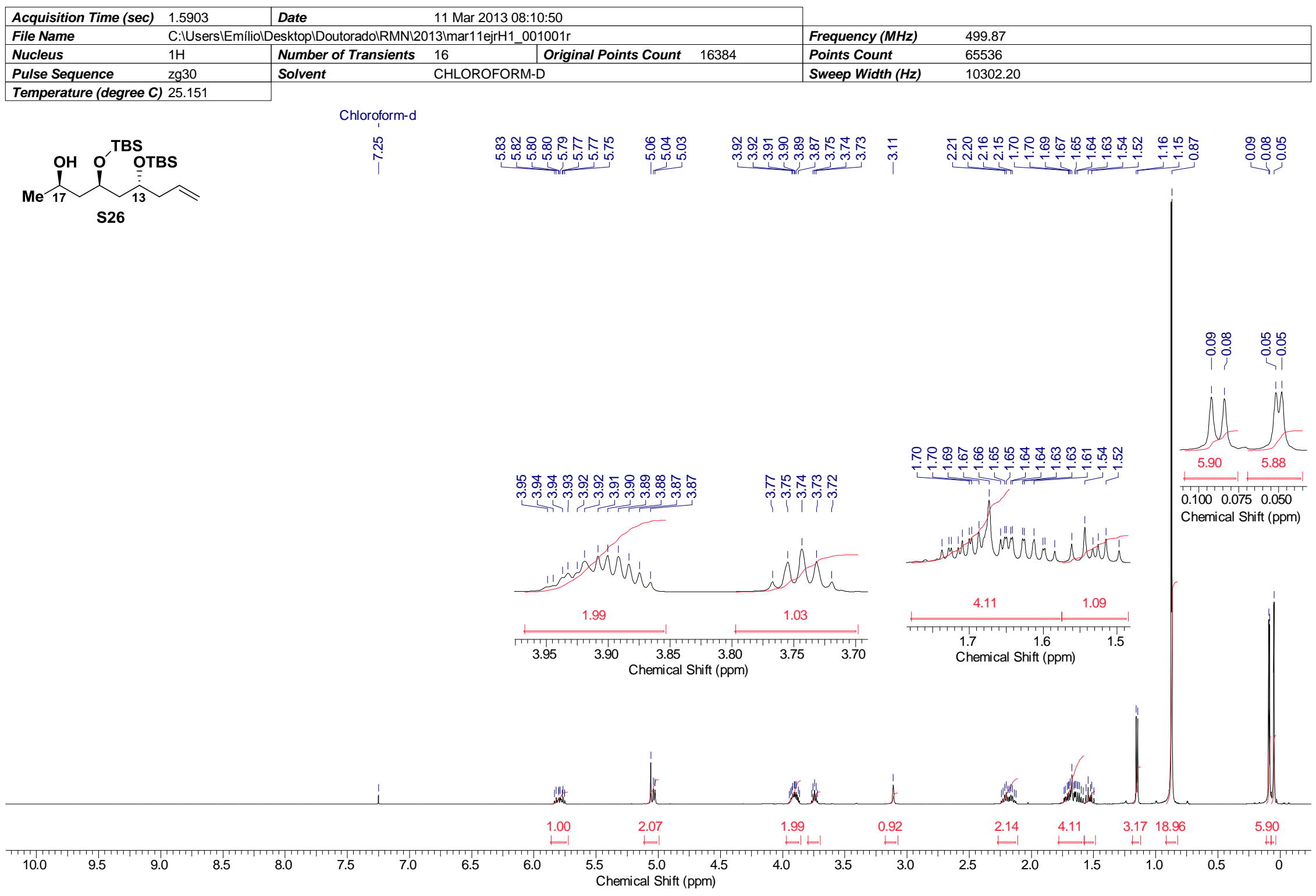

Figure 94: ${ }^{1} \mathrm{H}$ NMR spectrum of compound $\mathbf{S} 26\left(500 \mathrm{MHz}, \mathrm{CDCl}_{3}\right)$. 


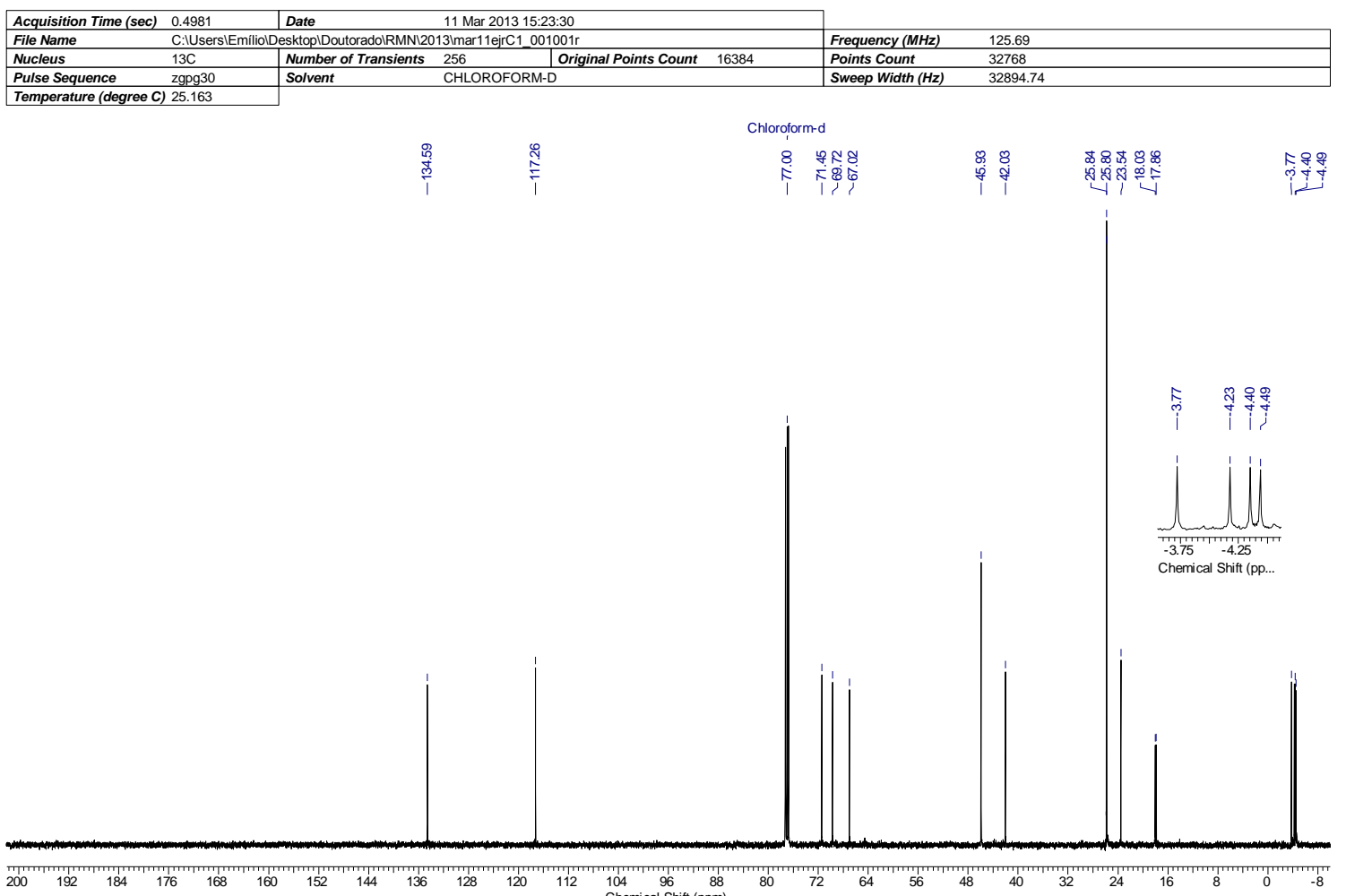

Figure 95: ${ }^{13} \mathrm{C}$ NMR spectrum of compound $\mathbf{S 2 6}\left(125 \mathrm{MHz}, \mathrm{CDCl}_{3}\right)$.

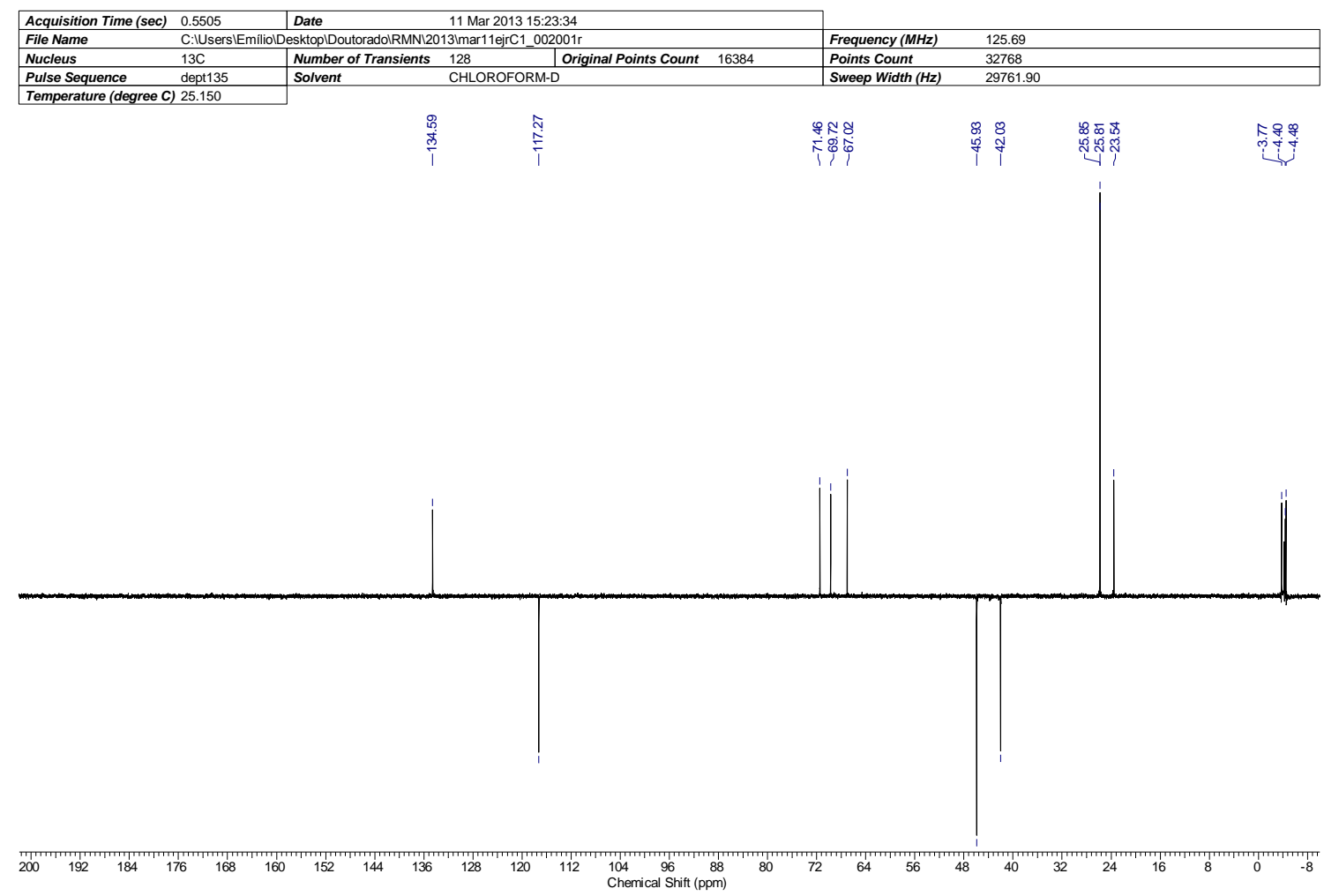

Figure 96: ${ }^{13} \mathrm{C}$ NMR (dept 135) spectrum of compound $\mathbf{S 2 6}\left(125 \mathrm{MHz}, \mathrm{CDCl}_{3}\right.$ ). 


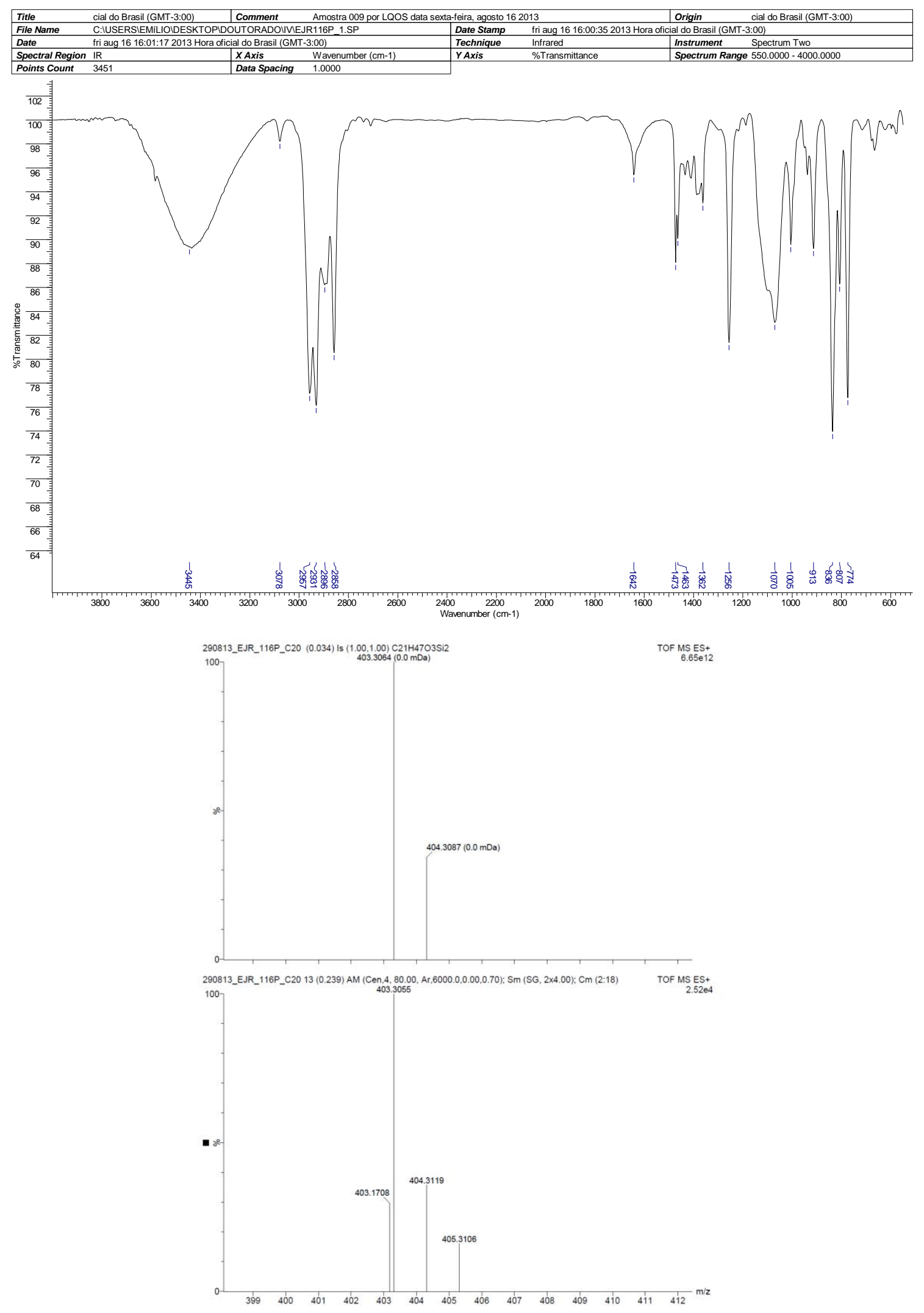

Figure 97: IR and HRMS (ESI TOF-MS) spectra of compound S26. 


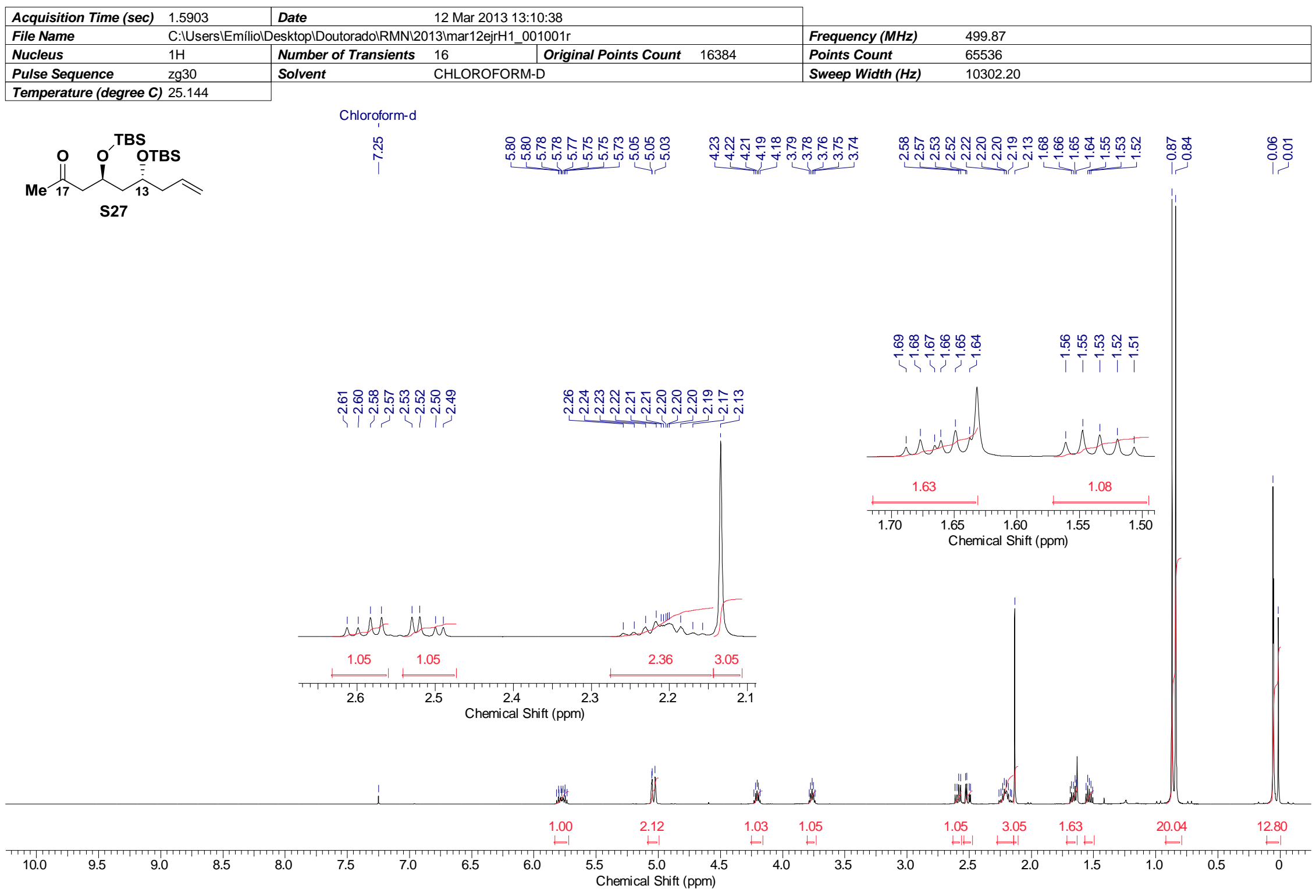

Figure 98: ${ }^{1} \mathrm{H}$ NMR spectrum of compound $\mathbf{S} 27\left(500 \mathrm{MHz}, \mathrm{CDCl}_{3}\right)$. 


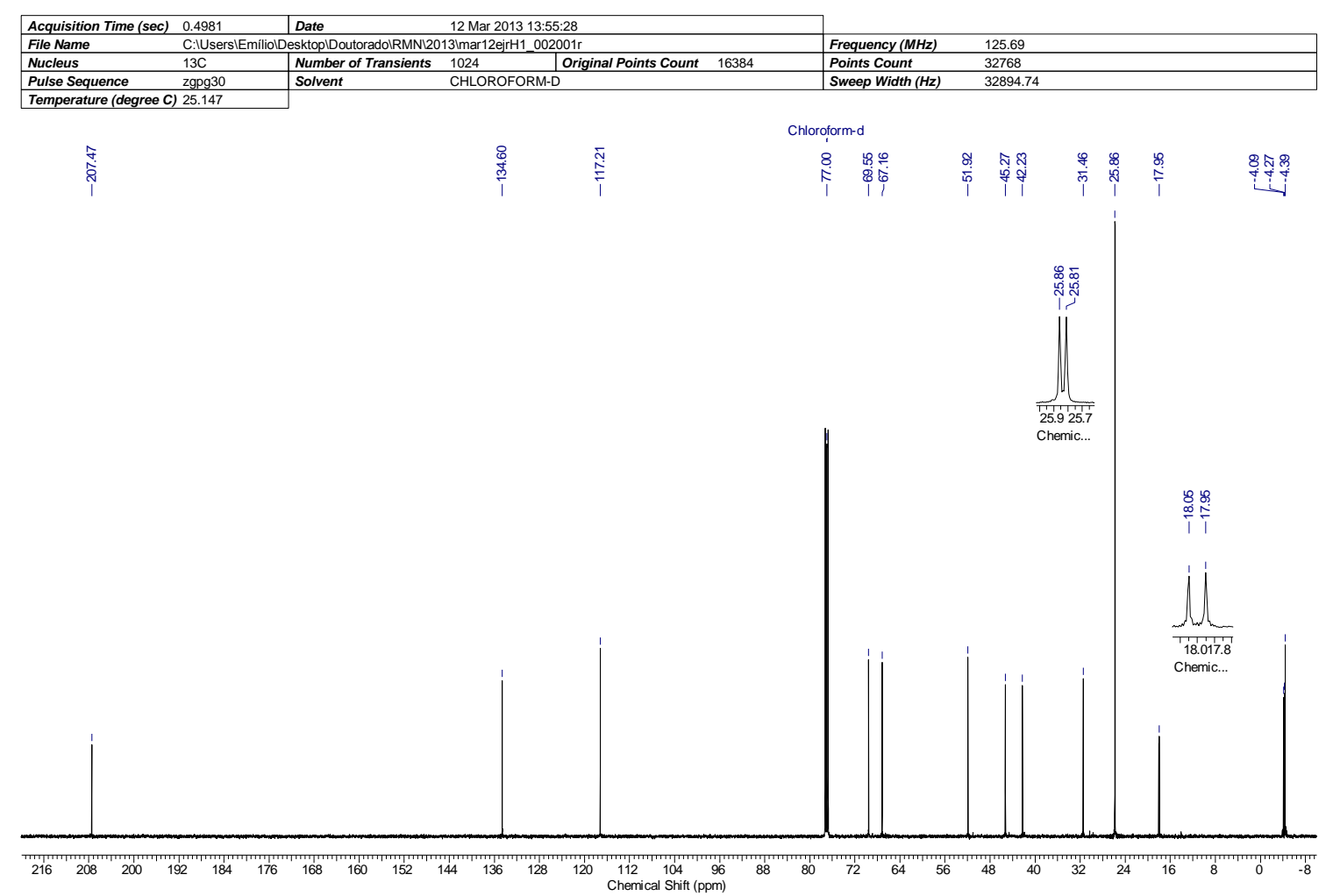

Figure 99: ${ }^{13} \mathrm{C}$ NMR spectrum of compound $\mathbf{S 2 7}\left(125 \mathrm{MHz}, \mathrm{CDCl}_{3}\right)$.

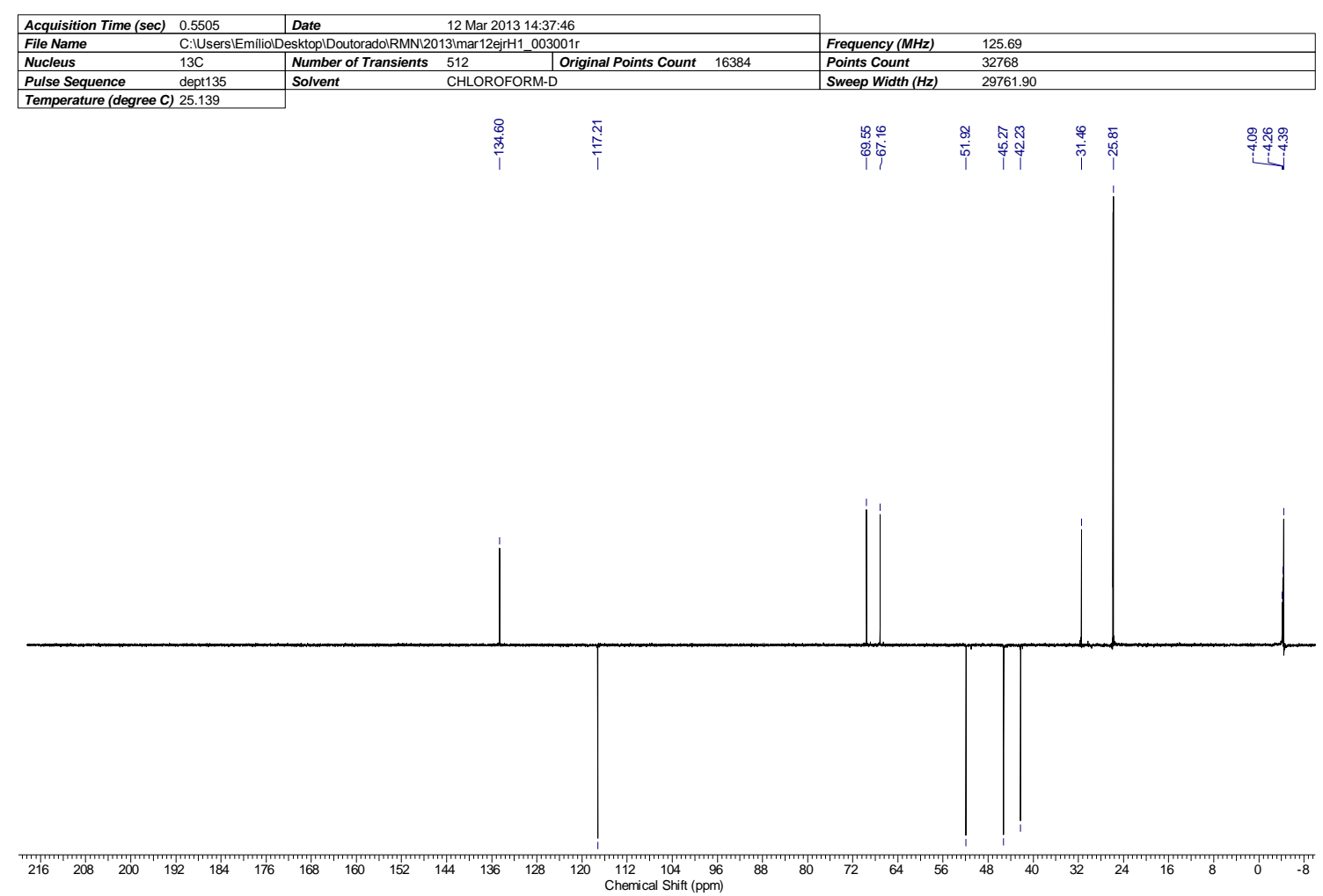

Figure 100: ${ }^{13} \mathrm{C}$ NMR (dept 135) spectrum of compound $\mathbf{S 2 7}\left(125 \mathrm{MHz}, \mathrm{CDCl}_{3}\right.$ ). 


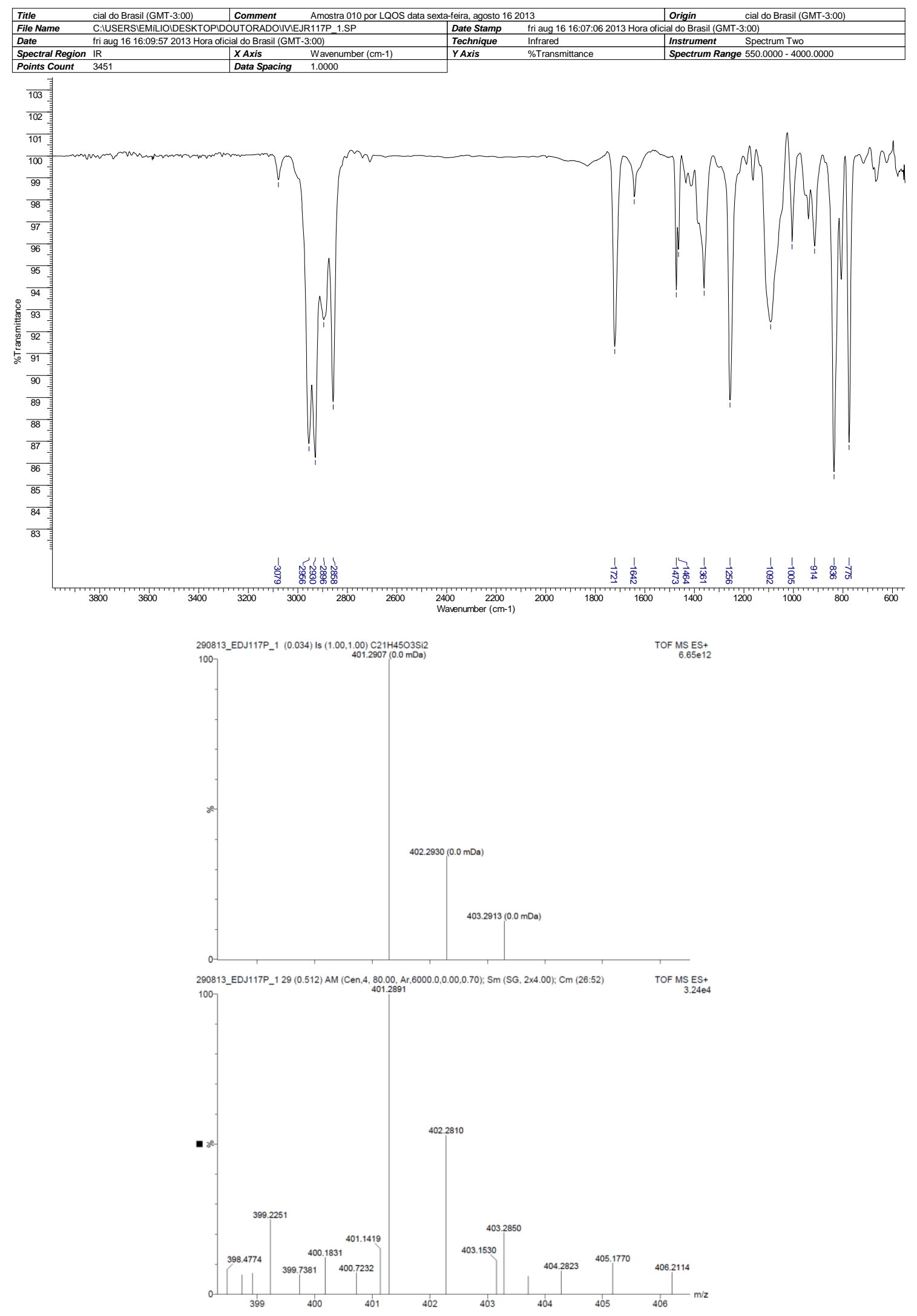

Figure 101: IR and HRMS (ESI TOF-MS) spectra of compound S27. 


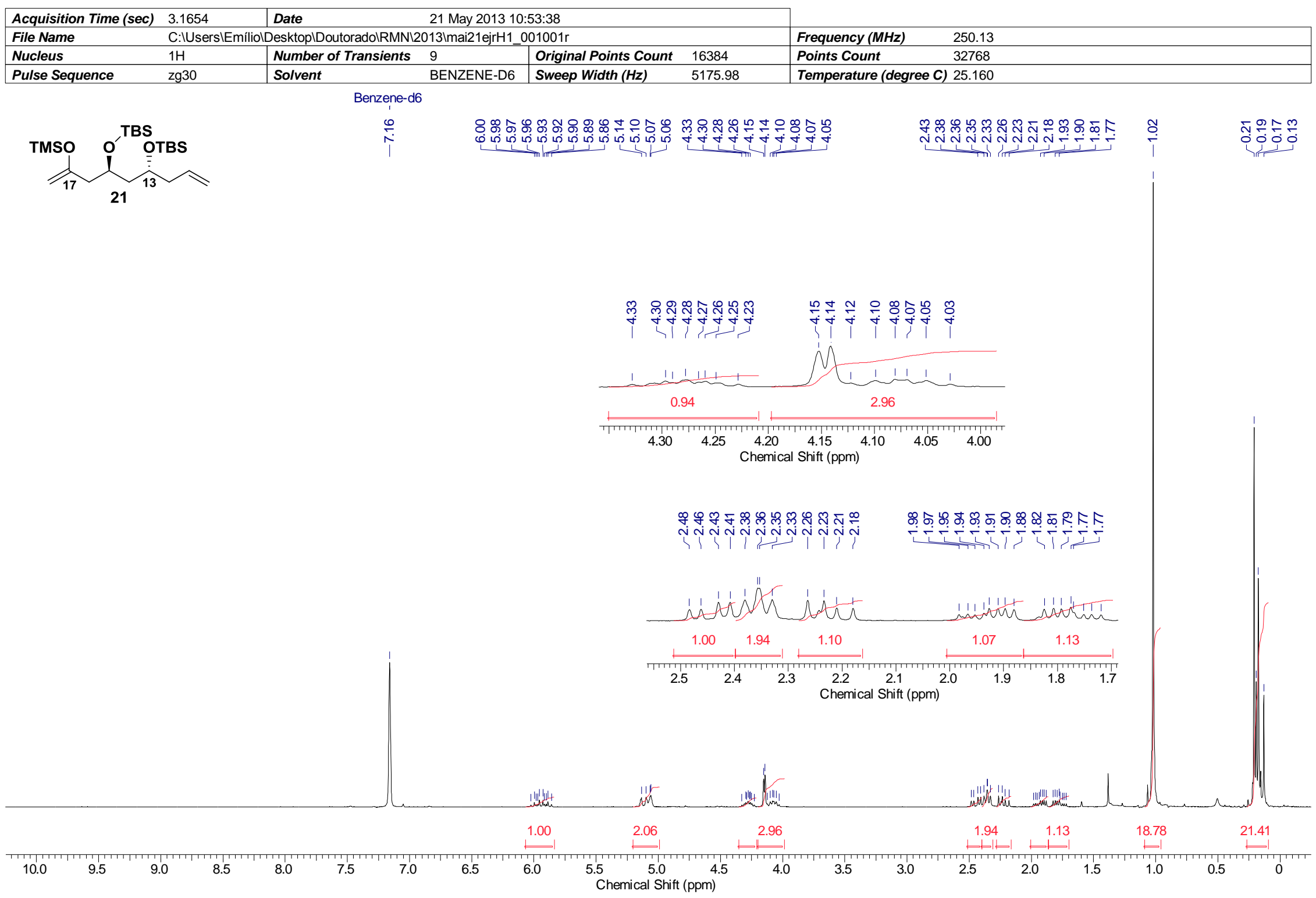

Figure 102: ${ }^{1} \mathrm{H}$ NMR spectrum of compound $21\left(250 \mathrm{MHz}, \mathrm{C}_{6} \mathrm{D}_{6}\right)$. 


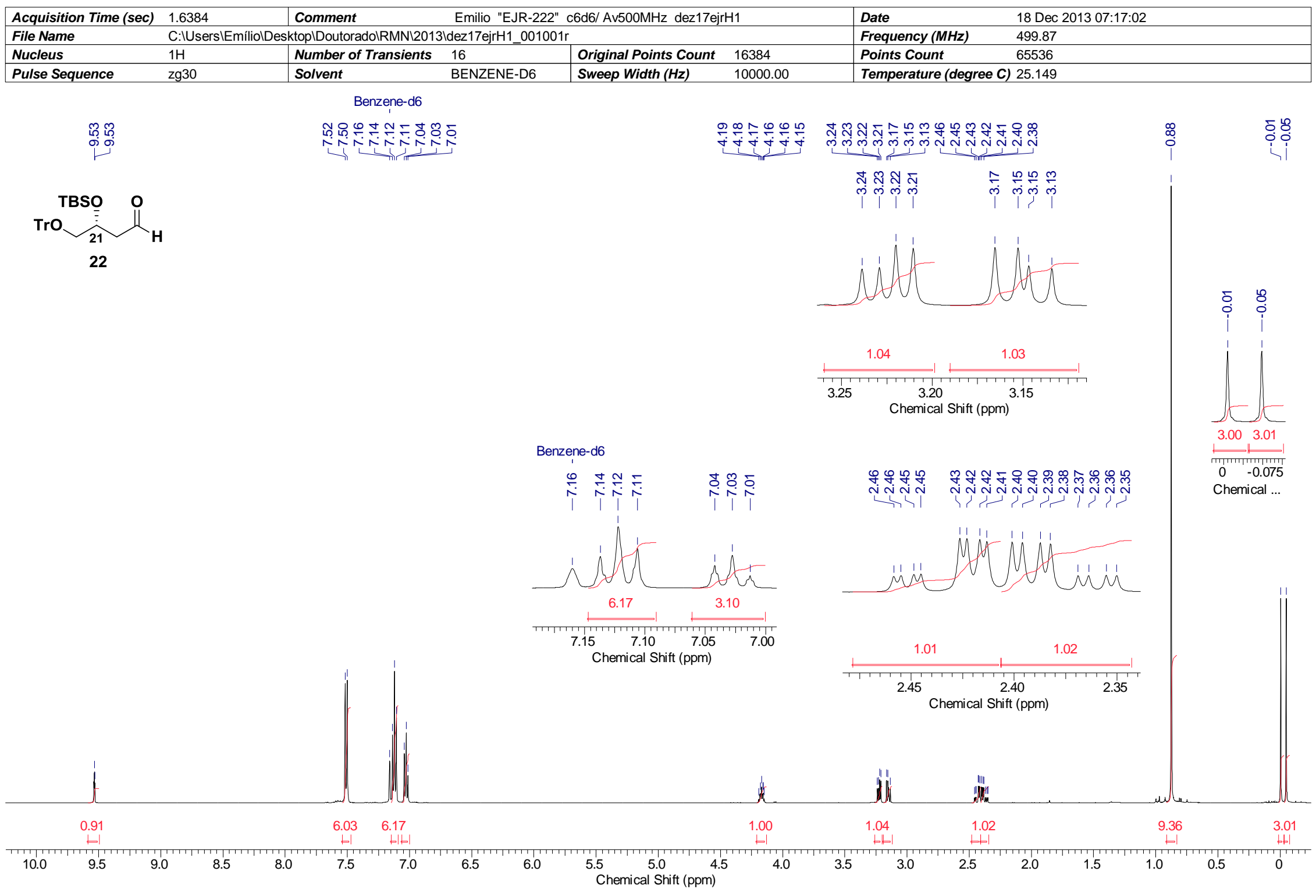

Figure 103: ${ }^{1} \mathrm{H}$ NMR spectrum of compound $22\left(500 \mathrm{MHz}, \mathrm{C}_{6} \mathrm{D}_{6}\right)$. 


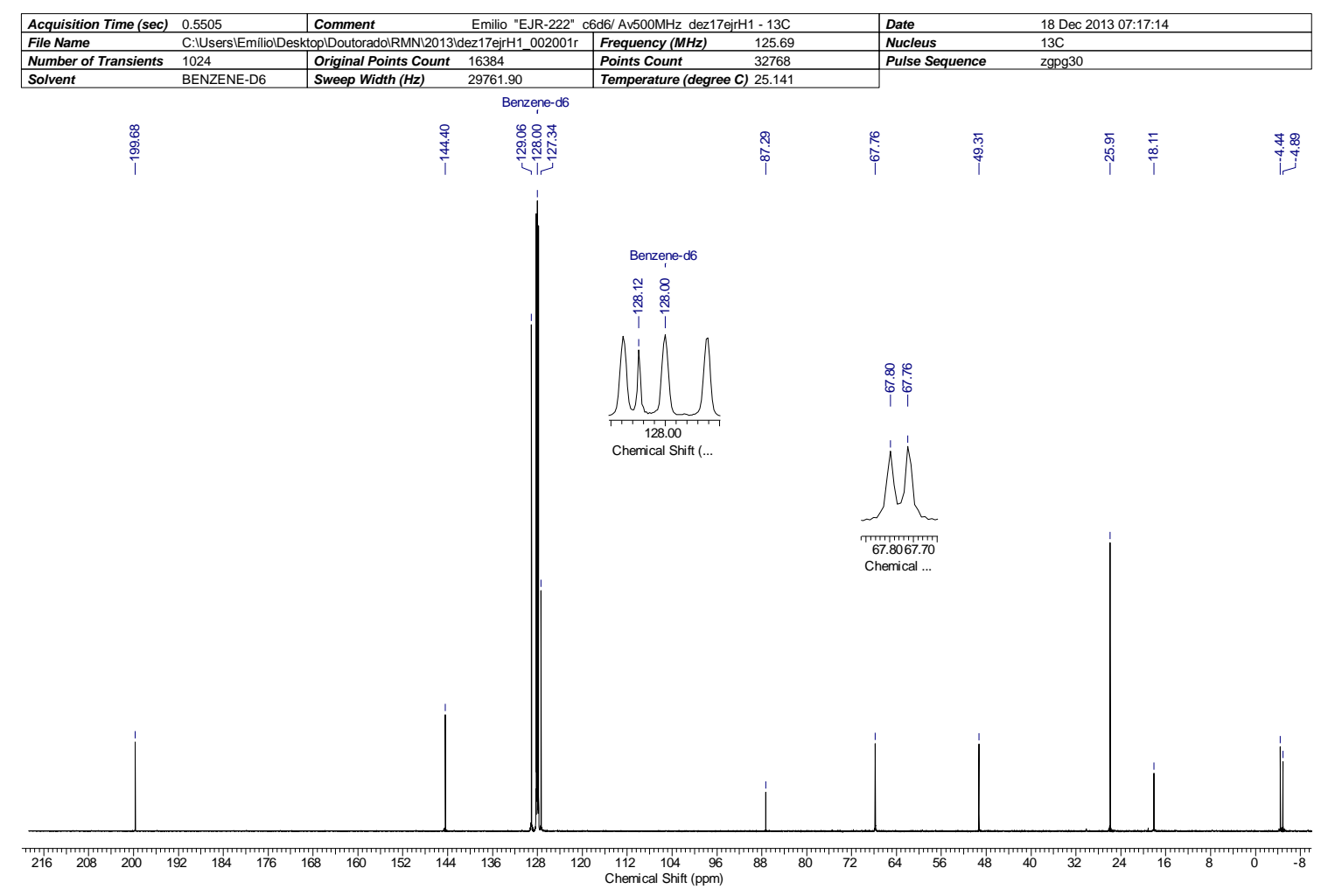

Figure 104: ${ }^{13} \mathrm{C}$ NMR spectrum of compound $22\left(125 \mathrm{MHz}, \mathrm{C}_{6} \mathrm{D}_{6}\right)$.

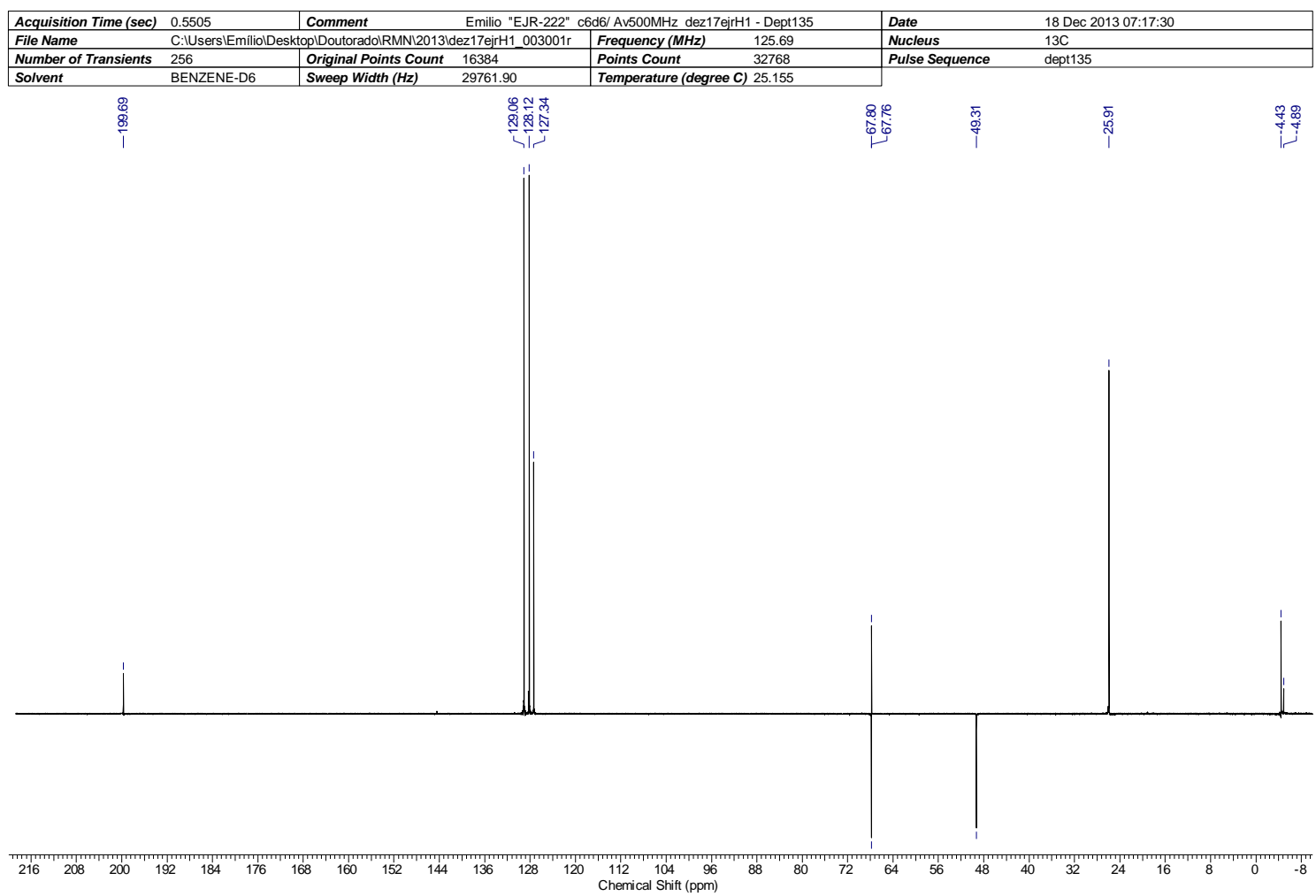

Figure 105: ${ }^{13} \mathrm{C}$ NMR (dept 135) spectrum of compound $22\left(125 \mathrm{MHz}, \mathrm{C}_{6} \mathrm{D}_{6}\right)$. 

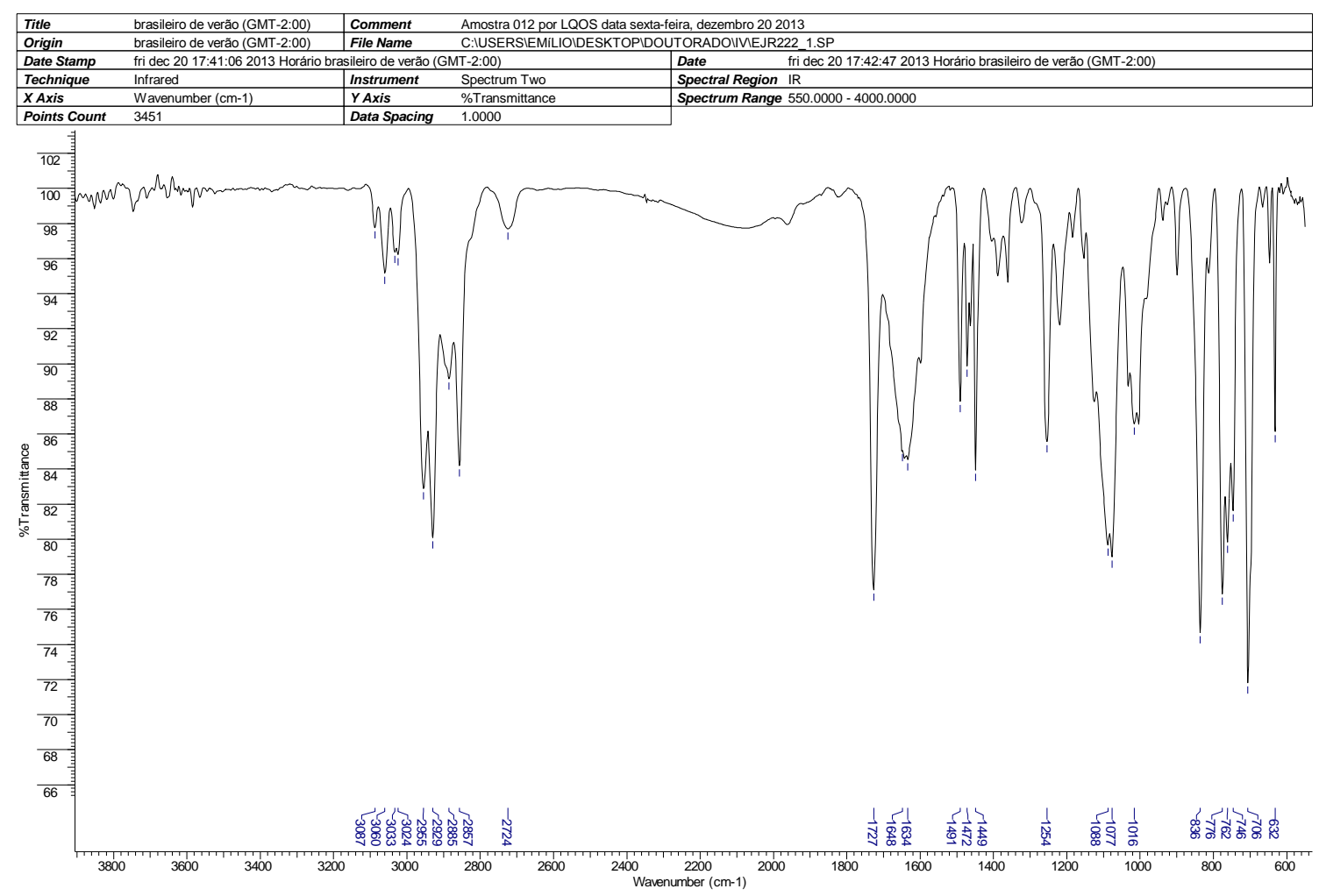

B001 \#23 RT: 0.35 AV: 1 NL: 1.44E8

T: FTMS + p ESI Full ms [150.00-1500.00]

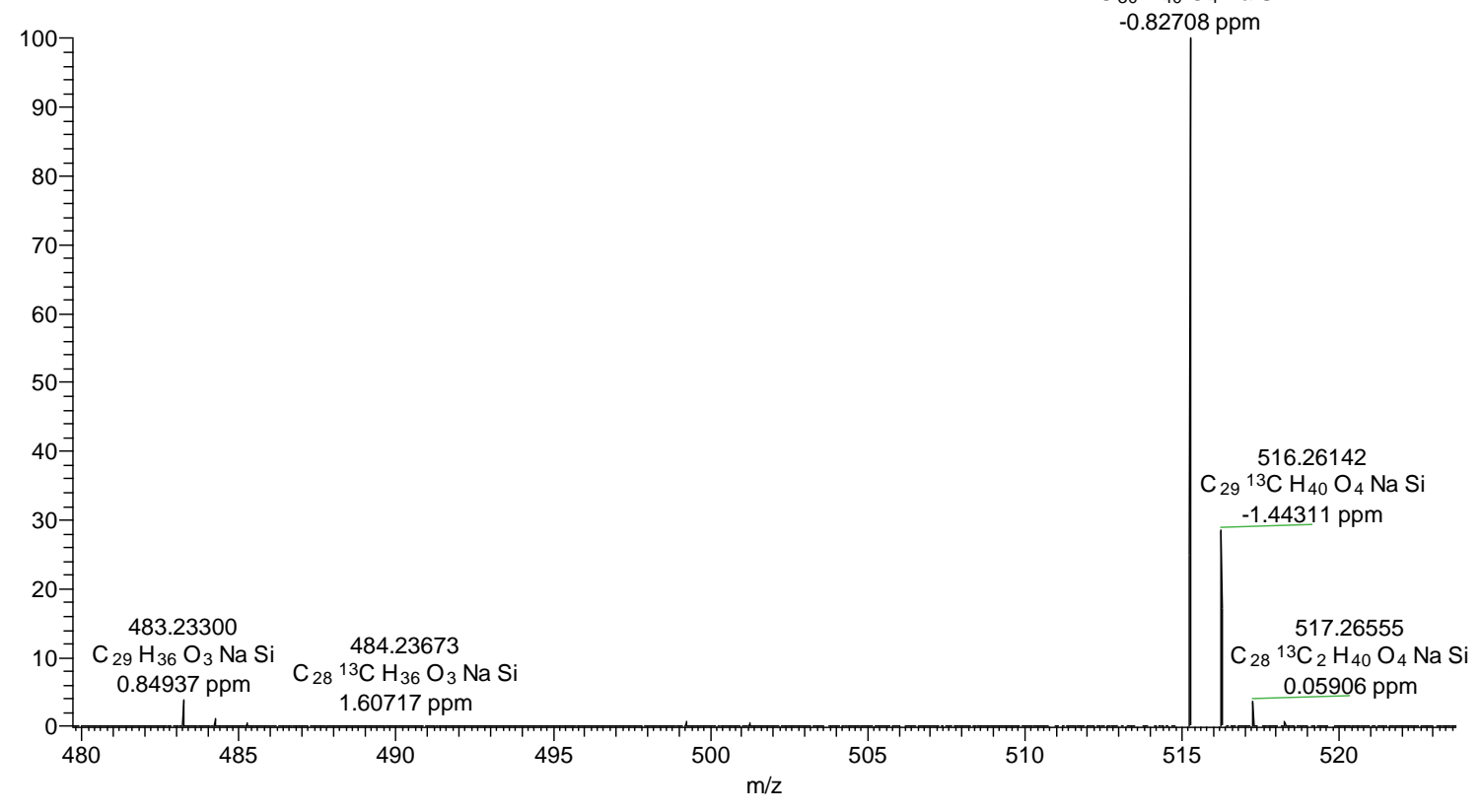

Figure 106: IR and HRMS (ESI FT-ICR-MS) spectra of compound 22. 


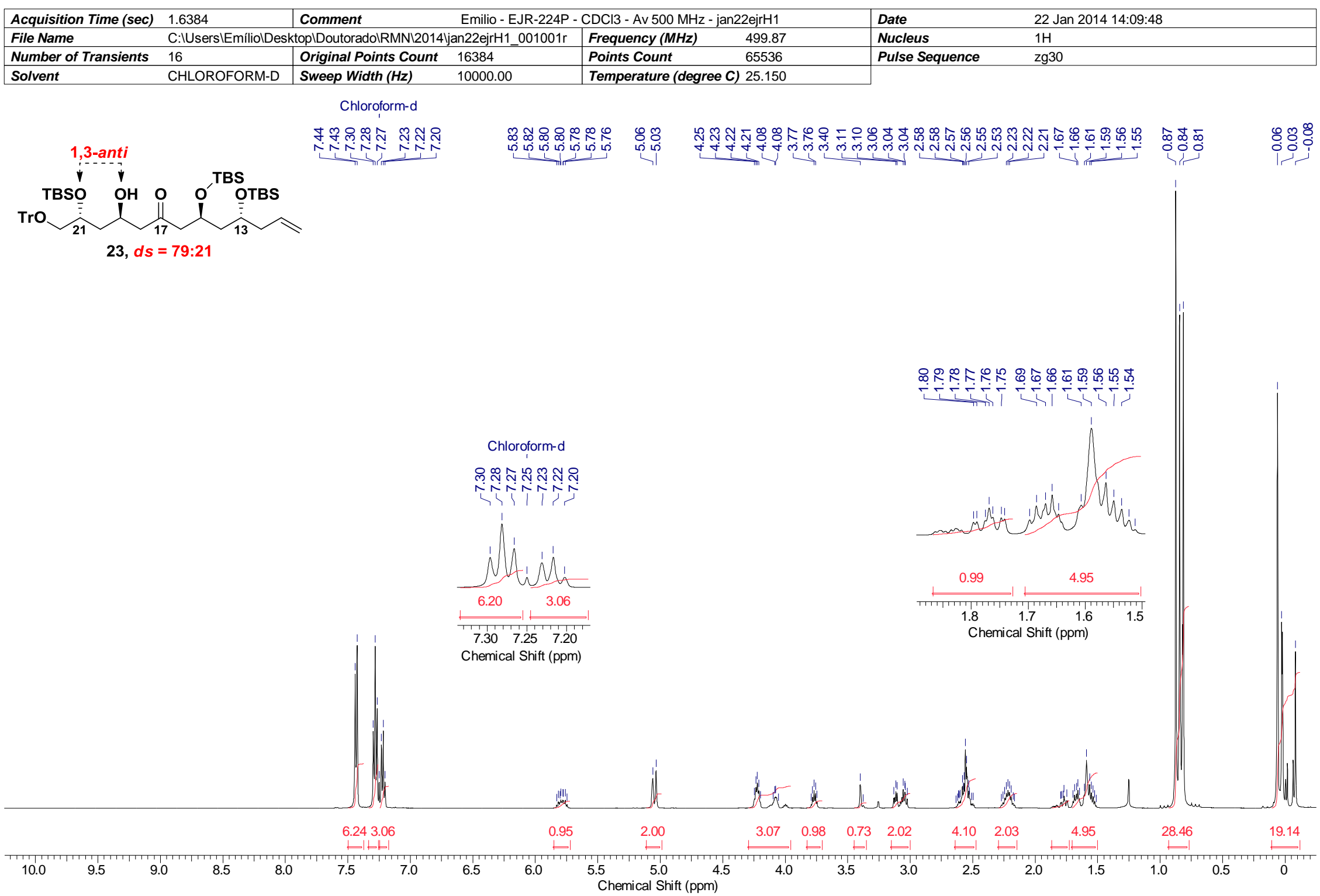

Figure 107: ${ }^{1} \mathrm{H}$ NMR spectrum of compound $23\left(500 \mathrm{MHz}, \mathrm{CDCl}_{3}\right)$. 


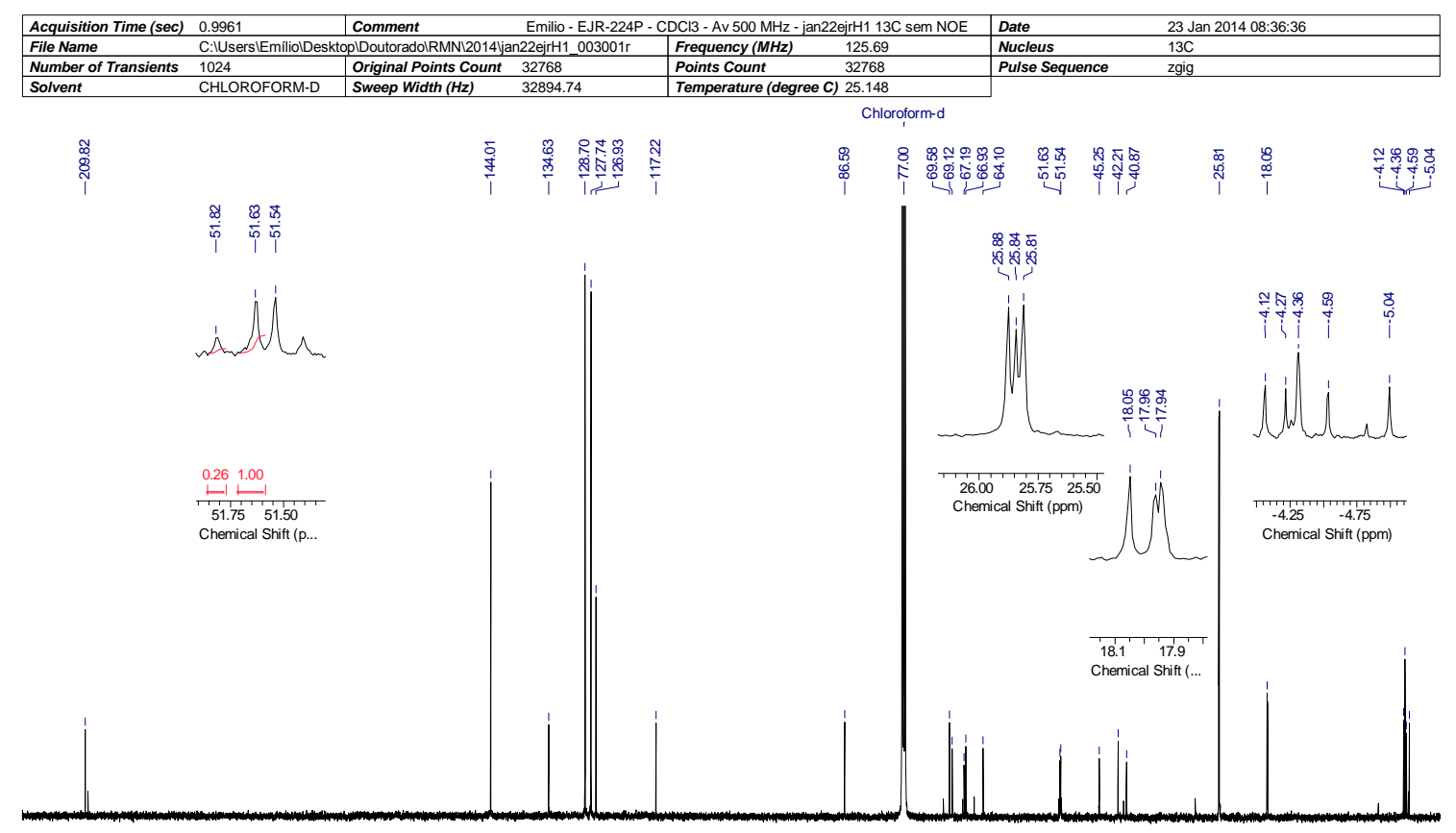

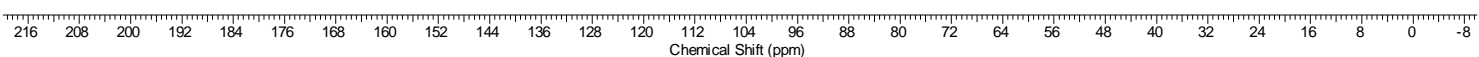

Figure 108: ${ }^{13} \mathrm{C}$ NMR spectrum of compound $23\left(125 \mathrm{MHz}, \mathrm{CDCl}_{3}\right)$.

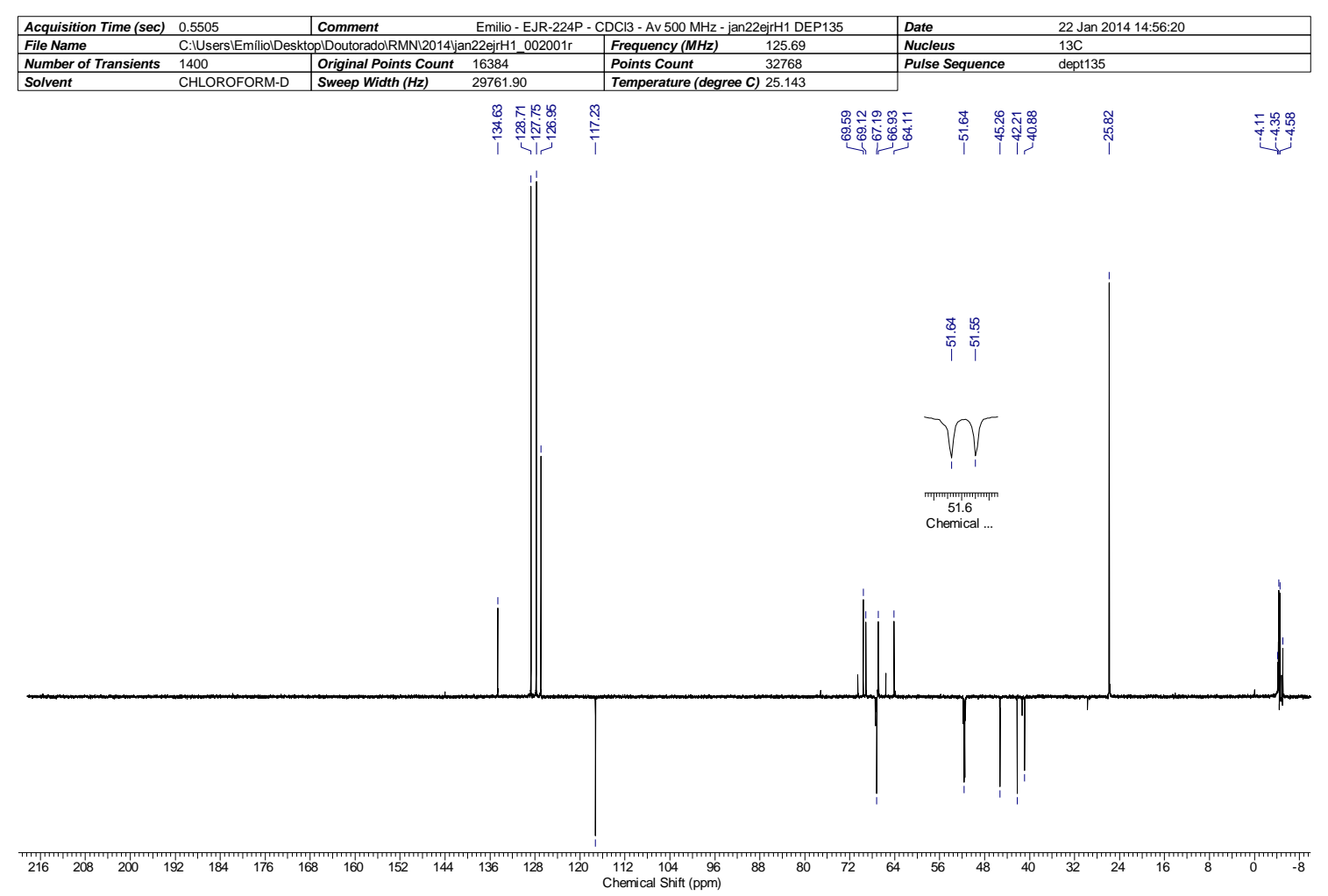

Figure 109: ${ }^{13} \mathrm{C}$ NMR (dept 135) spectrum of compound $23\left(125 \mathrm{MHz}, \mathrm{CDCl}_{3}\right)$. 


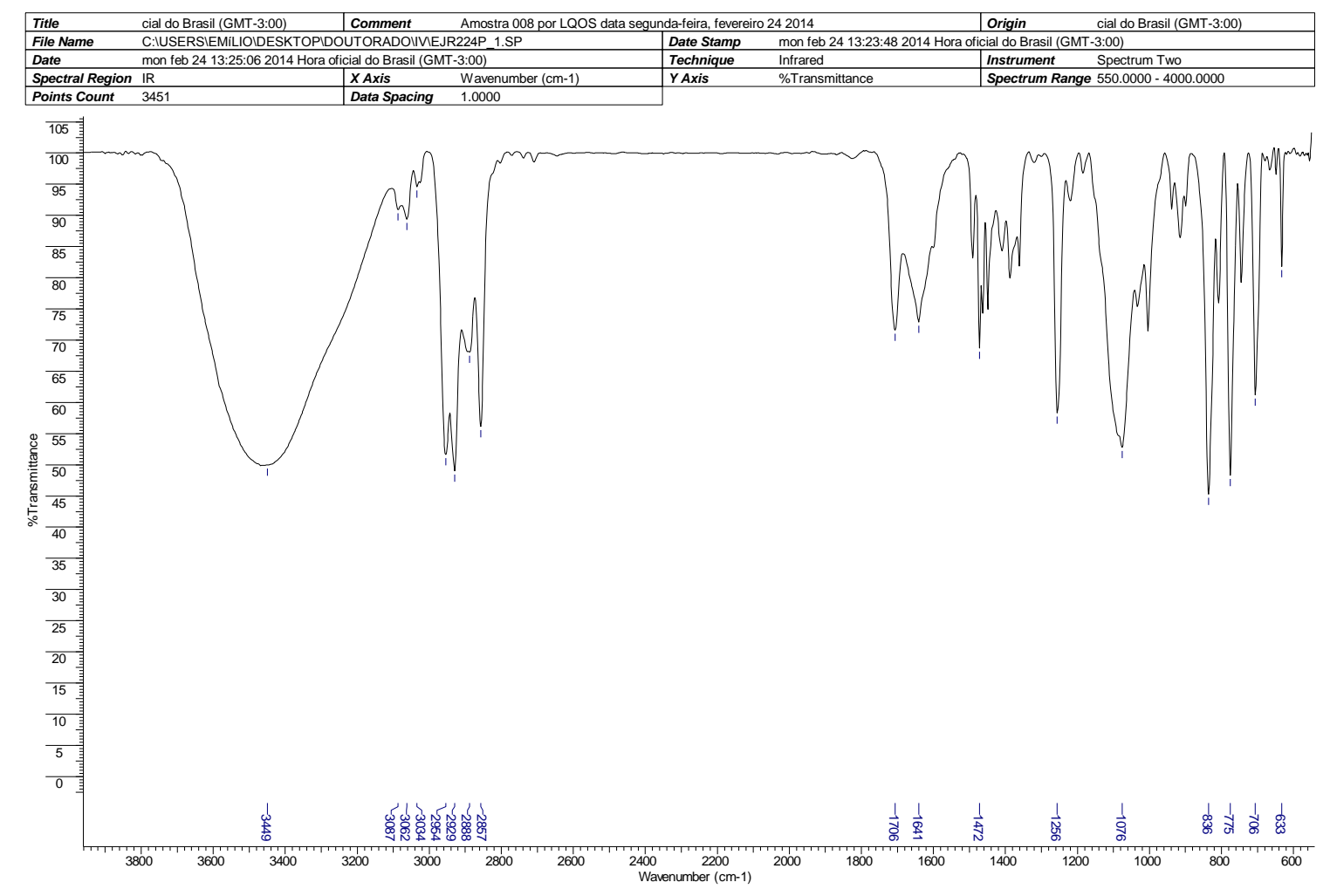

B002 \#27 RT: 0.41 AV: 1 NL: 4.55E7

T: FTMS + p ESIFull ms [150.00-1500.00] 883.51519

$\mathrm{C}_{50} \mathrm{H}_{80} \mathrm{O}_{6} \mathrm{Na} \mathrm{Si}_{3}$
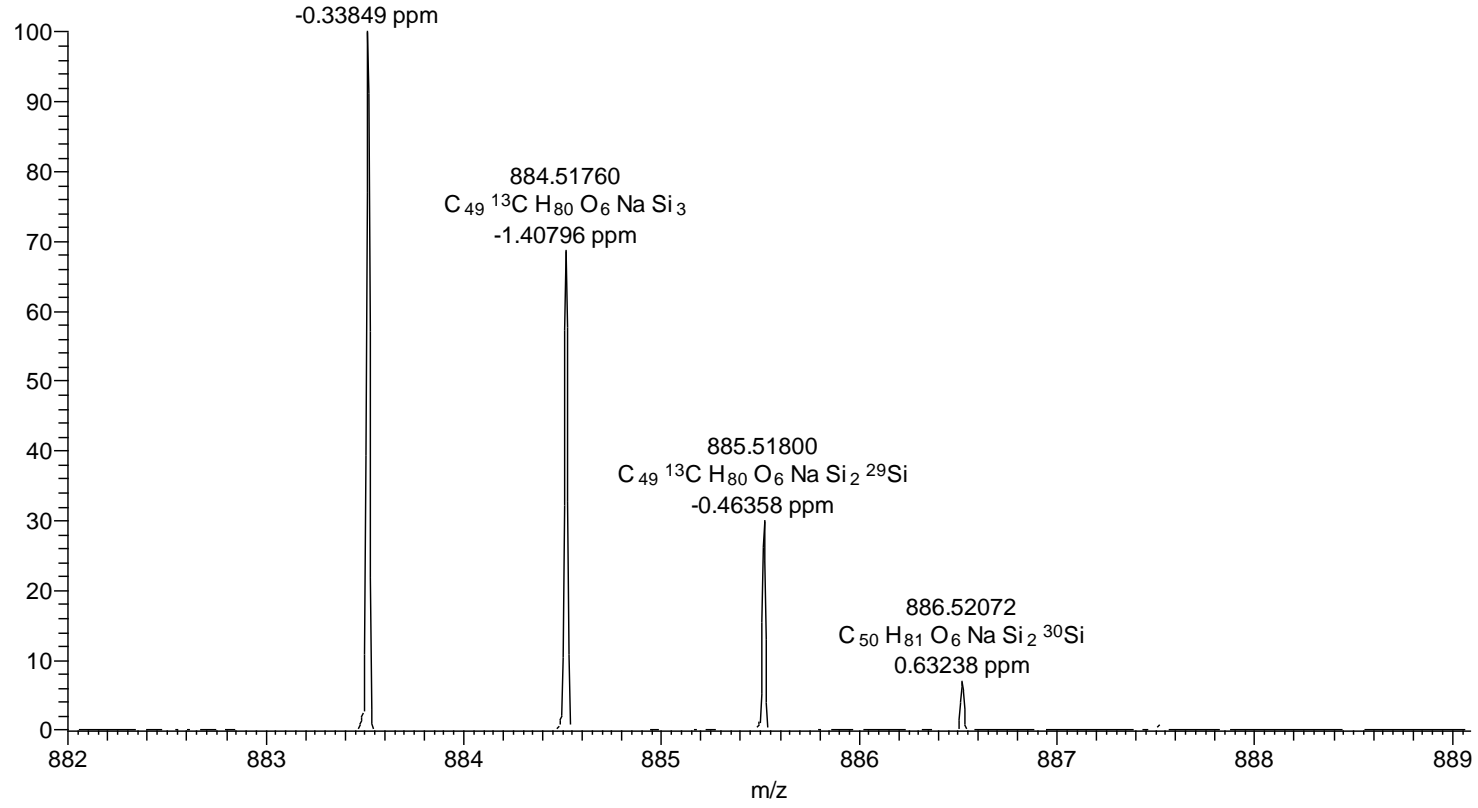

Figure 110: IR and HRMS (ESI FT-ICR-MS) spectra of compound 23. 


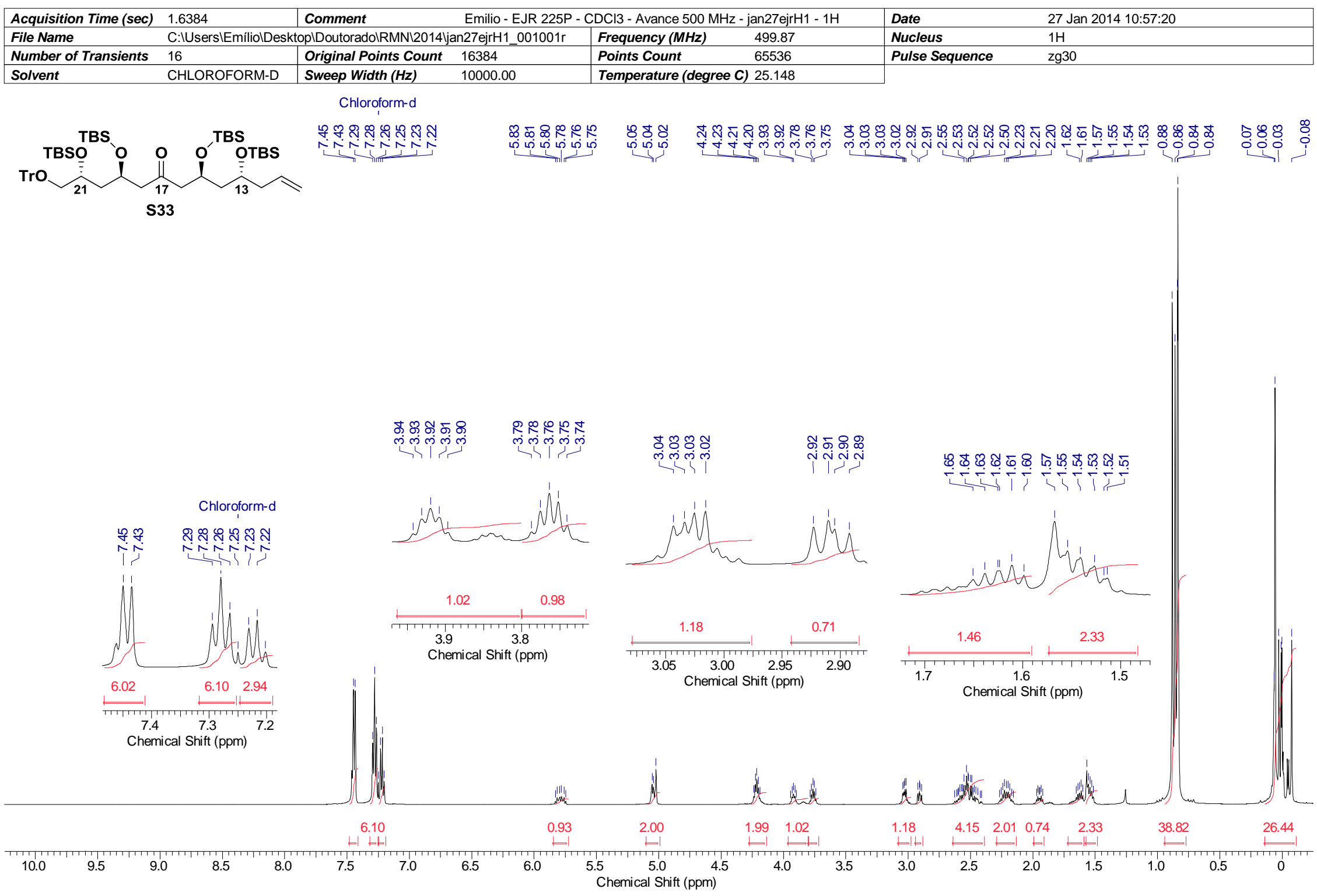

Figure 111: ${ }^{1} \mathrm{H}$ NMR spectrum of compound $\mathbf{S} 33\left(500 \mathrm{MHz}, \mathrm{CDCl}_{3}\right)$. 


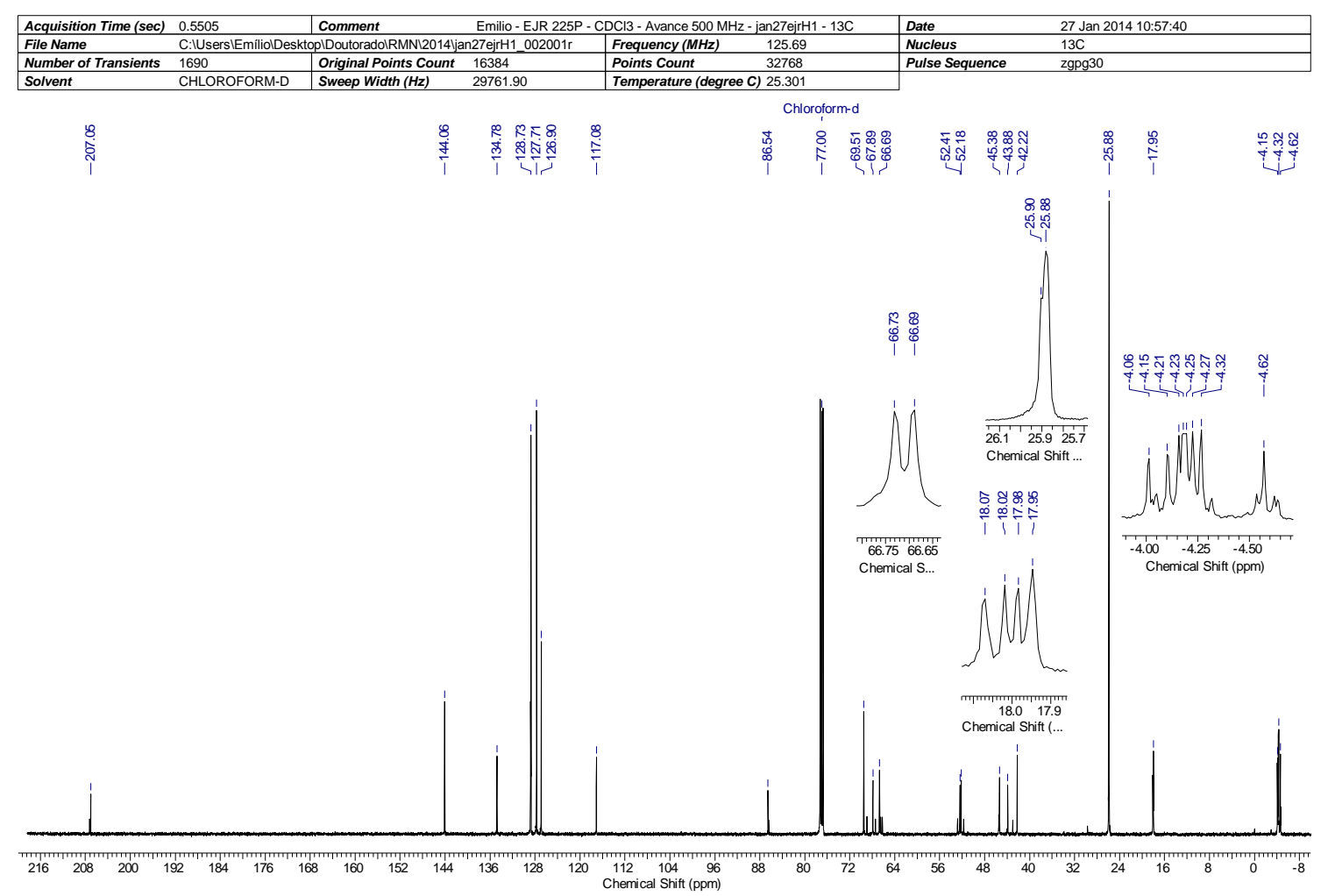

Figure 112: ${ }^{13} \mathrm{C}$ NMR spectrum of compound $\mathbf{S} 33\left(125 \mathrm{MHz}, \mathrm{CDCl}_{3}\right)$.

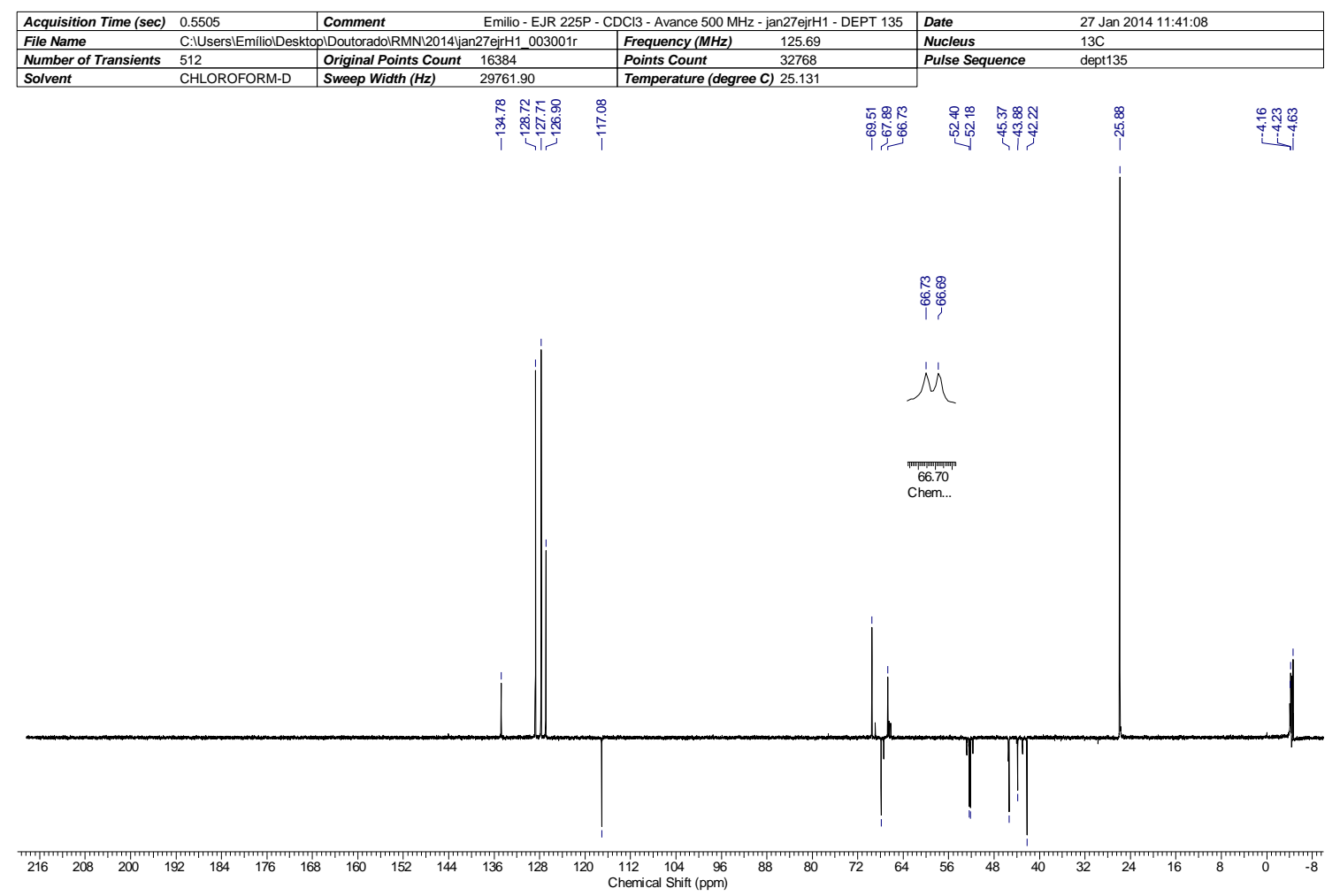

Figure 113: ${ }^{13} \mathrm{C}$ NMR (dept 135) spectrum of compound $\mathbf{S} 33\left(125 \mathrm{MHz}, \mathrm{CDCl}_{3}\right)$. 

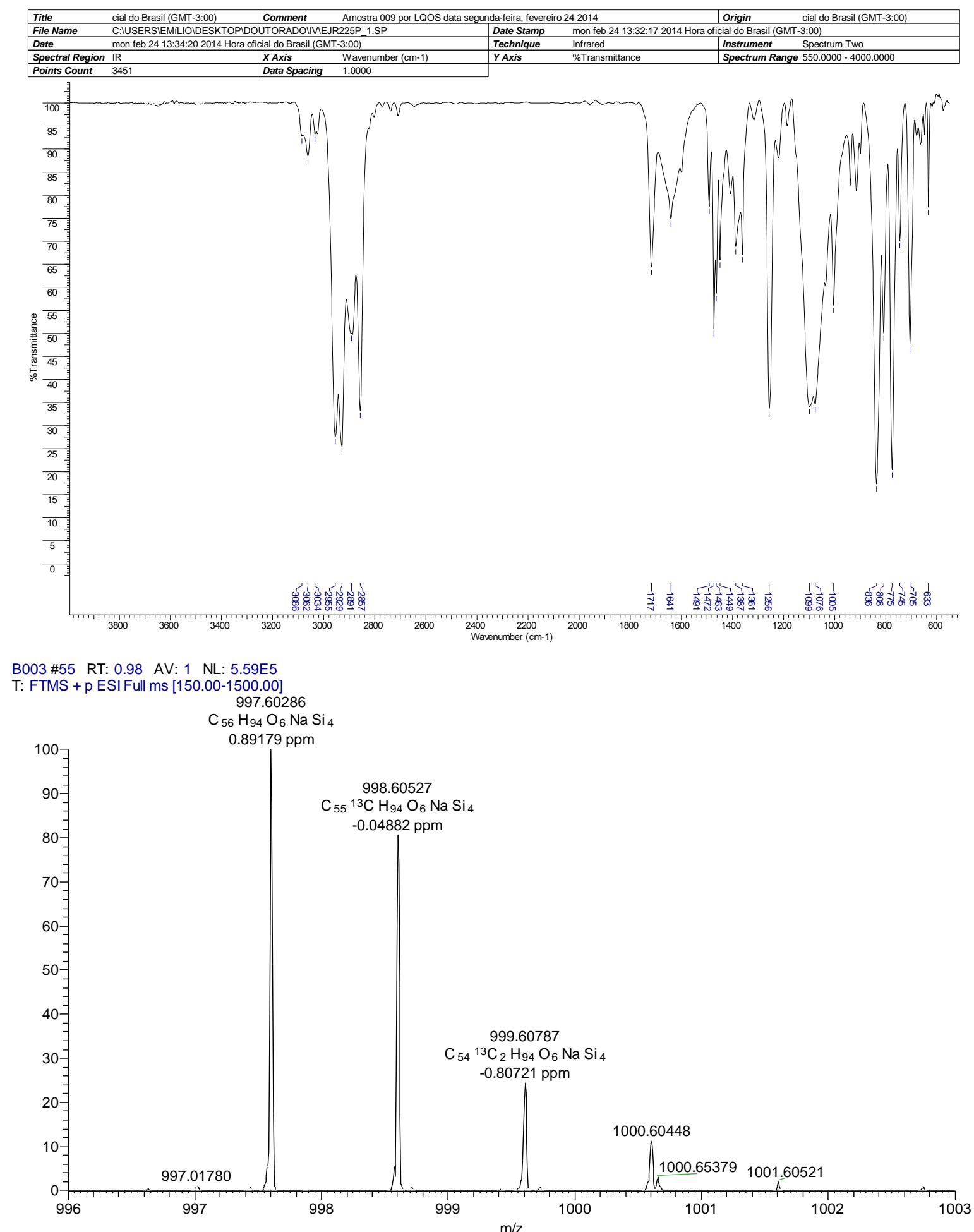

Figure 114: IR and HRMS (ESI FT-ICR-MS) spectra of compound S33. 


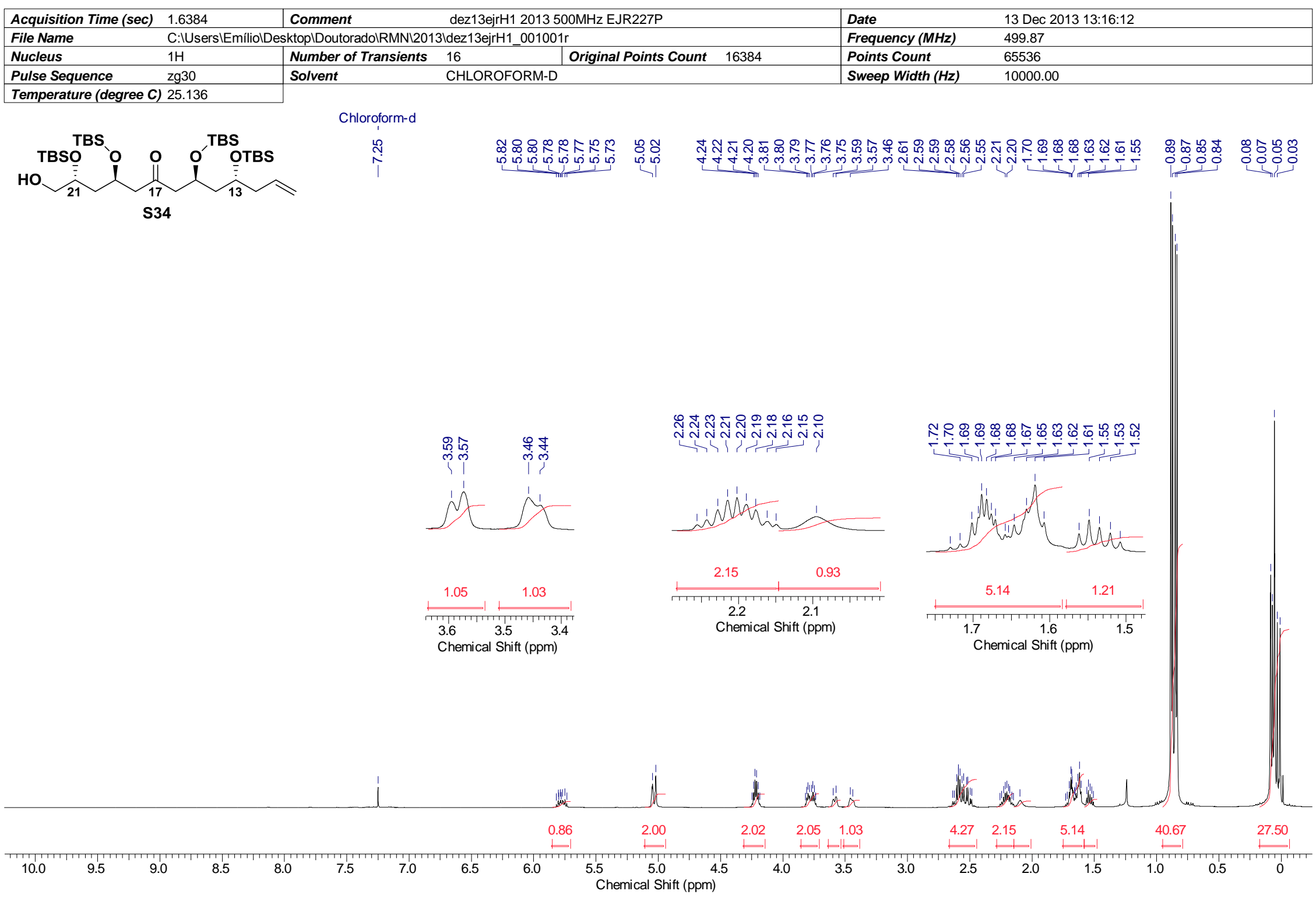

Figure 115: ${ }^{1} \mathrm{H}$ NMR spectrum of compound $\mathbf{S} 34\left(500 \mathrm{MHz}, \mathrm{CDCl}_{3}\right)$. 


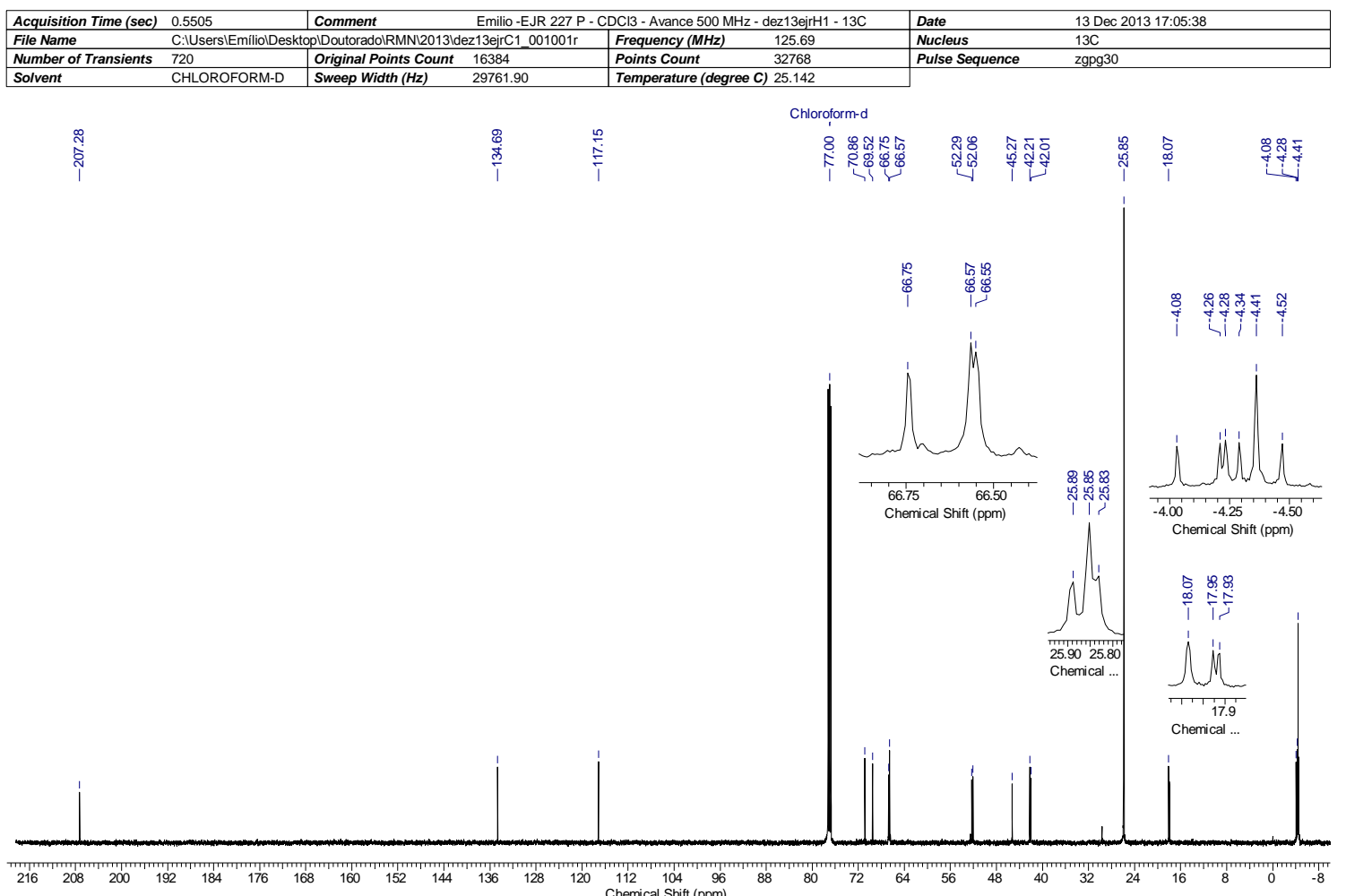

Figure 116: ${ }^{13} \mathrm{C}$ NMR spectrum of compound $\mathbf{S} 34\left(125 \mathrm{MHz}, \mathrm{CDCl}_{3}\right)$.

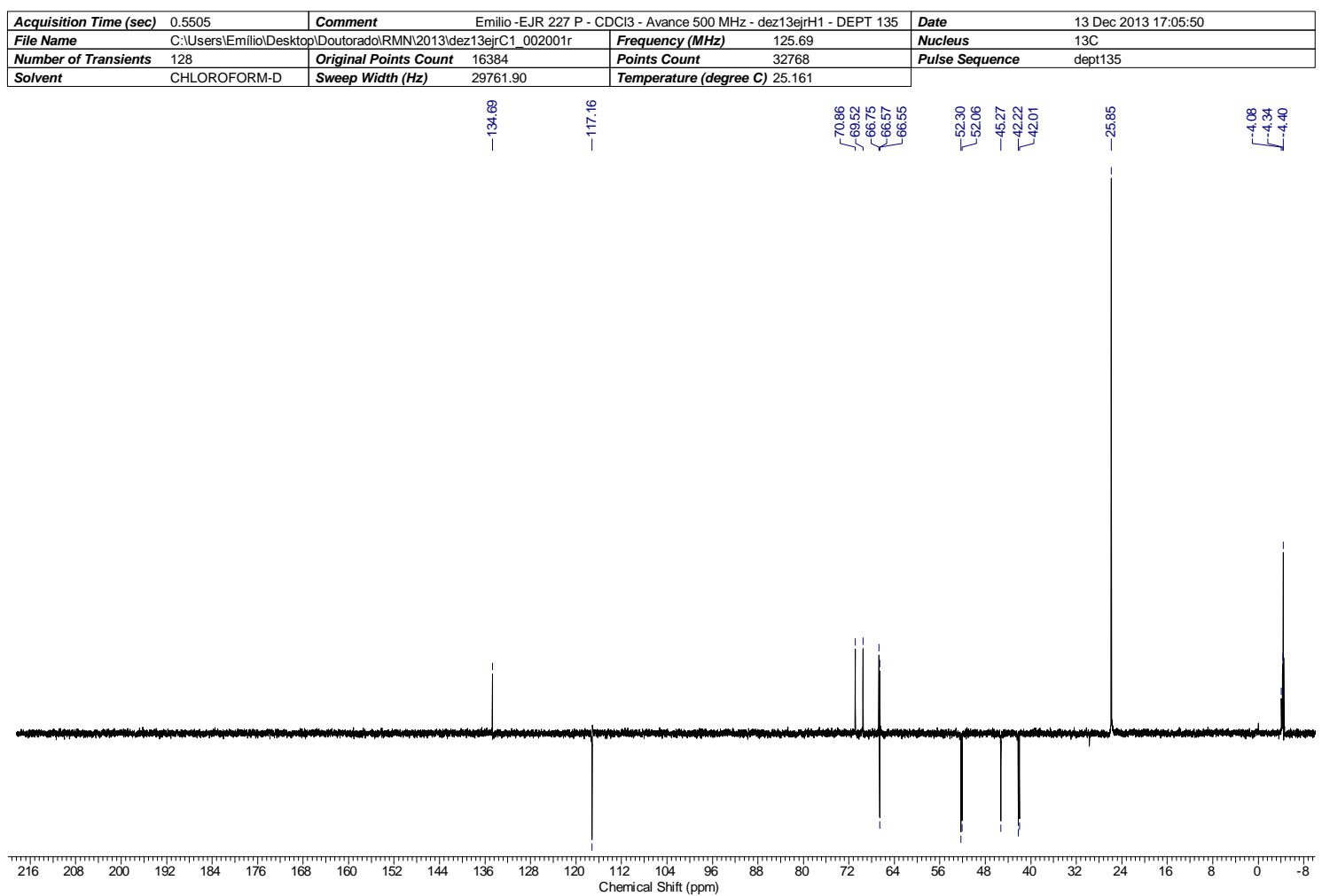

Figure 117: ${ }^{13} \mathrm{C}$ NMR (dept 135) spectrum of compound $\mathbf{S} 34\left(125 \mathrm{MHz}, \mathrm{CDCl}_{3}\right)$. 


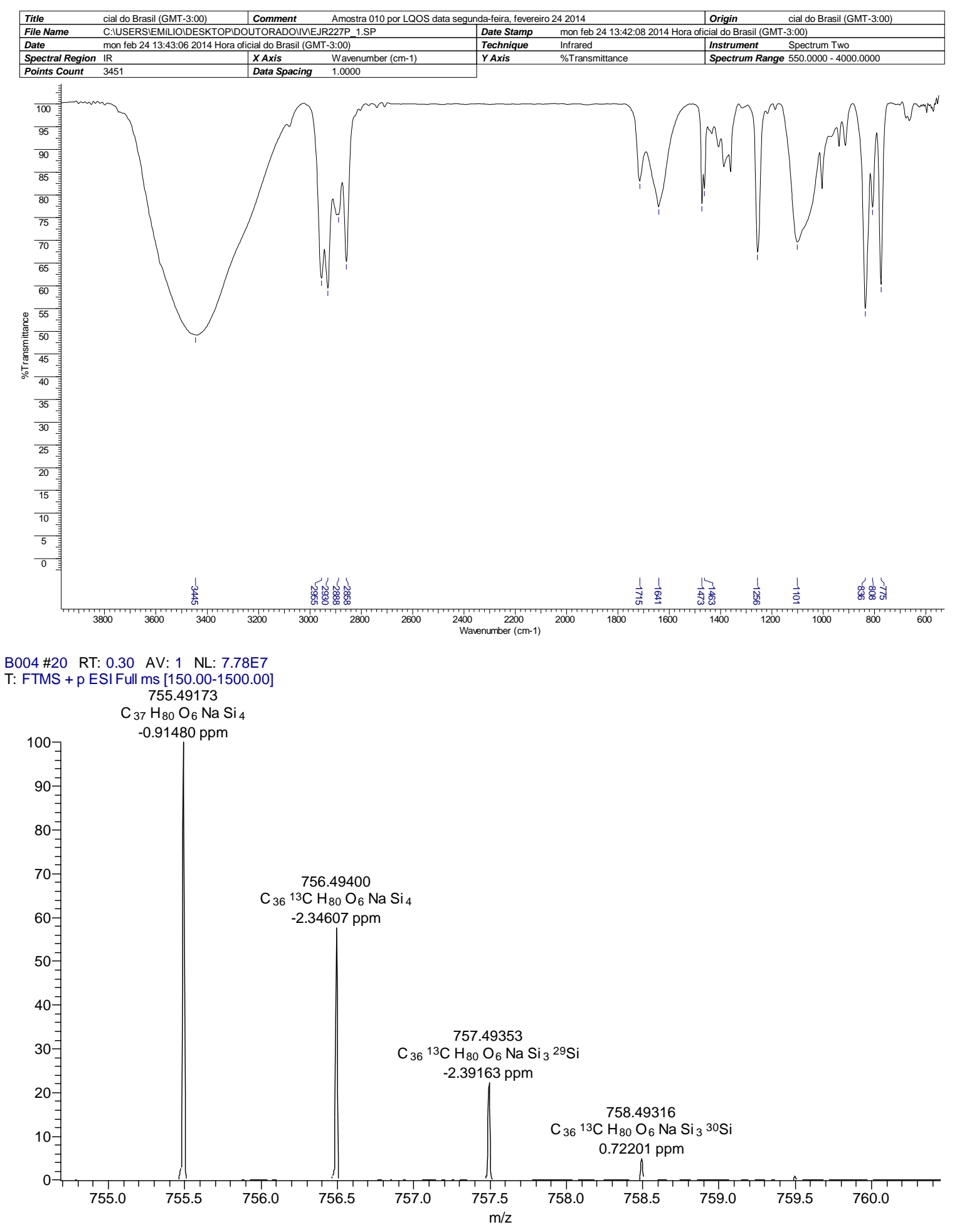

Figure 118: IR and HRMS (ESI FT-ICR-MS) spectra of compound S34. 


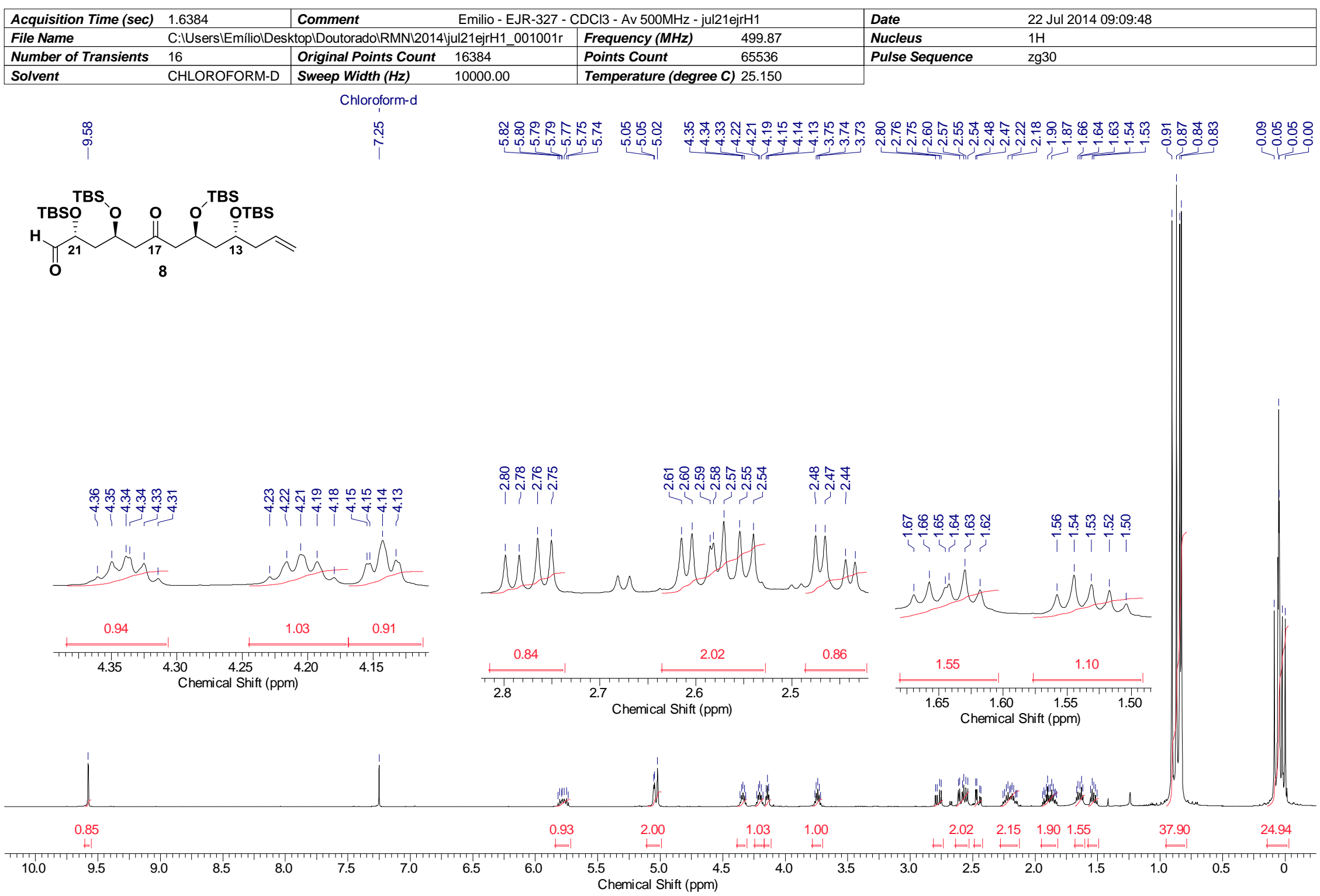

Figure 119: ${ }^{1} \mathrm{H}$ NMR spectrum of compound $8\left(500 \mathrm{MHz}, \mathrm{CDCl}_{3}\right)$. 


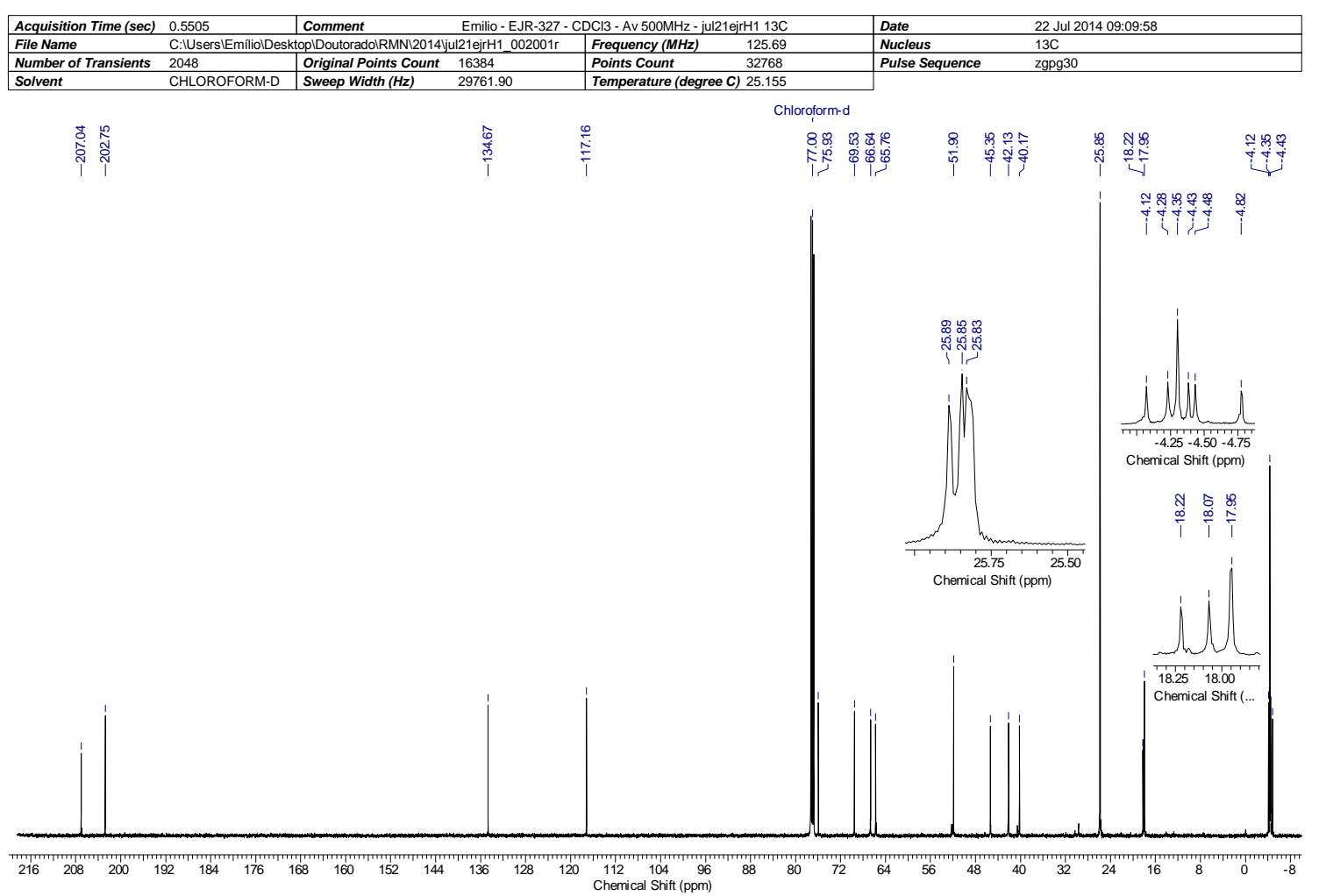

Figure 120: ${ }^{13} \mathrm{C}$ NMR spectrum of compound $8\left(125 \mathrm{MHz}, \mathrm{CDCl}_{3}\right)$.

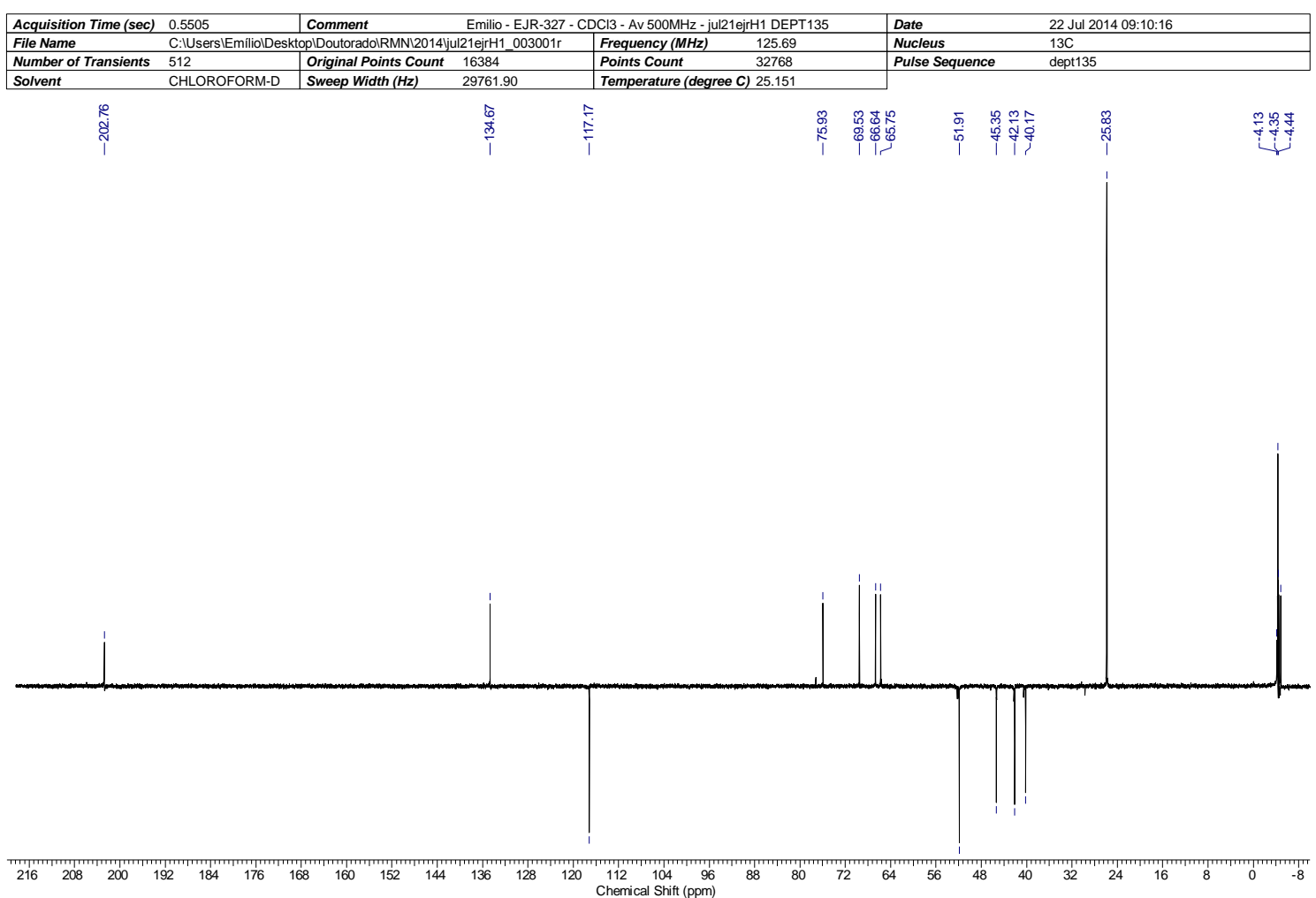

Figure 121: ${ }^{13} \mathrm{C}$ NMR (dept 135) spectrum of compound $8\left(125 \mathrm{MHz}, \mathrm{CDCl}_{3}\right)$. 


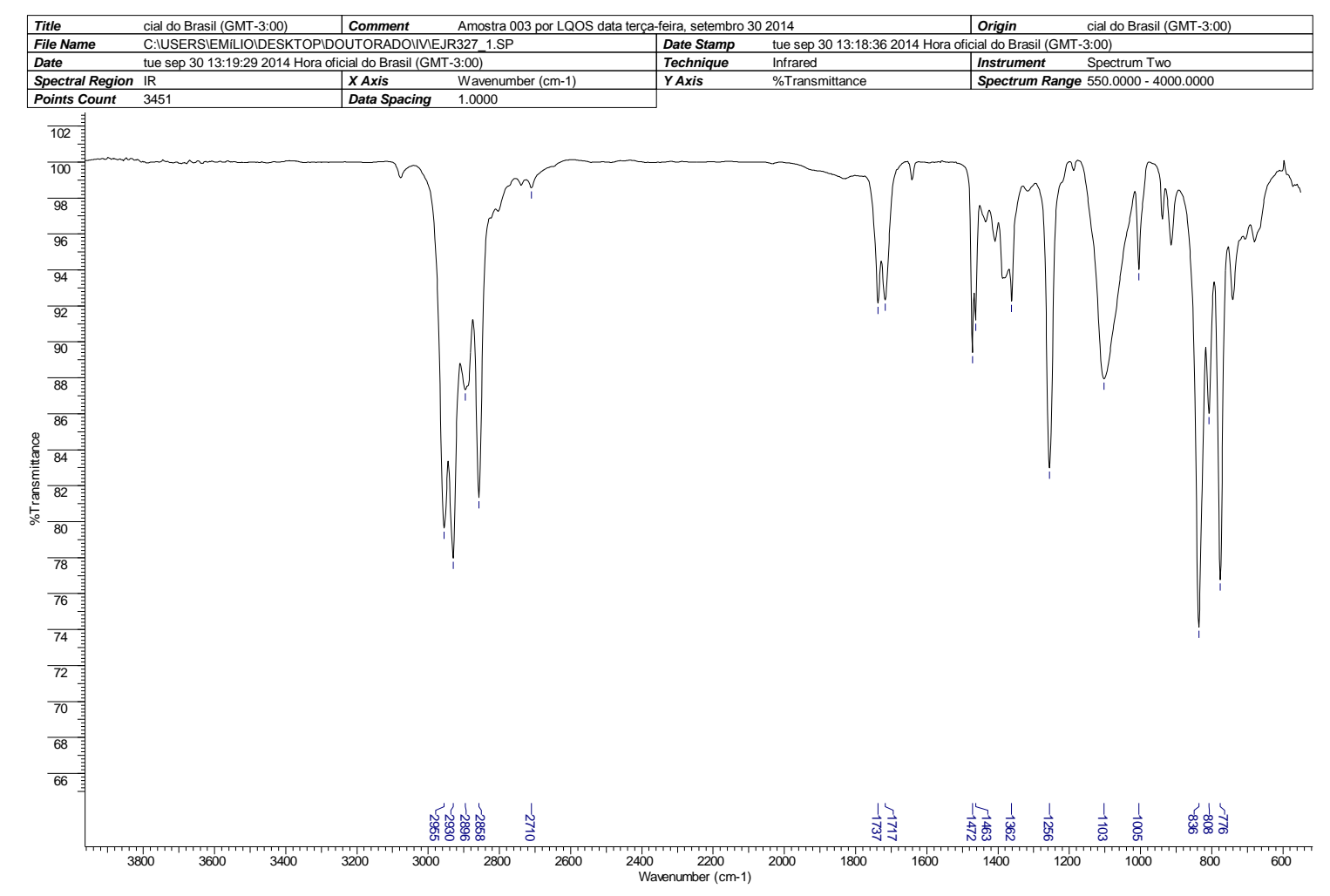

B005 \#30 RT: 0.45 AV: 1 NL: 7.89E5

T: FTMS + p ESIFull ms [150.00-1500.00]

753.47682

$\mathrm{C}_{37} \mathrm{H}_{78} \mathrm{O}_{6} \mathrm{Na} \mathrm{Si}_{4}$

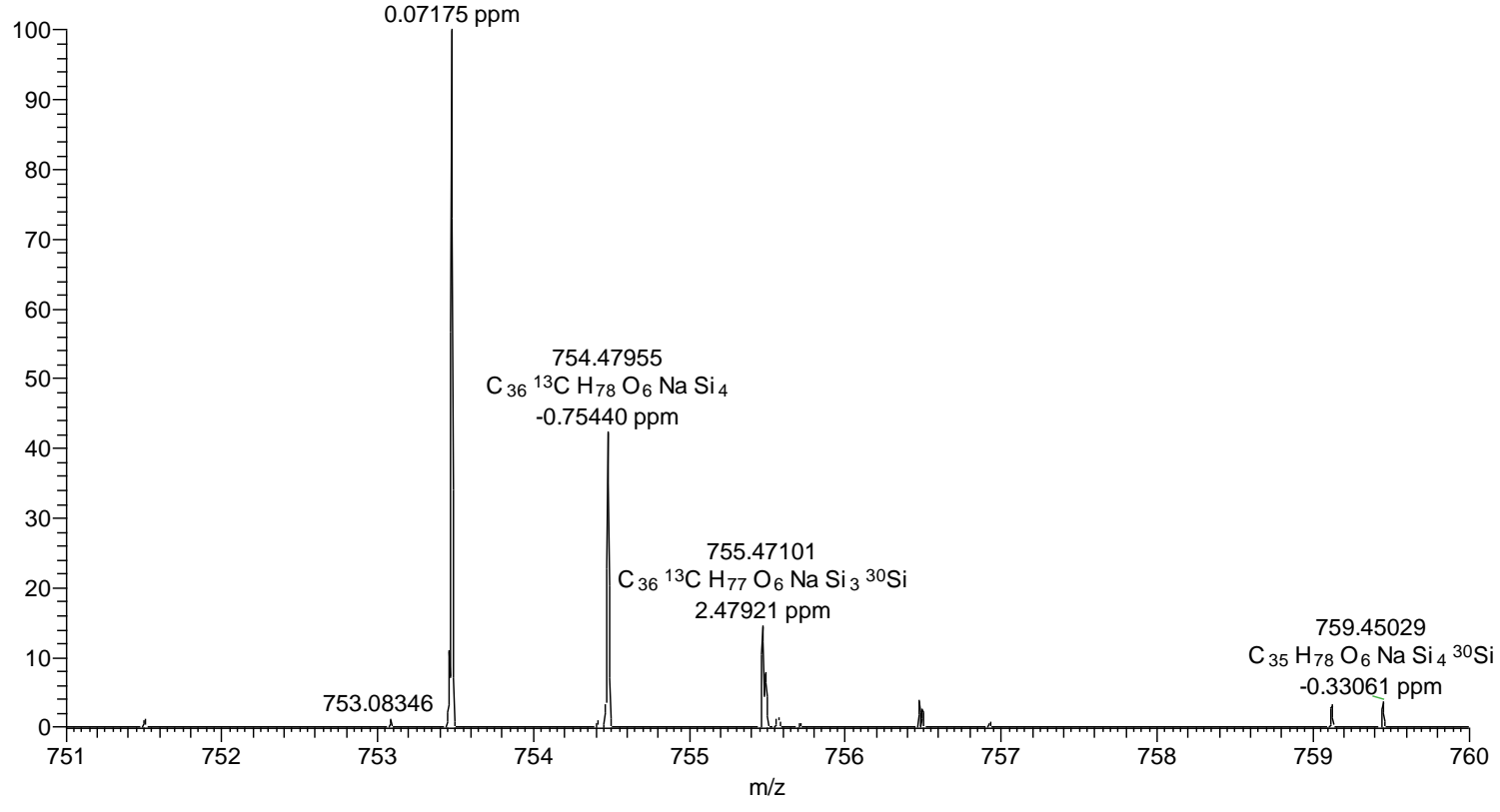

Figure 122: IR and HRMS (ESI FT-ICR-MS) spectra of compound 8. 


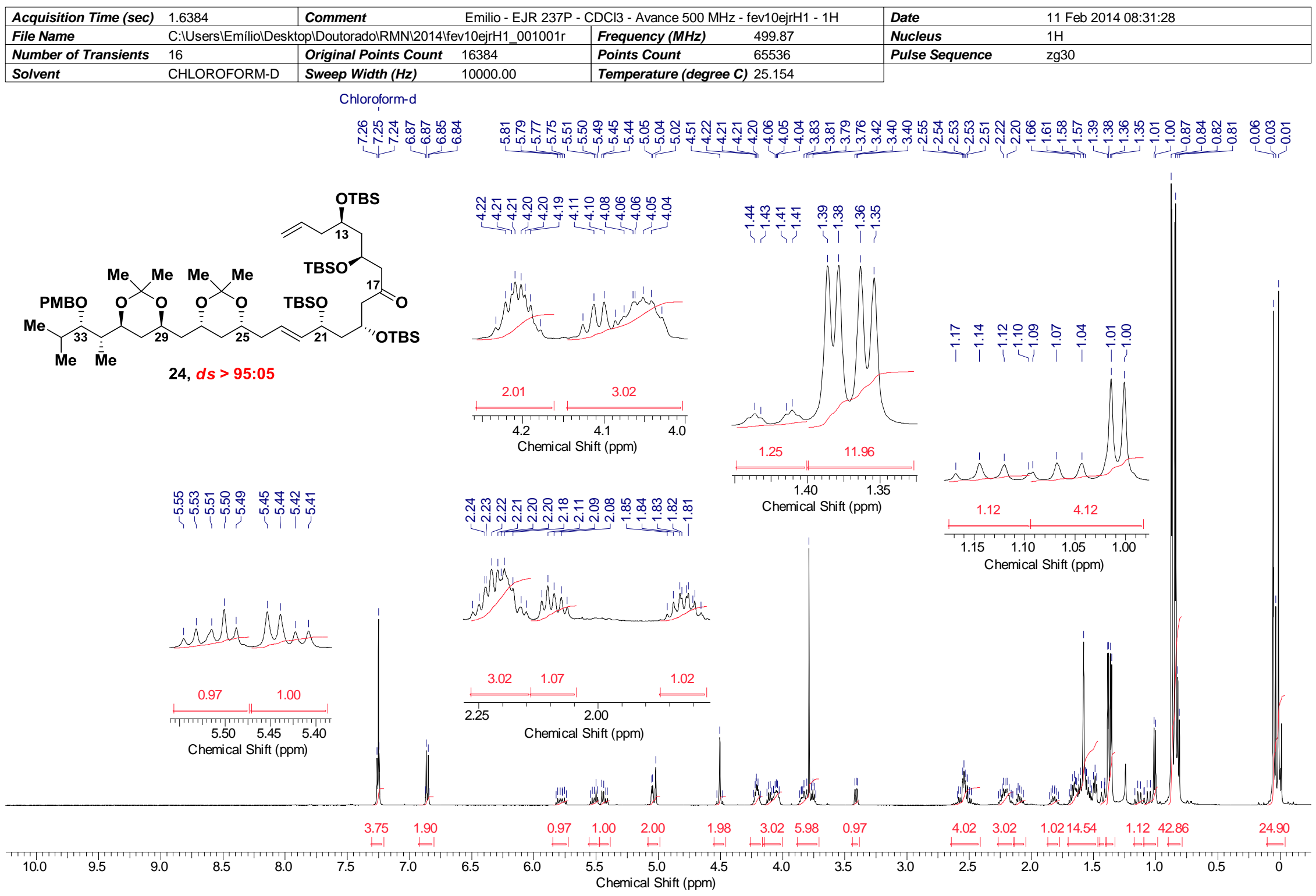

Figure 123: ${ }^{1} \mathrm{H}$ NMR spectrum of compound $24\left(500 \mathrm{MHz}, \mathrm{CDCl}_{3}\right)$. 


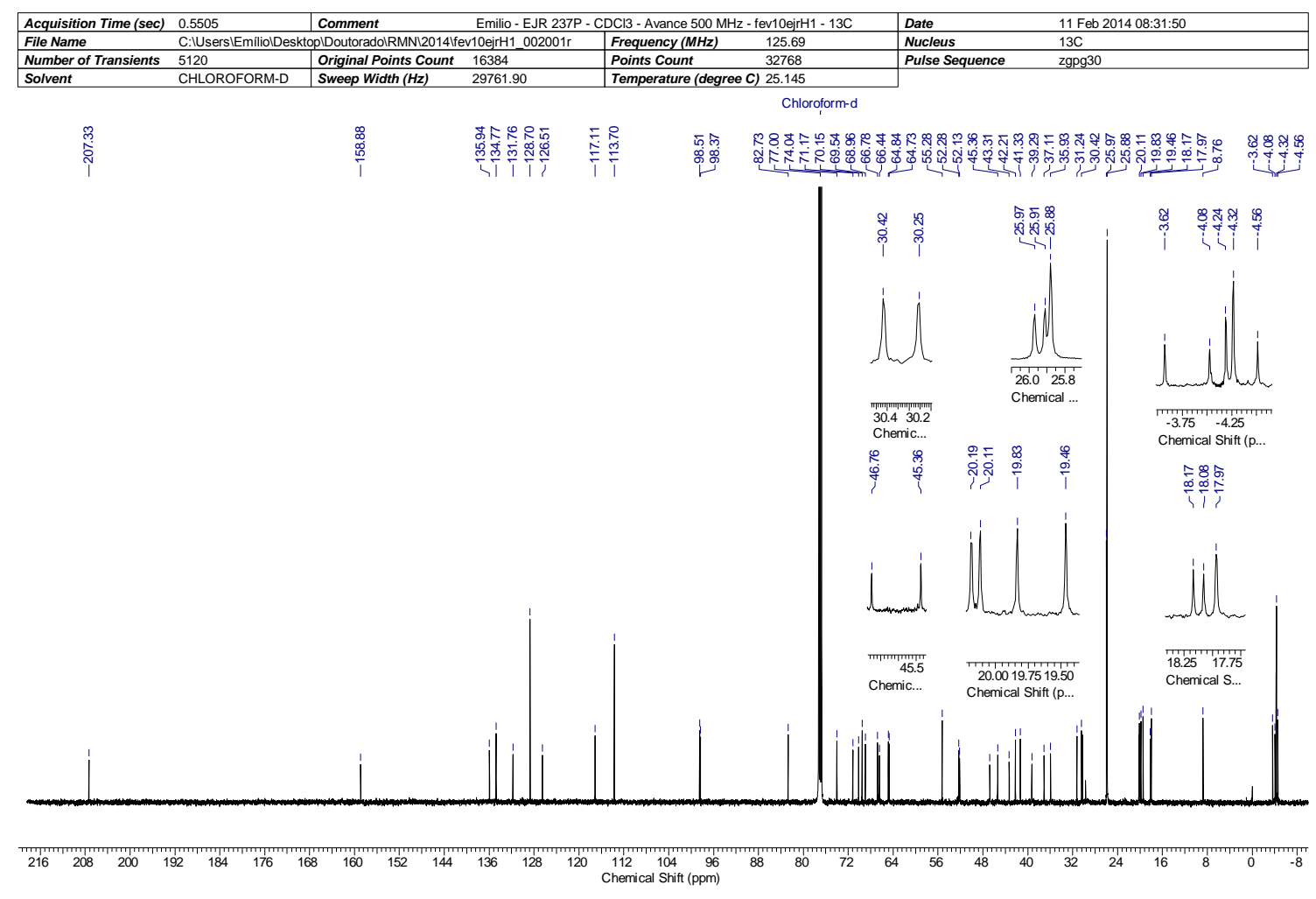

Figure 124: ${ }^{13} \mathrm{C}$ NMR spectrum of compound $24\left(125 \mathrm{MHz}, \mathrm{CDCl}_{3}\right)$.

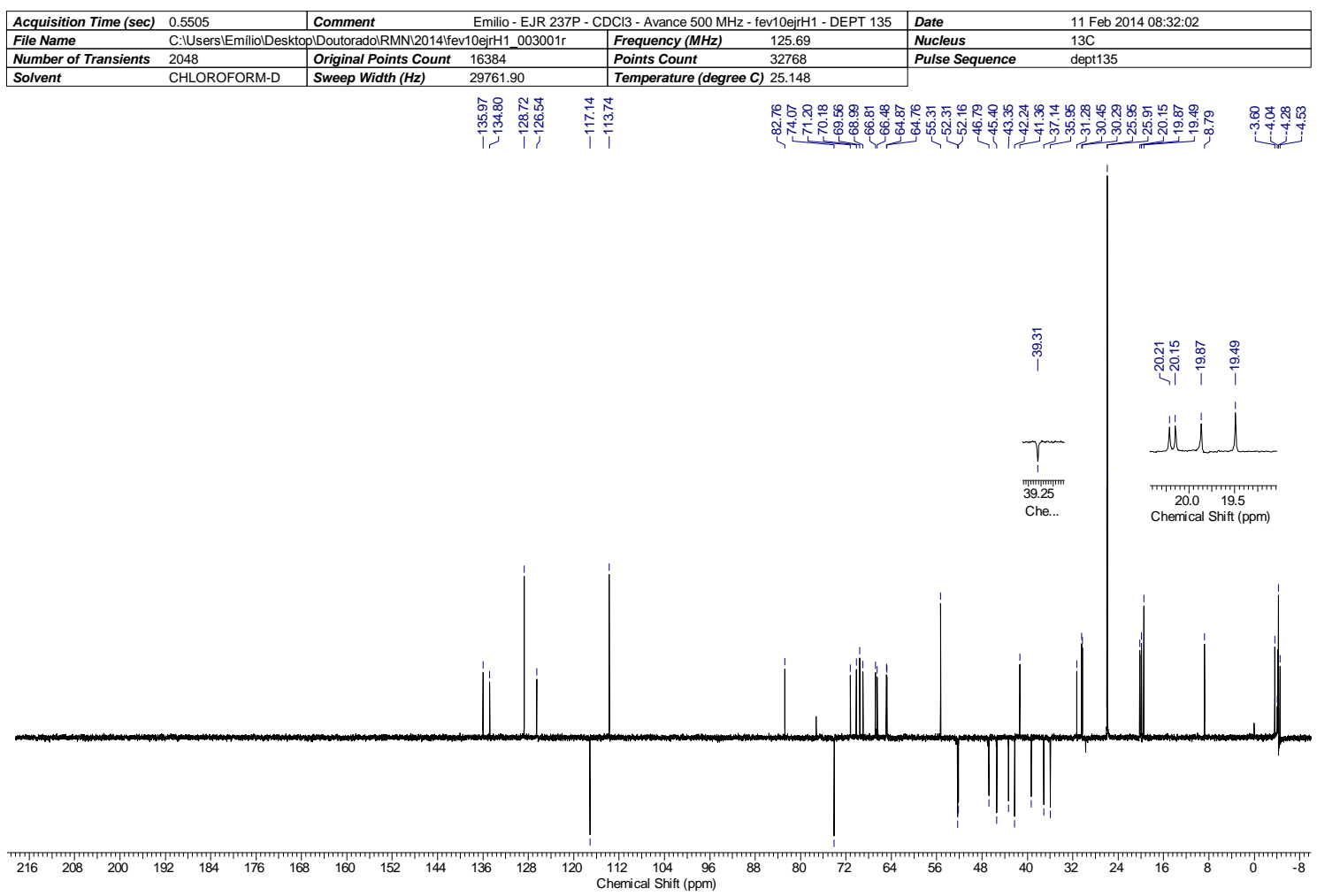

Figure 125: ${ }^{13} \mathrm{C}$ NMR (dept 135) spectrum of compound $24\left(125 \mathrm{MHz}, \mathrm{CDCl}_{3}\right)$. 


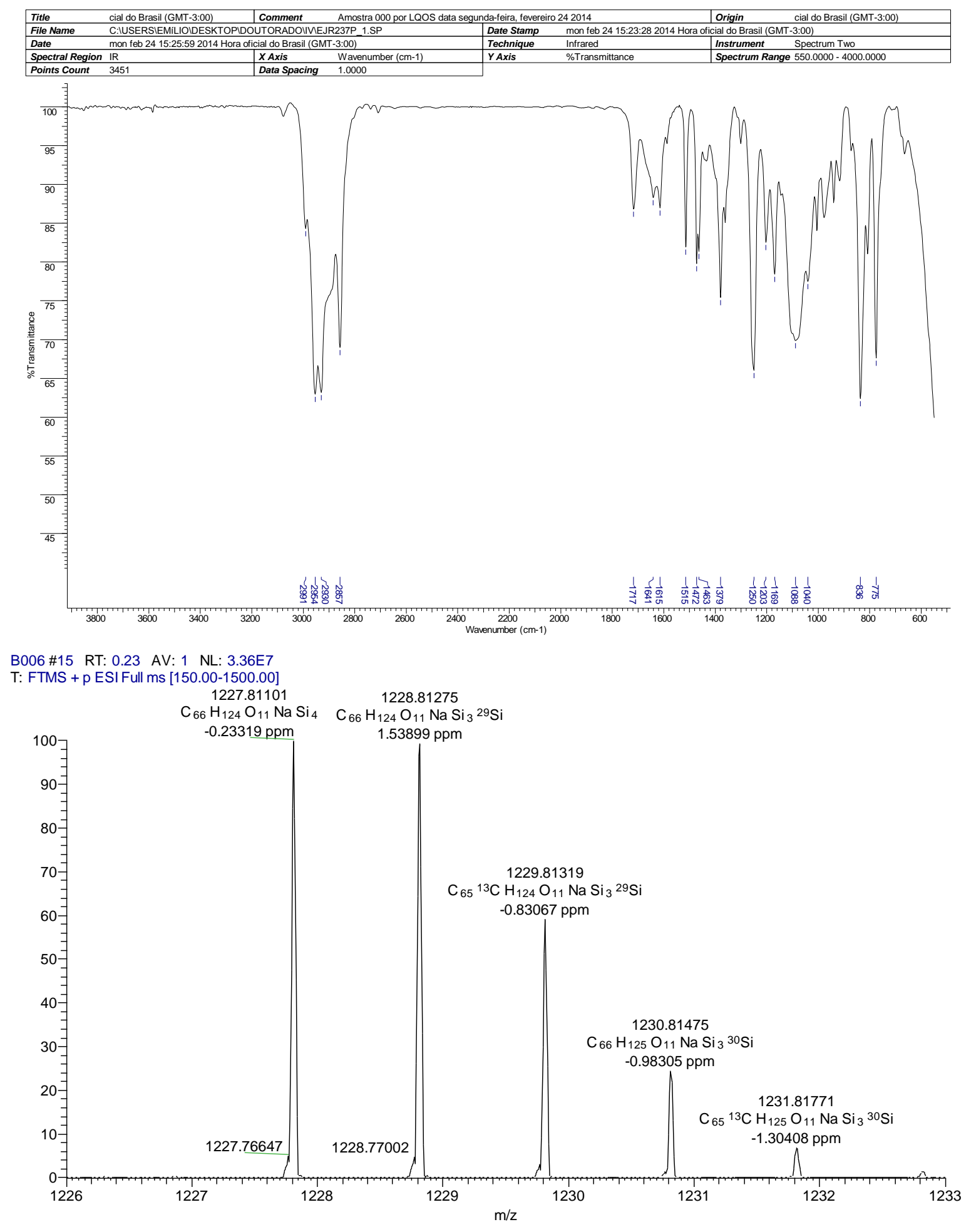

Figure 126: IR and HRMS (ESI FT-ICR-MS) spectra of compound 24. 


\begin{tabular}{|c|c|c|c|c|c|c|}
\hline \multirow{3}{*}{$\begin{array}{l}\text { Acquisition Time (sec) } \\
\text { File Name } \\
\text { Nucleus }\end{array}$} & \multicolumn{4}{|c|}{14 Mar 2014 16:56:56 } & \multirow{2}{*}{\multicolumn{2}{|c|}{$\begin{array}{ll}\text { Frequency }(\mathrm{MHz}) & 499.87\end{array}$}} \\
\hline & C:IUser & esktop/DoutoradoIRMNI2 & $114 \mathrm{In}$ & $1001 \mathrm{r}$ & & \\
\hline & $1 \mathrm{H}$ & Number of Transients & 16 & Original Points Count 16384 & Points Count & 65536 \\
\hline Pulse Sequence & $\mathrm{zg} 30$ & Solvent & & & Sweep Width $(\mathrm{Hz})$ & 10302.20 \\
\hline
\end{tabular}

Temperature (degree $C$ ) 25.15

Chloroform-d

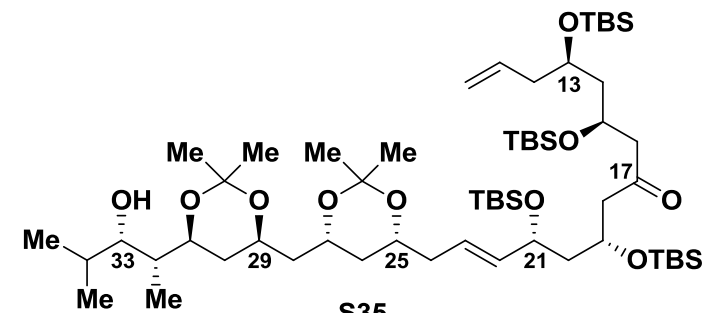

S35
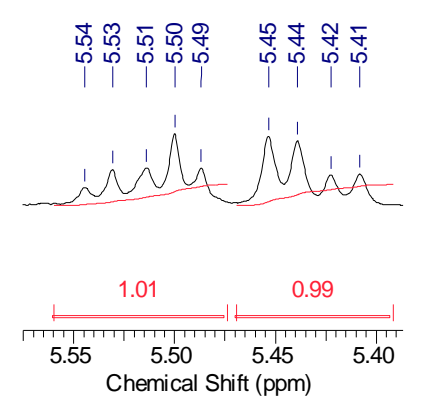

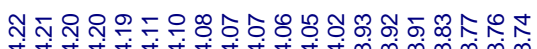

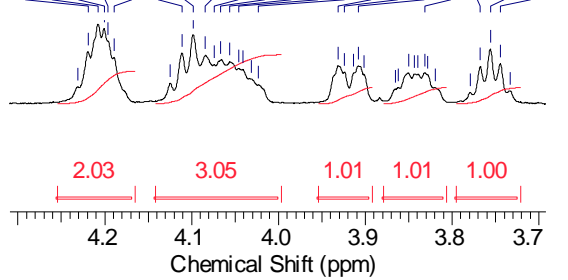

Chemical Shift (ppm)

$\begin{array}{ccc}5.55 & 5.50 & 5.45 \\ \text { Chemical Shift (ppm) }\end{array}$

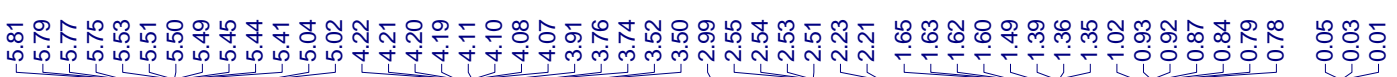
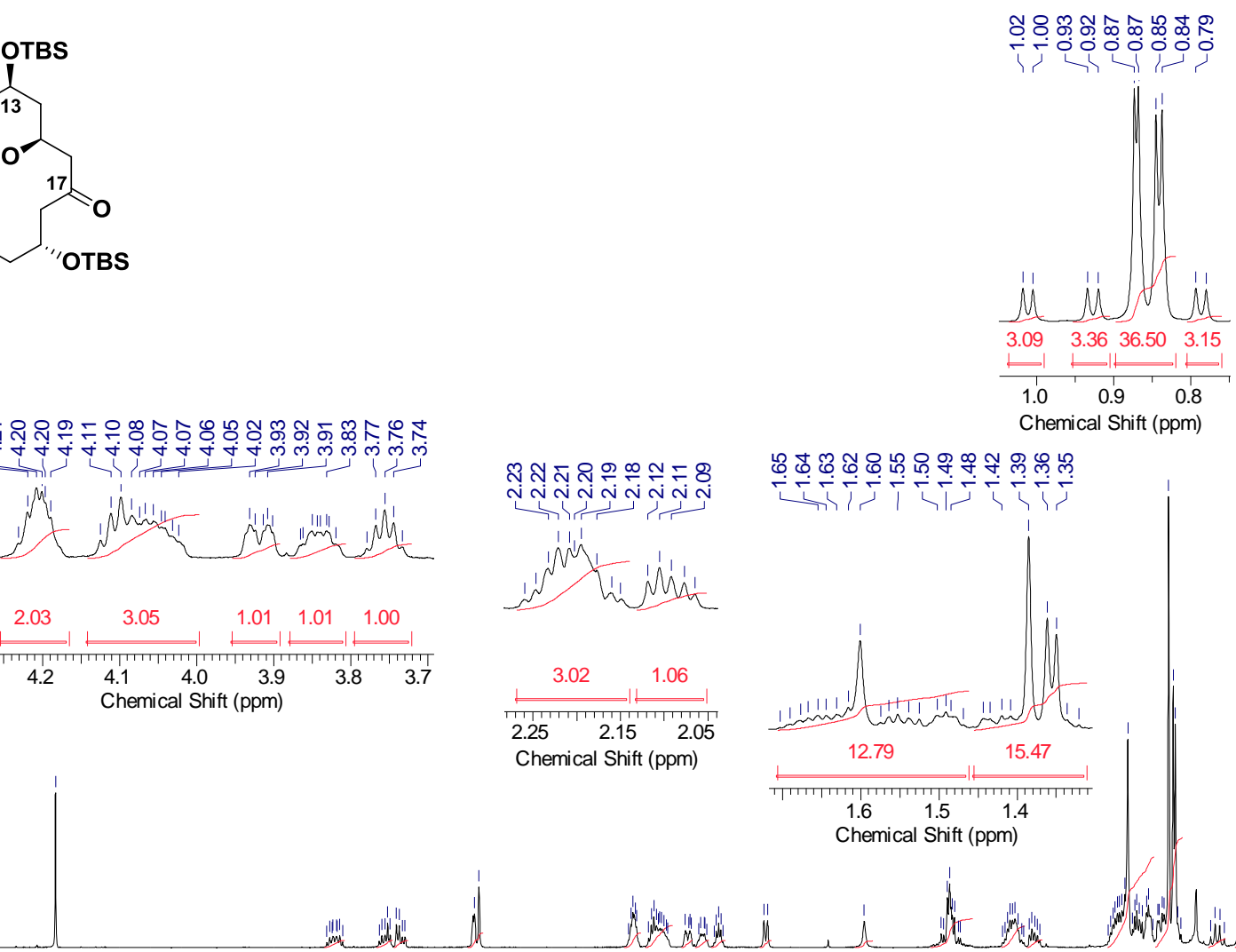

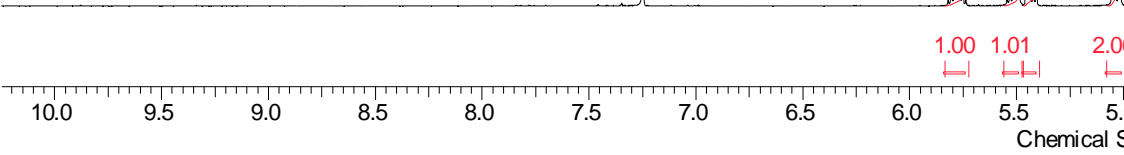

Figure 127: ${ }^{1} \mathrm{H}$ NMR spectrum of compound $\mathbf{S} 35\left(500 \mathrm{MHz}, \mathrm{CDCl}_{3}\right)$. 


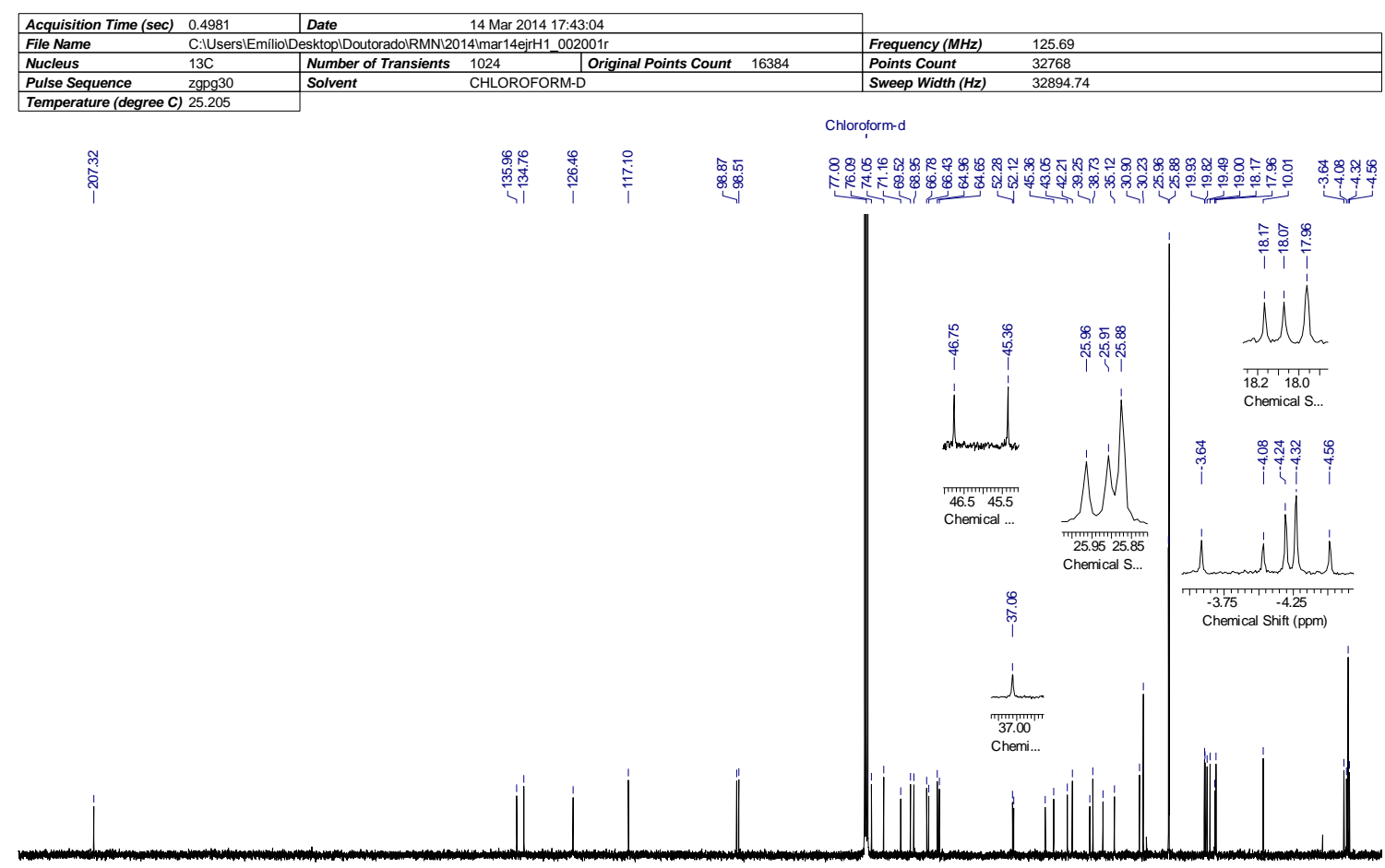

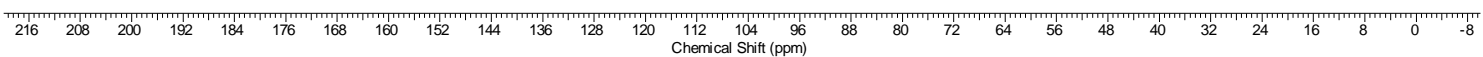

Figure 128: ${ }^{13} \mathrm{C}$ NMR spectrum of compound $\mathbf{S} 35\left(125 \mathrm{MHz}, \mathrm{CDCl}_{3}\right)$.

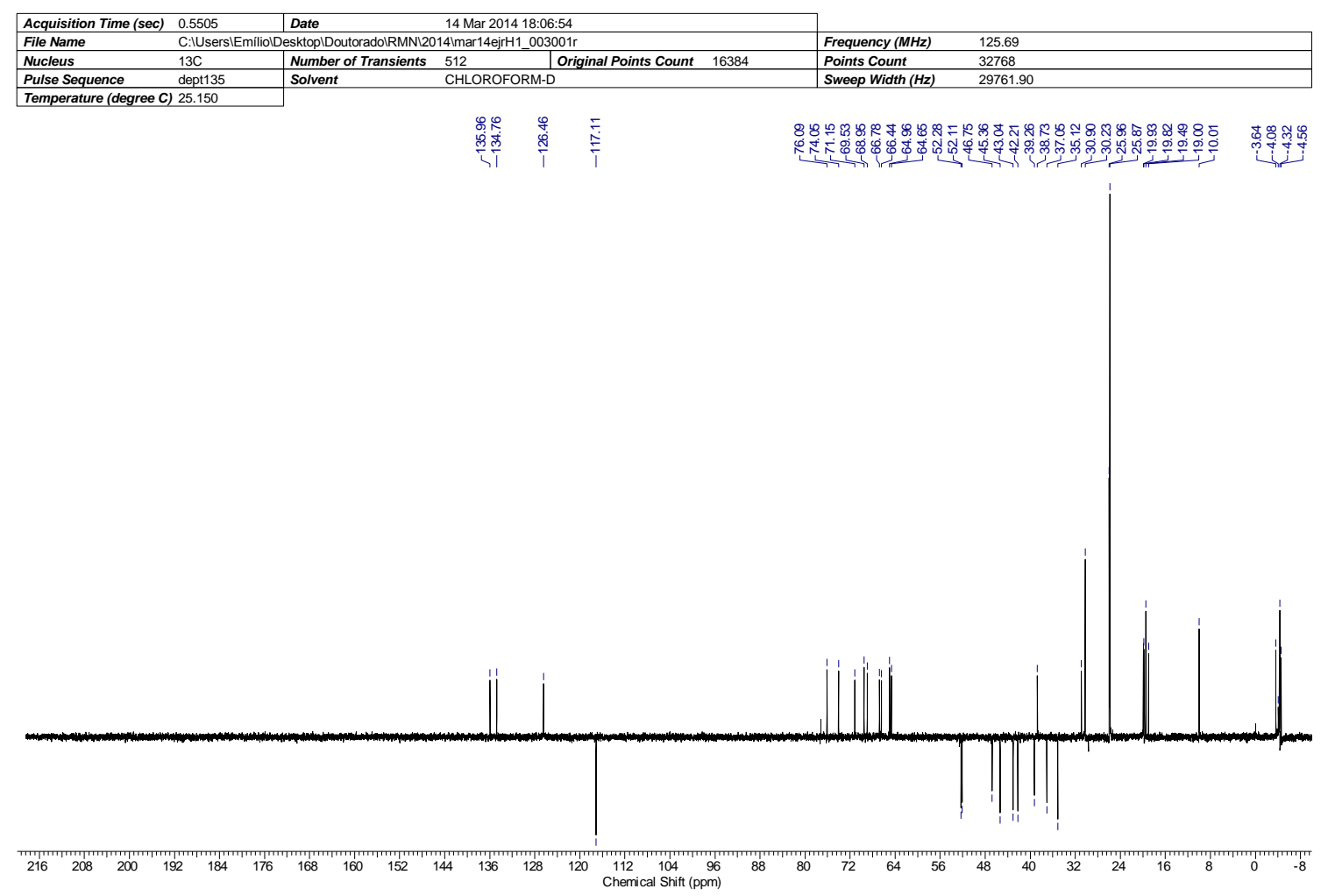

Figure 129: ${ }^{13} \mathrm{C}$ NMR (dept 135) spectrum of compound $\mathbf{S} 35\left(125 \mathrm{MHz}, \mathrm{CDCl}_{3}\right)$. 


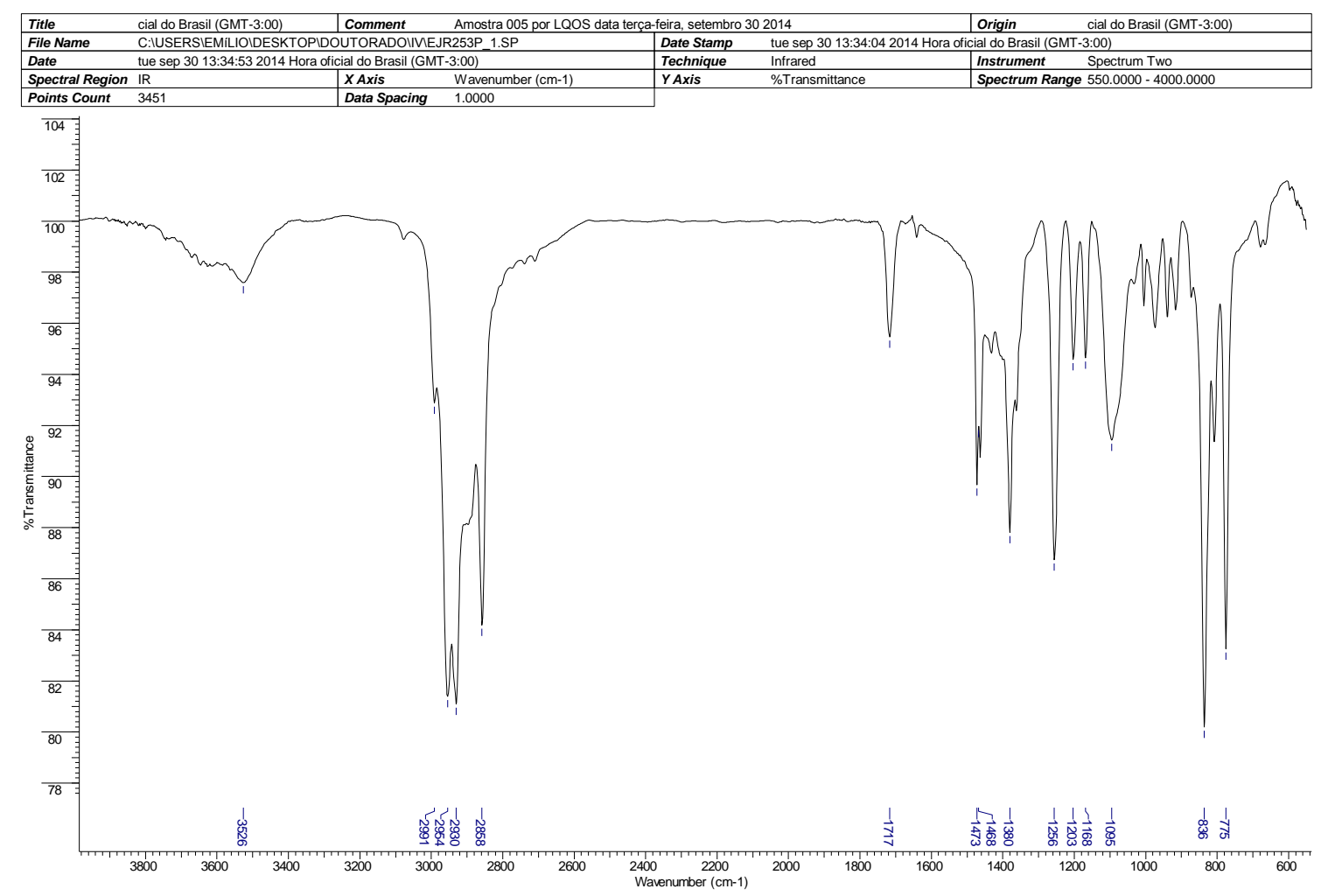

B007 \#22 RT: 0.35 AV: 1 NL: 8.61E6

T: FTMS + p ESI Full ms [150.00-1500.00]

1107.75339

$\mathrm{C}_{58} \mathrm{H}_{116} \mathrm{O}_{10} \mathrm{Na} \mathrm{Si}_{4}$

100 马 1108.75574

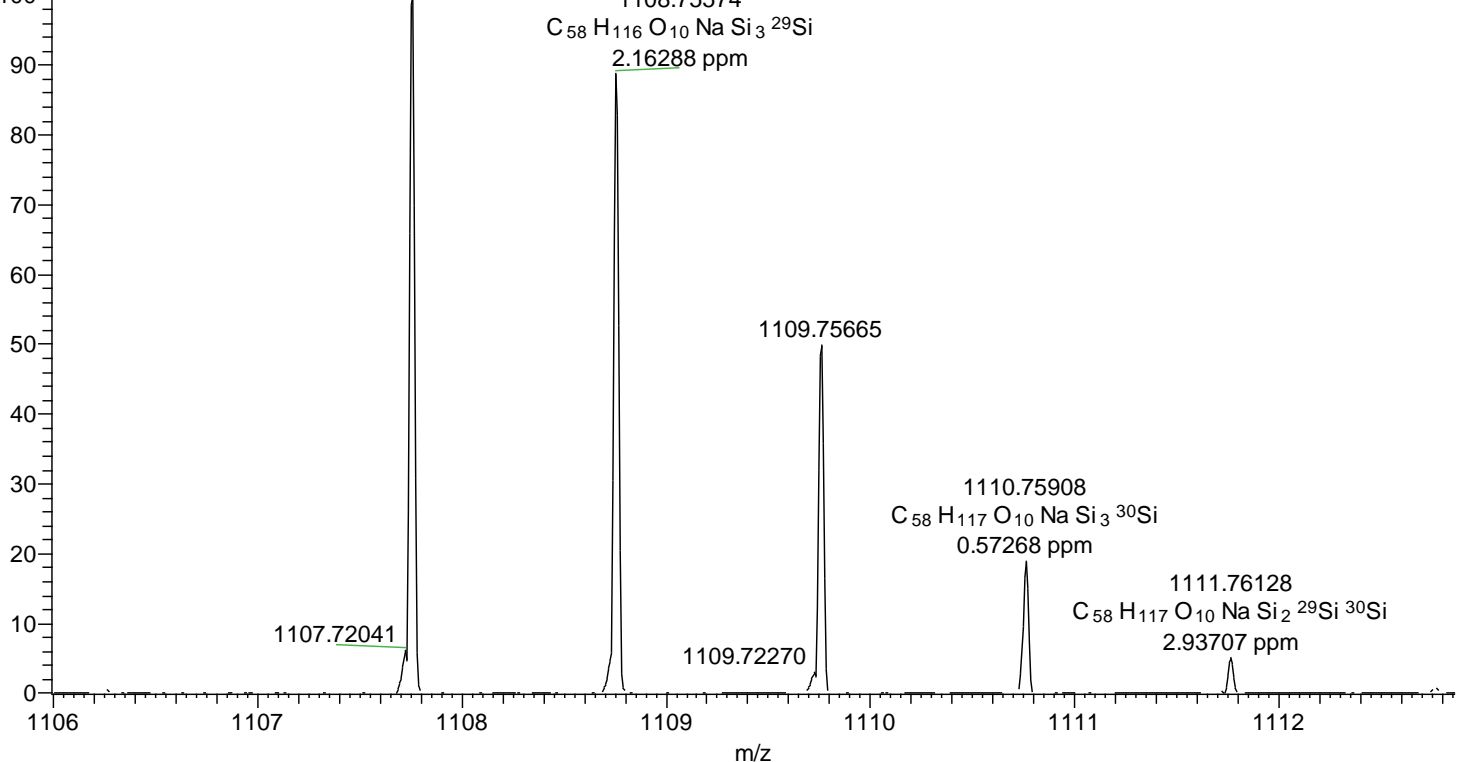

Figure 130: IR and HRMS (ESI FT-ICR-MS) spectra of compound S35. 


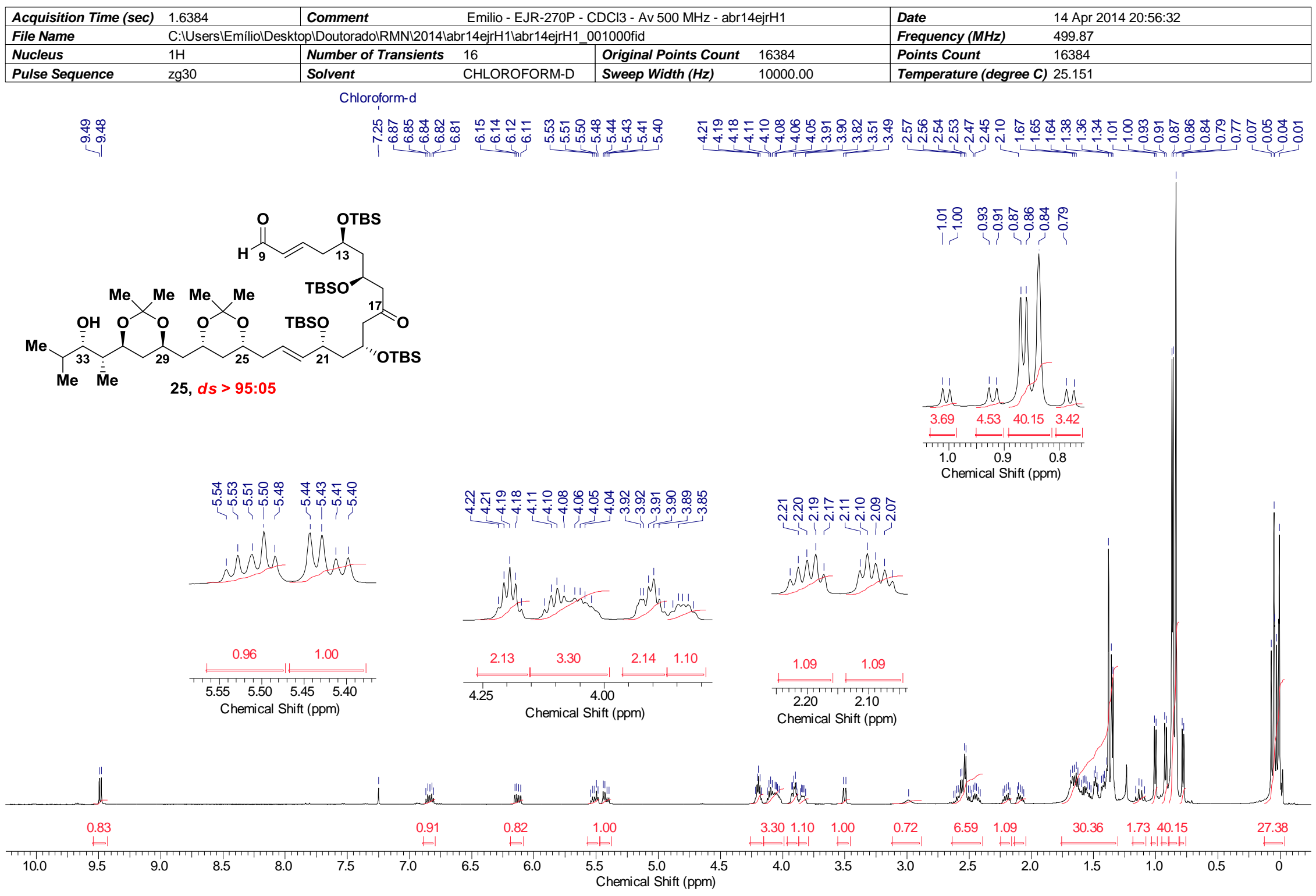

Figure 131: ${ }^{1} \mathrm{H}$ NMR spectrum of compound $25\left(500 \mathrm{MHz}, \mathrm{CDCl}_{3}\right)$. 


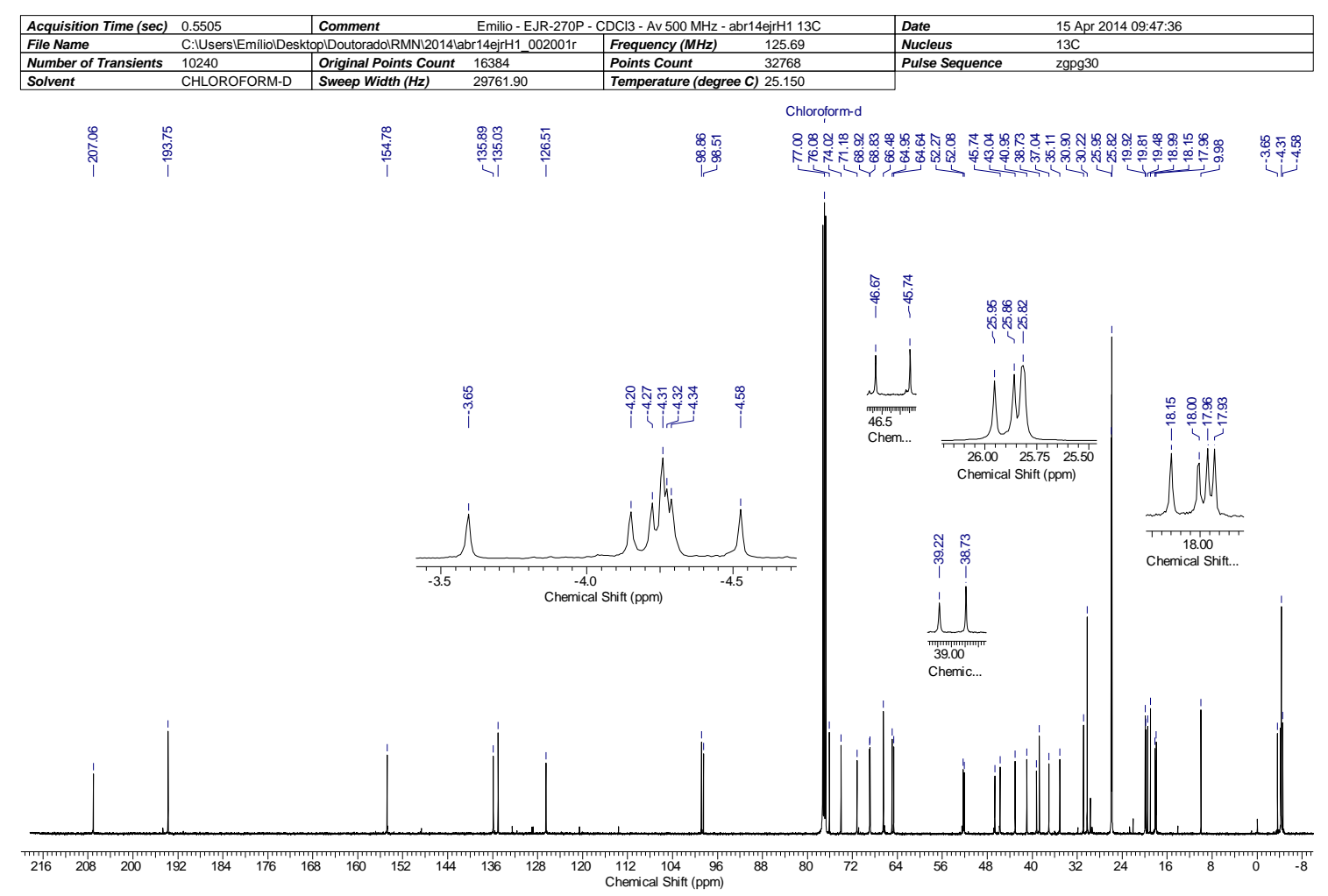

Figure 132: ${ }^{13} \mathrm{C}$ NMR spectrum of compound $25\left(125 \mathrm{MHz}, \mathrm{CDCl}_{3}\right)$.

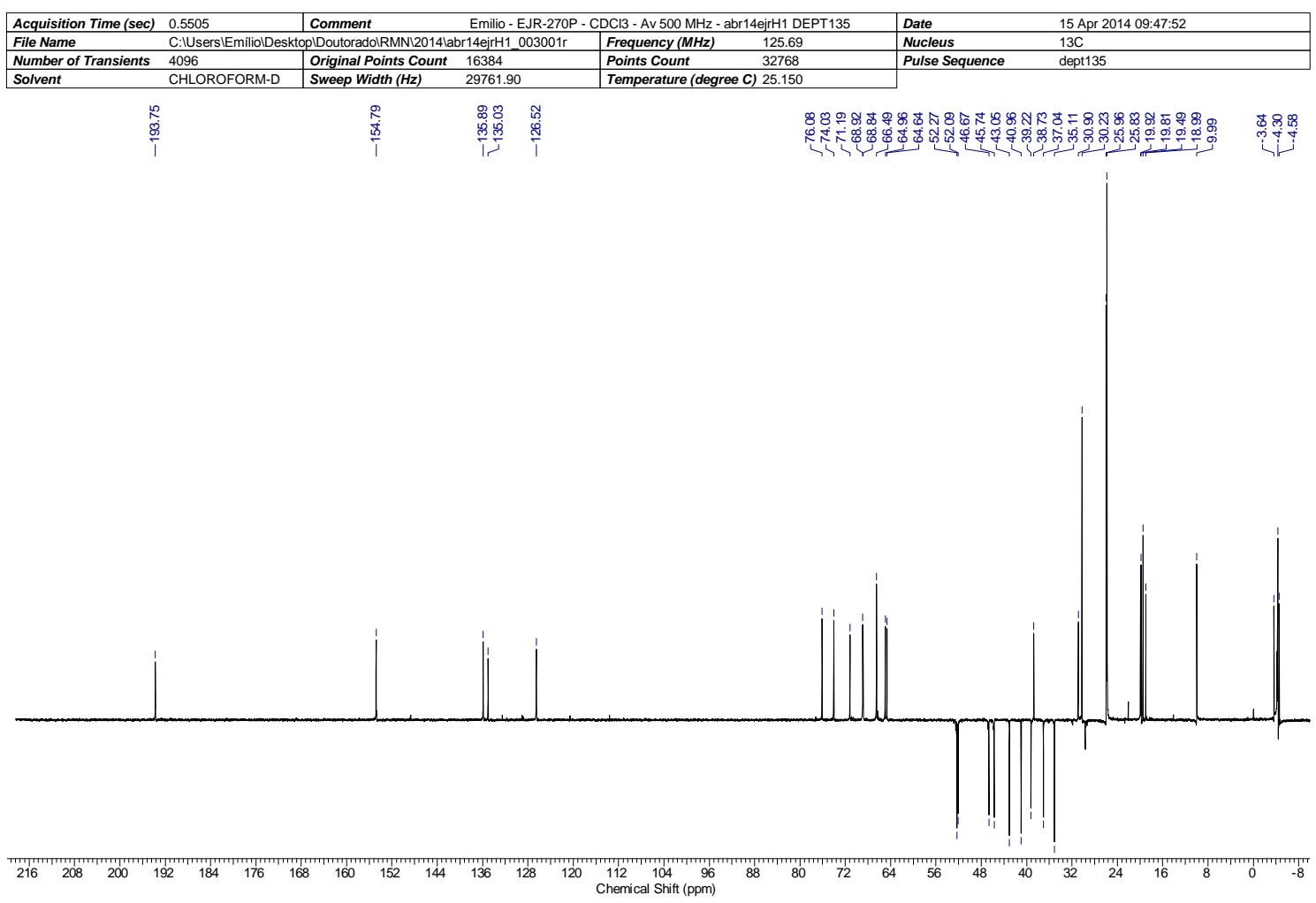

Figure 133: ${ }^{13} \mathrm{C}$ NMR (dept 135) spectrum of compound $25\left(125 \mathrm{MHz}, \mathrm{CDCl}_{3}\right)$. 


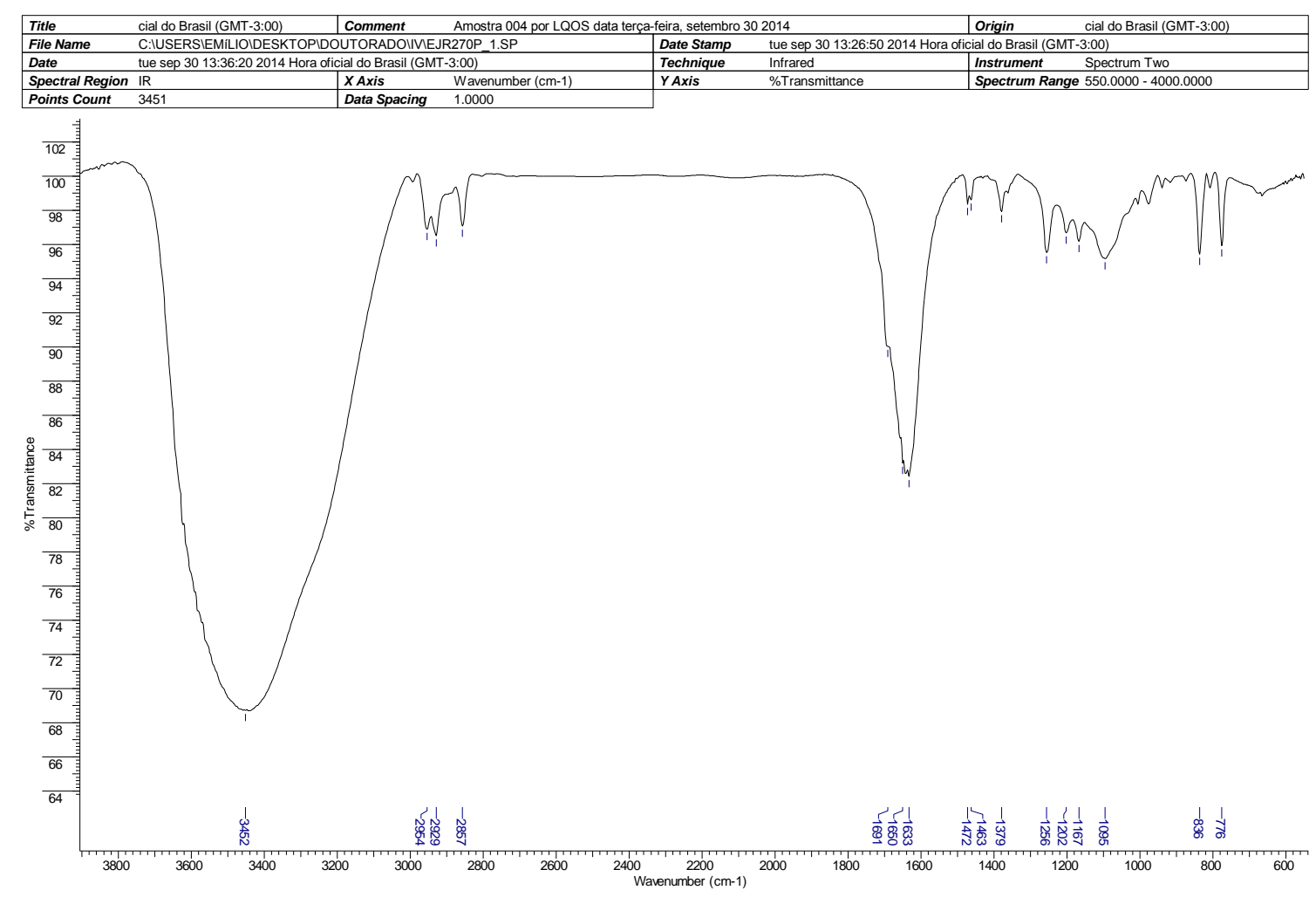

B008 \#21 RT: 0.32 AV: 1 NL: $4.29 E 6$

T: FTMS + p ESI Full ms [150.00-1500.00]

1135.74884

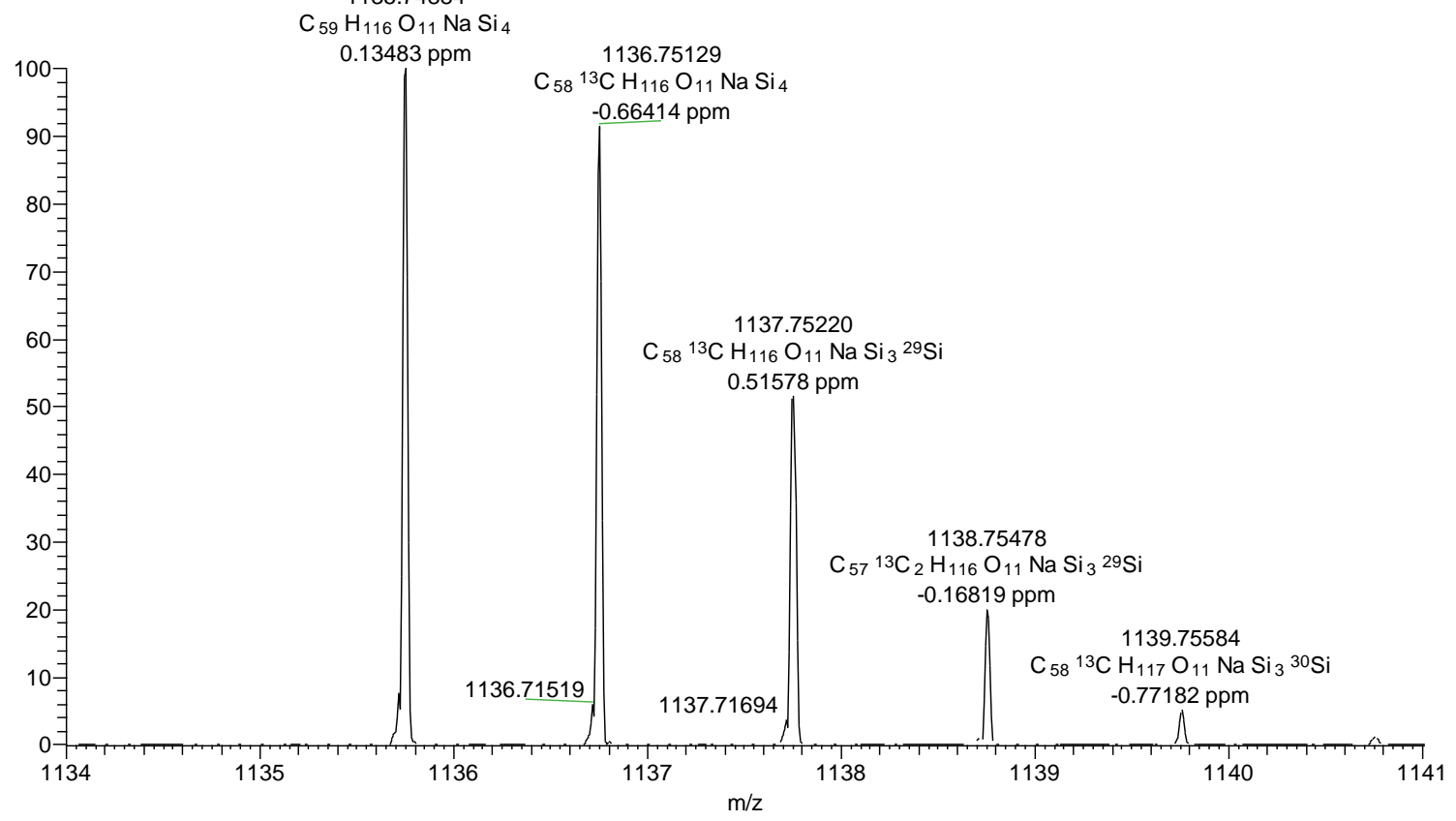

Figure 134: IR and HRMS (ESI FT-ICR-MS) spectra of compound 25. 


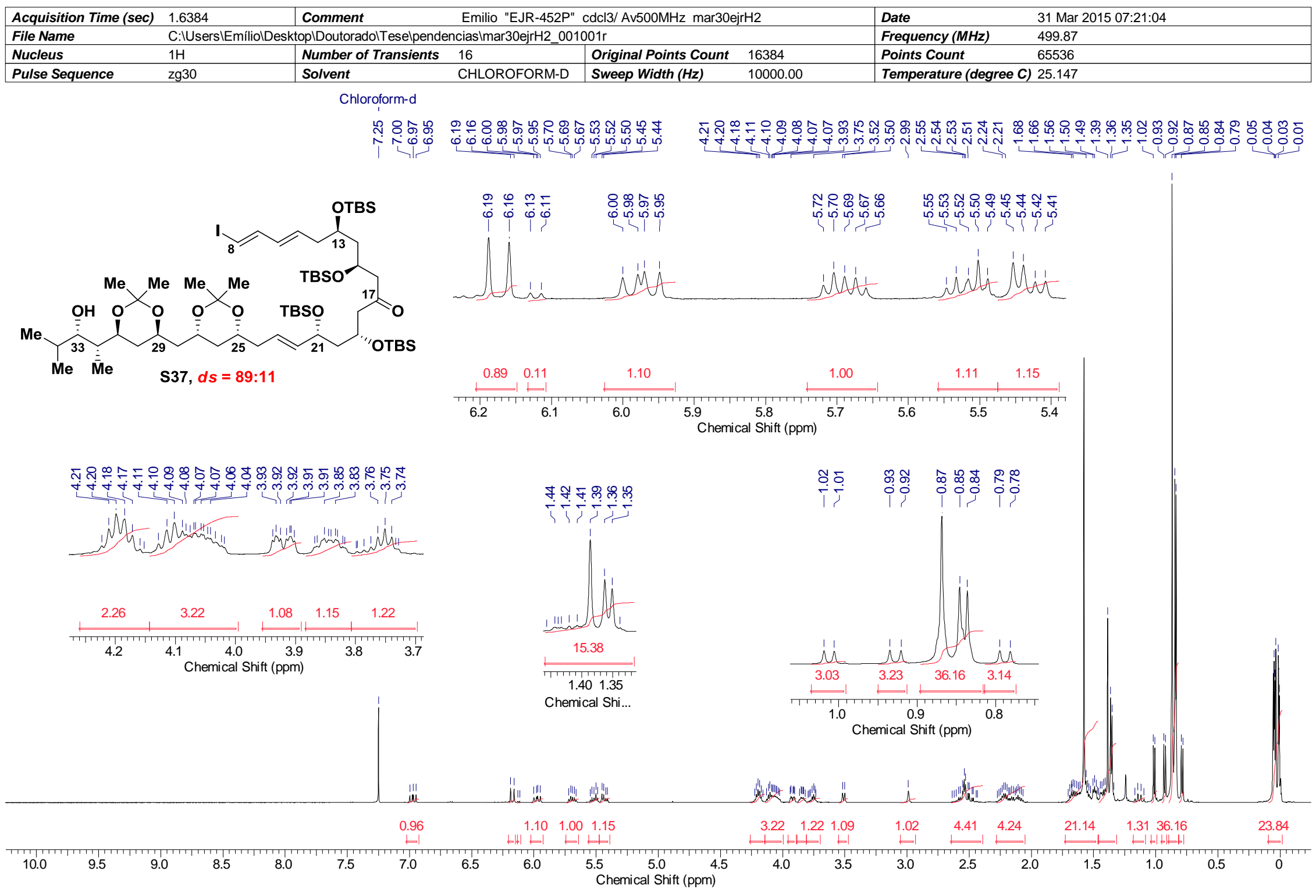

Figure 135: ${ }^{1} \mathrm{H}$ NMR spectrum of compound $\mathbf{S} 37\left(500 \mathrm{MHz}, \mathrm{CDCl}_{3}\right)$. 


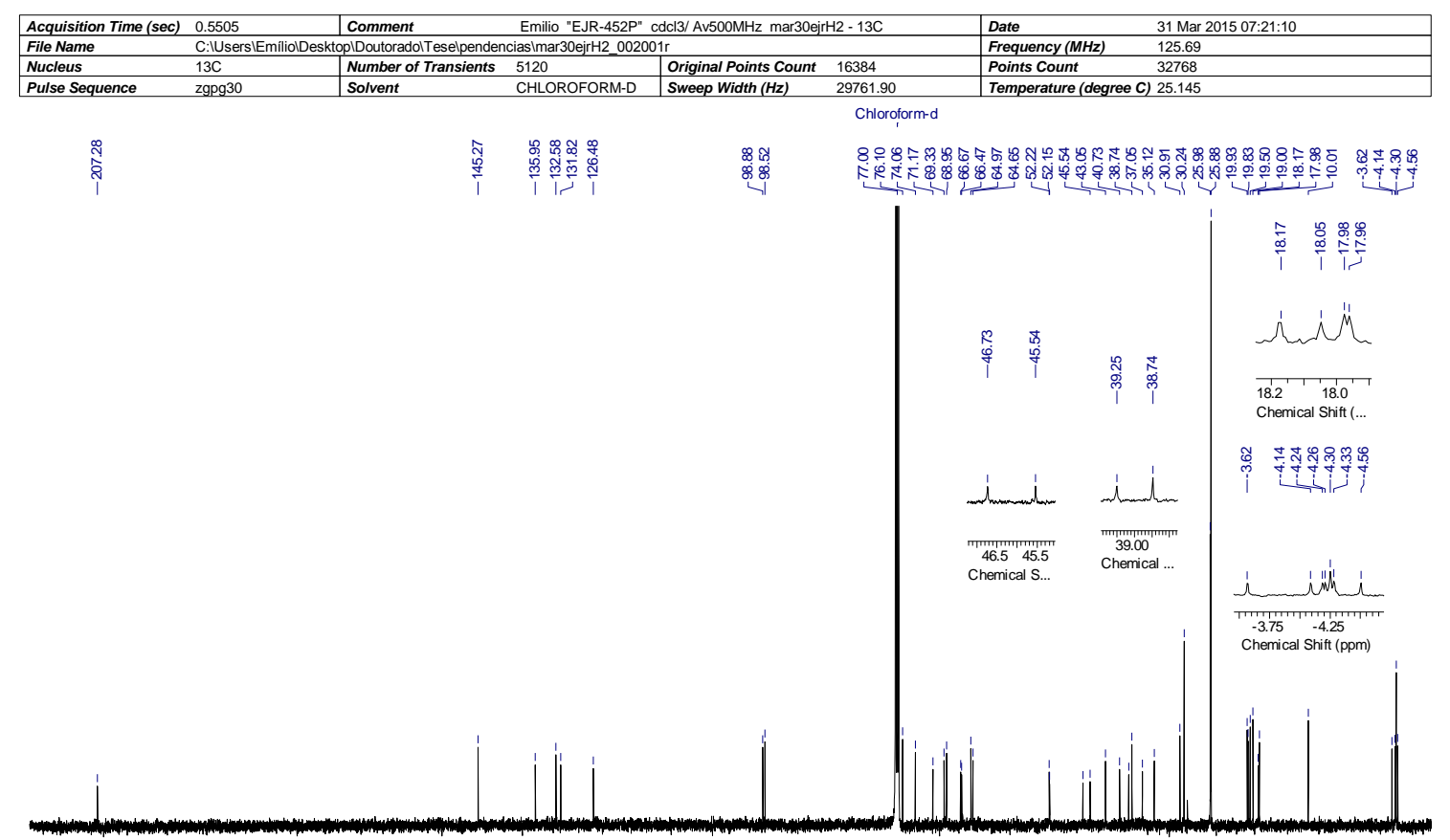

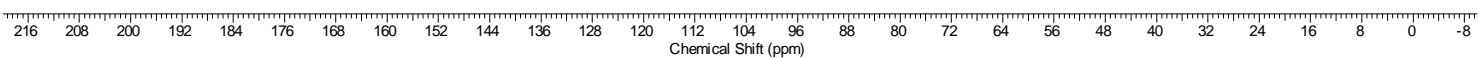

Figure 136: ${ }^{13} \mathrm{C}$ NMR spectrum of compound $\mathbf{S} 37\left(125 \mathrm{MHz}, \mathrm{CDCl}_{3}\right)$.

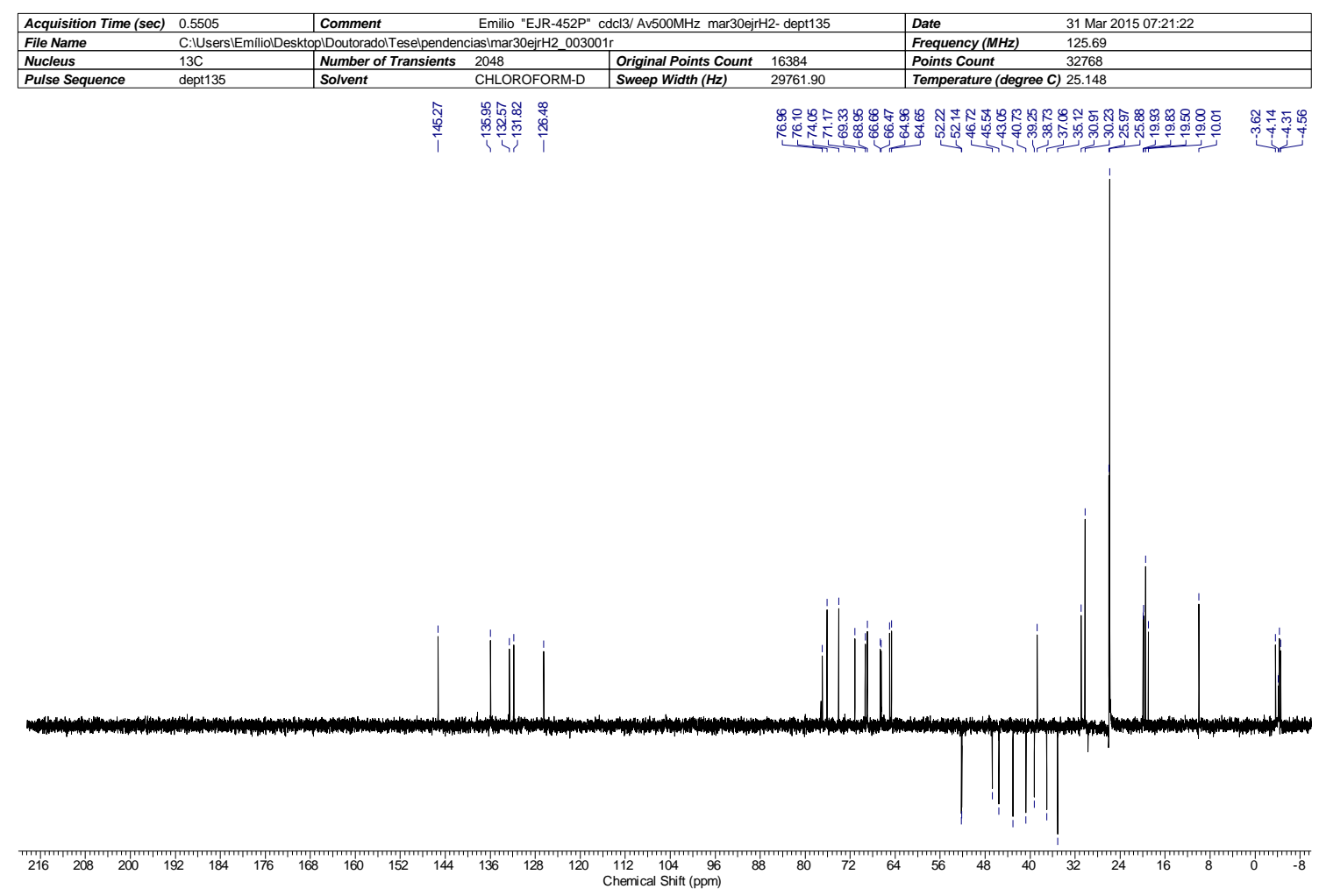

Figure 137: ${ }^{13} \mathrm{C}$ NMR (dept 135) spectrum of compound $\mathbf{S} 37\left(125 \mathrm{MHz}, \mathrm{CDCl}_{3}\right)$. 


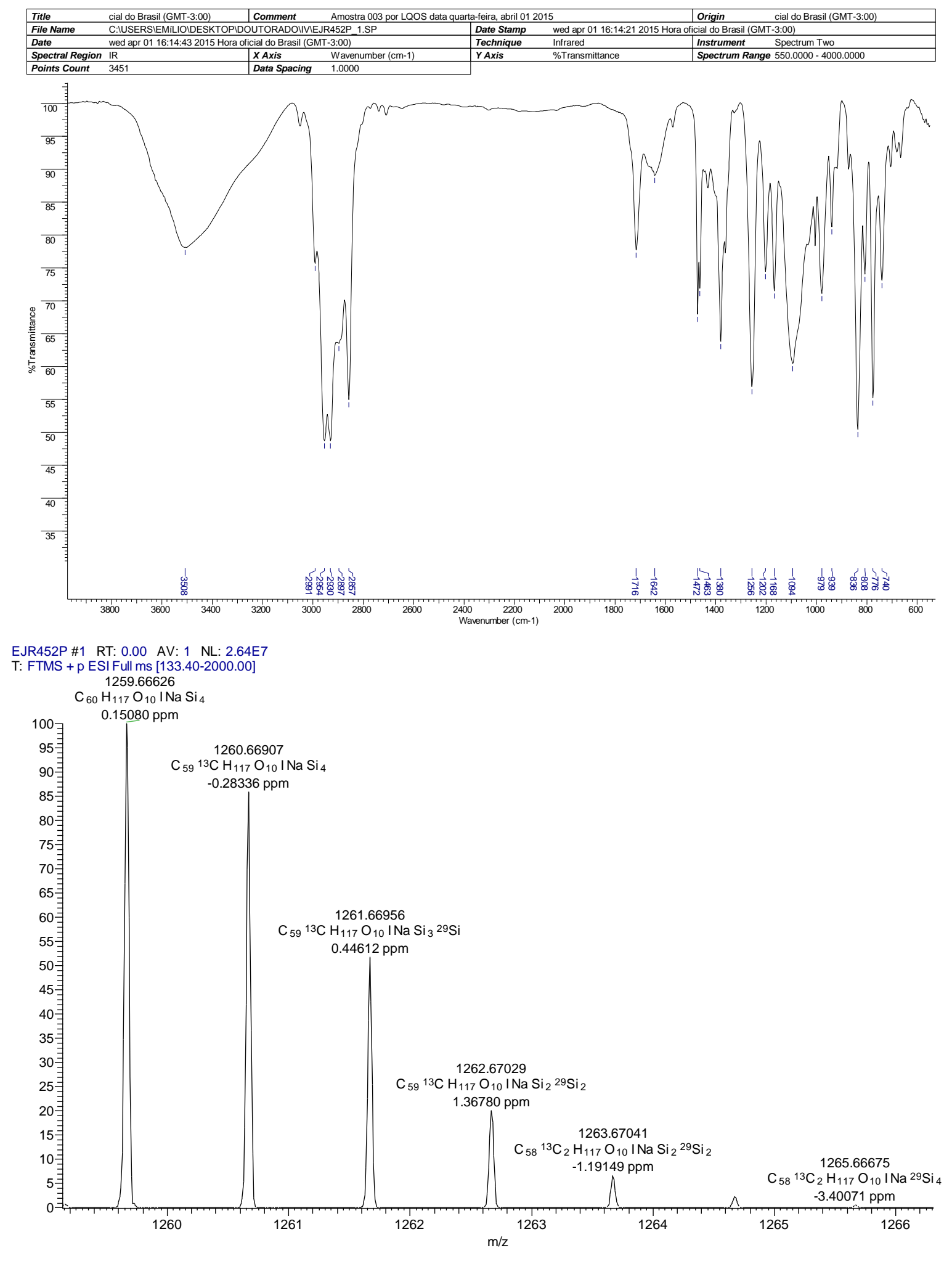

Figure 138: IR and HRMS (ESI FTMS) spectra of compound S37. 


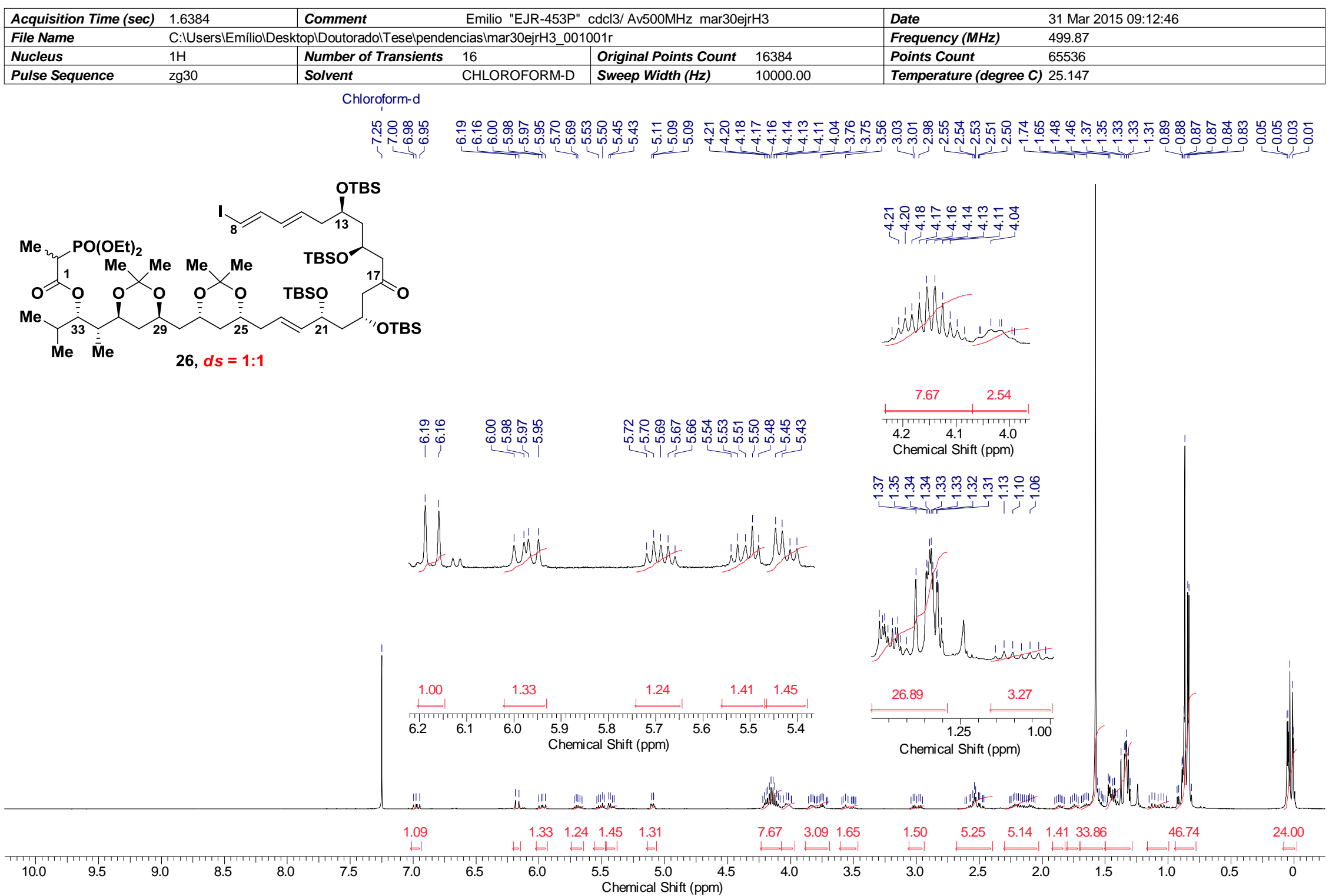

Figure 139: ${ }^{1} \mathrm{H}$ NMR spectrum of compound $26\left(500 \mathrm{MHz}, \mathrm{CDCl}_{3}\right)$. 


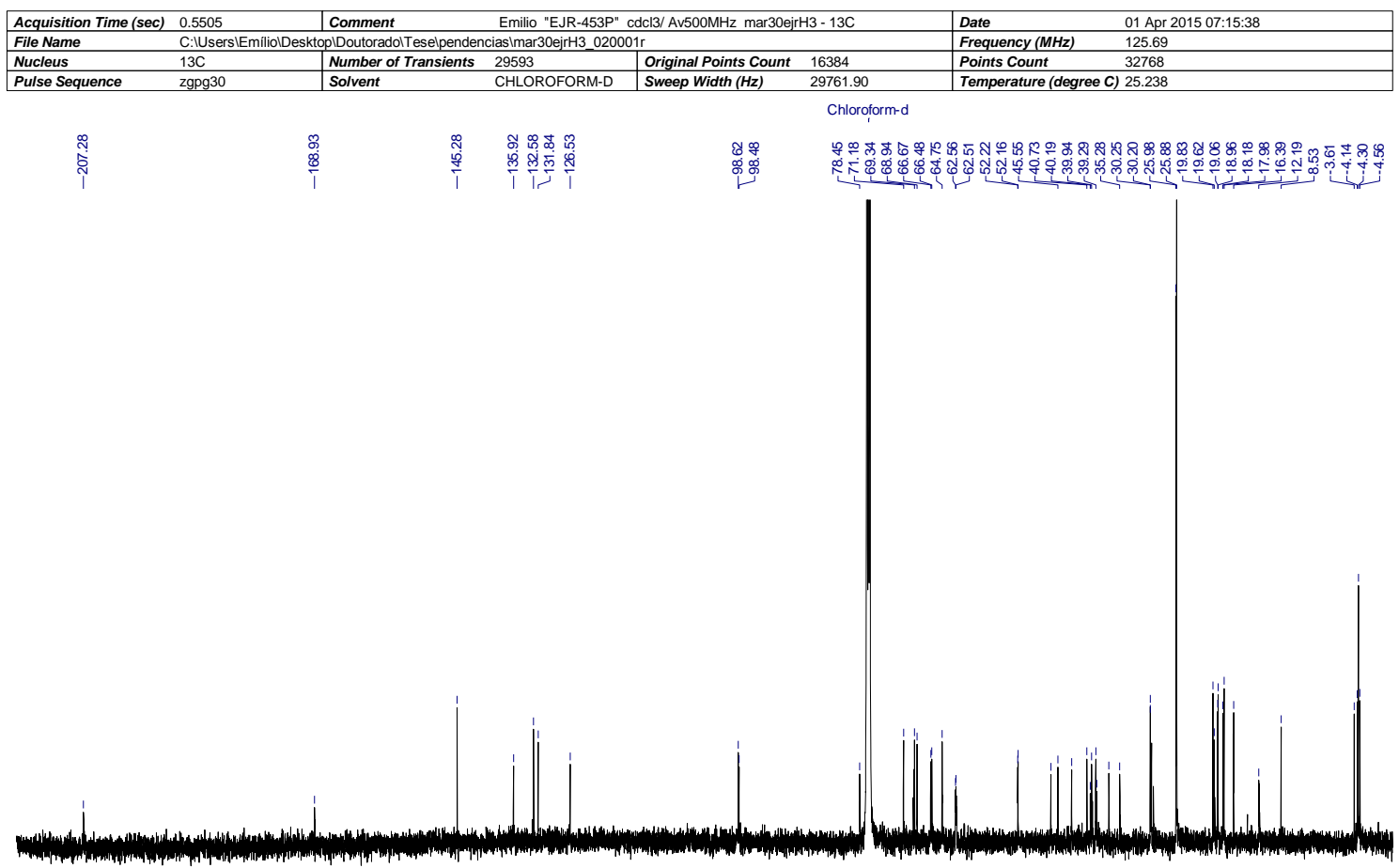

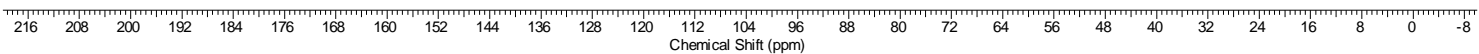

Figure 140: ${ }^{13} \mathrm{C}$ NMR spectrum of compound $26\left(125 \mathrm{MHz}, \mathrm{CDCl}_{3}\right)$.

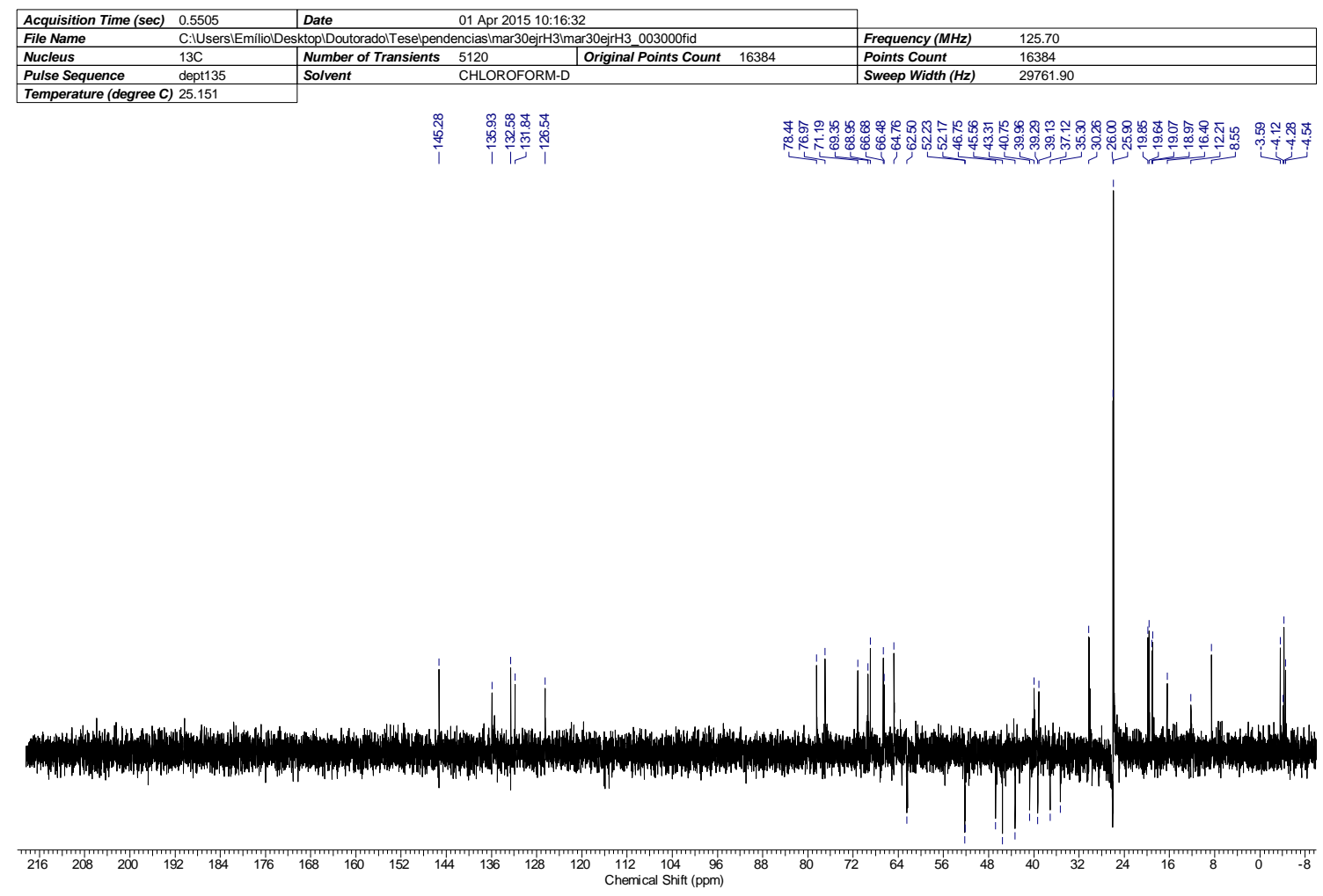

Figure 141: ${ }^{13} \mathrm{C}$ NMR (dept 135) spectrum of compound $26\left(125 \mathrm{MHz}, \mathrm{CDCl}_{3}\right)$. 

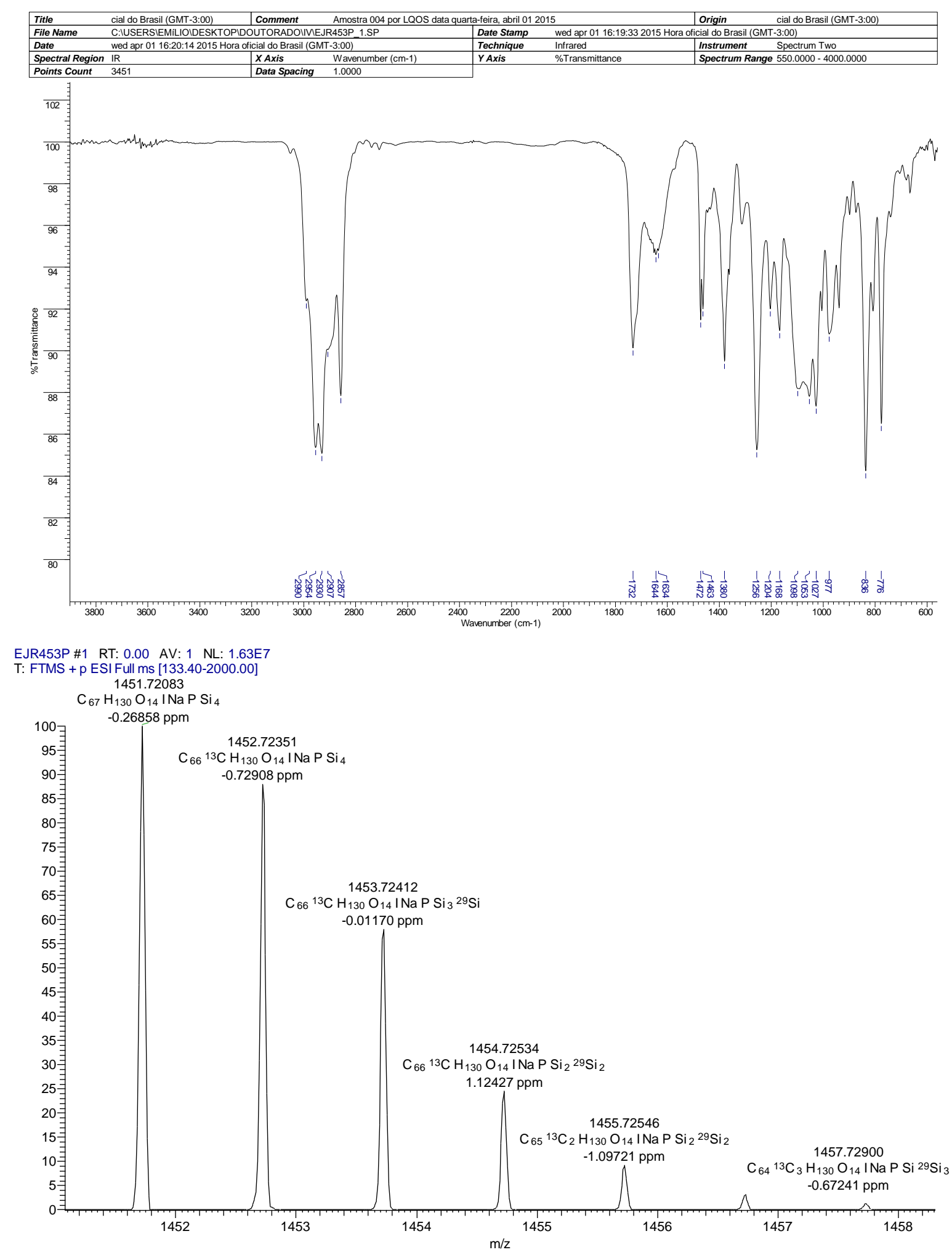

Figure 142: IR and HRMS (ESI FTMS) spectra of compound 26. 


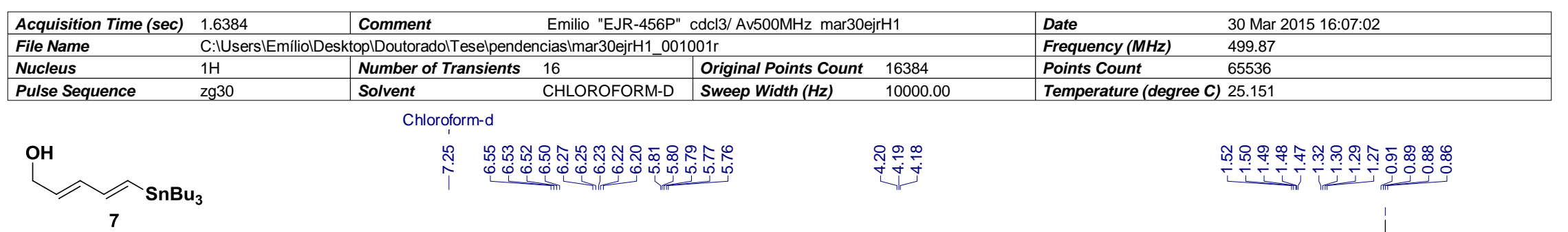

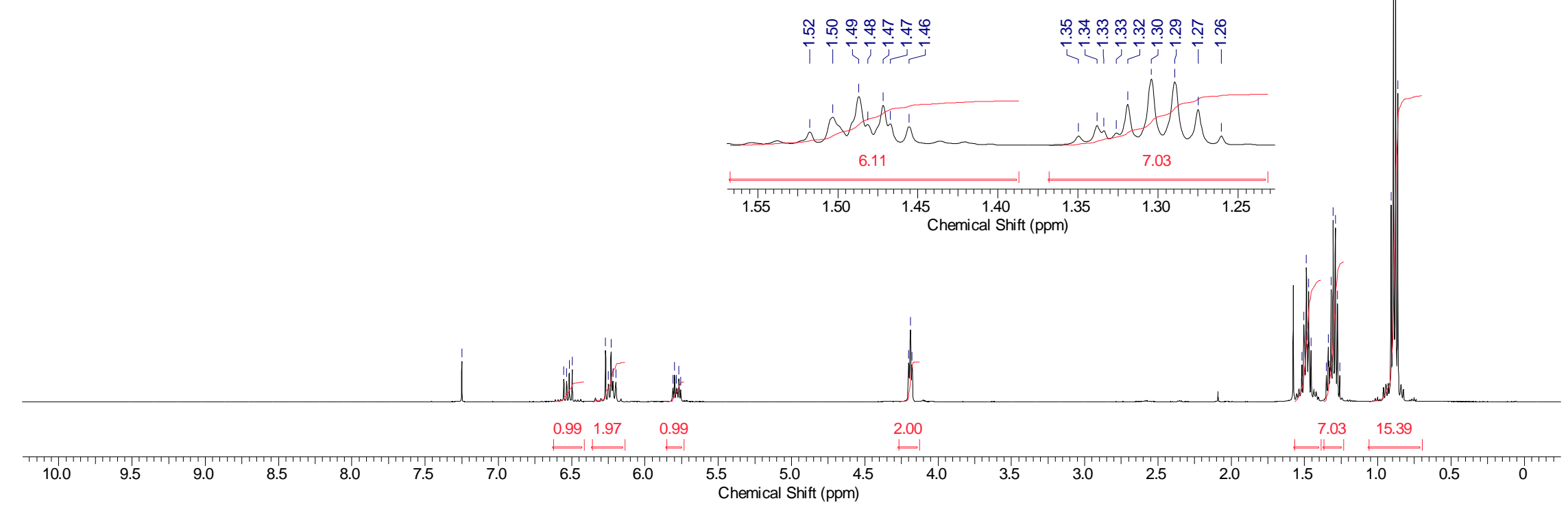

Figure 143: ${ }^{1} \mathrm{H}$ NMR spectrum of compound $7\left(500 \mathrm{MHz}, \mathrm{CDCl}_{3}\right)$. 


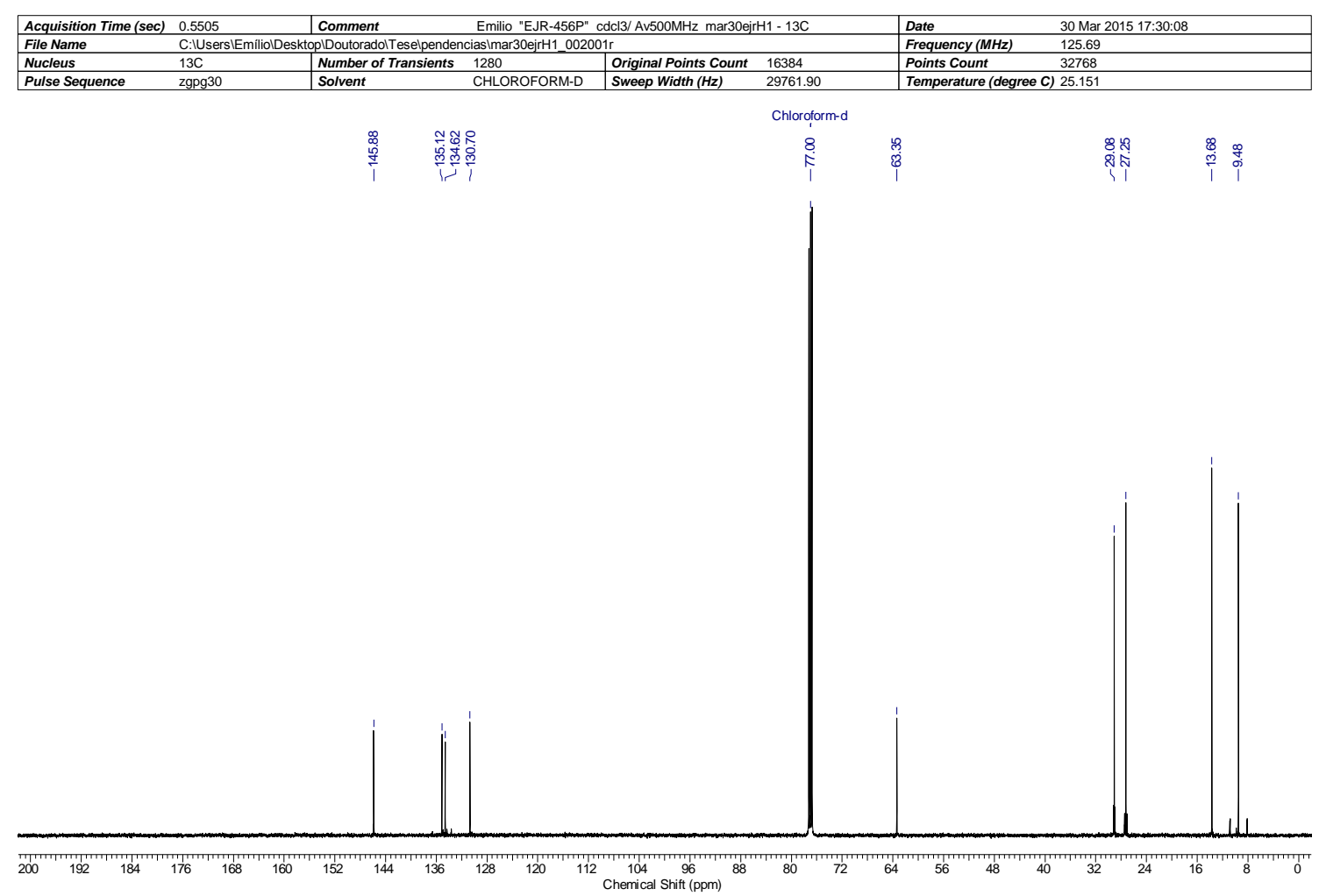

Figure 144: ${ }^{13} \mathrm{C}$ NMR spectrum of compound $7\left(125 \mathrm{MHz}, \mathrm{CDCl}_{3}\right)$.

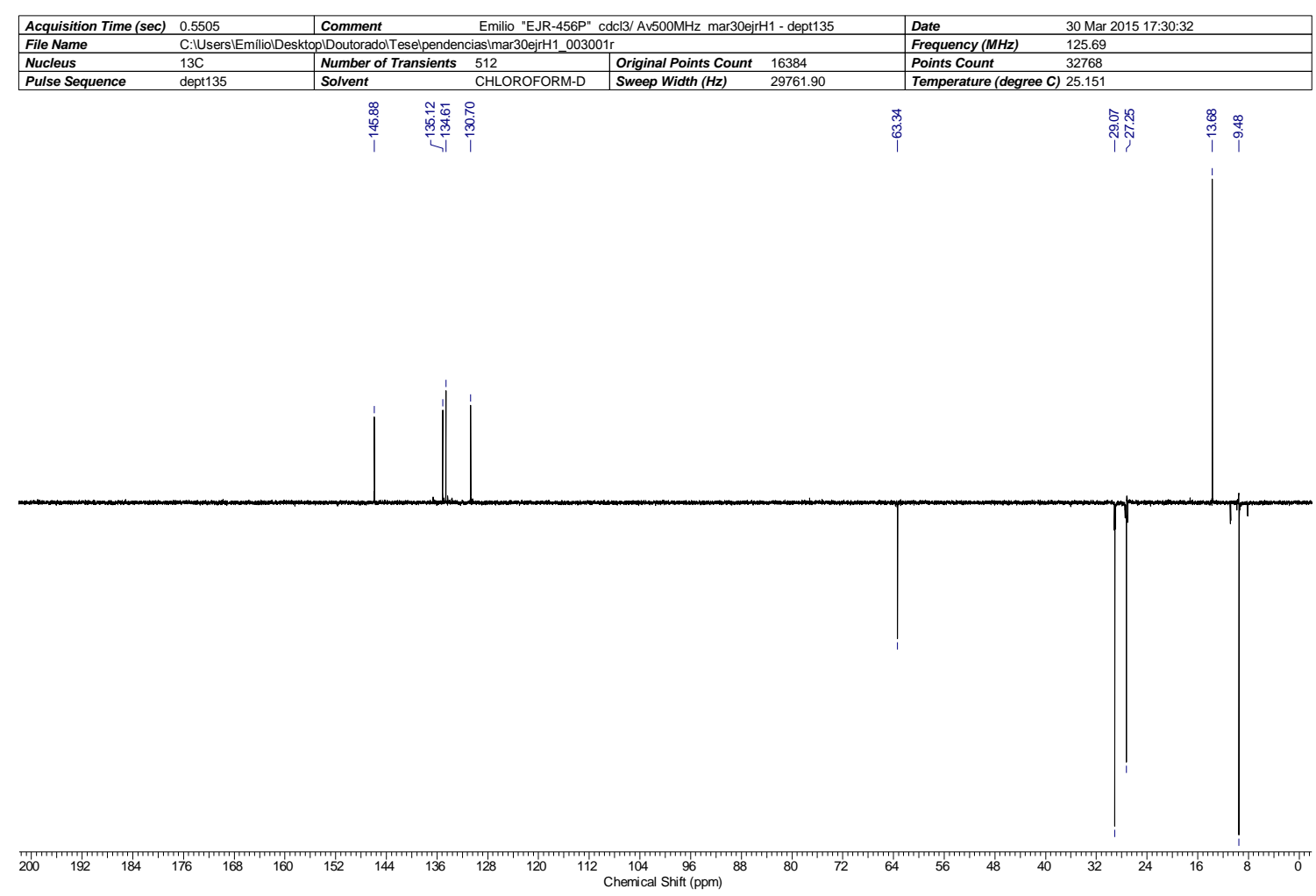

Figure 145: ${ }^{13} \mathrm{C}$ NMR (dept 135) spectrum of compound $7\left(125 \mathrm{MHz}, \mathrm{CDCl}_{3}\right.$ ). 


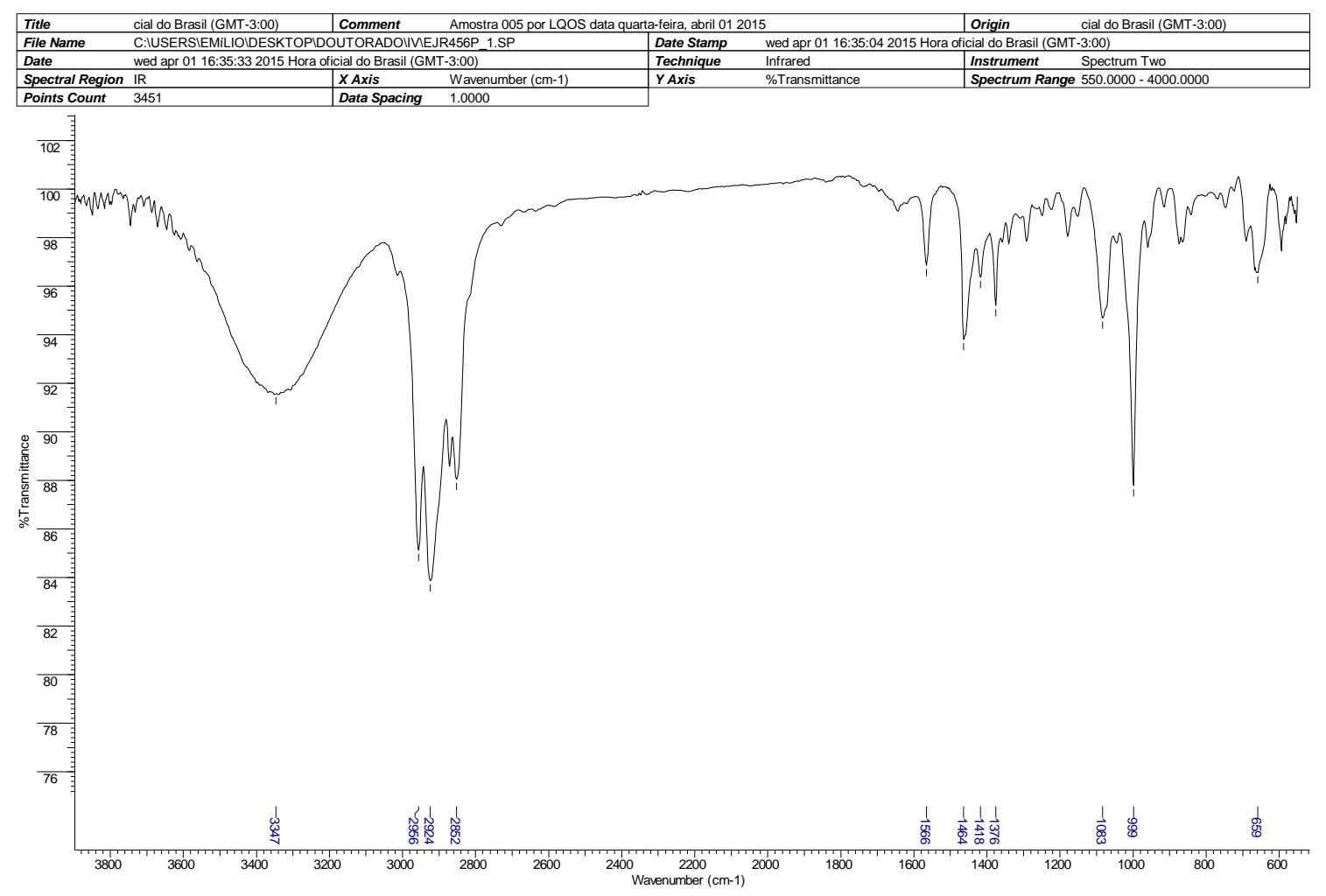

Figure 146: IR spectrum of compound 7. 


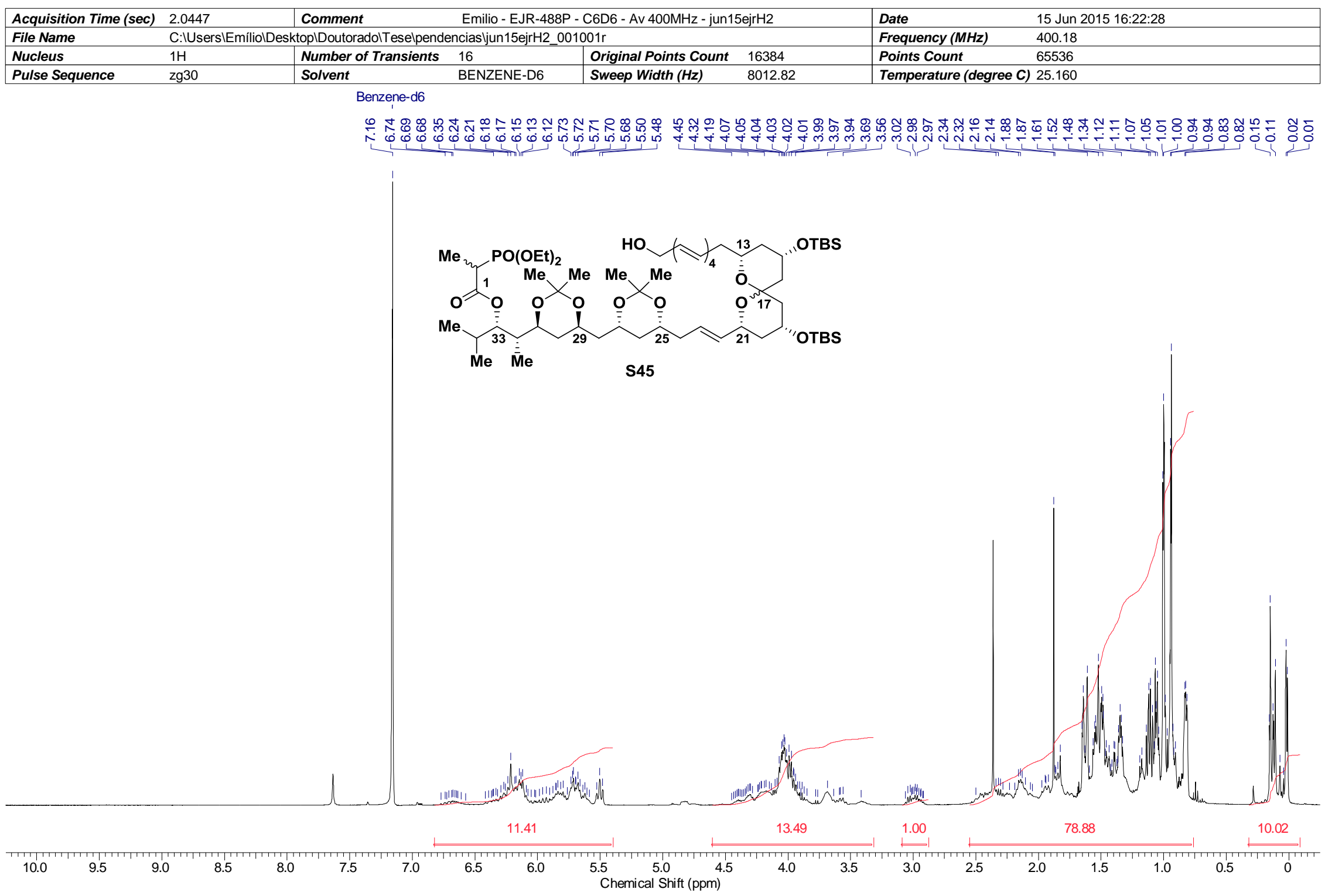

Figure 147: ${ }^{1} \mathrm{H}$ NMR spectrum of compound $\mathbf{S} 45\left(400 \mathrm{MHz}, \mathrm{C}_{6} \mathrm{D}_{6}\right)$. 


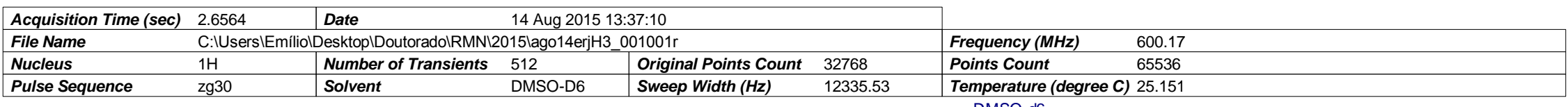

Pulse Sequence

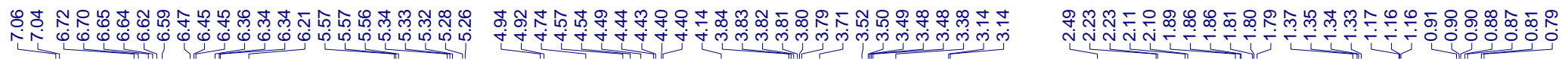

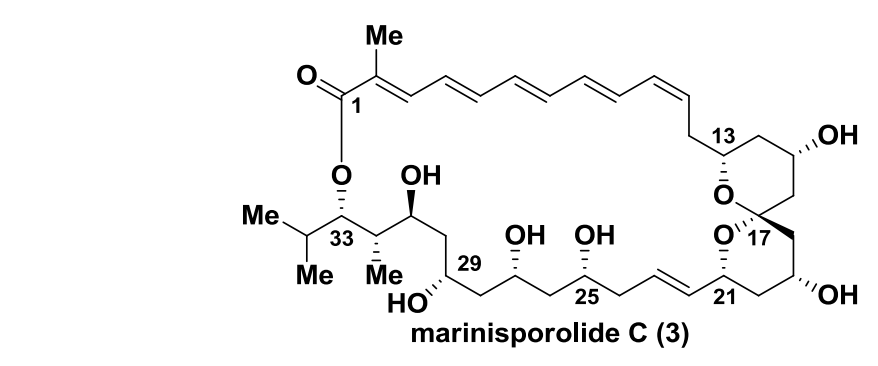

marinisporolide $C$ (3)

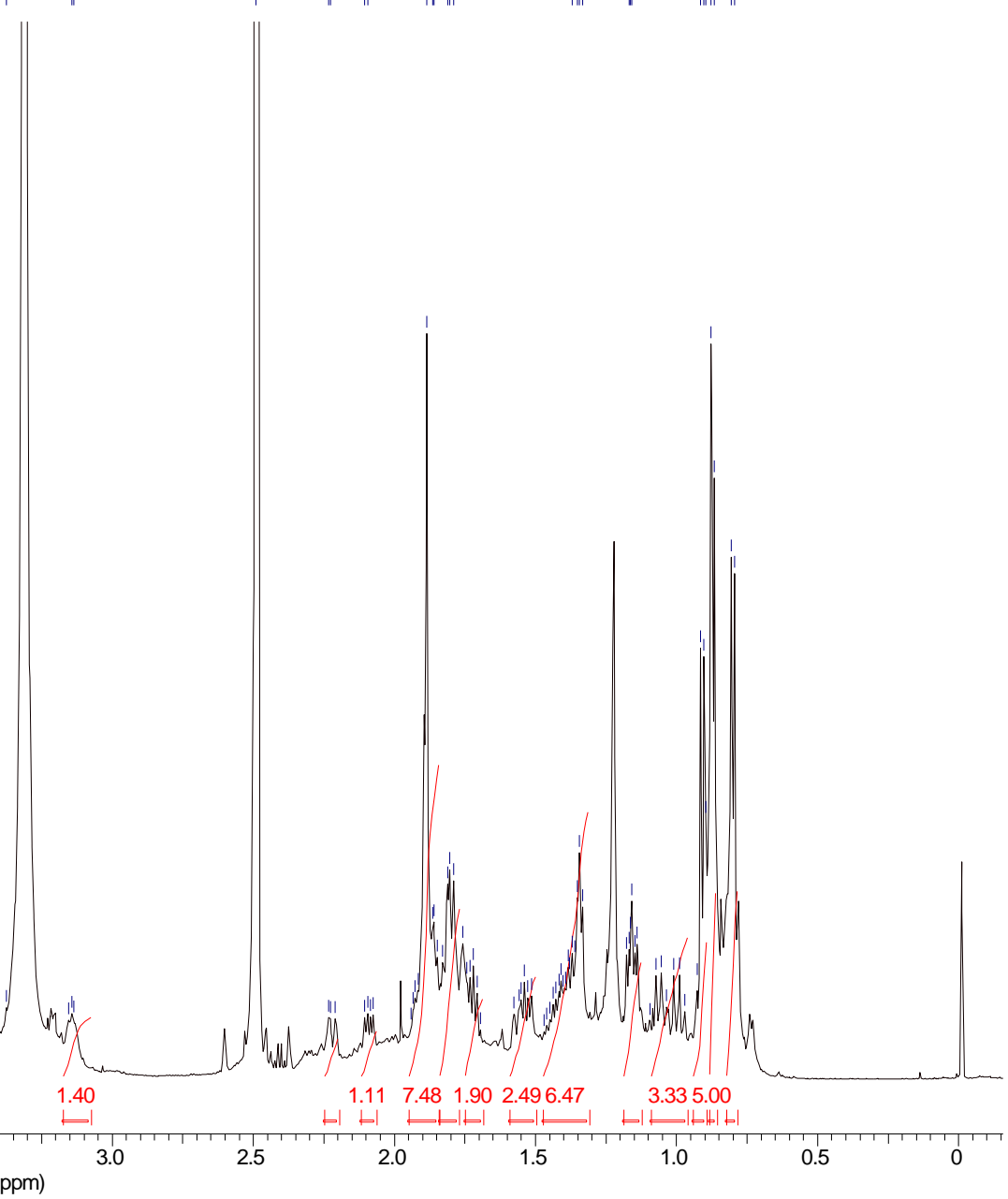

Figure 148: ${ }^{1} \mathrm{H}$ NMR spectrum of marinisporolide $\mathrm{C}(3)\left(600 \mathrm{MHz}\right.$, DMSO- $\left.d_{6}\right)$. 


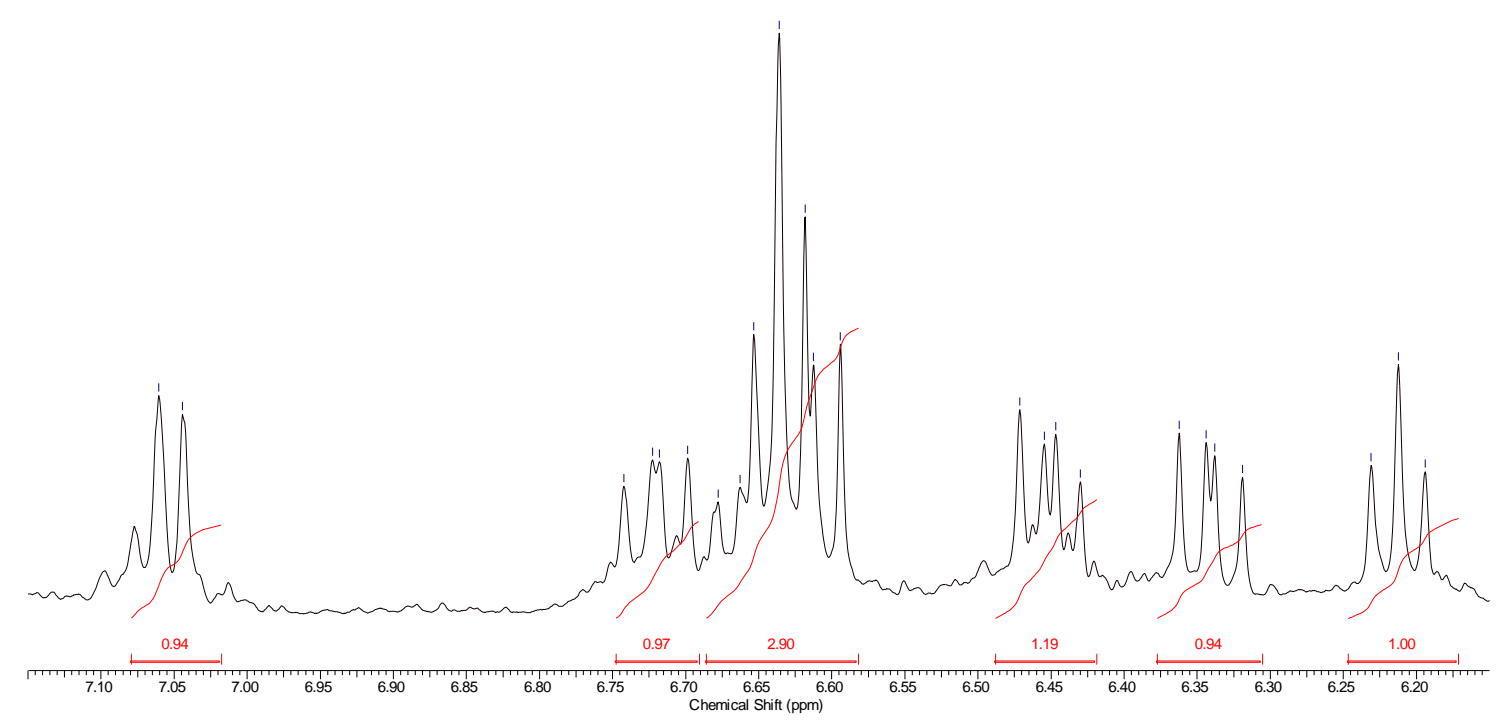

Figure 149: ${ }^{1} \mathrm{H}$ NMR spectrum of marinisporolide $\mathrm{C}(3)$ : $6.15-7.15$ region.

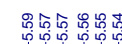

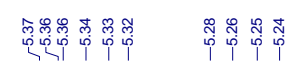

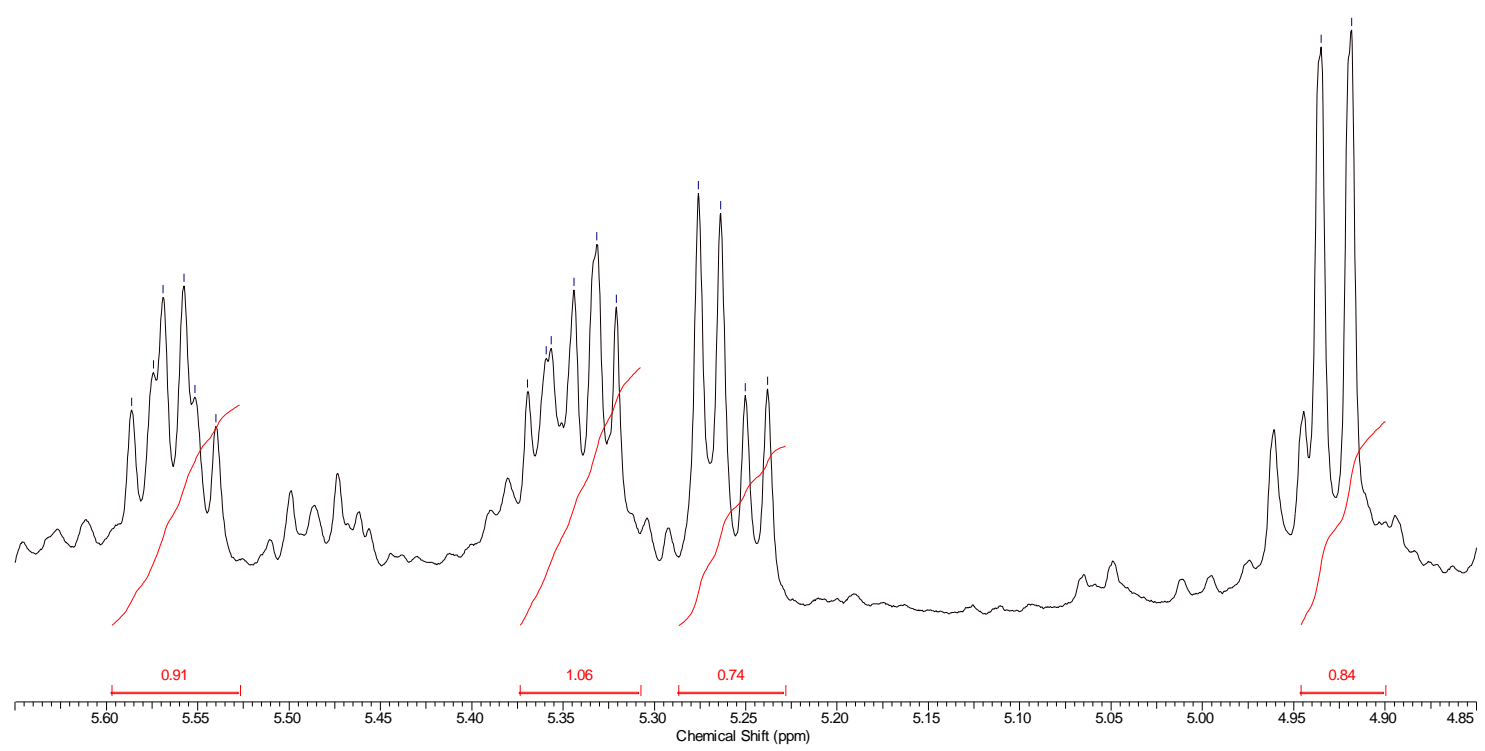

Figure 150: ${ }^{1} \mathrm{H}$ NMR spectrum of marinisporolide $\mathrm{C}(3)$ : 4.85-5.65 region. 


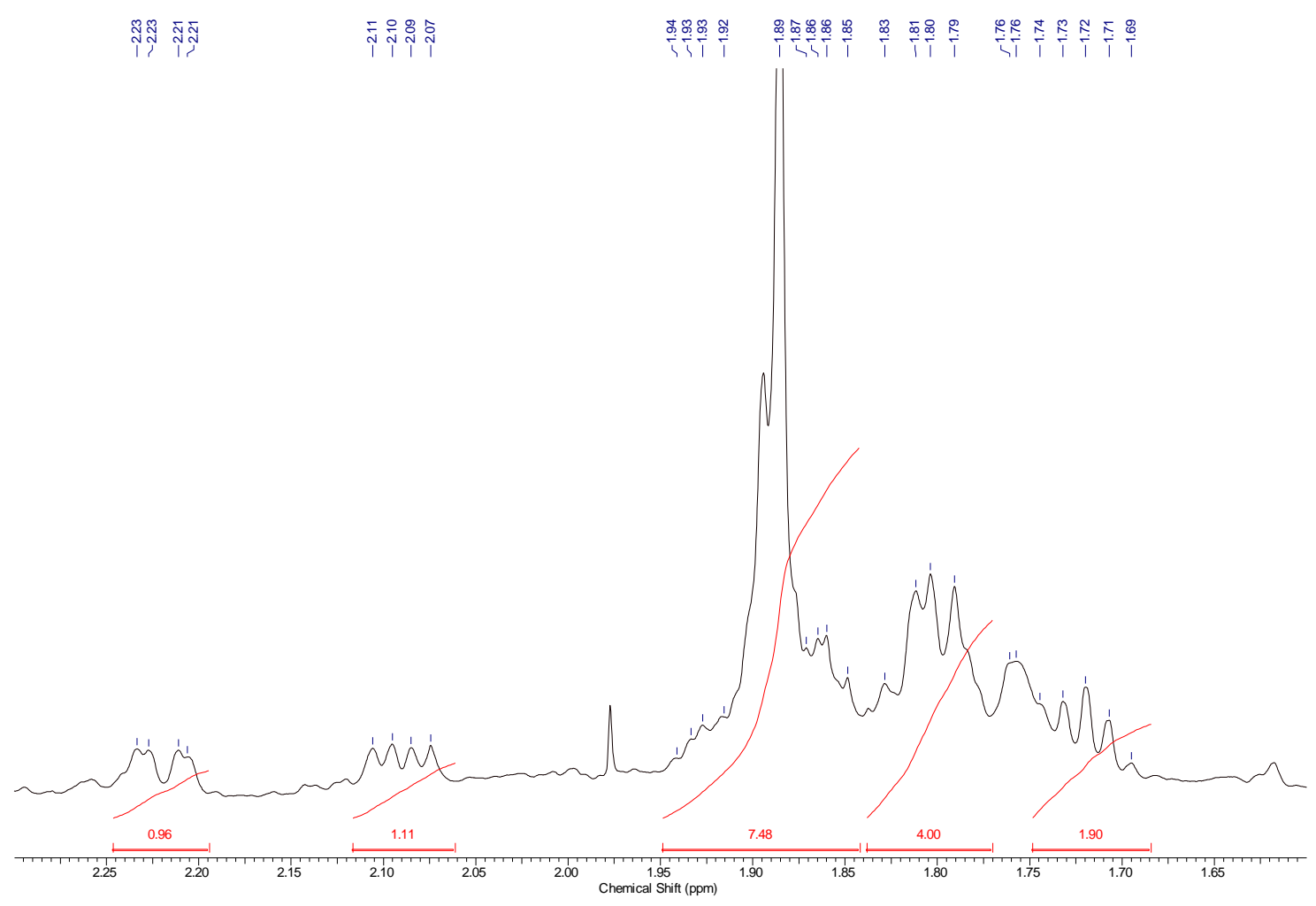

Figure 151: ${ }^{1} \mathrm{H}$ NMR spectrum of marinisporolide $\mathrm{C}(3)$ : $1.60-2.30$ region.

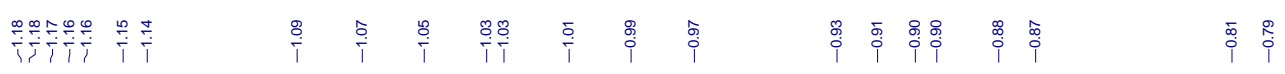

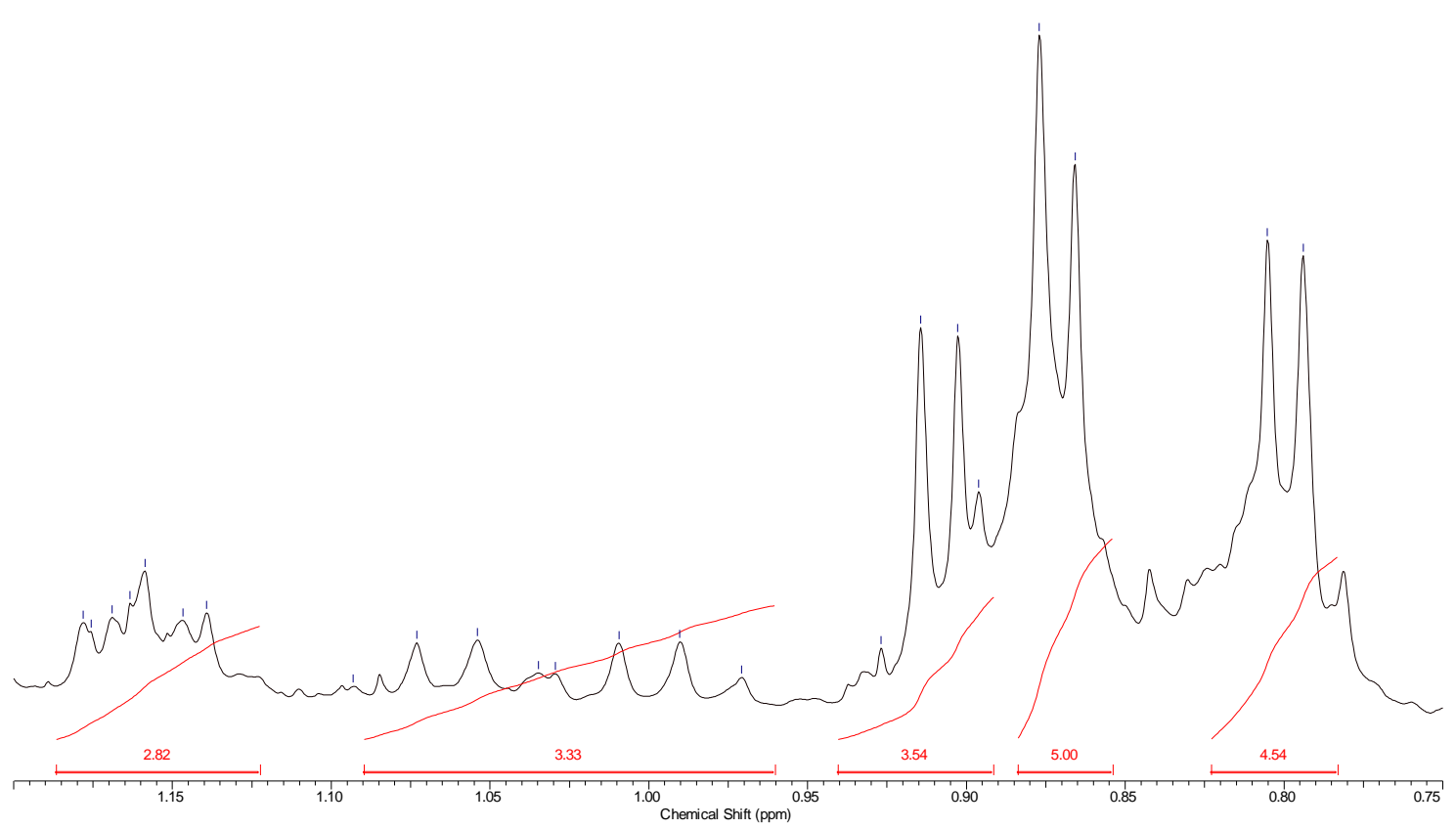

Figure 152: ${ }^{1} \mathrm{H}$ NMR spectrum of marinisporolide $\mathrm{C}(3)$ : 0.75-1.20 region. 


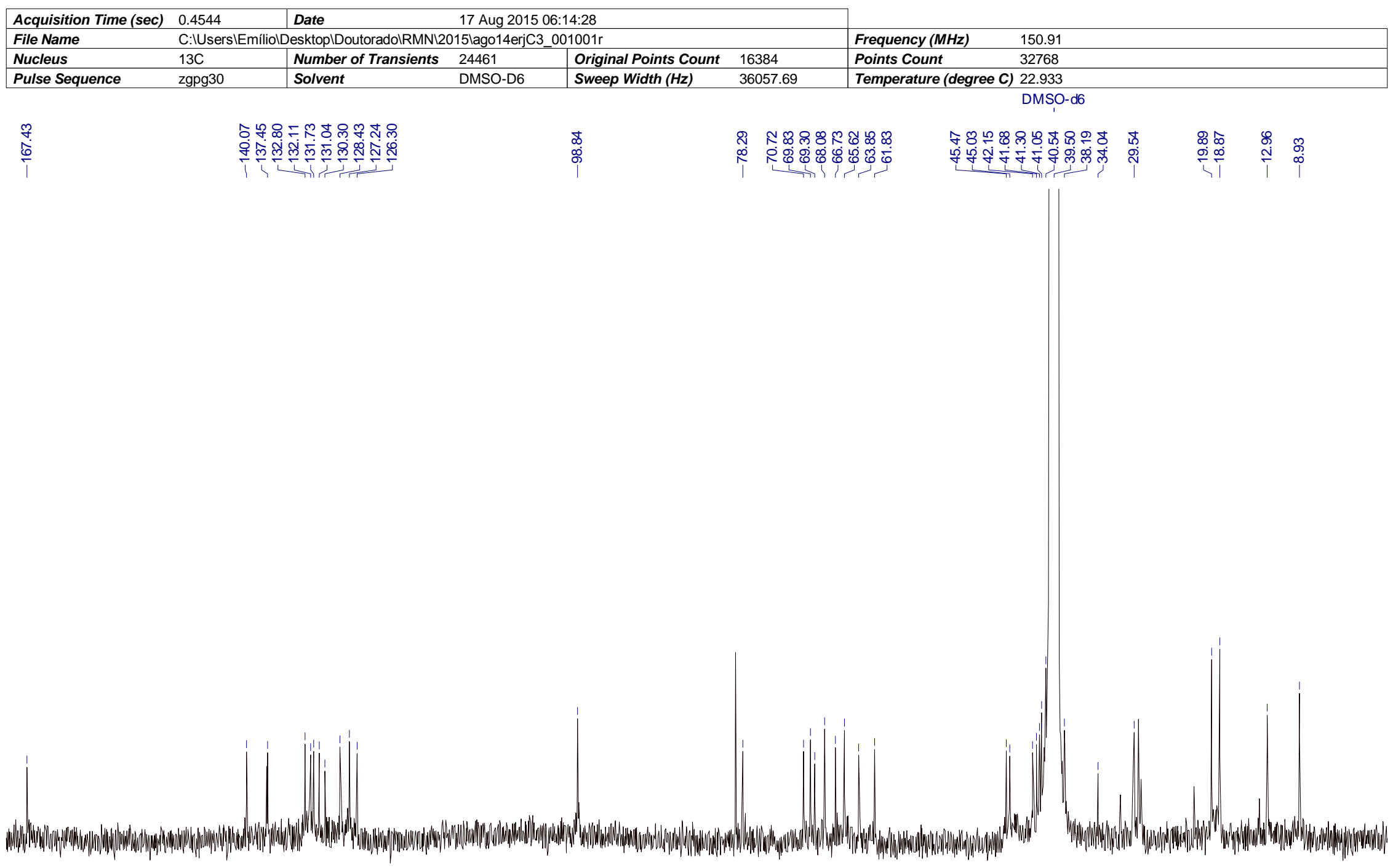
168
$160 \quad 152$
$144 \quad 136$
128
$120 \quad 112 \quad 104$
$88 \quad 80$
$72 \quad 64 \quad 56$
48
$40 \quad 32$
$24 \quad 16880$

Figure 153: ${ }^{13} \mathrm{C}$ NMR spectrum of marinisporolide C (3) $\left(150 \mathrm{MHz}\right.$, DMSO- $\left.d_{6}\right)$. 


14 i 1111

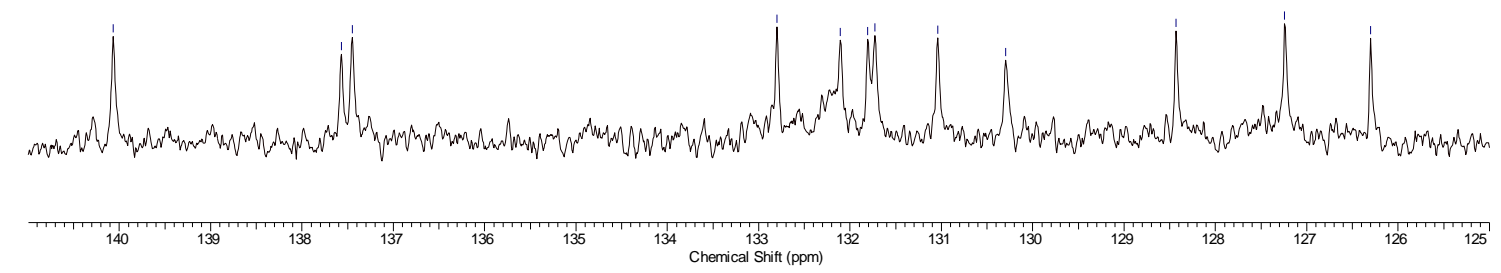

Figure 154: ${ }^{13} \mathrm{C}$ NMR spectrum of marinisporolide $C(3)$ : 125.0-141.0 region.

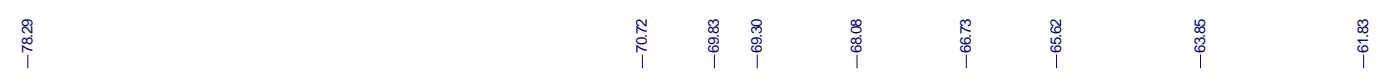

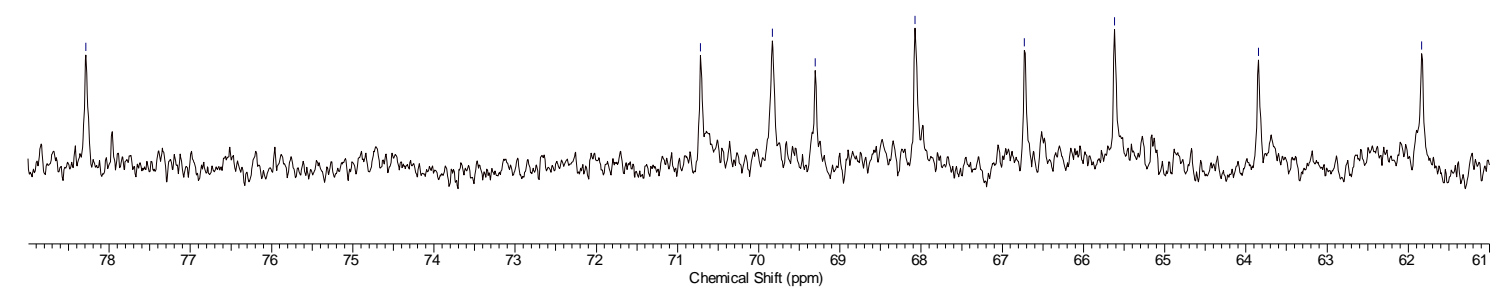

Figure 155: ${ }^{13} \mathrm{C}$ NMR spectrum of marinisporolide $\mathrm{C}(3)$ : $61.0-79.0$ region. 


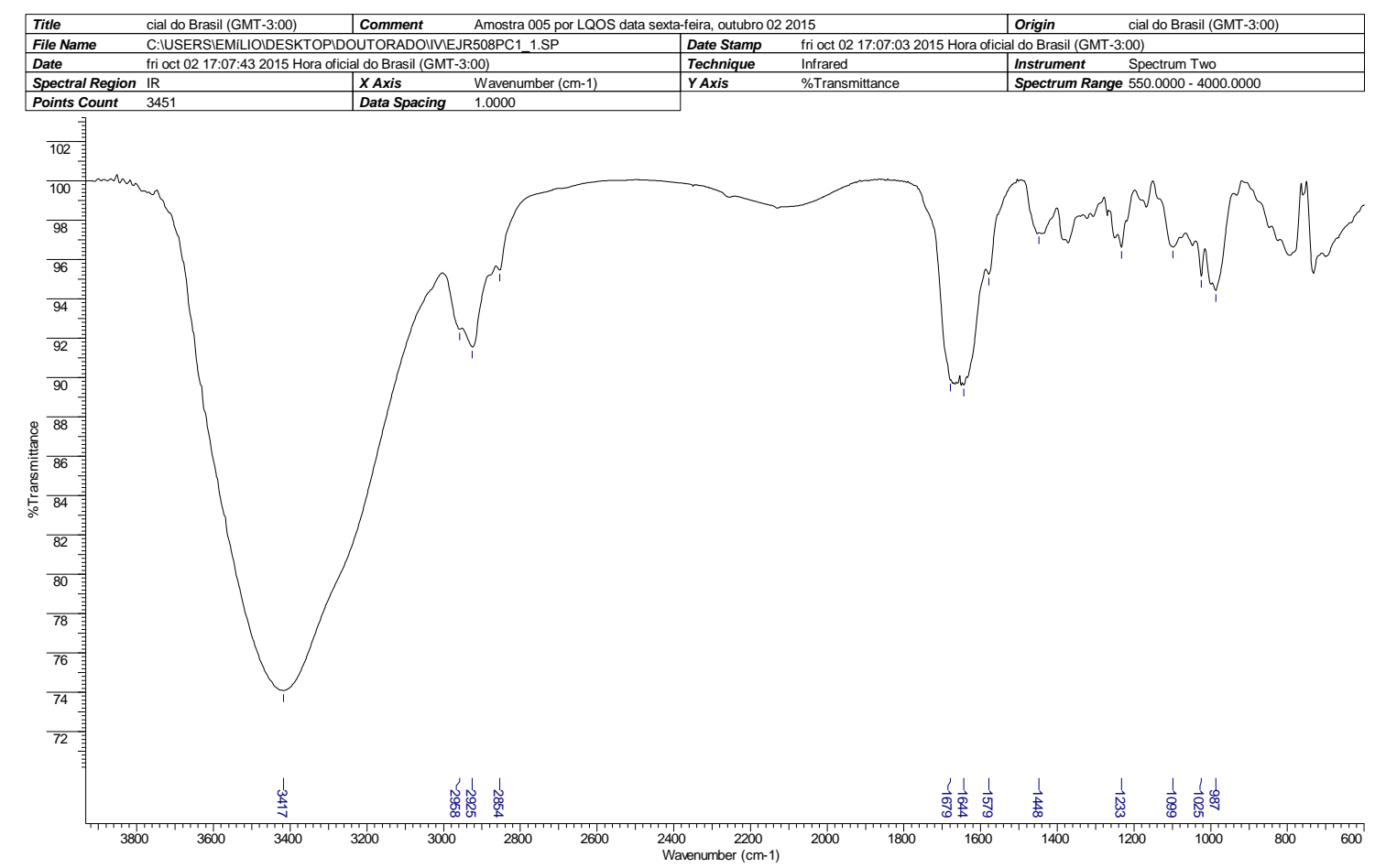

EJR-508PC \#1 RT: 0.01 AV: 1 NL: $3.37 E 5$

T: FTMS + p ESI Full ms [500.00-1000.00]

697.39233
$\mathrm{C}_{38} \mathrm{H}_{58} \mathrm{O}_{10} \mathrm{Na}$

$100 \quad 0.16482 \mathrm{ppm}$

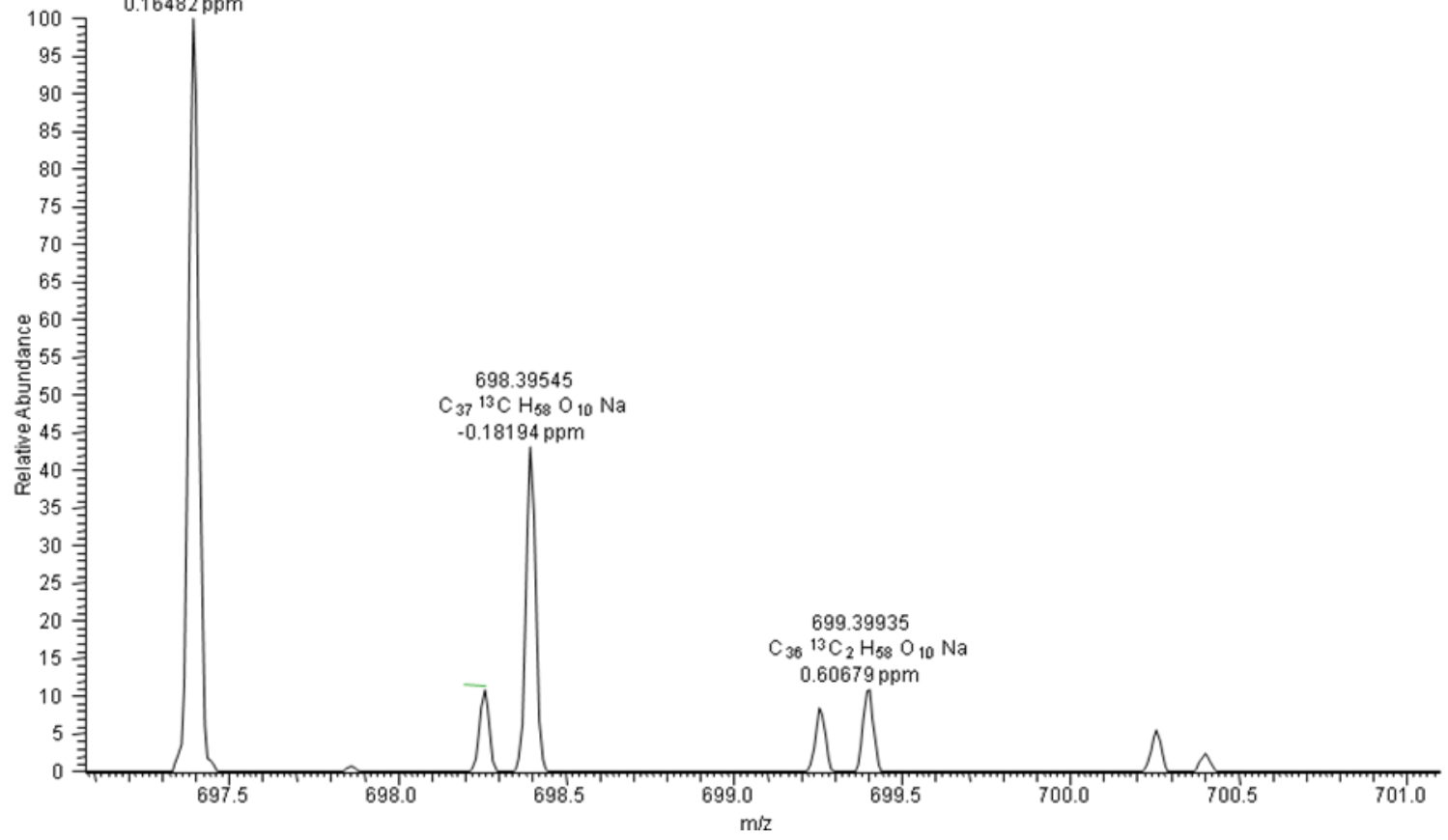

Figure 156: IR and HRMS (ESI FTMS) spectra of marinisporolide C (3). 


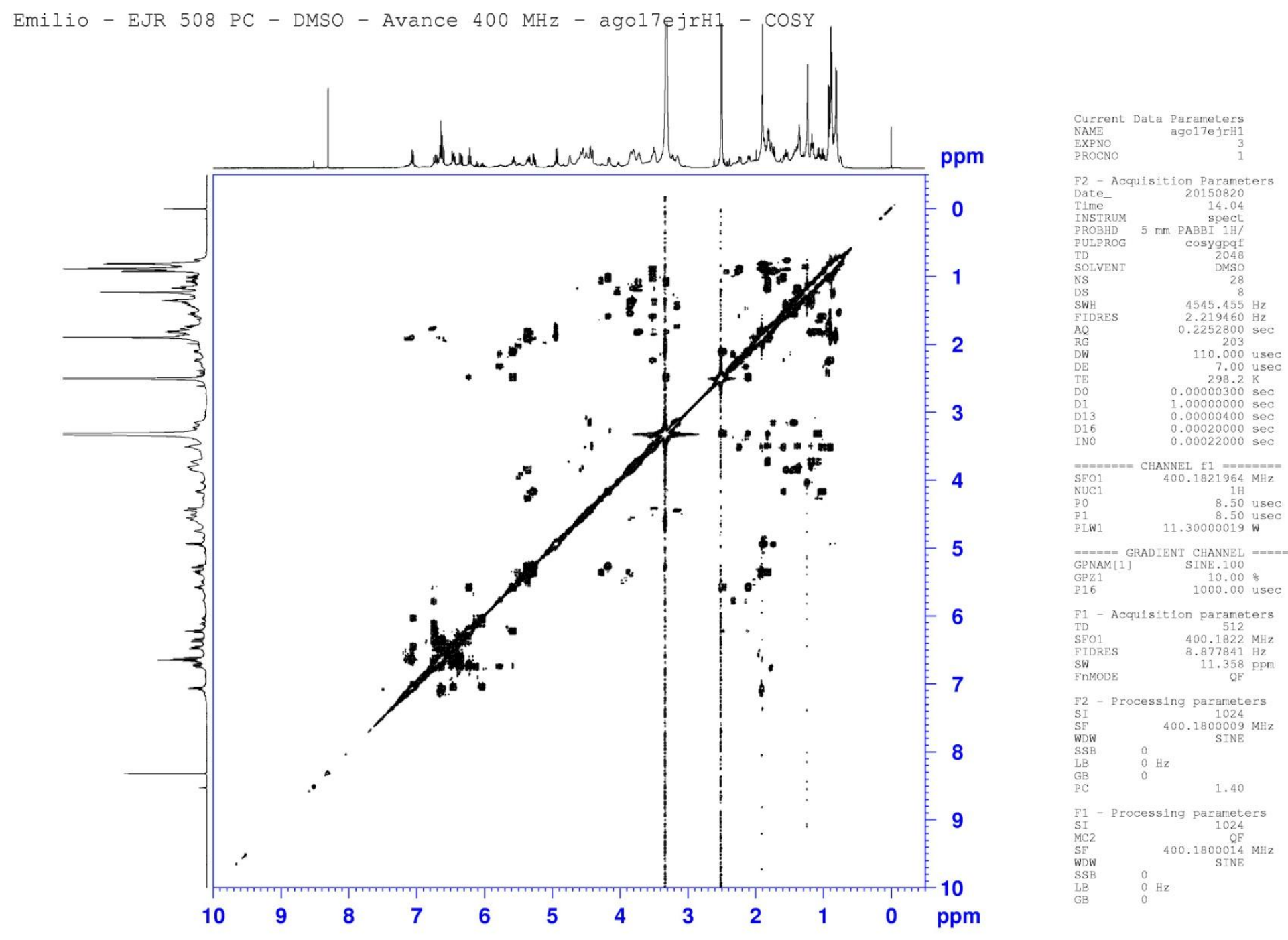

Figure 157: COSY contour map of marinisporolide C (3) $\left(400 \mathrm{MHz}\right.$, DMSO- $\left.d_{6}\right)$. 


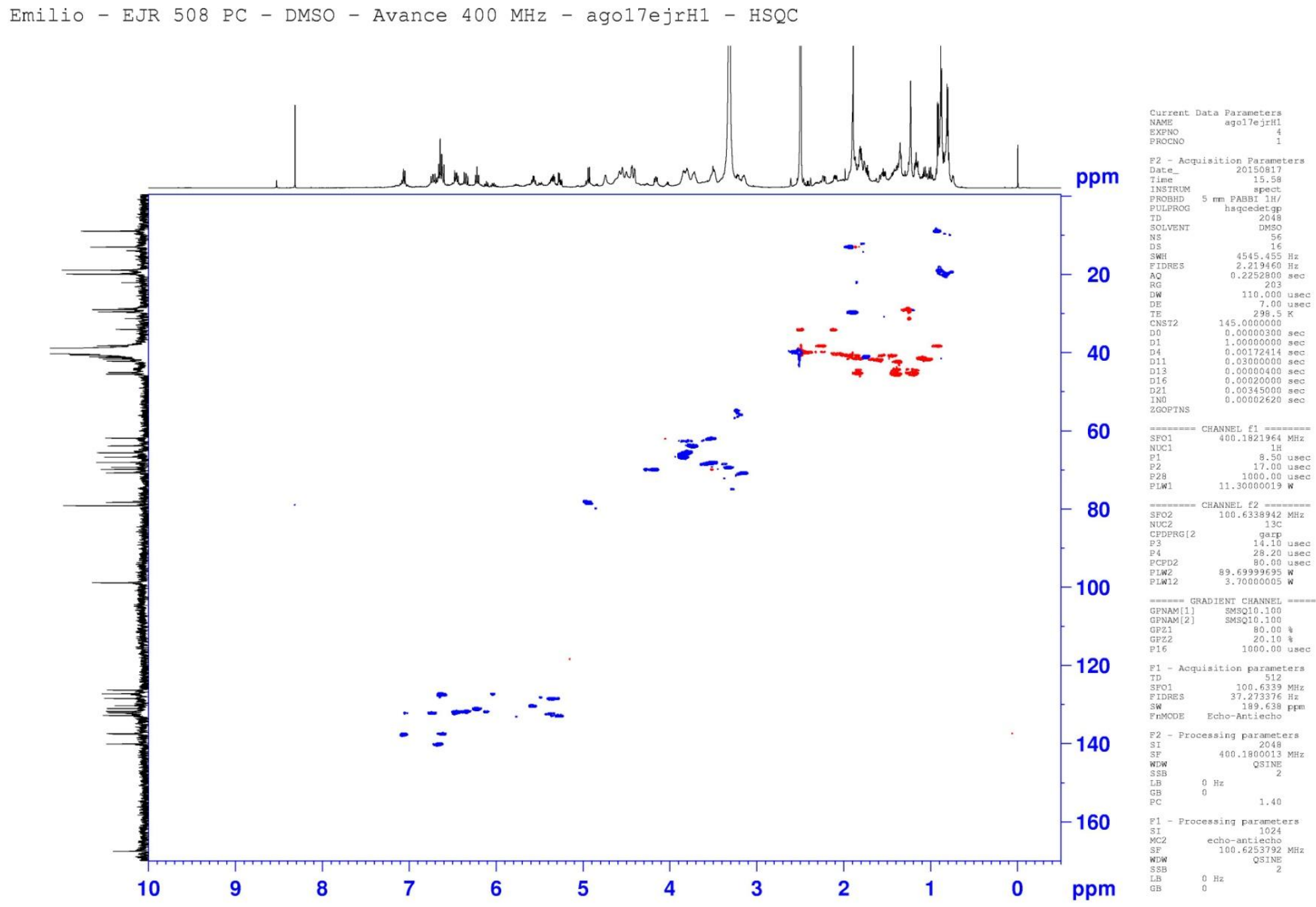

Figure 158: HSQC contour map of marinisporolide C (3) $\left(400 \mathrm{MHz}\right.$, DMSO- $\left.d_{6}\right)$. 
Emilio - EJR 508 PC - DMSO - Avance $400 \mathrm{MHz}$ - agol7ejrH1 - HMBC

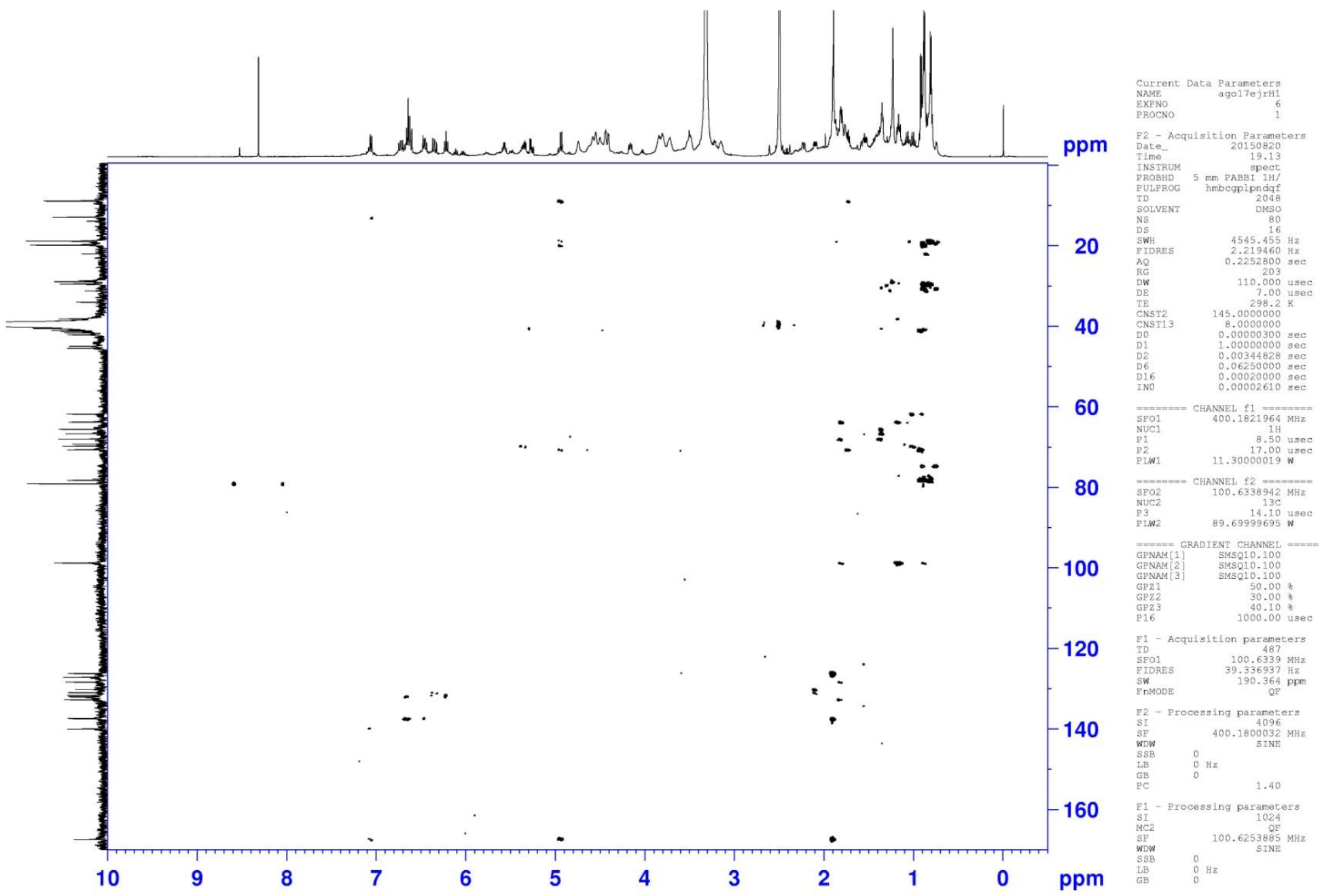

Figure 159: HMBC contour map of marinisporolide C (3) $\left(400 \mathrm{MHz}\right.$, DMSO- $\left.d_{6}\right)$. 


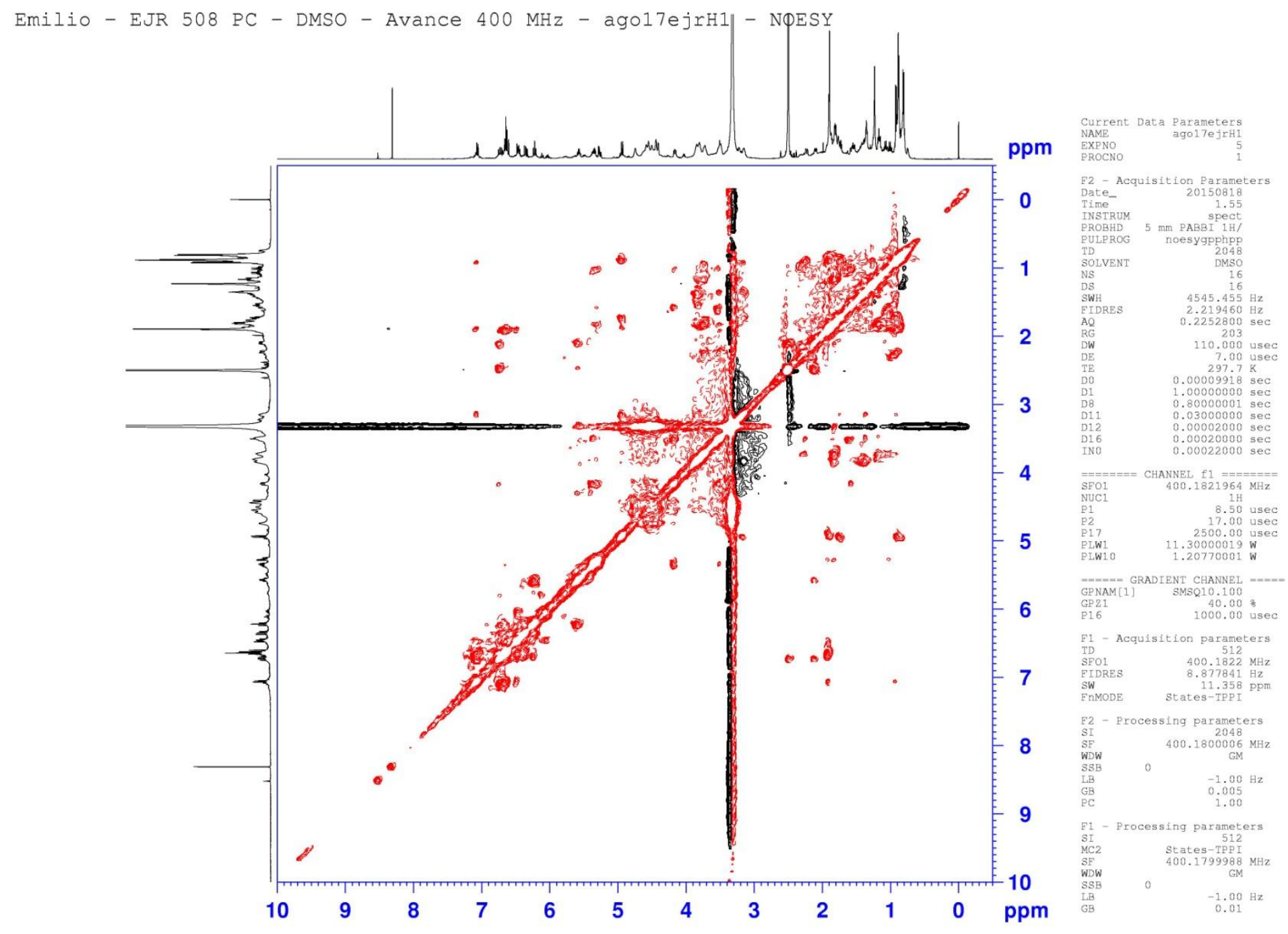

Figure 160: NOESY contour map of marinisporolide C (3) $\left(400 \mathrm{MHz}\right.$, DMSO- $\left.d_{6}\right)$. 


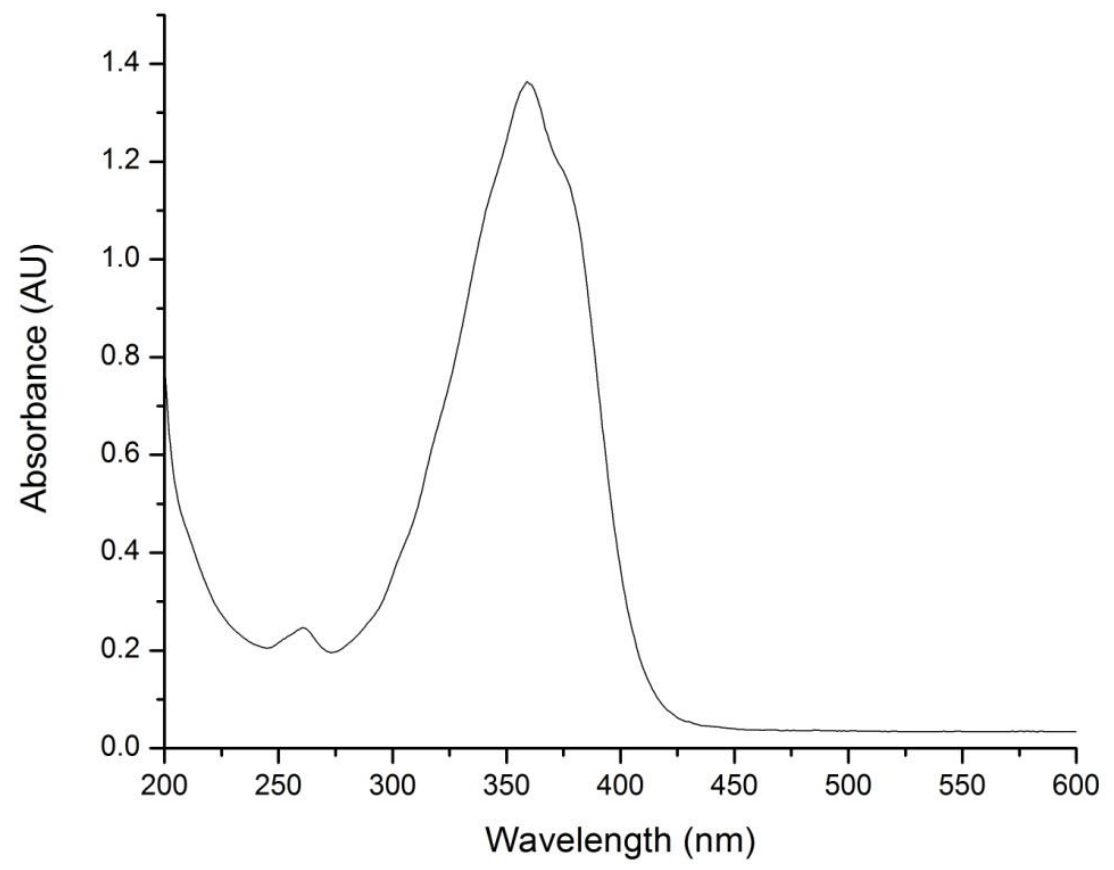

Figure 161: UV-Vis spectrum of marinisporolide $\mathrm{C}(3)(\mathrm{MeOH})$.

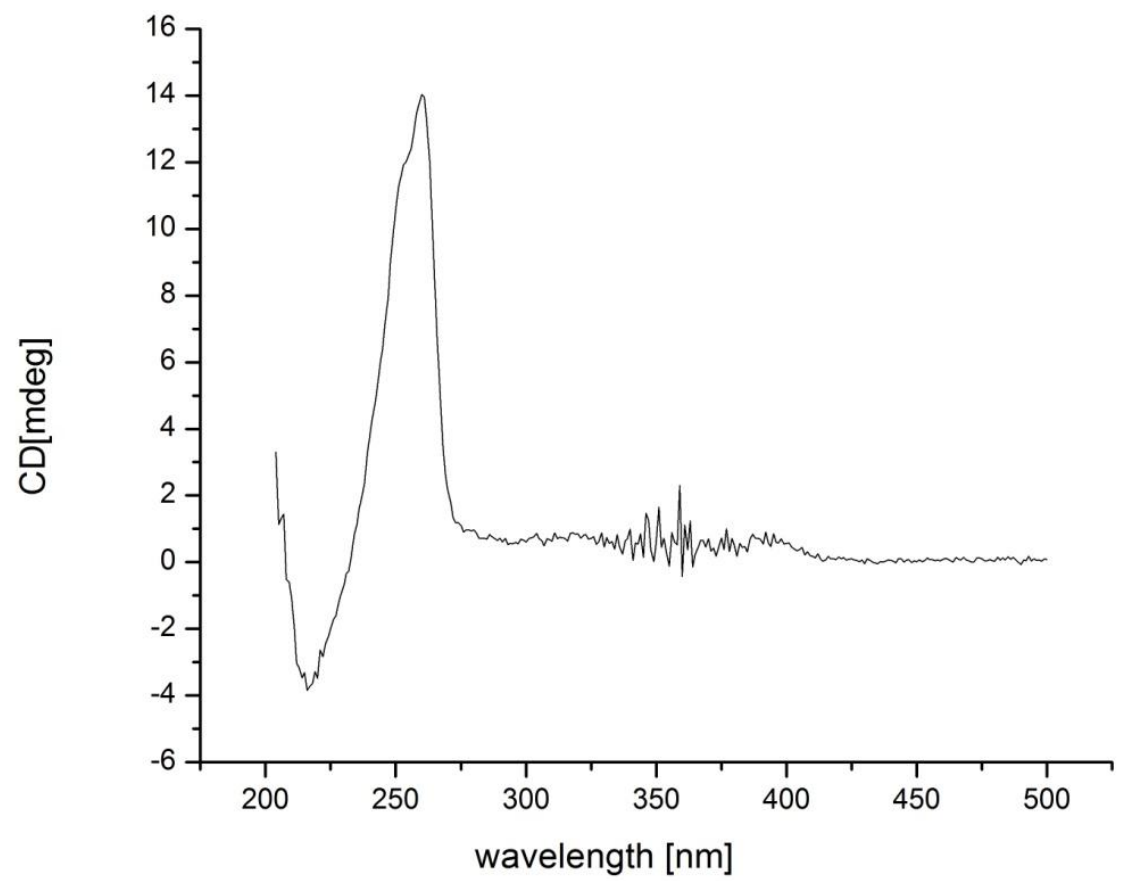

Figure 162: Circular Dichroism spectrum of marinisporolide C (3) $(\mathrm{MeOH})$. 